Hans P. Fischer

Die Finanzierung des
Umweltschutzes im

Rahmen einer rationalen

Umweltpolitik 
Hans P. Fischer

\section{Die Finanzierung des Umweltschutzes im Rahmen einer rationalen Umweltpolitik}

In den ersten drei Kapiteln werden die notwendigen Voraussetzungen einer rationalen Umweltpolitik formuliert. Im vierten Kapitel untersucht der Verfasser dann wie unter diesen Bedingungen eine zielorientierte Umweltpolitik betrieben werden kann, und zwar unter der Nebenbedingung, daß das öffentliche Budget möglichst wenig belastet wird.

Hans P. Fischer, 34, studierte von 1965 bis 1970 Wirtschaftswissenschaften in Mainz. Im Frühjahr 1970 legte er die Diplomprüfung ab und promovierte danach bei Professor Dr. Kurt Schmidt am Institut für Finanzwissenschaft der Johannes Gutenberg-Universität Mainz. Er ist seit 1970 am selben Institut als wissenschaftlicher Mitarbeiter tätig. 
Die Finanzierung des Umweltschutzes im Rahmen einer rationalen Umweltpolitik 


\section{Finanzwissenschaftliche Schriften}

Herausgegeben

von den Professoren

Albers, Krause-Junk, Littmann, Oberhauser, Pohmer, Schmidt

\section{Band 9}

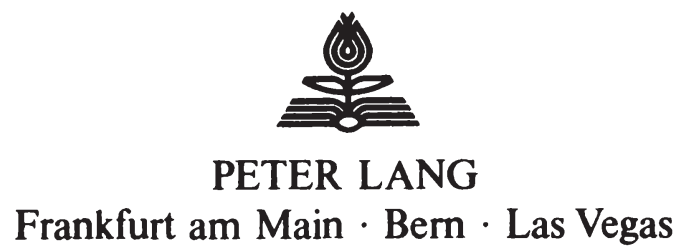


Hans P. Fischer

\section{Die Finanzierung des \\ Umweltschutzes im Rahmen einer rationalen Umweltpolitik}

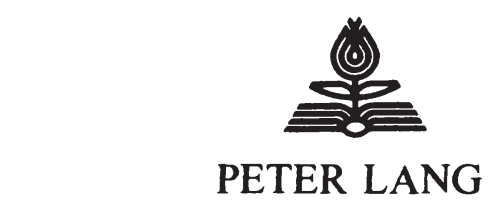

Frankfurt am Main - Bern - Las Vegas 
CIP-Kurztitelaufnahme der Deutschen Bibliothek

\section{Fischer, Hans P.:}

Dic Finanzierung des Umweltschutzes im Rahmen einer rationalen Umweltpolitik. - Frankfurt am Main, Bern, Las Vegas: Lang, 1978.

(Finanzwissenschaftliche Schriften; Bd. 9) ISBN 3-261-02660-X

Open Access: The online version of this publication is published on www.peterlang.com and www.econstor.eu under the international Creative Commons License CC-BY 4.0. Learn more on how you can use and share this work: http://creativecommons. org/licenses/by/4.0.

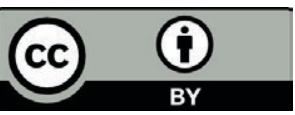

This book is available Open Access thanks to the kind support of ZBW - Leibniz-Informationszentrum Wirtschaft.

Gedruckt mit freundlicher Unterstützung der

„Freunde der Universitït Mainz e. V.“.

ISBN 3-261-02660-X

ISBN 978-3-631-75188-6 (eBook)

(C) Verlag Peter Lang GmbH, Frankfurt am Main 1978

Alle Rechte vorbehalten.

Nachdruck oder Vervielfältigung, auch auszugsweise, in allen Formen wie Mikrofilm, Xerographie, Mikrofiche, Mikrocard, Offset verboten.

Druck: Fotokop Wilhelm Weihert KG, Darmstadt

Titelsatz: Fotosatz Aragall, Wolfsgangstraße 92, Frankfurt am Main. 


\section{Vorwort}

Umweltschutz ist zweifellos eine interdisziplinäre und multidisziplinäre Aufgabe. Eine wissenschaftliche Arbe1t über den Umweltschutz kann das meines Erachtens nicht ignorieren. Beim Verfassen einer Dissertation steht man dabei vor dem Dilemma, keine Teamarbelt schreiben $z u$ dürfen. Ich habe mich deshalb $z u$ dem - für die Sache und auch für mich - weniger befriedigenden Weg entschieden, mich so welt in die außerokonomischen Geblete des Umweltschutzes einzuarbeiten und die dabel erlangten Kenntnisse so welt zu berlicksichtigen, wie mir dies für die Untermauerung der ökonomischen Analyse notwendig schien. Ich bin bewust die Gefahr des Dilettantismus eingegangen, um die - wie ich meine - schlimmere Alternative zu vermeiden: die Ignoranz der Interdependenzen, die zwischen Okologie und Okonomie bestehen.

Die Arbeit wurde im Frühjahr 1977, das Literaturstudium bis auf einige Ausnahmen berelts Mitte 1976 beendet. Sie wurde vom Fachbereich Rechts- und wirtschaftswissenschaften der Johannes Gutenberg-Universität Mainz Im Sommersemester 1977 als Dissertation angenomen.

Die Arbeit entstand während meiner Tätigkeit als wissenschaftlicher Mitarbelter von Herrn Professor Dr. Kurt Schmidt am Lèrstuhl für Volkswirtschaftslehre und Finanzwissenschaft der Johannes Gutenberg-Universität Mainz. Sollte die Arbelt zu erkennen geben, daB ihr Entstehen nicht nur auf "knowledge of the book" sondern auch auf "knowledge of the practice" beruht, so wäre dies auf den hellsamen Einflub von Herrn Professor Schmidt zurückzuführen. Dafür wăre $1 \mathrm{ch}$ thm dann ebenso zu Dank verpflichtet, wie ich es für die Geduld bin, die er bel Entstehung der Arbeit aufgebracht hat. Herrn Professor Dr. Rlaus Rose danke ich für die Bereitschaft, das Koreferat ubernommen zu haben. Für die Mühe, dle mit dem Schreiben des Manuskripts verbunden war, möchte ich Frau Elke Pfennig danken. 

Vorwort

Abkürzungsverzeichnis

Einleitung

I. Umweltbelastung und Budgetbelastung 11

II. Disposition

Hauptteil

I. Zur systematischen Sammlung von Daten der Umweltbelastung 17 A. Zur Notwendigkeit umweltrelevanter Informationen 17

B. Zur Diagnose der Umweltsituation

1. Zur Diagnose von Art und Umfang der Umweltbelastung

a) Zur Notwendigkeit einer Umweltstatistik

b) Der institutionelle Rahmen

c) Arten der Umweltbelastung

aa) Erfassungs - und Einteilungskriterien

bb) Arten der Luftbelastung

cc) Arten der Gewässerbelastung

dd) Arten der Bodenbelastung

ee) Arten der Pflanzen- und Tierbelastung

ff) Arten der Lebensmittelbelastung

gg) Arten des Lärms

d) Die katastermäßige Erfassung der umweltbelastenden Schadstoffe

aa) Zur Aufstellung von Immissionskatastern

bb) Zur Aufstellung von Emissionskatastern

cc) Zur statistischen Aufbereitung der Katasterdaten

2. Zur Diagnose der Schadstoffirkungen 58

a) Zur statistischen Erfassung der Schadstoffwirkungen

b) Zur Systematisierung der Schäden 59

c) Die Wirkungen der Schadstoffe 61

aa) Die Wirkungen der Schadstoffe auf die Umwelt der Menschen

(1) Ókologische Wirkungen

(2) Wirkungen auf die materielle Umwelt (Sachgüter)

bb) Die Wirkungen der Schadstoffe auf die menschliche Gesundheit

(1) Zur Definition der menschlichen Gesundheit

(2) Probleme bei der Erfassung der Schads to $f$ fwirkungen

(3) Methoden zur Erfassung der Schadstoffwirkungen auf die menschliche Gesundhelt 83

(4) Systematisierung der Schadstoffwirkungen auf die menschliche Gesundheit 88

(5) Darstellung von Schadstoffwirkungen auf die menschliche Gesundheit

(6) Zur Beachtung der toxikologischen Gesamtsituation 
(7) Zur Aufstellung eines Wirkungskatasters

3. Zur Diagnose der Ursachen der Umweltbelastung

a) Probleme und Ansätze der Ursachendiagnose

b) Zur fatalistischen Ursachendiagnose

c) Zur ideologisch geprägten Ursachendiaqnose

d) Zur pragmatischen Ursachendiagnose

e) Fazit der Ursachendiagnose

C. Zur Prognose der Umweltsituation

I. Ziele der Umweltpolitik

A. Umweltschutz als gesellschaftspolitisches Ziel

1. Zur Notwendigkeit eines umweltpolitischen Zielsystems

2. Umweltschutz - ein Produkt der Reformeuphorie?

3. Zur Stellung des Umweltschutzes in der Hierarchie der gesellschaftspolitischen Ziele

B. Zur Operationalisierung der Ziele des Umweltschutzes

1. Zur Ableitung unschädlicher Immissionskonzentrationen (Immissionsarenzwerte)

2. Zur Ableitung von Immissionsstandards

a) Zur Festlegung optimaler Immissionskonzentrationen

b) Zur Festlegung der optimalen Umweltqualität

c) Zur Eignung von Kosten-Nutzen-Analysen bei der Ableitung optimaler Immissionskonzentrationen einzelner Schadstoffe

d) Grenzen der Anwendbarkeit von KostenNutzen-Analysen bei der Bestimmung optimaler Immissionskonzentrationen

e) Zur Festlegung von Immissionsstandards durch politische Entscheidung

aa) Von der optimalen Immissionskonzentration zum Immissionsstandard

bb) Implikationen der Notwendigkeit politischer Entscheidungen

cc) Zum Charakter der Immissionsstandards

C. Zur Ableitung von Immissionsstandards für die einzelnen Umweltbereiche

1. Immissionsstandards für schadstoffe in der Luft

2. Immissionsstandards für die Gewässer

3. Immissionsstandards für den Boden

4. Toleranzgrenzen der Lebensmittelbelastung

5. Immissionsstandards für den Lärm

III. Grundlagen elner rationalen Planung des Umweltschutzes

A. Forderungen an eine rationale Umweltschutzplanun

B. Die Maßnahmen des Umweltschutzes

1. Zur Systematisierung der Umweltschutzmaßnahmen

2. Darstellung der UmweltschutzmaBnahmen

a) Nicht-technische Maßnahmen des Umweltschutzes

aa) Die Förderung der Umweltforschuna

bb) Ausbildung von Umwelt (schutz) fachleuten

cc) Geburtenkontrolle 
dd) Die Schaffung eines (neuen) Umweltbewußtseins

ee) Die Kontrolle des technischen Fortschritts

b) Raumordnung als Maßnahme des Umweltschutzes

c) Technische Maßnahmen des Umweltschutzes

aa) Technik und Umweltschutz

bb) Ansatzpunkte des technischen Umweltschutzes

cc) Konkrete Maßnahmen des präventiven technischen Umweltschutzes

dd) Beurteilung der technischen Maßnahmen des präventiven Umweltschutzes

C. Ansätze einer optimalen Planung des Umweltschutzes

1. Planung des Umweltschutzes nach Gossen?

2. Das Optimierungsmodell von Thoss

3. Darstellung eines einfachen Planungsansatzes

D. Grenzen einer rationalen Planung des Umweltschutzes

IV. Träger und Instrumente einer rationalen Umweltpolitik

A. 'Zur Allokation der Umweltschutzaufgabe zwischen privatem und öffentlichem Bereich

1. Zur Aufgabe einer rationalen Umweltpolitik

2. Umweltschutz durch Privatinitiative?

a) Mögliche Träger privater Umweltschutzinitiativen

b) Möglichkeiten privater Umweltschutzinitiativen

c) Grenzen des Umweltschutzes durch Privatinitiativen

3. Die umweltpolitischen Aufgaben des Staates

a) Zur Einteilung der umweltpolitischen Aufgaben des Staates

b) Zur Schaffung und Verbesserung der umweltpolitischen Rahmenbedingungen

c) Strategien zur Durchsetzung der Umweltpolitik

B. Instrumente zur Durchsetzung der Umweltpolitik

1. Zur Einteilung und Bewertung der umweltpolitischen Instrumente

2. Darstellung und kritische Würdiguno der umweltpolitischen Instrumente

a) Die Instrumente zur Durchsetzung des

Verursacherprinzips

aa) Zur Internalisierung negativer externer Effekte durch eine

Pigou'sche Steuer

bb) Auflagen als Instrument zur Durchsetzung des Verursacherprinzips

(1) Gebote

(2) Verbote

(3) Standards

(a) Produktstandards

(b) Emissionsstandards 
cc) Marktwirtschaftliche Instrumente zur Durchsetzung des Verursacherprinzips

(1) Die Besteuerung der Produktmenge

(2) Die Besteuerung von Produktionsfaktoren

(3) Die Emissionsabgabe 355

(4) Emissionszertifikate 380

dd) Benutzungsqebühren als Instrument des Verursacherprinzips

b) Die Instrumente des Gemeinlastprinzips

aa) Subventionen

bb) Allgemeine Deckungsmittel

C. Mögliche Auswirkungen einer rationalen Umweltpolitik auf das öffentliche Budget

1. Einteilung der budgetären Wirkungen einer rationalen Umweltpolitik

2. Ein möglicher Weg zur Ermittlung der budqetären Auswirkungen einer rationalen Umweltpolitik

3. Versuch einer Tendenzaussage über die budgetären Konsequenzen des Verursacher- und Gemeinlastprinzips 
In das Abkürzungsverzelchnis haben wir nur Abkürzungen aufgenommen, die mehrmals vorkommen und von denen wir annehmen, da $\beta$ sie dem Leser unbekannt sein könnten.

$\mathrm{Ab} f \mathrm{G}$

BauNvo

BBaug

BImSchG

$\mathrm{BSB}_{5}$

c.p.

CSB

DFG

E.P.A.

EStDV

GewO

GWB

H.M.S.O.

IAWR

InvZulG

J.E.C.

MIK

MKRO

$\mathrm{NZZ,} \mathrm{FA}$

ppm

ROG

RSU

StBFG

StPO

StVzo

TAL

U.S.C.E.Q.
Abfallbeseitigungsgesetz

Baunut $z$ ungsverordnung

Bundesbaugesetz

Bundesimmissionsschutzgesetz

Biochemischer Sauerstoffbedarf in funf Tagen

ceteris paribus

Chemischer Sauerstof fbedarf

Deutsche Forschungsgemeinschaft

Environmental Protection Agency

Einkommens teuer-Durch fuhrungsverordnung Gewe rbeordnung

Gesetz gegen Wettbewerbsbeschränkungen Her Majesty's stationary Office

Internationale Arbeltsgemeinschaft der Wasserwerke am Rhein

Investitionszulagengesetz

Joint Economic Committee

Maximale Immissionskonzentration

Ministerkonferenz für Raumordnung

Neue zürcher Zeitung, Fernausgabe

parts per million

Raumordnungsgesetz

Der Rat von Sachverständigen für Umwe ltfragen

Städtebauforderungsgesetz

strafprozeBordnung

straßenverkehrszulassungsordnung

Technische Anleitung zur Reinhaltung der Luft

United States Council on

Environmental Quality 
U.S.G.P.O.

UStatG

UWG

VDI

WHO

WMO
United States Government Printing Office

Umweltstatistikgesetz

Gesetz gegen unlauteren Wettbewerb

Verein Deutscher Ingenieure

World Health Organization

World Meteorological Organization 
I. Umweltbelastung und Budgetbelastung

Die Belastung der Umwelt mit Schadstoffen aller Art und die damit verbundenen Gefahren für die menschliche Gesundheit und das ökologische Gleichgewicht haben zu Beginn der siebziger Jahre vor allem in den Industriestaaten zur Aufstellung von Programmen geführt, die einen Schutz der Umwelt und damit des Menschen vor den Gefahren der Umweltbelastung gewährleisten sollen. Die Aufstellung der Umweltschutzprogramme wurde in den meisten Ländern durch Kostenschätzungen ergänzt ${ }^{1)}$.

Je nach dem Stand der Umweltbelastung in den einzelnen Ländern und der strenge der ziele, die durch die Realisierung der jeweiligen Umweltprogramme erreicht werden sollten, ergaben sich unterschiedich hohe finanzielle Belastungen. Setzt man die für unterschiedliche zeiträume geschätzten durchschnittlichen Umweltschutzaufwendungen pro Jahr zum jeweiligen Bruttosozialprodukt in Beziehung, so erhält man z.B. für die USA 2,28 , für die Bundesrepublik Deutschland 1,88 , für die Niederlande 1 bis $1,5 \&$ und für Italien 0,68 , wobei der Anteil im Laufe der Jahre steigt ${ }^{2}$ ?

In absoluten 2 ahlen wurden für die Bundesrepublik Deutschland durch das Umweltprogramm entstehende Ausgaben in Höhe von 36 Mrd. DM für die Jahre von 1971 bis 1975 geschätzt, wobei 18,9 Mrd. DM (also ungefähr 55 \&) auf den staat entfallen sollten. Das bedeutete eine durchschnittliche jährliche Belastung der öffentlichen Haushalte von $3,8 \mathrm{Mrd}$. DM, wovon rund 83 \& (also ungefähr

1) Vgl. OECD-Environment Directorate, Survey of Pollution Control Cost Estimates Made in Member Countries, Paris 1972. Für die Bundesrepublik Deutschland schätzten Ackermann, Geschka und Karsten die voraussichtliche Gesamtbelastung der Volkswirtschaft durch das Umweltprogramm der Bundesregierung; vgl. Materialienband zum Umweltprogramm der Bundesregierung (zu BT-Drucksache VI/2710), Bonn 1971, S. $593 \mathrm{ff}$.

2) Vgl. OECD-Environment Directorate, Analysis of Costs of Pollution Control, Paris 1973, S. 4 ff. 
3,1 Mrd. DM) auf die Kommunen entfallen sollten 3) Dies schienen in den Jahren, in denen die Steuereinnahmen noch reichlich flossen, keine zu hohen Mehrbelastungen der öffentlichen Haushalte zu sein, wenn auch für die Gemeinden Finanzierungsprobleme vorauszusehen waren. Nach der Verabschiedung des Umweltprogramms verschlechterte sich indessen die Haushaltslage aus verschiedenen Gründen. Die Finanzlerung der im Umweltprogramm vorgesehenen öffentlichen Ausgaben wurde mit der Abschwächung der Konjunktur schwieriger, da die steuereinnahmen nicht mehr in genügendem Umfang stiegen. Infolge der రlkrise kam es zu einer teilweisen Substitution der Energieträger (vor allem von leichtem zu schwerem Heizöl), was die Belastung der Luft mit Schwefeloxiden erhöhte; die langfristig geplante substitution fossiler Brennstoffe durch die Kernenergie wurde in verstärktem Umfang in Angriff genommen. Die zunehmende Luftbelastung und die stelgende Gefahr der radioaktiven Umweltbelastung führte nun bei einer gegenüber Umweltfragen sensibilisierten Bevölkerung zur Forderung nach mehr Umweltschutz. Die im Umweltprogramm vorgesehenen Maßnahmen erwiesen sich - sowelt sie liberhaupt durchgeführt wurden - auch auf dem Gebiet des Gewässerschutzes und des Lärmschutzes als unzureichend - nicht nur, weil die objektiv meBbaren Belastungen stiegen, sondern auch weil die umweltbewuster gewordene Bevölkerung strengere Maßstäbe bel der Beurteilung der Umweltsituation anlegte.

Es gilt nun das Dilemma zu lösen, den gestiegenen Ansprüchen der Bevölkerung an die Umweltqualität bel der angespannten Lage der öffentlichen Haushalte in der Bundesrepublik Deutschland gerecht $z u$ werden. Eine Umweltpolitik, die diese Aufgabe lösen soll, muß die umweltpolitischen ziele zu erreichen versuchen, ohne den budgetpolitischen Bogen $z u$ überspannen, oder anders formuliert: Die Umweltpolitik muß bei der Verfolgung der umweltpolitischen ziele die budgetpolitischen Restriktionen beachten. Elner weite-

3) Vgl. Geschka, H./Lichtwer, L., Schätzungen der monetären Aufwendungen für Umweltschutz in der Bundesrepublik Deutschland für den Zeitraum 1971-1975, in: Battelle-Informationen 14, 1972 , S. 4 . 
ren Belastung der offentlichen Budgets durch die Erfüllung umweltpolitischer Aufgaben sind in der Bundesrepublik Deutschland durch die Hohe der Staatsquote, aber auch der steuer- und Kreditfinanzierungsquote enge Grenzen gesetzt ${ }^{4}$ ! Aktzeptiert man die Meinung, das die Staatsquote nicht weiter steigen darf, so können uberproportional zu den Steuereinnahmen steigende öffentliche Ausgaben für den Umweltschutz nur dann getätigt werden, wenn andere Ausgaben unterproportional steigen (Umstrukturierung der Ausgaben). Da eine solche umstrukturierung wegen der gesetzlich oder vertraglich fixierten Höhe vieler öffentlicher Ausgaben und wegen des Einflusses von Interessengruppen nur in engen Grenzen möglich ist, muB in diesem Fall die Devise lauten, daß das Budget möglichst von Umweltschutzausgaben frelgehalten werden soll.

Läßt man eine Erhöung der Staatsquote zu, bietet sich zu deren Finanzierung eine Erhöhung der Abgabenquote und/oder der Kreditfinanzierungsquote an. Eine weltere Erhöhung der Kreditfinanzierungsquote 1st wegen der hohen Neuverschuldung des staates in den letzten Jahren äußerst problematisch. Da auch die steuerbelastung in der Bundesrepublik Deutschland hoch ist, empfiehlt sich auch kelne Erhöhung der Steuerquote; was bleibt, ist eine Erhöhung der Gebührenquote. Daraus kann man die Forderung ableiten, daß der staat seine Umweltschutzausgaben in möglichst großem Umfang durch die Erhebung von Gebühren finanzieren soll. Zusammenfassend kann man bis hierher sagen, daB eine den budgetpolitischen Restriktionen gerecht werdende Umweltpolitik möglichst wenige offentliche Ausgaben und für die unbedingt notwendigen offentlichen Umweltschutzausgaben nach Möglichkeit eine Gebührenfinanzierung vorsehen soll.

Eine solche vordergrüdig auf Minimierung der Budgetbelastung bedachte Umweltpolitik kann allerdings so hohe budgetwirksame Folgekosten hervorrufen, daB die Gesamtbelastung der öffentlichen Budgets gröBer wird, als wenn der staat selbst gleich

4) Vgl. Sachverständigenrat zur Begutachtung der gesamtwirtschaftlichen Entwicklung (SVR), Vor dem Aufschwung, Jahresgutachten 1975/76, Stuttgart und Mainz, 1975, S. 124 f. und S. $166 \mathrm{ff}$. 
alle notwendigen Umweltschutzmaßnahmen finanziert hätte. Offenbar gibt es hinsichtlich der Budgetbelastung ein Optimum, das dort liegt, wo die Summe aus direkten öffentlichen Umweltschutzausgaben und den Folgekosten, die durch die Art der Finanzierung dieser Umweltschutzausgaben entstehen, ein Minimum 1st.

Neben der Belastung der öffentlichen Haushalte kann es aber auch zu einer Entlastung infolge des Umweltschutzes kommen, dann nämlich, wenn der Umweltschutz eines seiner Hauptziele, den Schutz der menschlichen Gesundheit, erreicht; die Folge kann eine spürbare Entlastung der Gesundheitsbudgets der offentlichen Gebietskörperschaften sein. Ein besserer Gesundheitszustand der Bevölkerung kann darüber hinaus $z u$ Produktivitätsfortschritten führen, die das Wachstum des Bruttosozialprodukts und damit der Steuereinnahmen begünstigen, so daß sich durch den Umweltschutz auch eine Verbesserung auf der Elnnahmenselte der öffentlichen Budgets ergeben kann. Zumindest langfristig ist es denkbar, daB sich die öffentlichen Umweltschutzausgaben in dem Sinne rentieren, daß sie in anderen öffentlichen Bereichen zu Minderausgaben und/oder zu allgemeinen Einnahmenerhöhungen führen, welche die ursprüglichen Umweltschutzausgaben überkompensieren. Die Reduzierung der Umweltbelastung muß also keineswegs eine dauerhafte Budgetbelastung zur Folge haben.

II. Disposition

Ziel dieser Arbeit ist $\mathrm{zu}$ untersuchen, wie sich eine rationale, d.h. zielorientierte, Umweltpolitik unter der Nebenbedingung gestalten läBt, daB der Gesamthaushalt von Bund, Ländern und Gemeinden möglichst wenig belastet wird.

Dazu müssen zuerst die Bedingungen einer rationalen Umweltpolitik aufgezelgt werden (I. bis III. Kapitel). Wie eine solche rationale Umweltpolitik aussehen kann, wollen wir anhand des folgenden Ablaufschemas mit Rückkopplung erläutern ${ }^{5)}$ :

5) Dieses Ablaufschema enthält einen Regelkreis. Die Umwelt stellt die Regelstrecke dar und die Reglerung den Regler. MeBgröBen sind die erm1ttelten MeBdaten der Umweltbelastung, FührungsgröBen die operational definierten ziele des Umweltschutzes und Stellgrößen die umweltpolitischen Maßnahmen, 


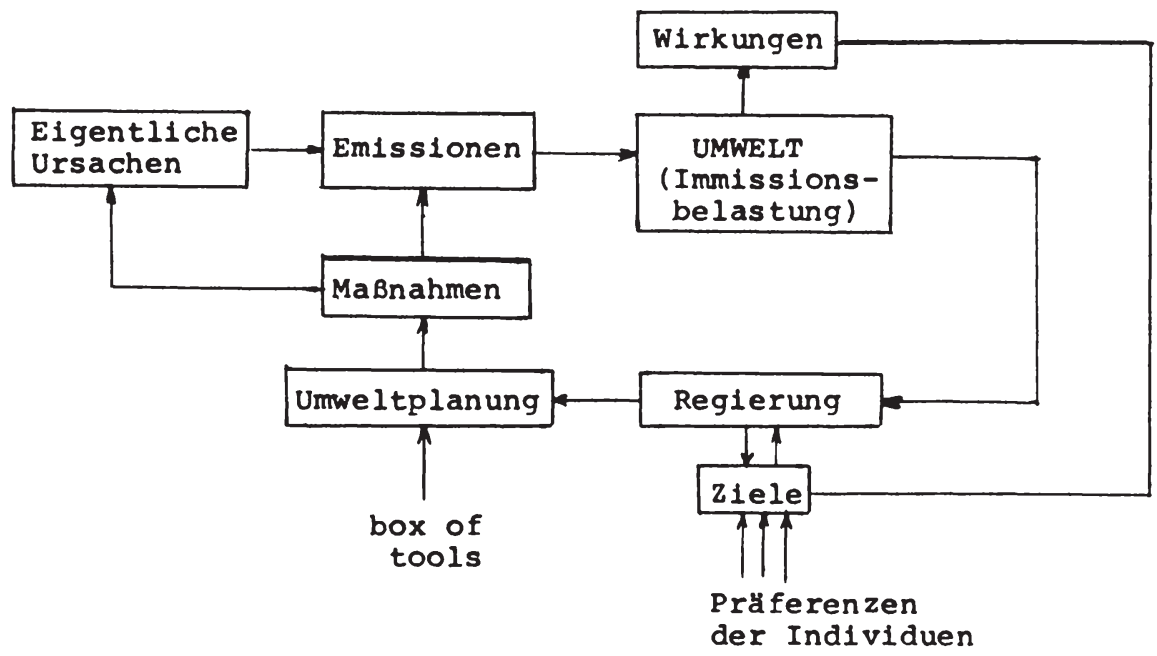

Abb. 1

Grundlage einer rationalen Umweltpolitik ist die systematische Sammlung von Informationen uber Art und Umfang der in der Umwelt vorkommenden Schadstoffkonzentrationen (der Immissionsbelastungen), 1hrer Wirkungen, 1hrer Herkunft (Emissionsquellen) und ihrer Ursachen (I. Kapitel).

Die Kenntnis der Schadstoffwirkungen ist notwendig, un die ziele der Umweltpolitik operational zu definieren; bel der Bildung der Umweltschutzziele sind auch Interdependenzen mit anderen zielen zu berücksichtigen (II. Kapltel).

Die Ermittlung von Art und Umfang der tatsăchlichen Schadstoffkonzentrationen 1st erforderlich, um sle mit den zulassigen Schadstoffkonzentrationen, den zielen, zu vergleichen. Dieser Soll-Ist-Verglelch ist zusammen mit der Identifikation der Emissionsquelien und der Ermittlung der eigentlichen Ursachen der Umweltbelastung Voraussetzung elner rationalen Umweltplanung, deren Aufgabe es 1st, die zur Verfügung stehenden MaBnahmen des technischen und nicht-technischen Umweltschutzes optimal zu

die ergriffen werden, wenn die Meßgrößen gröBer als die FührungsgröBen sind. 
kombinieren (III. Kapitel).

Im IV. (und letzten) Kapitel untersuchen wir, wie die geplanten Umweltschutzmaßnahmen so durchgeführt werden können, daß sie zur Realisierung der Umweltschutzziele führen und dabei die öfentlichen Haushalte möglichst wenig belasten.

Zunächst werden wir prüfen, inwieweit der Umweltschutz elne öffentliche Aufgabe ist und inwieweit dies fur die Allokation der Umweltschutz a u $\mathrm{g}$ a $b$ e $n$ zwischen privatem und öffentlichem Sektor relevant ist.

Sodann werden wir die Instrumente der beiden Finanzierungsprinzipien des Umweltschutzes, also des Verursacher- und Gemelnlastprinzips darstellen und kritisch würdigen. Wir werden dabei besonderen Wert auf die Untersuchung der Allokationseffizienz der Instrumente sowie ihre Informations- und Administrationskosten legen.

Sodann untersuchen wir, inwleweit das offentliche Budget durch die Anwendung der alternativen Instrumente des Verursacher- und Gemeinlastprinzips be- und entlastet werden kann. Wir berücksichtigen dabei sowohl die direkten als auch die indirekten wirkungen auf das Budget; letztere entstehen dadurch, daß andere Ziele als der Umweltschutz durch die umweltpolitischen Maßnahmen tangiert werden 6 ?

Eine zusammenfassende Beurteilung der umweltpolitischen Instrumente hinsichtlich ihrer Eignung, die umweltpolitischen ziele im Rahmen einer marktwirtschaftlichen ordnung möglichst effizient $\mathrm{zu}$ erreichen, beendet das Kapitel.

6) Hier zelgt sich eine Redundanz - nicht nur bei der Behandlung des Themas, sondern auch eine in der politischen Realität vorhandene - zwischen der Formulierung der Umweltschutzziele und den Wirkungen der diese Ziele anstrebenden Umweltpolitik. Der Verfasser steht hier vor dem gleichen Dilemma wie die Politiker und Wissenschaftler, die bei der Zielbildung mitwirken. Es müssen die ziele formuliert werden, ohne daß man die Wirkungen kennt, die von der Realisierung dieser Ziele auf die Erreichung anderer Ziele ausgehen, wodurch die Erreichung der Umweltschutzziele selbst gefährdet werden kann. Die Konsequenz kann nur sein, daß die Zielsetzung in einem iterativen Proze $B$ erfolgen muß. 
Hauptteil

I. Zur systematischen Sammlung von Daten der Umweltbelastung

A. Zur Notwendigkelt umweltrelevanter Informationen

Grundlegende Voraussetzung für einen ökonomischen Umweltschutz sind qualitativ und quantitativ ausreichende Informationen uber die tatsächliche (gegenwärtige) und die in der zukunft erwartete Umweltbelastung, ihre Wirkungen und ihre Ursachen. Ohne solche Informationen ist keine rationale Umweltplanung und -politik möglich 1' Ohne : ationale Umweltplanung ist elner ökonomischen Kriterien genuged.den Finanzierung des Umweltschutzes die Grundlage entzogen - sowohl im öffentlichen wie auch im privaten Berelch: Es würde entweder zuviel oder zuwenig für den Umweltschutz ausgegeben. Eln beredtes Belspiel für eine solche Fehlallokation - eben auf mangelnde Informationen zurïckzufuhren liefert eln anderer Bereich, nämlich der Bildungssektor. So wie heute der zustand der Umwelt wurde in der zweiten Hălfte der sechziger Jahre der Zustand des Bildungswesens als katastrophal

1) Zum allgemeinen Zusammenhang zwischen Information, Planung und rationaler Wirtschaftspolitik vgl. Wille, E., Planung und Information, Berlin 1970, S. $24 \mathrm{ff}$. und S. $113 \mathrm{ff}$. Auf die speziellen Erfordernisse der Umweltplanung zugeschnittene Informationen werden in zwel Studien EDV-Anlagen produzlerender Unternehmen dargestellt: vgl. Günther, U. u.a., UmweltschutzInformations- und Steuerungssystem, IBM-DV-Anwendung, 0.0. , 1972; Birkle, M. u.a.. Informationssystem Umwelt. Konzept eines MeB- und Planungsinformationssystems für UmweltmaBnahmen, hrsg. von der Slemens AG, München 1973. Diese Studien erfolgten wohl im Hinblick darauf, dem Bundesinnenministerium bzw. dem Umweltbundesamt die hard ware fur das inzwischen im Aufbau befindliche Umwelt- Planungs- und Informationssystem (UMPLIS) verkaufen zu können; vgl. Kunz, W./Rittel, H., Projekt UMPLIS - Aufgaben und Aufbau, in: Umwelt, 1973, 3, S. $43 \mathrm{ff.}$; Kitschler, W. , Aufbau eines Informationssystems für Umweltplanung, in: Allg. Stat. Archiv, Bd. 59, 1975, S. 25 ff. 
bezeichnet 2) Ohne ausreichende Analyse der Situation wurden die falschen Maßnahmen ergriffen. Die finanziell stark geforderte akademische Ausbildung führte $z u$ elnem Uberangebot an Akademikern; die berufliche Ausbildung wurde dagegen stark vernachlässigt. Damit die Ressourcen und finanziellen Mittel nicht auch auf dem Gebiet des Umweltschutzes fehlgeleitet werden, bedarf es einer auf möglichst gesicherten Daten über die Umweltsituation und thre Entwicklung fuBenden Analyse.

Es ist nun keineswegs so, $\mathrm{daB}$ es uberhaupt an Diagnosen und Prognosen der gegenwärtigen und zukünftigen Umweltsituation fehlt 3) Das Gegenteil ist der Fall: Eine Flut von Presseartikeln, Funk- und Fernsehsendungen, populärwissenschaftlichen Büchern, Broschüren von Unternehmensverbänden, Gewerkschaften, Kirchen, Naturschutzverbänden liefern ebenso Informationen wie wissenschaftliche (oder doch diesen Ausspruch erhebende) Publikationen von einzelnen Wissenschaftlern (vom Theologen bis $z$ um Futorologen) und Forschungsinstituten. Fast alle diese Informationen unterscheiden sich voneinander in ihren Aussagen, selbst wenn sie sich auf die allgemeine situation und Entwicklung der Umwelt beziehen. Einlge Unternehmerverbände verharmlosen die Umweltsituation, elnige Naturschutzverbände dramatisieren sie und einige Futurologen prophezeien den Weltuntergang durch Umweltverschmutzung. Aufgrund solcher Informationen läßt slch ebensowenig eine Umweltplanung und Umweltpolitik aufbauen wie aufgrund fehlender Informationen. Es kommt darauf an, möglichst umfassende objekt1ve Daten über Art und Umfang der Umweltbelastung zu erhalten, und dies kann nur durch Messungen der

2) Vgl. Ronge, V., Die Umwelt im kapitalistischen System, in: Glagow, M. (Hrsg.), Umweltgefahrdung und Gesellschaftssystem, München 1972 , S. 99.

3) Sogar die amtliche Statistik sammelte in der Bundesrepublik Deutschland auch bisher schon Daten uber die Umweltbelastung, meistens allerdings nur in der Form indirekter Indikatoren; vgl. Bartelmus, P., Probleme der Entwicklung eines umweltstatistischen Systems, in: Statistische Hefte, N.F., 14. Jg., 1973, S. 140; Baltes, H./Nowak, W., Umweltstatistik - Ein Instrument der Umweltplanung, in: Wirtschaft und Statistik, 1974,4 , S. 237 . 
Umweltdaten und deren Erfassung in elner Umweltstatistik errelcht werden. "Monitoring is the key to effective environmental protection programs" 4 )

4) U.S. Environmental Protection Agency, Research and Monitoring, U.S. Government Printing office (G.P.O.), Washington, D.C., 1972, S. 12. Monitoring bedeutet auch Uberwachen. Darin zeigt sich die doppelte Funktion der Messung: Sie soll Daten der Umweltbelastung für die Planung liefern und den Erfolg der aufgrund der Planung ergriffenen Maßnahmen kontrollieren. 
"Der Mensch ist mit dem Verstande und der schöpferischen Kraft begabt, um das, was 1 hm gegeben 1st, zu vermehren; bis jetzt aber hat er nicht geschöpft, sondern nur vernichtet.

Die wälder werden immer seltener und seltener, die Flüse versiegen, das Wild ist verschwunden, das Klima verdorben und mit jedem Tag wird die Erde immer ärmer und häßlicher".

(Anton Tschechow, Onkel Wanja)

\section{B. Zur Diagnose der Umweltsituation}

1. Zur Diagnose von Art und Umfang der Umweltbelastung

a) Zur Notwendigkeit einer Umweltstatistik

Fur eine rationale Umweltplanung müsen die Daten uber Art und Umfang der Umweltbelastung möglichst vollständig sein. Die Grenze 1st dort zu ziehen, wo die Kosten der Datenerfassung größer werden als deren Nutzen fur die Umweltplanung und damit für die Umweltpolitik. Natürlich ist die Bestimmung dieser Grenze äußerst schwlerig. Sie scheint uns jedoch um ein vielfaches höher $z u$ liegen als die durchschnittlich rund.sechs Millionen DM pro Jahr, welche für die Durchführung des Umweltstatistikgesetzes der Bundesrepublik Deutschland veranschlagt wurden 5); hier muB man allerdings soglelch hinzufügen, daB dieses Gesetz keinesfalls den Anforderungen genügt, die an eine Umweltstatistik, die fur Zwecke der Umweltplanung dienen soll 6) gestellt werden muß. Ganz im Gegensatz dazu wollen wir nun versuchen, ein sehr anspruchsvolles system der Umweltstatistik zu entwerfen, wohl wissend, daB wir damit sehr wahrscheinlich die oben aufgezeigte Grenze uberschreiten. Es erscheint uns jedoch

5) Vgl. Entwurf elnes Gesetzes uber Umweltstatistiken, BTDrucksache 7/988, S. 12 .

6) Vgl. $§ 1$ Umweltstatistikgesetz (UStatG) 
besser, ein solch umfassendes umweltstatistisches system zunächst einmal voll durchzustrukturieren und dann - kostenbedingte - Abstriche zu machen.

b) Der institutionelle Rahmen

In diesem Abschnitt befassen wir uns nur mit der statistik der mengenmäBigen Umweltbelastungen 7). Wir orientieren uns dabei an dem Weg, den die einzelnen Schadstoffe zurücklegen. Diesen Weg kann man in drei Teile glledern: Emission, Transport und Immission der Schadstoffe ${ }^{8}$. Fur die Wirkungen der Schadstoffe auf die natürliche und materielle Umwelt sowie vor allem auf den Menschen ist die Immissionskonzentration der Schadstoffe maBgebend. Es ist also besonders wichtig, die Konzentration der einzelnen schadstoffimmissionen $z \mathrm{u}$ messen. Ein vorbeugender Umweltschutz - und dies sollte Umweltschutz, wenn Immer möglich, sein - benötigt dagegen vor allem Daten darüber, woher die einzelnen Schadstoffe kommen, also über die Emissionsquellen. Denn nur Maßnahmen im Berelch des Emittenten - seien sie technischer, juristischer oder ökonomischer Natur - können letztlich zu hohe Immissionskonzentrationen via Reduktion der Emissionen verhindern. Theoretisch ist es nun möglich, aufgrund der Immissionskonzentration die Emissionskonzentration und auch die Emissionsquelle mit Hilfe komplizierter mathematischer Modelle zu ermitteln 9 ). Dies geschieht z.B. bel der Luftbelastung unter zugrundelegung meteorologischer und topologischer Daten. Man versucht quasi den Weg der Schadstoffe von ihrer Immission zur Emisstonsquelle zurückzuverfolgen. Zumindest wenn viele Emittenten und viele Schadstoffe vorliegen, ist dies jedoch ein ziemlich aussichtsloses Unterfangen 10 ?

7) Zur Statistik der Wirkungen siehe unten s. $61 \mathrm{ff}$.

8) Vgl. Bartelmus, P., Probleme der Entwlcklung elnes umweltstatistischen Systems, a.a.0., S. 124.

9) Vgl. Günther, U. u.a., Umweltschutz- Informations- und Steuerungssystem, a.a.o., s. $32 \mathrm{ff}$.

10) Vgl. Halbritter, G., Abgabenprinzip und Bewertungsproblematik, in: Zur Problematik des Verursacherprinzips. Berlin 1972 , S. 36 . 
Der elnzige zuverlässige Weg, dieses zurechnungsproblem in den Griff zu bekommen, ist eine Messung der Emissionsmengen je Zeiteinhelt direkt an der Emissionsquelle oder in den Fällen, wo dies - wegen der Vielzahl der Emissionsquellen wie bei privaten Haushalten und Kraftfahrzeugen - aus kostengründen nicht möqlich ist, elne Berechnung der Emissionsmengen mit Hilfe sogenannter Emissionsfaktoren 11)

Die Begriffe Emission und Immission sollen hier im weitesten Sinne gebraucht werden. Ublicherweise spricht man nur bel luftbelastenden Schadstoffen und Lärm von Emission und Immission, manchmal auch bel Belastungen der Gewässer. Wir wollen diese Begriffe auch ausdehnen auf Belastungen des Bodens. Emissionsquellen sind in der Regel Punktquellen: Schornsteine (Luftbelastung), Radiolautsprecher (Lärm), Abflußrohre (Gewässerbelastung), Fabriktore (Abfall) Giftspritzen (Pestizide) sind einige typische Beispiele. Der Ort der Emissionsmessung ist damit eindeutig bestimmt. Anders verhäl es sich mit den Immissionen. Die Immissionen finden in dem gesamten Umweltmedium statt. So stellt sich die Frage, wo die Immissionen gemessen werden sollen. Um einen zuverlässigen Uberblick uber die Immissionen zu erlangen, wird nichts anderes ubrigbleiben, als das betreffende Umweltmedium mit einem MeBstellennetz zu uberziehen 12) Je dichter die Maschen dieses Netzes sind; um so informativer ist die Messung der Immissionsbelastung.

Das folgende Schema faßt diese Ausfuhrungen noch einmal zusammen :

11) Vgl. Dreyhaupt, F.J., Das Emissionskataster, in: Schultze, H. (Hrsg.), Umwelt-Report, Frankfurt 1972, S. 213. Näheres hierzu siehe unten $S .51 \mathrm{ff}$.

12) Vgl. U.S. Environmental Protection Agency, Research and Monitoring, a.a.0.. s. 12. Sehr detaillierte Vorstellungen zur Messung der Luftbelastung unterbreiten Günther, U. U.a., Umweltschutz-Informations - und steuerungssystem, a.a.o., und Birkle, M. u.a., Informationssystem Umwelt, a.a.o. 
Es bedeuten: EQ (Emissionsquellen),

EM (Emissionsmessung),

die Punkte stellen ImmissionsmeBstellen

des jeweiligen Netzes dar.

$L M_{1}$ (Luft- und Lärmmeßstationen), $1=1, \ldots, m$

$\mathrm{GM}_{1}$ (GewässermeBstationen) $; 1=1, \ldots, \mathrm{n}$

$\mathrm{BM}_{1}$ (BodenmeBstationen) $i=1, \ldots, \mathrm{r}$
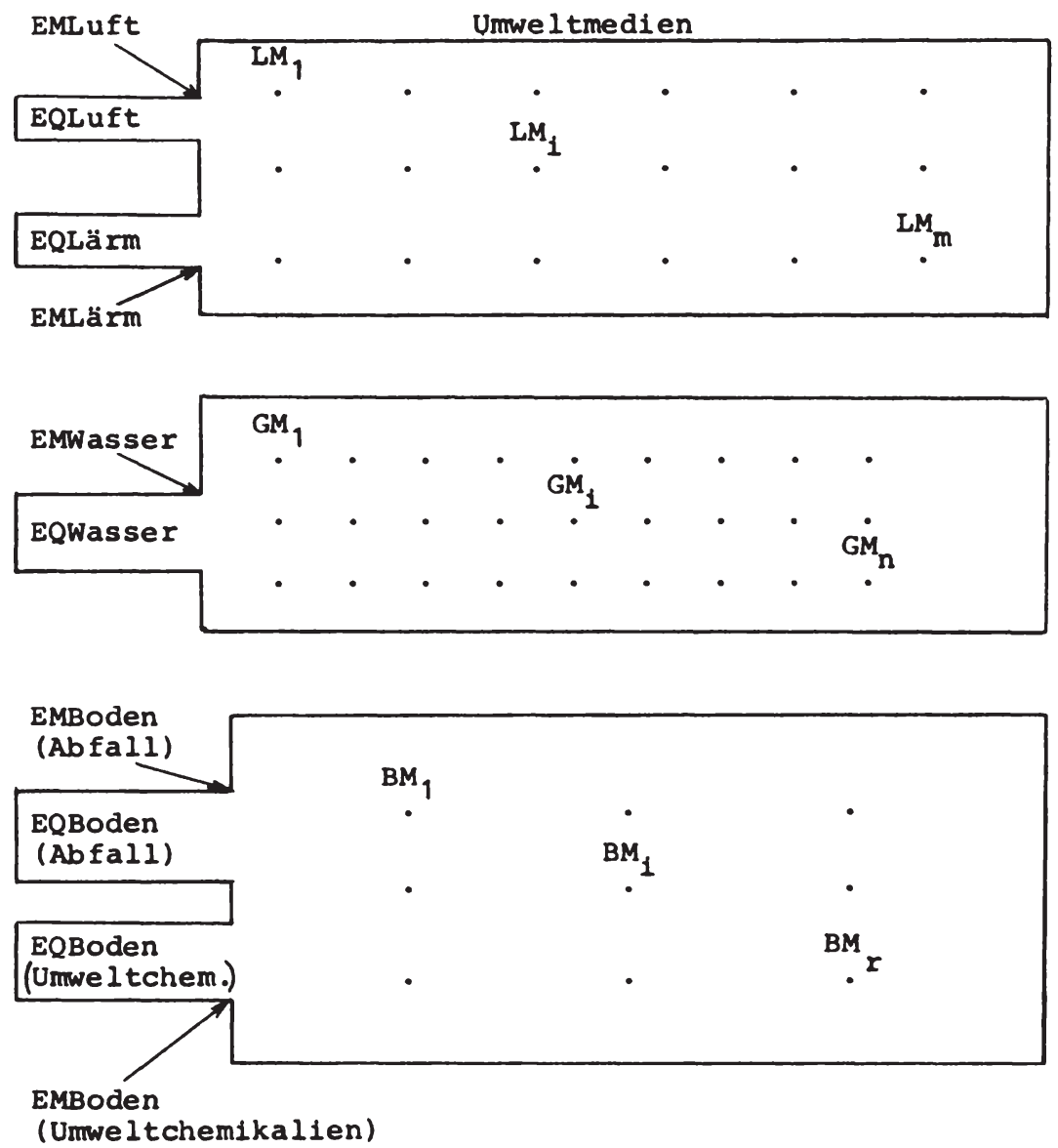

Abb. 2 
Hiermit ist der institutionelle Rahmen der Messung abgesteckt. Es gilt nun noch $z u$ entscheiden, in welchen Zeitabständen die Messungen vorgenommen werden sollen und welche Schadstoffe $z u$ erfassen sind. Grundsätzlich können die Messungen kontinulerlich, zu regelmäBigen oder unregelmäBigen Zeitpunkten oder während bestimmter Zeitabschnitte erfolgen. Das vollständigste B1ld uber die Umweltbelastung (Immissionen) und ihre Ursachen (EQ) erhielte man bel elner kontinuierlichen Messung aller Emissionen und Immissionen 13). Eine solche Messung ist nicht nur

13) Technisch ist eine kontinuierliche Messung vor allem der Luftbelastung und des Lärms, aber auch der Gewässerbelastung, heute kaum noch ein Problem, besonders seit die naBchemischen MeBverfahren immer mehr durch MeBgeräte ersetzt werden, die auf die physikalischen und physikochemischen Eigenschaften der Schadstoffe ansprechen. Welche Entwicklung die Umwelt-MeBtechnik in den letzten zehn Jahren genommen hat, wird klar, wenn man eine Untersuchung der OECD aus dem Jahre 1964 (OECD, Methods of Measuring Air Pollution, Paris 1964) mit einer Monographie von Katz, M., Measurement of Air Pollutants. Guide to the Selection of Methods, Genf 1969, oder gar der neuesten Entwicklung vergleicht, uber die ausfuhrilch in vielen Beiträgen der Zeitschriften "Umwelt" (hrsg. vom VDI) und "U - das technische Umweltmagazin" berlchtet wird. Besonders durch die Verbindung der Gaschromatographie mit Flammenionisations-, Massen- oder Atomabsorptionsspektrometern als Dedektoren können mehrere Schadstoffe in der Luft gleichzeitig kontinulerlich und automatisch gemessen werden; vgl. dazu Morgan, G.B./Ozolins, G./Tabor, E.C., Air Pollution Surveillance System, in: Science, vol. 170, 1970, S. 289-296; Ball, D.F., The Identification and Measurement of Gaseous Pollutants, in: International Journal of Environmental Studies, vol. 1 , 1971, S. 267 ff.; Becker, Herbert, Gleichzeitig mehrere Stoffe messen, in: Umwelt, 1973, 6, S. 25 ff.; Günther, P., Exakte Messung - reinere Luft, in: U, 1972, 4, S. 34. Im Berelch des technisch möglichen 1st auch bereits heute eine kontinulerliche Erfassung der Schadstoffkonzentration in der luft durch Satelliten. Der Vorteil einer solchen Fernerkundung besteht darin, daß die gleichzeitige Beobachtung ganzer Gebiete elne grobere Aussagekraft hat als die punktuelle Messung; vgl. dazu Lichtwer, L./Schaude, G.R., Nutzen der Fernerkundung der Erde, in: Battelle-Information 18, Frankfurt, 1974, s. $34 \mathrm{ff}$. Noch im Entwicklungsstadium befindet sich die Laser-Lidar-Technik zur Messung der Luftbelastung. Zwar ist schon heute eine Identifikation vieler Schadstoffe möglich: für die quantitative Messung kleiner Konzentrationen sind die Laser-Lidar-Geräte 
aus Kostengründen utopisch, sie ist auch gar nicht notwendig, da manche Belastungen - vor allem des Bodens - diskontinuierlich erfolgen, wenn sie auch kontinuierlich fortwirken. Darüber hinaus stellt sich bel der Messung der Bodenbelastung auch noch ein technisches MeBproblem. Es gibt keine die Bodenbelastung kontinuierlich messenden Geräte wie sie für die Belastung der Luft und der Gewässer sowie des Lärms zur Verfügung stehen. Es müssen immer erst Bodenproben genommen und dann in einem Labor untersucht werden. Eine kontinuierliche Messung der Luft- und Gewässerbelastung sowie des Lärms ist heute emissions- und immissionsseitig kaum noch ein technisches, doch ein Kostenproblem. Zumindest die wegen ihrer hohen Konzentration oder ihrer Toxizität für die Umwelt und den Menschen besonders gefährlichen Schadstoffe müssen jedoch U.E. bereits heute emissionsund immissionsseitig kontinuierlich gemessen werden. Welche Schadstoffe dies sind, ist von Region $z u$ Region verschieden und $1 \mathrm{~m}$ Einzelfall zu entscheiden. Für die nächsten Jahre ist aber auf alle Fälle elne kontinuierliche Messung möglichst vieler Schadstoffemissionen und-immissionen in Luft und Wasser sowie des Lärms anzustreben 14)

Als letztes muß nun noch entschieden werden, welche schadstoffe gemessen werden sollen und wie sie für die statistische Erfassung einzutellen sind. Diese Kategorisierung der Schadstoffe bildet zusammen mit einer katastermäBigen Registrierung 15) der Emissionen und Immissionen die Grundlage für ein umweltstatistisches System.

jedoch noch nicht weit genug entwickelt; vgl. dazu Kuper, G., Laser-Lidar, was kann es wirklich?, in: Umwelt, 1972, $1, \mathrm{~S}$. $40 \mathrm{ff}$. und Becker, Herbert, Der Nachweis von Lufteremastoffen mit abstimmbaren Lasern, in: Umwelt, 1972, 1, S. 45 f. Einen beeindruckenden Uberblick über das Angebot deutscher Unternehmen auf umweltmeBtechnischem Gebiet gibt die vom verband der Deutschen Feinmechanischen und Optischen Industrie herausgegebene Broschüre "UmweltmeBtechnik, Instrumente der Feinmechanik und Optik", Köln 1974.

14) Vgl. Rat von Sachverständigen für Umweltfragen (RSU), Umweltgutachten 1974, Stuttgart und Mainz 1974, S. 39.

15) Vgl. Baltes, H./Nowak, W., Umweltstatistik - ein Instrument der Umweltplanung, a.a.0., s. 238. 
c) Arten der Umweltbelastung

aa) Erfassungs- und Einteilungskriterien

Keiner der in die Umwelt gelangenden stoffe ist per se schädlich; es kommt immer auf die Konzentration dieser stoffe in

Luft, Wasser und Boden an, ob sie zu Schadstoffen werden. Manche Stoffe können in relativ groben Mengen emittlert werden, ohne die Qualität von Schadstoffen $z$ u erlangen, sei es weil sie sich schnell verflüchtigen (etwa Abgase), durch die Selbstreinigungskraft, die allen Umweltmedien mehr oder weniger elgen ist, beseitigt oder ganz einfach abgebaut werden (wie etwa manche Umweltchemikalien) oder well die Umwelt und die Menschen relativ hohe Konzentrationen dieser stoffe schadlos aufnehmen können (z.B. $\mathrm{CO}_{2}$ ). Andere Schadstoffe sind dagegen bereits in kleinsten Konzentrationen äuBerst gefährlich für die Umwelt und den Menschen, sei es wegen ihrer Toxizität (z.B. Schwermetalle und lhre Verbindungen) oder weil sie sich nur langsam abbauen (z.B. DDT). Wann die für den Menschen und die Umwelt schädliche Konzentration eines Schadstoffs erreicht ist, kann nur wieder durch Messungen in den elnzelnen Umweltmedien festgestellt werden. Es kann nun nicht Aufgabe eines Meßstellennetzes sein, alle uberhaupt nur denkbaren Schadstoffe zu messen, gleichgültig wie weit ihre Konzentration von der Schädlichkeitsgrenze 16) entfernt ist. Dies muß im Rahmen spezieller Messungen erfolgen, die zwar auch von den Stationen des MeBstellennetzes vorgenommen werden könnten, sofern sie die Einrichtungen dafür haben, besser jedoch von speziellen "MeBtrupps", die mit einem mobilen MeBlabor 17) ausgerüstet sind, in bestimmten Zeitabständen durchgeführt werden sollten 18). Die festen stationen des MeBstellennetzes sollten

16) Zur Bestimmung der Schädlichkeitsgrenzen siehe unten S.137 ff.

17) Vgl. O.V., Fahrendes Meslabor für Umweltdaten, in: Umwelt, 1971,6, S. 45 und Schütz, H. Umweltschutz-MeBwagen, Elnzelbericht L $3474 \mathrm{zu}$ Hartmann \& Braun-MeBwerte, 0.0. und J.. S.3.

18) Hinweise darauf, wann Messungen neuer Schadstoffe aufzunehmen sind, können bestimmte Indikatoren liefern. Als besonders billige und doch zuverlässige Indikatoren haben sich Pflanzen (besonders Flechten) und Tiere erwiesen, vgl. Hawksworth, D. L., Lichens as Litmus for Air Pollution: A Historical Review, 
sich allein auf die (möglichst kontinuierliche) Messung der Schadstoffe beschränken, die etwa eine bestimmte prozentuale Marge unterhalb der Schädlichkeitsgrenze uberschritten haben. Ist die schädlichkeitsgrenze fur einen luftbelastenden stoff z.B. $1 \mathrm{mg} / \mathrm{m}^{3}$ Luft, so kann man ihn dann in den MeBkatalog aufnehmen, wenn er bei elner Routinemessung - etwa mit dem mobilen MeBlabor - eine Konzentration von 0,01 oder 0,001 oder 0,0001 usw. $\mathrm{mg} / \mathrm{m}^{3}$ erreicht hat. Ob die sicherheitsmarge, ab der man eine laufende Messung vornehmen wird 100, 1000, 10000 oder gar noch mehr Prozent unter der Schädlichkeitsgrenze liegt, hängt von der Gefährlichkeit des stoffes und auch von den Kosten der Messung ab. Bei radioaktiven Substanzen z.B. wird man schon bei weit unter der Schädlichkeltsgrenze festgestellten Konzentrationen elne laufende Messung einfuhren. Was die Immissionsseite betrifft - und damit das MeBstellennetz - können wir resümieren, daB alle die Schadstoffe laufend (möglichst kontinuierlich) gemessen werden sollen, die eine, je nach Gefährlichkeit der Stoffe verschiedene Marge unterhalb der Schädlichke1tsgrenze überschritten haben. Das setzt natürlich voraus, daß die Schädlichkeitsgrenzen bekannt sind. Ist das nicht der Fall, sollte eine weitere prozentuale sicherheitsmarge für die Aufnahme in den Schadstoffkatalog des MeBstellennetzes eingefuhrt werden. Emissionsseitig sind zunächst auf jeden Fall die stoffe $z u$ messen, die auch immissionsseitig gemessen werden, anders wäre

in: International Journal of Environmental Studies, vol. 1, 1971, S. 281 ff.; Georgi1, H.-W.., Die lufthygienisch-meteorologische Modelluntersuchung im Untermaingebiet, in: Schultze, H. (Hrsg.), Umweltreport, a.a.0., S. 220; van Haut, H./Guderian, R., Pflanzen als Indikatoren der Luftverschmutzung, in: Probleme der Umweltforschung, Berlin 1973, S. 36 f.; Jürging, P., Flechten - Bioindikatoren der Luftverunreinigung?, in: Steubing, L./Kunze, C./Jäger (Hrsg.), Belastung und Belastbarkeit von Okosystemen. Tagungsbericht der Gesellschaft für రkologie, Giessen 1972, S. 145; Hahn, J./Aehnelt, E., Die Fruchtbarkeit der Tiere als biologischer Indikator für Umweltbelastungen, ebenda, S. $49 \mathrm{ff}$; Antweller, H., Tiere als Indikatoren der Luftverschmutzung, in: Probleme der Umweltforschung, a.a.0., S. $46 \mathrm{ff}$.; Meyl, A.H., Abwässer in Küstennähe, in: DFG-Mittellungen $2 / 74,5,28$. 
- wie bereits erwähnt - eine zurechnung der eventuell eintretenden Schäden auf die Verursacher kaum möglich. Elnige der immissionsseitig gemessenen Stoffe können allerdings emissionsseitig gar nicht gemessen werden, da sle erst durch chemische Reaktionen nach der Emission entstehen (z.B. Ozon). Es sind darüber hinaus aber auch all jene Emissionen $z u$ messen, die noch nicht in den Katalog der Immissionsseitig zu messenden Schadstoffe aufgenommen wurden, jedoch potentiell schădlich sind.

Nach diesen allgemeinen Vorbemerkungen wollen wir nun vorschlagen, welche Arten der Umweltbelastung in einem umweltstatistischen System, das dem Anspruch, Grundlage elner rationalen Umweltplanung $z u$ werden, genügt, berücksichtigt werden müBten. Wir sind uns der Willkür unseres Vorschlags bewußt. Mit gutem Grund können sicher weitere Schadstoffe zur Erfassung vorgeschlagen werden, mit wenlger gutem Grund allerdings die Streichung Irgendeines von uns vorgeschlagenen Schadstoffes. Wir stützen uns bei unserem Vorschlag auf die Kataloge der Umweltbelastungsarten vor allem im Umweltprogramm der Bundesregierung 19) dem ersten Gutachten des Rates von Sachverständigen für Umweltfragen (RSU) ${ }^{20}$ ) und der Reports des U.S. Council on Environmental Quality 21), aber auch auf eine Fulle spezieller Untersuchungen ${ }^{22}$ ) über die verschiedenen Arten der Umweltbelastung.

Als Einteilungskriterium wählen wir das jeweils belastete Um-

19) Vgl. Materialienband zum Umweltprogramm der Bundesregierung, a.a.o.

20) Vgl. RSU, Umweltgutachten 1974, a.a.0.

21) Seit 1970 erscheint jährlich ein Report, herausgegeben vom U.S.G.P.O., Washington, D.C. $1970 \mathrm{ff}$.

22) Diese speziellen Untersuchungen befassen sich entweder allgemein mit den verschledenen Arten der Umweltbelastung oder sle beziehen sich konkret auf bestimmte Länder, Lufträume und Gewässer. Jede Literaturauswahl - und nur eine solche käme bel der Fülle der Veröffentlichungen in Frage - wäre hier willkurlich, weshalb wir ganz darauf verzichten wollen. 
weltmedium, also die Luft, die Gewässer und den Boden 23) ferner die Pflanzen- und Tierwelt sowie die Kontamination der Lebensmittel; hinzu kommt der Lärm als ein Faktor, der den Menschen direkt schädigt, wenn sich auch der Schall der Luft als Transportmittel bedient.

bb) Arten der Luftbelastung

Für eine laufende (möglichst kontinuierliche) immissionsseitige Messung schlagen wir folgende luftbelastenden stoffe vor:

Stäube

Schwe feloxide $\left(\mathrm{SO}_{2}, \mathrm{SO}_{3}\right)$

Stickoxide

Kohlenmonoxid $\left(\mathrm{NO}_{2}, \mathrm{NO}_{3}\right)$

Chlorwasserstoff (CO)

Fluor und Fluorwasserstoff

Blei

$(F, F H)$

Kadmi um

Geruchss tof fe

Radioaktivität

( $\mathrm{H}_{2} \mathrm{~S}$, Merkaptane)

cc) Arten der Gewässerbelastung

Für den zustand der Gewässer spielen nicht nur Art und Konzentration der eingeleiteten Schadstoffe eine Rolle sondern auch die durch die Schadstoffe hervorgerufenen biologischen, chemischen und physikalischen Veränderungen. Deshalb ist es notwendig, neben der Konzentration der Schadstoffe auch den biologischen, chemischen und physikalischen zustand der Gewässer mit Hilfe bestimmter Kennziffern zu messen.

Die Schadstoffe kann man noch einmal unterteilen in primär ökologisch schädliche und primär gesundheitsschädliche stoffe.

23) Vgl. Hueting, R., The Set-Up of the Statistical system within which the Deterioration of the Human Environment will be Estimated, in: OECD (Hrsg.), Problems of Environmental Economics, Paris 1972, S. 99; Bartels, H., Statistik als Hilfsmittel der Umweltpolitik, in: Allgemeines Statistisches Archiv, Bd. 59, 1975, S. 12. 
Wir schlagen vor, folgende Schadstoffe bzw. Schadstoffgruppen laufend (mögllchst kontinuierlich) zu messen:

(1) Primär obkologisch schädliche stoffe

Phosphorverbindungen ( $u . a$. menschliche und tierische Exkremente, Detergentien, Düngemittel)

stickstoffverbindungen (u.a. Düngemittel und Abfälle)

Mineralo1 (produkte)

feste Abfälle (u.a. Schrott, Giftfässer)

(2) Primär gesundheitsschädliche stoffe

Verbindungen von Schwermetallen wie Quecksilber (Hg),

Ble1 $(\mathrm{Pb})$, Kadmium ( $\mathrm{Cd})$

Pestizide (u.a. chlorierte Kohlenwasserstoffe wie

DDT, Phosphorsaure wie E 605)

andere chlorlerte Kohlenwasserstoffe (z.B. PCB)

Aromatische Kohlenwasserstoffe (Benzpyren, Phenole)

Pathogene Mikroorganismen (Bakterien, Viren, Wurmeler)

Weiterhin schlagen wir vor, die folgenden den biologischen, chemischen und physikalischen zustand der Gewässer kennze1chnenden Größen $\mathrm{zu}$ messen:

Blochemischer Sauerstoffbedarf (BSB) 24)

Chemischer Sauerstoffbedarf (CSB) 25)

pH-Wert 26)

24) Der biochemische Sauerstoffbedarf ist "eine MeBzahl fur den Anteil fäulnisfähiger organischer Substanzen im Abwasser; meist als 5-tägiger biochemischer Sauerstoffbedarf, $\mathrm{BSB}_{5}$ ' angegeben; das ist die Menge Sauerstoff in $\mathrm{mg} / \mathrm{l}$, die die Bakterien benotigen, um die organische Substanz in 5 Tagen be1 $20^{\circ}$ C zu zersetzen (Sauerstoffzehrung)" (Herder-Lexikon Umwelt, Freiburg 1973, S. 40).

25) Der chemische Sauerstoffbedarf entspricht dem "Kallumdichromatverbrauch $\left(\mathrm{K}_{2} \mathrm{Cr}_{2} \mathrm{O}_{7}\right)$ als $\mathrm{MaB}$ für die Gesamtmenge oxydierbarer organischer Verbindungen; er liegt 1 mmer hoher als der BSB" (Herder-Lexikon Umwelt, a.a.0., S. 53).

26) Der pH-Wert "bezeichnet die Wasserstoffionenkonzentration als Maß für neutrale, saure oder alkalische Reaktionen von wăssrigen Lösungen: neutrales Wasser hat einen pH-Wert von 7 , saures kleiner als 7, alkalisches Wasser gröBer als 7 (bis 14)" (Umwelt - ABC, Wiesbaden 1972, S. 114). 
Temperatur

Trübung

Radioaktıvität

dd) Arten der Bodenbelastung

Die Arten der Bodenbelastung sind äuBerst heterogen. Elne scharfe Abgrenzung der verschiedenen Arten ist kaum möglich, da fast jede Art der Bodenbelastung Merkmale hat, nach denen man sie auch in andere Kategorien einteilen könnte. Wir wollen die Bodenbelastungsarten in zwel Hauptgruppen einteilen 27): (1) primär quantitative Bodenbelastungen und (2) primär qualitative Bodenbelastungen.

ad (1): Zu den primär quantitativen Bodenbelastungen zählen

(a) die Uberbauung des Bodens mit

(aa) siedlungen 28)

(aaa) Wohnsiedlungen

(bbb) Industrie- und Gewerbesiedlungen

(bb) Verkehrsbauten

(aaa) Straßen, Parkplätze

(bbb) Flugplätze

(ccc) Gle1skörper

(cc) Freizelt- und Erholungsstätten

(b) Eingriffe in die Bodensubstanz

(aa) Abbau von Bodenschätzen

(bb) Erosion

ad (2): $\mathrm{Zu}$ den primär qualitativen Bodenbelastungen gehören

(a) Eingriffe in die Bodenfruchtbarkeit durch Umwelt-

27) Ähnliche Einteilung bei Olschowy, G./Mrass, W./Kullmer, H.J./Bürger, K., Zur Belastung der Landschaft, in: Schriftenreihe für Landespflege und Naturschutz, Heft 4, Bonn-Bad Godesberg, 1969, S. 16.

28) Eine Belastung des Bodens stellt hier nicht nur die Uberbauung als solche dar (volumenmäBige Belastung); hinzu kommt noch eine strukturelle Belastung durch eine "Bebauung am falschen Ort" (Zersiedlung, Bebauung landschaftlich reizvoller Geblete und ähnliches); vgl. dazu Olschowy, G., Zersiedlung der freien Landschaft, in: Olschowy, G. (Hrsg.), Belastete Landschaft - gefährdete Umwelt, München 1971, S. 327. 
chemikalien und 81

(aa) Düngemittel

(aaa) organische Dünger

(bbb) anorganische Dünger (Stickstoff- und Phosphorverbindungen, Kalium, Calzium Magnesium, u.a.)

(bb) Pestizide (Herbizide, Fungizide, Insektizide) (cc) 81

(b) die Deponie von Abfällen 29)

(aa) Hausmüll- und hausmüllähnliche Abfälle

(bb) Industriemüll

(cc) Abfalle aus der Viehhaltung und Schlachtung

(dd) Verpackungsmaterial (Papier, Kunststoffe)

(ee) Abfälle aus dem medizinischen Berelch

(ff) Inertmaterial (Bauschutt u.ä.)

(gg) Autowracks und Altrelfen

(hh) Klärschlamm

(1i) Radioaktive Abfälle

Manche der Bodenbelastungsarten wie die Uberbauung des Bodens, der Abbau von Bodenschätzen und die Verwendung von Umweltchemikalien wie Düngemittel und Pestizide haben einen ganz anderen Charakter als die anderen Arten der Umweltbelastung. Die Schadstoffe, welche Luft und Gewässer belasten, der Abfall und der

29) Eine Systematisierung der Abfallarten ist wohl eine der schwierigsten Vorarbeiten für eine Abfallstatistik (vgl. Baltes, H./Nowak, W., Umweltstatistik - ein Instrument der Umweltplanung, a.a.O., S. 242 und Bartels, H., Statistik als Hilfsmittel der Umweltpolitik, a.a.o., s. 17 f.); uber weitere Klassifizierungsversuche vgl. U.S. Council on Environmental Quality, Environmental Quality, The First Annual Report, Washington, D.C., 1970, S. $107 \mathrm{ff.;}$ Brennpunkt Müllproblem, hrsg. vom Presse- und Informationsamt der Bundesreglerung in Zusammenarbelt mit dem Bundesministerium für Gesundheitswesen, Bamberg 1968; Müller, H. -J.., Stadtreinigung im Rahmen des Umweltschutzes, in: Der stätetag, 1971, 7, S. 405; 0lschowy, G., Bilanz des Abfallproblems, in: Olschowy, G. (Hrsg.), Belastete Landschaft gefährdete Umwelt, a.a.o., S. $217 \mathrm{ff.;} \mathrm{Höfken,} \mathrm{F.,} \mathrm{Pro-}$ bleme der Abfallbehandlung, ebenda, S. $224 \mathrm{ff}$. 
Lärm sind - wenn wir von ihrer mutwilligen Erzeugung absehen ungewollte Nebenprodukte, die bei der Produktion oder dem Konsum anfallen. Der Bau von Siedlungen, Verkehrsanlagen, Freizeitund Erholungsstätten, der Abbau von Bodenschätzen, die Verwendung von Pestiziden und Düngemitteln sind dagegen bewußte Maßnahmen von z.T. lebenswichtiger Bedeutung. Ohne Düngemittel und Pestizide wäre der Hunger in der Welt noch größer. Ohne den Bau von Wohnungen wäre kein menschenwürdiges Leben denkbar. Ohne den Abbau von Bodenschätzen, den Bau von Industrie- und Verkehrsanlagen wäre der wohlstand (ausgedrückt durch das reale Bruttosozialprodukt) pro kopf der Bevölkerung viel geringer. Diese Arten der Umweltbelastung haben also einen besonders ambivalenten Charakter, da sie es selbst sind, die auch einen Nutzen stiften, während es bel den als Nebenprodukte bezeichneten Abgasen, Abwissern und Abfällen die jeweiligen Kuppelprodukte sind, welche den Nutzen stiften.

ee) Arten der Pflanzen- und Tierbelastung

Die Belastungen der Pflanzen- und Tierwelt kann man in indirekte und direkte Belastungen einteilen.

$\mathrm{Zu}$ den indirekten Belastungen gehören die Belastungen, die via Kontakt der Pflanzen und Tiere mit den belasteten Umweltmedien (Luft, Wasser, Boden) erfolgen. Insofern sind dies bereits (ökologische) Wirkungen der verschiedenen Belastungen der Umweltmedien und werden weiter unten behandelt 30 )

Direkte Belastungen der Pflanzen- und Tierwelt sind dagegen solche, die gezielt auf eine Vernichtung ausgerichtet sind, wie die Abholzung von Wäldern, die Jagd nach Tieren und die Verwendung von Bioziden (u.a. Insektizide, Herbizide, Fungizide), die durch Vernichtung bestimmter Pflanzen- und Tierarten andere Pflanzen- und Tierarten schützen sollen.

30) Siehe unten S. $61 \mathrm{ff}$. 


\section{ff) Arten der Lebensmittelbelastung}

Wir wollen unterscheiden zwischen einer indirekten und einer direkten Belastung der Lebensmittel. Indirekt werden die Lebensmittel dadurch belastet, daB die "Rohprodukte" Pflanzen und Tiere - wie oben geschildert - durch den Umweltkontakt Schadstoffe aufnehmen, die bis zu ihrer Verarbeitung als Nahrungsmittel nicht vollständig abgebaut werden (Rückstände). Insoweit gehört die Behandlung der Lebensmittelbelastung in das Kapitel über die (gesundheitsschädlichen) Wirkungen der Belastung der verschiedenen Umweltmedien, pflanzen und Tiere Neben dieser unbeabsichtigten Kontamination der Lebensmittel durch nicht oder nur schwer abbaubare stoffe wie Insektizide (u.a. DDT, Aldrin, Dieldin, Lindan) Herbizide, Fungizide, Schwermetalle (u.a. Blei, Kadmium, Quecksilber), künstliche Radionuklide (vor allem Caesium 137, Strontium 90 und Jod 131 letzteres ist allerdings relativ schnell abbaubar -), speziellen Produkten wie Salmonellenkeimen, $\mathrm{PCB}$ und $\mathrm{HCB} 32$ ) und Rückständen aus Düngemitteln (vor allem stickstoffverbindungen) werden Nahrungsmittel auch bewuBt mit Fremdstoffen belastet, und zwar in Form von zusatzstoffen - in erster Linie zwecks Konservierung (vor allem Nitrite und Nitrate) - und Zusätzen zum Tierfutter (Antibiotika, hormonwirksame Verbindungen, Psychopharmaka) zwecks Verbesserung der Fleischqualität der geschlachteten Tiere 33)

\section{gg) Arten des Lärms}

Lärm wird allgemein als Schall, der von Menschen als störend oder belästigend empfunden wird, definiert ${ }^{34}$ ). Die Einteilung der Lärmarten erfolgt in der Regel nach den verursachenden Schallquellenbereichen 35 ?

31) Siehe unten S. $88 \mathrm{ff}$.

32) Vgl. Deutsche Gesellschaft für Ernährung e.V., Ernährungsbericht 1972, Frankfurt/M., 1973, S. $130 \mathrm{ff.}$

33) Vgl. RSU, Umweltgutachten $1974, \mathrm{a} . \mathrm{a.0.,} \mathrm{S.} 66 \mathrm{f}$.

34) Vgl. ebenda, S. 77.

35) Vgl. ebenda, S. $83 \mathrm{ff}$. 
Man untersche1det:

(1) Industrielărm

(2) Baulärm

(3) Verkehrslärm (Kfz, Schlene, Flugzeuge, Schiffe)

(4) Wohnlärm

d) Die katastermäbige Erfassung der umweltbelastenden Schadstoffe aa) Zur Aufstellung von Immissionskatastern

(1) Aufgaben der Immissionskataster

Wenn feststeht, welche Arten der Umweltbelastung in welchen Zeitabständen und an welchen Orten gemessen werden sollen und die notwendigen MeBvorrichtungen installiert sind, kann man den Umfang der Umweltbelastung in sogenannten Immissionskatastern tabellarisch 36) oder kartographisch fur jeden Okofaktor, die Lebensmittel und den Lärm getrennt darstellen. Je dichter das Meßstellennetz 1st und je hăufiger gemessen wird, um so luckenloser sind die Informationen uber die Belastung des betreffenden Umweltmediums. Die Kenntnis der răumlichen Vertellung der Immissionskonzentrationen ist Voraussetzung fur die Ermittlung der Wirkungen der verschiedenen Arten der Umweltbelastung und damit auch für deren Bewertung.

\section{(2) Das Immissionskataster "Luft"}

(a) Die Luftbelastungsmatrix

Die katastermăbige Erfassung der Luftbelastung erfolgt auforund der Messungen der Schadstoffkonzentrationen in den verschiedenen Stationen des MeBstellennetzes. Bei $m$ MeBstellen $j$ und $n$ Schadstoffen 1 kann man in einer $m x n$ - Matrix die Luftbelastung des gesamten Mesgebletes tabellarisch sehr übersichtlich darstellen. Würde man für ein Geblet eine Maschenwelte des Luftmeßstellen-

36) Solche Umweltbelastungsmatrizen haben in der L1teratur ganz unterschiedliche Namen. Sie helBen z.B. 8kologische InputOutput-Tabellen (vgl. Anno 709 p.R., Schlußberlcht der Prospektivkonferenz der Neuen Helvetischen Geselischaft, Aarau und Frankfurt/M. 1973, S. 270 f.) oder Umweltbilanzen (vgl. Häfele, W., Stoffbilanzen, in: Zur Problematik des Verursacherprinzips, Berlin 1972, S. 23). 
netzes von $r \mathrm{~km}$ wählen, so ergäbe sich bel elner Gesamtfläche des Gebietes von $s \mathrm{~km}^{2}$ für $\mathrm{m}=\frac{\mathrm{s}}{\mathrm{r}^{2}}$ 37) Für die Bundesrepublik Deutschland mit einer Fläche von $\frac{r^{2}}{\text { rund }} 250000 \mathrm{~km}^{2}$ ergăbe sich bei einer Maschenwelte von $50 \mathrm{~km}(25 \mathrm{~km})$ eine Anzahl von 100 (400) MeBstationen 38)

Die Luftbelastungsmatrix hätte allgemein folgendes Aussehen:

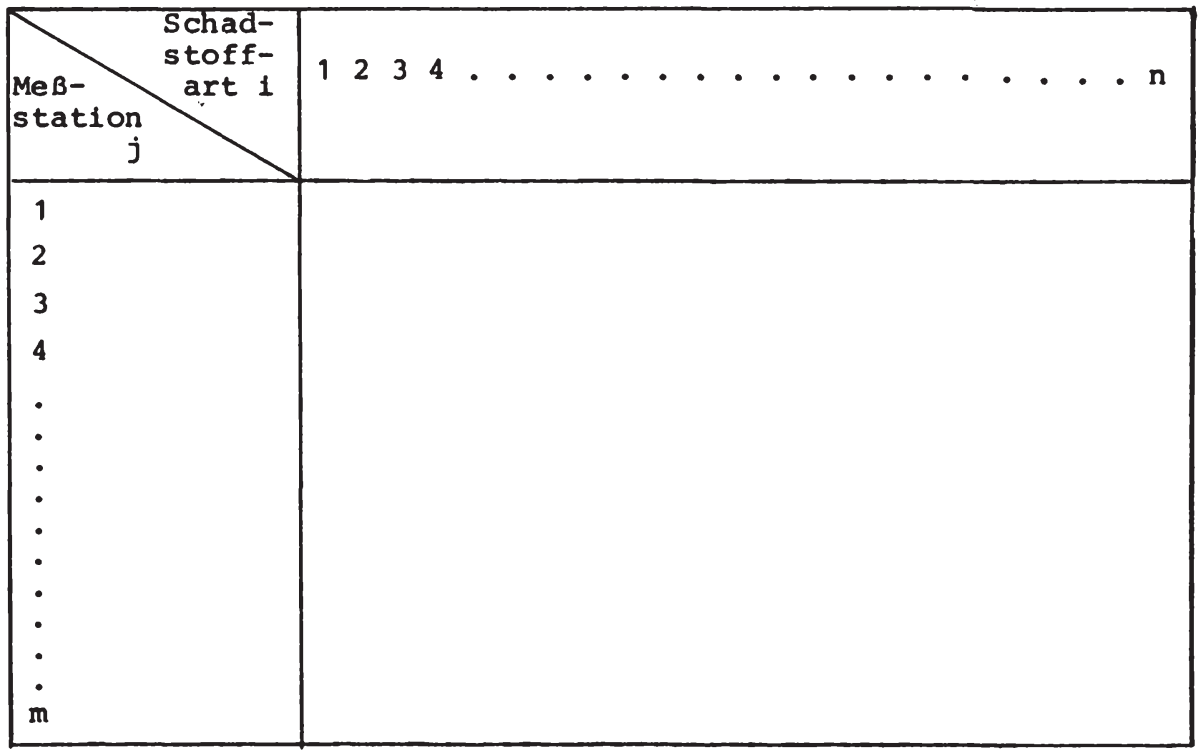

$\mathrm{Abb} \cdot 3$

37) Die Niederlande haben ein MeBstellennetz für die Luftbelastungsmessung geplant, das im Endausbau eine Maschenweite von $28 \mathrm{~km}$ haben soll (vgl. Sandscheper, G. , Luftüberwachungsnetz für die Niederlande, in: Umwelt, 1971, 6, S. 35).

38) In der Bundesrepublik Deutschland haben die Bundesländer mit dem Bau eigener MeBstellennetze begonnen. In Bayern sollen im Endausbau 85 feste automatisierte Luftmeßstationen kontinuierlich die Luftbelastung messen (vgl. o.V., Initiative für Bayerns Luft, in: $U, 1973,5, S .46)$. In Baden-Württemberg baut siemens ein automatisches kontinuierlich messendes Netz von 14 Stationen. Die Immissionen werden zusammen mit den Wetterdaten gemessen, um so Rückschlüsse auf die Emittenten zu ermöglichen (vgl. Siemens AG., Forschung und Entwicklung, München $0 . \mathrm{J}$.$) . Auch in den anderen Bundesländern 1st$ der Bau oder zumindest die Planung von Meßstellennetzen be- 
Die Messung der Schadstoffkonzentrationen in der Luft erfolgt am besten in der heute ublichen Dimension $\mathrm{mg} / \mathrm{m}^{3}$ oder bei sehr $\mathrm{klei}$ nen Konzentrationen in $\mu \mathrm{g} / \mathrm{m}^{3}$.

\section{(b) Luftbelastungskarten}

Recht anschaulich kann man die Luftbelastung auch kartographisch darstellen. Jedem Schadstoff kann man eine bestimmte Farbe (z..B. $\mathrm{SO}_{2}$ rot, $\mathrm{Co}$ blau, usw.) und jeder schadstoffkonzentration eine andere Tönung ( $\mathrm{z}$. B. wei $B$, hell-, mittel- und dunkelrot für unbedeutende, niedrige, mittlere und hohe $\mathrm{SO}_{2}$-Konzentrationen bzw. weiB, hell-, mittel- und dunkelblau für unbedeutende, niedriqe; mittlere und hohe Co-Konzentrationen) zuordnen. In einer $\mathrm{SO}_{2}-\mathrm{Be}-$ lastungskarte erhält nun jedes Maschenquadrat jene Farbtönung, die der dort gemessenen $\mathrm{SO}_{2}$-Konzentration entspricht. Einen recht guten, wenn auch nicht so genau wie in der Matrix quantifizierten Uberblick uber die gesamte Luftbelastung erhält man, wenn man die Luftbelastungskarten für alle Schadstoffe auf durchsichtiges Material druckt und dann übereinanderlegt. Die bei Durchsicht oder Durchleuchtung hellen stellen sind die reineren Gebiete; je undurchsichtiger die Maschenquadrate sind um so belasteter ist dagegen die Luft in diesem Gebiet. Kartographisch kann man die verschiedenen Konzentrationen eines Schadstoffes auch mit Hilfe von Isobelastungslinien darstellen (vgl. Abbildung 4) 39?

reits weit fortgeschritten. Leider besteht die Gefahr der mangelnden Koordination, denn nicht nur die Standorte müssen aufeinander abgestimmt werden, sondern auch die Meßgeräte, da die MeBergebnisse bei unterschiedlichen Geräten zum Teil stark differieren (vgl. Jessel, U., Seven Years Experience in Air Pollution Monitoring in Germany, in: DFG-Mitteilungen, 2/74, S. 43). Man kann nur hoffen, daB die Verwaltungsvorschriften uber Meßobjekte, MeBverfahren und-geräte, bei der $\mathrm{Zahl}$ und Lage der Meßstelien $\mathrm{zu}$ beachtende Grundsätze und die Auswertung der MeBergebnisse, die der Bundesminister des Innern zur einheitlichen Beurteilung der Meßdaten nach $\S 45$ BImSchG erlassen kann, nicht zu spät kommen.

39) Vgl. Günther, U. u.a., Umweltschutz-Informations- und steuerungssystem, a.a.0.. S. 36 ; Birkle, M. u.a., Informationssystem Umwelt, a.a.0., s. 69 . 


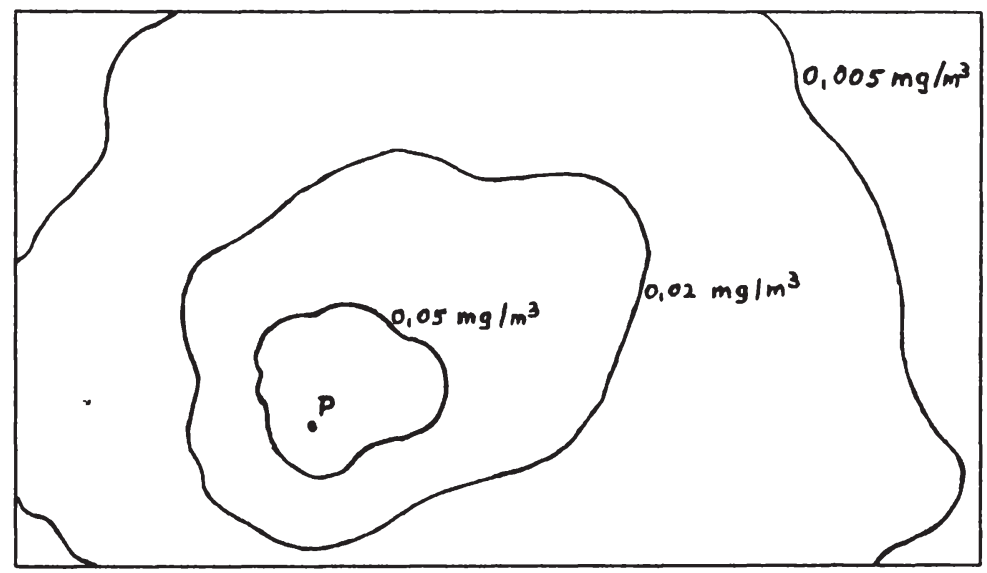

Abb. 4

In Abbildung 4 sind Isobelastungsinien für $\mathrm{SO}_{2}$ angegeben. Sie bezeichnen die Verteilung unterschiedlicher Immissionskonzentrationen, die durch die $\mathrm{SO}_{2}$-Emission aus einer Punktquelle $\mathrm{P}$ verursacht wurden.

\section{(c) Verfeinerungen des Immissionskatasters "Luft"}

Das bisher dargestellte Immissionskataster beruht auf einem sehr starren Meßstellennetz. Dies hat den Vorteil, daß auch belastungsarme Gebiete, der sogenannte Background ${ }^{40}$ ) bel der Messung berulcksichtigt werden; es hat allerdings den Nachteil, daB in stark belasteten Gebleten unter Umständen nicht genügend Messungen vorgenommen werden. Will man die Gesamtzahl der Meß-

40) Die Messung in den Reinluftgebieten, dem sogenannten Background, ist deshalb besonders wichtig, weil auch niedrige Konzentrationen von luftbelastenden Schadstoffen langfristia schädlıch werden können (Langzeltwirkung, schleichende Gefahrl: vgl. dazu de Haar, U., Das MeBstellenprojekt "Reinhaltung der Luft", in: Umschau in Technik und Wissenschaft, 1970, Heft 23, S. 746; Rönicke, G., Messung der Luftverunreinigung im Rahmen eines Netzes von Backgroundstationen, in: Olschowy, G., Belastete Landschaft - gefährdete Umwelt, a.a. O., S. 107; Erikson, E., The Importance of Investigating Global Background Pollution, in: Meteorological Aspects of Air Pollution, WMO-Technical Note No. 106, Genf 1970, S. 32. 
stationen unverändert lassen, kann man in solchen Fällen, die Background-Stationen zugunsten zusätzllcher MeBstationen in stark belasteten Gebleten verringern. Will man auf kelne Backgroundstationen verzichten, kann man zusätzliche Messungen in den Belastungsgebieten nur durch Einrichtung weiterer MeBstationen (eventuell auch mobiler Art) vornehmen.

Bisher haben wir noch nichts uber den Standort der Messtationen innerhalb der "Maschenquadrate" des Meßstellennetzes gesagt. EIne starre Lösung wăre es, wenn die MeBstationen genau in der Mitte des jeweiligen Maschenquadrates errichtet würden. Dies ist in vielen Fällen aus den verschiedensten Grüden sicher nicht möglich, darüber hinaus aber auch gar nicht empfehlenswert. Der beste standort innerhalb der Maschenquadrate wäre dort, wo die Belastung der Luft im allgemeinen am grobten 1st. Diesen Standort könnte man z.B. mit einer mobilen MeBstation ermitteln. Doch stellen sich auch dem meist Schwierigkeiten entgegen. Dann sind Neherungslösungen zu suchen. Es muB auch darauf geachtet werden, daB sich benachbarte MeBstationen nicht zu nahe rucken, etwa well die gröBte Luftbelastung gerade auf der Grenze zwischen den beiden Meßstellengebieten besteht. Allgemein kann man sagen, daB die standortwahl der MeBstationen nur so varilert werden soll, daB die Vollständigkelt und Vergleichbarkeit der MeBdaten erhalten bleibt. Der standort der MeBstationen sollte sich in erster Linie nach sachlichen Gesichtspunkten richten und weniger nach Verwaltungs- oder Ländergrenzen 41)

\section{(3) Das Immissionskataster "Gewässer"}

(a) Einteilung der Gewässerarten

Im Gegensatz zur Luft, die sich uber dem gessamten Land befindet, sind Gewässer an bestimmte Grenzen gebunden. Fur die katastermäBige Erfassung der Gewässerbelastung empfiehlt sich daher zunächst eine Einteilung der Gewässerarten.

Wir wollen folgende Elntellung vornehmen:

41) Vgl. Birkle, M. u.a., Informationssystem Umwelt, a.a.o., S. 24 . 
(1) Oberflächengewässer

(a) Meer

(aa) offenes Meer

(bb) Küstengewăsser

(b) Seen

(c) Talsperren

(d) Flüsse und Kanäle

(2) Grundwasser

Jedes dieser Gewässer wirft spezifische MeBprobleme auf, nicht zuletzt in bezug auf den Standort der MeBstationen. Soll auf der Wasseroberfläche oder am Gewässergrund ${ }^{42}$, am Ufer oder auf dem offenen Gewässer die Belastung gemessen werden? Entscheidungen darliber sind ebenso wichtig wie daruber, ob kontinuierlich oder nur in bestimmten oder unbestimmten zeltabständen der $\mathrm{Z}$ ustand der Gewässer gemessen werden soll.

AuBerdem ist es nur sinnvoll, sich auf ganz bestimmte Gewässer der jeweiligen Gewässerart $z u$ beziehen (die Nordsee, die Ostseeküste, den Bodensee, die Edertalsperre, den Rhein, den RheinHerne-Kanal); es 1st mit anderen Worten ein Immissionskataster wiederum in Form einer Matrix oder einer Karte für jedes konkrete Gewässer aufzustellen. Das MeBgeblet wird durch die Ufer der Gewässer bestimmt. Die MeBstationen sollten moglichst dicht und gleichmäBig vertelit sein, so dab sie die Gewässerqualität umfassend widergeben ${ }^{43}$ ?

42) Der $\mathrm{BSB}_{5}$ sollte z.B. mögllchst auf dem Gewässergrund gemessen werden, da dort der Sauerstoffverbrauch am grobsten ist, also das "Umkippen" beginnt.

43) Die Fa. Siemens AG baut in Belgien ein MeBstellennetz für die Gewässer, das Im Endausbau 240 MeBstationen umfassen soll: vgl. Siemens AG, Forschung und Entwicklung, a.a.0., S. 41. In den USA gibt es rund 900 dem Bund unterstehende MeBstationen sowie rund 1000 Langze1t- und 5000 Kurzze1tMeBstationen der Bundesstaaten (vgl. U.S. Environmental Protection Agency, Research and Monitoring, a.a.0.. S. 15). In der Bundesrepublik Deutschland sind die Länder dabel, ihr Gewässer-Meßstellennetz auszubauen (vgl. O.V.,Gewăsserüberwachung in Hessen, in: U, 1973, 2, S. 26). Die Gesamtsituation eines Gewăssers kann besonders gut beurtelit werden, wenn die stationären Messungen durch eine Uberwachung aus 
(b) Gewässerbelastungsmatrix

Die Belastungsmatrix für ein Gewässer, z.B. den Rhein, enthält nicht nur die Schadstoffe, sondern auch die Daten, die den biologischen, chemischen und physikalischen zustand widergeben.

Bel $p$ Mebstationen am Rhein und $q$ zu messenden Schadstoffen und $s$ zu messenden Eigenschaften erhält man folgende Matrix:

\begin{tabular}{|c|c|c|c|c|c|}
\hline \multirow[b]{2}{*}{$\begin{array}{l}\text { MeB- } \\
\text { station }\end{array}$} & \multirow{2}{*}{\multicolumn{2}{|c|}{ Schads toffe }} & \multicolumn{3}{|c|}{ Eigenschaften } \\
\hline & & & $\begin{array}{l}\text { biolog. } \\
123\end{array}$ & $\begin{array}{lll}\text { chem. } & \\
4 & 5 & 6\end{array}$ & $\begin{array}{l}\text { physik. } \\
\begin{array}{lllll}7 & 8 & 9 & \ldots\end{array}\end{array}$ \\
\hline $\begin{array}{l}1 \\
2 \\
3 \\
4 \\
5 \\
\cdot \\
\cdot \\
\cdot \\
\cdot \\
\cdot \\
\cdot \\
\cdot \\
\end{array}$ & & & & & \\
\hline
\end{tabular}

Abb. 5

(c) Gewässerbelastungskarten

Für jedes Gewässer kann auch elne Belastungskarte angefertigt werden 44 ) Sie kann wieder in verschiedenen Farbtonen die Be-

der Luft (aus Flugzeugen oder Satelliten) ergänzt werden (vgl. Rokosch, P., Mit Flugzeug und Kamera, in: U, 1972, $5,5.34)$.

44) Ein relativ kostengüstiges Verfahren zur Herstellung solcher Gewässergütekarten ist die Fotographie der Gewässer aus Satelliten oder Flugzeugen (vgl. Lichtwer, L./Schaude, G.R.. Nutzen der Fernerkundung der Erde, a.a.0., S. 41). 
lastungsgrade des Gewässers durch einen Schadstoff darstellen oder aber bestimmte Elgenschaften blologischer, chemischer oder physikalischer Natur. Man kann auch zunächst auf Grund der Gewässerbelastung durch Schadstoffe und die dadurch bedingten Anderungen der Qualität des Gewässers Güteklassen bilden und jeder Güteklasse eine Farbe zuordnen ${ }^{45}$ ?

\section{(4) Das Immissionskataster "Boden"}

Bel manchen Arten der Bodenbelastung fallen Emission und Immission zusammen, da kein Transport der "Schadstoffe" im weitesten Sinne stattfindet. Dies gilt besonders für die primär quantitat1ve Bodenbelastung durch Uberbauung und Eingriffe in die Bodensubstanz. Bel Umweltchemikalien und Abfall kann man dagegen durchaus zwischen Emission und Immission unterscheiden.

(1) Die Erfassung der Flächennutzung

Die primär quantitative Belastung des Bodens kann durch elne statistik der Flächennutzung erfabt werden 46). Sie soll in bestimmten Zeitabständen daruber Auskunft geben, wie der gesamte Boden eines Landes genutzt wird: zur Bebauung, land- und forstwirtschaftlichen Nutzung, Abbau von Bodenschätzen, Freize1t und Erholung oder gar nicht (Sozlalbrache). Die Darstellung kann in Tabellenform oder - übersichtlicher - kartographisch erfolgen. Elne solche Flächennutzungskarte kann wertvolle Hinweise darauf geben, wo bestimmte für die Bodennutzung typische Arten der Belastung der Okosysteme, der Lebensmittel sowie durch Lärm zu erwarten sind 47)

(2) Das Immissionskataster "Umweltchemikalien und 81 "

Die Bodenbelastung durch Umweltchemikalien und 81 kann nur durch die Analyse von Bodenproben ermittelt werden. Hieruber ein einigermaßen voliständiges Bild $z u$ gewinnen, 1st äuBerst aufwendig. Eine Beschränkung der Bodenanalysen auf die landwirtschaft-

45) Vgl. RSU, Umweltprobleme des Rheins, Mainz u.a. 1976, Kartenbeilagen.

46) Eine solche Erfassung wird bereits vom Statistischen Bundesamt durchgefuhrt; vgl. dazu die Statistischen Jahrbulcher.

47) Insofern kann die Flächennutzungskarte als Grundlage für ein Emissionskataster dienen. 
liche Nutzfläche scheint allerdings vertretbar ${ }^{48}$ ) Die Bodenproben sind möglichst an den am stärksten mit Umweltchemikalien belasteten Stellen der landwirtschaftlichen Nutzfläche in bestimmten Zeltabständen (etwa vor dem Säen oder Pflanzen und kurz vor der Ernte) vorzunehmen 49) Die Erfassung kann wiederum in einer Matrix und mit Hilfe von Karten erfolgen.

(3) Das Immissionskataster "Abfall"

Art und Menge (in Gewichts- und Volumeneinheiten) des Abfalls sowie dessen Verbleib werden im Immissionskataster "Abfall" erfaBt. Es ist anzustreben, daB die endgultige Ablagerung des $A b-$ falls nur noch in geordneten Deponien erfolgt ${ }^{50)}$; solange dies nicht der Fall ist, mulssen auch die wilden Deponien erfast werden 51). Die Immissionsmessung erfolgt in den Deponien. In den geordneten Deponien wird genau Buch geführt über Art und Menge des Abfalls 52) In den wilden Deponien müssen spezielle Untersuchungen der Abfallzusammensetzung erfolgen. Da die verschiedenen Abfallarten ganz unterschiedlich behandelt und gelagert werden müssen, empfiehlt es sich, Deponien für spezielle Abfallarten elnzurichten, also etwa jewells Deponien für Industrie-

49) In den USA z.B. wird jährlich in 3000 stationen der Pestizldgehalt des Bodens gemessen (vgl. U.S. Environmental Protection Agency, Research and Monitoring, a.a.0., s. 15).

50) Wenn dieses ziel erreicht ist, kann man den Abfall in die Kategorle der primär quantitativen Bodenbelastung einordnen, denn eine geordnete Deponie sollte kelne qualitativen Auswirkungen auf den Boden (und das Grundwasser) haben.

51) Vgl. U.S. Environmental Protection Agency, Research and Monitoring, a.a.0., S. 16 .

52) Dies wird insbesondere bel giftigen Abfällen durch die Bestimmung des $\S 11$ AbfBG in Verbindung mit der Abfallnachweisverordnung erleichtert. Nach $\S 11$ (3) AbfBG kann von Unternehmen die Fuhrung von Nachweisbüchern verlangt werden, in denen Art und Umfang giftiger Abfalle sowie deren Beseltigung erfabt sein mulssen. Der Transport solcher Abfälle wird mit Hilfe von Begleitscheinen ubberwacht, die vom Empfänger der Abfälle in der Deponie zu quittieren sind (vgl. Bulletin der Bundesreglerung Nr. 94, v. 13.8.1974, S. 974 und Frey, P. , Giftmull-Produzenten werden erfaBt, in: Umwelt, 1974, 1, S. 20). Die emissionsseitige Erfassung der Abfälle in Verbindung mit dem Begleitscheinverfahren ermöglicht also auch die immissionsseitige Feststellung (den Verbleib) von Abfallart und -menge. 
müll ("Sondermüll"), Hausmüll und radioaktive Abfälle. Dies gewahrleistet nicht nur eher eine sachkundige Behandlung des Abfalls, es erlelchtert auch die Erfassung.

Die Deponien und die in thnen gelagerten Abfallarten und -mengen können sowohl tabellarisch als auch kartographisch erfast werden.

(5) Die katastermäßige Erfassung der Pflanzen- und Tierweltbelastung

Die indirekten Arten der Pflanzen- und Tierweltbelastung müssen in den Wirkungskatastern für das jeweilige Umweltmedium erfaßt werden 53) während die verschiedenen direkten Arten tabellarisch (2.B. Zählung des Tierbestandes) oder kartographisch (z.B. in Form von Vegetationskarten) erfabt werden können.

(6) Die katastermäßige Erfassung der Lebensmittelbelastung Für die katastermäBlge Erfassung der Lebensmittelbelastung empfiehlt sich zunächst elne Eintellung der Lebensmittelarten. Man kann unterteilen in 54)

(A) Lebensmittel tierischer Herkunft

(1) Fleisch

(a) von Säugetieren

(aa) Haustieren

(bb) Wildtieren

(b) von Geflügel

(aa) Hausgeflügel

(bb) Wildgeflügel

(c) von Fischen und anderen Wassertieren

(a) aus Binnengewässern

(bb) aus dem Meer

(2) andere Tierprodukte

(a) Milch

53) Siehe unten S. $61 \mathrm{ff}$.

54) Zusammengestellt nach: Deutsche Gesellschaft fur Ernährung e.V., Ernährungsbericht 1972, Frankfurt/M. 1973, S. $129 \mathrm{ff}$. 

(b) Fette
(c) Eier
(d) Honig

(B) Lebensmittel pflanzlicher Herkunft

(1) Getreide und Getreideprodukte

(2) Obst und Obstprodukte

(3) Gemüse und Gemuseprodukte

In einer Matrix können diese Lebensmittelarten - eventuell nach weiteren Untergliederungen - mit den verschiedenen Arten der Schadstoffrilckstände und den Nahrungsmitteln absichtlich beigegebenen Stoffen kombiniert dargestellt werden.

\section{(7) Das Immissionskataster "Lärm"}

Lärm schädigt außer der Tierwelt keinen bkofaktor, sondern führt direkt $z u$ einer Schädigung der menschlichen Gesundhe1t; deshalb empiehlt sich eine Messung des Lärms vor allem dort, wo der Mensch in erster Linie von ihm belastet werden kann, also in den Arbelts-, Wohn-, Freizeit- und Erholungsgebieten. Die Messungen sollten möglichst kontinuierlich erfolgen. Das MeBstellennetz sollte vor allem in Ballungsgebieten möglichst dicht sein, da die Lärmquellen sehr zahlreich sind, ihre Wirkung jedoch je nach Siedlungsdichte und -höhe auf einen mehr oder weniger kleinen Raum beschränkt ist.

Die Darstellung der Lärmbelastung kann wiederum tabellarisch oder kartographisch ${ }^{55}$ ) erfolgen.

(8) Die Gesamtbelastung der Umwelt

Im Rahmen der Suche nach einem neuen Wohlstandsindikator, der das Bruttosozialprodukt als MaB für den Wohlstand eines Landes ablosen und die sogenannte Lebensqualitat zum Ausdruck bringen

55) Vgl. Dreyhaupt, F.J., Die modellmäBige Ermittlung und Darstellung der Lärmverteilung in Stadtstrukturen, in: Deutscher Arbeltsring für Lärmbekämpfung e.v. (Hrsg.), Verkehrslärmtagung in Bonn-Bad Godesberg vom 19./20. April 1971, S. 13. Die Darstellung in den Lärmkarten kann durch Linien glelcher Schallpegel z.B. in $5 \mathrm{~dB}(\mathrm{~A})$-Abstufungen erfolgen (vgl. RSU, Umweltgutachten 1974, a.a.0., S. 96). 
soll, versucht man, einen Gesamtindikator (bzw. Totalindex) für die Umweltqualităt zu finden 56 ?

Man könnte daher so vorgehen, das man die mengenmäBige Belastung der verschiedenen Umweltmedien, der Pflanzen- und Tierwelt, der Lebensmittel sowle den Lärm in elner Region durch ziffern kennzeichnet, die den Charakter informativer Indikatoren 57) haben.

Das Hauptproblem bel der Erfassung der Gesamtbelastung ist die Aggregation der einzelnen Indikatoren $z u$ einem Umweltindikator - vor allem wegen einer adăquaten Gewichtung der einzelnen Indikatoren 58). Trotz der groben Anstrengungen bei der suche nach einem Umweltindikator möchten wir bezweifeln, daB es in absehbarer Zeit gelingt, elnen aussagekräftigen Indikator fur die gesamte Umweltqualităt zu finden 59! Das Beispiel der USA, wo fast jede gröBere stadt einen elgenen Luftgütelndex (AIr Pollution Index) hat, der in einer Kennziffer oder verbalen Bezelchnung täglich veroffentlicht wird und Auskunft liber die

56) Vgl. Cazes, B., Environmental Quality Indicators and Social Indicators, in: OECD, Problems of Environmental Economics, a.a.0., S. 80 f.; Krengel, R., Die Messung der Umweltverschmutzung - ein neuer Wohlstandsindikator, in: Gumpel, W./ Keese, D. (Hrsg.), Probleme des Industrialismus in Ost und West, Festschrift für Hans Raupach, München 1973, S. 436; von Borries, D.F.W., zur Konstruktion von Umweltindizes, in: Allgemeines Statistisches Archiv, Bd. 59, 1975, S. 47; RSU, Umweltgutachten 1974, a.a.0., S. 219.

57) Vgl. Lelpert, C., Soziale Indikatoren, Uberblick úber den Stand der Diskussion, in: Konjunkturpolitik, 19. Jg., 1973, S. 221 .

58) Vgl. ebenda, S. 230; RSU, Umweltgutachten 1974, a.a.0., S. 204; Hansmeyer, K. $-H .$, Anforderungen der Umweltpolitik an die amtliche Statistik, in: Allgemeines statistisches Archiv, Bd. 59, 1975, S. 9; Scherrer, H.U., Gesamtbewertung der Umweltbelastung, in: Neue Züricher Zeltung (NZZ), Fernausgabe (FA) Nr. 131 vom 14.5.1972.

59) Optimistischer sind hier u.a. Lelpert, C., Sozlale Indikatoren, a.a.0., S. 242 und Creutz, G., Bericht über die Diskussion der vortrăge zum Thema "Umweltschutz und Statistik" auf der 45. Jahreshauptversammlung der Deutschen statistischen Geselischaft am 26.9.1974 in Dortmund, in: Allgemeines Statistisches Archiv, Bd. 59, 1975, S. 70. 
herrschende Belastung der Luft mit verschiedenen Schadstoffen geben soll, zelgt, wie wenig aussagekrätig solche Indizes im Grunde sind 60) - und bel Ihnen handelt es sich nur um den Gesamtbelastungsindex elnes Umweltmediums und nicht einen solchen der gesamten Umwelt.

bb) Die Aufstellung von Emissionskatastern

(1) Aufgaben der Emissionskataster

Während die Aufstellung von Immissionskatastern wichtig für die Feststellung der Wirkungen der Umweltbelastung und damit für deren Bewertung ist, dienen Emissionskataster via Ermittlung der Emissionsquellen primär der Feststellung der Verursacher sowie der Art und Hohe der Emission ${ }^{61}$. Wir wollen uns hier nur kurz dem Problem zuwenden, ob die Emittenten tatsächlich auch die Verursacher der Umweltbelastung sind. Die emittierenden Produzenten behaupten, ihre Emissionen wären die Folge davon, daB sle die Nachfrage nach Gutern befriedigen wollen. Um die nachgefragten Güter anbleten zu können, müBten sie sie produzieren und das sel mit Emissionen verbunden. Die elgentlichen Verursacher ihrer Emissionen seien somit die Endnachfrager. Die emittierenden Haushalte behaupten, daB ihre Emissionen (z.B. $\mathrm{SO}_{2}$, Kunststoffabfalle) Folgen des Angebots der Produzenten (Heizol, Verpackungsmaterial) selen; folglich seien diese die elgentlichen Verursacher. Gegen die Argumentation der emittierenden Produzenten läBt sich elnwenden, daB wir heute uberwlegend Verkäufermärkte haben und die Werbung immer mehr dahin wirkt, das sich die Nachfrage nach dem Angebot richtet. Die Argumentation der emittierenden Haushalte wäre nur dann stichhaltig, wenn es keine zumutbaren emissionsfreien oder -ärmeren

6o) Vgl. Thom, G.C.lott, W.R., Air Pollution Indices, A Compendium and Assessment of Indices Used in the United States and Canada, U.S.G.P.O., Washington, D.C., 1975.

61) Vgl. Dreyhaupt, F.J., Luftreinhaltung als Faktor der Stadtund Regionalplanung, Aachen 1970, S. 101; Günther, U. u.a.., Umweltschutz-Informations- und Steuerungssysteme, a.a.o.., S. 28 . 
Alternativen $z u$ den Gutern gäbe, die bel ihnen Emissionen hervorrufen.

Wollte man sich in jedem Einzelfall darauf einlassen, festzustellen, ob ein Kăufermarkt oder Verkäufermarkt besteht und ob Alternativen $\mathrm{zu}$ den Emissionen bewirkenden Glitern bestehen und ob diese zumutbar sind, würde die suche nach dem Verursacher zu einem kostspieligen Unterfangen. Wir wollen als Verursacher deshalb einfach diejenigen bezeichnen, die im juristischen sinne Eigentümer der Emissionsquellen sind.

Dem Versuch, mit Hilfe einer Verflechtungsanalyse 62) den eigentlichen (ökonomischen) Verursacher einer Umweltbelastung festzustellen, messen wir nur akademische Bedeutung bei. Es ist zwar bereits gelungen, elne solche Verflechtungsanalyse fur $\mathrm{so}_{2}$ in der Bundesrepubl1k Deutschland durchzufuhren 63). Will man jedoch auf der Grundlage der durch eine Verflechtungsanalyse ermittelten 8konomischen Verursacher das Verursacherprinzip durchsetzen, so 1st - un Wettbewerbsverzerrungen $z u$ vermeiden eine solche Verflechtungsanalyse zumindest fur die wichtigsten Schadstoffe notwendig - ein unter Kostengesichtspunkten zu aufwendiges Verfahren, zumal es andere Möglichkeiten gibt, die ökonomischen Verursacher zu treffen, nämlich via Uberwälzung von Steuern, die irgendwo in der Verursachungskette auf den (Faktor-) Input oder (Güter-) Output erhoben werden 64) Berulcksichtigt man, daß die Uberwälzungschancen (neben den Angebotselastizitäten) von den Nachfrageelastizitaten abhängen und da $\beta$ in den Nachfrageelastizitäten die Bedürfnisintensität nach den Gütern zum Ausdruck kommt - je starrer die Nachfrage ist, um so mehr wird das Gut begehrt und un so mehr kann ceteris paribus von der steuer uberwalzt werden -, so 1st dies dem Markt uberlassene Verfahren der Identifizierung der ökonomischen Verursacher der Verflechtungsanalyse bel weltem uberlegen. Das Hauptverdienst der Verflechtungsanalyse ist u.E., deutlich

62) Vgl. RSU, Umweltgutachten 1974, a.a.0., S. $229 \mathrm{ff}$.

63) Vgl. ebenda, S. $234 \mathrm{ff.}$

64) Slehe unten S. $341 \mathrm{ff}$. 
gemacht zu haben, daß manche scheinbar umweltfreundliche Sektoren (auch Endnachfragesektoren), well sie selbst kaum Schadstoffe emittieren, indirekt - via Bezug von Vorleistungen, deren Produktion sehr emissionsintensiv ist - beträchtlich zur Umweltbelastung beitragen 65 ? Neben der Emissionsquelle und damit dem Verursacher muB das Emissionskataster aber auch Art und Menge der Emissionen erfassen, denn die Höhe der Emissionen bestimmt neben anderen Einflubfaktoren die Hobe der Immissionen und der durch sie hervorgerufenen Schäden 66) Die Messung der Emissionen wird damit zur Grundlage für die Heranzlehung der Emittenten (Verursacher) zur Behebung des Schadens.

\section{(2) Einteilung dirr Emittenten}

Unter ökonomisch nn und auch speziell finanzwirtschaftlichen Gesichtspunkten scheint eine institutionelle Gliederung der Emittenten wie in der volkswirtschaftlichen Gesamtrechnung in die Sektoren Unternehmen, private Haushalte, Staat und Ausland sinnvoll 67). In all diesen sektoren befinden sich Betreiber emittierender stationärer und mobiler Anlagen. Da mobile Emissionsquellen (Verkehrsmittel) besondere Probleme bei der katastermäBlgen Erfassung aufwerfen, wollen wir sle aus der Institutionellen Gliederung herausnehmen und als elgenen Sektor Verkehr zusammenfassen. Da wir in dieser Arbelt von Problemen

65) Vgl. RSU, Umweltgutachten 1974, a.a.0., S. 236.

66) Mit Hilfe von Diffusionsmodellen kann man, ausgehend von den gemessenen Emissionswerten, die Immissionskonzentration berechnen (vgl. Dreyhaupt, F.J., Emissionskataster als Hilfsmittel zur Luftreinhaltung, in: Institut fur gewerbliche Wasserwirtschaft und Luftreinhaltung e.v. (Hrsg.), Umweltschutz - eine Aufgabe unserer Ze1t, K8ln 1971, S. 50.

67) Naturwissenschaftlich-technisch orlentierte Untersuchungen unterscheiden meist zwischen Industrle, Haushalt und Kleingewerbe sowle Verkehr; vgl. dazu Georgi1, H.W., Die lufthygienisch-meteorologische Modelluntersuchung im RheinMain-Geblet, a.a.0., S. 217 f. und Dreyhaupt, F.J., Luftrelnhaltung als Faktor der Stadt- und Regionalplanung, a.a.o., s. 1 . 
der nationalen Grenzen uberschreitenden Umweltbelastung absehen 68), betrachten wir im folgenden die vier Emittentengruppen Unternehmen, Haushalte, Staat und Verkehr. Diese E1ntellung ist jedoch noch viel zu grob. Vor allem der sektor Unternehmen muß tiefer gegliedert werden. Hier bletet sich zunächst eine weltere Untergliederung nach Branchen an, da viele Arten der Umweltbelastung branchentypisch sind 69) Wir empfehlen für die Gliederung des Unternehmenssektors diejenige der amtlichen Statistik 70) In vielen Fällen, besonders in Ballungsgebieten, wird es jedoch notwendig sein, bis zu den einzelnen Unternehmen - ja u.U. gar bis zu den emittierenden Anlagen bestimmter Unternehmen zu gliedern. Je nach der mit der Emissionserfassung einhergehenden Problemstellung können dann die Mengen gleicher Schadstoffe, die aus verschiedenen Quellen emittiert wurden, wieder aggreglert werden - U.U. auf Branchenebene oder gar für den gesamten Unternehmenssektor.

Bel den privaten Haushalten halten wir eine weltere Untergliederung nicht für sinnvoll.

Der staat kommt aus verschiedenen Gründen als Emittent in Frage. Zum einen 1st er ebenso wie Unternehmen und private Haushalte Elgentümer von Emissionsquellen (z.B. bei offentlichen Unternehmen), zum anderen 1st er qua Gesetz verantwortlich fur die Vermeidung oder Beseltigung bestimmter Emissionen (z.B. für die

68) Zur Feststellung, welche Länder bel grenzuberschreitender Luftbelastung Verursacher sind, hat die OECD MeBstationen in ganz Europa aufgebaut; vgl. O.V., Who "Exports" and who "Imports" Pollution?, in: OECD-Observer, No. $70,1974, \mathrm{~S}$. 12 f.; $z u$ weiteren Problemen der grenzuberschreitenden Umweltbelastungen vgl. OECD (Hrsg.), Problems in Transfortier Pollution, Paris 1974.

69) Vgl. Krengel, R., Die Messung der Umweltverschmutzung - ein neuer Wohlstandsindikator, a.a.0., S. 431. Für die Fluorund die Fluorwasserstoffemission ist z.B. Uberwiegend die Aluminiumindustrie verantwortlich, für die Emission von Pestiziden die Landwirtschaft usw.

70) Von dieser Gliederung geht z.B. auch der RSU aus, wenn er den Beitrag der verschiedenen Sektoren zur $\mathrm{SO}_{2}$-Belastung untersucht, vgl. RSU, Umweltgutachten 1974, a.a.0., S. $237 \mathrm{f}$. 
Klärung kommunaler Abwässer) 71) Kommt z.B. eine öffentliche Gebietskörperschaft dieser gesetzlichen Verpflichtung nicht nach, so wollen wir nicht den Sektor aus dem die Emissionen stammen, sondern diese Gebletskorrperschaft als eigentlichen Emittenten bezelchnen. Wir nehmen elne Untergliederung des Sektors Staat in öffentliche Gebletskörperschaften (und hier wiederum in Bund, Länder und Gemeinden) sowie in öffentliche Unternehmen (und hier wiederum gemaß Branchen 72 ) sowie Bundesbahn und Bundespost) vor.

Die Einteilung des Sektors Verkehr erfolgt am sinnvolisten nach den Verkehrswegen Straße, Schiene, Wasser und Luft. Emissionsquellen sind also Kraftfahrzeuge und Motorräder, Bahnen, Schiffe und Flugzeuge.

(3) Zur Erfassung der Emissionsquelien

Die Erfassung von Emissionen wirft wegen der großen Dichte bestimmter Emissionsquellen Probleme auf. Dies soll am Beispiel der Emissionen luftbelastender stoffe verdeutlicht werden. Die Emissionsquelien im Bereich des Sektors Unternehmen (und hier vor allem im industriellen sektor und weniger im kleingewerblichen Sektor und Dienstleistungsbereich) sind - selbst in Ballungsgebieten - räumlich relativ weit verteilt und somit relativ einfach zu identifizieren; thre Anzahl ist relativ gering. Man spricht in diesem Fall von Punktquellen. An thnen kann die Emissionsmenge relativ leicht gemessen werden. Anders verhält es sich dagegen mit sehr dicht verteilten Emissionsquellen wie wir sie in den Sektoren "private Haushalte" (Wohnhäuser) und "Verkehr" (Kraftfahrzeuge) vorfinden. Im Fall der Hausbrandemissionen aus Wohnsiedlungen spricht man von

71) Gemeinde- und Landkrelsordnungen der Lănder bestimmen $2 . B$. , daB die Gemeinden für die Abwasserbeseitigung zuständig sind (vgl. Bock, J., wasser, Abwasser und Mull heute und morgen, in: Wasser und Boden, Bd. 22, 1970, Heft 5, S. 118). Auch der ehemalige Bundesminister Genscher spricht in einem Interview von Gemeinden als Verursachern von Umweltbelastunaen (vgl. Die Wirtschaftswoche, Nr. 23, vom 1.6.1973).

72) Besonders wichtig ist hier sicher die Energiebranche. 
Flächenquellen, im Fall der Kfz-Emissionen von Linienquellen

Be1 Flächen- und Linlenquelien ist zwar auch eine Messung der Emissionen an jeder einzelnen Quelle (Schornstein, Auspuff) mög$11 \mathrm{ch}$, aus Kostengründen jedoch kaum durchführbar. An die Stelle der Messung tritt hier eine Berechnung der Emissionen. Bei der Berechnung der Hausbrandemissionen mussen zunăchst die verwendeten Brennstoffarten und der Brennstoffverbrauch ermittelt werden. Beldes kann man z.B. durch eine Reprăsentativumfrage feststellen 74) Eine andere Methode zur Ermittlung des Brennstoffverbrauchs 1st die stereoskop1sche Luftbildauswertung, mit der man das Volumen der fotographierten Gebăude bestimmen und daraus Rückschlüsse auf den Brennstoffverbrauch zlehen kann 75! M1t Hilfe von Emissionsfaktoren, die angeben, wieviel $\mathrm{kg}$ Schadstoffe bel Verbrennung von $1 t$ Helzmaterial emittiert werden, kann man nun die Emissionsmengen berechnen 76 )

Schwieriger ist die Berechnung der Abgasemissionen von Kraftfahrzeugen 77) Die Emissionsmengen je Zeiteinhelt hăngen ab von Art und Menge des Kraftstoffverbrauchs und vom Fahrverhalten. Der Kraftstoffverbrauch wlederum ist abhängig von der Anzahl der Kraftfahrzeuge und von den Fahrzeugtypen (Hubraum, Nutzlastklasse). Das Fahrverhalten ist vor allem elne Funktion der Verkehrsdichte. Fur die Bestimmung der Emissionsfaktoren ist es

73) Vgl. Dreyhaupt, F.J., Das Emissionskataster, a.a.0., s. 212.

74) Vgl. ebenda, S. 213 und derselbe, Luftreinhaltung als Faktor der Stadt- und Regionalplanung, a.a.0., S. 111 ; Güther, U. u.a., Umweltschutz- Informations - und steuerungssystem, a.a.0., S. $28 \mathrm{f}$.

75) Vgl. Dreyhaupt, F.J., Luftreinhaltung als Faktor der stadtund Reglonalplanung, a.a.0., s. 111.

76) Vgl. ebenda, S. 112. Auch für industrielle Branchen kann man elne Berechnung der Emissionen vornehmen, und zwar mit Hilfe branchenspezifischer Emissionskoeffizienten (vgl. Krengel, R., D1e Messung der Umweltverschmutzung - ein neuer Wohlstandindikator, a.a.0., S. $433 \mathrm{f.l}$

77) Zum folgenden vgl. Dreyhaupt, F.J.. Luftrelnhaltung als Faktor der Stadt- und Reglonalplanung, a.a.0.. S. $113 \mathrm{ff}$. und ausfuhrlicher bel May. H./Plassmann, E.. Abgasemissionen von Kraftfahrzeugen in GroBstädten und industrielien Ballungsgebleten, Koln 1973, s. $68 \mathrm{ff}$. 
notwendig, Anzahl und Art der Kraftfahrzeuge pro Zelteinheit und Einheitsfläche oder Einheitsstrecke zu bestimmen. Das kann durch Auszählung oder Luftbildaufnahmen geschehen. AuBerdem muB das Fahrverhalten registriert werden. Dies kann durch den Einsatz von Testfahrzeugen erfolgen, welche die in Frage kommenden straben zu verschiedenen zelten abfahren, oder durch die Fahrt auf Prüfstănden mit der simulation verschiedener Verkehrssituationen.

Die hier geschilderten Möglichkelten der emissionsseitigen katastermäbigen Erfassung umweltbelastender Schadstoffe durch Messung oder Berechnung bestehen auch für die Emissionen in anderen Umweltmedien und für den Lărm, so das es grundsätzlich kelne unuberwindbaren Schwierigkelten glbt, Emissionskataster für die Berelche Luft, Gewăsser, Boden sowie den Lărm aufzustellen. Gesetzlich geregelt sind in der Bundesrepublik Deutschland die katastermäBige Erfassung luftbelastender Emissionen ( $\$ 46$ BImSchG) und giftiger Abfalle ( $\$ 11 \mathrm{AbfBG}$ ). Am fortgeschrittensten sind sicher die theoretischen 78) und praktischen 79) Arbeiten bel der Aufstellung von Emissionskatastern für den Be-

78) Vgl. z.B. die Schriftenrelhe Umweltschutz des TUV Rheinland e.V., Bd. 1-5, in denen die theoretischen Grundlagen für die katastermäBige Erfassung luftbelastender stoffe allgemein (Bd. 1 und 2) und für die Sektoren Straßenverkehr (Bd.3), Industrieunternehmen (Bd. 4) und private Haushalte sowie. kleingewerbliche Betriebe (Bd. 5) konkret herausgearbeitet werden.

79) Um prakt1sche Erfahrungen für die Aufstellung von Emissionskatastern zu gewinnen, wurde in der Region Untermain ein Modellversuch für die emissionsseitige Erfassung luftbelastender Stoffe speziell für Ballungsgeblete gestartet (vgl. BT-Drucksache VI/2036). Elnige Städte, z.B. Köln, haben bereits Emissionskataster aufgestellt (vgl. May, H./Plassmann, E.. Abgasemissionen von Kraftfahrzeugen in Großstädten und industriellen Ballungsgebieten, a.a.0.). Auch einzelne Unternehmen erfassen bereits freiwillig bestimmte Schadstoffe in Emlssionskatastern (vgl. Haberle, M., Zur Erstellung eines Geruchskatasters der BASF Ludwigshafen, in: Umwelt 1973,6, S. $39 \mathrm{ff.l}$ 
relch der Luftbelastung ${ }^{80}$ ) Bel analoger Anwendung des $\S 46$ BImSchG auf den Lărm wird auch die Erfassung aller Lărmquellen in einem flächenbezogenen Lärmkataster, wie sle der RSU fordert 81) möglich sein. Für die emissionsseitige Erfassung der Gewässer- und Bodenbelastung (auBer durch giftige Abfälle und Bebauung) fehlen bisher die gesetzlichen Grundlagen.

\section{cc) Zur statistischen Aufbereitung der Katasterdaten}

Die in den Emissions- und Immissionskatastern gesammelten Daten geben bei hinreichender Vollständigkelt ein differenziertes B1ld über Art, Umfang und Herkunft der umweltbelastenden Stoffe; sie müssen jedoch noch aufbereltet werden, um statistischen Anforderungen $2 u$ genügen.

Vor allem die Analyse der Wirkungen der Umweltbelastung erfordert Angaben daruber, wie groB die Belastung einer bestimmten Region zu elnem bestimmten Zeitpunkt oder in einem bestimmten Zeitraum durch bestimmte schadstoffe ist.

Der amtlichen statistik obliegt es also, die Daten aus den Emissions- und Immissionskatastern $\mathrm{zu}$ regionalisieren ${ }^{82}$ ) mit einer zeltdimension $\mathrm{zu}$ versehen und $\mathrm{zu}$ aggregieren.

\section{(1) Regionalisierung der Daten}

Das Emissionskataster "Luft" gibt u.a. Auskunft uber den Standort und die Anzahl der Emissionsquellen. Nehmen wir an, es welse

80) Abgesehen natürlich von der schon lange bestehenden katastermäBigen Erfassung der Grundstücke elnes ganzen Landes bel den Kataster- oder Vermessungsämtern der Gemeinden. Hier kann man sowohl die Art der Bodenbelastung (Bebauung oder nicht) als auch den Eigentümer (Emittent im weiteren Sinne) der jewe1ligen Katasterparzellen feststellen.

81) Vgl. RSU, Umweltgutachten 1974, a.a.0., s. 96.

82) Vgl. Bartelmus, P., Probleme der Entwicklung elnes umweltstatist1schen Systems, a.a.O., S. 130 und S. 140; Krengel, R., Die Messung der Umweltverschmutzung - ein neuer Wohlstandindikator, a.a.0.. S. 433; Entwurf elnes Gesetzes uber Umweltstatistiken, a.a.0., s. 8 ; Häfele, W., Stoffbilanzen, a.a.0., S. 23; Hansmeyer, K. -H., Anforderungen der Umweltpolitik an die amtliche Statistik, a.a.0., s. 7 f. 
die Schornsteine des Kraftwerkes $A$ und der Erdölraffinerie $B$ - in beiden Fällen Punktquellen - und die siedlung $C$ (eine Flächenquelle) als Emittenten von $\mathrm{SO}_{2}$ aus (vgl. Abb.6). Das Immissionskataster "Luft" zeige in den Maschenquadraten c11, c12, d11, d12, e 11 und e12 eine besonders hohe $\mathrm{so}_{2}$-Konzentration. Unter Berücksichtigung dieser Daten sowie der meteorologischen und topologischen Verhältnisse können wir nun eine $\mathrm{SO}_{2}$-Belastungsregion bilden 83 ? Nehmen wir an, es herrsche in dem in Frage kommenden Gebiet vor allem Nordwestwind und es werde im Osten durch den Kamm $K$ eines Höhenzuges begrenzt, so könnten wir das schraffiert gekennzeichnete Gebiet als Belastungsregion bezelchnen.

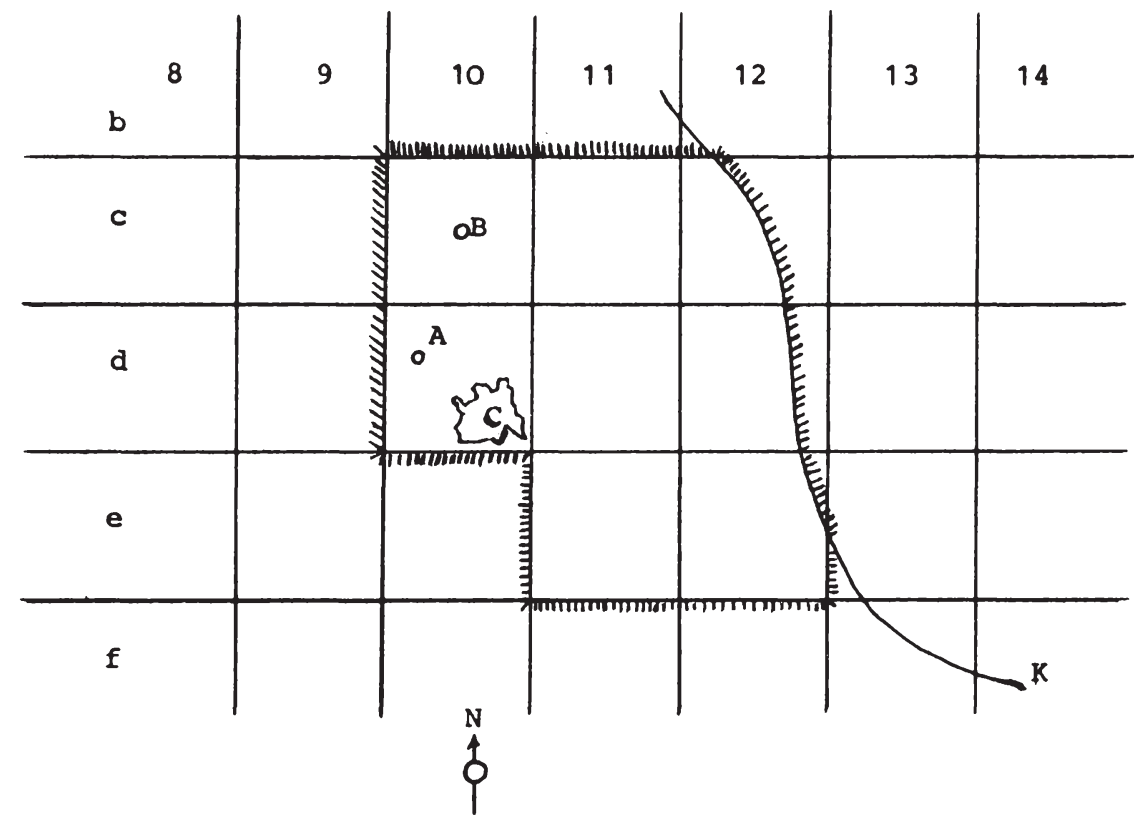

Abb. 6

83) In den USA werden "air basins" gebildet; das sind nicht an Bundesstaatengrenzen gebundene Geblete, die unter dem Gesichtspunkt der Immissionsbelastung sowie topologischer und meteorologischer Gegebenheiten abgegrenzt werden (vgl. Blair, R.D., The Clean Air Act of 1970, in: Land Economics, vol. 49, 1973, S. 262). 
Wir beziehen die Emissionsquellen mit in das Belastungsgebiet ein - obwohl an ihrem Standort in c10 und d10 keine hohe $\mathrm{SO}_{2}{ }^{-}$ Konzentration gemessen wird (Schornsteine von A und B sind sehr hoch, C liegt an der Ostgrenze von d10, starke Luftbewegung) -, damit sich Verursacher und Träger der $\mathrm{SO}_{2}$-Belastung in der gleichen Region befinden. Es lassen sich nun für alle schadstoffe und alle Umweltmedien solche Belastungsregionen bilden. Diese Belastungsreglonen werden sich mehr oder weniger überlagern und es wird Gebiete geben, in denen alle Umweltmedien durch eine Vielzahl von Schadstoffen belastet sind. Solche Vielfach-Belastungsregionen können $z u$ neuen Schwerpunktregionen der Umweltbelastung zusammengefaBt werden. Zur Bildung solcher Umweltregionen können grundsätzlich die gleichen statistischen Verfahren angewandt werden wie bei der Bildung von Wirtschaftsräumen ${ }^{84}$ ?

\section{(2) Die zeitliche Dimensionlerung der Daten}

Die Zeitdimension, mit der die Daten in die Emissions- und Immissionskataster eingetragen werden, ist in der Regel meBtechnisch bedingt und genügt oft nicht den Ansprulchen einer Wirkungsanalyse der Umweltbelastung. Bei nicht kontinuierlicher Messung aber kontinuierlicher Emission bzw. Immission müssen z.B. Emissions- und Immissionsdaten zeitlich hochgerechnet werden; umgekehrt müssen bei kontinuierlicher Messung aber diskontinuierlicher Emission oder Immission Zeiten der Spitzenbelastung statistisch festgehalten und nicht nur gemittelt werden. Es ist für jeden Schadstoff festzulegen, ob selne Emission oder Immission entweder in Sekunden-, Minuten-, Stunden-, Tages-, Monats- bzw. Jahresdurchschnittswerten oder ob auch Maxima der Emissionsraten und Immissionskonzentrationen angegeben werden sollen.

84) Vgl. z.B. Fischer, A., Die struktur von wirtschaftsräumen. Ein Beitrag zur Anwendung statistischer Methoden in der Reglonalforschung, Wiesbaden 1969; Schulze, P.M., Regionsund Informationssystem, unveröffentlichte Habilitationsschrift, Mainz 1975; Bartelmus;, P., Probleme der Entwicklung eines umweltstatistischen Systems, a.a.0., S. 143. 
(3) Die Aggregation der Daten

Manche Schadstoffe, wie 2.B. Blei, wirken über alle drei Umweltmedien auf die menschliche Gesundheit. Die Gesamtbelastung einer Region durch Blei kann man also nur feststellen, wenn man die Bleikonzentration aus den Immissionskatastern Luft, Gewässer und Boden aggregiert. 
"Das Bekannte ist darum, daB es bekannt ist, noch nicht erkannt".

(G.W.F. Hegel, Phänomenologie des Geistes)

2. Zur Diagnose der Schadstoffwirkungen

a) Zur statistischen Erfassung der Schadstoffwirkungen Die Kenntnis der Wirkungen der Schadstoffe auf die Umwelt und den Menschen 1st nicht nur - wie bereits erwähnt - Voraussetzung für eine Bewertung der Schäden, sondern auch für die konkrete Formulierung der umweltpolitischen ziele ${ }^{85}$ ). Deshalb 1st eine Statistik der eingetretenen Schäden 86 ) ebenso wichtig wie die Statistik der (emittierten und immittierten) Schadstoffmengen. Die regionalisierte statistik der schadstoffkonzentrationen ist dabei Voraussetzung für die Schadensstatistik, denn nur wenn Art und Menge der Schadstoffe in der Umwelt bekannt sind, lassen sich eventuell Beziehungen zwischen thnen und den von ihnen bewirkten Schäden messen. Um die Wirkungen der Schadstoffe auf die menschliche Gesundheit zu messen, könnten bereits vorhandene Statistiken des Gesundheitswesens herangezogen werden, wenn sie durch eine umweltrelevante Krankheitsgliederung verbessert und durch Korrelationsrechnungen ergänzt würden 87? Daneben müBten auch die Wirkungen der Schadstoffe auf die natürliche oder materielle Umwelt erfabt werden ${ }^{88}$ ? Die gröBte

85) Vgl. dazu unten S. $137 \mathrm{ff}$.

86) Der RSU spricht von einem wirkungskataster, das neben Emissions- und Immissionskataster die dritte wesentliche Informationsquelle für den Umweltschutz sein soll (vgl. RSU, Umweltgutachten 1974 , a.a.0., S. 18).

87) Vgl. Bartelmus, P., Probleme der Entwicklung eines umweltstatistischen Systems, a.a.O., S. 139 ff.; Bartels, H., Statistik als Hilfsmittel der Umweltpolitik, a.a.0., s. 22 .

88) Vgl. Bartelmus, P., Probleme der Entwicklung eines umweltstatistischen Systems, a.a.0., S. 142 . 
Schwierigkeit bei der Erfassung der Schadstoffwirkungen auf die Umwelt und den Menschen dürfte darin liegen, einen elndeutigen Ursache-Wirkungs-Zusammenhang zwischen Schadstoffimmission und dem Schaden nachzuweisen 89) Das liegt u.a. daran, daB z.B. eine Krankheit mehrere Ursachen haben kann. Eine starke Korrelation zwischen einer hohen Schadstoffkonzentration und einer Krankheit, die durch diesen Schadstoff hervorgerufen werden kann, ist daher noch kein Beweis für die tatsächliche Verursachung. Nur selten - und dies in der Regel bei "Katastrophen" - wurde ein eindeutiger Ursache-Wirkungs-Zusammenhang nachgewiesen. Das heiBt nun keineswegs, daB man auf Korrelationsrechnungen verzichten soll: Wenn es häufig und an verschledenen orten $z u$ starken Korrelationen zwischen einer bestimmten Schadstoffkonzentration und einer bestimmten Krankhelt kommt, so können u.U. Regressionsanalysen elnen ursächlichen zusammenhang feststellen. Um eine aussagekräftige statistik der durch die schadstoffe bewirkten Schäden aufstellen zu können, ist - neben der Statistlk der Schadstoffmengen - eine möglichst vollständige Kenntnis der Schadstoffwirkungen sowie eine Systematislerung der Schäden notwendig.

b) Zur Systematisierung der Schäden

Die in die Umwelt emittierten Schadstoffe bewirken in der Regel zunächst elne Belastung der Umwelt selbst und dann über diese eine Belastung des Menschen 90) Manche Schadstoffe und der Lärm

89) Vorausgesetzt man we1 $B$, daB ein solcher $\mathrm{zusammenhang} \mathrm{über-}$ haupt bestehen kann. Bei vielen Schadstoffen $v$ e $r \mathrm{~m} u t e t$ man bestenfalis eine gesundheitsschadliche Wirkung oder umgekehrt formuliert: Bel bestimmten Krankheiten vermutet man als Ursache umweltbelastende Schadstoffe.

90) Umweltschutz ist also Menschenschutz; vgl. Goldsmith, E./ Allen, R., Planspiel zum Uberleben, Stuttgart 1972, S. 15; Schaefer, H., Die Okologie als Problem des Umweltschutzes, 1n: Schaefer, H. (Hr.sg.), Folgen der Zivilisation, Therapie oder Untergang?, Frankfurt/M. 1974, S. 98; Bauer, R.K., Methodische Grundprobleme der Umweltstatistik, in: Allgemeines Statistisches Archiv, Bd. 59, 1975, S. 33; van Belle, G./Schneiderman, M., Some Statistical Aspects of Environmental Pollution and Protection, in: International Statistical Review, vol. 41, 1975, S. 319. 
führen jedoch direkt $z u$ einer Gesundheitsschädigung beim Menschen. Tellt man die Umwelt in eine natürliche und materielle Umwelt ein, so kann man noch unterschelden zwischen b̈kologischen Schäden und Sachschäden. Die bkologischen Schäden unterteilt man am besten noch nach den betroffenen Umweltmedien Luft, Wasser und Boden. Okologische Schäden können an den Umweltmedien selbst hervorgerufen werden und/oder an den in thnen lebenden Tieren und Pflanzen. Ferner kann es $\mathrm{zu}$ Beeinflussungen des Kllmas via ökologischen Schäden an Luft, Wasser, Boden und Pflanzenwelt kommen. Auch Schäden in einem Umweltmedium können Schäden in den anderen Umweltmedien bewirken. Alle diese 8kologischen Schäden können ebenso wie die Sachschäden die menschliche Gesundheit beeinflussen. Die möglichen Wirkungen sind noch einmal in dem folgenden schema zusammengefaBt:

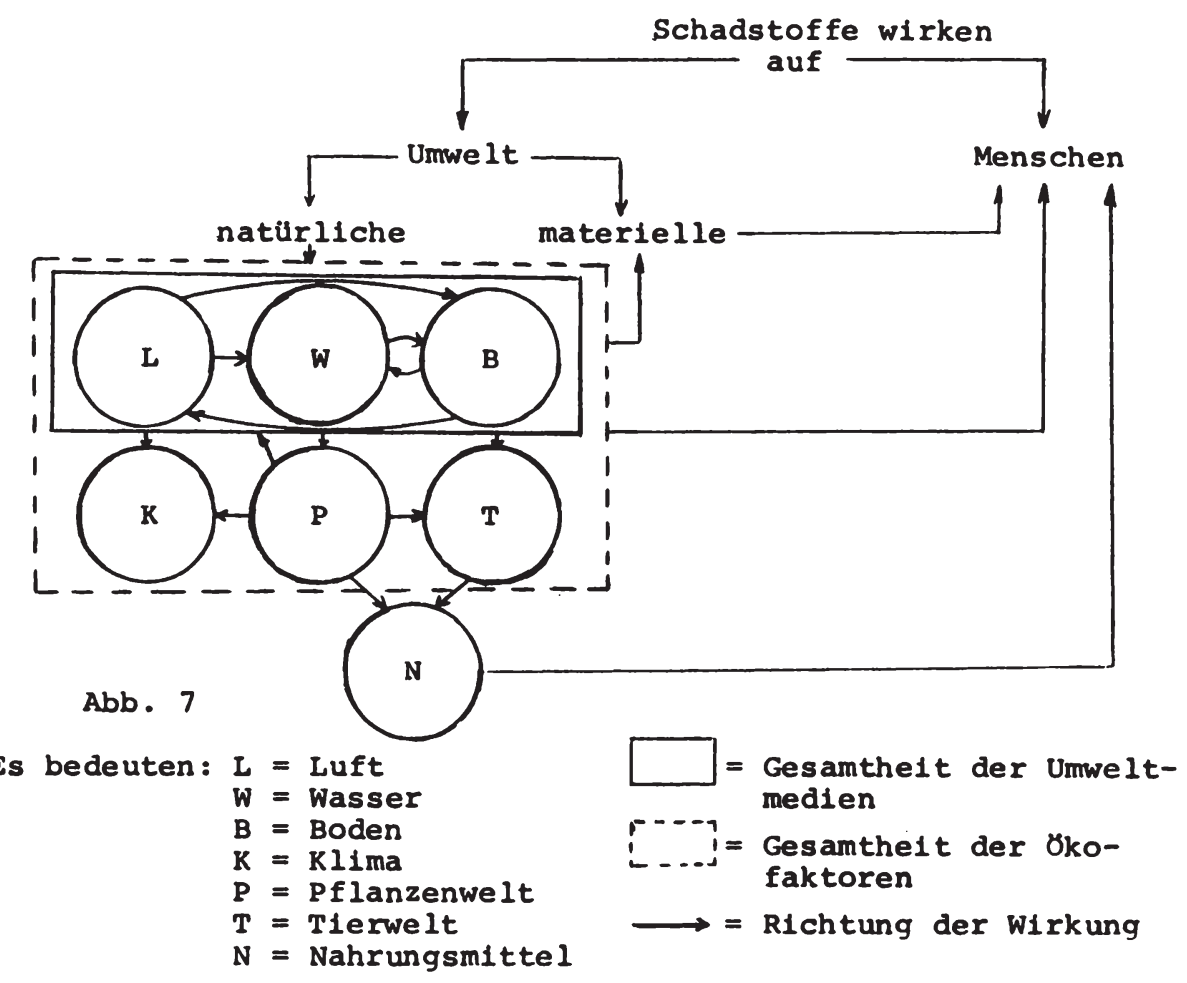


Diesem Gliederungsschema der Schadstoffwirkungen folgend, wollen wir die wirkungen der Schadstoffe 91) darstellen.

c) Die Wirkungen der Schadstoffe

aa) Die Wirkungen der Schadstoffe auf die Umwelt der Menschen

(1) Okologische Wirkungen

(a) Störungen des ökologischen Gleichgewichts

Die Okologie kann man als die Wissenschaft von der struktur und Funktion der bkosysteme bezeichnen 92) Diese Okosysteme bestehen aus den రkofaktoren Luft, Wasser und Boden (Biotop) der Pflanzenund Tierwelt (Biozönose) sowie dem Klima; sle bilden zusammen die Biosphäre. Innerhalb der రkosysteme und zwischen den రkosystemen besteht ein natürliches Gleichgewicht, das sogenannte bkologische (oder biologische) Gleichgewicht 93). Es ist dadurch gekennzeichnet, daß die jährliche Produktion an organischer Masse gleich dem Gesamtverbrauch an organischer Masse ist. Gelangen nun Schadstoffe in ein bkosystem, so kann qua Behinderung der Produktion (oder Uberproduktion) organischer Masse und/oder durch zusätzlichen (verminderten) Verbrauch organischer Masse das b̈kologische Gleichgewicht dieses రkosystems, aber auch der gesamten Blosphäre 94) gestört werden. Da die natürliche Umwelt

91) Es handelt sich dabel zumindest un die potentielien wirkungen der Schadstoffe, denn ob sie tatsächlich schädlich sind, hängt naturlich von ihrer konzentration ab.

92) Vgl. dazu und den folgenden Ausführungen dieses Abschnittes Odum, E.P., Okologie, München-Basel-Wien 1967, S. 11. Zur Komplexität der das obologische Gleichgewicht verkörpernden Kreisläufe vgl. McHale, J., Der ökologische Kontext, Frankfurt/M. 1974, S. 51 ff. Eingriffe in diese Krelsläufe können zur Storrung des gesamten ökologischen Gleichgewichts fuhren (vgl. ebenda, S. 94).

93) Vgl. Schäfer, G., Kybernetische Modelle als Hilfsmittel zur Darstellung ökologischer $\mathrm{Z}$ usammenhänge, in: Steubing, L./ Kunze, C./Jäger, J.. Belastung und Belastbarke1t von bkosystemen, a.a.0.. S. 181 ff.; Klausewitz, W./Schäfer, W./ Tobias, W., Umwelt 2000, Kleine Senckenberg Rethe, Bd. 3, Frankfurt/M. 1971, S. $18 \mathrm{ff}$.

$94) \mathrm{Vgl}$. Stockmann, H.-U., Die natürlichen Faktoren in der Planung, in: Institut für.Raumforschung, Informationen, Bd. 20, 1970 , S. 365 . 
jedoch zum einen anpassungsfähig ist (man spricht deshalb von einem dynamischen ökologischen Gleichgewicht ${ }^{95)}$ ) und zum anderen Selbstreinigungskräfte entwickelt, führt nicht jede Schadstoffmenge, sondern erst eine bestimmte schadstoffkonzentration zu einer Störung des ökologischen Gleichgewichts. Die Okosysteme sind bis zu bestimmten Grenzen belastbar ${ }^{96}$ ? Nur die uber diese Belastungsgrenzen hinausgehenden Wirkungen wollen wir als rkologische Schäden bezeichnen.

(b) Okologische Wirkungen der Luftbelastung

Die Belastung des Umweltmediums Luft kann $(\alpha)$ zu Schäden an diesem selbst führen, indem es seine natürliche Zusammensetzung über die Belastungsgrenzen hinaus verändert; darüber hinaus kann die Belastung $(\beta)$ Schäden in anderen Umweltmedien hervorrufen; ferner kann sie $(\gamma)$ Schäden bei den vom Lebensraum Luft abhängigen Pflanzen und Tieren bewirken; weiterhin sind $(\delta)$ Klimaveränderungen denkbar.

(d) Die untere Luftschicht, die Troposphäre, besteht in reinem Zustand aus rund 778 Stickstoff und 21 sauerstoff; der Rest besteht aus Wasser, Edelgasen und Kohlendioxid.

Eine der von Okologen meist diskutierten Fragen ist nun, ob die ständig steigende Verbrennung fossiler Brennstoffe $\mathrm{zu}$ elnem nicht mehr reparablen Schaden am Sauers tof fhaushalt - einem $\mathrm{O}_{2}$-Defizit - der Troposphäre führen kann 97) Während einige Autoren diese Gefahr eines Sauerstoffdefizits in der Atmosphäre als gering betrachten ${ }^{98}$ ), halten andere sie be-

95) Vgl. Schaefer, H., Die Ukologie als Problem des Umweltschutzes, a.a.0., s. 118.

96) Nur Schadstoffkonzentrationen, die über diese Grenzen - mit deren Bestimmung wir uns unten befassen (vgl. S. $137 \mathrm{ff.}$ ) hinausgehen, stellen Umweltbelastungen dar; vgl. dazu Ellenberg, H., Belastung und Belastbarke1t von Okosystemen, in: Steubing, L./Kunze, C./Jäger, J., Belastung und Belastbarkeit von okosystemen, a.a.0., S. $19 \mathrm{ff}$.

97) Vgl. Olschowy, G./Mrass, w./Kullmer, H.J./Bürger, K., Zur Belastung der Landschaft, a.a.0., S. 34.

98) Vgl. Schaefer, H., Die Okologie als Problem des Umweltschutzes, a.a.0., s. 108. 
reits für akut 99! Bei differenzierter Betrachtung des Problems haben beide Selten recht: Global ist in absehbarer Ze1t nicht die Gefahr der Sauerstoffverarmung vorhanden, regional und vor allem lokal besteht jedoch die Gefahr eines belastenden Sauerstoffverbrauchs. So soll in den USA im Jahre 1966 die Sauerstoffproduktion (durch Photosynthese) weniger als 608 des Sauerstoffverbrauchs (durch Atmung der Lebewesen und Verbrennung fossiler Brennstoffe) betragen haben; dieses Sauerstoffdefizit wurde jedoch durch Luftzirkulation aus dem pazifischen Raum ausgeglichen 100), es wurde mit anderen worten Sauerstoff (kostenlos) in die USA 1mportiert. Erst wenn ein solcher Ausgleich der Sauerstoffbilanz nicht mehr mög$11 \mathrm{ch}$ 1st, kommt es zu einer Sauerstoffkrise 101) Uber der Troposphäre befindet sich als nächste Luftschlcht die stratosphäre. Durch die No ${ }_{x}$-Emissionen von Uberschallverkehrsflugzeugen (SST), die in Stratosphärenhöhe fllegen, kann es zu elner schädigung des die stratosphäre umgebenden Ozonschildes kommen, der die Erdoberfläche vor der schadlichen UV-Strahlung schützt 102)

( $\beta$ ) Die Belastung der Luft mit Schadstoffen kann dadurch zu Belastungen der belden anderen Umweltmedien Wasser und Boden führen, daB die Schadstoffe in der Luft mit dem Niederschlag in die Gewăsser und den Boden gelangen.

So fuhrt die $\mathrm{SO}_{2}$-Belastung der Luft $\mathrm{zu}$ elner Versäuerung der Niederschlăge und damit der Oberflächengewässer und des Bodens: der pH-Wert in den Gewässern und im Boden sinkt. Wird

99) Vgl. LaMont Cole, C., A Race for Survival, in: Pole, N. (Hrsg.), Environmental Solutions, Cambridge 1972, S. $19 \mathrm{f}$.

100) Vgl. ebenda.

101) Vgl. Egl1, E., Natur in Not, Gefahren der Z1vilisationslandschaft, 2. Aufl., Bern und Stuttgart 1970, S. 18 ff.

102) Vgl. World Meteorological Organization (WMO), Meteorology and the Human Environment, WMO-No. 313, Genf 1971, S. 32. Man stellt sich vor, daß das emittierte $\mathrm{NO}$ bzw. $\mathrm{NO}_{2} \mathrm{mit} \mathrm{O}_{3}$ $\mathrm{zu}$ NO ${ }_{2}$ bzw. $\mathrm{NO}_{3}$ oxidiert und $\mathrm{O}_{2}$ freisetzt (vgl. SchmidtBurbach, G.M., klimaveränderungen im urbanen Bereich, in: U, 1973, 4, S. 24 . 
der pH-Wert der Gewässer kleiner als 5,5 sterben Edelfische, sinkt er unter 4, so ist organisches Leben nur noch bedingt möglich 103) Eine Senkung des pH-Werts im Boden führt $z u$ einer störung des Stickstoffkreislaufs und letztlich zur Auswaschung für das Pflanzenwachstum notwend1ger Spurenelemente 104) Auch staubtelle und an diese gebundene Schadstoffe wie Bleipartikel gelangen mit dem Regen auf die Erdoberfläche und führen so zu elner zusatzlichen Belastung der Gewăsser und des Bodens.

( $\gamma$ ) $\mathrm{Zu}$ den akutesten ökologischen Wirkungen der Luftbelastung gehören die Schäden an der Pflanzen- und Tierwelt.

$\left(\gamma_{1}\right)$ Für die pflanzenwelt am gefăhrlichsten 1st, da am we1testen verbreitet, die $\mathrm{SO}_{2}$-Belastung der Luft 105) Besonders gefahrdet 1st durch $\mathrm{SO}_{2}$ der Nadelholzbestand 106) Nicht wenlger gefährlich sind jedoch Fluorwasserstoff, Chlorwasserstoff, der photochemische smog mit seinen Komponenten PAN (Peroxyacetylnitrat) und ozon 107) sowie stăube. Die Wir-

103) Vgl. Rönicke, G., Die Atmosphäre, ihre Entwicklung und gegenwärtige situation. Änderungen durch den Einflus des Menschen, in: Sioli, H. (Hrsg.), Okologle und Lebensschutz in internationaler S1cht, Freiburg 1973, S. $49 \mathrm{ff}$.

$104)$ Vgl. RSU, Umweltgutachten 1974, a.a.0., s. 120.

105) Vgl. Guderian, R., Wirkungen von Luftverunreinigungen auf Pflanzen, in: Schultze, H. (Hrsg.), Umweltreport, a.a.o., S. 206 .

106) Vgl. Dreyhaupt, F.J., Luftreinhaltung als Faktor der Stadtund Regionalplanung, a.a.0., s. 42 und Lahmann, E., Literaturstudie über die ökonomischen Konsequenzen der Schăden und Belästigungen, die durch die Luftverschmutzung durch Schwefeldioxid sowohl bel Materialien und der Vegetation als auch bei Mensch und Tier hervorgerufen werden, 0.0 .. 1974 , S. 31 .

107) Die B1ldung von photochemischem Smog stellt ein Beispiel für die Kombinationswirkung luftbelastender stoffe - hier von $\mathrm{NO}_{2}$ und ungesattigten Kohlenwasserstoffen - dar; unter dem ElnfluB des Sonnenlichtes (Photolyse) bilden sich dabel u.a. Ozon und PAN; besonders letzteres stellt ein starkes Pflanzengift dar (vgl. Becker, K.H./Schurath, U., Photochemie der Luftverschmutzung, in: Schultze, H. (Hrsg.), Umweltreport, a.a.0., S. $230 \mathrm{f}$. und RSU, Umweltgutachten 1974, a.a.0., s. 119). 
kungen auf die Pflanzen sind sich ähnlich: In allen Fällen wird die Photosynthese gestort; es kommt zu Nekrosen, Blattfall, Wachstumsbehinderungen und schlleßllch dem Absterben der Pflanzen 108)

$\left(\gamma_{2}\right)$ Tiere sind am meisten durch Fluor- und Blelimmissionen gefährdet, und zwar weniger durch Elnatmung als durch die Aufnahme via Niederschlăge mit Fluor- oder Bleiverbindungen kontaminierter Nahrung. Es kann zu Blelvergiftungen und zur sogenannten Fluorose, einer Knochenveränderung, kommen 109)

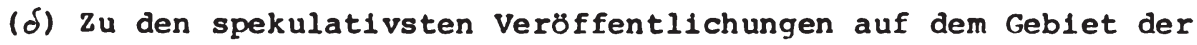
Umweltbelastung zăhlen diejenigen uber elne Modifikation des Klimas durch die Belastung der Luft mit Schadstoffen. Die Prognosen einiger Futurologen oder futurologisch argumentierender jkologen reichen vom globalen Hitzetod bzw. einer Uberschjemungskatastrophe bis zu einer bevorstehenden Elsze1t 110). Die "H1tzetod"- bzw. "Uberschwemmungskatastrophenvertreter" argumentieren folgendermaßen 111):

108) Vgl. Guderian, R., Wirkungen von Luftverunreinigungen auf Pflanzen, a.a.0.. S. 206 ff.: Dreyhaupt, F.J., Luftreinhaltung als Faktor der Stadt- und Reglonalplanung, a.a.o., S. 45 f.; RSU, Umweltgutachten 1974, a.a.0., S. 21 f. und S. 119; WMO, Air Pollutants, Meteorology, and Plant Injury, Technical Note No. 96, Genf 1968, S. 6 ff.; Materialienband zum Umweltprogramm der Bundesreglerung, a.a.0., S. 208; Olschowy, G., Katalog 8kolog1scher Noxen und der von thnen bedingten Schäden, in: Schaefer, H. (Hrsg.), Folgen der Zivilisation, a.a.0., s. 160.

109) Vgl. RSU, Umweltgutachten 1974, a.a.0., s. 21; Dreyhaupt, F.J.. Luftreinhaltung als Faktor der Stadt- und Regionalplanung, a.a.0., S. 42; Olschowy, G./Mrass, W./Kullmer, H. J./Bürger, K., Zur Belastung der Landschaft, a.a.0., s. 34 ; Egl1, E., Natur in Not, a.a.0., S. 40.

110) Vgl. z.B. Taylor, G.R., Das Selbstmordprogramm, Frankfurt/M. 1971 , S. $60 \mathrm{ff.}$

111) Vgl. Fritsch, B., Umwelt und Grenzen des Wachstums, in: Mitte1lungen der List-Gesellschaft, Fasc. $8(1973 / 74)$, Nr. 5, S. 92 ff.; McHale, J., Der ökolog1sche Kontext, a.a.0., S. $74 \mathrm{ff.;}$ Rönlcke, G., Die Atmosphäre, Ihre Entwicklung und gegenwärtige Situation, a.a.0., S. 45 f.; Schaefer, H., Die రkologie als Problem des Umweltschutzes, a.a.0., S. $108 \mathrm{f.:}$ WMO, Meteorology and the Human Environment, a.a.0., S. 23 . 
Der steigende Energleverbrauch in der Welt äußert sich wegen der Verbrennung fossiler Brennstoffe in einem exponentiellen Anstieg von $\mathrm{CO}_{2}$ in der Atmosphäre; da $\mathrm{CO}_{2}$ die nächtliche (relativ langwellige) Wärmeabstrahlung von der wăhrend des Tages durch die (relativ kurzwellige) Sonneneinstrahlung aufgeheizten Erdoberfläche absorblert, vermindert ein Anstieg von $\mathrm{CO}_{2}$ in der Atmosphäre zunehmend die Rückstrahlung von wärme an den Weltraum; es kommt also $z u$ einem allmählichen Anstieg der Temperatur auf der Erde ("Trelbhauseffekt") mit der Folge, daB die polaren Eisflächen und Gletscher in den Gebirgen schmelzen, wodurch der Wasserspiegel der Meere ansteigt und weite Landstriche uberschwemmt; es kommt zu einer polwärts gerichteten globalen Verlagerung der Klimazonen, was zu einer Versteppung z.B. der Mittelmeerländer und Kaliforniens führen würde 112) Gegen diese Folgen des $\mathrm{CO}_{2}$ spricht, daB die fossilen Brennstoffe erschopft sein werden, bevor die geschilderten Folgen eintreten können 113). Die Energie wird in zukunft immer mehr durch Kernkraftwerke erzeugt werden. Dadurch hat sich das Problem der Aufwärmung der Atmosphäre jedoch nur verlagert, denn die Kühlung der Kernreaktoren führt ebenfalls $z u$ einer thermischen Belastung der Atmosphäre 114) - entweder direkt via Abwärme der Kühltürme oder indirekt via thermische Belastung der Gewässer. Damit verlagert sich das Problem der Klimabeeinflussung allerdings auch von der globalen auf die

112) Vgl. Flohn, H./Fraedrich, K., Eingriffe in das Klima, in: Umwelt, 1973, 5, S. 21.

113) Vgl. Schaefer, H., Die Okologie als Problem des Umweltschutzes, a.a.0., S. 108.

114) Vgl. Kiese, O., Die Rolle künstlich erzeugter wärme in der Atmosphäre, in: Steubing, L./Kunze, C./ Jäger, J. (Hrsq.), Belastung und Belastbarke1t von Okosystemen, a.a.0., S. 178. 
lokale oder höchstens regionale Ebene ${ }^{115}$ ), nämlich auf die Umgebung der Kernkraftwerke: Es kann durch die Abwärme der Kernkraftwerke zur Beeinflussung des Mikroklimas kommen. Die Prognostiker einer neuen Eiszeit argumentieren, daß die Belastung der Luft mit Stäuben und die dadurch bedingte Dunstbildung $z u$ einer verringerten Sonneneinstrahlung und damit $z$ u einer Senkung der Tagestemperatur führt 116); dem steht entgegen, daB gerade dieser Dunst in der Nacht die Wärmeabstrahlung von der Erde behindert, so das die nächtliche Temperatur steigt. Andererselts stellen die Staubpartikel aber auch Kondensationskerne dar, die zu Nebel, Wolkenbildung und damit schließlich zu Niederschlägen führen 117), was sich temperatursenkend auswirkt.

Zusammenfassend kann man wohl sagen, daß in absehbarer $\mathrm{Zu}-$ kunft höchstens reglonale Modifikationen des Klimas durch die Belastung der Luft mit Schadstoffen (einschl. Abwärme) zu erwarten sind und die Art der Klimaveränderunq (Temperatur, Niederschlagshäufigkeit) von den regionalen bzw. lokalen Luftbelastungsverhältnissen abhängt.

(c) రkologische Wirkungen der Gewässerbelastung Die ökologischen Wirkungen, die von der Gewässerbelastung mit Schadstoffen ausgehen, hängen sehr stark vom Gewässertyp ab, vor allem vom Gewässervolumen, der Gewässertiefe und der Strömung. Ein kleines, flaches, stehendes Gewässer (z.B. ein Telch) wird

115) Vgl. Kiese, D., Die Rolle künstlich erzeugter wärme in der Atmosphäre, in: Steubing, L./Kunze, C./Jäger, J. (Hrsq.), Belastung und Belastbarke1t von Okosystemen, a.a.0., s. 178; Flohn, H., Klimaschwankungen und Klimamodifikation: Fakten und Probleme, in: Universitas, 28. Jg., 1973, S. 1297; Budyko, M.I., Das Klima der zukunft, in: Forschung 173, Frankfurt/M. 1972, S. 93; Landsberg, H.E., Man-made Climatic Changes, in: Sclence, vol. 170, 1970, Nr. 3964, S. 1268.

116) Vgl. Rönlcke, G., Die Atmosphäre, 1hre Entw1ckluna und gegenwärtige Situation, a.a.0., S. 48 f.; WMO, Meteoroloqy and the Human Environment, a.a.O., S. 32 .

117) Vgl. Landsberg, H.E., Man-made Climatic Chanqes, a.a.O., S. 1272 . 
von einer bestimmten Schadstoffart und-menge ceteris paribus stärker belastet als eln - um das andere Extrem zu nennen - Meer mit einer großen Tiefe und starken strömung, da die Selbstreinigungskraft eines Gewässers mit dessen Tlefe und strömung zunimmt 118)

Man kann die obologischen wirkungen der Gewässerbelastung in dre1 Gruppen eintellen: $(\alpha)$ in sauerstoffverzehrende wirkungen, ( $\beta$ ) In toxische wirkungen auf Pflanzen- und Tierwelt der Gewässer und $(\gamma)$ in Wirkungen auf das Klima. Die zwelte Gruppe von Gewässerbelàstungen führt wiederum zu elnem Sauerstoffverzehr via zersetzung der getöteten Pflanzen und Tiere 119)

(d) Die Sauerstoffbilanz elnes Gewässers ist ausgeglichen, wenn die Sauerstoffproduktion (z.B. durch die Photosynthese des Phytoplanktons) und die Sauerstoffzufuhr (aus der Luft) dem Sauerstoffverzehr (z.B. durch das Zersetzen toter Wasserbewohner) entsprechen. Dieses Gleichgewicht kann gestört werden, indem $\left(\alpha_{1}\right)$ der sauerstoffverzehr erhöht und/oder $\left(\alpha_{2}\right)$ die Sauerstoffproduktion bzw. die $\left(\alpha_{3}\right)$ sauerstoffzufuhr behindert werden. Alle drei Falle wollen wir anhand typischer Beispiele erläutern.

$\left(\alpha_{1}\right) \mathrm{zu}$ einer Erhohung des Sauerstoffverbrauchs kommt es in den Gewässern vor allem durch die Belastung mit stickstoffund Phosphorverbindungen. Stickstoffverbindungen gelangen in erster Linie durch Abschwemmung landwirtschaftlich genutzter Böden, die mit stickstoff gedungt wurden, in die Gewăsser; Phosphorverbindungen kommen ebenfalls via $\mathrm{Ab}-$ schwemmung phosphorgedüngter Böden in die Gewässer, daneben aber auch durch die Fäkalien und waschmittelabwässer der

118) Vgl. Llebmann, H., Die Wasserqualităt der oberbayerischen Seen, in: Olschowy, G. (Hrsg.), Belastete Landschaft - qefährdete Umwelt, a.a.0., S. 66 .

119) Vgl. U.S. Department of the Interior/Federal Water Pollution Control Administration. The Economics of Clean Water, Bd. 2, U.S.G.P.O., Washington, D.C., 1970, S. xi. 
Haushalte 120?

$\mathrm{N}$ - und P-Verbindungen stellen eine wichtige Nährstoffanrelcherung der Gewässer dar und führen vor allem $z u$ einem starken Wachstum des Phytoplanktons und damit aber auch der (tierischen) Lebewesen, die sich von dem Phytoplankton ernähren. Die Zunahme des Phytoplanktons hat zunächst einen durchaus positiven Einfluß auf den Sauerstoffhaushalt der Gewässer, denn das Phytoplankton sorgt ja durch Photosynthese für die Sauerstoffproduktion. Zum gesteigerten Sauerstoffverzehr kommt es erst durch die Zersetzung der gestlegenen Anzahl an Lebewesen 121) Reicht der Sauerstoff nicht mehr aus, um die Lebewesen $\mathrm{zu}$ zersetzen, sinken sie $z$ um Grund des Gewässers und beginnen dort zu faulen (anaerobe Zersetzung), wobel slch giftige Gase wie Schwefelwasserstoff $\left(\mathrm{H}_{2} \mathrm{~S}\right)$, Ammoniak und Methan bilden, welche wiederum $z u$ einem Sterben der Lebewesen im Wasser führen können 122) Diesen ganzen Vorgang bezelchnet man als Eutrophierung 123) Sie kann im Extremfall zur Verlandung eines Gewässers führen 124): "das Gewässer stirbt".

120) Vgl. U.S. Council on Environmental Quality, Environmental Quality, The First Annual Report, a.a.0., s. 31; Liebmann, H. , Die Wasserqualität der oberbayerischen Seen, a.a.0., S. 67; RSU, Umweltgutachten 1974, a.a.O., S. 55 f.; Materialienband zum Umweltprogramm der Bundesregierung, a.a. O., S. 99 f. und S. 129; Simonis, W., Eutrophieruna, in: Schaefer, H. (Hrsg.), Folgen der Zivilisation, a.a.0.,. S. 171 .

121) Vgl. Elster, H.-J., Okologische Probleme der Binnengewăsser, In: Forschung'73, a.a.0., S. 208; Schwoerbel, J., Blologie des Wassers, in: Schröder, B. (Hrsg.), Wasser, Frankfurt/M. 1970 , S. 234.

122) Vgl. Flrnhaber, R.B., Patient Bodensee. Wie lange noch Trinkwasserspeicher?, in: Umwelt, 1971, 3, S. 27; Klee, 0., Eine Klärwerke-Kur für den Bodensee, in: Kosmos, 1971, 12, S. 508; Olschowy, G. ,/Mrass, W./Kullmer, H.J./Bürger, K., Zur Belastung der Landschaft, a.a.0., S. 27.

123) Vgl. neben der $z u$ diesem Problemkreis angegebenen Literatur in den Fußnoten 118 bis 122 vor allem die ausführliche Untersuchung der OECD (Hrsg.). Eutrophication in Large Lakes and Impoundments, Uppsala Symposium, Report Prepared by C.P. Milway, Paris 1970.

124) Vgl. Simonis, W., Eutrophierung, a.a.0., S. 172. 
Neben diesem biochemisch bedingten Sauerstoffverzehr durch Eutrophierung gibt es noch den rein physikalisch bedingten Sauerstoffverbrauch in Gewässern durch deren thermische Belastung mit Kuhlwasser aus Kernkraftwerken 125); aber auch der biochemische Sauerstoffbedarf steigt durch die höhere thermische Belastung 126). Andererseits kann die Aufwärmung der Gewässer im Winter ökologisch positıve Wirkungen haben, da sie ein zufrieren der Gewässer verhindert 127)

$\left(\alpha_{2}\right)$ Die Sauerstoffproduktion in den Gewässern kann durch Zerstörung der an der sauerstoffproduktion beteiligten organismen erfolgen. Es muB nicht unbedingt eine Belastung der Sauerstoffproduzenten durch toxische stoffe sein 128); auch feste Stoffe, die in Gewässer abgelagert werden, können durch Zerstörung der Bodenfauna die Sauerstoffproduktion behindern 129)

$\left(\alpha_{3}\right)$ Die Sauerstoffzufuhr aus der Luft kann durch die Belastung eines Gewässers mit 81 verhindert werden 130 ).

$(\beta)$ Die Pflanzen- und Tierwelt der Gewăsser wird, wie berelts dargestellt, durch die Eutrophlerung und auch die thermische Belastung, die beide zu einem Sauerstoffverzehr führen, gefährdet. Die sauerstoffzehrende Belastung der Gewässer hat vor allem eine Gefährdung der Fische zur Folge ${ }^{131}$ )

125) Vgl. Könlg, H.-W., Thermlsche Belastung der Fließgewässer, in: Olschowy, G. (Hrsg.), Belastete Landschaft - gefährdete Umwelt, a.a.0., S. 57.

126) Vgl. ebenda, S. 58.

127) Vgl. The Economy, Energy, and the Environment. A Background Study prepared for the Use of the J.E.C., U.S.G.P.O., Washington, D.C., 1970, S. 96.

128) Vgl. Schwoerbel, J., Blologie des Wassers, a.a.0., S. 235.

129) Vgl. Materialienband zum Umweltprogramm der Bundesreglerung, a.a.0., S. 183 .

130) Vgl. Ant, H., Olverschmutzung der Meere und ihre Folgen, in: Olschowy, G. (Hrsg.), Belastete Landschaft - gefährdete Umwelt, a.a.0., s. 90 .

131) Vgl. The Economy, Energy, and the Environment, a.a.0., S. 95; Kön1g, H.-W., Thermische Belastung der Flleßgewăsser, a.a.0., S. 58 und RSU, Umweltgutachten 1974, a.a.0., S. 58 . 
Die ökologischen Wirkungen einer toxischen Belastung äußern sich in erster Linie in einer Ausrottung einzelner Arten und damit einer Verschlebung der Blozönosen; Schädlinge können überhand nehmen 132) Vor allem Mineralölprodukte gefährden die Biozönosen der im Wasser lebenden Tiere und Pflanzen 133)

Die Ausrottung kann auf verschiedene Weise vor sich gehen. Am spektakulärsten 1st die als రlpest bekannte Belastung von Seevögeln mit $\measuredangle 1$, das die Gefleder verklebt und so allmählich den Tod der Tiere herbelfuhrt 134) Neben dieser "mechanischen" Art der Tötung, können $\delta l$ und Mineralolprodukte aber auch toxisch wirken 135) Aufsehen erregten ferner akute Vergiftungen von Fischen, belspielsweise die zu einem großen Fischsterben fuhrende Belastung des Rheins durch das Insektizid Endosulfan (Thiodan) im Jahre 1969 136) Subtiler und gefährlicher sind allerdings chronische Vergiftungen etwa durch Pestizide oder Schwermetallverbindungen, die über die Nahrungskette $z u$ einer allmählichen Ansammlung der Giftstoffe bzw. Ihrer Metabolismen im Körper der Tiere führen und so entweder thren Tod (Seehunde, Seevögel, F1sche) oder Fortpflanzungsschäden bewirken. Am bekanntesten ist das Beispiel des DDT und seines Metaboliten DDE. Das in allen Weltmeeren nachgewiesene Pflanzenschutzmittel DDT wird vom

132) Vgl. Ant, H., Verschmutzung der Fließgewässer und ihre Folgen am Beispiel der Lippe und des Rheins, in: Olschowy, G. (Hrsg.), Belastete Landschaft - gefährdete Umwelt, a.a.o., S. $46 \mathrm{f}$.

133) Vgl. RSU, Umweltgutachten 1974, a.a.0., S. 56.

134) Vgl. Gerlach, S.A., Auswirkungen der Meeresverschmutzung auf das Leben im Meer und die Nahrungsketten, in: Schultze, H. (Hrsg.), Umweltreport, a.a.O., S. 156.

135) Vgl. Ant, H., blverschmutzung der Meere und ihre Folgen, a.a.0., S. 87 .

136) Vgl. Ant, H., Blologische Probleme der Verschmutzung und akuten Vergiftung von Fliebgewässern, unter besonderer Berücksichtigung der Rheinvergiftung im Sommer 1969, in: Schriftenreihe für Landespflege und Naturschutz, Heft 4 , Bonn-Bad Godesberg 1969, S. $97 \mathrm{ff}$. 
Phytoplankton, dem ersten Glied der Nahrungskette, im Meer aufgenommen; dieses wird vom zooplankton gefressen; das zooplankton dient kleineren Meerestieren, die wiederum von den Fischen gefressen werden, als Nahrung. Die Fische schließlich sind die Hauptnahrung von Seevögeln, Pinguinen, Robben. In jedem Glied der Nahrungskette sammelt sich das nur langsam abbaubare DDT (bzw. DDE) an, so daß schließlich für die höheren Lebewesen so starke Konzentrationen entstehen, daß sle vergiftet werden oder - wie z.B. bei den Kalifornischen Pelikanen - die Ansammlung von DDT in den Vogeleiern führt zu einer derartigen Verdünnung der Eierschalen, daß sie bei der Brut zerbrechen 137). Die Anwendung schnell abbaubarer Insektizide wie etwa des Phosphorsäureesters E 605 hatte zwar nicht diese negativen ökologischen Wirkungen; aber es wirkte zu undifferenziert und vernichtete auch Nutzinsekten 138 ?

( $\gamma$ ) Von der Eutrophierung mit der thermischen Belastung der Gewässer können Wirkungen auf das (Mikro-) Klima ausgehen. Die Eutrophierung kann via Verlandung eines Gewässers die Verringerung des Niederschlags bewirken; die thermische Belastung der Gewässer führt dagegen via stärkere Verdunstung zur größeren Nebel- und Niederschlagsbildung 139 ?

(d) Ökologische Wirkungen der Bodenbelastung Wir wollen die ökologischen Wirkungen der Bodenbelastung $(\alpha)$ in Wirkungen auf den Boden selbst, $(\beta)$ in Wirkungen auf die beiden anderen Umweltmedien Luft und Gewässer, ( $(\gamma)$ in Wirkungen auf die Pflanzen- und Tierwelt und $(\delta)$ in Wirkungen auf das Klima ein-

137) Vgl. Gerlach, S.A., Auswirkungen der Meeresverschmutzung auf das Leben im Meer und die Nahrungsketten, a.a.O., S. 157.

138) Vgl. Klausewitz, W./Schäfer, W./Tobias, W., Umwelt 2000, a.a.0., s. 40.

139) Vgl. The Economy, Energy, and the Environment, a.a.o., S. 96 . 
teilen.

(a) Die größte Belastung für den Boden selbst erfolgt durch den Eingriff des Menschen in den natürlichen Stickstoffkreislauf durch die Düngung mit stickstoff. Die Folge ist eine Humusverarmung des Bodens verbunden mit einer Nitratauswaschung 140), was durch eine weitere stickstoffdüngung kompensiert werden soll. Es kommt $\mathrm{zu}$ einem kumulativen Proze $\beta$ der Humusvernichtung, der bel nicht rechtzeitigem Eingreifen mit der Erosion des Bodens 141) und damit einer Abschwemmung der in der Humusdecke befindlichen pflanzlichen Nährstoffe, vor allem von Stickstoff, Phosphor und Kalium endet 142 )

( $\beta$ ) Damit sind wir bereits bei den ökologischen Wirkungen der Bodenbelastung in anderen Umweltbereichen. Wir haben oben bereits dargestellt, wie Stickstoff- und Phosphorverbindungen durch Abschwemmung gedüngter Böden zur Entrophierung der Gewässer beltragen. Auch andere auf oder im Boden befindliche Schadstoffe wie Abfälle 143) und Pestizide 144) können durch Abschwemmung in die Oberflächengewässer und durch Versickerung ins Grundwasser gelangen, und dies um so mehr, je dünner die Humusdecke ist, die eine Filterung und

140) Vgl. Borneff, J., Schadstoffe im Wasser: Herkunft, Bedeutung und Beseitigung, in: Zentralblatt für Bakteriologie, Parasitenkunde, Infektionskrankheiten und Hygiene, I. Abt. Orig. B., Bd. 155, Heft 3, 1971, S. 222.

141) Vgl. Preuschen, G., Der Einfluß der Landwirtschaftsformen auf die Anderung in der natürlichen Umwelt, in: Sioli, $H$. (Hrsg.), Okologie und Lebensschutz in internationaler Sicht, a.a.0., s. 273.

142) Vgl. Kovda, V.A., Boden, Mensch und Biosphäre, in: Aufgabe zukunft - Qualität des Lebens, Frankfurt/M. 1972, Bd. 4, S. 120.

Wir verkennen dabei nicht die positive Wirkung der Düngung auf die Nahrungsmittelproduktion.

143) Vgl. Olschowy, G./Mrass, W./Kullmer, H.J./Bürger, K., Zur Belastung der Landschaft, a.a.0., s. 17 und 53.

144) Vgl. Domsch, K.H., Zum Problem der Biozide, in: Olschowy, G. (Hrsg.), Belastete Landschaft - gefährdete Umwelt, a.a. O., S. 144 . 
Umwandlung vieler Schadstoffe vornimmt 145)

(y) In erster Linie wirkt sich die Bodenbelastung jedoch auf das Pflanzenwachstum aus 146), entweder via Schädigung der Humusdecke 147) oder via Belastung der Bodenfauna mit Pestiziden und die folgende Aufnahme der mikrobiellen wirkstoffmetaboliten durch die Pflanzen 148)

Die flächenmäßlge Belastung des Bodens durch Baumaßnahmen aller Art geht natürlich auch $z$ u Lasten der Vegetation, aber ebenso (wegen der Biotopvernichtung) $\mathrm{zu}$ Lasten der Tierwelt 149). Auch der Straßenverkehr führt zum Tod vieler Tiere 150)

$(\delta)$ Beeinflussungen des (Mikro-)klimas gehen von der flächenmäBigen Belastung des Bodens durch Bebauung aus. So wird das Windfeld durch die künstliche "Oberflächenrauhigkeit" beeinflußt, die durch die Bebauung der Landschaft mit siedlungen entsteht 151) Die Baumasse (Häuser, Straßen) speichert die Sonnenwärme 152) Die durchschnittliche Temperatur ist in städtischen Gebieten zum einen deshalb, aber auch wegen der

145) Vgl. Ulrich, B., Die Filterfunktion von Böden, in: Steubing, L./Kunze, C./Jäger, J. (Hrsg.), Belastung und Belastbarke1t von Okosystemen, a.a.0., S. 169; Benecke, P., Okologische Probleme des Wasserhaushalts auf dem Lande, in: Probleme der Umweltforschung, a.a.0., S. 114.

146) Vgl. Olschowy, G., Zur Belastung des Bodens, in: Olschowy, G. (Hrsg.), Belastete Landschaft - gefährdete Umwelt, a.a.0., S. 141 .

147) Vgl. Preuschen, G., Der Einfluß der Landwirtschaftsformen auf die Anderung in der natürlichen Umwelt, a.a.0., s. $273 \mathrm{f}$.

148) Vgl. Schaefer, H., Die Okologie als Problem des Umweltschutzes, a.a.O., S. 119; Kovda, V.A., Boden, Mensch und Biosphäre, a.a.0., s. $130 \mathrm{f}$.

149) Vgl. RSU, Umweltgutachten 1974, a.a.O., S. 133 f.; Olschowy, G., Katalog ökologischer Noxen und der von thnen bedingten Schäden, a.a.0., S. 165; Olschowy, G./Mrass, W./Kullmer, H. $\mathrm{J} . /$ Bürger, K., Zur Belastung der Landschaft, a.a.0., S. 43.

150) Vgl. ebenda, S. 43; Olschowy, G., Katalog ökologischer Noxen und der von ihnen bedingten Schäden, a.a.0., S. $166 \mathrm{f}$.

151) Vgl. Schmidt-Burbach, G.M., Klimaänderung im urbanen Bereich, a.a.0., S. 24 .

152) Vgl. ebenda. 
dort vorhandenen künstlichen wäremquellen und des geringeren Windes größer und schwankt zwischen Tag und Nacht weniger als in ländlichen Gebieten 153)

(e) Ökologische Wirkungen der Vegetations- und Tierweltbelastungen

Fauna und Flora werden indirekt durch die oben geschilderten Belastungen der Umweltmedien Luft, Wasser und Boden geschädigt. zu unmittelbaren Schäden an Pflanzen und Tieren kommt es durch die Verwendung von Pflanzenschutzmitteln. Obwohl diese Mittel - wie ihr Name sagt - den Schutz der Pflanzen zur Aufgabe haben, führen sie bel undifferenziertem Einsatz 154) zu Pflanzen- und Tierschädigungen ${ }^{155)}$ und damit $(\alpha)$ zu einer beträchtlichen Störung des ökologischen Gleichgewichts, $(\beta)$ zu Auswirkungen auf die Umweltmedien Luft, Wasser und Boden und $(\gamma)$ zur Beeinträcht1gung des Klimas.

(d) Das ökologische Gleichgewicht kann durch Ausrottung oder Schädigung einer Pflanzen- oder Tlerart gestört werden, weil diese Ausrottung $z u$ einem Uberhandnehmen (Schädlinge) oder der Belastung der Pflanzen- und Tierarten (wegen Nahrungsentzugs) führen kann, so daß wieder korriglerende Maßnahmen notwendig sind, die ihrerselts wiederum ökologisch nachteilige Folgen haben können ${ }^{156}$ ) Die nicht beabsichtigte Schä-

153) Vgl. McCormick, R.A., Meteorological Aspects of Air Pollution in Urban and Industrial Districts, in: WMO, Meteorological Aspects of Air Pollution, a.a.O., s. 7.

154) Besonders negativ wirkt sich die Besprühung von Wäldern mit Herbiziden zwecks Unkrautvernichtung aus (vgl. Wellenstein, G., Chemie aus dem Hubschrauber, in: U, 1974, 2, S. $36 \mathrm{f.})$.

155) Zu den Klassikern der Umweltschutzliteratur zählt das Buch von Rachel Carson, Der stumme Frühling, München 1970, das besonders eindrücklich auf die Gefahren der pflanzenschutzmittel für die Tierwelt hinweist.

156) Zu einigen Beisplelen vgl. RSU, Umweltgutachten 1974, a.a.0., S. $144 \mathrm{ff}$.; Kurir, A., Chemische Pflanzenbekämpfung - Gefahr für Mensch und Tier, Teil $I$, in: $U, 1974,4, S .23$; Olschowy, G./Mrass, W./Kullmer, H.J./Bürger, K., Zur Belastung der Landschaft, a.a.0., s. $43 \mathrm{ff}$. 
digung von Tieren durch die Verwendung von Pestiziden muß nicht durch direkte Aufnahme des Schadstoffes erfolgen, sondern kann auch wieder über die Nahrungskette geschehen 157) Die Liste der in den letzten 300 Jahren ausgerotteten Säugetier- und Vogelarten (durch alle Arten der Belastung) ist beeindruckend 158) Sicher nicht so spektakulär, aber vielleicht b̈kologisch nicht minder schädlich ist die Gefährdung vieler tausender von Pflanzenarten 159) denn das Verschwinden jeder Tier- oder Pflanzenart bedeutet eine Verringerung der Stabilität der Biosphäre: "Der Wert eines రkosystems ist eng mit seiner vielfalt verbunden"160)

( $\beta$ ) Die Ausrottung von Pflanzen oder gar ganzen wäldern hat auch Auswirkungen auf die Umweltmedien. Pflanzen und vor allem Wälder üben eine beträchtliche Filterfunktion in bezug auf die staubbelastung der Luft und auch den Lärm aus 161) Besonders Waldboden ist gut zur Spelcherung von Regenwasser geelgnet 162) er sorgt damit für eine glelchmäßige wasservertellung: In Regenzelten werden Uberschwemmungen verhindert, in Trockenzeiten dient er als Wasserreservolr 163). Pflanzen sind die Voraussetzung der Humusbildung, denn Humus ist

157) Vgl. Olschowy, G., Katalog ökologischer Noxen und der von thnen bedingten Schäden, a.a.0., S. 165; Passino, R. , Industrie und Landschaft, in: Offner, H. (Hrsg.), Die Zukunft der Landschaft in Europa, München 1971, S. 44; Maldague, M.E., Landwirtschaft und Forstwesen, in: Offner, H. (Hrsg.), Die Zukunft der Landschaft in Europa, a.a.0., S. 94 .

158) Vgl. Klausewitz, W./Schäfer, W./Toblas, W., Umwelt 2000 , a.a.0., S. $10 \mathrm{ff}$.

159) Schmid, R., Gefährdete Pflanzen in der heutigen Welt, in: Universitas, 28. Jg. 1973, S. $1351 \mathrm{ff}$.

160) Maldague, M.E., Landwirtschaft und Forstwesen, a.a.O., S. 103.

161) Diese Schutzfunktion darf natürlich nicht überstrapaziert werden, well sonst die wälder selbst Schaden nehmen, vgl. o. S. $64 \mathrm{f}$.

162) Vgl. Preuschen, G., Der EinfluB der Landwirtschaftsformen auf die Anderung in der natürlichen Umwelt, a.a.0., S. 272 .

163) Vgl. Olschowy, G., Katalog ökologischer Noxen und der von thnen bedingten Schäden, a.a.O., S. 159 . 
nichts anderes als der Rückstand (noch) nicht abgebauter Pflanzen 164). Die Ausrottung von Pflanzen oder gar ganzen Wallern bedeutet also letztlich die Vernichtung der Humusschicht mit der Folge der Erosion 165)

( 5 ) Die Vegetationsbelastung etwa in Form der Vernichtung eines Waldgebletes kann zu einer Veränderung der klelnklimatischen Verhältnisse führen 166). Die Abholzung eines waldes mit der nachfolgenden Erosion ist der klassische Fall lokaler Kl1mabeeinflussung 167). Die Folgen sind Temperaturerhöhung, Rückgang der Luftfeuchtigkeit und Niederschläge und eine Erhöhung der Windgeschwindigkeit an der Erdoberfläche ${ }^{168}$ ?

(2) Wirkungen auf die materielle Umwelt (Sachgüter)

(a) Systematisierung der Wirkungen

Die Belastung aller drel Umweltmedien kann zu schädlichen Wirkungen auf Sachgüter (von der Kleidung über das Auto bis zum Kunstdenkmal) führen. Wir wollen diese Wirkungen getrennt für die einzelnen Umweltmedien untersuchen.

(b) Wirkungen der Luftbelastung auf die gebaute Umwelt Am vielfältigsten und auch am meisten untersucht sind die wirkungen der Luftbelastung auf eine Reihe von Sachgütern. Wir können unterscheiden zwischen Wirkungen auf Bauwerke, wobei h1storische Bauwerke oder auch Kunstdenkmäler eine besondere

164) Vgl. Preuschen, G., Der EinfluB der Landwirtschaftsformen auf die Anderung in der natürlichen Umwelt, a.a.o., S. 272.

165) Vgl. Kovda, V.A., Boden, Mensch und Biosphäre, a.a.O., S. 125; Olschowy, G./Mrass, W./Kullmer, H.J./Bürger, K., zur Belastung der Landschaft, a.a.O., S. 41; Benecke, P., Ckologische Probleme des Wasserhaushalts auf dem Lande, a.a. O., S. 41.

166) Vgl. Olschowy, G./Mrass, W./Kullmer, H.J./Bürger, K., Zur Belastung der Landschaft, a.a.0., s. 41.

167) Vgl. Landsberg, H.E., Man-made Climatic Changes, a.a.O., S. 1269 .

168) Vgl. ebenda und Budyko, M.I., Das Klima der Zukunft, a.a.O., S. 87 . 
Beachtung verdienen, Metalle und Textilien 169)

Als Ursache für das Brüchigwerden von Natursteinbauten und die Korrosion von Metallen (z.B. bei Kraftfahrzeugen) wird die Belastung der Luft vor allem mit Schwefeldioxid, Chlorwasserstoff und katalytisch wirkenden schwermetallpartikeln angegeben 170? Besonderes Aufsehen haben in letzter zeit die Schäden an unwiederbringlichen historischen Bauwerken wie der Akropolis 171), dem Kölner Dom 172) oder dem Freiburger Münster 173) durch die $\mathrm{SO}_{2}$-Belastung erregt. Man stellt sich den Mechanismus der Zerstörung so vor, daB das $\mathrm{SO}_{2}$ mit dem Kalkstein, Marmor, Schiefer oder Mörtel an den Gebäuden reagiert und zu sulfat wird, das dann durch den Regen fortgewaschen w1rd 174). Die Ursache für den Zerfall von Bauwerken ist natürlich nicht nur die Luftbelastung mit Schadstoffen; auch die witterung spielt dabel eine Rolle, und es ist zumindest sehr schwer, wenn nicht gar unmöglich zu sagen, wieviel von der zerstörung auf die Luftbelastung und wieviel auf die Verwltterung zurückzuführen ist 175)

169) Vgl. Materialienband zum Umweltprogramm der Bundesreglerung, a.a.0., S. 209; Dreyhaupt, F.J., Luftreinhaltung als Faktor der Stadt- und Regionalplanung, a.a.0., S. $46 \mathrm{ff}$.

170) Vgl. ebenda, S. 47; RSU, Umweltgutachten 1974, a.a.0., S. 22; Lahmann, E., Literaturstudie über die ökonomischen Konsequenzen ...., a.a.0., S. $24 \mathrm{f}$.

171) Vgl. o.V., Krebs zerstört die Akropolis, in: U, 1974, 4, S. 12 .

172) Vgl. o.v., Der Dom im Wettlauf mit der Zeit, in: Umwelt, $1972,6, \mathrm{~S} .38 \mathrm{f}$.

173) Vgl. Rönicke, G., Die Atmosphäre, ihre Entwicklung und gegenwärtige Situation, a.a.0., S. $35 \mathrm{ff}$.

174) Vgl. Lahmann, E., Literaturstudie über die ökonomischen Konsequenzen ..., a.a.0., S. 25 .

175) Von manchen Autoren wird bestritten, daß die $\mathrm{SO}_{2}$-Belastung der Luft für den Zerfall von Bauwerken verantwortlich ist; sie glauben thn allein auf die Verwitterung zurückführen zu können; vgl. dazu Riederer, J., Plädoyer für die Verwitterung. Steinschäden auch ohne Rauchgas, in: Umwelt, 1974, $1,5.42 \mathrm{f}$. und Frey, P., Streit um den Zerfall von Baudenkmälern: Luftverunreinigung oder Verwitterung?, in: Umwelt, 1973, 2, S. 22 f. 
Bei den Metallen sind vor allem Zink, Nickel und Eisen wegen der $\mathrm{SO}_{2}$-Belastung einer verstärkten Korrosion ausgesetzt ${ }^{176}$ ) Aber auch andere Schadstoffe in der Luft wie Chlor- und Fluorwasserstoff und Stickoxide greifen Metalle an 177).

Textilien und auch Leder werden ebenfalls von der $\mathrm{SO}_{2}$-Belastung aber auch $z$.B. von Stäuben beschädigt ${ }^{178}$ )

(c) Wirkungen der Gewässerbelastung auf Sachgüter

D1e thermische Belastung der Gewässer führt zu einer Erhöhung der Korrosionsgeschwindigkeit ${ }^{179}$ ) Das kann vor allem zu Schäden an Schiffen und Unterwasserleitungen führen.

(d) Wirkungen der Boden- oder Vegetationsbelastung auf Sachgüter Boden- oder Vegetationsbelastungen wie etwa die Vernichtung eines Waldes mit nachfolgender Erosion können vor allem 1m Hochgeb1rge zu Schäden an Sachgütern führen, denn wälder sind ein guter Schutz gegen Schneelawinen ${ }^{180)}$; Erosionen begünstigen Steinlawinen.

bb) Die Wirkungen der Schadstoffe auf die menschliche Gesundheit (1) Zur Definition der menschlichen Gesundhe1t Für elne Bewertung der Schadstoffwirkungen ist es notwendig, den Begriff der menschlichen Gesundhelt zu definieren. Wir wollen die Definition der World Health Organisation (WHO) übernehmen, die auch nach Aussage von Medizinern "Leitlinie ökologischer

176) Vgl. Lahmann, E., Literaturstudie über die ökonomischen Konsequenzen ..., a.a.0., S. 24 .

177) Vgl. Dreyhaupt, F.J., Luftreinhaltung als Faktor der Stadtund Regionalplanung, a.a.0., S. 47.

178) Vgl. Lahmann, E., Literaturstudie über die ökonomischen Konsequenzen ..., a.a.0., S. 24 .

179) Bei elner Erhöhung der Wassertemperatur um $10^{\circ} \mathrm{C}$ verdoppelt sich die Korrosionsgeschwindigkeit (vgl. König, H.-W., Thermische Belastung der Fließgewässer, a.a.0., S. 59).

180) Vgl. Olschowy, G., Katalog ökologischer Noxen und der von ihnen bedingten Schäden, a.a.O., S. 159 . 
Forderungen werden (muß)" 181): "Die Gesundhe1t ist der Zustand des vollständigen körperlichen, geistigen und sozialen Wohlbefindens und nicht nur das Freisein von Krankhelt und Gebrechen" 182) Diese Definition scheint auf den ersten Blick sehr anspruchsvoll zu sein, da sle neben dem körperlichen wohlbefinden auch das - sehr subjektiv bedingte - geistige (besser vielleicht seelische) und soziale wohlbefinden fordert. Damit wird ausgesagt, daB auch die seelisch labilen und sensiblen Menschen sich wohlfühlen sollen. Dies schelnt vor allem deswegen gerechtfertigt, da störungen des seelischen und sozlalen Wohlbefindens zu körperlichen Krankheiten bel den Betroffenen führen können und auch - etwa via Aggresivität - zu Störungen des wohlbefindens der Mitmenschen.

Mag es noch relativ einfach sein, physisches Wohlbefinden zu definteren, so 1st es sehr schwierig ein Maß für seelisches oder gar soziales Wohlbefinden zu bestimmen 183) Gerade letztere können aber durch verschiedene Arten der Umweltbelastung hervorgerufen werden 184) Da es auch von individuelien Faktoren (körperliche und seelische Konstitution, Erziehung u.v.a.m.) abhängt, ob sich ein Mensch gesund fühlt, läBt sich kein "gesunder standardmensch" definieren - Gesundheit nach der Defination der wHO ist ein relativer Begriff 185). Die gesundheltliche Belastbarkeit durch Schadstoffe hängt - auf physische Faktoren beschränkt z.B. von Alter, Geschlecht, Konstitution und Disposition des

181) Klosterkotter, w., Lärmwirkungen und Lebensqualităt, in: U. 1973, 3, S. 19 .

182) Satzung der wно, Präambel, abgedruckt in: Dokumente, Heft 6 , hrsg. von der Forschungsstelle für völkerrecht und ausländisches öffentliches Recht der Universität Hamburg, Frankfurt 1952 .

183) Vgl. WHO, Measurement of Levels of Health, Technical Report Series No. 137, Genf 1957, S. 17.

184) Vgl. dazu unten S. $90 \mathrm{ff}$.

185) Vgl. Lüth, P., Was 1st Gesundheit? - Was ist Krankhe1t?. In: Die Neue Gesellschaft, 20. Jg., 1973, S. 832 . 
einzelnen Menschen ab

Die Menschen sind mit anderen Worten gegenüber gewissen Umweltbelastungen - Individuell unterschied$11 \mathrm{ch}$ - adaptionsfähig 187). Diese Anpassungsfähigkeit ist jedoch begrenzt. Auch sehr geringe, aber hăufig auftretende Belastungen können $\mathrm{zu}$ irreparablen Gesundheitsschäden führen 188) . Für konkrete gesundheitspolitische Maßnahmen - und zu ihnen gehört der Umweltschutz! - genügt diese allgemelne Definition der Gesundheit nicht. Eine Operationalisierung des Gesundheitsbegriffs läßt sich jedoch erst vornehmen, wenn die wirkungen der schadstoffe auf die menschliche Gesundhelt bekannt sind. Bevor wir uns jedoch den einzelnen schadstoffwirkungen auf die Gesundheit zuwenden, wollen wir uns mit einigen methodischen problemen der Feststellung dieser Schadstoffwirkungen befassen.

(2) Probleme bei ler Erfassung der Schadstoffwirkungen Der Nachweis eines Zusammenhangs zwischen einer Krankheit und elner Immissionsbelastung ist ein großes ätiologisches Problem 189) dessen Lösung aber u.a. dann erforderlich 1st, wenn man das Verursacherprinzip bei der Finanzierung des Umweltschutzes anwenden 190) oder aber gar den Umweltschutz überhaupt rechtfertigen will. Die Schwierigkeiten entstehen dadurch, daß es meist mehrere Ursachen für die glelche Krankhelt gibt (multifactorial etiology) 191) Der Nachwe1s, daß gerade die Immis-

186) Vgl, auch die Definition von Krankheit, In: Zetkin/ Schaldach, Wörterbuch der Medizin, 5. Aufl., Bd. 2, München 1974, S. 758 .

187) Lowenstein, F.W.. Man's Health as Depending on His Environment, in: Sioli, H. (Hrsg.), Okologie und Lebensschutz in internationaler sicht, a.a.O., S. 245.

188) Vgl. Dubos, R., Man, Medicine, and Environment, Harmondsworth 1970, S. 123.

189) Die Atiologie 1st die Lehre von den krankheitsverursachenden Faktoren; vgl. Borneff, J., Hyglene, Ein Leitfaden für Studenten und Ärte, Stuttgart 1971, S. 9 und Lüth, P., Was ist Gesundheit? - Was ist Krankhe1t?, a.a.O., S. 834 .

190) Vgl. Repenning, K., Das Verursacherprinzip, in: Zur Prolematik des Verursacherprinzips, a.a.0., s. 9.

191) Vgl. Dubos, R., Man, Medicine, and Environment, a.a.0., S. $105 \mathrm{f}$. 
sionsbelastung fur die Krankheit verantwortlich 1st, wird daher kaum möglich sein. Hinzu kommt, daß in der Regel mehrere Schadstoffe gleichzeltig auf den Menschen einwirken, so daß die Identifikation des krankheitsverursachenden Faktors schwierig ist ${ }^{192}$ ) Noch komplizierter wird die Feststellung der krankheltsauslösenden Schadstoffe bei Kombinationswirkungen oder gar bei synerglstischen Effekten 193) Kaum einfacher ist der Nachweis chronischer Wirkungen der Umweltbelastung 194), da die verursachende Immissionsbelastung und das Auftreten der Krankheit oft Jahrzehnte - bel Erbschäden gar Generationen! - auseinanderliegen können 195) Umgekehrt ist der Nachweis relativ einfach, wenn Immission und Krankhelt oder gar Tod zeltlich dicht zusammenliegen, also bel akuten Schäden 196) Deshalb werden auch vor allem die Smog-Katastrophen von Maastal (1930), Donora (1948) und London (1952) zitiert, wenn es un den Nachweis geht, daß Umweltbelastungen zu Krankheit und gar Tod führen können 197)

192) Vgl. Cassell, E.J., The Health Effects of Air Pollution and their Implications for Control, in: Law and Contemporary Problems, vol. 33, 1968, S. $198 \mathrm{f}$.; Olschowy, G. , Auswirkung der Luftverunreinigung auf den Menschen, in: Olschowy, G. (Hrsg.), Belastete Landschaft - gefährdete Umwelt, a. a.0., S. 127 .

193) Vgl. Schnelder, Wolfgang, Verursacherprinzip oder die Belastung aller?, in: Gumpel, W./Keese, D. (Hrsg.), Probleme des Industrialismus in Ost und West, a.a.O., S. 473. Ein synergistischer Effekt liegt vor, wenn der kombinierte Effekt der Schadstoffe größer ist als die summe der Einzeleffekte (vgl. ebenda).

194) Vgl. Schaefer, H., Der Mensch in der technisch veränderten Welt, in: Schaefer, H. (Hrsg.), Folgen der Zivilisation, a.a.O.. S. 34; Dubos, R., Promises and Hazards of Man's Adaptability, in: Jarrett, H. (Hrsg.), Environmental Quality in a Growing Economy, 3. Aufl., Baltimore und London 1971 , S. 25 .

195) Vgl. Marquardt, H., Die Auslösung von Erbschäden und Krebs durch Umweltfaktoren, in: Universitas, 28. Jg., 1973, S. 1315 .

196) Vgl. ebenda, S. 1314 f.; Schlipköter, H.-W./Dolgner, R., Epidemiologische Untersuchungen im Zusammenhang mit lufthygienischen Fragen, in: Zentralblatt für Bakteriologie.... a.a.0., S. 274 .

197) Vgl. Cassell, E.J.., The Health Effect of Air Pollution and their Implications for Control, a.a.0., S. 204. 
M1t den soeben skizzierten Problemen hat vor allem die epidemiologische Methode, die im folgenden Abschnitt dargestellt wird, zu kämpfen. Andere Methoden zum Nachweis eines Ursache-Wirkunaszusammenhangs zwischen Immission und Krankheit sind Laboratoriumsversuche mit Tieren und die Heranzlehung gewerbetoxikologischer bzw. arbeitsmedizinischer Untersuchungen. Auch sie sollen im nächsten Abschnitt dargestellt und 1hre Vor- und Nachtelle gegenüber der Epidemiologie abgewogen werden.

(3) Methoden zur Erfassung der Schadstoffwirkungen auf die Gesundhe1t

(a) Epidemiologische Studien

Mit Hilfe von epidemiologischen studien wird versucht, einen $\mathrm{zu}-$ sammenhang zwischen Morbiditäts-bzw. Mortalitätsraten elnerseits und Immissionsbelastung andererseits nachzuweisen. Man bedient slch dabel statistischer Methoden von der - sicher unaeelgneten - einfachen Korrelationsrechnung bis hin zur multiplen Regression und Diskriminanzanalyse 198). Die Untersuchungen basieren auf den Morbiditäts- und Mortalitätsstatistiken, die tellweise erhebliche Mängel aufwelsen. Die Morbiditätsstatistik welst u.a. wegen Selbstbehandlungen grobe Lücken auf 199) und die Mortalitätsstatistik, in der die Todesursachen erfaBt werden, ist problematisch, da oft mehrere Krankheiten vorhanden sind, die den Tod herbeigefüht haben können 200) Ob die Daten über die Immissionskonzentration verschledener schadstoffe quantitat $1 \dot{v}$ und qualitativ den Ansprüchen elner glaubwürdigen epidemiolo-

198) Vgl. Lawther, P.J/Martin, A.E./Wilkins, E.T., Epidemioloay of Alr Pollution, Report on a Symposium, WHO Public Health Papers, No. 15, Genf 1962; Gardner, M.J.; Using the Environment to Explain and Predict Mortality, in: Journal of the Royal statistical Society, vol. 136, 1973, S. 428; Lave, L.B./Seskin, E.P., Health and Air Pollution, in: Swedish Journal of Economics, vol. 73, 1971, S. $76 \mathrm{ff}$.

199) Vgl. Borneff, J., Hyglene, a.a.O., s. 3.

200) Vgl. ebenda, S. 7. 
gischen studie genügen, möchten wir ebenfalls bezweifeln 201) $\mathrm{Ob}$ der Nachwels gesundheitsschädlicher Wirkungen der Umweltbelastung anhand von Veränderungen der Morbiditätsrate oder der Mortalitätsrate erfolgen soll, ist umstritten. Lave und Seskin z.B. verwenden in 1 hrer Untersuchung die Mortalitătsrate 202) was von einigen Autoren deshalb befurwortet wird, we1l die Morbiditässtatistiken unbefriedigend sind 203) Andere Autoren halten dagegen die Morblditatsrate fur eine bessere GröBe, da die Mortalitătsrate etwa infolge irgendeiner kurzfristig hohen Immissionskonzentration beträchtlich uber den langfristigen Trend ansteigt, bald danach aber ebenso beträchtlich unter diesen langfristigen Trend sinkt, so daß der Tod infolge der Immissionsbelastung nur etwa 24-48 stunden früher elngetreten ist 204) Ob man in solchen Fallen noch von einem ursächlichen Zusammenhang zwischen Tod und Immissionsbelastung sprechen kann, scheint zumindest zweifelhaft. Die bisher geschilderten Schwierigkeiten epidemiologischer studien lassen slch durch elne Verbesserung der Mortalitäts- und Morbiditätsstatistiken einerselts und die Einfuhrung von Immissionskatastern andererseits zumindest stark vermindern. Die eigentliche Crux besteht darin, daB eine Vielzahl von Schadstoffen gleichzeltig auf die Gesundheit wirken und so auf Grund epidemiologischer studien bestenfalls unspezifische Wirkungen feststellbar sind, etwa der Art, daß mit dem Anstelgen der Luftverschmutzung die sterblichkelt oder die Haufigkeit einer be-

201) Hier wird die Bedeutung der Aufstellung von Immissionskatastern besonders klar. Vgl. auch van Belle, G./Schnelderman, M. , Some Statistical Aspects of Environmental Pollution and Protection, a.a.0., S. 321 .

202) Vgl. Lave, L.P./Seskin, E.P., Health and Air Pollution, a.a.0., S. 76 ff.

203) Vgl. Schaefer, H., Hinwelse auf Umweltschäden aus Lebenserwartung, spezifischen Sterblichkeiten, Sterbeziffern und Krankheitshăufigkeiten, in: Schaefer, H. (Hrsg.), Folgen der Zivilisation, a.a.0., s. 73.

204 ) Lawther, P.J./Martin, A.E./Wilkins, E.T., Epidemiology of A1r Pollution, a.a.O., S. 24 . 
stimmten Krankhelt steigt 205) Damit ist jedoch noch keineswegs der Tod oder Krankhelt verursachende Schadstoff bestimmt 206) Um auf Grund epidemiologischer Untersuchungen Aussagen der Art machen zu können, daB ein bestimmter schadstoff in elner bestimmten Konzentration eine bestimmte Krankheit hervorruft, müBten die Morbiditätsraten zweier völlig homogener Bevölkerungsgruppen, die sich nur im Grad der Belastung durch diesen Schadstoff unterschelden, verglichen werden 207) Solche "idealen" Voraussetzungen lassen sich in der Realität jedoch kaum finden. Es ist zwar mögl1ch, bestimmte störfaktoren (z.B. Z1garettenrauchen) auszuschalten, indem man besonders geeignete Beobachtungsobjekte (z.B. Kinder) wăhlt 208), aber noch so große Verfeinerungen der epidemiologischen studien können nichts daran ändern, daß sie nicht geelgnet sind, einen eindeutigen Nachweis für einen Ursachen-Wirkungszusammenhang zwischen bestimmten Immissionskonzentrationen und Mortalitäts-bzw. Morbiditätsraten zu liefern; die festgestellten zusammenhănge haben lediglich Wahrscheinlichkeitscharakter 209)

\section{(b) Laborversuche mit Tieren}

Tierversuche ermöglichen das, was bel epidemiologischen studien praktisch ausgeschlossen 1st: die isolierte Betrachtung der Wirkung eines Schadstoffs mit variabler Dosierung und Konzentra-

205) Vgl. Schaefer, H., Hinweise auf Umweltschäden ..., a.a.0., S. 83 .

206) Vgl. Hettche, H.0., Gesundheit und Großstadtluft, in: Staub, Bd. 21, 1961, S. 50 .

207) Vgl. Cassell, E.J., The Health Effect of Air Pollution and their Implications for Control, a.a.0., S. $200 \mathrm{f}$.: Lawther, P.J./Martin, A.E./Wilkins, E.T., Epidemiology of Air Pollution, a.a.0., S. $18 \mathrm{f}$.

208) Vgl. Schlipköter, H.-W., Belastungen des Menschen durch die Umweltverschmutzung, in: Probleme der Umweltforschung, a.a. O..S. 58 ff.; Schlipköter, H.-W./Dolgner, R., Epidemiologische Untersuchungen im $\mathrm{Z}$ usammenhang mit lufthygienischen Fragen, a.a.0., S. 278 .

209) Vgl. Schaefer, H., Der Mensch in der technisch verănderten Welt, a.a.0., S. $26 \mathrm{f}$. 
tion 210) - allerdings nur auf die Gesundhelt eines Versuchstieres, womit auch gleich der Nachtell dieser Methode genannt 1st. Denn die Ergebnisse aus Tierversuchen sind nur sehr bedingt auf den Menschen ubertragbar 211) da z.B. bel Mensch und Tier elne unterschledilche Entglftung der Schadstoffe möglich ist 212). Ein weiterer Nachteil 1st, daß fast nur akute Wirkungen eines Schadstoffs auf das Versuchtstier festgestellt werden können; Tierversuche liefern also vor allem den "toxikologischen Zugang" zum Problem des Nachweises von Schadstoffwirkungen 213) Laborversuche mit Menschen würden zwar elndeut1ge Aussagen uber den Ursache-Wirkungszusammenhang verschiedener Schadstoffe zulassen, verbleten sich jedoch aus ethischen Gründen. Mehr oder wenlger freiwlilig stellt der Mensch jedoch an manchen Arbeltsplätzen, wo er der Belastung durch bestimmte Schadstoffe ausgesetzt ist, eine Art "Versuchskaninchen" dar. Diesen Umstand macht sich die nun $z u$ schildernde dritte Methode der Feststellung von Schadstoffwirkungen auf die menschliche Gesundheit zunutze.

(c) Heranziehung arbeitsmedizinischer und gewerbetoxikologischer Untersuchungen

Durch Beobachtungen und Untersuchungen von Menschen, die an ihrem Arbeltsplatz Schadstoffbelastungen ausgesetzt sind, Iassen sich relatıv zuverlässig die Wirkungen dieser Schadstoffe auf die

210) Vgl. Antweiler, H./Pott, F., Tierexperimentelle Ergebnisse uber die Wirkung partikel- und gasförmiger Luftverunre1n1gungen, in: Zentralblatt für Bakteriologie ..., a.a.o., S. 264 .

211) Vgl. Schlipköter, H.-W./Dolgner, R., Epidemiologische Untersuchungen Im Zusammenhang mit lufthygienischen Fragen, a.a. 0., S. 273; Borneff, J.., Hyglene, a.a.0., S. 12 f.; Cassell, E.J.. The Health Effects of Air Pollution and their Implications for Control, a.a.O., S. 204; van Belle, G./ Schneiderman, M., Some Statistical Aspects of Environmental Pollution and Protection, a.a.0., s. 323.

212) Vgl. Henschler, D., Versuch einer Zuordnung von chemischen Noxen und Schäden beim Menschen, in: Schaefer, H. (Hrsg.), Folgen der Zivilisation, a.a.0., s. 47:

213) Vgl. Schaefer, H., Hinwelse auf Umweltschăden ..., a.a.0., s. 73 . 
menschliche Gesundheit feststellen 214). Das gilt sowohl für akute Wirkungen wie Vergiftungen als auch für chronlsche Erkrankungen. Methodisch von Nachteil ist hier, daß manche Stoffe, welche die Umwelt belasten, am Arbeitsplatz nicht vorkommen. Eine Ubertragung der arbeitsmedizinischen Untersuchungen auf die Umweltbelastung ist allerdings oft nicht möglich, weil die Personen am Arbeltsplatz nur während der Arbeitszeit der Schadstoffbelastung ausgesetzt sind, also bis zum nächsten Arbeltstag elne gewisse Erholungszeit haben, während die Schadstoffbelastung in der Umwelt permanent sein kann.

(d) Vergleich der Methoden

Aus der Darstellung der Methoden ist ersichtlich, daß kelne Methode allein voll befriedigende Ergebnisse über den Zusammenhang zwischen Umweltbelastung und Krankheit liefern kann. Manche Autoren geben jedoch epidemiologischen Untersuchungen eindeutig den Vorzug 215), u.a. deswegen, weil Langzeiteffekte von Schadstoffen (chronische Wirkungen) nur mit Hilfe epidemiologischer Untersuchungen festgestellt werden können 216) Zweifellos bedarf es jedoch der Ergänzung der epidemiologischen studien durch tierexperimentelle Forschungen im Labor 217)

214) Vgl. Coenen, R. U.a., Alternativen zur Umweltmisere, Raubbau oder Partnerschaft, München 1972, S. 109; Valentin, H., Arbeitsmedizinische Untersuchungen von Berufstätigen, in: Der Städtetag, 1971, Heft 3, S. 177; Henschler, D., Versuch einer zuordnung von chemischen Noxen und Schäden beim Menschen, a.a.0., S. $58 \mathrm{f}$.

215) Vgl. Borneff, J., Hygiene, a.a.O., S. 12 f.; Lawther, P.J./ Martin, A.E./Wilkins; E.T., Epldemiology of Air Pollution, a.a.0., S. 26 .

216) Vgl. Schlipköter, H.-W./Dolgner, R., Epidemiologische Untersuchungen im Zusammenhang mit lufthygienischen Fragen, a.a.0., s. 273 f.

217) Vgl. Schlipköter, H.-W., Die Luftverunreinigung als gesundheitliches Problem, in: Schultze, H. (Hrsg.), UmweltReport, a.a.0., S. 200; Henschler, D., Versuch einer $\mathrm{Zu}-$ ordnung von chemischen Noxen und Schäden beim Menschen, a.a.O.. S. 49; Lawther, P.J./Martin, A.E./Wilkins, E.T., Epidemiology of Air Pollution, a.a.O., S. 15. 
(4) Systematislerung der Schadstoffwirkungen auf die menschliche Gesundheit

Für die systematische Erfassung der Schadstoffwirkungen in einem Wirkungskataster ${ }^{218)}$ ist eine Einteilung der Schadstoffwirkungen nach gemelnsamen Merkmalen notwendig. Es sind verschiedene Gliederungsmöglichkeiten denkbar.

(a) Ausgehend von dem Schema der gesamten Schadstoffwirkungen 219) könnte man die gesundheitsschädichen wirkungen in direkte und indirekte unterteilen 220). Die direkt gesundheitsschädlichen Wirkungen wären diejenigen, die von Schadstoffkonzentrationen ausgelöst werden, die per se keine ökologischen Schäden hervorrufen können (z.B. Lärm, Gerüche) bzw. unter der ökologisch schädlichen Immissionskonzentration liegen. Demgemä $B$ sind die indirekten gesundheitsschädlichen Wirkungen diejenigen, die über eine Belastung der ökofaktoren auf den Menschen wirken, z.B. durch Reduzierung der Nahrungsmittelreserven (etwa infolge der Erosion), die Verschandelung der Landschaft oder die Verseuchung des Trinkwassers 221)

(b) Des weiteren 1st eine Eintellung der gesundheitsschädlichen Wirkungen nach den Wegen der Schadstoffaufnahme möglich. Man kann hierbel zunächst einmal zwischen der Aufnahme durch Einatmung, Nahrung (einschl. Trinkwasser) und Hautkontakt unterscheiden 222) Da wir von der Gesundheitsdefinition der wHO ausgehen und demzufolge auch berelts Beeinträchtigungen des Wohlbefindens als gesundheltsschädlich bezelchnen, können wir auch die Sinnesorgane Gehör, Nase und Auge als Aufnahmeorgane gesundheitsschädlicher Umweltbelastungen, nämlich

218) Vgl. dazu unten S. $111 \mathrm{f}$.

219) Vgl. oben S. 60.

220) Vgl. Coenen, R. u.a., Alternativen zur Umweltmisere, a.a. O.. S. 155.

221) Vgl. ebenda.

222) Bär, F./Grunow, w., Hygienisch-toxikologische Aspekte der Umweltsituation, in: Schultze, H. (Hrsg.), Umwelt-Report, a.a.0. S. 287; Henschler, D., Versuch einer Zuordnung von chemischen Noxen und Schäden beim Menschen, a.a.0., S. 46. 
von Lärm, Gerüchen und ästhetischen Beelnträcht1gungen bezeichnen.

(c) Von der Definition der wHO ausgehend kann man zwischen physischen, psychischen und sozialpsychischen wirkungen der Umweltbelastung unterscheiden. Vor allem den letzten beiden Wirkungen wird noch $z u$ wenig Beachtung geschenkt 223).

(d) Legt man die zeit, die zwischen Immissionsbelastung und Krankheitsentstehung vergeht als Unterscheidungsmerkmal zugrunde, so kann man zwischen akuten, chronischen, teratogenen und mutagenen wirkungen unterscheiden 224). Bei akuten Schäden können der zeltpunkt der Immissionsbelastung und der des Gesundheitsschadens sehr dicht beleinander liegen (z.B. bei Kohlenmonoxidvergiftungen von Verkehrspolizisten). Chronische Schäden treten dagegen erst nach Jahren oder Jahrzehnten auf; zu ihnen gehören auch die kanzerogenen Schäden, Teratogene Wirkungen sind embryonale MiBbildungen, betreffen also die nächste Generation. Mutagene Wirkungen, also Erbschäden, können noch spätere Generationen treffen.

(e) Schließlich bietet sich noch eine Gliederung der gesundheitsschädlichen Wirkungen nach den రkofaktoren oder Umweltmedien an, deren sich die Schadstoffe als Träger bedienen; man kann also unterteilen in gesundheitsschädliche Wirkungen der Luft-, Gewässer-, Boden-, Pflanzen-, Tierwelt- und Lebensmittelbelastung. Als spezielle gesundheitsschädliche Immission kommt noch der Lärm hinzu. Welche der fünf aufgezeigten Gliederungsmöglichkeiten man wählt, hängt vom jewelligen Untersuchungsziel ab. Auch Kombinationen der Gllederungsarten sind denkbar. Für unsere Zwecke scheint die

223) Vgl. Picht, G., Umweltschutz und Politik, in: von Welzsäcker, E. (Hrsg.), Humanökologie und Umweltschutz, Studien zur Friedensforschung, Bd. 8, stuttgart und Munchen 1972, S. 83; Friedman, S., Facing Man and Society: The Challenge, in: Tsuru, S. (Hrsg.), Proceedings of the International Symposium on Environmental Disruption, Tokio 1970 , S. 33 ff.

224) Vgl. Bär, F./Grunow, W., Hygienisch-toxikologische Aspekte der Umweltsituation, a.a.0., s. 287 f.; Marquardt, H. , Die Auslösung von Erbschäden und Krebs durch Umweltfaktoren, a. a.O., S. $1314 \mathrm{f}$. 
letzte Gliederung am besten geelgnet zu sein. Wir wollen sie für die nun folgende Darstellung der Schadstoffwirkungen auf die menschliche Gesundhelt zugrunde legen. Vollständigkeit ist dabei im Rahmen dieser Arbeit nicht möglich.

(5) Darstellung von Schadstoffwirkungen auf die menschliche Ges undheit

(a) Gesunheitsschädliche Wirkungen der Luftbelastung Wie bereits erwähnt, sind die Ergebnisse epidemiologischer studien über den Zusammenhang zwischen Morbiditäts-bzw. Mortal1tätsrate einer Krankheit einerseits und Luftbelastung durch Schadstoffe andererseits recht unspezifischer Natur; sie sagen also nichts konkretes darüber aus, welche Schadstoffe für die jeweilige Krankheit oder den Tod verantwortlich sind 225) Die Ergänzung epidemiologischer studien durch Tierexperimente und arbeitsmedizinische Erkenntnisse haben jedoch Ergebnisse von hohem Wahrscheinlichkeitscharakter erbracht. Uber die wichtigsten dieser Ergebnisse wird im folgenden berichtet:

$(\alpha)$ staubwirkungen

Staub ist zum einen an sich gesundheitsschädlich, zum anderen deswegen, well er als "Schlepper" für andere schädliche substanzen vor allem in die Atemwege und die Lunge dient. Direkt gesundheitsschädlich 1st der staub zunächst deshalb, weil er - in der Atmosphäre verteilt - zu einer Verringerung der UVStrahlung führt, was bel Kindern $z u$ Rachitis führen kann 226)

225) Vgl. Lave, L.B./Seskin, E.P., Air Pollution and Human Health, a.a.0., S. $367 \mathrm{ff}$. Hier werden Zusammenhänge zwischen der Mortalitätsrate infolge Bronchitis, Lungenkrebs, HerzgefäBerkrankungen, Atemwegserkrankungen und der Säuglingssterblichkeit einerseits sowie der Luftbelastung andererseits hergeleitet.

226) Vgl. Coenen, R. U.a., Alternativen zur Umweltmisere, a.a. O.. S. 126; Schlipköter, H. -W. bel der öffentlichen Anhörung des Innenausschusses des Deutschen Bundestages, abgedruckt in: Zur Sache 3/72, Umweltschutz (II), Bonn 1972, S. 18; Olschowy, G./Mrass, W./Kullmer, H.J./Bürger, K., $\mathrm{Z}$ ur Belastung der Landschaft, a.a.0., S. 34; Hötker, D./ Wierling, L., Umweltschutz im Ruhrgeblet, in: Bundesbaublatt, Bd. 20, 1971, S. 325 . 
Staubteilchen mit einem Durchmesser unter 5/1000 mm können in die kleinsten Verästelungen der Lunge (die Alveolen) vordringen und dort die Schleimhäute schädigen 227) Besonders schädlich sind stäube jedoch deswegen, weil sie als Transportmittel für andere Schadstoffe fungieren. In Verbindung mit $\mathrm{SO}_{2}$, das an den Staubpartikeln kondensieren und so via schwefelige Säure $\left(\mathrm{H}_{2} \mathrm{SO}_{3}\right) \mathrm{zu}$ Schwefelsäure $\left(\mathrm{H}_{2} \mathrm{SO}_{4}\right)$ werden kann, können stäube chronische Bronchitis und chronische Erkrankungen der Lunge begünstigen 228). Asbeststaub kann z.B. in Verbindung mit 3,4Benzpyren zu Lungenkrebs führen 229) Ohne Bindung an Asbeststaub wird 3,4-Benzpyren dagegen in der Lunge ziemlich schnell abgebaut 230 )

( $\beta$ ) so $_{x}$-Wirkungen

$\mathrm{SO}_{2}$ und $\mathrm{SO}_{3}$ können neben den nur in Verbindung mit stäuben auftretenden gesundheltsschädlichen Wirkungen zu Reizerscheinungen an den Augen und in den Luftwegen führen 231) und das Knochen-

227) Vgl. Coenen, R. u.a., Alternativen zur Umweltmisere, a.a.0., S. 112. Besonders gefährlich sind wegen ihrer Feinhelt und Struktur Asbestfasern und Metallstäube (vgl. Grefen, K., Feinstäube gefährden den Städter, in: Umwelt, 1974, 4, S. 22 ).

228) Vgl. RSU, Umweltgutachten 1974, a.a.0., S. 18; Cassell, E.J., The Health Effects of Air Pollution and their Implications for Control, a.a.0., S. 205 f.; Villiers, A.J. de, The Effects of Air Pollution on Health, in: Irving, R.M./ Priddle, G.B. (Hrsg.), Cris1s, London 1971, S. 119. Von weltaus gröBerer Bedeutung für das Entstehen dieser Erkrankung scheint jedoch das Rauchen von zigaretten $z u$ sein (vgl. z.B. Cassell, E.J., The Health Effects of AIr Pollution and their Implications for Control, a.a.0., S. 208; Schmidt, Ferdinand, Krebsursache: Abgas oder Tabakrauch, in: Umwelt, 1971, 6, S. $28 \mathrm{ff.l}$. Inwieweit bel Rauchern der Tabakrauch oder die Luftverschmutzung für Bronchitis, Lungen- und Atemwegserkrankungen bis hin zum Krebs verantwortlich sind, ist umstritten und die Diskussion darüber tellweise ldeologisch gefärbt.

229) Vgl. Olschowy, G., Auswirkungen der Luftverunrelnigung auf den Menschen, a.a.0., S. 129; Beck, E.G., Fasermengen sind schwer zu messen, in: Umwelt, 1975, 4, S. 31.

230) Vgl. Schlipkbter, H.-W., Die Luftverunreinigung als gesundheitliches Problem, a.a.0., S. $203 \mathrm{f}$.

231) Vgl. Materiallenband zum Umweltprogramm der Bundesregierung, a.a.0., S. 209. 
wachstum bel Kindern verzögern 232)

( $\gamma$ ) No ${ }_{x}$-W1rkungen

Stickoxide können, ăhnlich wie so $x^{\prime}$ elne Relzung der Augen und Luftwege bewirken 233) Gefährlicher ist jedoch die durch $\mathrm{NO}_{2}$ hervorgerufene Verhinderung der Infektionsresistenz gegenüber Bakterien 234). Auch hier ist es eher eine Art Katalysatorfunktion, die schădich ist und wenlger der stoff selbst.

(d) co-wirkungen

CO 1st vor allem deswegen gesundheitsschädlich, weil es mit Hämoglobin, das für den Transport des Sauerstoffs aus dem Blut in die Gewebe verantwortlich ist, zu Carboxyhämoglobin (COHb) reaglert 235); das so gebundene Hämoglobin ist nicht mehr zum Sauerstofftransport geelgnet, wodurch sich vor allem die Durchblutung der Herzmuskulatur und des Gehirns verschlechtert 236) Die Wirkungen relchen je nach der Konzentration und Einwirkungsdauer von $C O$ von Beeinträchtigungen der Aufmerksamkelt und einem dadurch bedingten Nachlassen des Reaktionsvermögens 237) b1s hin zu akuten Vergiftungen. Auch chronische Wirkungen in Form einer Beeinträchtigung der Funktionen des zentralnervensytems und des Herz- Krelslauf-Apparates sind mögl1ch 238). Selbst sehr geringe co-Konzentrationen können gesundheitsschädlich sein,

232) Vgl. Hötker, D./Wierling, L., Umweltschutz im Ruhrgebiet, a.a.o., S. 325 .

233) Vgl. Materiallenband zum Umweltprogramm der Bundesregierung, a.a.0., S. 209 .

234) Vgl. Schlipköter, H.-W., Krank von der Stadtluft, in: U, 1973, 2, S. 21; RSU, Umweltgutachten 1974, a.a.O., S. 19.

235) Vgl. Jermini, C., Die gesundheitlichen Gefährdungen durch Kohlenmonoxid, in: Universitas, 28. Jg., 1973, S. 1239.

236) Vgl. Schlipköter, H. W. , Auswirkungen von Autoabgasen auf die Stadtbevölkerung, in: Forschung'74, Frankfurt/M. 1973, S. 30; derselbe, Die Luftverunreinigung als gesundheltliches Problem, a.a.0., S. 202.

237) Vgl. ebenda: RSU, Umweltgutachten 1974, a.a.0., S. 19.

238) Vgl. Jermini, C., Die gesundheitlichen Gefährdungen durch Kohlenmonoxid, a.a.O., s. 1240. 
wenn sie über einen längeren zeltraum auf den Menschen wirken. Die Wirkung $w$ ist in folgender Form von der co-konzentration $c$ und der Wirkungsdauer $t$ abhängig: $W=c . t$. Die Wirkung ist die glelche, unabhängig davon, ob z.B. 40 ppm 60 Minuten lang, 80 ppm 30 Minuten lang oder 2400 ppm elne Minute lang wirken 239? (E) Bleiwirkungen

Im Gegensatz zu den bisher behandelten in der Atmosphäre vorkommenden Schadstoffen, die fast ausschlieBlich durch die Atemluft aufgenommen werden, kann aus der Luft stammendes Blel auch mit der Nahrung aufgenommen werden 240). Die akute Bleivergiftung ist als Folge der Luftbleibelastung sehr unwahrscheinlich, da Blel tellweise durch den Darm und die Nieren wieder ausgeschieden wird. Ein Teil des mit der Nahrung aufgenommenen Bleis wird in der Leber verarbeitet und dann ausgeschieden. Die Bleiverarbeitungskapazität der Leber 1st jedoch beschränkt, und es besteht die Gefahr des "Uberlaufens", so daB Blel in den Blutkreislauf gelangt und damit den Blutbleispiegel erhöh 241) Zu einer langfristigen Lagerung und Kumulierung von Blel kann es in den Knochen kommen 242). Aus diesen Bleldepots kann es im Alter durch Knochenumbauprozesse zu einer Freisetzung der angesammelten Bleimenge kommen, was zu elner starken Erhöhung des Blutbleispiegels führen würde 243)

Mit der Atemluft aufgenommener staub kann eine zellschädigende Wirkung haben, vor allem auf die für den 3,4-Benzpyrenabbau in

239) Vgl. Malorny, G., Allgemeiner Uberblick über die Wirkung von Kohlenmonoxid auf den Menschen, in: VDI (Hrsg.), Kohlenmonoxid. Entstehung, Messung und Wirkungskriterien, Düsseldorf 1972, S. 48 .

240) Vgl. Materialienband zum Umweltprogramm der Bundesreglerung, a.a.0., S. 95; Klausew1tz, W./Schäfer, W./Toblas, W., Umwelt 2000, a.a.0., s. 44 und die Ausführungen von Hoschek in: Blel und Umwelt, Berlin 1972, S. 82.

241) Vgl. ebenda.

242) Vgl. ebenda; Schlipköter, H.-W., Auswirkungen von Autoabgasen auf die stadtbevollkerung, a.a.0., s. 31.

243) Vgl. ebenda. 
der Lunge verantwortlichen Alveolarmakrophagen 244). Auch Störungen des zentralen Nervensystems 245) und Enzymschädigun-

gen 246) werden genannt. Mutagene und teratogene Wirkungen 247) werden vermutet. Darüber hinaus erhöht Ble1 die Infektionsanfäll1gke1t 248).

(५) Kadmi umwirkungen

Kadmium, das in der Luft vorkommt, gelangt wie Blel auch via Vegetationsaufnahme mit der Nahrung in den menschlichen Körper. Es kann zu einer Entmineralisierung der Knochen führen, der sogenannten Itai-Itai-Krankheit 249). Ferner ist eine kanzerogene wirkung möglich 250).

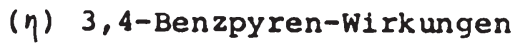

Die kanzerogene Wirkung von 3,4-Benzpyren ist wohl unbestritten 251); es bedarf jedoch - wie bereits geschildert - des Vor-

244) Vgl. Schl1pköter, H.-W./Beck, E.G., Die zellschädigende Wirkung von Blei, in: Umwelt, 1972, 5, S. 52.

245) Vgl. RSU, Umweltgutachten 1974, a.a.0., S. 19 f.

246) Vgl. Materialienband zum Umweltgropramm der Bundesregierung, a.a.0., S. 96 .

247) Vgl. ebenda und die Diskussionsbeiträge in: Blel und Umwelt, a.a.0., S. $84 \mathrm{ff}$.

248) Vgl. Schlipköter, H.-W./Beck, E.G., Die zellschädigende Wirkung von Blei, a.a.0., S. 52 .

249 ) Vgl. RSU, Umweltgutachten 1974, a.a.O., S. 19 f.; Klausewitz, W./Schäfer, W./Tobias, W., Umwelt 2000, a.a.0., S. 50; Hötker, D./Wierling, L., Umweltschutz im Ruhrgebiet, a.a.O., S. 325; Materialienband zum Umweltprogramm der Bundesregierung, a.a.0., S. 97 f.; Stelte, W., Die Kontamination von Lebensmitteln mit schädlichen Stoffen aus der Umwelt, in: Schaefer, H. (Hrsg.), Folgen der Zivilisation, a.a.0., S. 64; Coenen, R. U.a., Alternativen zur Umweltmisere, a.a.0., S. 132 f. Die Itai-Itai-Krankhe1t wurde allerdings durch Kadmium im Trinkwasser hervorgerufen.

250) Vgl. Borneff, J., Schadstoffe Im Wasser, a.a.O., S. 228.

251) Vgl. Grăf, W. , Umweltgefährdung durch kanzerogene Substanzen, in: Steubing, L./Kunze, C./Jäger, J. (Hrsg.), Belastung und Belastbarkeit von Ökosystemen, a.a.0., s. 116 f. und Schlipköter, H. W. bel der Anhörung vor dem Innenausschuss des Deutschen Bundestages, a.a.0., s. 21. 
handenselns felner Stäube, welche die Alveolen am Abbau des 3,4Benzpyrens in der Lunge hindern 252)

(I) Ozon-Wirkungen

Ozon kann zu Reizungen der Augen und der oberen Luftwege fuhren. Darüber hinaus schwächt es die Resistenz gegenüber Infektionen 253)

\section{(i) Geruchsstoff-Wirkungen}

Geruchsintensive stoffe wie Merkaptane oder Schwefelwasserstoff $\left(\mathrm{H}_{2} \mathrm{~S}\right)$ führen $\mathrm{zu}$ subjektiv bedingten Belästigungen 254) stellen also kelne direkte Gesundheitsgefährdung dar 255) sondern nur elne Beeinträchtigung des Wohlbefindens 256) Sie können jedoch psychosomatische Schäden auslösen, die lhrerselts $z u$ "echten" Krankhe1ten führen 257)

(x) Wirkungen radioaktiver Strahlen

Der Mensch kann den radioaktiven Strahlen direkt oder auf dem Umweg über die Nahrungskette ausgesetzt seln. Im zwe1ten Fall gelangen die Radionuklide in den Boden und das Wasser, wo sie von Pflanzen und Tieren aufgenommen werden, die als Nahrungsmittel des Menschen dienen 258) Die Gefahr akuter Schäden, etwa in Form des Strahlensyndroms 259) , 1st wegen der hohen Kraftwerk-

252) Vgl. oben S. 91 und S. $93 \mathrm{f}$.

253) Vgl. Schlipkoter, H.-W., Auswirkungen von Autoabgasen auf die stadtbevolkerung, a.a.0., s. 32 .

$254)$ Vgl. Fodor, G.G./Winneke, G., Belästigung durch geruchsintensive Stoffe, 1n: Zentralblatt für Bakteriologie ..., a.a.0., S. 292 .

255) Vgl. Haberle, M., Zur Erstellung eines Geruchskatasters der BASF Ludwigshafen, a.a.0., S. 39.

256) Vgl. Materiallenband zum Umweltprogramm der Bundesrealeruna, a.a.0., S. 209:

257) Vgl. Fodor, G.G./Winneke, G., Belästigung durch geruchsintensive Stoffe, a.a.0., S. 290.

258) Vg1. Klausew1tz, W./Schäfer, W./Toblas, W., Umwelt 2000 , a.a.0., S. $76 \mathrm{f}$. und die Ausfuhrungen von D. Simonis be 1 der öffentlichen Anhörung des Innenausschusses des Deutschen Bundestages, abgedruckt in: Zur Sache 2/75, Umweltschutz (IV), Bonn 1975, S. 27.

259) Vgl. Graeub, R., Die sanften Mörder, Atomkraftwerke demaskiert, Frankfurt/M. 1974, S. $78 \mathrm{f}$. 
slcherheit sehr gering; diese Gefahr stelgt allerdings bel gestörtem Betrieb infolge von Unfällen, Sabotage oder Krieg 260) Unter "normalen" Bedingungen können Lonislerende Strahlungen zu Krebs- und Leukämieerkrankungen (somatische Wirkungen), Mutationen in den Keimzellen (genetische Wirkungen) und embryonalen MiBbildungen (teratogene Wirkungen) fuhren 261) Auch kleinste Dosen radioaktiver Belastung können gefährlich sein, da manche radioaktive Substanzen sich im körper ablagern, kumulieren und sehr langsam abbauen; am bekanntesten ist in dieser Beziehung wohl das Stront1um 90, das sich in den Knochen ansammelt, und die Radiojod-Aufnahme durch die Schilddrüse von Kleinkındern über die Nahrungskette Weide-Kuh-Milch 262)

(b) Gesundheitsschädliche Wirkungen der Gewässerbelastung Eine Reihe von Schadstoffen, die in die Atmosphäre emittiert werden, gelangen auch in die Gewässer, entweder durch direkte Einleitung oder - aus der Atmosphäre - mit dem Regenwasser 263) Die gesundheitsschädliche Wlrkung dieser Schadstoffe ist in der Regel die gleiche wie die direkte, nur daB sie mit der Nahrung oder dem Trinkwasser aufgenommen werden. In diesem Abschnitt können wir deshalb auf die Darstellung der Schadstoffwirkungen verzichten, die auch uber die Atmosphäre die menschliche Gesundheit beeinflussen.

(ג) Quecksilberwirkungen

Die gesundhe1tsschădlichen Wirkungen des Quecksilbers wurden welt-

260) Vgl. die Ausführungen von E.-H. Graul bel der öffentlichen Anhörung vor dem InnenausschuB des Deutschen Bundestages, abgedruckt in: Zur Sache 2/75, a.a.0., S. 19.

261) Vgl. die Ausführungen von K. Aurand, ebenda, S. 25; Materialienband zum Umweltprogramm der Bundesregierung, a.a.0., S. 115; Klausewitz, W./Schäfer, W./Tobias, W., Umwelt 2000, a.a.0.. S. 76 f.; Wachsmann, F., Gefahren durch Ionisierende Strahlungen, in: Schultze, H. (Hrsg.), Umwelt-Report, a.a.0.. S. 336; Walser, E., Sind Atomreaktoren gefahrilch?, in: NZZ, FA Nr. $134 \mathrm{v}, 18.5 .1971$.

262) Vgl. die Ausführungen von W. Jacobl bel der öffentlichen Anhörung vor dem InnenausschuB des Deutschen Bundestages, In: Zur Sache $2 / 75$, a.a.0., S. 35 .

263) Schwoerbel, J., Blologie des Wassers, a.a.0., S. 237. 
weit durch den Tod von 43 japanischen Fischern bekannt, welche mit Methylquecksilber belastete Fische aus der Bucht von Minamata gegessen hatten 264). Diese als Minamata-Krankheit bezelchnete gesundheitsschädliche Wirkung des Quecksilbers ăußert sich in Störungen des zentralen Nervensystems, die zu Lăhmungen und in schweren Fällen zum Tod fuhren können 265)

Das Quecksilber gelangt über die Nahrungskette Phytoplankton Zooplankton - Fische (bzw. Krebse, Muscheln) in den menschlichen Organismus 266) Innerhalb der einzelnen Glieder der kette kommt es zu Anlagerungen des Quecks1lbers. Im Phytoplankton wurden Konzentrationen bis zum 100000 fachen der Konzentration im Wasser nachgewiesen 267) Von Quecksilber können auch teratogene W1rkungen ausgehen 268) und es besteht der verdacht mutagener 269) und kanzerogener ?70) Effekte.

264) Vgl. Coenen, R. U.a.. Alternativen zur Umweltmisere, a.a.0., S. 134; Materialienband zum Umweltprogramm der Bundesreglerung, a.a.0., S. 93; Klausewitz, W./Schäfer, W./Toblas, W., Umwelt 2000, a.a.0., S. 47 f.; Kazantz1s, G., The Poison Chain for Mercury in the Environment, in: International Journal of Environmental Studies, vol. 1, 1971, S. 303.

265) Vgl. Coenen, R. u.a., Alternativen zur Umweltmisere, a.a.0., S. $134 \mathrm{ff.;}$ Materialienband zum Umweltprogramm der Bundesreglerung, a.a.0., S. 93; Klausewitz, W./Schäfer, W./Tobias, W.. Umwelt 2000, a.a.0., s. $47 \mathrm{f}$.

266) Vgl. Materiallenband zum Umweltprogramm der Bundesreglerung, a.a.0., S. 182; Kazantzis, G., The Poison Chain for Mercury in the Environment, a.a.0., S. 304; Caspers, H., Sieben Jahre Forderung des Schwerpunktprogramms "Litoralforschung Abwässer in Kulstennähe", in: DFG-Mitte1lungen 2/73, S. 65; Borneff, J., Schadstoffe im wasser, a.a.O., S. 228 .

267) Vgl.Klausewitz, W./Schäfer,W./Toblas, W., Umwelt 2000, a.a.O., S. 46; Caspers, H., Sleben Jahre Förderung des Schwerpunktprogramms "Litoralforschung - Abwässer in Küstennähe", a.a.0., S. 65 .

268) Vgl. Kazantz1s, G., The Polson Chain for Mercury in the Environment, a.a.0., S. 303; Schneider, wilhelm, Verschmutzende Saubermacher, Bedrohliche Phosphatverschmutzung unserer Gewăsser, in: Umwelt, 1971, 3, S. 27; Degenhardt, K. -H., Teratologische Probleme der Umweltverschmutzung, in: Schultze, H. (Hrsg.), Umwelt-Report, a.a.O., S. 293.

269) Vgl. Kazantzis, G., The Polson Chain for Mercury in the Environment, a.a.0., S. 305.

270) Vgl. Borneff, J., Schadstoffe im Wasser, a.a.O., S. 228. 


\section{( $\beta$ ) Pestizidwirkungen}

\section{$\left(\beta_{1}\right)$ DDT-Wirkungen}

DDT 1st die Abkürzung fur Dichlor-Diphenyl-Trichloräthylen; es handelt slch also um einen chlorierten Kohlenwasserstoff. DDT 1st das wohl bekannteste, wirksamste und zumindest früher am weitesten verbreltete schädingsbekämpfungsmittel. Mit seiner H1lfe 1st es gelungen, die Malaria so gut wle auszurotten - ein offenbar sehr positiver gesundheitlicher Effekt, den man nicht vergessen sollte (zumal er absolut sicher ist), wenn man die (noch?) nicht bewlesenen gesundheitsschädlichen Effekte des DDT betrachtet 271) DDT gelangt bel der Anwendung in alle drel Umweltmedien Luft, Wasser und Boden. Die Belastung des Menschen erfolgt uber die verschiedenen Nahrungsketten. Dieser Vorgang wird in dem folgenden Schema dargestellt 272):

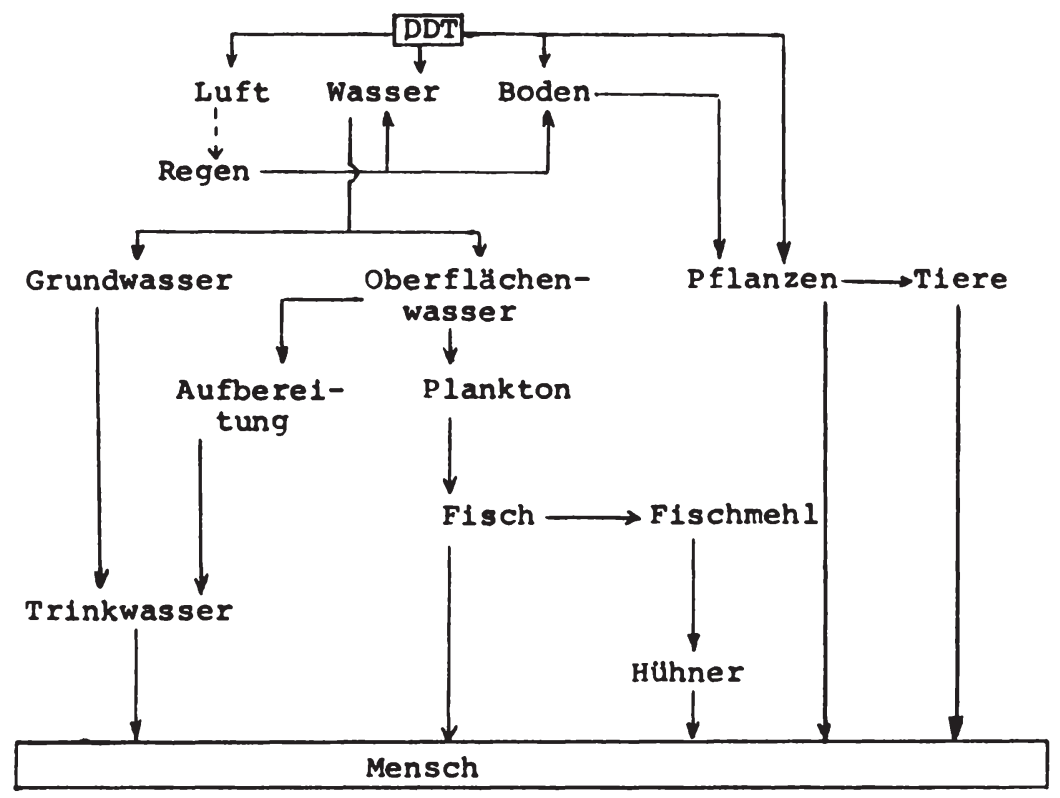

Abb. 8

271) Vgl. Umweltschutz als Mythos, in: NZZ, FA Nr. 308 v. 10.11. 1971.

272) Zusammengestellt nach Borneff, J., Hygiene, a.a.0., S. 73 f. und S. 221 . 
Etwa 708 des DDT gelangen durch tierische Produkte, 258 durch pflanzllche Nahrung und der Rest uber das Wasser (Fische und Grundwasser) in den menschlichen Körper 273). Die mögliche Gefährlichke1t des DDT beruht darauf, daB es slch nur langsam abbaut (über $20 \mathrm{Jahre),} \mathrm{sich} \mathrm{in} \mathrm{den} \mathrm{einzelnen} \mathrm{Gliedern} \mathrm{der} \mathrm{Nah-}$ rungskette anrelchert und schlleblich im Körperfett des Menschen anlagert 274); es sind also auch geringe Konzentrationen wegen der mögllchen Kumulation im Verlauf der Jahre potentiell schäd11ch. Akute Erkrankungen sind nicht zu erwarten, chronische Schäden können dagegen nicht ausgeschlossen werden. Vermutet werden kanzerogene 275) und teratogene 276) wirkungen. Solange die Möglichkeit dieser schäden nicht widerlegt ist, scheint es uns gerechtfertigt, DDT unter die primär gesundheitsschädlichen stoffe zu subsumieren, auch wenn die ökologischen schäden bereits offensichtlich sind 277 ?

$\left(\beta_{2}\right)$ Phosphorsäureester-Wirkungen

Den Nachtell der großen Persistenz von DDT versuchte man durch die Entwicklung und Anwendung schnell abbaubarer Insekt1zide zu umgehen; man fand organische Phosphorverbindungen, etwa vom Typ des Phosphorsäureesters E 605, die nur eine kurze Lebensdauer hatten. Dafür sind diese Insekt1zide aber akut toxisch - E 605 ist ein beliebtes Mord- und Selbstmordmittel - und fuhren bei entsprechender Dosis zur Atemlähmung und in geringeren Mengen $\mathrm{zu}$ chronischen Magen-Darm-Beschwerden 278)

273) Vgl. Borneff, J., Schadstoffe im Wasser, a.a.0., S. 225.

274) Vgl. Coenen, R. u.a., Alternativen zur Umweltmisere, a.a.0., S. $142 \mathrm{ff.;}$ Simonis, W., Pestizide und ihre Akkumulation in den "Nahrungsketten", in: Schaefer, H. (Hrsq.), Folqen der Zivilisation, a.a.0., S. 258 f.; Klausewitz, W./Schäfer, W./Toblas, W., Umwelt 2000 , a.a.0., S. $36 \mathrm{ff}$.

275) Vgl. ebenda, S. 39.

276) Degenhardt, K.-H., Teratologische Probleme der Umweltverschmutzung, a.a.0., S. 292.

277) Der RSU betrachtet DDT primär aus ökologischen Gründen als schädlich (vgl. RSU, Umweltgutachten 1974, a.a.O., S. 66).

278) Vgl. Borneff, J., Hyglene, a.a.o., S. 73; Coenen, R. u.a., Alternativen zur Umweltmisere, a.a.0., S. 145. 
( $\gamma$ ) PCB-Wirkungen

PCB ist die Abkürzung für Polychlorid-Biphenyl; es handelt sich also wie beim DDT um einen chlorierten Kohlenwasserstoff. Seine gesundheitsschădliche Wirkung ist wie die des DDT noch nicht nachgewiesen 279) Dies düfte auch noch schwieriger sein als beim DDT, da PCB noch langsamer abgebaut wird.

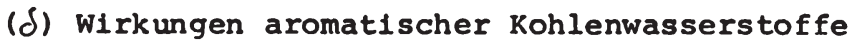

Die kanzerogene wirkung des 3,4-Benzpyrens wurde bere1ts geschildert 280 ?

Daneben gelten vor allem Phenole als gesundheitsschädlich, weil sie als Aktivatoren für krebserzeugende Verbindungen dienen können 281) die Krebsentstehung durch andere stoffe also begünst1gen.

(E) Wirkungen pathogener Mikroorganismen

Die gesundheitsschädichen Wirkungen, die von pathogenen Mikroorganismen verursacht werden, gehören zu den klassischen Wirkungen der Gewässerbelastung. Die Bakterien, Viren und Wurmeler stammen vor allem aus dem Fäkalabwasser der Haushalte. Bakterien fuhren u.a. zu Typhus, Paratyphus, Ruhr und Cholera 282); Viren sind die Erreger der Poliomyelitis, Hepatitis und Meningitis 283) (ら) Wirkungen eutrophierter Gewässer

Eutrophiertes, also sauerstoffarmes Wasser, beelnträchtigt dessen Nutzung als Trinkwasser nicht nur wegen dessen schlechtem Geruch und Geschmack - was nach der wHO-Definition bereits aus-

279) Vgl. RSU, Umweltgutachten 1974, a.a.0., s. $66 \mathrm{f}$.

280) Vgl. S. 94. Im Wasser gelöstes 3,4-Benzpyren ist allerdings weltaus ungefăhrlicher für die Karzinombildung als das in der Atmosphäre vorkommende (vgl. Borneff, J., Hygiene, a.a.0., S. 68).

281) Vgl. Borneff, J., Schadstoffe Im Wasser, a.a.0., S. 228.

282) Vgl. Materialienband zum Umweltprogramm der Bundesregierung, a.a.0., S. 183; Böhnke, B., Was vertragen überhaupt unsere Binnengewăsser an Belastungen und welcher Relnigungsgrad läBt sich hieraus fúr Kläranlagen ableiten, in: Schultze, H. (Hrsg.), Umwelt-Report, a.a.0., S. 96 .

283) Vgl. Materiallenband zum Umweltprogramm der Bundesregierung, a.a.0., S. 183; FuB, K., Gewässerschåden - Gewăsserschutz, in: Der Landkre1s, 1970 , Heft $8 / 9$, s. 328. 
relchen würde, es als gesundheitsschädlich $z$ u bezelchnen sondern auch wegen der infolge der Eutrophierung entstehenden Phytoplanktontoxine 284)

$(\eta)$ Wirkungen weichen Wassers

Eine geringe Wasserhärte kann - wie epldemiologische Untersuchungen vermuten lassen - $z u$ Koronarinfarkten führen, da bel welchem Wasser Spurenstoffe gelöst werden 285)

(v) Wirkungen der thermischen Belastung

Die thermische Belastung der Gewässer fuhrt zu einer Vermehrung der Blaualgen und der pathogenen und apathogenen Bakterien, die alle hochtoxische Stoffe an das wasser abgeben, die auch bel der Trinkwasseraufbereitung nicht zu beseitigen sind 286 )

(i) Gesundheitsschädliche Wirkungen der stickstoffdungung Durch die Düngung mit Stickstoffverbindungen (vor allem Nitrate) kommt es zu einer Nitratanreicherung in den Pflanzen (z.B. im Spinat) und durch Abschwemmung der Boden sowle Vers1ckerung von Regenwasser unter Umständen auch im Grundwasser ${ }^{287}$ ) Voraussetzung für eine gesundheltsschädliche Wirkung ist die Umwandlung des Nitrats in Nitrit. Nitrit kann bel sauglingen zu Blausucht fuhren 288). Eine kanzerogene wirkung kann Nitrit beim $2 u-$ sammentreffen mit bestimmten sekundären Aminen im Magen-DarmTrakt haben 289 ?

$284)$ Vgl. Simonis, w., Eutrophierung, a.a.0., S. 170; Borneff, J., Hyglene, a.a.0., S. 103.

285) Vgl. ebenda, S. 50.

286) Vgl. die Ausführungen von $K$. Holl bel der öffentllchen Anhörung vor dem Innenausschuß des Deutschen Bundestages, in: Zur Sache 2/75, a.a.0., S. 145.

287) Vgl. Borneff, J., Schadstoffe im Wasser, a.a.0., S. 222; Sander, J., Ernährung und Krebs, in: Schultze, H. (Hrsg.), Umwelt-Report, a.a.o., S. 303 f.; Materlallenband zum Umweltprogramm der Bundesreglerung, a.a.0., S. 99; RSU, Umweltgutachten 1974, a.a.O., S. 121.

288) Vgl. Borneff, J., Schadstoffe im Wasser, a.a.o., S. 222; derselbe, Hyglene, a.a.0., s. $61 \mathrm{f}$.

289) Vgl. ebenda; Sander, J., Ernahrung und Krebs, a.a.0., S. $301 \mathrm{ff.;}$ RSU, Umweltgutachten 1974, a.a.0., S. 121. 
(c) Gesundhe1tsschädl1che Wirkungen der Bodenbelastung

( $\alpha$ ) Wirkungen der primär quantitativen Bodenbelastung

Die Bebauung des Bodens und der Abbau von Bodenschätzen können ebenso wie die Erosion langfristig zu einer Existenzbedrohung der Menschheit werden, da hierdurch - ceteris paribus - die Nahrungsmittelproduktion elngeengt und die Rohstoffreserven allmahlich erschöpft werden. Wir wollen uns hier auf die gesundheltsschädlichen wirkungen beschränken, die vor allem durch die Art der Bebauung des Bodens mit Siedlungen und Verkehrsbauten hervorgerufen werden, den sogenannten affekt1ven Emissionen 290). Da ist zunächst einmal die visuelle Beelnträchtigung zu nennen, die bestimmte Gebăude, siedlungen und Verkehrsbauten hervorrufen, Indem sie stadt- und Landschaftsbilder zerstören. Solche das ästhetische Empfinden beeinträchtigende Bauten beeinflussen zweifellos das Wohlbefinden 291) und sind somlt gesundhe1tsschädlich. Von der Architektur der stadte können aber auch psychisch und sozial schädliche Wirkungen ausgehen. Neurosen, Aggressionen, Depressivităt und andere psychische und soziale Schäden sind als Folgen der kommunikationsfeindilchen Architektur empirisch nachgewiesen 292). Ein beredtes Beispiel sind die psychischen Krankheitssymptome bel vielen Bewohnern des Märkischen Viertels in Berlin 293)

$(\beta)$ Herbizid-Wirkungen

Herbiz1de können elne Relhe von Gesundhe1tsschäden hervorrufen;

290) Vgl. Steckeweh, P., Umweltschutz und Stădtebau, in: Bundesbaublatt, Bd. 20, 1971, Heft 2, S. 71.

291) Vgl. Schaefer, H., Die రkologie als Problem des Umweltschutzes, a.a.O., S. 127; OECD, Effects of Traffic and Roads on the Environment in Urban Areas, Paris 1973, S. $9 \mathrm{ff}$. und S. $39 \mathrm{ff}$.; Benthem, R.J., Stădtische Ballungen, in: Offner, H. (Hrsg.), Die Zukunft der Landschaft in Europa, a.a.0., S. 19.

292 ) Vgl. Piperek, M., Bauen als Umweltzerstörung. Eine visuelle Bankrotterklärung, in: Umwelt, 1974, 1, S. 28 f.; Friedman, S., Facing Man and Soclety: The Challenge, a.a.o., s. 33 f.; Aurand, K., Umweltschutz und Gesundheit, in: Aufgabe Zukunft - Qualität des Lebens, Bd. 4, a.a.0., S. 55.

293) Vgl. Schultz, U., Umwelt aus Beton oder Unsere unmenschlichen Stădte, Reinbek 1971, S. 7; Steckeweh, P., Umweltschutz und städtebau, a.a.0., s. 71. 
sie können $z u$ Potenzstörungen Leukämieanfälligkeit steigern togene 295) und genetische 296) Effekte haben.

(r) Wirkungen der Abfälle

Welche gesundheltsschädlichen Wirkungen von den Abfällen ausgehen können, hängt natürlich von der Art der Abfallstoffe ab. Besonders Industriemüll ("Sondermüll") und Abfälle aus dem medizinischen Berelch können hochtoxische Schadstoffe enthalten; Klärschlamm ist erheblich mit Bakterien angerelchert 297) Besonders wenn die Abfälle ungeordnet depontert werden, locken sie Krankheitsüberträger wie Fliegen, Mücken und Ratten an, so daB sie zu seuchenhygienischen Gefahrenherden werden 298) und daruber hinaus eine visuelle und geruchsintensive Beeinträcht1gung darstellen 299?

(d) Gesundhe1tsschädliche Wirkungen der Pflanzen- und Tierweltbelastung

Neben den in den vorangegangenen Abschnitten geschilderten Gesundheltsgefahrdungen infolge der mit der Nahrung verbundenen Aufnahme schadstoffbelasteter Pflanzen und Tiere sind noch andere gesundhe1tl1che Beeinträchtigungen denkbar.

Die Vernichtung von Waldgebleten im Gebirge hat z.B. zur Folge, daB die dort lebenden Menschen verstärkt der Gefahr von Lawinen

294) Vgl. Kurir, A., Chemische Pflanzenbekämpfung - Gefahren für Mensch und Tier, Teil 1, a.a.0., S. 23 f. und Teil 2, in: $\mathrm{U}, 1974,5, \mathrm{~S} .34$.

295) Degenhardt, K.-H., Teratolog1sche Probleme der Umweltverschmutzung, a.a.O., S. 292.

296) Vgl. Schaefer, H., Die Okologie als Problem des Umweltschutzes, a.a.0., S. 119 f.

297) Vgl. Böhnke, B., Was vertragen uberhaupt unsere Binnengewässer an Belastungen und welcher Reinlgungsgrad läBt sich hieraus für Kläranlagen ableiten?, a.a.0., S. 97.

298) Vgl. Presse- und Informationsamt der Bundesregierung (Hrsg.), Brennpunkt Müllproblem, Bonn 1968, S. 9; Olschowy, G./Mrass, W./Kullmer, H.J./Bürger, K., Zur Belastung der Landschaft, a.a.0., S. 53.

$299)$ Olschowy, G., Bllanz des Abfallproblems, a.a.O., S. 216. 
ausgesetzt sind, und die zerstörung von wäldern in der Nähe von Ballungsgebieten beraubt die Bevolkerung einer wichtigen Möglichkeit der Naherholung 300)

Die Dezimierung oder gar vollständige Ausrottung von Pflanzenund Tierarten fuhrt zu einer Verarmung der Natur, die nicht nur negative ökologische Auswirkungen hat 301), sondern auch das Wohlbefinden der Menschen negativ beeinflussen kann. Die ökologischen Schäden, wie die Zunahme von Schädlingen, können darüber hinaus ebenfalls gesundheitsgefahrdend oder gar existenzbedrohend sein - denken wir nur an die zunahme von Heuschreckenschwärmen infolge der Vernichtung der natürlichen Feinde der Heuschrecken und die damit verbundene Vernichtung ganzer Ernten.

(e) Gesundhe1tsschädl1che Wirkungen der Lebensmittelbelastung In diesem Abschnitt wollen wir uns kurz mit den gesundheitsschädlichen Folgen der direkten Lebensmittelbelastung durch $\mathrm{Zu}-$ satzstoffe zwecks Konservierung und zusätze zu Futtermitteln befassen; die gesundheitsschädlichen Wirkungen der indirekten Arten der Lebensmittelbelastung, die via Nahrungskette entstehen können, wurden bereits in den vergangenen Abschnitten behandelt. Konservierungsmittel sind vor allem Nitrite und Nitrate. Wie wir bereits wissen, kann Nitrit im Magen-Darm-Trakt mit bestimmten Aminen Nitrosamine bilden, die Krebs hervorrufen 302 ) Als Zusätze zum Tierfutter werden unter anderem Antibiotika und andere Pharmaka verwendet. Folgen können neben toxischen auch allergische Reaktionen und eine Antibiotika-Resistenz sein 303)

300) Vgl. Olschowy, G., Katalog ökologischer Noxen und der von thnen bedingten Schäden, a.a.0., S. 159.

301) Siehe oben S. 75 ff.

302) Vgl. oben S. 101 und RSU, Umweltgutachten 1974, a.a.0., S. 67; Preussmann, R., Analytik und Entstehung von N-NitrosoVerbindungen, in: DFG-Mittellungen $2 / 74, \mathrm{~S} .21 \mathrm{f}$.

303) Vgl. RSU, Umweltgutachten 1974, a.a.0., s. 67. 
(f) Gesundheitsschädliche Wirkungen des Lärms

$\mathrm{Ob}$ Lärm gesundheitsschädlich ist, hängt von objektiven Tatbeständen $a b$ - wie der Stärke des Lärms, der Frequenz bzw. der Höhe der Töne und dem zeitlichen Auftreten 304) - und von der individuell verschiedenen Lärmempfindlichkeit der von der Lärmimmission Betroffenen: Lärm wird also subjektiv unterschiedlich empfunden 305) Die Lärmempfindlichkeit ist unter anderem altersbedingt. Altere Menschen sind lärmempfindlicher als jüngere, allerdings nicht gegenuber Tönen mit hoher Frequenz, da sie diese nicht mehr wahrnehmen können 306) Ein weiterer, subjektiver Faktor, der die gesundheltsschädliche Wirkung des Lärms mitbestimmen kann, ist die Einstellung des Individuums zum Lärm an sich, der Lärmquelle und dem Lärmverursacher 307) Klosterkötter/Gono folgend, wollen wir die Lärmwirkungen in $(\alpha)$ aurale und $(\beta)$ extraaurale Lärmwirkungen unterteilen; letztere werden noch einmal gegliedert in $\left(\beta_{1}\right)$ physiologische

304) Vgl. Olschowy, G./Mrass, W./Kullmer, H.J./Bürger, K., Zur Belastung der Landschaft, a.a.0., S. 55; Grandjean, E./ Gilgen, A./Bättig, K., Die Fluglärmbelastung, in: Städtehyglene, Bd. 20, 1969, Nr. 4, S. 74; Lüscher, E., Gesundheltsschädliche wirkungen des Lärms und deren Ursachen, in: Zeitschrift für Präventivmedizin, 12/1957, S. 447 f.

305) Vgl. Nitschke, E., Lărm, in: Schultz, U. (Hrsg.), Lebensqualität, Frankfurt/M. 1975, s. 143; Committee on the Problem of Noise, Noise, Final Report, H.M.S.O., Reprint, London 1971, S. 2 und 8; Klosterkotter, W. bei der offentlichen Anhörung vor dem InnenausschuB des Deutschen Bundestages, in: Zur Sache 4/73, Umweltschutz (III), S. 29.

306) Vgl. N1tschke, E., Lärm, a.a.0., S. 152; Klosterkötter, w., Lärmforschung und Lärmbekämpfung, 1n: Universitas, 28. Jg., 1973, S. 891; Hösel, G., Umweltkrise und Gesundheitsgefahren, in: Der Städtetag, Bd. 24, 1971, 2, S. 75 .

307) Vgl. Lủscher, E., Gesundheitsschädliche Wirkungen des Lärms und deren Ursachen, a.a.0., S. 451; von Eiff, A.W., Mensch und Lärm, in: DFG-Mittellungen von der Jahresversammlung 1971, Bonn 1971, S. 33; Hörmann, H. bel der offentlichen Anhörung vor dem InnenausschuB des Deutschen Bundestages, in: Zur Sache 4/73, a.a.0., S. 38; DFG (Hrsg.), Fluglärmwirkungen. Eine Interdisziplinäre Untersuchung über die Auswirkungen des Fluglärms auf den Menschen, Bd. 1, Boppard 1974 , S. 238 . 
und $\left(\beta_{2}\right)$ psychische Lärmwirkungen 308)

(ג) Aurale Lärmwirkungen

Die klassiche Lärmwlrkung ist der Gehörschaden, der über Schwerhörigkeit bis hin zur Taubheit führen kann 309) Be1 Gehörschäden hervorrufenden Lärmintensitäten (über 90 DIN-Phon) führt kont1nuierlicher Lärm eher zu Gehörschäden als diskontinuierlicher Lärm, da das Innenohr dabei keine Erholungsphasen hat. Bel kontinuierlichem Lärm besteht die Gefahr einer dauerhaften Hörschwellenverschiebung (PTS = Permanent Threshold Shift), bei diskontinuierlichen Lärm dagegen nur die einer vorübergehenden (TTS = Temporary Threshold Sh1ft) 310) Gehörschäden sind fast ausschlieblich die Folge von Lärm am Arbeltsplatz 311) Wahrscheinlich sind jedoch Mikroschädigungen des Innenohrs durch den Alltagslärm ("Sociocusis") eine wesentliche Teilursache des Altershörverlustes 312) Auch Kommunikationsstörungen durch Lärm zählen Klosterkötter/Gono zu den auralen Lärmwirkungen 313)

308) Vgl. Klosterkötter, W./Gono, F., Quellen und gesundheltliche Wirkung des Lärms, in: Zentralblatt für Bakteriologie ...., a.a.0., s. $307 \mathrm{ff}$.

309) Vgl. von Elff, A.W., Mensch und Lärm, a.a.O., S. 36; U.S. Environmental Protection Agency, Effects of Noise on People, U.S.G.P.O., Washington, D.C. 1971, S. 6 ff.; RSU, Umweltgutachten 1974, a.a.0., S. 77; Fleischer, K., Schall als Umweltnoxe, in: Schaefer, H. (Hrsg.), Folgen der Zivilisation, a.a.0., S. 68; Klosterkötter, W. , Lärmwirkungen auf den Menschen, in: Schultze, H. (Hrsg.), Umwelt-Report, a.a.O., S. 262; U.S. Department of Commerce, The Noise Around Us, U.S.G.P.O., Washington, D.C., 1971, S. 12; Bryan, M./Tempest, W., Cause for Concern - Noise Pollution of the Work Environment, in: International Journal of Environmental Studies, vol. 1, 1971, S. 100; Hösel, G., Umweltkrise und Gesundheitsgefahren, a.a.0., S. 75 .

310) Vgl. die Ausführungen von A.W. von Elff bel der öffentlichen Anhörung vor dem InnenausschuB des Deutschen Bundestages, in: Zur Sache 4/73, a.a.0., S. 40; Fleischer, K., Schall als Umweltnoxe, a.a.0., S. 69.

311) Vgl. die Ausfuhrungen von $W$. Klosterkötter bel der offentlichen Anhörung vor dem InnenausschuB des Deutschen Bundestages, a.a.0., S. 34 .

312) Vgl. ebenda.

313) Vgl. Klosterkbtter, W./Gono, F., Quellen und gesundheltliche Wirkungen des Lărms, a.a.0., S. 307; Klosterkötter, W., Lärmwlrkungen auf den Menschen, a.a.0., S. 262; Bryan, 
Sie führen jedoch nicht (zumindest nicht direkt) zu physischen Gesundheitsschäden - wenn man von elner möglichen Uberanstrengung des Stimmorgans zur Ubertönung des Lärms absieht -, sondern infolge der vermehrten Konzentration $z u$ einer psychischen Anspannung und somit eventuell $\mathrm{zu}$ einer Beelnträchtigung des Wohlbefindens 314?

\section{( $\beta$ ) Extraaurale Lärmwirkungen}

\section{$\left(\beta_{1}\right)$ Physlologische Lärmw1rkungen}

Die wichtigste physiologische Lärmwirkung, die bereits ab einer Lautstärke von 60 DIN-Phon einsetzen kann 315), ist die Aktivierung des zentralen und vegetativen Nervensystems wirkungen auf den gesamten Organismus haben kann 316) was Aus317) besonders auf Blutdruck und stoffwechsel 318). Möglicherweise ist Lärm ein Risikofaktor für die Entstehung der essentlellen Hypertonie

M./Tempest, W., Cause for Concern - Noise Pollution of the Work Environment, a.a.0., s. 100; Committee on the Problem of Noise, Nolse, a.a.0., S. 11; U.S. Environmental

Protection Agency, Effects of Noise on People, a.a.0., S. $43 \mathrm{ff.;}$ RSU, Umweltgutachten 1974, a.a.0., S. 77; DFG (Hrsg.), Fluglärmwl rkungen, Bd. 1, a.a.0., s. 240.

314) Vgl. die Ausführungen von $W$. Klosterkötter bel der bffentlichen Anhorung vor dem InnenausschuB des Deutschen Bundestages, a.a.0., s. 33 .

315) Vgl. Olschowy, G./Mrass, W./Kullmer, H.J./Bürger, K., Zur Belastung der Landschaft, a.a.0., S. 56 .

316) Vgl. die Ausführungen von w. Klosterkotter bel der öffentlichen Anhorung vor dem Innenausschus des Deutschen Bundestages, a.a.0.. s. $30 \mathrm{ff.;}$ Klosterkbtter, W./Gono, F., Quellen und gesundheitliche Wirkungen des Lärms, a.a.o., S. 308 f.; von Eiff, A.W., Mensch und Lärm, a.a.0., S. 39 ; Fleischer, K., Schall als Umweltnoxe, a.a.0., S. 70; Grandjean, E./Gilgen, A./Bättig, K., Die Fluglärmbelastung, a.a.0., S. 73; Lüscher, E., Gesundheitsschädliche Wirkungen des Lärms und deren Ursachen, a.a.0., S. 450; RSU, Umweltgutachten 1974, a.a.0., s. 77; Klosterkötter, W., Lärmwirkungen auf den Menschen, a.a.0., S. 262 .

317) Vg1. Drob11, M., Der Lärmschutz in der Raumplanung, In: Mitteilungen des österreichischen Instituts für Raumplanung, $1970,143 / 1-4$, S. 11.

318) Vgl. Hösel, G., Umweltkrise und Gesundheitsgefahren, a.a.0., S. 75; DFG (Hrsg.), Fluglärmwirkungen, Bd. 1, a.a.0., s. $421 \mathrm{f} . ;$ Klosterkötter, W., Lärmwirkungen auf den Menschen, a.a.o., s. 263 . 
Die am weitesten verbreitete Folge der Aktivierung des zentralen und vegetativen Nervensystems ist jedoch die storung von Schlaf, Entspannung und Erholung 320) Sowohl schlafdauer als auch Schlafqualität (Schlaftiefe) können gestört werden, so daB ein Schlafdefizit entsteht 321) das physische und psychische schäden verursachen kann 322)

$\left(\beta_{2}\right)$ Psychische Lärmwirkungen

Psychische Lärmwirkungen können berelts bel Lärm unterhalb von 60 DIN-Phon eintreten 323) Der Lărm wird dann elnfach als lăstig empfunden und kann Ärger, Zorn, Aggresivität, unertrăgliche Spannung, Nervosität und Schreckreaktionen auslösen 324) Diese psychischen Lărmwirkungen - der "Lărmstress" 325) - können

319) Vgl. DFG (Hrsg.), Fluglärmwirkungen, Bd. 1, a.a.0., S. 315.

320) Vgl. die Ausführungen von $\mathrm{w}$. Klosterkötter bel der öffentlichen Anhörung vor dem InnenausschuB des Deutschen Bundestages, a.a.0., s. $30 \mathrm{ff.;}$ derselbe, Lărmwirkungen auf den Menschen, a.a.0., S. 262; RSU, Umweltgutachten 1974, a.a.0., S. 77; Committee on the Problem of Nolse, Nolse, a.a.0., S. 7; Hösel, G. , Umweltkrise und Gesundheitsgefahren, a.a.0., s. 75 .

321) Vgl. die Ausfuhrungen von $w$. Klosterkötter bel der öffent11 chen Anhörung vor dem InnenausschuB des Deutschen Bundestages, a.a.O., S. $30 \mathrm{ff.;}$ Flelscher, K., Schall als Umweltnoxe, a.a.0., s. 71 .

322) Vgl. U.S. Environmental Protection Agency, Effects of Noise on People, a.a.O., S. 78 und die Ausfuhrungen von U.J. Jovanovic bei der offentlichen Anhörung vor dem InnenausschuB des Deutschen Bundestages, in: Zur Sache 4/73, a.a.0., S. 42 ff.

323) Vgl. Olschowy, G./Mrass, W./Kullmer, H.J./Bürger, K., Zur Belastung der Landschaft, a.a.0., s. 56.

$324)$ Vgl. Klosterkötter, W./Gono, F., Quellen und gesundheltliche Wirkungen des Lärms, a.a.0., S. 309 ; U.S.

Environmental Protection Agency, Effects of Noise on People, a.a.0., S. 101 und S. 134 sowle die Aus führungen von A.W. von Eiff bel der öffentlichen Anhörung vor dem Innenausschuß des Deutschen Bundestages, a.a.0., S. 40 sowle von $W$. Klosterkobter, ebenda, S. $29 \mathrm{f}$.

$325)$ Vgl. Klosterkötter, W./Gono, F., Quellen und gesundhe1tliche Wirkungen des Lärms, a.a.0., S. 309. 
wiederum negative Auswirkungen auf das vegetative Nervensystem haben 326) und zu Einschlafstörungen, Schlafunterbrechungen und einer Minderung der Schlafqualität führen 327) Bel den hier relevanten Lautstärken von unter 60 DIN-Phon hat ungleichmäßiger Lărm elne gröBere störwirkung als kontinulerlicher Lärm. Schlafstörungen werden vor allem durch elnen Wechsel des Gerăuschpegels bewirkt 328 )

Eine extraaurale Lärmkrankheit ist bisher nicht bekannt; Lărm ist jedoch zweifellos ein stressfaktor und wirkt als solcher am Entstehen von Krankheiten mit 329) Geht man - wie wir das getan haben - von der Gesundheitsdefinition der wHO aus, sind jedoch auch bereits die Belästigungen durch geringeren Lărm gesundheltsschädlich 330 ?

(y) Möglichkeiten der Lärmgewöhnung

An Lărmstärken, die $z u$ einem Gehörschaden füren, gibt es keine Gewohnung. Ob die physiologischen Lärmwirkungen mit der Zeit nachlassen, 1st umstritten. Während einige Autoren meinen, Schäden des vegetativen Nervensystems könnten nicht vermieden werden 331) halten andere elne Gewöhnung bis zu einer bestimmten Lautstärke für möglich. Von Elff meint, daß bis zu 90 dB bei den

326) Vgl. Lüscher, E., Gesundhe1tsschädliche Wirkungen des Lărms und deren Ursachen, a.a.0., S. 450.

327) Vgl. Klosterkotter, W./Gono, F., Quellen und gesundheltliche Wirkungen des Lärms, a.a.0., s. 311.

328) Vgl. Flelscher, K., Schall als Umweltnoxe, a.a.0., S. 71; Klosterkötter, W. , Lärmforschung und Lärmbekămpfung, a.a.0., S. 898 und U.J. Jovanovic bel der offentlichen Anhörung vor dem InnenausschuB des Deutschen Bundestages, a.a.0., S. 42 .

329) Vgl. Klosterkötter, W./Gono, F., Quellen und gesundhe1tliche Wirkungen des Lärms, a.a.0., S. 313; Bryan, M./ Tempest, W.. Cause for Concern - Nolse Pollution of the Work Environment, a.a.0., S. 100.

330) Vgl. Committee on the Problem of Nolse, No1se, a.a.0., S. 7; U.S. Department of Commerce, The No1se Around U's, a.a.0., s. 13 .

331) Vgl. Fleischer, K., Schall als Umweltnoxe, a.a.0., s. 70; Drob11, M., Der Lärmschutz in der Raumplanung, a.a.0.. S. 12; Olschowy,. G./Mrass, W./Kullmer, H.J./Blurger, K., Zur Belastung der Landschaft, a.a.0., S. 56 . 
meisten Menschen eine Gewöhnung eintritt, allerdings nicht bel der peripheren Gefabreaktion, wo er die Grenze bel etwa 70 dB sieht 332). Anpassungen an geringen Lärm gelten allgemein als mögllch, hängen jedoch von der individuellen Lärmempfindlichkeit und anderen subjektiven Faktoren ab, wie etwa der Einstellung zum Lärmverursacher 333) Auch objektive Faktoren wie Intensität und Regelmäßigkeit des Lärmgeräusches bestimmen die Adaptionsmöglichkeiten 334) Andererselts ist zu bedenken, daB auch eine Sensibilisierung möglich ist ${ }^{335}$ ) und die jeweiligen Anpassungsvorgänge den Organismus belasten können 336)

\section{(6) Zur Beachtung der toxikologischen Gesamtsituation}

In den vorangegangenen Abschnitten haben wir fast ausschlieBlich die gesundheitsschädlichen wirkungen jewells eines Schadstoffes in einem Medium betrachtet. Häufig kommt der gleiche Schadstoff aber in mehreren Medien gleichzeitig vor, so daB die jeweilige Schadstoffbelastung des Menschen gröBer ist als die Betrachtung der Belastung elnes Mediums vermuten läßt 337 ) Schwerwiegender für die menschliche Gesundheit ist allerdings, daB der Mensch nicht nur einem Schadstoff ausgesetzt ist, sondern gleichzeltig von einer Fülle von Schadstoffen belastet wird. Die gesundheltsschädlichen Wirkungen der Schadstoffe

332) Vgl. die Ausfuhrungen A.W. von Elffs bei der öffentlichen Anhörung vor dem InnenausschuB des Deutschen Bundestages, a.a.0., S. $40 \mathrm{ff}$. und derselbe, Mensch und Lärm, a.a.0., S. $40 \mathrm{f}$.

333) Vgl. Committee on the Problem of Nolse, Nolse, a.a.O., S. 10; RSU, Umweltgutachten 1974, a.a.0., S. 79 und die Ausfuhrungen von $W$. Klosterkotter bei der öffentlichen Anhörung vor dem InnenausschuB des Deutschen Bundestages, a.a.O., S. 37 sowle von H. Hörmann, ebenda, S. 39.

334) Vgl. die Ausführungen von U.J. Jovanovic bei der öfentlichen Anhörung vor dem InnenausschuB des Deutschen Bundestages, a.a.0., S. 47.

335) Vgl. die Ausführungen von W. Klosterkötter, ebenda, S. 37.

336) Vgl. die Ausführungen von H. Hörmann, ebenda, S. 39.

337) Vgl. Bär, F., Die toxikologische situation in der modernen Zivilisation, in: Sioli, H. (Hrsg.), రkologie und Lebensschutz in internationaler sicht, a.a.0., s. 172 . 
- man spricht von Kombinationswirkung - können sich addieren oder neutralisieren 338). Auch synergismen können vorkommen. Darunter versteht man, daß die Gesamtwirkung der Schadstoffe größer 1st als die summe ihrer Elnzeleffekte 339) Manche in der Umwelt vorkommende stoffe sind an sich nicht gesundheltsschädlich, sondern haben nur eine Katalysator- oder Schlepperfunktion für Schadstoffe 340) Manchmal sind es auch erst die Abbauprodukte (Metaboliten) der (Schad)stoffe, die gesundheltsgefährdend wirken 341). Erst die Beachtung all dieser Faktoren, der sogenannten toxikologischen Gesamtsituation 342), erlaubt eine realistische Einschätzung der Gesundheltsgefahren, die von der Umweltbelastung ausgehen können.

\section{(7) Zur Aufstellung eines Wirkungskatasters}

Zuverlässige Informationen über die toxikologische Gesamtsituation kann man nur durch die systematische Erfassung der Schadstoffwirkungen auf den Menschen und seine natürliche und materlelle Umwelt gewinnen 343) Das könnte am besten in einem wirkungskataster geschehen, das neben Emissions- und Immissionskataster die dritte wesentliche Informationsquelle für die Um-

338) Vgl. Băr, F., Die toxikologische situation in der modernen Zivilisation, in: Siol1, H. (Hrsg.), Okologie und Lebensschutz in internationaler sicht, a.a.0., s. 172; Schl1pköter, H.-W., Auswirkungen von Autoabgasen auf die stadtbevölkerung, a.a.0., s. 33.

339) Vgl. Bär, F./Grunow, W., Hygienisch-toxikologische Aspekte der Umweltsituation, a.a.O., S. 290; Cassell, E.J., The Health Effects of Air Pollution, a.a.0., S. 214.

340) Vgl. Bär, F., Die toxikologische situation in der modernen Zivilisation, a.a.0., S. 185 .

$341)$ Vgl. Marquardt, H., Umweltbedingte Mutagenităt, in: Schultze, H. (Hrsg.), Umwelt-Report, a.a.0., S. 297.

342) Vgl. Bär, F./Grunow, W., Hygienisch-toxikologische Aspekte der Umweltsituation, a.a.0., S. 289 ; Coenen, R. u.a., Alternativen zur Umweltmisere, a.a.0., S. 109.

343) Vgl. Entwurf der gesundheltspolitlschen Leltsatze, vorgelegt vom Gesundheitspolitischen Ausschuß beim SPD-Vorstand, 1n: Langze1tprogramm 1, Bonn-Bad Godesberg 1972, S. 195. 
weltplanung und die Umweltpolitik darstellen sollte 344) Die Aufstellung eines solchen wirkungskatasters erfordert einerseits ein Immissionskataster, das (möglichst kontinuierlich) aufgezeichnete Daten uber die Immissionsbelastung einer Region enthält und andererselts gegenüber dem gegenwärtigen $\mathrm{Z} u s t a n d$ verbesserte Morbiditäts - und Mortalitätsstatistiken, welche zuverlässige epidemiologische studien erlauben. Die Schadstoffwirkungen können auch mit Hilfe von exponierten Wirkungsobjekten (Bloindikatoren) ermittelt werden, die zu diesem zweck im Immissionsgebiet verteilt werden 345 ?

344) Vgl. RSU, Umweltgutachten 1974, a.a.0., S.17 f.; Stratmann, H. Emissionsüberwachung und Immissionsschutz, Vortrag beim IBM Seminar "Umweltschutz und Datenverarbeltung" vom 7.-9. März 1973 in Bad Liebenzell, 0.0. und J.. S. 14 ff.

345) Vgl. RSU, Umweltgutachten 1974, a.a.0., S. $17 \mathrm{f} ;$; von Borries, D.F.W., Zur Konstruktion von Umweltindizes, a.a.0., S. 62 . 
"Die überhandnehmende Technik quält und ängstigt mich - es wälzt sich herum wie ein Gewitter, langsam, langsam; aber es hat seine Richtung genommen, es wird kommen und treffen".

(J.W. von Goethe, Wilhelm Meisters
Wanderjahre)

3. Zur Diagnose der Ursachen der Umweltbelastung

a) Probleme und Ansätze der Ursachendiagnose

Elne langfristig wirksame Therapie der Umweltbelastung, die mehr als ein Kurieren von symptomen sein will, muß die eigentlichen Ursachen $c \geqq r$ Umweltbelastung bekämpfen 346). Dazu bedarf es aber zunächst siner Diagnose der Ursachen, und zwar nicht nur der mehr oder weniger offensichtlichen (und vordergründigen) Ursachen wie der Emission von Schadstoffen, sondern der hinter diesen steckenden tieferen Ursachen 347) Daruber was diese tieferen Ursachen sind, gibt es verschiedene Hypothesen. Man kann sie in vier Gruppen einteilen, von denen wir die erste nur kurz erwähnen wollen. Das ist die Gruppe, welche das (angeblich) durch die christliche Religion geprägte Verhältnis des Menschen zur Natur als elgentliche Ursache der Umweltbelastung ansieht 348) Die "orthodox christliche Arroganz

346) Vgl. Hödl, E., Umweltpolitik, Die Scheuklappe der "Okonomisten", in: Die Wirtschaftswoche, 26. Jg., 1972, Heft 8, S. 39 ; Kade, G., Marktwirtschaft und Umweltschutz, in: gdi-toples $4 / 71$, S. 10.

347) Vgl. von Walterskirchen, K.P., Bemerkungen zur Umweltpol1tik, in: Horn, C. u.a. (Hrsg.), Umweltpolitik in Europa, Frauenfeld 1973, S. 13 ff.; Forrester, J.W., Der teufliche Regelkreis, stuttgart 1972, S. 15 und S. 36; Tschumi, P.A., Bevölkerungswachstum, Wirtschaft und Umweltschutz, in: NZZ, FA Nr. 236 vom 29.8.1971, S. 37.

348) Vgl. White, jr, L.T., Die historischen wurzeln unserer ökologischen Krise, in: Lohmann, M. (Hrsg.), Gefährdete Zukunft - Prognosen angloamerikanischer Wissenschaftler, München 1970, S. $20 \mathrm{ff}$; Blanke, F., Der Christ und die Erde, In: Natur geplündert oder gehegt. Schrlften der Neuen 
gegenüber der Natur" 349) 1st nach dieser Theorie zusammen mit einer gewissenlosen Ausnutzung der wissenschaftlichen und technischen Entdeckung Ursache für eine bedenkenlose Nutzung der Natur 350) Unseres Erachtens kann es nicht Gegenstand einer wirtschaftswissenschaftlichen Arbelt seln, diese Theorle auf ihren Erklärungswert $z u$ untersuchen. Wir wollen uns deshalb im folgenden den drel anderen - einer ökonomischen Beurtellung $z u-$ gänglichen - Ursachendiagnosen zuwenden.

Die erste dieser dre1 Gruppen glaubt als Ursachen der Umweltbelastung vor allem das Bevölkerungswachstum und die damit einhergehende Bevblkerungsdichte sowie den stelgenden Wohlstand diagnostizieren zu können. Diese vor allem von Nicht-రkonomen gestellte Ursachendiagnose wollen wir als fatalistische Ursachendiagnose bezeichnen.

Die zwe1te Gruppe von Erklarungsversuchen 1st 1deologisch geprägt. Ihre Verfechter fuhren die Umweltbelastung auf das marktwirtschaftliche system und das damit verbundene Gewinnstreben zurück. Wir nennen sle 1 deologisch geprăgte Ursachendiagnose. Die dritte Gruppe schlieblich sieht die Ursache der Umweltbelastung überwiegend in elnem Versagen des staates, der nicht rechtzeitig Umweltschutzmaßnahmen beschlossen und vor allem durchgesetzt hat - obwohl dies grundsätzl1ch mögl1ch gewesen

Helvetischen Geselischaft, Heft 9, Horgen 1961, S. 11 ff.; Cort1, W.R., Mensch und Natur, ebenda, S. 53 ff.; Rade, G., Ckonomische und gesellschaftspolitische Aspekte des Umweltschutzes, in: Gewerkschaftliche Monatshefte, 22 . Jg., 1971 , S. 259 .

349) White, jr., L.T., Die historischen Wurzeln unserer 8kologischen Krise, a.a.0., S. 29.

350) Vgl. Huber, G., Gelstige Implikationen der Umweltverănderung, in: Leibundgut, H. (Hrsg.). Schutz unseres Lebensraums, München-Bern-Wien 1971, S. $118 \mathrm{ff.;} \mathrm{Picht,} \mathrm{G.,} \mathrm{Um-}$ weltschutz und Politik, a.a.0., S. 86 . Widerspruch findet diese Ursachendiagnose u.a. bei Moncrief, L.W. The Cultural Basis for Our Environmental Crisis, in: Dorfman, R./Dorfman, N.S. (Hrsg.), Economics of the Environment, New York 1972, S. 285 f. 
wăre. Wir wollen hier von einer pragmatischen Ursachendiagnose sprechen 351 )

\section{b) Zur fatalistischen Ursachendiagnose}

Diese Ursachendiagnose der Umweltbelastung bezeichnen wir deshalb als fatalistisch, well sie die zunehmende Umweltbelastung mit quas1 naturgesetzlicher GewlBhelt auf das Bevölkerungswachstum und die damit steigende Bevolkerungsdichte und Urbanisierung einerseits und den steigenden Wohlstand in Form einer durch den technischen Fortschritt ermöglichten real stelgenden Pro-KopfProduktion bzw. eines real steigenden Pro-Kopf-Konsums andererseits zurückfuhrt 352). In erster Linie dem technischen Fortschritt, der erst den stelgenden Wohlstand bel zunehmender Bevölkerung ermöglicht, werden viele Arten der Umweltbelastung zugeschrieben. Vor allem scheint der Dualismus zwischen Natur und

351) Die Bezelchnungen der Ursachendiagnose enthalten zweifellos eine Wertung; der Leser wird zurecht vermuten, daß wir uns der letzten Ursachendlagnose anschlieBen werden.

352) Vgl. Forrester, J.W., Der teufliche Regelkreis, a.a.0., S. 74 f.; Goldsmith, E./Allen, R., Planspiel zum Uberleben, a.a.0., S. 10; Schaefer, H., Die Okologie als Problem des Umweltschutzes, a.a.O., S. 139; Dubos, R., Promises and Hazards of Man's Adaptability, a.a.0., S. 32; Meadows, D. u.a., Die Grenzen des Wachstums, Bericht des club of Rome zur Lage der Menschheit, Stuttgart 1972, S. 83; Hardin, G., Die Traglk der Allmende, in: Lohmann, M. (Hrsg.), Gefährdete $\mathrm{Zukunft}$ - Prognosen angloamerikanischer Wissenschaftler, a.a.0., S. 38; Lord Llewellyn-Davies/Cowan, P., Stadtplanung und Wissenschaft, ebenda, S. 118; Benthem, R. J., Städtische Ballungen, a.a.0., S. 11; Passino, R., Industrie und Landschaft, a.a.O., S. 40 f.; Moncrlef, L.W., The Cultural Basis of Our Environmental Crisis, a.a.0., S. $287 \mathrm{f.;}$ Fisher, J.L., Impact of Population on Resources and the Environment, in: American Economic Review, vol. 61, 1971, Papers and Proceedings, S. 395 ff.; Basler, E., Umweltprobleme aus der Sicht der technischen Entwicklung, in: NZZ, FA Nr. 132 vom 16.5.1971; Tschum1, P.A., Bevölkerungswachstum, Wirtschaft und Umweltschutz, a.a.0.; Meyervon Gonzenbach, R., Die Beanspruchung der Umwelt durch die Besiedlung, in: Leibundgut, H. (Hrsg.), Schutz unseres Lebensraumes, a.a.0., S. 65 ff.; Istock, C.A., Modern Environmental Deterioration as a Natural Process, in: International Journal of Environmental Studies, vol. 1, 1971 , S. 151 ff. 
Technik als so sicher zu gelten 353) daß man die Im technischen Fortschritt liegenden Möglichkeiten des Umweltschutzes nicht sieht 354). Die entgegengesetzte Position, wie sie vor allem von Ingenleuren und Technikern vertreten wird, kommt am besten in folgendem zitat zum Ausdruck: "In a technological society, technology itself can be used creatively to help solve the human problems technology causes" 355) Der darin sichtbare Fortschrittsglaube elnes Technikers ist allerdings kaum weniger naiv als die Fortschrittsfeindlichkelt einiger Blologen. Gerade eine solche Fortschrittsgläubigkelt kann Ursache von einem ungezügelten - d.h. hier hinsichtlich der Umweltbelastung rücksichtslosen technischen Fortschritt werden 356) Bei nüchterner Betrachtung ergibt sich, daB der technische Fortschritt an sich nicht umweltfeindlich 1st, sondern nur einzelnen Richtungen, in die er

353) Vgl. Commoner, B., Die Bedeutung der Blosphäre, in: Lohmann, M. (Hrsg.), Gefährdete Zukunft - Prognosen angloamerikanischer Wissenschaftler, a.a.0., S. 101; Mislin, H., Zerstörung des Lebensraumes und Umweltverantwortung, in: Lelbundgut, H. (Hrsg.), Schutz unseres Lebensraumes, a.a.O., S. 19; Henke, w., Umwelt, Technik, Natur, Kultur, in: Recktenwald, H.C. (Hrsg.), Das Umweltproblem aus ökonomischer und juristischer sicht, Göttingen 1975, S. 31.

354) Vgl. Beckerman, W., Environmental Pollcy Issues: Real and Fictitious, in: OECD (Hrsg.), Problems of Environmental Economics, a.a.0., S. 22; Zuckerman, S., Technologie und Gesellschaft - Eine Herausforderung an die Privatwirtschaft, Grundsatzbericht vor dem XXIII. Kongress der IHK Wien, 0.0., 1971, S. 8; Schăr, M., Der Mensch als NutznleBer und Opfer der technischen Entwicklung, in: Leibundgut, H. (Hrsg.), Schutz unseres Lebensraumes, a.a.0., S. $28 \mathrm{ff}$.; Egl1, E., Die Erhaltung der Landschaft, in: Natur geplündert oder gehegt, a.a.O., s. 19 und 27; derselbe, Natur in Not, a.a.0.. S. $11 \mathrm{ff.}, 55 \mathrm{f}$. und 117; Balke, S., Umweltbedingungen als Lebensgrundlage. Schriftenrethe der Vereinigung Deutscher Gewässerschutz EV-VTG, Nr. 28, Bonn-Bad Godesberg 1970, S. 5 ff.; Menke-Gluckert, P., Umweltschutz - Achillesferse oder Herausforderung für die Industriegesellschaft, In: Die Neue Gesellschaft, 1971, 8, S. 581.

355) Smith, R.E., Polltical Control of Technology to Preserve Human Values, in: The Engineering Economist, vol. 15 (4), 1970 , S. 224 .

356) Moncrlef, L.W., The Cultural Basis for Our Environmental Crisis, a.a.0., s. 291. 
gelenkt worden ist 357). Ebensowenig ist auch der stelgende Wohlstand an sich nicht Ursache der Umweltbelastung. "It is the forms and methods of production and consumption to which our soclety has become accustomed that cause the damage" 358 )

Damit erhalten stelgender Wohlstand und technischer Fortschritt, aber auch das Bevölkerungswachstum nur noch den Charakter bestenfalis sekundärer Ursachen der Umweltbelastung 359! Die tieferen Ursachen der Umweltbelastung liegen in der falschen Lenkung des technischen Fortschritts und der damit zusammenhängenden Art der Produktionsverfahren (ProzeBinnovation) und Produkte (Produktinnovation) sowie in den Konsumgewohnheiten

c) Zur ideologisch geprägten Ursachendiagnose

Vor allem Neomarxisten sehen die Ursachen der falschen Lenkung des technischen Fortschritts in Form umweltbelastender ProzeBund Produktinnovationen und der Konsumgewohnheiten in Form des "Ex und Hopp" in dem marktwirtschaftlichen System selbst begründet; die Umweltbelastung in sozialistischen Lăndern sind dagegen ein Erbe des kapitalistischen Systems und Folge der Notwendigkeit einer raschen (nachholenden) Industrialisierung ${ }^{361 \text { ) }}$ Vor allem im Proflmotiv als Motor des marktwirtschaftlichen Systems wird die elgentliche Ursache fur die Umweltbelastung

357) Vgl. Jacoby, N.H., The Polluters: Industry or Government?, The Institute of Economic Affairs, London 1972, S. $14 \mathrm{f}$.

358) Ebenda, S. 13.

359) Vgl. dazu unten S. $126 \mathrm{f}$.

360) Vgl. Glagow, M., Umweltgefahrdung und Gesellschaftssystem, a.a.0., S. 16; Dahmén, E., Umweltschutz und ökonomische Systeme, in: Glagow, M. (Hrsg.), Umweltgefährdung und Gesellschaftssystem, a.a.0., S. 62 f.; Kade, G., Marktwirtschaft und Umweltschutz, a.a.0., S. 10; Schwarzes Kollekt1v, Okologie und Macht, in: Kursbuch 33, Berlin 1973, S. $113 \mathrm{ff}$.

361) Vgl. Höhmann, H.-H./Seidenstecher, G./Vajna, T., Umweltschutz und okonomisches System in Osteuropa, StuttgartBerlin-Köln-Mainz, 1973, S. 9 f. 
gesehen 362) In den Berelch krimineller Delikte gehorrende Umweltbelastungen (wie im Falle des Reeders Bernhold) werden als Folge des profitmotivs gedeutet und damit dem marktwirtschaftl1chen System angelastet 363)

Der Profit als Differenz zwischen Erlos und Kosten kann (auf der Produktionsseite) durch Kostensenkungen und (auf der $\mathrm{Ab}-$ satzselte) Erlosstelgerungen erhöht werden. Die durch das Profitmotiv bestimmte kapitalistische Produktionsweise zelchnet sich nach Ansicht der Neomarxisten und ihren Anschauungen nahestehenden'Vertretern durch eine ungezügelte Verwendung der natürlichen Ressourcen aus ("Verschwendungskap1talismus") 364) vor allem der unterbewerteten, wenn nicht gar kostenlosen Produktionsfaktoren Luft, Wasser und Boden, die ebenso wie die "anarchische Dezentralislerung des Produktionsprozesses" 365) fur die Umweltbelastung verantwortlich gemacht werden. Auf der Absatzselte äuBert slch das profitmotiv vor allem in der durch Marketing und Werbung herbelgeführten stelgerung der Konsumbe-

$362)$ Vgl. Schwarzes Kollektiv, రkologie und Macht, a.a.o., S. 119; Kade, G., Durch das Profitmotiv in die Katastrophe, In: Die Wirtschaftswoche, 1971, Heft 40, S. 39 ff.; Zschocke, H. Ungelöstes Problem der Umweltgestaltung in Westdeutschland, in: Glagow, M. (Hrsg.), Umweltgefăhrdung und Gesellschaftssystem, a.a.0., S. $149 \mathrm{ff}$. Ahnlicher Ansicht ist auch z.B. Sicco Mansholt (vgl. dessen Beltrag in Kursbuch 33, 1973, S. 137); vgl. ferner: Kneese, A.V., Pollution and the Profit Motive, in: Wolozin, H. (Hrsg.), The Economics of Pollution, Morristown, N.J., 1974, S. 221 .

363) Vgl. Uhrhammer, C. u.a., Umweltverschmutzung durch Profitinteressen?, In: Dörge, F.W. (Hrsg.), Qualităt des Lebens, Opladen 1973, S. 69 ff.

$364)$ Vgl. Vilmar, F., Vergeudungskapitalismus oder Wirtschaftsdemokratie, in: Kapp, K.W./Vilmar, F. (Hrsg.), Sozlal1slerung der Verluste?, München 1972, s. 12 ff.; Schwarzes Kollektiv, Okologie und Macht, a.a.O., S. 119; Kade, G., Okonomische und gesellschaftspolitische Aspekte des Umweltschutzes, a.a.0., S. 263; Kapp, K.W.. 'Recycling' in Contemporary China, in: Kyklos, Bd. 27, 1974, S. 297; Salgo, H., The Obsolescence of Growth: Capitalism and the Environmental Crisis, in: Review of Radical Political Economics, vol. 5 (3), 1973, S. 27.

365) Ronge, V., Die Umwelt im kapitalistischen System, a.a.0., S. 107 . 
dürfnisse, die in Verbindung mit der geplanten Obsoleszenz der angebotenen Güter für die zum Absatz der Güter notwendige Nachfrage sorgt 366). Die geplante Obsoleszenz und der Kauf "nicht notwendiger" Güter steigern das Abfallproblem im Konsumbereich. Neben dem Profitmotiv wird vor allem die im kapitalistischen System institutionalisierte "private Verfügungsmacht uber den Einsatz der produktiven Faktoren" 367) für die Umweltbelatung verantwortlich gemacht.

Die Verfechter der marktwirtschaftlichen ordnung wehren sich gegen diese Argumentation auf zweierlel Weise. Sie weisen zum einen auf die ihrer Ansicht nach nicht minder großen Umweltbelastungen in den Zentralverwaltungswirtschaften Osteuropas hin und versuchen zum anderen aufzuzelgen, das das marktwirtschaftliche System nicht als solches für die Umweltbelastung verantwortlich ist, sondern nur einige mit mehr oder weniger marktkonformen Maßnahmen korrigierbare Funktionsmängel 368 ) Die Ideologen beider Selten geraten allzu leicht in die Gefahr das von ihnen verteidigte Wirtschaftsordnungssystem in seiner idealtypischen Form dem realtypischen System der anderen Wirtschaftsordnung gegenüberzustellen. Die Marxisten vergleichen die konkrete situation in kapitalistischen Ländern mit dem "Idealzustand der elgenen Utopie" 369), oder aber die "Marktwirtschaft wird mit unbegrenzter Freiheit und laissez faire

366) Vgl. Kade, G., Okonomische und gesellschaftspolitische Aspekte des Umweltschutzes, a.a.0., s. 263.

367) Ebenda, S. 269.

368) Vgl. Erhard, L./Müller-Armack, A., Soziale Marktwirtschaft, Frankfurt-Berlin-Wien, 1972, S. 298 ff.; Goldman, M.I., The Convergence of Environmental Disruption, in: Dorfman, R./Dorfman, N.S. (Hrsg.), Economics of the Environment, a.a.0., S. 294 f.; derselbe, Environmental Disruption in the Soviet Union, in: Tsuru, S. (Hrsg.), Proceedings of the International Symposium on Environmental Disruption, a.a.0., S. $171 \mathrm{ff}$.

369) Issing, 0., zerstörung der Umwelt - ein Versagen des marktwirtschaftlichen Systems?, in: Recktenwald, H.C. (Hrsg.), Das Umweltproblem aus ökonomischer und juristischer Sicht, a.a.O., S. 129 ; Frey, R.L., Umweltschutz als wirtschaftspolitische Aufgabe, in: Schweizerische Zeitschrift für Volkswirtschaft und Statist1k, $108 \mathrm{Jg} ., 1972$, S. 473. 
assozilert" 370) was - wie auch Marktwirtschaftler kaum leugnen werden - zweifellos Umweltbelastungen begünstigen würde. Die Apologeten der marktwirtschaftlichen Ordnung stellen dagegen ein funktionsfähiges marktwirtschaftliches system elnem durch Bürokratismus und egoistischem Funktionärsdenken gekennzeichneten zentralverwaltungswirtschaftlichen system gegenüber 371)

Argumentationen auf dieser Ebene führen zu keiner wissenschaftlich fundierten Ursachendiagnose. Zulassig ist nur ein Vergleich der jeweiligen idealtypischen oder realtypischen Formen der Wirtschaftsordnungen 372) Beim Vergleich der idealtypischen Wirtschaftsordnungen kann man wohl zu dem Ergebnis kommen, daß die Marktwirtschaft - zumindest dann, wenn ihre Laissez faire-Komponente überwlegt - Ursache der Umweltbelastung ist; der auf Eigennutz ("Profit") bedachte Unternehmer wird durch den Konkurrenzmechanismus gerade zur Externalisierung der Umweltkosten gezwungen. Je schlechter seine umweltbezogene Grenzmoral ist, um so größer 1st der daraus gezogene Grenzgewinn. Der Staat, dessen wirtschaftspolitische Akt1vitäten so gering wie möglich sind, kann daran kaum etwas ändern 373) . In der heutigen zeit ist es jedoch nicht mehr vertretbar, den Idealtypus der Marktwirtschaft mit einer "Laissez-faire-Wirtschaft" in einem "Nachtwächterstaat" zu identifizieren. Der Idealtypus einer s 0 z 1 a 1 e $n$ Marktwirtschaft kann unseres Erachtens nur mit einem wirtschaftssystem gleichgesetzt werden, in dem der Staat auch für die Internalisierung der externen Effekte sorgt.

370) Möller, H., Zum Begriff der Umweltschăden Insbesondere im offentlichen Sektor, in: Gumpel, W./Keese, D. (Hrsg.), Probleme des Industrialismus in Ost und West, a.a.0., s. 453.

371) Vgl. Erhard, L./Müller-Armack, A., Soziale Marktwirtschaft, a.a.0., S. 295 ff.

372) Vgl. Issing, 0., Zerstorrung der Umwelt - ein Versagen des marktwirtschaftlichen Systems?, a.a.0., s. 129.

373) Vgl. Hansmeyer, K.-H./Rürup, B., Umweltgefährdung und Geselischaftssystem, in: Wirtschaftspolitische Chronik, 1973, Heft $2,5.11$ ff. 
In der idealtypischen Zentralverwaltungswirtschaft können planexterne Effekte deshalb nicht vorkommen, well dort die mit allen Informationen versehene, stets das Allgemeinwohl beachtende zentrale Verwaltungsbehörde umweltfreundlich plant, die notwendigen Befehle zur Vermeldung von Umweltbelastungen ertellt und durchsetzt.

Ein Vergleich der realtypischen wirtschaftsordnungssysteme zeigt, daB in belden die Umweltbelastung Ausmaße angenommen hat, die Gegenmaßnahmen erfordern. In den marktwirtschaftlich orlentierten Industriestaaten ist es offenbar (noch) nicht gelungen, die hinsichtlich der Umweltbelastung vorhandenen Măngel im Allokationsmechanismus Markt hinreichend zu beseitigen - d.h. konkret: die externen Effekte $z u$ internalisieren -, und in den Industriestaaten mit elner zentralverwaltungswirtschaft erfolgte keine (genügende) Ex ante-Internalisierung der Umweltkosten durch deren Berücksichtigung im Wirtschaftsplan. Diese (bisher) ungenügende Internalisierung der Umweltkosten Im Preis bzw. Plan durch den Staat kann man als eigentliche Ursache der Umweltbelastung bezeichnen 374). Die Gründe hierfür können im markt- und planwirtschaftlichen System - wenn auch mit unterschledilcher Gewichtung - die gleichen sein: (1) die fehlende oder ungenügende Bereitschaft des Staates, etwas gegen die Umweltbelastung zu unternehmen, (2) das Unvermögen, die Internalisierung durchzusetzen - vorausgesetzt, die Bereltschaft dazu ist vorhanden. Elne Diagnose der Ursachen, die frel von Fatallsmus und

374) Vgl. Jacoby, N.H., The Polluters: Industry or Government?, a.a.o., S. $19 \mathrm{ff.;}$ The Economics of National Priorities, Hearings before the Subcommittee on Priorities and Economy in Government of the Joint Economic Committee, U.S.G.P.O., Washington, D.C., 1971, S. 221; Schultze, C.I. U.a., Setting National priorities, The 1972 Budget, Washington, D.C., 1971, S. 238; Würgler, H., Okonomische und pol1tische Ursachen der Umweltveränderungen, in: Leibundgut, H. (Hrsg.), Schutz unseres Lebensraumes, a.a.0., S. $96 \mathrm{ff.;}$ Materialien zum Bericht zur Lage der Nation 1974, BTDrucksache $7 / 2423$, S. 393; Dahmen, E., Umweltschutz und ökonomische Systeme, a.a.O., S. 65; U.S. Council on Environmental Quality, First Annual Report, a.a.o., s. 12; derselbe, Second Annual Report, Washington, D.C., 1971, s. 102 . 
ideologischen Scheuklappen ist - wir bezelchnen sie als pragmatisch -, muB diese systemindifferenten Faktoren als eigentliche Ursachen der Umweltbelastung 1dentifizieren. Wir wollen diese Faktoren im folgenden Abschnitt näher betrachten.

\section{d) Zur pragmatischen Ursachendiagnose}

Die mangelnde Bereltschaft des Staates, Maßnahmen gegen die Umweltbelastung $z u$ ergreifen oder $z u$ inftileren, kann wiederum mehrere Ursachen haben. Eine Ursache kann das fehlende ProblembewuBtsein der Verantwortlichen in Parlament und Regierung einerselts und der Bevölkerung andererselts sein; es können ihnen Informationen über Art, AusmaB und Wlrkungen der Umweltbelastung fehlen 375), so daB niemand eine Veranlassung sieht, etwas gegen die Umweltbelastung zu unternehmen. Folgende vier Fälle von vorhandenem bzw. fehlendem ProblembewuBtsein bei Bevölkerung und staat sind denkbar:

\begin{tabular}{|l|c|c|}
\hline $\begin{array}{c}\text { Bevölke- } \\
\text { rung hat }\end{array}$ & $\begin{array}{l}\text { Praat" } \\
\text { hat }\end{array}$ & $\begin{array}{l}\text { Problembe- } \\
\text { wuBtsein } \\
\text { problembewuBtsein }\end{array}$ \\
\hline $\begin{array}{l}\text { kein Pro- } \\
\text { blembe- } \\
\text { wuBtsein }\end{array}$ & 1 & 3 \\
\hline $\begin{array}{l}\text { Problem- } \\
\text { bewuBtsein }\end{array}$ & 2 & 4 \\
\hline
\end{tabular}

375) Vgl. RSU, Umweltgutachten 1974, a.a.0., S. 53; Höhmann, H.H./Seidenstecher, G., Umweltschutz und ökonomisches System in Osteuropa, a.a.O., s. 28 f.; Seidenfus, H.S., Umweltschutz, politisches System und wirtschaftilche Macht, in: Schnelder, H.K./Watrin, C. (Hrsg.), Macht und bkonomisches Gesetz, Bd. 2, Berlin 1972, S. 815; Schmidt, Helmut, Umweltzerstorrung und Sozialkosten, in: Kapp, W./Vilmar, F. (Hrsg.), Sozlalisierung der Verluste?, a.a.0., s. 100. 
Der erste Fall kommt - zumindest in den Industriestaaten kaum noch als Ursache der Umweltbelastung in Frage. Fall zwel düfte vor allem in einigen zentralverwaltungswirtschaftich organisierten Staaten Osteuropas anzutreffen sein. Hier 1st bei den Regierenden das ProblembewuBtsein durchaus vorhanden und Umweltbelastungen werden auch nicht geleugnet 376), andere Aufgaben als Umweltschutz aber für wichtiger gehalten. Gegenüber der Bevölkerung können in den mehr oder wenlger totalitären staaten Informationen über die Umweltbelastung zurückgehalten oder die daraus entstehenden Gefahren verharmlost werden 377), so daB bel der Bevollkerung höchstens ein ungenügendes Problembewußtsein entstehen kann.

Der dritte Fall ist nur bel elner Regierung denkbar, die völlig den Kontakt zu lhrer Bevölkerung verloren hat. Auch das wird kaum in einer parlamentarischen Demokratie der Fall sein, unter Umständen dagegen in elnem totalitären Staat. Es 1st allerdings auch denkbar, daB die Bürger in elner parlamentarischen Demokratie ihr Problembewustsein in bezug auf die Umweltbelastung nicht artikulieren, weil sie andere Aufgaben als den Umweltschutz für wichtiger halten.

Selbst im vierten Fall, wo sowohl dem staat als auch der Bevolkerung das Problem der Umweltbelastung bewußt 1st, mus es nicht zur Berücksichtigung des Umweltschutzes bei der Aufstellung des nationalen zielkatalogs kommen, weil - wie in den Fällen zwel und drei - entweder die elne oder die andere Seite oder aber gar beide Seiten gemeinsam dem Umweltschutz nicht genügend Priorität beimessen. Fehlendes problembewubtsein kann also keineswegs

376) Vgl. Bora, G., Planwirtschaft als Voraussetzung elner wirksamen Umweltpolitik, in: Horn, C. U.a.. (Hrsg.), Umweltpol1t1k in Europa, a.a.O., S. 246; Höhmann, H.-H./Se1denstecher, G./Vajna, T., Umweltschutz und okonomisches System in Osteuropa, a.a.O.., S. 9; Fedorenko, N./Gofman, K., Problems of Optimization in the Planning and Control of the Environment, in: Problems of Economics, vol. 15 (12), 1973, S. 38.

377) Vgl. Erhard, L./Müller-Armack, A., Sozlale Marktwirtschaft, a.a.0., s. 298 . 
die alleinige Ursache der Umweltbelastung sein - oder umgekehrt: Problembewubtsein ist nur die notwendige, aber nicht die hinreichende Bedingung fur einen wirksamen Umweltschutz. Konflikte mit anderen (wirtschafts-)politischen zielen können eine weitere Ursache für einen (ungenügenden) Umweltschutz, also zu große Umweltbelastung sein 378) Nur wenn das ProblembewuBtsein vorhanden ist und dem Umweltschutz die Prioritat innerhalb der politischen Hierarchie elngeräumt wird, die - eventuell zu Lasten anderer Ziele - notwendig 1st, um die Umweltbelastungen zu begrenzen, 1st die Grundlage für einen effizienten Umweltschutz vorhanden. Die angemessene Berücksichtigung des Umweltschutzes im nationalen Zlelkatalog ist jedoch auch nicht mehr als eine notwendige Bedingung. Noch so hehre Umweltschutzziele bleiben Makulatur, wenn es am Willen und der Fähigkelt zur Durchsetzung der Maßnahmen fehlt, die zur Erreichung der Umweltschutzziele notwendig sind 379) Am fehlenden willen zur Durchsetzung zu zweifeln, hieBe die Politiker der Heuchelel zu zeihen - eine nicht unbedingt wirklichkeitsfremde Annahme, von der wir jedoch nicht ausgehen wollen: Ursache der Umweltbelastung wäre dann der fehlende Wille, die notwendigen UmweltschutzmaBnahmen zu ergreifen. Von gröBerer Bedeutung sind unseres Erachtens die Fäle, in denen es zwar nicht am willen aber an der Fähigkeit der Politiker mangelt, die Umweltschutzmaßnahmen durchzusetzen.

378) Besonders in Staaten mit elnem Nachholbedarf an Konsum scheint dem wirtschaftlichen wachstum (noch) eine so groBe Bedeutung zuzukommen, daB dort von Regierung und Bevölkerung bewußt große Umweltbelastungen in Kauf genommen werden (vgl. Höhmann, H.-H./Seidenstecher, G./Vajna, T., Umweltschutz und bkonomisches system in Osteuropa, a.a.O., S. $10 \mathrm{ff}$. und S. 168; Ui Jun, Der japan1sche Kapitalismus als Vorhut der ökologischen Katastrophe, in: Kursbuch 33 , Berlin 1973, S. 164; Issing, O.. Zerstörung der Umwelt - ein Versagen des marktwirtschaftlichen systems?, a.a.0., s. $129 \mathrm{f}$.; Hansmeyer, K.-H./Rürup, B., Umweltgefahrdung und Gesellschaftssystem, a.a.0., S. 15 und S. 21 f.)

379) Vgl. Hohmann, H.-H./Seldenstecher, G./Vajna, T., Umweltschutz und Ökonomisches System in Osteuropa, a.a.O., S. 12 und S. 31 ff.; Moncrief, L.W., The Cultural Basis for Our Environmental Crisis, a.a.0., S. 291. 
Die Durchsetzung der zur Errelchung der Umweltschutzziele notwendigen Maßnahmen kann bereits im (vor-)parlamentarischen Raum an dem EinfluB von Interessengruppen scheltern. Es werden dann zwar unter Umständen Umweltschutzgesetze verabschiedet, doch sind die darin enthaltenen Vorschriften derart verwassert oder unvollständig, daß sie zu keinem ausreichenden Umweltschutz fuhren 380) Als Ursache der Umweltbelastung kann man dann den zu starken EinfluB der Interessengruppen auf die Gesetzgebung oder umgekehrt die zu geringe Autorität der Reglerung und des Parlaments bezeichnen 381) Oberflächlich betrachtet können auch technische und finanzielle schwierigkelten die Durchsetzung notwendiger UmweltschutzmaBnahmen verhindern. Das ist jedoch nur vordergründig der Fall, da sie durch rechtliche Maßnahmen (wie Verbote bestimmter umweltbelastender Akt1vitäten) substitulert werden können, was allerdings zu einer Verschärfung der zielkonflikte führt.

Die Durchsetzung der als notwendig erachteten Umweltschutzmaßnahmen kann aber nicht nur im Rahmen des Gesetzgebungsverfahrens torpediert werden, die Durchsetzung des selbst alle notwendigen Maßnahmen vorsehenden Umweltschutzgesetzes kann völlig oder zumindest tellweise beim Vollzug scheitern. Ursache der Umweltbelastung ist dann das sogenannte Vollzugsdefizit. Dafür kann - wie im Falle ungenügender Durchsetzungsfähigke1t im (vor-) parlamentarischen Raum - die mangelnde Autorität des Staates Ursache sein. Am Vollzugsdefizit können jedoch neben dem

380) Ein Beispiel dafür ist das von allen Parteien des Bundestages verabschledete Abwasserabgabengesetz; die dort vorgesehene Abgabenhöhe je Schadstoffeinheit ist viel zu niedrig, um eine Internalisierung der Umweltkosten zu bewirken. Für die Wasserverschmutzer ist es viel billiger - und für den staat fiskalisch ergiebiger! - die Abwasserabgabe zu zahlen als eine Abwasserreinigung vorzunehmen. Siehe dazu unten S. $370 \mathrm{ff}$.

381) Vgl. Forsthoff, E., Der Staat der Industriegesellschaft, München 1971 , S. 120 ff. 
fehlenden Willen zur Kontrolle

objektive Schwierigkeiten bei der Kontrolle wie Erfassungs- und Zurechnungsprobleme aber auch unklare Kompetenzverteilungen schuld sein ${ }^{383}$ ). Je strenger die Sanktionen sind, die im Falle elnes Verstoßes gegen die Umweltschutzgesetze vorgesehen und ergriffen werden, um so mehr erubrigen sich allerdings Kontrollen. Wenlger mangelhafte Kontrolle als ungenügende Sanktionen sind daher die elgentliche Ursache des Vollzugsdefizits beim Umweltschutz. Es kommt dabei nicht nur darauf an, daB solche Sanktionen im Gesetz vorgesehen sind, sondern vor allem darauf, daB sie in voller strenge ergriffen werden.

\section{e) Fazit der Ursachendiagnose}

In den vorangegangenen Ausfuhrungen wurde gezeigt, daB das Bevolkerungswachstum und die damit elnhergehende stelgende Bevolkerungsdichte sowie stelgendes Produktionswachstum und zunehmender Wohlstand verbunden mit bestimmten konsumgewohnheiten keine eigentlichen Ursachen der Umweltbelastung sind, da sie nicht zwangsläufig zu Umweltbelastungen füren. Es gibt vielmehr technische, juristische und okonomische Maßnahmen des Umweltschutzes, welche diese negativen Auswirkungen via Internalisierung der ex-

382) Vor allem auf kommunaler Ebene scheuen sich manchmal die Behörden, umweltbelastende Unternehmen, die potente Gewerbesteuerzahler sind, zu kontrollieren oder gar zur Rechenschaft zu ziehen; daruber hinaus tragen oft die Gemeinden selbst zur Umweltbelastung bei; vgl. dazu Ewringmann, D./Zimmermann, K., Kommunale Wirtschaftsforderung und Umweltschutz, in: Archiv für Kommunalwissenschaften, 12. Jg., 1973, Bd. 2, S. 293; Steckert, U., Umweltschutz als kommunale Aufgabe, in: Umwelt, 1971, 6, $S$. 15 f.; Bragdon, C., The Community Nolse Problem: Factors Affecting its Management, in: Natural Resources Journal, vol. 10,1970 , S. $694 \mathrm{ff}$.

383) Lorenz, G., Umweltschutz für eine Grosstadt?, in: Umweltschutz - aber wie? Rechtliche Hindernisse, rechtliche Moglichkeiten. Schriften der Evangelischen Akademie in Hessen und Nassau, Heft 95, Frankfurt/M. 1972, S. 55; U.S. Counc1l on Environmental Quality, Environmental Quality, First Annual Report, a.a.O., S. 15. 
ternen Effekte auf die Umwelt verhindern können 384). Damit wird das Nichtergreifen dieser Masnahmen zur eigentlichen Ursache der Umweltbelastung. Die fehlende oder unzurelchende Internalisierung der Umweltexternalitaten selbst hat nun wiederum mehrere Ursachen, nicht - wie Ideologen behaupten - dem marktwirtschaftlichen system immanente Măngel, sondern systemIndifferente Faktoren institutioneller Art wie fehlendes oder zu geringes Problembewubtsein infolge unzulänglicher Informationen uber Art, AusmaB und wirkungen der Umweltbelastungen. Das fuhrt zu elner zu geringen Prioritat fur den Umweltschutz in der Hierarchle der politischen ziele und/oder zu Durchsetzungsproblemen auf Gesetzgebungs- und/oder Vollzugsebene infolge des Einflusses von Interessengruppen und $z u$ lascher Durchfuhrung von Kontrolien durch die Behörden sowie zu zaghafter Ergrelfung von Sanktionen durch die Gerichte. Die Therapie der Umweltbelastung, also der Umweltschutz, muß vor allem diese institutionellen und systemindifferenten Ursachen bekămpfen.

C. Zur Prognose der Umweltsituation

Fur eine langfristige Umweltplanung als Grundlage einer rat1onalen Umweltpolitik ist neben elner Diagnose der gegenwărtigen auch eine Prognose der zukunftig erwarteten Umweltbelastung erforderlich.

384) Die Autoren, die melnen, Bevölkerungswachstum und stelgender Konsum seien die primären Ursachen der Umweltbelastung und die Internalisierung der externen Effekte durch okonomische und rechtliche Masnahmen nur ein Kurieren an den Symptomen (vgl. z.B. Scherrer, H.U., Gesamtbewertung der Umweltbelastung, a.a.o.), da wachsende Bevölkerung und steigender Konsum den Wirkungsgrad dieser Maßnahmen bald wieder zunichte machen würden, ubersehen, das bel $k$ o $n-$ $s$ e $q u$ e $n t e r$ Durchsetzung der Internalislerung der externen Effekte quasi automatisch via den Marktmechanismus Im Falle elner zu stark wachsenden Bevollkerung und $z u$ stark stelgendem Konsum in Form stelgender Preise Krafte ausgehen, welche Bevölkerungswachstum und Konsumstelgerung bremsen. 
Da die zukünftige Umweltbelastung nicht nur von der Entwicklung physischer Faktoren wie Bevölkerungswachstum, Nahrungsmittelund Rohstoffangebot sowle $\mathrm{Z}$ unahme und Zusammensetzung des Kapitalstocks abhängt, sondern auch von der Entwicklung der Technologie sowle der Wandlung sozialer und b̈konomischer Faktoren, 1st elne Prognose der zükünftigen Umweltbelastung ăußerst schwierig 385)

Es genügt nicht, etwa eine elnfache Extrapolation der jeweiligen Schadstoffemissionen in Abhänglgkelt von der (geschätzten) Entwlcklung bestimmter physischer Indikatoren (z.B. dem Bevölkerungswachstum, dem wachstum des realen Bruttosozlalprodukts und des Energleverbrauchs einschließlich allfälliger Substitutionen der Energleträger 386 ) oder der Entwicklung des Kraftfahrzeugverkehrs 387), vorzunehmen. Es müssen daruber hinaus Prognosen uber die Entwicklung der Technologie hinsichtlich threr positiven und negativen Einflüsse auf die Umwelt abgegeben werden. Es mübte $z$. B. vorausgesagt werden, wann ein (serienreifer) umweltfreundlicher Automotor entwickelt sein wird. Verschiedene Methoden der technologischen Prognose (technological forecasting) versuchen solche Fragen zu beantworten 388) S1e haben dabel mit der kaum uberwindbaren Schwierigkelt zu kämpfen, Richtung und Umfang kreativer Prozesse vorauszusagen, die Voraussetzung für technologische Entwicklungen sind 389) Nahezu

385) Vgl. U.S. Council on Environmental Quality, Environmental Quality, Third Annual Report, Washington, D.C., 1972, S. $54 \mathrm{ff}$.

386) Vgl. Die Energiepol1t1k der Bundesreglerung, BT-Drucksache 7/1057, S. 4, wo eine Prognose der Energleentwicklung vorgenommen wird. Auf die Bedingtheit einer solchen Prognose weist u.a. der RSU hin (vgl. RSU, Umweltgutachten 1974, a.a.O., S. 147 ff.).

387) Vgl. Entwicklungstendenzen des Kraftfahrzeugverkehrs in der Bundesrepublik Deutschland bis 1980.

Schriftenreihe des Verbandes der Automobilindustrie e.V., Nr. 5, Frankfurt/M. 1970.

388) Vgl. Jantsch, E., Technological Forecasting in Perspective, Par1s 1967; Blohm, H./Steinbuch, K. (Hrsg.), Technische Prognosen in der Praxis, Düsseldorf 1972, S. 1 ff.

389) Vgl. U.S. Council on Environmental Quality, Environmental Quality, Third Annual Report, a.a.0., S.69. 
aussichtslos dürfte es auch sein, soziale und okonomische Faktoren, welche die Umweltbelastung positiv oder negativ beeinflussen, zutreffend zu prognostizieren 390). Man muste Fragen der folgenden Art beantworten können: Welche Maßnahmen wird die Regierung in zukunft zum Schutz der Umwelt ergrelfen, und wie werden die Betroffenen darauf reagieren? Wie werden sich die Konsumentenprä ferenzen ändern, wenn die Konsumenten umweltbewuBter werden? Es bedarf wohl keiner welteren Erläuterungen, daß solche Prognosen nur außerst spekulativ sein können. Darüber hinaus haben Prognosen mit ähnlichen Schwierigkeiten wie Diagnosen zu kämpfen: Es fehlen Informationen über die Wirkungen mancher Schadstoffe, vor allem über Langzeltwirkungen, deren Kenntnis gerade im Rahmen elner Prognose der zukünftigen Umweltsituation sowohl in bkologischer Hinsicht (z.B. langfristige Klimave:änderungen) als auch im Hinblick auf gesundheltsschädliche Folgen (z.B. teratogene und mutagene Schäden) von besonderer Bedeutung 1st. Auch mangelnde Kenntnisse uber die Anpassungsfähigke1t ökologischer Systeme und des mensch11chen Organismus an Umweltbelastungen erschweren eine Prognose der zukunftigen Umweltsituation, well (langfristige) Prognosen der Umweltentwicklung also offenbar mit einer Vielzahl von Problemen behaftet sind, ist zwangsläufig die langfristige Umweltplanung äuBerst schwlerig. Sie sollte auf alle Fälle so vorgenommen werden, daß sie an Anderungen der tatsächlichen oder geschätzten Daten schnell und elnfach angepaßt werden kann. Dies wird bei einer flexiblen Rahmenplanung eher möglich sein als bel einer starren Detallplanung. Die langfristige Umweltplanung sollte also möglichst flexibel sein und sich möglichst wenig auf Einzelheiten beziehen.

390) Vgl. U.S. Council on Environmental Quality, Environmental Qual1ty, Th1rd Annual Report, a.a.0., S. 72. 
"Das ziel muß man früher kennen als die Bahn"

(Jean Paul, Levana oder Erzieh-Lehre)

II. Ziele der Umweltpolitik

A. Umweltschutz als geselischaftspolitisches ziel

1. Zur Notwendigkelt elnes umweltpolitischen Zielsystems Die Diagnose (und Prognose) der Umweltbelastung, 1hrer Ursachen und Wirkungen ist die erste Voraussetzung fur eine rationale Umweltschutzplanung. Eine rationale Planung muß zielorlentiert seln; nur operational definierte ziele für die einzelnen Berelche des Umweltschutzes können als MaBstab fur die Therapie der Umweltbelastung - eben den Umweltschutz dienen ${ }^{1)}$ : Erst ein Verglelch der diagnostizierten Umweltbelastungen mit den Umweltschutzzielen gibt Anhaltspunkte dafür, wo und mit welcher Dringlichkelt Umweltschutzmaßnahmen ergriffen werden müssen 2). Die Ableitung operationaler $\mathrm{Zlele}$ des Umweltschutzes ist somit neben den Informationen über die Umweltsituation die zweite Voraussetzung für elne rationale Umweltpolitik.

2. Umweltschutz - ein Produkt der Reformeuphorie?

Vor 1970 existierte unseres Wissens das Wort Umweltschutz nicht, wenn auch die Tatbestände, die wir heute darunter zusammenfassen, wie Immissionsschutz, Gewăsserschutz, Abfallbeseitigung, Tierschutz, Pflanzenschutz, Natur- und Landschaftsschutz,

1) Vgl. Kapp, K.W., Zur Praxis der Umweltpolitik und der Umweltplanung, in: Kapp, K.W./Vilmar, F. (Hrsg.), Sozialisierung der Verluste?, a.a.0., S. 160.

2) Vgl. Frey, R.L., Umweltschutz als wirtschaftspolitische Aufgabe, in: Schweizerische Zeitschrift für Volkswirtschaft und Statist1k, $108 \mathrm{Jg} ., 1972$, S. 453. 
Lebensmittelhygiene, Lärmschutz u.a. nicht nur dem Worte nach bekannt waren, sondern auch - mit niedrigerem stellenwert als heute - politische Aufgaben darstellten. Den Rang eines nationalen zieles ${ }^{3)}$ erlangte der Umweltschutz - nahezu weltweit erst zu Beginn der 70er Jahre. Dies war in der Bundesrepublik Deutschland eine zelt, in der - manche meinen allzu euphorisch innere Reformen in grobem Umfang angekundigt wurden. Dieses Zusammentreffen der "Umweltwoge" mit dem (angeblichen) Reform(uber)elfer der damaligen Bundesregierung - der Umweltschutz spielt eine wichtige Rolle in der Regierungserklärung von $1969^{4)}$ - legt die Vermutung nahe, daB der Umweltschutz ein Produkt dieser (angeblichen) Reformeuphorie war. DaB das jedoch nicht der Fall war, zeigten die ungefahr zur gleichen Zeit einsetzenden Bemühungen der me1sten Regierungen - zumindest in den hochindustrialisierten Lăndern -, dem Umweltschutz elne höhere Priorität in der Rangskala der politischen ziele elnzurăumen 5) Interessant ist, daB gerade internationale Organisationen wie die OECD, die bis dahin ein besonderes Falble für möglichst hohe

3) Vgl. Schultze, C.L./Fried, E.R./Rivlin, A.M./Teeters, N.H. (Hrsg.), Setting National Priorities, The 1973 Budget, Washington, D.C., 1972, S. 367; Vaughan, R.D., Environmental Quality - A National Goal, in: Gluckman, L.A. (Hrsa.), Planning for Solld Waste Management, U.S.G.P.O., Washington, D.C. 1971, S. $111 \mathrm{f}$; Dürr, E., Wirtschaftspolitische Ziele - Ein historischer Uberblick, in: Recktenwald, H.C. (Hrsg.), Das Umweltproblem aus okonomischer und juristischer sicht, a.a.0., S. 9; Osswald, A., Grundprobleme des Umweltschutzes, 1n: Hessen - Informationen, Schriftenrelhe der Hessischen Landesreglerung, Heft 2, Wiesbaden 0.J., S. 37 .

4) Vgl. die Reglerungserklärung des Bundeskanzlers vor dem Deutschen Bundestag vom 28.10.1969, in: Bulletin des Presseund Informationsamtes der Bundesregierung, Nr. 132 vom 29 . 10.1969 .

5) Vgl. die von der Akademie für Raumforschung und Landesplanung als Manuskript vervielfätigten "Materialien Umweltschutz und Raumordnung", Hefte 2 und 3: Ubersicht uber Ziele und Bestrebungen in Europa, Hannover 1971 und 1972, sowle Heft 7: Ziele und Bestrebungen in hochindustrialisierten außereuropä1schen Ländern, Hannover 1973. 
Wachstumsraten hatte 6) und die NATO 7) deren eigentliche Aufgabe die Gewăhrleistung der äuBeren sicherheit ihrer Mitgliedsländer ist, sich mit dem Umweltschutz zu befassen begannen, obwohl sie an thren ursprünglichen Zielen Abstriche machen müssen, wenn dem Umweltschutz mehr Gewicht elngerăumt wird.

Die Gründe dafür liegen wohl darin, daB in der zunehmenden Umweltbelastung - zumindest langfrist1g - eine Gefahr gesehen wird, welche die Realisierung anderer ziele so stark zu beeintrăchtigen droht, das dieser - auch auf kosten eines (kurz- und mittelfristig) niedrigeren zielerreichungsgrades bei den traditionellen zielen - Einhalt geboten werden muß. Mögen diese Erkenntnisse der Umweltgefahrdung zunăchst auch nur wenige Verantwortliche in Politik, Wirtschaft und Wissenschaft gehabt haben und somit das plötzliche Auftauchen des Umweltschutzes als nationales ziel ursprünglich auf elnen lead ${ }^{8)}$ zurückzuführen sein, so war es eine, man wăre beinahe geneigt zu sagen "glückl1che" Hăufung von Katastrophen, wie das Auseinander-

6) Vgl. OECD at Work for Environment, Paris 1971; Act1vities of the OECD in 1972, Paris 1973, S. $59 \mathrm{ff}$; OECD and the Environment, in: OECD-Observer Nr. 53, 1971, S. $19 \mathrm{ff.;}$ OECD's Programme on the Environment, in: OECD-Observer No. 50, 1971, S. 7 f.; Environment: A New Programme of International Co-operation, in: OECD-Observer, No, 58, 1972 , S. $27 \mathrm{f.}$; Some Recent OECD Initiatives in the Field of the Environment, in: OECD-Observer, No. 62,1973 , S. $8 \mathrm{ff}$.

7) Vgl. Nagel, S., Umweltschutz: Neue Aufgabe der NATO, in: Umwelt 1971,4, S. 25 ff.; Randers, G. . NATO's International Governmental Cooperation on Environmental Managemant, in: Kneese, A.V./Rolfe, S.E./Harned, J.W. (Hrsg.), Managing the Environment, International Economic Cooperation for Pollution Control, 2. Aufl.., New York-Washington-London 1973, s. $343 \mathrm{ff}$. DaB sich der Zweck der NATO in absehbarer Zeit erübrigen könnte, well der Umweltschutz in allen Lăndern so hohe Ausgaben erfordert, daB es zu einer allmählichen Abristung kommt, scheint nicht mehr als das Wunschdenken einiger Friedensforscher zu sein (vgl. FitzRoy, F.R./von Weizsalcker, E., Einige politisch-8konomische Fragen im Umweltschutz, in: von Weizsăcker, E. (Hrsg.), Studien zur Friedensforschung a.a.0., S. 120).

8) Vgl. Schmidt, Kurt, Zur politischen Reaktion auf Nachfragewogen in der staatswirtschaft, in: Finanzarchiv, N.F., Bd. 33, 1974, S. 42 . 
brechen des Tankers Torrey Canyon, spektakuläre Fischsterben Infolge der Verschmutzung von Gewässern und die Menschenleben kostende Minamata- und Ital-Itai-Krankheit 9) welche die Umweltbelastung so stark Ins BewuBtsein der Bevölkerung brachten, daß die Basis für den Umweltschutz auch von deren selte her zumindest gestärkt, wenn auch noch nicht fest verankert wurde. Das Bedurfnis nach elner sauberen Umwelt war geweckt 10! Mit Umweltschutz ließ sich nun Politik machen; Umweltschutz fand Eingang in die Programme der Partelen, well sie sich erhofften, dadurch wählerstimmen zu gewinnen - und das zu recht, wie eine von der Bundesreglerung in Auftrag gegebene Umfrage zeigt: Bel der Frage nach den Aufgabenbereichen auf dem Gebiet der inneren Reformen, um die sich die Bundesregierung in zukunft mehr kümmern soll, wurde der Umweltschutz an erster stelle genannt 11) Ob das Bedürfnis nach Umweltschutz bei der Bevölkerung oder den Politikern fest verankert oder nur eine Modeerscheinung war, muBte sich in den Jahren des konjunkturellen Abschwungs und der Energiekrise zelgen, in denen die im Umweltprogramm der Bundesregierung verkündeten Maßnahmen für Private und staat ausgabenwirksam werden sollten. Würden abnehmende Beschäftigung, sinkende Wachstumsraten, steigende Energiekosten und sinkende Steuereinnahmen Arbeltnehmer, Unternehmer und staat den Umweltschutz vergessen lassen?

In der Bundesrepublik Deutschland und auch anderen Ländern war dies nicht der Fall: Staat, Wirtschaftsverbănde und Gewerkschaften betonten das gemeinsame Interesse an einem wirksamen Umweltschutz, der unabhängig von konjunkturellen Schwankungen

9) Vgl. The Torrey Canyon, Report of the Committee of Scientists on the Scientific and Technological Aspects of the Torrey Canyon Desaster, H.M.S.O., London 1967; vgl. ferner oben die Seiten $71,94,97$.

10) Vgl. Frey, B.S., A Dynamic Theory of Public Goods, in: Finanzarchiv, N.F., Bd. 32, 1973/74, S. 187 f.

11) Vgl. Infas, Die inneren Reformen im splegel der Bevölkerungsmelnung, Bonn 1971; Menke-Glückert, P., Das Umweltprogramm der Bundesregierung, in: von Weizsäcker, E. (Hrsq.), Studien zur Friedensforschung, a.a.O., S. 125. 
und Energiekrisen betrieben werden soll 12). Die konjunkturelle Krise hatte somit für den Umweltschutz zunächst elnmal die Funktion eines Prufstelnes der Ernsthaftigkeit der Bemuhungen um eine saubere Umwelt. Ebenso wichtig war aber, daB - durch Konjunktur- und Energlekrise bedingt - die ersten konkreten UmweltschutzmaBnahmen nicht in elne zeit der vollen Kassen bei Staat und Privaten fielen und daB so nicht der Formulierung von Maximalzielen im Hinblick auf elne saubere Umwelt Vorschub geleistet, sondern das durchaus spürbar wurde, das Umweltschutz teuer 1st und zumindest kurzfristig EinbuBen bel der Erreichung anderer Zlele erfordert. Nach sechs Jahren "Up and Down with Ecology" 13) kann man wohl feststellen, daß der Umweltschutz fest in der Hierarchie der gesellschaftspolitischen ziele der Bundesrepublik Deutschland verankert und, wie Gesetzesinitiativen, Umweltschutzinvestitionen und Bürgerinitiativen zeigen, ein Bestandtell der Alltagsbeschäftigung von Politikern und Bürgern geworden 1 st.

3. Zur Stellung des Umweltschutzes in der Hierarchie der gesellschaftspolitischen ziele

"Maßstab jeder Umweltpolitik ist ... der Schutz der Wurde des Menschen, die bedroht ist, wenn seine Gesundhe1t und sein Wohlbefinden ... gefahrdet werden" 14) Aus dieser Formulferung, die

12) Vgl. Malhofer, $W .$, Umweltpolitik in der Bewahrung, in: U 1976, 2, S. 12; Genscher, H.-D., Kein Abstrich beim Umweltschutz, in: U 1974, 1, S. 8; Hartkopf, G., Umweltpolitik nach Gymnich, 1n: U 1975, 4, S. 8; Die Energiepolitik der Bundesreglerung, BT-Drucksache, 7/1057, S. 6 und S. 17; van Schaik, R.J., The Impact of the Economic Situation on Environmental Policies, in: OECD-Observer No. 79, 1976, S. 25.

13) Downs, A., Up and Down with Ecology - The "Issue-attentioncycle", in: Public Interest, vol. 28, 1972, S. $38 \mathrm{ff.;}$ vgl. auch Frey, B.S., A Dynamic Theory of Public Goods, a.a.0., S. 187 ff.

14) Menke-Glückert, P., Das Umweltprogramm der Bundesregierung, a.a.0.. S. 127; vgl, auch RSU, Umweltgutachten 1974, a.a.0., S. 5; Genscher, H.-D., Gesellschaftspol1t1sche Aufgabe des Umweltschutzes, in: Bulletin der Bundesreglerung, Nr. 178 vom 18.12.1970, S. 1945; DFG, Umweltforschung, Bonn-Bad Godesberg 1971, S. 11. 
in ähnlicher Form im Umweltprogramm der Bundesregierung steht ${ }^{15)}$, kann man schließen, daB oberstes $\mathrm{ziel}$ des Umweltschutzes die Erhaltung bzw. Wiederherstellung der Menschenwürde via Erreichung des Zwischenziels Gesundheit ist. Das Gesundheitsziel erfordert - ausgehend von der Definition der WHO

- nicht nur das Freisein von Krankheit, sondern auch Wohlbefinden. Umweltbelastungen können, wie wir aus dem ersten Kapitel wissen, direkt $z u$ Krankheiten führen (z.B. via toxische Belastung des Trinkwassers), aber auch unser Wohlbefinden stören (z.B. durch die Verschandelung der Landschaft mit Verkehrsbauten), wodurch indirekt Krankheiten hervorgerufen werden können. Umweltschutz als Unterziel des Gesundheltsziels hat also zwei Komponenten: den Schutz vor Krankheiten hervorrufenden und den Schutz vor das Wohlbefinden störenden Umweltbelastungen. Die Zlelhierarchie hat also folgendes Aussehen:

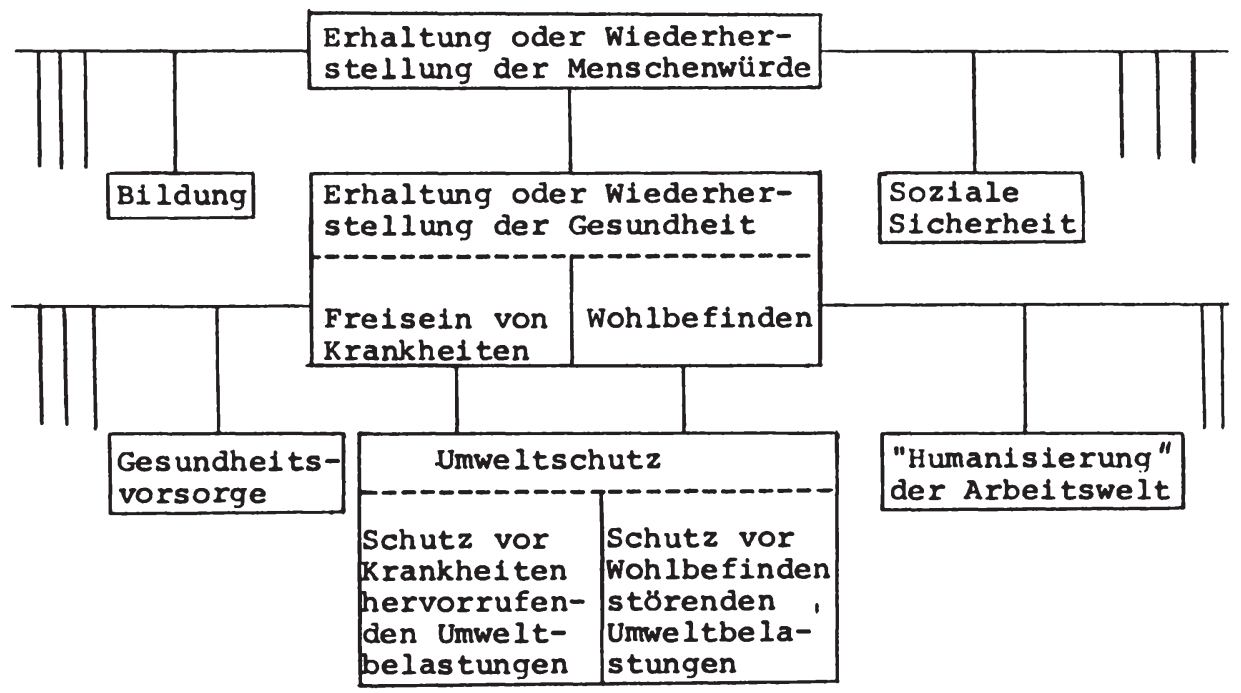

15) Vgl. Das Umweltprogramm der Bundesregierung, 3. Auflage, Stuttgart-Köln-Berlin-Mainz 1973, S. 28.

16) Siehe oben S. 80. 
Das Gesundheitsziel gehört zu den Hauptzielbereichen (primary goal areas), die in Hauptziele (social concerns) wie Umweltschutz zerlegt werden; daraufhin erfolgt eine weitere Untertellung in Unterzlele (sub-concerns), die allerdings noch keineswegs operational sein müssen 17)

Eine Untertellung des Hauptziels Umweltschutz kann zunächst nach den verschiedenen Umweltbereichen erfolgen 18), also in: Schutz vor Luft-, Gewässer- und Bodenbelastungen, Schutz der Pflanzen- und Tierwelt, Schutz der Lebensmittel vor schadstoffen und Lărmschutz. Weiterhin kann - je nach dem bereits vorhandenen Belastungsniveau der Umweltbereiche - zwischen der Vermeidung und der Beseitigung der Belastungen unterschieden werden, die in den jewelligen Umweltbereichen vorhanden sind ${ }^{19}$ ? AuBerdem kann man noch zwischen den Komponenten Gesundheitsschutz und bkosystemschutz differenzieren, obwohl letzterer kein Schutz um der Okosysteme selbst willen ist ${ }^{20)}$, sondern auch im Hinblick zumindest auf das Wohlbefinden der Menschen vorgenommen wird 21) Das ökologische ziel könnte lauten: Erhaltung oder Wiederherstellung eines funktionsfähigen bkosystems (oder ökologischen Gleichgewichts) 22) Konkretisiert

17) Vgl. Leipert, C., Soziale Indikatoren, a.a.0., s. $241 \mathrm{f}$.

18) Vgl. RSU, Umweltgutachten 1974, a.a.0., S. 9.

19) Vgl. ebenda und Malhofer, W., Umweltpolit1k in der Bewährung, a.a.0., S. 12; Schäfer, Friedrich, Das Verursacherprinzip in der Umweltschutzpolitik, in: Umwelt, 1972, 2, S. 4 .

20) Etwas anderer Ansicht ist Frey, R.L., Umweltschutz als wirtschaftspolitische Aufgabe, a.a.0., S. 454.

21) Der RSU schlägt vor, die ökologischen ziele nach den verschiedenen ökologischen Bereichen (Stadt-, Agrar- und natürliche (kosysteme) zu differenzieren; in Stadtökosystemen wäre dann das obologische ziel Menschenschutz, in Agrarökosystemen Nahrungsmittelschutz und in natürlichen Okosystemen Naturschutz (vgl. RSU, Umweltgutachten 1974, a.a.0., S. 112). Der Nahrungsmittelschutz, langfristig auch der Naturschutz sind natürlich ebenfalls "Menschenschutz".

22) Vgl. RSU, Umweltprobleme des Rheins, a.a.0., S. 185; RSU, Umweltgutachten 1974 , a.a.0., s. 13 . 
könnten die Unterziele des Umweltschutzes helBen: Iuft-, Gewässer-, Boden-, Pflanzen-, Tierwelt- und Lebensmittelbelastung sowle Lärm sollen so gering sein, daß sle zusammen ke1ne Gesundheitsschäden, ökologische Schäden oder Schăden an materielien Gütern hervorrufen können. Von diesen konkretislerten Unterzielen des Umweltschutzes kann man ausgehen, um operationale ziele - teilweise in quantifizierter Form, tellwelse lediglich qualitativ - abzuleiten 23)

\section{B. Zur Operationalisierung der Z1ele des Umweltschutzes}

1. Zur Ableitung unschädlicher Immissionskonzentrationen (Immissionsgrenzwerte)

Ausgangspunkt für eine Operationalisierung der Unterziele des Umweltschutzes ist die Ermittlung der Immissionskonzentration eines jeden Schadstoffs, die mit sicherheit keine Krankheit hervorrufen, das Wohlbefinden stören, okologische Schäden oder Schäden an Sachgütern herbeiführen kann. Voraussetzung fur die Bestimmung solcher Immissionskonzentrationen ist die Kenntnis der Schadstoffwirkungen 24) Wie wir aus dem ersten Kapitel wissen, können die Wirkungen der Schadstoffe auf die mensch11 che Gesundheit in Tierversuchen, mit Hilfe epidemiologischer Studien und durch die Heranziehung gewerbetoxikologischer Untersuchungen ermittelt werden, womit auch grundsätzlich die Moglichkelt besteht, die unschädliche Dosis jedes Schadstoffs zu bestimmen 25). Es 1st dabel zu beachten, daß die Ergebnisse von Tierversuchen nicht ohne weiteres auf den Menschen ubertragbar sind, daB epidemiologische studien Wahrscheinlichkeitscharakter haben und daB die Bedingungen für eine schadstoffbelastung des Menschen am Arbeitsplatz andere sind als in der freien Umwelt.

23) Vgl. RSU, Umweltgutachten 1974, a.a.0., S. 9.

24) Vgl. Präambel der VDI-Richtlinien zur Reinhaltung der Luft, abgedruckt in: RSU, Umweltgutachten 1974, a.a.O., S. 15.

25) Vgl. Lange, G., Die Suche nach der gefahrlichen Dosis, in: Umwelt, 1973, 5, s. 18. 
Dem 1st durch Risikozuschläge bei der Bestimmung der gesundheitsunschädlichen Grenzwerte Rechnung zu tragen 26) Die unschädliche Immissionskonzentration für einen Schadstoff wird in der Regel unter der Annahme abgeleitet, daß nur dieser den Mensch belastet. Die toxikologische Gesamtsituation mit den möglichen kumulativen und synergistischen Effekten bleibt in den meisten Fällen der Grenzwertbestimmung unberücksichtigt 27) Im allgemeinen ist die toxikologische Gesamtsituation um so gefährlicher, je dichter besiedelt und industrialisiert ein Geblet 1st. Dem könnte man bei der Immissionsgrenzwertbestimmung dadurch Rechnung tragen, daB man je nach Bevölkerungsdichte und Industrlalisierungsgrad Belastungszonen ${ }^{28}$ ) definiert und für diese unterschiedliche pauschale Abschläge von den Immissionsgrenzwerten vornimmt. Be1 dre1 Bevölkerungsdichteklassen und dre1 zu definierenden Graden der Industrialisierung (etwa Emissionsintensităt der ansässigen Industriebranchen) ergäben sich neun Belastungszonen:

\begin{tabular}{|l|c|c|c|}
\hline $\begin{array}{l}\text { Ind. Einw. je qkm } \\
\text { grad }\end{array}$ & $\begin{array}{r}\text { unter } \\
100\end{array}$ & $\begin{array}{c}\text { zwischen } \\
100 \text { und 200 }\end{array}$ & $\begin{array}{l}\text { über } \\
200\end{array}$ \\
\hline I (gering) & 1 & 2 & 3 \\
\hline II (mittel) & 4 & 5 & 6 \\
\hline III (stark) & 7 & 8 & 9 \\
\hline
\end{tabular}

26) Vgl. Präambel der VDI-Richtlinien zur Reinhaltung der Luft, a.a.0., s. 16 .

27) Die Präambel der VDI-Richtlinlen zur Reinhaltung der Luft fordert die Berulcksichtigung kumulativer Wirkungen bei der Festlegung der MIK-Werte (vgl. a.a.0., S. 15); bei der DFG laufen Untersuchungen zur Ermittlung von Belastungsgrenzen bei Schadstoffkombinationen in Gewässern (vgl. Meyl, A.H., Abwässer in Kủstennähe, in: DFG-Mitteilungen 2/74, S. 29); vgl. auch Kapp, K.W., Zur Praxis der Umweltpolitik und der Umweltplanung, a.a.0., S. 162 .

28) Vgl. § 44 (2) Bundestmmissionsschutzgesetz (BImSchG), wonach Belastungsgebiete unter anderem wegen des dort besonders stark vermuteten kumulativen Zusammenwirkens verschiedener Schadstoffe festgelegt werden können; vgl. ferner Binswanger, H.-C., Eine umweltkonforme Wirtschaftsordnung, in: von Walterskirchen, M.P. (Hrsg.), Umweltschutz und Firtschaftswachstum, a.a.0., s. 139 . 
MiBt man der Bevölkerungsdichte bei der Verursachung der Umweltbelastung eine geringere Bedeutung bel als dem Industrial1slerungsgrad, so ergibt sich eine horizontal verlaufende Durchnummerierung der Belastungsklassen. In Gebleten, die zur höchsten Belastungsklasse 9 gehören, sind die Immissionsgrenzwerte am stärksten $z u$ reduzieren.

Neben dieser räumlichen ist auch eine zeltliche Differenzierung der Immissionsgrenzwerte möglich, da die Einwirkungsdauer der Schadstoffe unterschiedlich sein kann. Die Immissionsgrenzwerte für kurz dauernde Belastungen können höher sein als die für ständ1ge Elnwirkungen. Man kann elnen Kurz- und Langzeltwert festlegen 29 ?

Schlleblich ist auch noch eine sachliche Differenzierung der Immissionsgrenzwerte denkbar. Wegen unterschledlicher Nutzungsansprüche an die jewelligen Umweltmedien können für die Nutzungen, bel denen in der Regel kein direkter Kontakt mit dem Menschen erfolgt, weniger strenge Immissionsgrenzwerte bestimmt werden 30 ?

Weiterhin ist $z u$ beachten, daß die Immissionsgrenzwerte nicht für elnen gesunden Erwachsenen festgelegt werden sollen, sondern für konstitutionell schwache ${ }^{31)}$ wie säuglinge, Greise, Schwangere, Kranke und Behinderte. Bel r Belastungsregionen, $n$ Nutzungsansprüchen und $t$ unterschiedlichen Elnwirkungszeiträumen erhlelte man $r \cdot n \cdot t$ Immissionsgrenzwerte für jeden Schadstoff, deren Elnhaltung gewährleistet, daB durch diesen Schadstoff die Gesundhelt nicht gefährdet wird (unschädliche Immissionskonzentration). Für den Schutz der Okosysteme selbst, die pflanzen und Tiere sowie den Schutz materieller Güter können

29) Vgl. Prăambel der VDI-R1chtlinien zur Reinhaltung der Luft, a.a.0., s. 16 .

30) Vgl. Goldman, M.I./Shoop, R., What is Pollution?, in: Goldman, M.I (Hrsg.), Ecology and Economics, a.a.o., S. 102; RSU, Umweltgutachten 1974, a.a.0., S.9.

31) Vgl. Präambel der VDI-Richtlinien zur Reinhaltung der Luft, a.a.0., s. 15 . 
ebenfalls Immissionsgrenzwerte abgeleitet werden 32)

Bel den Stoffen, bei denen gesundheitsschädliche oder ökologisch schädliche wirkungen (noch) nicht nachgewiesen werden konnten, jedoch ein mehr oder weniger starker Verdacht auf Schädlichkeit besteht, sind - besonders wenn ein Verdacht auf karziogene, teratogene oder mutagene wirkungen vorliegt - sehr niedrige Immissionsgrenzwerte festzulegen 33 ? die mehr den Charakter von vorläufigen Richtwerten haben und mit zunehmender Wirkungskenntnis modifiziert werden können 34 ) Der Wunsch, für alle Umweltbereiche und alle Schadstoffe Immissionsgrenzwerte festzulegen, wird wohl nie erfüllt werden können - wenlger deshalb, weil noch viele Wirkungskenntnisse fehlen, als vielmehr aus dem Grund, daB manche Schadstoffwirkungen nicht quantifizierbar sind.

2. Zur Ableitung von Immissionsstandards

a) Zur Festlegung optimaler Immissionskonzentrationen Die im vorangegangenen Abschnitt abgeleiteten Immissionsgrenzwerte implizieren, daB bel Ihrer Elnhaltung keine schädlichen Wirkungen auf den Menschen, seine natürliche und materielle Umwelt ausgehen können. Würden diese "slcheren" Immissionsqrenzwerte $z u$ zielen des Umweltschutzes erklärt, würde ihre Realisierung einen sehr hohen Aufwand - auch in Form von Opportunitätskosten - erfordern 35! Aber nicht nur unter bkonomischen

32) Vgl. Präambel der VDI-Richtlinien zur Reinhaltung der Luft, a.a.0., s. 16 .

33) Vgl. ebenda, S. 15; von Elff., A.W., Mensch und Lärm, in: DFG-Mittell ungen, Jahresversammlung 1971, S. 44; Gräf, W. , Umweltgefährdung durch kancerogene Substanzen, in: Steubing, L./ Kunze, C./Jäger, J. (Hrsg.), Belastung und Belastbarkeit von Ökosystemen, a.a.0., S. 116.

34) Vgl. RSU, Umweltgutachten 1974, a.a.0., S. 221.

35) Vgl. Hazelton, J.E., Effluents and Affluence, in: Goldman, M.I. (Hrsg.), Ecology and Economics, a.a.0., S. 135;

Schneider, Wolfgang, Verursacherprinzip oder die Belastung aller?, In: Gumpel, W./Keese, D. (Hrsg.), Probleme des Industrialismus in Ost und West, a.a.0., s. 473, Fußnote 13. Nach Ansicht einiger Autoren sollen bel der Festlegung von 
Aspekten sondern auch wegen allgemeiner Risikoüberlegungen scheint eine so hoch gesteckte zielsetzung beim Umweltschutz übertrleben $\mathrm{zu}$ sein: Der Mensch ist bereit - auch im Hinblick auf seine Gesundheit - bestimmte Risiken einzugehen; er trinkt Alkohol und riskiert dabei eine Leberzirrhose, er raucht zigaretten und riskiert dabei eine Lungenkrebserkrankung, er iBt soviel, daB durch sein tbergewicht Herz- und Kreislauferkrankungen beglinstigt werden, er fährt Ski und riskiert Beinbrüche, usw. Warum sollen nun gerade an die Umweltqualität so hohe Anforderungen gestellt werden, daB von ihr keine Gesundheitsgefahren ausgehen? Dies ist nicht einsichtig, denn die Umweltbelastung ist ebenso wie das Rauchen, Alkoholtrinken und übermäßige Essen nur ein Risikofaktor unter vielen für die menschliche Gesundheit 37) Allerdings besteht hinsichtlich der Umweltbelastung ein gewichtiger Unterschied zu den anderen Risikofaktoren: Der Einzelne kann nicht selbst entscheiden, ob und in welchem Umfang er das Risiko der Umweltbelastung tragen will. Uber den Risikofaktor Umweltbelastung, dem er ausgesetzt ist, entscheiden andere. Deshalb ist zu fordern, daß bel diesen zielsetzungsentscheidungen Vertreter möglichst vieler Interessenrichtungen vertreten sind und auf keinen Fall diejenigen majorisiert werden können, die vor allem die Umweltbelastungen $z u$ tragen haben 38 )

Immissionsstandards keine ökonomischen Uberlegungen berücksichtigt werden (vgl. Knelson, J.H., Luftqualitätskriterien und Immissionsgrenzwerte für Kohlenmonoxid in den Vereinigten Staaten, in: VDI (Hrsg.), Kohlenmonoxid, a.a.O., S.98).

36) Vgl. Comar, C.L./Thompson Jr., J.C., Social, Environmental, and Health Costs, in: Summary Report of the Cornell Workshop on Energy and the Environment, a.a.0., s. $30 \mathrm{ff}$.

37) Vgl. Schäfer, Hans, Hinweise auf Umweltschäden aus Lebenserwartung, spezifischen Sterblichkeiten, Sterbeziffern und Krankheitshäufigkeiten, in: Schäfer, Hans (Hrsg.), Folgen der Zivilisation, a.a.0., S. 90 ff.; Kowalski, E., Strahlengefährdung und Umweltschutz, in: NZZ, FA Nr. 66 vom 7.3.1972.

38) Vgl. Fox, I.K., Institutional Mechanisms, in: Summary Report of the Cornell Workshop on Energy and the Environment, a.a.0., S. $167 \mathrm{ff}$. 
Ausgangswerte bei der Fixlerung von verbindlichen Umweltqualitätsnormen in Form von Immissionsstandards sollten die im letzten Abschnitt geschilderten unschädlichen Immissionskonzentrationen sein; von thnen sind nach Anhörung von Wissenschaftlern, Vertretern von Wirtschaftsverbänden und Umweltschutzorganisationen kostenbedingte Abstriche zu machen 39) wobel für alle das dadurch entstehende Risiko für den Gesundhe1tszustand der Bevölkerung sichtbar gemacht werden muß. Die optimale Immissionskonzentration fuir jeden Schadstoff ist dort, wo die Nutzenverluste durch weitere Entfernung vom gesundheitsunschädlichen Immissionsgrenzwert gleich den Kostenersparnissen durch diese "Aufwelchung" sind 40) oder umgekehrt - von einer starken Belastung durch einen Schadstoff ausgehend - formuliert: Die Immissionskonzentration hat dann die optimale Höhe erreicht, wenn die Kosten für eine Senkung der Schadstoffimmission um eine Einheit (z.B. $1 \mu \mathrm{g} / \mathrm{m}^{3}$ ) gleich dem Nutzen dieser Senkung sind, wenn also die Grenzkosten gleich dem Grenznutzen der Immissionsreduktion sind 41) Diese zunăchst leerformelhaft erscheinende Optimumbedingung kann man auch graphisch darstellen (vgl. Abb. $9 \mathrm{a}, \mathrm{b}$ ). Geht man von den realistischen Annahmen aus, daß die Kosten der Schadensvermeidung $\mathrm{K}_{V}$ mit stei-

39) Vgl. Häberle, M., Wo sind die wirklichen Grenzen?, in: Umwelt, $1976,1, \mathrm{~S} .25$.

40) Wir gehen nicht so weit wie Möller, der meint, daß man dann nlcht mehr von Umweltschäden sprechen kann, wenn Umweltbelastungen als kalkuliertes Risiko in kauf genommen werden (vgl. Möller, H., Zum Begriff der Umweltschäden Insbesondere im öffentlıchen Sektor, in: Gumpel, W./Keese, D., Probleme des Industrialismus in Ost und West, a.a.0., S. 439 ff.). Eine chronische Bronchitis infolge der Luftbelastung ist jedoch unseres Erachtens nicht deshalb kelne Krankheit mehr, weil sie - etwa zur Erhaltung unseres Arbeitsplatzes - von uns in Kauf genommen wird.

41) Vgl. Thomas, H.A., Ein mathematisches Modell zur Diskussion von Umweltgütenormen, in: Umwelt, 1973, 2, S. 39; Fox, I.K./ Wible, L.F., Information Generation and Communication to Estabilsh Environmental Quality Objectives, in: Natural Resources Journal, vol. 13, 1973, S. 135 f.; U.S.C.E.Q., Environmental Quality, The Second Annual Report, U.S.G.P.O., Washington, D.C., 1971, S. 114; Jacoby, N.H., The Polluters: Industry or Government?, a.a.0., s. 24 . 
gender (potentieller) Immissionskonzentration $s$ elnes bestimmten Schadstoffes uberproportional steigen ${ }^{42}$ ), also gilt $\frac{d K_{v}}{d s}>0$ und $\frac{d^{2} K_{v}}{d s^{2}}>0$, und $d a \beta$ der Nutzen $N_{V}$ der Schadensvermeldung mit zunehmender (potentieller) Immissionskonzentration nur unterproportional steigt (mit abnehmender Immissionskonzentration überproportional sinkt), also gilt $\frac{d N v}{d s}>0$ und $\frac{d^{2} N_{v}}{d s^{2}}<0$, so erhält man $z . B$. den verlauf der $\mathrm{K}_{\mathrm{V}}$ - und $\mathrm{N}_{\mathrm{V}}$ - Kurven in Abbildung 9a:

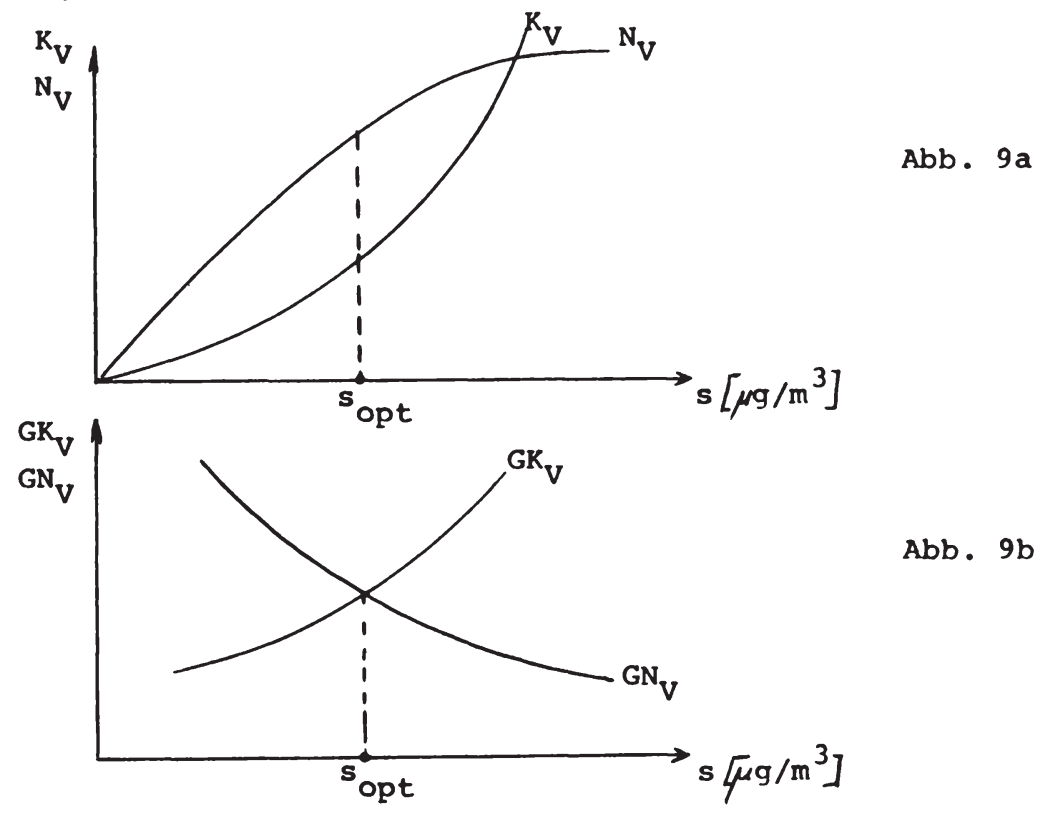

42) Vgl. Ackermann, K./Geschka, H./Karsten, D., Die wirtschaftspolitische Lösung: Verursacherprinzip, in: Umwelt, 1972, 2, S. 31; Schultze, C.L./Fried, E.R./Riviin, A.M./Teeters, N.H., Setting National Priorities, a.a.0., S. 370; Tax Foundation, Pollution Control: Perspectives on the Government Role, A Summary, New York 1971, S. 8; Kohn, R.E., A CostEffectiveness Model for Air Pollution Control with a single Stochastic Variable, in: Journal of the American Statistical Assoclation, vol. 67, 1972, S. 21; Schrelber, M., Kosten der Luftreinhaltung, in: Wasser, Luft und Betrieb, Bd. 15, 1971, 4. S. 146 . 
Die optimale Immissionskonzentration ist dann bel sopt' wo die Differenz zwischen den Vermeidungskosten und dem Nutzen der Schadensvermeldung am grobten ist oder mit anderen worten, wo die Grenzkosten der Schadensvermeldung $\mathrm{GK}_{\mathrm{V}}$ gleich deren Grenznutzen $\mathrm{GN}_{\mathrm{V}}$ ist ${ }^{43}$ ) (vgl. Abb. 9b). Eine formal etwas andere und differenziertere Ableitung der optimalen Immissionskonzentration eines Schadstoffs wollen wir nun noch in Anlehnung an Ridker vornehmen 44) In Abb. 10a stellt $\mathrm{K}_{\mathrm{R}}$ die Kosten der Immissionsreduktion dar. Die Immissionskonzentration in Höhe von $s_{\max }$ ist diejenige, die realisiert wird, wenn keine Maßnahmen zur Immissionsreduktion ergriffen werden. Die Kosten der Immissionsreduktion steigen nun mit abnehmender Immissionskonzentration uberproportional an. Die Kurve $K_{B}$ gebe die Kosten der Immissionsbelastung durch die jeweiligen Immissionskonzentrationen an. Bel einer Immissionskonzentration in Höhe von $s_{u}$ sollen noch keine schäden entstehen (sie entspricht also der oben abgeleiteten unschädichen Immissionskonzentration), danach steigen die schadenskosten mit zunehmender Immissionskonzentration uberproportional an. Die optimale Immissionskonzentration sopt liegt nun dort, wo die Gesamtkostenfunktion $\mathrm{K}=\mathrm{K}_{\mathrm{R}}+\mathrm{K}_{\mathrm{B}}$ ihr Minimum hat, oder anders formuliert, wo die Grenzkosten der Immissionsreduktion glelch den Grenzkosten der Immissionsbelastung sind (vgl. $\mathrm{Abb}$. 10b).

Diese formal eleganten, marginalanalytischen Methoden $\mathrm{zur} B e-$ stimmung der optimalen Immissionskonzentration verschleiern jedoch die Schwierigkeiten, die in der Realität bel der Bestimmung eines solchen optimums bestehen. Voraussetzung ist

43) Marburger, E.-A., Die ökonomische Beurtellung der stădtischen Umweltbelastung durch Automob1labgase. Methoden und Quantifizlerungsversuche, Düsseldorf 1974, S. 254; Randall, A./Ives, B./Eastman, C., Bidding Games for Valuation of Aesthetic Environmental Improvements, in: Journal of Environmental Economics and Management, vol. 1, 1974, S. 133; Fry, C.L., Pollution Control. Proper Policies Could Improve Functioning of Market Economy, in: Federal Reserve Bank of Dallas, Business Review, Oct. 1973, S. 1.

$44)$ Vgl. Ridker, R.G., Economic Costs of Air Pollution. Studies in Measurement, New York, Washington, London 1967, S. 5. 


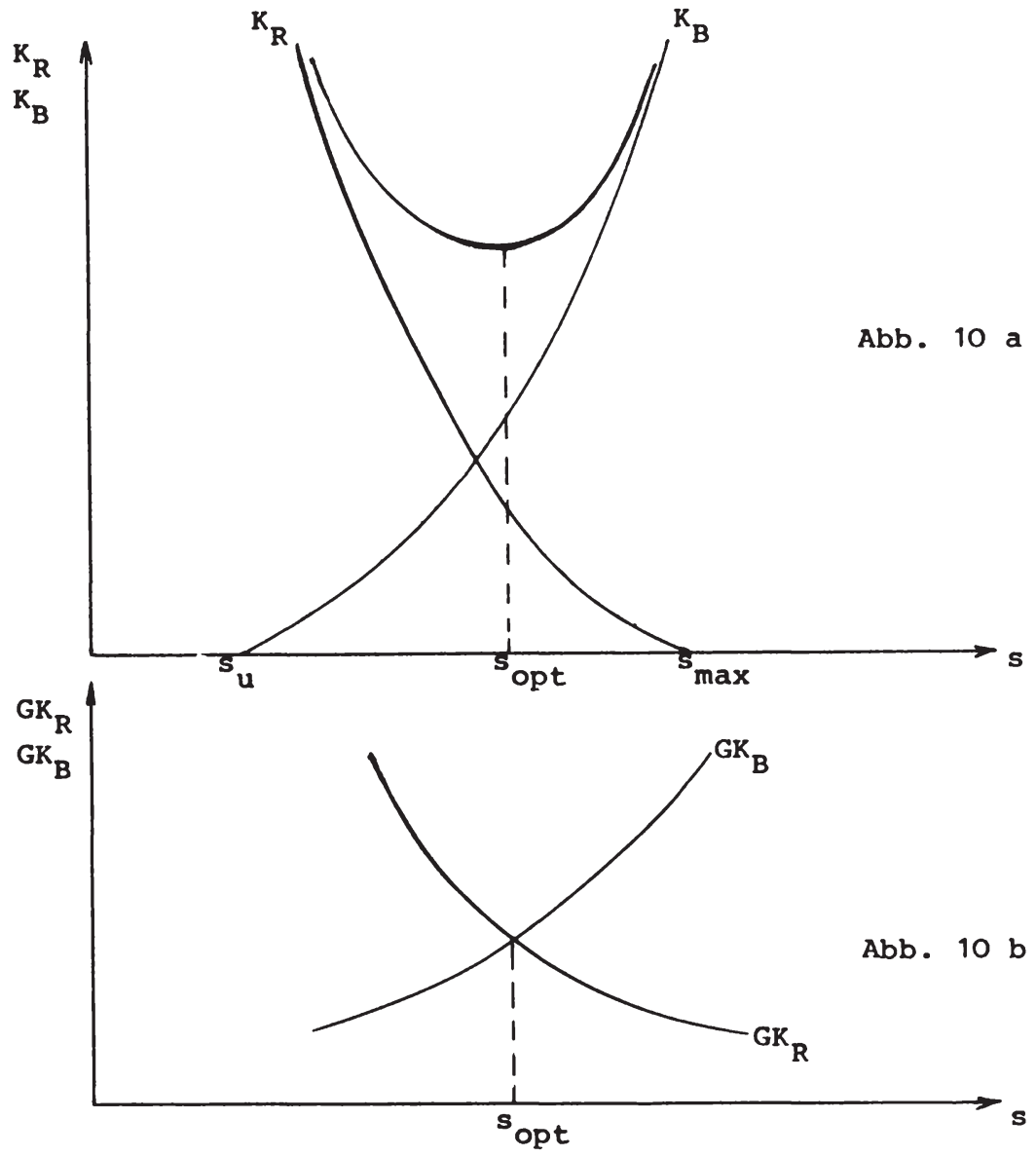

nämlich, daß die Verlăufe der Kosten- und Nutzenfunktion bzw. der Grenzkosten- und Grenznutzenfuntion, letztere zumindest im Berelch ihres Schnittpunkts, bekannt sind. Die Möglichkeit, die optimale Immissionskonzentration fur jeden einzelnen Schadstoff oder gar das optimale Niveau der gesamten Umweltbelastung mit Hilfe solcher Kosten-Nutzen-Uberlegungen zu bestimmen, hänqt also davon ab, Inwiewelt kosten und Nutzen der Immissionsreduktion eines schadstoffes bzw. des gesamten Umweltschutzes ermittelt werden können. 


\section{b) Zur Festlegung der optimalen Umweltqualität}

Rein formal ist es relativ einfach, eine optimale Gesamtbelastung der Umwelt $z u$ bestimmen. Geht man davon aus, daB die gesamte Umweltbelastung $U$ einer Periode von der in dieser Periode produzierten Gütermenge $X$ abhängt, also gilt $U=U$ (X), und unterstellt ferner die Existenz elner sozialen Wohlfahrtsfunktion $W=W(U, X)$, so kann die optimale Umweltbelastung (und Güterproduktion), wie in Abbildung 11 dargestellt, abgeleltet werden 45)

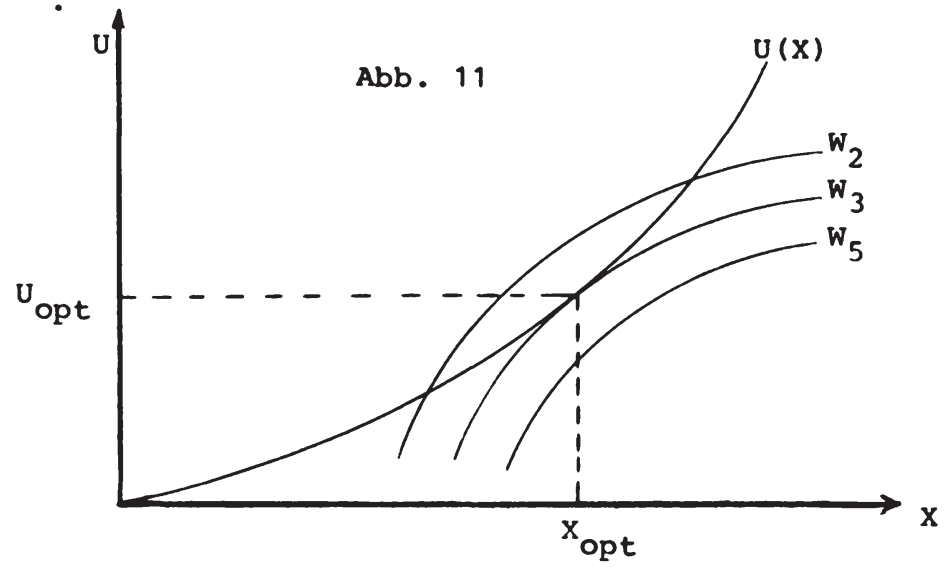

Es ist hier unterstellt, daB die Umweltbelastung mit stelgender Güterproduktion uberproportional zunimmt. Die gesellschaft11 chen Indifferenzkurven haben den in Abbildung 11 gezelgten Verlauf, well z.B. auf $w_{5}$ die gesellschaftliche Wohlfahrt be 1 stelgender Umweltbelastung nur glelch bleibt, wenn auch die Güterproduktion stelgt (Erklärung der positiven stelgung); eine höhere Umweltbelastung läßt außerdem nur bel überproportional

45) Dies ist elne von uns modifizierte Darstellung von Stein, J.L., The 1971 Report of the President's Council of Economic Advisers: Microeconomic Aspects of Public Pollcy, in: American Economic Review, vol. 61, 1971, S. 533; vgl. auch Beckerman, W., Environmental Policy Issues: Real and Fictitious, a.a.0., S. 20 f: und Frey, R.L., Umweltschutz als wirtschaftspolitische Aufgabe, a.a.0., S. 458. 
stelgender Produktion die gesellschaftliche Wohlfahrt unverändert (Erklärung der Krümmung). Die gesellschaftliche Wohlfahrt stelgt von links oben (hohe Umweltbelastung, geringe Produktion) nach rechts unten (geringe Umweltbelastung, hohe Guterproduktion) an. Die optimale Gesamtbelastung der Umwelt liegt bel U opt und damit bei einer Produktion in Hobe von $x_{\text {opt }}$, also dort, wo eine gesellschaftliche Indifferenzkurve (hier $W_{3}$ ) die Umweltbelastungsfunktion $U(X)$ tangiert.

Diese Bestimmung des optimalen Umweltbelastungs- und damit Umweltschutzniveaus ist aus zwel Gründen nicht operational. Zum einen ist es (bisher) nicht gelungen, eine soziale Wohlfahrtsfunktion aufzustellen, zum anderen existiert (noch) kein Umweltindikator, der die gesamte Umweltqualität wiederzugeben in der Lage wäre. An letzterem scheltert auch der Versuch, die optimale Gesamtbelastung der Umwelt mit Hilfe einer Kosten-Nutzen-Analyse zu bestimmen. Die Aussage, daß das optimale Umweltbelastungsniveau dort liegt, wo die sozialen Grenzkosten des Umweltschutzes gleich dem sozialen Grenznutzen des Umweltschutzes sind 46) wird nicht (erst) deshalb leerformalhaft, weil die Bewertung der sozialen Grenzkosten und Grenznutzen Schwierigkeiten bereltet, sondern (schon) deshalb, well die verschiedenen Arten der Umweltbelastung nicht auf einen Nenner gebracht werden können.

Wie zuversichtlich man sein kann, mit Hilfe einer Kosten-NutzenAnalyse die optimale Immissionskonzentration einzelner Schadstoffe zu bestimmen 47), soll im nächsten Abschnitt untersucht werden.

46) In Abb. 9b auf Seite 143 wären die Grenzkosten- und Grenznutzen-Kurve durch die soziale Grenzkosten- und die soziale Grenznutzenkurve und die Belastung $s$ durch einen Schadstoff durch einen Umweltindikator zu ersetzen (vgl. Hyman, D.N., The Economics of Governmental Activity, New York u.a., 1973, S. $61 \mathrm{f.l}$.

47) In Japan wurde z.B. mit Hilfe einer Kosten-Nutzen-Analyse ein $\mathrm{SO}_{2}$-Immissionsstandard festgeleat (vgl. OECD Environment Directorate, Instances, if any, where Cost-Benefit-Analyses has been applied to environmental problems - Japan -, Paris 1972 , S. 5 f.). 
c) Zur Eignung von Kosten-Nutzen-Analysen bei der Ableitung optimaler Immissionskonzentrationen einzelner Schadstoffe Interpretieren wir die Ridkerschen Kurven der Abbildungen 10 a und $b$ als soziale Kosten- und Grenzkostenkurven, so gibt $S_{R}$ die sozialen Kosten der Immissionsreduktion an und $\mathrm{SK}_{\mathrm{B}}$ die sozialen Kosten der Immissionsbelastung. Diese sozialen Kosten der Immissionsbelastung können wir als den sozialen Nutzen der Immissionsreduktion $\mathrm{SN}_{R}$ bezeichnen ${ }^{48}$ ). Die optimale Immissions-

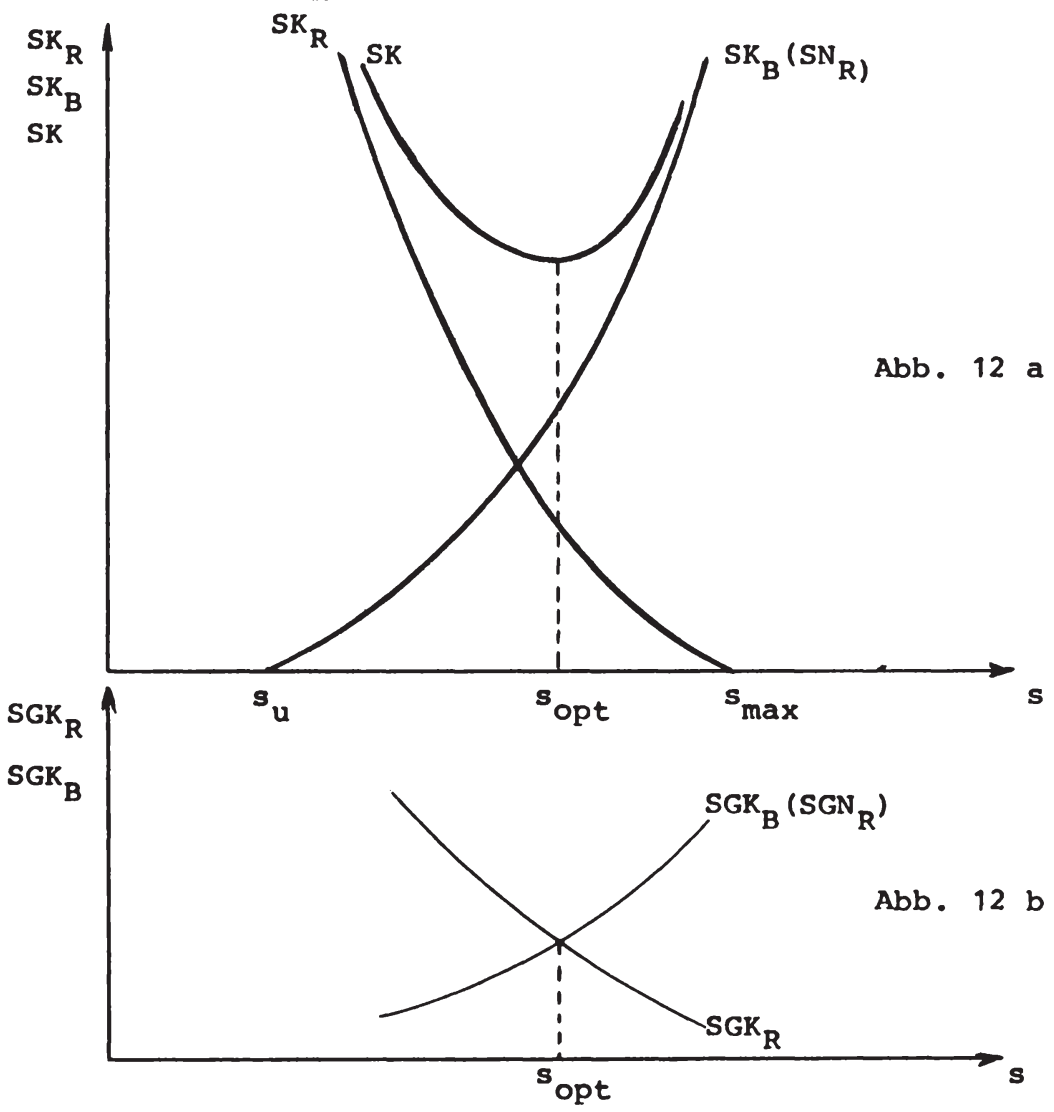

48) Vgl. Lave, L.B./Seskin, E.P., Air Pollution and Human Health, In: Dorfman, R./Dorfman, N.S. (Hrsg.), Economics of the Environment, a.a.0., S. 357. 
konzentration sopt liegt dort, wo die gesamten sozialen Kosten $\mathrm{SK}=\mathrm{SK}_{\mathrm{R}}+\mathrm{SK}_{\mathrm{B}}$ ihr Minimum haben ${ }^{49)}$ oder wo die sozialen Grenzkosten der Immissionsreduktion $\mathrm{SGK}_{\mathrm{R}}$ den sozialen Grenzkosten der Immissionsbelastung $\mathrm{SGK}_{\mathrm{B}}$ bzw. den sozialen Grenznutzen der Immissionsreduktion $S_{R}$ gleich sind (vgl. $\mathrm{Abb}$. 12 a und b).

Die Schwierigkeiten bei der Bestimmung dieses Optimums bestehen in der Ermittlung a 11 e r sozialen (Grenz-) Kosten und a 1 e $r$ sozialen (Grenz-)Nutzen; werden nicht alle sozialen Kosten und Nutzen erfaßt, ist sopt nicht die tatsächliche optimale Immissionskonzentration 50 )

Wir wollen uns bel der Elnteilung der Kosten und Nutzen an die Terminologie des U.S. Counc1l on Environmental Quality (CEQ) anlehnen, der zwischen "abatement costs" (wir wollen von Umweltschutzkosten im engeren Sinne sprechen), Transaktionskosten, Schadenskosten und Ausweichkosten unterscheidet 51). Dabei zählen die "abatement costs" und die Transaktionskosten $z u$ den sozialen Kosten der Immissionsreduktion und die Schadens- und Ausweichkosten $z u$ den sozialen Kosten der Immissionsbelastung, die den sozialen Nutzen der Immissionsreduktion entsprechen. Die gesamten sozialen Kosten der Immissionsreduktion erhält man jedoch erst, wenn man auch noch die Opportunitätskosten der Immissions-

49) Was 1dentisch ist mit der Annahme, daß der Nettonutzen maximiert wird (vgl. U.S.C.E.Q., Environmental Quality, The Fourth Annual Report, Washington, D.C., 1973, S. 109 und Fußnote 132).

50) Vgl. Ridker, R.G., Economic Costs of A1r Pollution, a.a.0., S. 10 .

51) Vgl. U.S.C.E.Q., Environmental Quality, The Fourth Annual Report, a.a.o., s. $76 \mathrm{f}$.

Beim U.S.C.E.Q. wird nicht ganz klar, ob er unter "abatement costs" nur die Kosten der Emissionsreduktion versteht und die Kosten, die für elne Vertellung der Schadstoffe oder die Immissionsreduktion aufgewendet werden, bereits als Ausweichkosten (avoidance costs) bezelchnen würde. Wir wollen als abatement costs die Kosten aller drei Umweltschutzmaßnahmen bezelchnen und uns den Begriff Ausweichkosten für die Kosten vorbehalten, die dadurch entstehen, daß Individuen oder Betriebe ein Immissionsgebiet zeltweise oder dauerhaft verlassen. 
reduktion berïcksichtigt ${ }^{52}$.

Bezeichnen wir die "abatement costs" mit $K_{U}$, die Transaktionskosten mit $K_{T}$, die Opportunitätskosten mit $K_{O}$, die Schadenskosten mit $K_{S}$ und die Ausweichkosten mit $K_{A}$, so gelten folgende Beziehungen:

$$
\begin{aligned}
& S K_{R}=K_{U}+K_{T}+K_{O^{\prime}} \\
& S K_{B}=K_{S}+K_{A}
\end{aligned}
$$

und für die gesamten sozialen Kosten der Immissionsreduktion und Immissionsbelastung gilt:

$$
\mathrm{SK}=\mathrm{SK}_{\mathrm{R}}+\mathrm{SK}_{\mathrm{B}}=\mathrm{K}_{\mathrm{U}}+\mathrm{K}_{\mathrm{T}}+\mathrm{K}_{\mathrm{O}}+\mathrm{K}_{\mathrm{S}}+\mathrm{K}_{\mathrm{A}} \cdot
$$

Dort wo diese Sozialkostenfunktion ihr Minimum hat, ist die optimale Immissionskonzentration sopt

Im folgenden wollen wir untersuchen, wie die einzelnen Kostenarten bestimmt werden können.

(1) Die Ermittlung der sozialen Kosten der Immissionsreduktion

(a) Kosten des Umweltschutzes Im engeren SInne (abatement costs)

Die sozialen Kosten, die durch Maßnahmen der Immissionsreduktion verursacht werden, bestehen zunächst aus den direkten Kosten der Maßnahmen selbst (Investitions- und Betriebskosten).-Diese Maßnahmen können entweder an der Emissionsquelle (Emissionsredukt1on), während der Ausbreitung des Schadstoffs oder im Immissionsgeblet durchgeführt werden (abatement costs). Diese durch technische Maßnahmen bedingten Umweltschutzkosten (z.B. für den Einbau von $\mathrm{SO}_{2}$-Abscheldern, den Bau von Kläranlagen, den Bau von Lärmschutzwällen) sind relativ leicht zu bestimmen, da für sie Marktpreise bestehen ${ }^{53}$ ? Be1 der Bewertung dieser Reduktions-

52) Vgl. Hyman, D.N., The Economics of Government Activity, a.a.o., s. $61 \mathrm{f}$.

53) Diese bel privaten Unternehmen und Haushalten, doch auch bel öffentlichen Gebletskörperschaften anfallenden Kosten für die Beschaffung und Unterhaltung von Umweltschutzanlagen im weltesten Sinne sind es vor allem, die bei den verschiedenen Untersuchungen über die gegenwärtige und zukünftige finan- 
kosten ist die kostengünstigste Maßnahme oder Maßnahmenkobination zugrundezulegen 54) Schwierigkeiten bei der Bewertung immissionsreduzierender Investitionen gibt es allerdings dann, wenn die Investitionen nicht allein dem Zweck der Immissionsreduktion dienen. Es kann sein, daB eine Investition, z.B. In

zielle Belastung durch den Umweltschutz erfaßt wurden. Vgl. dazu Ackermann, K./Geschka, H./Karsten, D., Gutachten zur Gesamtbelastung der Volkswirtschaft durch das Umweltprogramm der Bundesregierung, in: Materialien zum Umweltprogramm der Bundesregierung, a.a.O., S. $606 \mathrm{ff}$.; U.S. Department of the Interior/Federal Water Pollution Control Administration, The Cost of Clean Water, vol. I, Summary Report, vol. II, Detailed Analyses, U.S.G.P.O., Washington, D.C., 1968, S. $106 \mathrm{f}$.; The Cost of Clean Air, Second Report of the Secretary of Health, Education and Welfare to the Congress of the United States, U.S.G.P.O., washington, D.C., 1970; The Economics of Clean Air, Annual Report of the Administrator of the Environmental Protection Agency to the Congress of the United States, U.S.G.P.O., Washington, D.C., 1971 und 1972 .

Diese Untersuchungen versuchen die Investitions- und $\mathrm{Be}-$ trlebskosten von Anlagen zu schätzen, welche eine so große Emissionsreduktion bewirken, daß die Immissionsstandards eingehalten werden können. Die Kostenberechnungen orientieren sich also an bereits formullerten zielen in Form von Immissionsstandards für die verschiedenen schadstoffe, während unsere Fragestellung - jedenfalls an dieser Stelle - eine andere ist: Wir wollen die Kosten für die Realisierung unterschiedlicher Immissionskonzentrationen ermitteln, um so eine Funktion der Immissionsreduktionskosten zu erhalten. Die Bewertungsprobleme sind jedoch bei beiden Fragenstellungen die gleichen.

Kostenschätzungen, die sich vor allem auf die betriebswirtschaftlichen Kosten beziehen, nehmen unter anderem vor: Sprenger, R.-U., Struktur und Entwicklung von Umweltschutzaufwendungen in der Industrie, Berlin und München 1975; U.S.C.E.Q. /Department of Commerce/Environmental Protection Agency, The Economic Impact of Pollution Control, U.S.G.P.O., washington, D.C., 1972 (deutsch: Betriebswirtschaftliche Kosten von Umweltschutzmaßnahmen und ihre $g$ amtwirtschaft11che Auswirkung, Berlin 1972).

Neben diesen umfassenden Kostenschätzungen gibt es eine Fülle von Spezialuntersuchungen über die Kostenbelastung bestimmter Branchen durch den Umweltschutz; jede Literaturauswahl wäre hier rein willkürlich und soll deshalb unterbleiben.

$54)$ Vgl. Foster, C.D./Mackie, P.J., Noise: Economic Aspects of Choice, in: Urban Studies, Bd. 7, 1970, H. 2, S. $124 \mathrm{ff}$. 
ein neues Produktionsverfahren, gar nicht aus Gründen der Immissionsreduktion (via Emissionsbegrenzung) durchgefuhrt wurde, diese also nur ein umweltfreundlicher Nebeneffekt ist. Dann besteht die Gefahr, daß die gesamte Investition als Umweltschutzinvestition deklariert wird, die Kosten des Umweltschutzes also $\mathrm{zu}$ hoch angesetzt werden. Ferner ist es denkbar, daß durch Maßnahmen der Emissionsreduktion Nebenprodukte anfallen, die verkauft werden können. Ebenso können durch Umweltschutzinvest1tlonen Produktivitätsfortschritte entstehen. Die Erlöse aus dem Verkauf der Nebenprodukte und die Kostenersparnisse durch die Produktivitätsfortschritte sind dann von den Kosten der emissionsreduzierenden Maßnahmen abzuziehen, damit die sozialen Kosten des Umweltschutzes korrekt bewertet werden 55)

(b) Transaktionskosten

$\mathrm{Zu}$ den Immissionsreduktionskosten kann man auch die Informationskosten (z.B. für die Messung der Immissionsbelastung) und die Kontrollkosten (z.B. für die tberwachung der Emissionen), aber auch die Forschungs- und Entwicklungskosten zählen 56) - sofern sie nicht in den Preisen der Umweltschutzanlagen enthalten sind -, die z.B. dadurch entstehen, daß die Unternehmen nach emissionsärmeren Produktionsverfahren suchen. W1r wollen, dem CEQ folgend, die Gesamtheit dieser Kosten als Transaktionskosten bezeichnen 57 ?

55) Vgl. U.S.C.E.Q., Environmental Quality, The Fourth Annual Report, a.a.O., S. 89 f.; Sprenger, R.-U., Struktur und Entwicklung von Umweltschutzaufwendungen in der Industrie, a.a.O., S. $107 \mathrm{ff.;}$ Baltes, H./Nowak, W., Umweltstatistik - Ein Instrument der Umweltplanung, a.a.O., S. 244.

56) Die meiste in Fußnote 46) zitierte Literatur enthält auch Schätzungen über die Kosten der Messung, Kontrolle und auch der Forschung und Entwicklung. Die Kosten für diese Maßnahmen sind in der Regel nicht auf die einzelnen Wirtschaftseinheiten zurechenbar und werden deshalb vom staat getragen (vgl. dazu unten S. 289 ff. und S. 395).

57) Vgl. U.S.C.E.Q., Environmental Quality, The Fourth Annual Report, a.a.0., s. $83 \mathrm{f}$. 


\section{(c) Opportunitätskosten}

Die Maßnahmen des Umweltschutzes, die zu abatement costs und Transaktionskosten führen, beanspruchen Ressourcen, die damit zur Realislerung anderer (wirtschafts-) politischer Ziele nicht mehr zur Verfügung stehen. Umweltschutz führt also zu Verzichtskosten 58), die bei der Bestimmung der optimalen Immissionsgrenzwerte berücksichtigt werden müssen. Um diese Verzichtskosten bestimmen zukönnen, 1st zunächst die Kenntnis der wirkungen des Umweltschutzes auf die anderen (wirtschafts-)politischen ziele notwendig: Es müssen Zielbeziehungsfunktionen 59) zwischen dem Umweltschutz und z.B. der Preisniveaustabilität, der vollbeschäftigung, dem wachstum des realen Bruttosozialprodukts und dem $\mathrm{Zahlungsbilanzgle1chgewicht}$ aufgestellt werden. Diese zielbeziehungen können komplementär, neutral oder inkompatibel sein. Nur im letzten Fall entstehen Opportunitäskosten in Form von Zlelverzichten. Selbst wenn es nun gelänge, diese zielverzichte quantitativ zu erfassen 60 ) z. B. in Form geringerer

58) Vgl. Hyman, D.N., The Economics of Government Activity, a.a.0., S. 61 f.; Siebert, H., Probleme von Nutzen-KostenAnalysen umweltschützender Maßnahmen, in: Wirtschaftsdienst, 53. Jg., 1973, S. 132; RSU, Umweltgutachten 1974, a.a.0., S. 167 .

59) Brösse, U., Ziele in der Regionalpolitik. Zielforschung und Probleme der Realisierung von Zielen, Berlin 1972, S. 61 ff.; RSU, Umweltgutachten 1974, a.a.0., S. 11.

60) In einem neoklassischen Wachstumsmodell kann z.B. gezeigt werden, wie sich der Umweltschutz im Verzicht auf entsprechende Mengen elnes Konsumgutes niederschlägt (vgl. Alessio, F.J., The Opportunity cost of Pollution Abatement in a steady-state Expanding Economy, in: The Annals of Regional Science, vol. 5, 1971, S. $87 \mathrm{ff}$.$) . Von weitaus$ gröBerer praktischer Bedeutung als diese modelltheoretische Untersuchung ist die Ermittlung der zielverzichte mit Hilfe ökonometrischer Modelle, wie sie in den USA durchgeführt wird (vgl. Evans, M.K., A Forecasting Model Applied to Pollution Control Costs, in: American Economic Review, vol. 63, 1973, papers and proceedings, S. 244 ff.; U.S.C.E.Q.I Department of Commerce/Environmental Protection Agency, The Economic Impact of Pollution Control, a.a.0., s. $11 \mathrm{ff}$.$) .$ Mit Hilfe der Input-Output-Analyse wurde geschätzt, wie das Preisniveau in den USA infolge der Emissionsbeschränkungen stelgt (vgl. The Economics of Clean Air, Report of the Administrator of the Environmental protection Agency to the 
Wachstumsraten des realen Bruttosozlalprodukts oder eines gestiegenen Preisniveaus, wäre noch nichts uber die Bewertung dieser zielverzlchte, also die Höhe der Opportunitätskosten ausgesagt, denn Wachstumsverluste, Preisniveausteigerungen, Beschäftigungseinbußen und Exportverluste können durchaus unterschiedlich bewertet werden. Im Gegensatz zu den "abatement costs" und den Transaktionskosten lassen sich die Opportunitätskosten nur subjektiv bewerten 61) Hansmeyer we1st z.B. darauf hin, daB die Bewertung der Opportunitätskosten "planungsträgerbezogen" 62) 1st: Je niedriger die Planungsebene 1st, um so höher werden die Opportunitätskosten einer bestimmten Umweltschutzmaßnahme bewertet, da auf unteren Ebenen wegen des dort vorhandenen geringeren zielspektrums die zielverzichte konkreter sichtbar werden 63) Daneben spielen vor allem unterschiedliche Präferenzen, Partel- und Verbandzugehörigkeit derjenigen elne Rolle, welche die Bewertung der Opportunitätskosten vornehmen. Bereits an dieser stelle wird deutlich, daß die Bestimmung einer optimalen Immissionskonzentration nicht allein aufgrund objektiver Kriterien erfolgen kann.

(2) Die sozialen Kosten der Immissionsbelastung Die sozialen Kosten der Immissionsbelastung fallen an, wenn kelne oder nur ungenügende Immissionsreduktionsmaßnahmen ergriffen werden. Umgekehrt kann man - wie wir bereits wissen die Vermeidung der sozialen Kosten der Immissionsbelastung (durch die Immissionsreduktion) als den sozlalen Nutzen der Immissionsreduktion bezelchnen. Dies setzt allerdings voraus, daß die Schäden der Immissionsbelastung direkt erfaBt und bewertet werden können. Gelingt das nicht oder nur unvollkommen,

Congress of the United States, U.S.G.P.O., Washington, D.C., 1971 , S. 4-128 ff.).

61) Vgl. Siebert, H., Probleme von Nutzen-Kosten-Analysen umweltschützender MaBnahmen, a.a.0., s. 133.

62) Hansmeyer, K.-H., Volkswirtschaftliche Kosten des Umweltschutzes, In: Giersch, H. (Hrsg.), Das Umweltproblem in ökonomischer sicht, Tübingen 1974, S. 103

63) Vgl. ebenda. 
muß man nach anderen Möglichkeiten der Nutzenbewertung suchen.

(a) Schadenskosten

Die direkte Ermittlung der Schadenskosten, also die monetäre Bewertung der physischen und psychischen Gesundheitsschäden beim Menschen, der ökologischen Schäden und der Schäden an Sachgütern und Kunstwerken, setzt voraus, daB die Wirkungen der Immissionsbelastung quantitativ oder zumindest qualitativ erfaßt werden können. Mit den Möglichkeiten, aber auch den Problemen der Quantifizierung der Wirkungen der Umweltbelastung haben wir uns Im I. Kapitel befaßt 64) Es zeigte sich, daß manche Schäden, wie Beeinträchtigungen des Wohlbefindens, nur qualitativ erfaßt werden können. So entstehen berelts bel der Aufstellung nicht monetärer Schadensfunktionen in vielen Fällen Bewertungsprobleme, die nur subjektiv lösbar sind 65) Sind aber einmal aufgrund objektiver Erfassung und subjektiver Bewertung Schadensfunktionen der Form

$$
D_{i}=f\left(s_{k}\right)
$$

aufgestellt 66 ) , wobei $D_{i}$ den quantifizierten Schaden $D$ des 1 -ten Objekts $(i=1, \ldots, m)$ durch unterschiedliche Immissionskonzentrationen $s$ des schadstoffs $k(k=1, \ldots, n)$ bedeutet, gilt es in einem nächsten Schritt diesen Schaden monetär zu bewerten 67 ?

64) Vgl. oben S. $58 \mathrm{ff}$.

65) Vgl. z.B. die oben S. 105 ff. geschilderte subjektiv unterschiedliche Bewertung der Lärmwirkungen auf das Wohlbefinden verschiedener Menschen.

66) Vgl. Ridker, R.G., Economic Costs of Air Pollution, a.a.0., S. $16 \mathrm{ff.;}$ Lave, L.B./Seskin, E.P., Air Pollution and Human Health, a.a.0., s. $358 \mathrm{ff}$.

Neben diesen "Klassikern" der Bewertung von Umweltschäden vgl. vor allem die Belträge in dem von der OECD herausgegebenen Sammelband "Environmental Damage Costs", Record of a Seminar held at the OECD in August 1972, Paris 1974.

67) Vgl. Hueting, R., The Set-Up of the Statistical system within which the Deterioration of the Human Environment will be Estimated, a.a.0., S. 94; Waller, R.A., Environmental Quality, its Measurement and Control, in: Regional studies, 4, 1970, S. 177; Lave, L.B., Alr Pollution Damage: Some Difficulties in Estimating the Value of Abatement, in: Kneese, A.V./Bower, B.T. (Hrsg.), Environmental Quality 
Die monetäre Bewertung der Schäden ist realtiv einfach, wenn sie sich in finanziellen Verlusten äußern bzw. die Verhinderung der Schäden durch Maßnahmen möglich 1st, für die ein Marktpreis besteht 68 ) So entstehen z.B. bel Ernteschäden infolge der Luftbelastung Elnnahmeausfälle in der Landwirtschaft; bel Schäden an Gebäuden infolge der $\mathrm{SO}_{2}$-Belastung entstehen Reparaturkosten; die Hellung von Gesundheitsschäden infolge der Luftbelastung führt zu Behandlungskosten und, wegen der verminderten oder zeltweise oder dauernd eingesteliten Arbeitsleistung der Erkrankten entstehen Produktionsausfälle, die sich in verminderten ErIösen (bei den Unternehmen) und Elnkommen (bel den Erkrankten) äußern 69) Einnahmenausfälle, Reparaturkosten, Behandlungskosten und Erlösminderungen können als Werte für den jewelligen Schaden eingesetzt oder zumindest bel dessen Bewertung herangezogen werden.

Weltaus schwieriger ist die monetäre Bewertung der nicht objektIv meßbaren Schäden, wie etwa Beeinträchtigungen des Wohlbefindens, Störungen des ökologischen Glelchgewichts oder Zer-

Analysis, Baltimore und London 1972, S. 215; 0.V., How to Estimate the Real Cost of Environmental Damage, in: OECDObserver, No. 75,1975 , s. $29 \mathrm{f}$.

68) Vgl. Hueting, R., The Set-Up of the Statistical System within which the Deterioration of the Human Environment will be Estimated, a.a.0., S. 96 ff.; Mc Kean, R.N., Some Problems of Criteria and Acquiring Information, in: Jarret, H. (Hrsg.), Environmental Quality in a Growing Economy, Baltimore und London 1971, S. 62; Ridker, R.G., Economic Costs of Air Pollution, a.a.0., S. 16.

69) Vgl. Lave, L.B./Seskin, E.P., Air Pollution and Human Health, a.a.0., S. 380 ff.; Lave, L.B.. Alr Pollution Damage: Some Difficulties in Estimating the Value of Abatement, a.a.O., S. 215; Ridker, R.G., Economic Costs of Air Pollution, a.a.O., S. 17; U.S.C.E.Q., Environmental Quality. The Fourth Annual Report, a.a.o., S. $74 \mathrm{ff}$. Paul, M.E., Can Aircraft Nolse Nulsance be Measured in Money?, in: Oxford Economic Papers, vol. 23, 1971, S. 321; Meyer-Ablch, K.M., Was kostet die Umweltverschmutzung?, in: Umweltschutz - aber wie?, a.a.O., S. 15 f. Der U.S.C.E.Q. versuchte die Schäden, die durch einzelne Schadstoffe in der luft hervorgerufen werden, zu schätzen (vgl. U.S.C.E.Q. Environmental Quality, The Second Annual Report, a.a.o., S. 104 ff.). 
störungen unersetzlicher Kunstwerke 70) Manche dieser schäden - wie Beeinträchtigungen des Wohlbefindens und manche ökologische Schäden - versucht man mit Hilfe indirekter Bewertungsmethoden zu erfassen. So kann man z.B. mit Hilfe der Willingnessto-pay-Methode versuchen, die Bereltschaft der Bevölkerung $z u$ ermitteln, für die Reduzierung der Immissionsbelastung $z u$ zahlen 71) Hier wird versucht, durch Umfragen den sozialen Nutzen der Immissionsreduktion festzustellen. Obwohl diese Methode schon einige praktische Bedeutung erlangt hat 72) ist sie doch nur unter großen Vorbehalten anwendbar, da die Möglichke1t nicht ausgeschlossen werden kann, daß die Befragten den Nutzen $z u$ hoch angeben, well sie sich in der Position des

70) Vgl. Fry, C.L., Pollution Control, a.a.0., s. 4; Elckel, K.H./Jud, S., Umweltkosten des Straßenverkehrs, in: Umwelt, 1974,4, S. 18; Watson jr., W.D., Costs and Benefits of Fly Ash Control, in: Journal of Economics and Business, vol. 26 (3), 1974, S. 178 f.; Nydegger, A., Die Social Costs des Umweltunglelchgewichts, in: von Walterskirchen, M.P. (Hrsg.), Umweltschutz und Wirtschaftswachstum, a.a.0., S. 149 f.; U.S. Environmental Protection Agency, The Economic Impact of Nolse, U.S.G.P.O., Washington, D.C., 1971, S. 2; U.S.C.E.Q., Environmental Quality, The Second Annual Report, a.a.0., s. 104.

71) Vgl. Reichardt, R., Approaches to the measurement of environment, in: International Social Science Journal, vol. 22, 1970, S. $666 \mathrm{f}$; Hueting, R., The Set-Up of the Statistical system within which the Deterioration of the Human Environment W111 Be Estimated, a.a.0., S. $96 \mathrm{ff.;}$ Ridker, R.G., Economic Costs of Air Pollution, a.a.0., S. 18 .

$\mathrm{Zu}$ den verschiedenen Versuchen, die $\mathrm{Z}$ ahlungsbereltschaft der Bevölkerung für efine saubere Umwelt - und damit den Nutzen des Umweltschutzes - zu ermitteln, vgl. vor allem Freeman III, A.M., On Estimating Air Pollution Control Benefits from Land Value Studies, in: Journal of Environmental Economics and Management, vol. 1, 1974, S. 75 ff.; Randall, A./Ives, B./Eastman, C., Bidding Games for Valuation of Aesthetic Environmental Improvements, a.a.0., S. $135 \mathrm{ff}$; Bohm, P., A Note on the Problem of Estimating Benefits from Pollution Control, in: OECD (Hrsg.), Problems of Environmental Economics, a.a.o., s. $87 \mathrm{ff}$.

72) Vgl. Paul, M.E., Can Aircraft Noise Nuisance Be Measured in Money?, a.a.o., S. 298. 
"free rider" glauben, oder aber ihn zu niedrig ansetzen, weil sie befürchten, zur Finanzierung herangezogen zu werden 73)

Raffinierte Befragungstechniken können daran im Prinzip nur wenig ändern 74)

Eine andere Methode versucht die Schäden dadurch $z u$ bewerten, daB sie die Wertminderungen von Grundstücken und Gebäuden (in Form gesunkener Preise und Mieten) in 1 mmissionsbelasteten Gebieten in Beziehung zur Immissionsbelastung zu setzen versucht 75) Man kann dabel so vorgehen, daß man die Wertände-

73) Vgl. Foster, C.D./Mackie, P.J., Nolse: Economic Aspects of Cholce, a.a.0., S. 132; Lave, L.B., Alr Pollution Damage: Some Difficulties in Estimating the Value of Abatement, a.a.O., S. 231; Bohm, P., A Note on the Problem of Estimating Benefits from Pollution Control, a.a.0., S. 88 .

74) Vgl. Randall, A./Ives, B./Eastman, C., Bidding Games for Valuation of Aesthetic Environmental Improvements, a.a.0., S. $135 \mathrm{f} . ;$ Bohm, P., A Note on the Problem of Estimating Benefits from Pollution Control, a.a.0., S. $88 \mathrm{f}$. An dieser stelle weist Bohm auch darauf hin, daß die offenbarte $\mathrm{Z}$ ahlungsbereitschaft nach den Einkommensgruppen, denen die Befragten angehören, gewlchtet werden mủsse, da die Bereltschaft, elnen bestimmten Betrag für Umweltschutzmaßnahmen $z u$ zahlen mit stelgendem Einkommen zunehmen würde; dies impliziert allerdings die Annahme elnes abnehmenden Grenznutzens des Elnkommens; vgl. dazu auch Paul, M.E., Can Alrcraft Nolse Nulsance be Measured in Money?, a.a.o., S. 311 und Rathjens, G.W., National Environmental Pollcy: Goals and Priorities, in: Grad, F.K./Rathjens, G.W./ Rosenthal, A.J. (Hrsg.), Environmental Control: Priorities, Policies, and the Law, New York und London 1971, S. 11.

75) Vgl. Ridker, R.G./Henning, J.A., The Determinants of Residential Property Values with Special Reference to Air Pollution, in: Review of Economics and Statistics, vol. 49, 1967, S. 246 ff.; Anderson jr., R.J./Crocker, T.D., Air Pollution and Residential Property Values, in: Urban Studies, vol. 8, 1971, S. 171 ff.: Freeman III, A.M., Air Pollution and Property Values: A Methodological Comment, in: Review of Economics and Statistics, vol. 53, 1971, S. $415 \mathrm{f}$.: Anderson jr., R.J./Crocker, T.D., Air Pollution and Property Values: A Reply, in: Review of Economics and Statistics, vol. 54, 1972, S. 470 ff.; Freeman III, A.M., Air Pollution and Property Values: A Further Comment, in: Review of Economics and Statistics, vol. $56,1974, \mathrm{~S}, 554 \mathrm{ff}$.; Wieand, K.F., Air Pollution and Property Values: A Study of the Saint Louls Area, in: Journal of Regional Science, vol. 13, 1973, S. 92 ff.; Randall, A./Ives, B./Eastman, C., Bidding 
rungen von Gebäuden und Grundstücken im Zeltablauf verfolgt oder daB man thre Preise in einem belasteten denen in einem vergleichbaren unbelasteten Gebiet gegenüber stellt. Die zeitl1chen Wertminderungen bzw. räumlichen Wertunterschiede kann man dann zur monetären Schadensbewertung heranziehen. Gegen diese Bewertungsmethode spricht, daß der Grundstücks- und Gebäudemarkt kein vollkommener Markt ist und daB in den Marktpreisänderungen nicht nur Nachfrageänderungen infolge der Umweltbelastung zum Ausdruck kommen 76) Den Nachfrage- bzw. Preiseffekt infolge der Umweltbelastung $\mathrm{zu}$ isolieren, dürte daher kaum möglich sein.

Die gröBten Bewertungsprobleme werfen zwe1 weitere Kategorien von Schäden auf, nămlich zum einen die irreversiblen schäden wie unhe1lbare Gesundheitsschäden, der Tod von Menschen, die Ausrottung von Tler- und Pflanzenarten und die Zerstörung unwiederbringlicher Kunstwerke und zum anderen die Langzeitschäden, die quasi irreversibel sind.

Langzeitschäden, besonders wenn sie erst zukünftige Generationen betreffen (wie genetische Schäden, weltwelte Klimaveränderungen) bergen die Tendenz in sich, in der Gegenwart unter-

Games for Valuation of Aesthet1c Environmental Improvements, a.a.O., S. $184 \mathrm{f}$; Pearce, D., The Economic Evaluation of Nolse-Generating and Nolse Abatement Projects, in: OECD (Hrsg.), Problems of Environmental Economics, a.a.O., S. 105 ff.: Ridker, R.G., Economic Costs of Alr Pollution, a.a.0., S. $24 \mathrm{f}$.

76) Vgl. Waller, R.A., Environmental Qual1ty, its Measurement and Control, a.a.O., S. 184 ; Foster, C.D./MacKie, P.J., Nolse: Economic Aspects of Cholce, a.a.0., S. $133 \mathrm{f}$; Lave, L.B., A1r Pollution Damage: Some Difficulties in Estimating the Value of Abatement, a.a.0., S. 214 und S. $235 \mathrm{f}$.; Pearce, D., The Economic Evaluation of Nolse-Generating and Nolse Abatement Projects, a.a.0., S. 109; Ridker, R.G., Economic Costs of Air Pollution, a.a.0., S. 25.

77) Vgl. Fisher, A.C./Krutilla, J.V., Valuing Long Run Ecologlcal Consequences and Irreversibilities, in: Journal of Environmental Economics and Management, vol. 1, 1974, S. $96 \mathrm{ff.;}$ U.S.C.E.Q. Environmental Quality, The Fourth Annual Report, a.a.0., S. $110 \mathrm{ff}$. 
bewertet $z u$ werden, da die Bewertung von Angehörigen elner Generation vorgenommen wird, die nicht unter diesen Schäden zu leiden haben wird ${ }^{78}$ ). Nur eine ethische Haltung, die auch die Verantwortung für zukünftige Generationen bejaht, kann hier zu einer, auch den zukünftigen Generationen gerecht werdenden Bewertung von Umweltbelastungen mit Langzeitwirkungen führen. Auf alle Fälle ist die Bewertung solcher Schäden äußerst subjektiv.

Der Bewertung im Rahmen einer Kosten-Nutzen-Analyse völlig unzugänglich sind die irreversiblen Schäden ${ }^{79)}$ - trotz der Versuche, etwa den Tod eines Menschen in Form von Bruttosozialproduktsverlusten zu bewerten - und dies nicht etwa nur deshalb, well dabel die intangiblen Verluste der Angehörigen des Toten unberücksichtigt bleiben, sondern weil es u.E. aus ethischen Gründen unzulässig 1st, das Leben bzw. den Tod von Menschen in Ökonomische Kalküle einzubeziehen.

Die Ausrottung elner Tierart oder die Zerstörung eines Okosystems ist ein unersetzbarer Verlust, den manche in Kauf $z u$ nehmen bereit sind, wenn sle dafür materielle Vorteile erhalten. Für andere mag dieser verlust durch nichts kompensierbar sein, so daß sich schon aus diesem Grund eine Anwendung der KostenNutzen-Analyse verbietet ${ }^{80}$ ). Zusammenfassend kann man sagen: Die monetäre Bewertung der Schäden ist nur zum Tell objektiv möglich; bel vielen Arten von Schäden kommt nur eine subjektive Bewertung in Frage; manche Schäden lassen sich überhaupt nicht

78) Vgl. Coddington, A./Opschoor, H./Pearce, D., Some Limitations of Benefit-Cost Analysis in Respect of Programmes with Environmental Consequences, in: OECD (Hrsg.), Problems of Environmental Economics, a.a.0., S. 120; Pearce, D., The Economic Evaluation of Nolse-Generating and Nolse Abatement Projects, a.a.0., s. 103.

79) Vgl. Pearce, D., The Limits of Cost-Benefit Analysis as a Guide to Environmental Policy, in: Kyklos, Bd. 29, 1976 , S. $97 \mathrm{ff}$.

80) Vgl. Mishan, E.J., Evaluation of Life and Limb: A Theoretical Approach, in: Journal of Political Economy, vol. 79,1971, S. $691 \mathrm{f}$. 
monetär bewerten. Der Anwendung der Kosten-Nutzen-Analyse sind also konzeptionelle (Bewertung irreversibler Schäden) und praktische (unzureichende subjektive Methoden der Nutzenmessung) Grenzen gesetzt.

(b) Ausweichkosten

Statt Maßnahmen zu ergreifen, welche die Immissionskonzentration eines Schadstoffs verringern (was $z u$ Reduktionskosten führt) oder den Schaden zu tragen (was Schadenskosten hervorruft) können die potentiell Geschädigten eines Immissionsgebietes auch der (zu hohen) Immissionskonzentration ausweichen, indem sie das Immissionsgebiet zeitweise (z.B. am Felerabend, an Wochenenden und im Urlaub) oder dauernd (Umzug, Standortverlegung) verlassen. Dadurch entstehen Ausweichkosten 81). Die Stratedie des Ausweichens ist allerdings unseres Erachtens eine denkbar schlechte, denn ile führt dazu, daß unter Umständen nichts gegen regional hohe Immissionskonzentrationen unternommen wird, weil man glaubt, ihnen auswelchen zu können. So entsteht allmähl1ch die Gefahr, daß die Möglichkeiten des Ausweichens vor Immissionsbelastungen - im eigenen Land und auch auf der Erde - immer geringer werden; da andere $P$ laneten wenigstens in naher zukunft als Ausweichmöglichkeiten nicht in Frage kommen, scheint uns das Ausweichen vor Umweltbelastungen keine akzeptable Lösung der Schadensvermeidung zu sein. Nachtellig an dieser "Schein"lösung 1st auch, daß der Auswelchvorgang selbst - via höheres Verkehrsaufkommen, überfüllte Erholungsgeblete und Zersledlung der Landschaft - $z u$ weiteren Schäden und damit Kosten führt ${ }^{82}$ ?

81) Vgl. U.S.C.E.Q.,Environmental Quality, The Fourth Annual Report, a.a.0., S. 74; Ackermann, K./Geschka, H./Karsten, D. , Gutachten zur Gesamtbelastung der Volkswirtschaft durch das Umweltprogramm der Bundesregierung, a.a.0., S. 601.

82) Vgl. Ackermann, K./Geschka, H./Karsten, D., Gutachten zur Gesamtbelastung der Volkswirtschaft durch das Umweltprogramm der Bundesregterung, a.a.0., s. 601. 
d) Grenzen der Anwendbarke1t von Kosten-Nutzen-Analysen bei der Bestimmung optimaler Immissionskonzentrationen

Neben den im letzten Abschnitt aufgezeigten Bewertungsproblemen, welche die praktische Elgnung der Kosten-Nutzen-Analyse stark begrenzen, entstehen dadurch weitere Probleme, daß zwischen den einzelnen Kostenarten trade-offs bestehen, so daß keine gesonderte Minimierung der elnzelnen Kostenarten vorgenommen werden darf, wenn das gesamtwirtschaftliche soziale Kostenminimum realisiert werden soll 83). Den positiven (d.h. hier: ungünstigen) trade-off zwlschen Auswelch- und Schadenskosten haben wir bere1ts erwähnt. Auch daß zwischen Auswelchkosten und "abatement costs" ein negativer trade-off bestehen kann, d.h. elne Erhöhung der Auswelchkosten kann temporär zu einer Senkung der abatement costs führen, wurde berelts angedeutet. Auf weitere trade-offs weist der CEQ hin 84) So kann eine Erhöhung der Transaktionskosten (z.B. infolge verstärkter kontrollen) zu einer überproportionalen Senkung der Schadenskosten oder (z.B. Infolge verstärkter Umweltforschung) auch $z u$ einer überproportionalen Senkung der abatement costs führen. Die wegen der tradeoffs erforderliche gesamtwirtschaftliche Kostenminimierung stöBt besonders in elner marktwirtschaftlich organisierten und dezentralisierten Volkswirtschaft auf große Schwierigkeiten, da hier in der Regel eine einzelwirtschaftliche Kostenminimierung erfolgt 85)

Die Anwendbarkeit der Kosten-Nutzen-Analyse zur Ableitung einer optimalen Immissionskonzentration wird weiterhin dadurch einge-

83) Vgl. U.S.C.E.Q. Environmental Quality, The Fourth Annual Report, a.a.O., S. $109 \mathrm{f}$.

84) Vgl. ebenda. Dafür, daß zwischen abatement costs und Schadenskosten auch ein positiver trade-off bestehen kann, gibt es viele Beisplele. So füht z.B. der Schutz vor Lärm durch Lärmschutzwälle zu "visuellen" Kosten (vgl. Foster, C.D./Mackie, P.J., No1se: Economic Aspects of Choice, a.a.O., S. 124).

85) Vgl. U.S.C.E.Q. Environmental Quality, The Fourth Annual Report, a.a.0., s. 110 . 
schränkt, daß die sozialen Kosten des Umweltschutzes von den verschiedenen Einkommensgruppen in unterschiedlicher Höhe getragen werden und die sozialen Nutzen des Umweltschutzes den verschiedenen Einkommensgruppen in unterschiedlichem Umfang zugutekommen. Träger der sozlalen Kosten und Empfänger der sozialen Nutzen sind häufig nicht 1dentisch. Umweltschutz verändert also die Elnkommensverteilung ${ }^{86}$ ). Die Realislerung unterschiedilcher Immissionskonzentrationen impliziert elne unterschiedliche Einkommensvertellung, so daß mit der Entscheldung für eine bestimmte Immissionskonzentration auch elne Entscheidung für elne bestimmte Elnkommens-(um)vertellung gefällt wird. Die Kosten-Nutzen-Analyse als Entscheidungshilfe der Allokationsabtellung im Sinne Musgraves ist uberfordert, wenn sie auch distributive Wirkungen beurteilen soll. Die Festlegung einer bestimmten Immissionskonzentration kann nicht allein aufgrund einer Kosten-Nutzen-Analyse, sondern letztlich nur durch eine politische Entscheidung erfolgen 87) Das soll keineswegs heiBen, daß man auf Kosten-Nutzen-Analysen verzichten soll; sie können bel der Vorbereitung der politischen Entscheldung eine wichtige Hilfe sein, indem sie den Entscheidungsspielraum eingrenzen ${ }^{88}$. Eine qualitative Analyse

86) Vgl. Lave, L.B., A1r Pollution Damage: Some Difficulties in Estimating the Value of Abatement, a.a.O., S. 238; Comar, C.L./Thompson jr., J.C., Social, Environmental and Health Costs, a.a.0., S. 36; Halbritter, G., Abqabenprinzip und Bewertungsproblematik, in: Zur Problematik des Verursacherprinzips, a.a.0., s. 36 .

87) Vgl. Siebert, H., Probleme von Nutzen-Kosten-Analysen umweltschützender Maßnahmen, a.a.0., S. 135; Waller, R.A., Environmental Quality, 1ts Measurement and Control, a.a.o., S. 186; Schultze, C.L./Fried, E.R./ Rivlin, A.M./Teeters, N.H., Setting National Priorities, The 1973 Budget, a.a.o., S. 374 f.; Seldenfus, H.S., Umweltschutz, politisches System und wirtschaftliche Macht, in: Schnelder, H.K./Watrin, C. (Hrsg.), Macht und okonomisches Gesetz, Schriften des Vereins für Socialpolitik, N.F., Bd. 74, Berlin, 1973, S. 821 .

88) Vgl. Ackermann, K./Geschka, H./Karsten, D., Gutachten zur Gesamtbelastung der Volkswirtschaft durch das Umweltprogramm der Bundesregierung, a.a.0., s. 603. 
nicht quantifizlerbarer Faktoren kann als weitere Entscheidungshilfe die Kosten-Nutzen-Analyse ergänzen ${ }^{89}$ ?

e) Zur Festlegung von Immissionsstandards durch politische Entscheidung

aa) Von der optimalen Immissionskonzentration zum Immissionsstandard

Die Nichtanwendbarkeit der Kosten-Nutzen-Analyse be 1 irreversiblen Umweltschäden und praktische Schwierigkeiten bei der Bewertung sozialer Kosten und Nutzen lassen uns zu dem Schluß kommen, daß die gesetzliche Festlegung eines Immissionsstandards für einen bestimmten schadstoff nur sehr bedingt von der im vorletzten Abschnitt abgeleiteten optimalen Immissionskonzentration ausgehen kann.

Eine Glelchsetzung von optimaler Immissionskonzentration und Immissionsstandard, wie sie explizit oder implizit einigen Autoren vorschwebt 90) halten wir auch deshalb nicht für sinnvoll, da sich die optimale Immissionskonzentration $z u$ häufig ändert 91) So kann die optimale Immissionskonzentration $z$. $B$. mit steigendem technischen Fortschritt sinken 92), was sich (infolge elner Verringerung der abatement costs und evtl. auch der Transaktionskosten) in elner Linksdrehung der sozialen Kostenfunktion aubert (vgl. Abb. $13 \mathrm{a}, \mathrm{b}$ ). Ebenso kommt es z.B. Infolge eines gestiegenen UmweltbewuBtseins, das sich wegen der Höherbewertung einiger Schäden in

89) Vgl. Ridker, R.G., Economic Costs of Air Pollution, a.a.0., S. 10; Nydegger, A., Die Social Costs des Umweltungleichgewichts, a.a.0., S. 150 .

90) Vgl. Jacoby, N.H., The Polluters: Industry or Government?, a.a.o.. S. 24; Ridker, R.G., Economic Costs of A1r Pollution, a.a.0., S. $10 \mathrm{f}$.

91) Vgl. Waller, R.A., Environmental Quality, its Measurement and Control, a.a.O., S. 187; Meyer-Abich, K.M., Was kostet die Umweltverschmutzung?, a.a.0., s. 10.

92) Vgl. Slebert, H., Probleme von Nutzen-Kosten-Analysen umweltschützender Maßnahmen, a.a.0., S. 135; Meyer-Abich, K.M., Was kostet die Umweltverschmutzung?, a.a.0., s. 10 . 

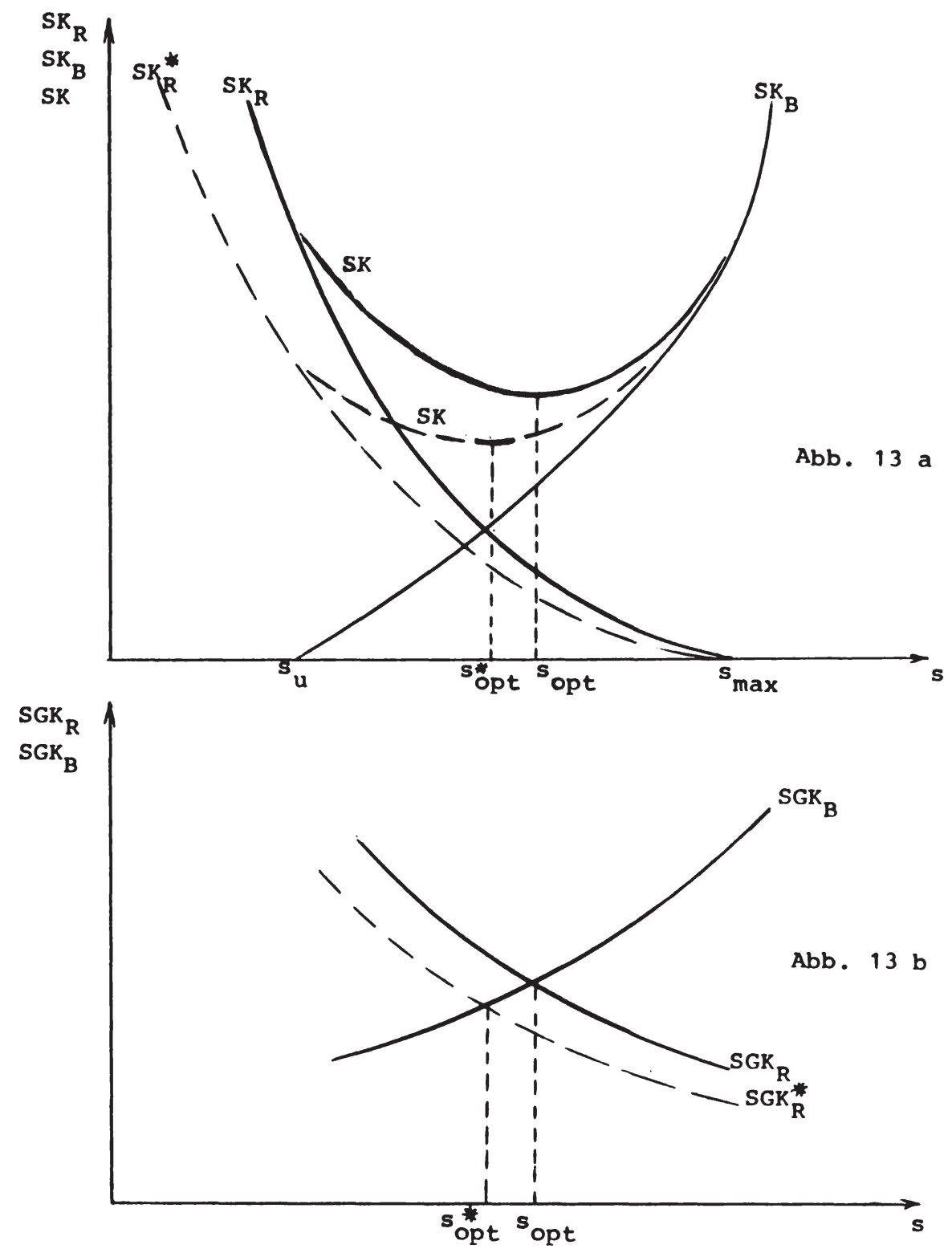
einer Linksdrehung der sozialen Nutzenfunktion äußert, zu einer Verringerung der optimalen Immissionskonzentration (vgl. Abb. $14 \mathrm{a}, \mathrm{b}$ ).

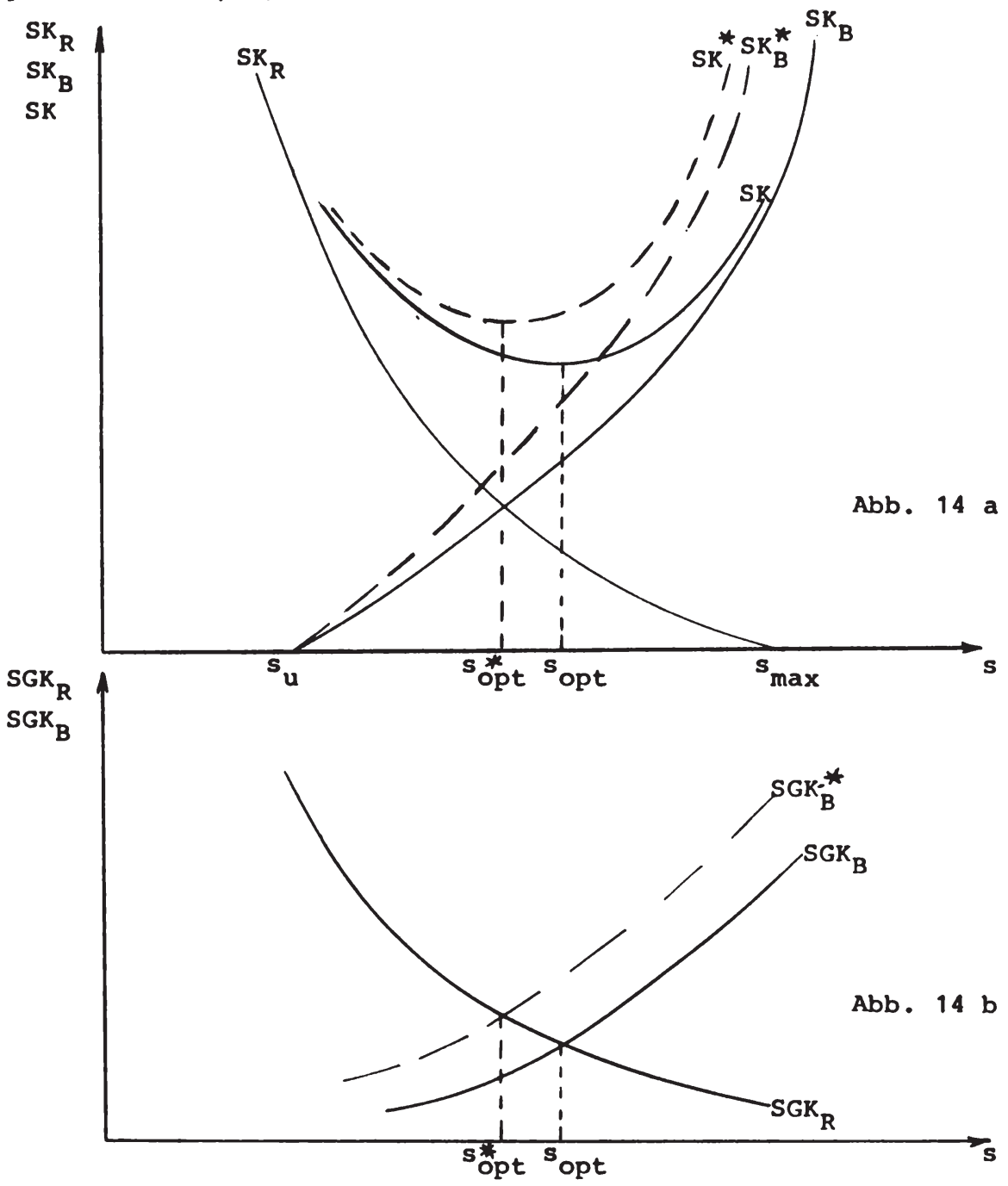

93) Vgl. Siebert, H., Probleme von Nutzen-Kosten-Analysen umweltschützender Maßnahmen, a.a.O., S. 135; Waller, R.A., Environmental Quality, its Measurement and Control, a.a.0., S. 188 . 
Würde der Immissionsstandard in Höhe der optimalen Immissionskonzentration fixiert, wäre eine ständige Anpassung des Immissionsstandards an die Veränderungen der optimalen Immissionskonzentration notwendig. Verwaltung und Legislative wäre nahezu ununterbrochen damit beschäftigt, neue Immissionsstandards zu ermitteln und $z u$ normieren. Dies würde nicht nur $z u$ einem unvertretbar hohen Aufwand führen, es widerspricht auch dem Charakter eines Immissionsstandards als nicht nur kurzfristlg gültigem Grenzwert, der zwar nicht über-, aber durchaus unterschritten werden darf. Er ist kein anzustrebendes ziel!

Es stellt sich zunächst die Frage, ob der festzulegende Immissionsstandard größer oder kleiner als die optimale Immissionskonzentration sein soll - falls eine solche uberhaupt ermittelt werden konnte. Auf den ersten Blick scheint es so, daß der Immissionsstandard zwischen $s_{\text {opt }}$ und $s_{\max }$ liegen sollte, um nicht die Realislerung der optimalen Immissionskonzentration $z u$ verhindern. Diese Lösung ist jedoch u.E. abzulehnen; sie birgt die Gefahr in sich, daß nicht das gesamtwirtschaftliche Optimum in sopt angestrebt wird, sondern - wegen der einzelwirtschaftichen Entscheldungsfreiheit - ein Punkt rechts davon, well dort die einzelwirtschaftlichen Immissionsreduktionskosten geringer sind.

Folgende Gründe sprechen U.E. dafür, den Immissionsstandard $z$ wischen $s_{u}$ und $s_{\text {opt }}$ festzulegen:

(1) Es besteht eine systematische Tendenz, die sozialen Kosten der Immissionsreduktion $z u$ überschätzen und die sozialen Kosten der Immissionsbelastung (den sozialen Nutzen der Immissionsreduktion) $z u$ unterschätzen ${ }^{94}$ ), so daB die ermittelte optimale Immissionskonzentration $z u$ hoch ist. Die Uperschätzung der sozialen Kosten der Immissionsreduktion rührt unter anderem wohl daher, das die abatement costs durch Umfragen bei den

$94)$ Vgl. Ackermann, K./Geschka, H./Karsten, D., Gutachten zur Gesamtbelastung der Volkswirtschaft durch das Umweltprogramm der Bundesreglerung, a.a.0., s. 600 ; Lave, L.B., Air Pollution Damage: Some Difficulties in Estimating the Value of Abatement, a.a.O., S. 238. 
Unternehmen ermittelt werden, was tendenziell dazu führt, daß sie $z u$ hoch angegeben werden 95 ! Außerdem werden wohl günstige trade-offs auBer acht gelassen; so wird z.B. nicht berücksichtigt, daB eine Erhöhung der Transaktionskosten zu einer überproportionalen Senkung der abatement costs führen kann. Die Unterschätzung der sozialen Kosten der Immissionsbelastung ist wohl vor allem darauf zurückzuführen, daß viele Schadstoffwirkungen noch unbekannt sind oder in ihrer Gefährlichkelt unterschätzt werden 96) Manche Methoden zur Bewertung der sozialen Nutzen, wie die Willingnes-to-pay-Methode, beinhalten geradezu die Tendenz zur Unterschätzung der sozialen Nutzen 97). Es ist also durchaus möglich, wenn nicht gar wahrscheinlich, daß das "richtige" sopt links von der aufgrund unzureichender Informationen und methodischer Schwierigkeiten ermittelten optimalen Immissionskonzentration liegt. Ein links vom ermittelten sopt liegender Immissionsstandard muB deshalb reallter keineswegs suboptimal sein.

(2) Mittel- und vor allem langfristig kann es infolge des technischen Fortschritts auf dem Gebiet des Umweltschutzes zu einer Senkung der abatement costs und der Transaktionskosten kommen, was sich in einer Linksdrehung der $\mathrm{SK}_{\mathrm{R}}$-Funktion und somit einer Reduzierung der optimalen Immissionskonzentration äußert. Den gleichen Effekt hat - wie wir bereits wissen - eine Stelgerung des Umweltbewußtseins - man kann auch sagen, eine mit steigendem Wohlstand sinkende Bereitschaft, Risiken durch die Umweltbelastung einzugehen -, was in einer Linksdrehung der sozialen Nutzenfunktion zum Ausdruck kommt. Im Zeitablauf bewegt sich die optimale Immissionskonzentration also wahrscheinlich in Richtung $s_{u}{ }^{98}$ ) Ein links vom ursprünglich er-

95) Vgl. U.S.C.E.Q., Environmental Quality, The Second Annual Report, a.a.0., s. 109.

96) Vgl. The Economics of Clean Air, a.a.0., s. 1-13 ff.; U.S.C.E.Q.,Environmental Quality, The Second Annual Report, a.a.0., S. 106 .

97) Vgl. oben S. $157 \mathrm{f}$. und Lave, L.B./Seskin, E.P., Health and Air Pollution, a.a.o., s. 383.

98) Wir wollen nicht verkennen, daß auch Tendenzen bestehen, 
mittelten sopt liegender Immissionsstandard kann also einer optimalen Immissionskonzentration eher näherkommen.

(3) Der wichtigste Grund dafür, daB der Immissionsstandard links vom ermittelten sopt liegen sollte, ist aber der, daß davon der Zwang ausgeht, alle Chancen (vor allem des technischen Fortschritts) zu nutzen, um auf möglichst effiziente Weise den Immissionsstandard einzuhalten oder ihn gar zur optimalen Immissionskonzentration werden zu lassen.

Während eine Festlegung des Immissionsstandards rechts von sopt die Tendenz in sich birgt, von dem gesamtwirtschaftlichen optimum wegzuführen (u.a. weil s opt im Zeitablauf wahrscheinlich nach links wandert), ist in der Festlegung des Immissionsstandards links von sopt geradezu eine Art Mechanismus eingebaut, der zur allmählichen Annäherung von sopt an den Immissionsstandard führt. Wenn beide übereinstimmen, stellt sich erneut die Frage, ob und wo eventuell ein neuer Immissionsstandard festgelegt werden sol1. Durch Neufestsetzung eines Immissionsstandards links vom alten, kann man allmählich die optlmale Immissionskonzentration Immer näher an die unschädliche Immissionskonzentration heranrücken.

Nachdem geklärt ist, daß der Immissionsstandard links von sopt liegen soll, ist zu bestimmen, wie weit links er von sopt (d.h. wie dicht bei $s_{u}$ ' festgelegt werden soll. Das hängt unter anderem davon $a b$, wie die politischen Entscheidungstrăger die Richtigkeit der Bewertung der sozialen Kosten und Nutzen einschätzen, wie sie die Entwicklung der Umwelttechnologie und des Umweltbewußtseins prognostizieren und welche allgemeine wirtschaftliche Entwicklung sie erwarten. Letzteres ist besonders deswegen von Bedeutung, well davon die Bewertung der opportunitätskosten abhängt. Werden z.B. Unterbeschäftigung und niedrigere Wachstumsraten erwartet, werden die opportunitätskosten des Umweltschutzes wahrscheinlich höher veranschlagt als wenn Vollbeschäftigung und viel Wachstum erwartet werden. Im ersten

die $S_{r}$-Funktion und damit $s_{\max }$ nach rechts $z u$ verschieben (vgl. Ridker, R.G., Economic Costs of Air Pollution, a.a.o., S. 10). 
Fall wird der Immissionsstandard weniger streng sein als im zweiten.

Die vorausgegangenen Ausführungen zeigten, daß die Festlegung eines Immissionsstandards letztlich eine politische Entscheidung ist, bei der naturwissenschaftliche und medizinische Erkenntnisse einerseits sowie ökonomische Uberlegungen andererseits helfen, den Entscheldungsspielraum einzugrenzen, indém sie als Untergrenze die unschädliche Immissionskonzentration $s_{u}$ und als weiteren Orientierungspunkt eine optimale Immissionskonzentration sopt festlegen, zwischen denen nach unserer Ansicht der Immissionsstandard festgelegt werden sollte.

bb) Implikationen der Notwendigkeit politischer Entscheidungen Die Notwendigkeit einer politischen Entscheldung über die Höhe der jewe1ligen Immissionsstandards impliziert, daß - besonders in einer pluralistischen Gesellschaft mit einer parlamentarischen Demokratie als Regierungsform - Interessen bei der Festlegung der Immissionsstandards mitspielen. Das können zum elnen die Interessen derjenigen seln, die unmittelbar zur Finanzierung des Umweltschutzes herangezogen werden sollen; sie sind an möglichst hohen Immissionsstandards interessiert. Es können zum anderen die Interessen derjenigen sein, die unter der Umweltbelastung leiden; sie sind für möglichst niedrige Immissionsstandards. Zur ersten Gruppe gehören vor allem die Industrie - vertreten durch ihre wirtschaftsverbände - und die Kommunen - in der Bundesrepublik Deutschland vertreten durch den Deutschen Städtetag. Zur zwelten Gruppe gehören vor allem die Bürger, die sich zwecks besserer Durchsetzung ihrer Interessen an elner sauberen Umwelt immer häufiger in Burgerinltiativen organisieren 99) Eine zwiespältige und manchmal auch eine zwielichtige stellung nimmt der staat als derjenige eln, der letztlich über die Höhe der Immissionsstandards entscheidet, wenn er selbst "Interessent" 1st. Das ist er z.B.

99) Vgl. Ewringmann, D./Z1mmermann, K., Kommunale Wirtschaftsförderung und Umweltschutz, a.a.0., s. $301 \mathrm{f}$. 
seit Bestehen des Bundesimmissionsschutzgesetzes, weil er danach eine Entschädigungspflicht für den Verkehrslärm auf öffentlichen straßen ubernommen hat ${ }^{100}$ ) Er ist als Träger der Lärmschutzkosten daran interessiert, die Immissionsstandards für den Straßenlärm möglichst hoch festzusetzen, um die Ausgaben für den Lärmschutz möglichst niedrig zu halten 101) Auch die Arbeitnehmer bzw. Ihre Gewerkschaften als ihre Vertreter befinden sich in einem Konflikt hinsichtlich der Höhe der Immisslonsstandards 102), da strenge Immissionsstandards Arbeitsplätze in emissionsintensiven Branchen gefährden können, während sie in der Umweltschutzindustrie neue Arbeitsplätze schaffen. Auch manche Wirtschaftszweige, wie etwa die Landwirtschaft und die Fischere1, können durchaus an strengen Immissionsstandards interessiert sein. Die Fronten pro und kontra strenge Immissionsstandards können also quer durch "traditionelle" Interessengruppen verlaufen.

$O b$ und welche Interessengruppen sich in welchem Umfang zu Lasten der Allgemeinheit bel der Festlegung der Immissionsstandards durchsetzen können, hängt von der Autorltät des Staates und der Machtverteilung zwischen den Interessengruppen ab. Ein um das Allgemeinwohl besorgter staat muB die Höhe der Immissionsstandards möglichst unabhänglg von den Gruppeninteressen und nicht zuletzt auch von den eigenen Interessen festlegen, was nicht heiBen soll, daß er nicht neben neutralen Sachverständigen (vor allem Medizinern, రkologen, Ingenieuren, Juristen und

100) Vgl. Nitschke, E., Lärm, a.a.0., s. 151.

101) Die jährlichen Lärmschutzkosten an Bundes-, Landes- und kommunalen Straßen würden bei einem Immissionsstandard von $75 \mathrm{~dB}(\mathrm{~A}) 171 \mathrm{M} 10 \mathrm{DM}$, bei elnem Immissionsstandard von 70 $\mathrm{dB}(\mathrm{A}) 478 \mathrm{MiO}$. DM, bel einem Immissionsstandard von 65 $\mathrm{dB}(\mathrm{A}) 1116 \mathrm{MiO}$. $\mathrm{DM}$ und bei einem Immissionsstandard von $60 \mathrm{~dB}(\mathrm{~A}) 2158 \mathrm{MiO}$. DM betragen, wobel auf die Kommunen die Hauptbelastung zukäme (vgl. Landesverband Bürgerinitiative Umweltschutz Nordrhein-Westfalen e.V., Wenlger Geld - mehr Lärm. Schutz der Bürger vor Straßenlärm ungenügend, in: U 1976,3, S. 42).

102) Vgl. O.V., Umweltschutz - Angst der Gewerkschaften, in: Wirtschaftswoche, Nr. 14 vom 30.3.1973, S. 26. 
Wirtschaftswissenschaftlern) auch Vertreter von Interessengruppen anhören soll, um sich so Entscheidungshilfen bei der Festlegung der Immissionsstandards $\mathrm{zu}$ verschaffen 103 ?

\section{cc) Zum Charakter der Immissionsstandards}

Immissionsstandards sind ebenso wie die unschädlichen Immissionskonzentrationen Grenzwerte; sie haben jedoch einen anderen Charakter. Während die unschädlichen Immissionskonzentrationen Grenzwerte sind, die aus medizinischen oder ökologischen Gründen nicht überschritten werden, sind die Immissionsstandards gesetzlich normierte Grenzwerte, die aus juristischen Gründen eingehalten werden müssen.

Dle Immissionsstandards können ebenso wie die korrespondierenden unschädlichen Immissionskonzentrationen nach regionalen und zeitlichen Gesichtspunkten sowie den Nutzungsansprüchen an das jewellige Umweltmedium modifiziert werden.

Die Immissionsstandards können über oder unter den unschädlichen Immissionskonzentrationen liegen. Liegen sie darüber, werden aus ökonomischen Gründen und allgemeinen Risikoüberlegungen bewußt Schäden in Kauf genommen. Solche Risiken wird man aber nur in den Fällen bereit sein elnzugehen, in denen man glaubt, daß von den Schadstoffen keine akuten Gesundheitsgefahren oder gar Irreversible Schäden ausgehen, also vor allem bel Beeinträchtigungen des Wohlbefindens, aber auch bel nur chronischen leichteren Erkrankungen. In den Fällen akuter Gefahren für das menschliche Leben oder irreversibler Schäden an der menschlichen Gesundheit oder dem ökologischen Gleichgewicht, also etwa in den Fällen radioaktiver Belastungen, nicht oder nur langsam abbaubarer

103) Auf diese Weise versuchten auch die Politiker in der Bundesrepublik Deutschland, sich Entscheidungshilfen zu verschaffen (vgl. die Hearings vor dem Innenausschuß des Deutschen Bundestages, in: Zur Sache $3 / 71,3 / 72,4 / 73$ und $2 / 75)$. $\mathrm{Zu}$ welchen Verzerrungen es allerdings kommen kann, wenn die Interessengruppen nicht glelchmäß1g stark vertreten sind, zelgte sich z.B. bel der Festlegung von Immissionsstandards für den Wisconsin-River (vgl. Fox, I. K./Wible, L.F.. Information Generation and Communication to Estabilsh Environmental Quality Objectives, a.a.0., S. 140 f.). 
Schadstoffe und toxischer Immissionsbelastungen, wird man die Immissionsstandards tellwelse welt unterhalb der Unschädlichkeitsgrenze festlegen. Dies gilt um so mehr, je abhängiger der Mensch von dem kontaminierten Umweltmedium ist (z.B. Trinkwasser und Nahrungsmittel).

Nach diesen allgemeinen Ausführungen über den Charakter der Immissionsstandards wenden wir uns im nächsten Abschnitt den Möglichkelten $z u$, in den verschiedenen Umweltberelchen Immissionsstandards für schadstoffe festzulegen.

C. Zur Ableitung von Immissionsstandards für die einzelnen Umwel tberei che

1. Immissionsstandards für Schadstoffe in der Luft Die in der Bundesrepublik Deutschland von der VDI-Kommission "Reinhaltung der Luft" abgeleiteten maximalen Immissionswerte in Form maximaler Immissionskonzentrationen (MIK-Werte) für elne Reihe von Schadstoffen 104), die die Luft belasten, entsprechen den von uns als unschädlich bezelchneten Immissionskonzentrationen. Sie enthalten einen Sicherheitsfaktor, um auch den Schutz der resistenzschwachen Bevölkerung zu gewährleisten ${ }^{105}$ ! Neben diesen MIK-Werten, die den Menschen auch vor Beeinträchtigungen des Wohlbefindens und vor allem vor chronischen Erkrankungen schützen sollen, sind noch Werte festzusetzen, ab denen eine toxische Wirkung auf den Menschen einsetzt 106). Für

104) Vgl. die Liste von MIK-Werten, die von der VDI-Kommission "Reinhaltung der Luft" vorgeschlagen werden, in: Umwelt, 1974,6, S. 34 f. und Schulte, H.J., Maximale Immissionskonzentrationen fủr 20 Stoffe erarbeltet, in: Umwelt, 1973 , 6. S. 38 .

105) Vgl. Schlipköter, H.-W., MIK-Werte dienen dem Schutz der Bevölkerung, in: Umwelt, 1973, 5, S. 36; derselbe, Die Luftverunreinigung als gesundheitliches Problem, a.a.o., S. 200 .

106) Vgl. Schlipköter, H.-W., MIK-Werte dienen dem Schutz der Bevollkerung, a.a.0., S. 36 .

Da Belästigungen durch Schadstoffe in der Luft, z.B. In Form von Gerüchen, schon bel sehr niedrigen Konzentrationen auftreten können, 1st es möglich, daß die Belăstiqungsgrenze 
die Schadstoffe, die kelne irreversiblen Schäden hervorrufen können, kann der Gesetzgeber die Immissionsstandards in der oben beschriebenen Weise zwischen den unschädlichen Immissionskonzentrationswerten (MIK-Werten) und den toxischen Immissionskonzentrationswerten festlegen 107) Stellt man die verschiedenen Grade der Umweltbeeinträchtigung durch Schadstoffe, die nicht zu irreversiblen Schäden führen wie in der skala auf S. 175 dar 108), so kann man sagen, daß eine gesetzliche Normierung eines Immissionsstandards dort beginnen kann, wo die Schwelle der Umweltverträglichkeit zur Umweltbelästigung überschritten wird; diese Schwelle entspricht unserer unschädlichen Immissionskonzentration. Unbedingt notwendig wird eine gesetzliche Normierung von Immissionsstandards dort, wo die Schwelle zwischen Umweltbelastung und Umweltgefährdung liegt (toxische Immissionskonzentration).

Bel den Schadstoffen, die zu irreversiblen Schäden führen können, müssen die Immissionsstandards - mit einem bestimmten Risikofaktor versehen - unterhalb der unschädlichen Immissionskonzentration, eventuell sogar nahe bei o festgelegt werden 109)

weit unterhalb der toxischen Belastungsgrenze liegt (vgl. Häberle, M., Zur Erstellung eines Geruchskatasters der BASF Ludwigshafen, a.a.O., S. 41; Fodor, G.G./Winneke, G. , Belästigung durch geruchsintensive Stoffe, a.a.O., S. 293.

107) Vgl. Schlipköter, H. -W., MIK-Werte dienen dem Schutz der Bevölkerung, a.a.0., S. 37.

In der Bundesrepublik Deutschland werden auf der Grundlage der MIK-Werte die Immissionsstandards, die in der Technischen Anleitung zur Reinhaltung der Luft (TAL) enthalten sind, abgeleitet (vgl. RSU, Umweltgutachten 1974, a.a.0., S. $15 \mathrm{ff.;}$ DreiBigacker, H.L./Surendorf, F./Weber, E., Zum Entwurf der Technischen Anleitung zur Reinhaltung der Luft, In: Umwelt, $1974,3, S .21)$. Besonders wichtig wäre, daß in die TAL auch Immissionsstandards für toxische Schwermetallstäube aufgenommen werden (vgl. Schlipköter, H.-W., MIKWert-Vorschläge für die Schwermetalle Blel, $\mathrm{Z}$ ink und Cadmium, in: Umwelt, 1973, 2, S. 12 ff.).

108) Vgl. Cmelka, D., Für eine sorgsame Umwelt-Terminologie, in: Umwelt, 1975, 5. S. 12 und Umwelt, 1976,1 . S. 33.

109) Das gilt z.B. für die Belastung der Menschen durch radioaktive Strahlen (vgl. Hahnemann, H.W., Strahlenschäden und Strahlenschutz, in: Umwelt, 1971, 5, S. 25), denn die Fest- 


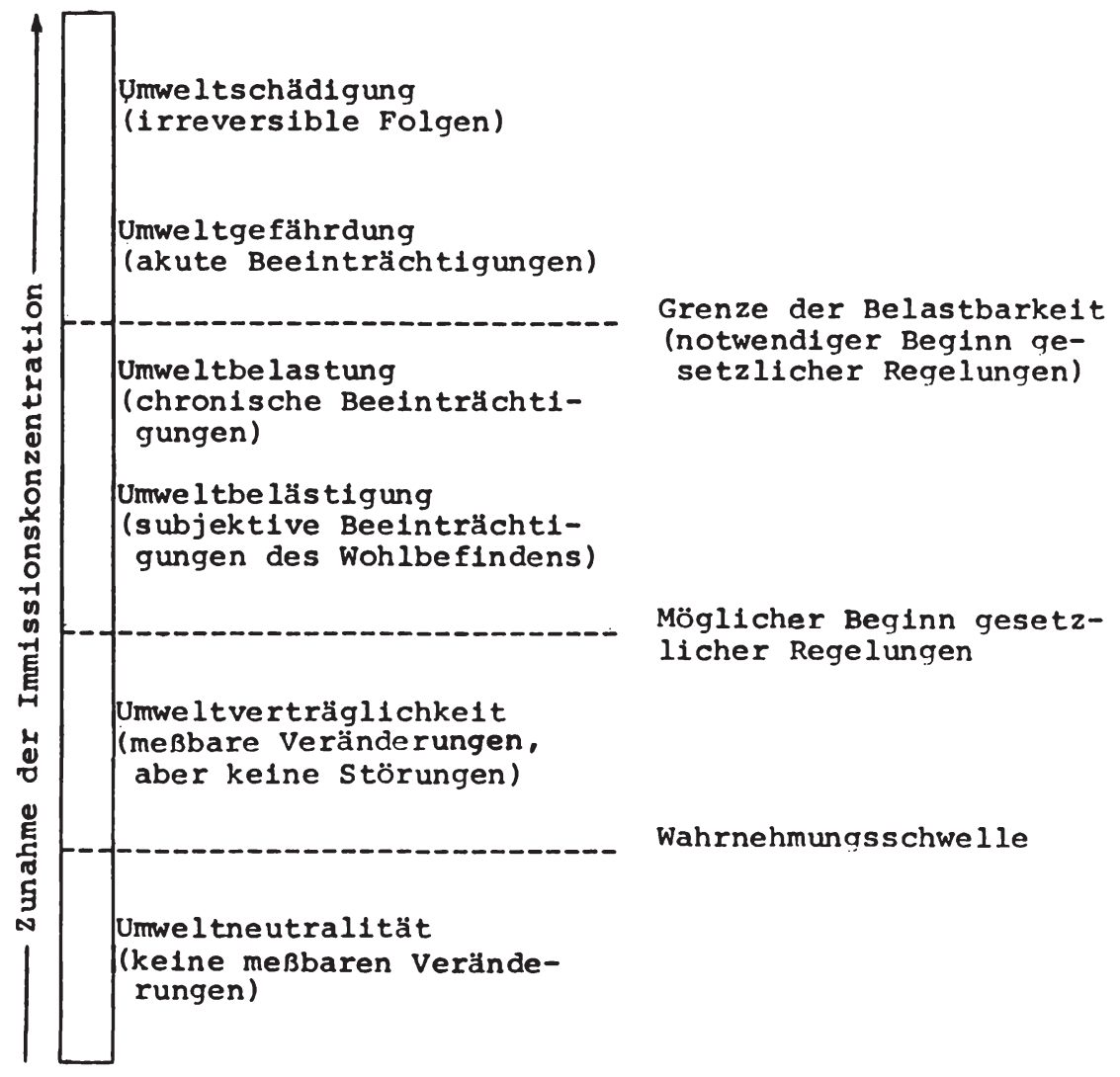

legung "elnes sogenannten Toleranzwertes ist in jedem Fall elne Vereinbarung uber Leben und Tod vieler ungefragter Menschen .... da die kleinste Dosis an der Zelle 1 rreparable Schäden auslösen kann" (B. Manstein bel der öffentlichen Anhörung vor dem Innenausschuß des Deutschen Bundestages, in: Zur Sache 2/75 (Umweltschutz IV), S. 22). Folgerichtig dürfen nach $\S 21$ der 1. Strahlenschutzverordnung "unbeschadet festgelegter Grenzwerte nur möglichst geringe Mengen radioaktiver stoffe in Luft und Wasser gelangen" (K. Aurand, ebenda, S. 25). 
Das große Manko der bisher in der Bundesrepublik Deutschland bestimmten MIK-Werte und der daraus abgeleiteten Immissionsstandards ist, daB sie unter der Annahme festgesetzt werden, daB nur der jeweilige schadstoff allein die Luft belastet 110 ! Dieser Mangel kann endgültig erst dann beseltigt werden, wenn genauere Kenntnisse uber Kombinationswirkungen und synergistische Effekte der Schadstoffe bekannt sind. Man kann diesem Mangel bel der Festlegung der Immissionsstandards hilfsweise dadurch Rechnung tragen, daß man die Immissionsstandards in Gebieten hoher Belastung niedriger festsetzt, da hier die Vermutung wohl berechtigt ist, daß besonders viele Schadstoffe die Luft belasten und so Kombinationswirkungen und kumulative sowie synergistische Effekte begünstigt werden.

Das ist ein Belspiel für die Notwendigkeit einer regionalen Differenzierung der Immissionsstandards 111) Auch eine zeit11che Differenzierung der Immissionsstandards für die luftbelastenden Schadstoffe 1st mögl1ch. Ze1tlich kann man elnmal nach der Zeitdauer der Schadstoffeinwirkung differenzieren. Die

110) Vgl. Coenen, R. U.a., Alternativen zur Umweltmisere, a.a.0., S. 109. Eine Ausnahme stellt der in den USA konstrulerte Air Pollution Index (API) dar, der die Komponenten Staub und $\mathrm{SO}_{2}$ enthält (vgl. van Belle, G./ Schneiderman, M., Some Statistical Aspects of Environmental Pollution and Protection, a.a.0., S. 318).

111) Vgl. Stehfest, H., Standards, in: Zur Problematik des Verursacherprinzips, a.a.O., S. 44; Binswanger, H.D., Eine umweltkon forme wirtschaftsordnung, a.a.0., S. 139. In den USA können die Bundesstaaten die Immissionsstandards für die verschiedenen "Alr Quality Control Regions" festlegen (vgl. The Cost of Clean Alr, The Second Report .... a.a.0., S. 8).

Es muß allerdings gewährleistet sein, daß sie nur niedriger festgelegt werden dürfen als die vom Administrator der Environmental Protection Agency $z u$ bestimmenden nationalen Immissionsstandards (vgl. Blair, R.D., The Clean Air Act of 1970, a.a.0.. S. 261).

Auch in Japan gibt es regional unterschiedliche Immissionsstandards (vgl. Janocha, P., Verschmutzungsgrenzen für jede Region, in: Umwelt $1975,4, \mathrm{~s} .54 \mathrm{ff.l}$. Ein regional unterschiedilcher Lebensstandard sollte unseres Erachtens allerdings nicht $\mathrm{zu}$ einer Differenzierung der Immissionsstandards führen, wie dies von der Tax Foundation vorgeschlagen wurde (vgl. Tax Foundation, Pollution Control; Perspectives on the Government Role, a.a.0., S. 6). 
Kurzzeit(mittel-) Werte (z.B. für eine halbe stunde) können dabei höher seln als die (Mittel-)Werte für elne Einwirkungsdauer von 24 Stunden oder gar diejenigen für ein Jahr 112) Ferner ist eine jahreszeitliche Differenzierung der Immissionsstandardas denkbar 113)

Auch elne Differenzierung nach den Nutzungsansprüchen an das jeweilige Immissionsgebiet ist möglich 114). So können die Immisslonsstandards z.B. für einen Luftkurort besonders niedrig festgesetzt werden.

Schließlich ist noch $z u$ bedenken, daß nicht nur die menschliche Gesundhelt sonder auch Pflanzen, Tiere und Sachgüter vor luftverunrelnigenden Schadstoffen geschützt werden sollen. Auf manche Arten der Luftbelastung - z.B. die $\mathrm{SO}_{2}$-Belastung - reagleren Pflanzen Lnd Sachgüter (vor allem Gebäude) empfindlicher als der Mensch 1.5) Man kann dabei so vorgehen wie in den USA, wo unterschiedliche Immissionsstandards für den Schutz der

112) Vgl. die Liste von MIK-Werten, In: Umwelt, 1974, 6, S. 34 f.: RSU, Umweltgutachten 1974 , a.a.0., S. 16; 0.V., Grundgesetz fur die Luftreinhaltung: die TA Luft, in: Umwelt, 1974,4, S. 12 .

Es ist allerdings nicht immer einfach, von den Kurzzeitwerten auf Langzeltwerte $z u$ schließen (vgl. Bauer, R.K., Methodische Grundprobleme der Umweltstatistik, a.a.o., S. 33).

113) Vgl. Tax Foundation, Pollution Control: Perspectives on the Government Role, a.a.0., s. 6 .

Elne solche Differenzierung wăre z.B. denkbar, well bestimmte Arten der Immissionsbelastung (z.B. photochemischer Smog) nur bei Inversionswetterlagen, die sich in bestimmten Jahreszeiten häufen, eintreten. Gegen eine solche Differenzlerung wendet sich Rathjens (vgl. Rathjens, G.W., National Environmental Policy: Goals and Priorities, a.a.0., S. 35 f.).

114) Vgl. Karsten, D., Umweltpolitik - Argumente für die marktwirtschaftliche Lösung, in: Wirtschaftswoche Nr. 20, 1972, S. 38 .

115) Vgl. Lahmann, E., Literaturstudie über die bkonomischen Konsequenzen ...., a.a.O., S. 21; Dreyhaupt, F.J., Luftreinhaltung als Faktor der stadt- und Reglonalplanung, a.a.O., S. 49; Olschowy, G., Katalog bkolog1scher Noxen und der von thnen bedingten Schäden, a.a.0., S. 160 . 
Menschen, Pflanzen und Sachgüter festgelegt werden 116). Das halten wir jedoch nicht für sinnvoll, da die betroffenen Menschen, Pflanzen und Sachgüter nicht lsoliert voneinander existieren. Die Konsequenz kann nur sein, sich bei der Festlegung des Immissionsstandards am empfindlichsten "Objekt" zu orientieren 117)

\section{Immissionsstandards für die Gewässer}

Die Gewăsserqualität wird nicht nur durch die verschiedenen Schadstoffkonzentrationen determiniert sondern auch durch bestimmte (bio-) chemische, physikalische und hygienische Eigenschaften, die threrseits allerdings wlederum von der Schadstoffbelastung abhängen können. Als Immissionsstandards kommen damit nicht nur gesetzlich zulässige schadstoffkonzentrationen in Frage sondern auch bestimmte biologische, chemische, physikal1sche und hygienische Größen und Kennziffern. Ein weiterer Unterschied zur Bestimmung von Immissionsstandards der Luftbelastung besteht darin, daß sich die Gewässer aus geogra-

116) Vgl. Bla1r, R.D., The Clean A1r Act of 1970, a.a.O., s. 261 ff.; Schultze, C.L./Fried, E.R./Rivlin, A.M./Teeters, N.H., Setting National Priorities, The 1973 Budget, a.a.O., S. 390 .

117) Vgl. Einbrodt, H.J., Grenzwerte für staubförmige Luftverschmutzungstoffe und andere Bekümpfungsmaßnahmen, in: Zentralblatt für Bakteriologie ...., a.a.O., S. 298. Das setzt allerdings voraus, daB auch für Pflanzen und Sachgüter MIK-Werte, also unschädllche Immissionskonzentrationen ermittelt werden (vgl. Schlipköter, H.-W., MIK-Werte dienen dem Schutz der Bevölkerung, a.a.o., s. $36)$.

Es 1st allerdings mindestens ebenso schwierig ökologische Belastungsgrenzen festzustellen wle MIK-Werte für den Schutz der menschlichen Gesundheit, da sowohl Widerstandsfähigkeit als auch Selbstreinigungskraft elniger రkofaktoren sehr stark von den differierenden abiotischen Standortfaktoren (Klima, geologische Verhältnisse) abhängen (vgl. RSU, Umweltgutachten 1974, a.a.0., S. 112; Ellenberg, H., Belastung und Belastbarkelt von Okosystemen, a.a.0., S. $24 \mathrm{f}$. ). Das ist jedoch ein welterer Grund dafür, daß Immissionsstandards regional unterschiedlich festgelegt werden können (vgl. RSU, Umweltgutachten 1974, a.a.o., S. 112). 
phischen Gründen besser nach Nutzungsansprüchen einteilen lassen als die Atmosphäre. Damit können die Immissionsstandards und Gütekennziffern auch besser nach Nutzungsansprüchen differenziert werden. Für die blologische Qualität eines Gewässers ist wenlger die Konzentration bestimmter Schadstoffe maßgeblich als vielmehr der sauerstoffgehalt 118) oder, umgekehrt formuliert, der Sauerstoffbedarf. Um den biologischen Zustand eines Gewässers beschreiben zu können, wurde das sogenannte Saprobiensystem entwickelt, das die (biologische) Gewăssergüte in vier Klassen einteilt 119).

Güteklasse 1 (oligosaprob): keine oder nur geringe Verunrein1gung, hoher sauerstoffgehalt

Güteklasse 2 (beta-mesosaprob): mäßıge Verunreinlgung, noch hoher Sauerstoffgehalt

Güteklasse 3 ( alpha-mesosaprob): starke Verunreinigung, niedriger Sauerstoffgehalt

Güteklasse 4 (polysaprob): übermäßige Verunrelnigung, sehr geringer sauerstoffgehalt.

Wie hoch der Sauerstoffgehalt eines Gewăssers 1st, hänat von

118) Dabel soll nicht verkannt werden, daß auch toxische substanzen das biologische Gleichgewicht stören können (vgl. Sontheimer, H., Untersuchungen zur Belastung des Rheins mit organischen stoffen, in: Gas- und Wasserfach, GWF, Wasser $111(1970), 8,5.426)$.

119) Vgl. Gräff, B./Spegele, H., Wörterbuch des Umweltschutzes, a.a.0., S. 115; Liebmann, H., Die Wasserqualität der oberbayerischen Seen, a.a.O., S. 64; RSU, Umweltgutachten 1974, a.a.0.. S. 48; Elster, H. -J., Forderungen an die Reinhaltung unserer Gewässer aus biologischer Sicht, in: Schultze, H. (Hrsg.), Umwelt-Report, a.a.O., S. $93 \mathrm{f}$.

Eine dem deutschen Saprobiensystem ähnliche Eintellung gibt es in der CSSR und eine zusätzliche Qualitätskriterien berücksichtigende Kategorisierung der Flüsse wird in der UdSSR vorgenommen (vgl. Litvinov, N., water pollution in the USSR and other Eastern European Countries, in: Bulletin of the WHO, vol. 26, 1962, S. $445 \mathrm{ff}$. ). Ahnllch differenzlerte Qualitätsanforderungen für die Gewässer in der Bundesrepublik Deutschland werden im Umweltgutachten des RSU zur Diskussion gestellt (vgl. RSU, Umweltqutachten 1974, a.a.O., S. 51). 
der Belastung des Gewässers mit sauerstoffzehrenden organischen Schadstoffen $a b$, aber auch von der Selbstreinigungskraft des Gewässers. Je höher die Belastung mit organischen Substanzen und je geringer die Selbstrelnigungskraft eines Gewăssers ist, um so hoher ist der biochemische sauerstoffbedarf in Form des $\mathrm{BSB}_{5}$. Die blologischen Qual1tătserfordernisse eines Gewässers können in Form der Güteklassen des Saproblensystems und durch den $\mathrm{BSB}_{5}$ angegeben werden.

Die Belastung eines Gewässers durch Chemikalien kann bel organischen Verbindungen durch den Kallumdichromat-Verbrauch, also den chemischen Sauerstoffbedarf (CSB), ermittelt werden; toxische Belastungen der Gewässer (z.B. durch Schwermetallverbindungen) müssen durch Messung der jeweiligen Schadstoffkonzentration ermittelt werden. Die chemischen Belastungsgrenzen können also durch den CSB und durch Immissionsstandards für toxische substanzen angegeben werden.

Die physikalischen Elgenschaften elnes Gewässers sind zum einen deswegen von Bedeutung, weil sie den blologischen und chemischen Zustand elnes Gewässers mitbestimmen (Temperatur), zum anderen deshalb, well sie für bestimmte ästhetische $\mathrm{Zustände} \mathrm{verantwort-}$ lich sind (Trübung, Farbe). Die physikalischen Belastungsgrenzen können also durch bestimmte Kennziffern (z.B. in ${ }^{\circ} \mathrm{C}$, Sichttiefe eines Gewässers) wiedergegeben werden.

Die Grenzen der Gewässerbelastung aus hygienischen Gründen können schließlich durch die Anzahl pathogener Mikroorganismen je Volumeneinhelt wasser aber auch durch bestimmte physiologische Merkmale (Geschmack, Geruch) festgelegt werden. Je nach den verschiedenen Nutzungsansprüchen können die biologischen, chemischen, physikalischen und hygienischen Immissionsstandards oder Gütekennziffern unterschiedlich hoch fixiert werden 120?

120) Vgl. dle Ausführungen von $W$. Gässler bel der öffentlichen Anhörung vor dem Innenausschuß des Deutschen Bundestages, in: Umweltschutz (I), Zur Sache 3/71, S. 48 und RSU, 
Die Nutzungsarten der Gewässer kann man wie folgt einteilen 121): Trinkwasser, Baden, Freizeit und Erholung (einschlieBlich Wassersport), Fischzucht, Bewässerung, Kühlwasser, industrielle Produktion, Wasserkraft, Schiffahrt und Abwassertransport. In einer Matrix (vgl. S. 182) lassen sich die Qualitätserfordernisse in Form der Immissionsstandards und der sonstigen die Gewässer kennzelchnenden $\mathrm{ziffern} \mathrm{mit} \mathrm{den} \mathrm{verschiedenen} \mathrm{Nutzungs-}$ arten kombinieren 122)

Die strengsten Qualitätsstandards sind zweifellos für die Gewässer festzulegen, die der Trinkwasserversorgung dienen 123), also vor allem für das Grundwasser; immer mehr sind es aber auch Oberflächengewässer, die als Trinkwasserreservoirs dienen 124).

Umweltgutachten 1974, a.a.O., S. 48. Nach $\S 36$ b Wasserhaushaltsgesetz ist eine nutzungsbezogene Festlegung von Immissionsstandards möglich, wenn die Bundesländer Bewirtschaftungspläne aufstellen (vgl. dazu Riegel, R., Gewässergütewirtschaft: Immissions- oder Emissionsstandards?, in: Umwelt, $1976,1, \mathrm{~S} .41 \mathrm{ff.}$ ). In den USA wird die nutzungsbezogene Festlegung von Immissionsstandards bereits praktiziert (vgl. Fox, I.K./Wible, L.F., Information Generation and Communication to Establish Environmental Quality Objectives, a.a.0., S. $137 \mathrm{f}$.$) .$

121) Vgl. Hazelton, J.E., Effluents and Affluence, a.a.O., S. 139 ; RSU, Umweltprobleme des Rheins, a.a.0., s. $88 \mathrm{ff}$. und S. 184; RSU, Umweltgutachten 1974, a.a.0., S. 48.

122) Vgl. Beckerman, W., Environmental Policy Issues: Real and Fictitious, a.a.O., S. 29; Schultze, C.L./Fried, E.R./ Rivilin, A.M./Teeters, N.H., Setting National Priorities, The 1973 Budget, a.a.O., S. 378 .

123) Die Qualitätsanforderungen für das Trinkwasser in der Bundesrepublik Deutschland finden sich in den "Leitsätzen für die Zentrale Trinkwasserversorgung" (DIN 2000), in: Wasserversorgungsnormen, DIN-Taschenbuch 12,2 . geänderte Aufl., hrsg. vom DNA, Berlin-Köln-Frankfurt 1971, s. 42 ff.; vgl. auch WHO, European Standards for Drinking-Water, 2. Aufl., Genf 1970, wo bakteriologische, virologische, radiologische, physikalische, chemische und physiologische Grenzwerte für das Trinkwasser festgelegt werden.

124) Aus diesem Grund müssen diese Oberflächengewässer mindestens eine Qualität haben, welche die Trinkwasseraufbereitung nicht zu sehr verteuert. Die Internationale Arbeitsgemeinschaft der Wasserwerke am Rhein (IAWR) hat Grenzwerte für sogenanntes Rohwasser, das nach Aufbereltung als Trinkwasser dienen kann, aufgestellt, und zwar einmal für den Fall eines 


\begin{tabular}{|c|c|c|c|c|c|c|c|c|c|}
\hline $\begin{array}{l}\text { Immis- } \\
\text { slons- } \\
\text { standard }\end{array}$ & 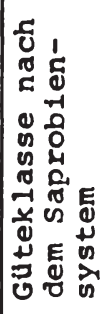 & $\infty_{\infty}^{n}$ & $\mathcal{c}^{\infty}$ & 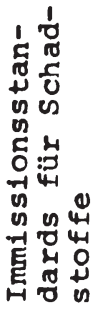 & 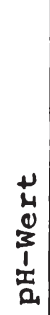 & $0^{0}$ & 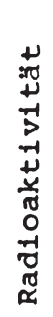 & 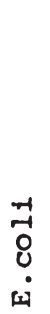 & 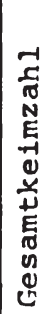 \\
\hline Trinkwasser & & & & & & & & & \\
\hline Baden & & & & & & & & & \\
\hline $\begin{array}{l}\text { Erholung, } \\
\text { Freizeit, } \\
\text { Sport }\end{array}$ & & & & & & & & & \\
\hline Fischzucht & & & & & & & & & \\
\hline Bewässerung & & & & & & & & & \\
\hline Küh lwasser & & & & & & & & & \\
\hline $\begin{array}{l}\text { Industrielle } \\
\text { Produktion }\end{array}$ & & & & & & & & & \\
\hline Wasserkraft & & & & & & & & & \\
\hline Schiffahrt & & & & & & & & & \\
\hline $\begin{array}{l}\text { Abwassertrans- } \\
\text { port }\end{array}$ & & & & & & & & & \\
\hline
\end{tabular}

einfachen und sodann auch für den eines technisch komplizierten und finanziell aufwendigen Aufbereltungsverfahrens (vgl. IAWR, Memorandum der IAWR, in: Gas-Wasser- Abwasser, 53. Jg., 1973, Nr. 6 und RSU, Umweltprobleme des Rheins, a.a.o.. s. $184 \mathrm{ff.}$ ). Grenzwertvorschläge für toxische Stoffe finden sich bei Borneff, J., Forderungen an die Reinhaltung unserer Binnengewässer aus hygienischer sicht, 1n: Schultze, H. (Hrsg.), Umweltreport, a.a.0., S. 90. 
Von minderer Qualität können dagegen die Gewässer sein, die ausschlleBlich der Schiffahrt und dem Transport von Abwässern vorbehalten sind. Die sonstigen Nutzungsarten verlangen zwischen diesen Extremen liegende Qualitätsstandards, wobel vor allem für die Nutzungen, bei denen der körperliche Kontakt mit dem Menschen groß ist (Baden, Freizeitaktivitäten) oder die der Nahrungsmittelproduktion dienen (Fischzucht, landwirtschaft11che Bewässerung, Nahrungsmittelindustrle) relativ strenge Qualitätsstandards festzulegen sind.

Eine regionale Differenzierung der Immissionsstandards ergibt sich bereits aus der unterschiedlichen Nutzung regional verteilter Gewässer. So kann eln See der Trinkwassergewinnung oder aber ausschließlich Bade-, Freizeit- und Erholungszwecken dienen, andere sind vor allem der Fischzucht vorbehalten; das Wasser der groBen Flüsse wird immer mehr zwecken der industriellen Produktion und der Schiffahrt dienstbar gemacht und manche Flüsse werden gar als Abwasserkanäle genutzt (Emscher). Problematisch wird elne regionale Differenzierung der Qualitätsanforderungen gemäß der unterschiedlichen Nutzungen dann, wenn sie sich auf das glelche Gewässer bezleht, etwa weil das Gewässer an verschiedenen stellen seines Laufes unterschiedllchen Nutzungsarten dient; dann müssen regional unterschiedliche - der jeweiligen Nutzungsart adäquate - Qualitätsanforderungen festgelegt werden. Das ist nur dann relativ unproblematisch, wenn die Reihenfolge der Nutzungen zwischen Quelle und Mundung so ist, daß die jeweils flußaufwärts liegende Nutzungsart die jeweils fluBabwärts folgende nicht gefährdet, also etwa das Wasser im Quellgebiet des Flusses der Trinkwassergewinnung dient, der dann folgende Oberlauf für Bade-, Freizeit-, Fischere1- und Bewässerungszwecke genutzt wird und erst anschließend bis zur Mündung wenlger Wasserqualitat erforderliche Nutzungsarten zugelassen werden 125). Ist eine solche Reihen-

125) Vgl. Elster, H.-J., Forderungen an die Reinhaltung unserer Binnengewässer aus biologischer sicht, a.a.0., s. 93 f. 
folge nicht gewährleistet, besteht die Gefahr, daß die Regionalisierung der Qualitätsstandards gemäß den Nutzungsarten dazu führt, daß flußabwärts liegende Regionen den Fluß z.B. zu Badezwecken nicht nutzen können, weil die Nutzung für den Abwassertransport flußaufwärts dies nicht mehr ohne Gesundheitsgefahren erlaubt 126)

Eine zeitliche Differenzierung der Qualitätsanforderungen ist lediglich in jahreszeitlicher Hinsicht denkbar; so könnte man In der kälteren Jahreszeit (also etwa von Oktober bis März) für organische Verschmutzungen der Gewässer höhere Immissionsstandards zulassen, da die Sauerstoffzehrung in der kälteren Jahreszeit, wegen der niedrigeren wassertemperatur, geringer ist.

\section{Immissionsstandards für den Boden}

Eine Operationalisierung der Bodenqualitätsziele in Form von Immissionsstandards ist äußerst schwierig. Zumindest die Umweltschutzliteratur hat sich nach unserer Kenntnis mit diesem Problem bisher kaum befaßt. Was die Bebauung des Bodens mit Siedlungen, Einzelgebäuden und Verkehrsbauten betrifft, könnte man die Vorschriften über den Standort und die Auflagen über das Aussehen derseiben ${ }^{127}$ ) als Immissionsstandards Im weitesten

126) Uber die praktischen Schwierigkeiten, die durch die regionale Differenzierung der Immissionsstandards infolge unterschiedlicher Nutzungen bei einem Gewässer entstehen können, vgl. Fox, I.K./Wible, L.F., Information Generation and Communication to Establish Environmental Quality Objectives, a.a.O., S. 143 .

1.27) Vgl. z.B. $\S 1$ (4) Bundesbaugesetz (BBauG), wonach sich die Bauleitpläne (Flächennutzungs- und Bebauungspläne) nach der Gesundhelt der Bevölkerung richten müssen. Durch ein Gesetz zur Änderung des BBaug vom 2.5.1975 (BGBI. I S. 1037) hat "die Bauleitplanung den Belangen des Natur- und Landschaftschutzes und der Gestaltung des Orts- und Landschaftsbildes zu dienen" ( $\S 1$ (5), Satz 1 BBaug). Im Bebauungsplan können u.a. Art und $\mathrm{MaB}$ der baulichen Gestaltung, Bauweise und Höhe der baulichen Anlagen festgelegt werden ( $\$ 9$ (1) BBauG). In der Baunutzungsverordnung (BauNvo) wird die Art der baulichen Nutzung in zehn Gruppen (Bauflächen und Baugebiete) unterteilt ( $\S 1$ BauNvo), und für jede Gruppe werden Vor- 
Sinne bezelchnen.

In ihrem Tourismus-Berlcht kündigt die Bundesregierung die Ermittlung von Belastungswerten und -grenzen für Urlaubs- und Freizeitgeblete an, und zwar besonders für Zelt- und Wohnwagenplätze 128)

Zum Schutz vor Bodenerosionen durch Oberflächenwasser kann je nach Bodenart die höchstzulässige Nelgung von Hängen festgelegt werden 129)

Die Abfallbeseitigungsvorschriften bezwecken auch einen Schutz des Bodens ${ }^{130}$ ) und können so als Bodenimmissionsstandards im weitesten sinne bezelchnet werden.

Uber die Höhe der Immissionsbelastung des Bodens mit Schadstoffen und Umweltchemikalien (Düngemittel und Pestizide) gibt es noch keine Vorschriften 131) - vielleicht deshalb, weil der Gesetzgeber glaubt, uber Grenzwertbestimmungen bei der Lebensmittelbelastung einen indirekten Einfluß auf die Bodenbelastung durch Umweltchemikalien und bestimmte Schadstoffe ausüben $z u$ können.

\section{Toleranzgrenzen der Lebensmittelbelastung}

Es müBte keine Vorschriften über die höchst zulässigen Konzentrationen von Schadstoffen in den Lebensmitteln geben, wenn in den Umweltbereichen Luft, Gewässer und Boden nur unschädliche Immissionskonzentrationen vorkommen würden. Da dies nicht der Fall ist und sich viele Schadstoffe via Nahrungskette in den als Nahrungsmittel dienenden Pflanzen und Tieren anlagern - man

schriften über das Maß der baulichen Nutzung erlassen ( $\S 16 \mathrm{ff}$. BauNvO).

128) Vgl. Tourismus in der Bundesrepublik Deutschland, a.a.0., S. 40 .

129) Vgl. Olschowy, G./Mrass, W./Kullmer, H.J./Bürger, K., Zur Belastung der Landschaft, a.a.0., S. 18 .

130) Vgl. $\S 2$, Satz 1, Ziffer 3 und Ziffer 5 sowie $\S 15$ (1) Abfallbeseitigungsgesetz ( $A b f G)$.

131) Nach $\S 15$ (2) AbfG kann u.a. die Aufbringung von Klärschlamm und Fäkalien auf landwirtschaftlich genutzte Böden beschränkt oder verboten werden. 
spricht von Rückständen -, müssen Höchstgrenzen für diese Schadstoffe in den Lebensmitteln festgelegt werden. In Tierversuchen wird zunächst die unwirksame Höchstdosis NEL (maximum No Effect Level) festgelegt, und zwar für den Fall lebenslanger Aufnahme durch das Tier 132). Daraus wird die höchste duldbare Tagesdosis für den Menschen, der ADI-Wert (Acceptable Daily Intake), unter Berücksichtigung eines Sicherheitsfaktor von 100 abgeleitet ${ }^{133}$ ). Die Dimension beträgt mg Schadstoff $/ \mathrm{kg}$ Körpergewicht pro Tag. Aus dem ADI-Wert erhält man unter Berücksichtigung der Verzehrgewohnheiten und des durchschnittlichen Körpergewichts des Menschen in Höhe von $70 \mathrm{~kg}$ die maximal duldbare Rückstandsmenge (permissible level) in $\mathrm{mg}$ Schadstoff $/ \mathrm{kg}$ Lebensmittel. Daraus hat der Gesetzgeber z.B. in der Bundesrepublik Deutschland die tatsächlich geduldete Rückstandskonzentration (permitted level), die sogenannte Toleranz abgeleitet, die oft weit unterhalb der maximal duldbaren Rückstandskonzentration liegt.

Wie die vorangegangenen Ausführungen zeigen, ist das Vorgehen bei der Festlegung von maximal zulässigen Rückstandskonzentrationen in Lebensmitteln regelrecht standardisiert. Das und die gesetzgeberische Tätigkeit ${ }^{134)}$ zeigen, daß diesem Bereich des

132) Vgl. dazu und $z u$ den folgenden Ausführungen Deutsche Gesellschaft für Ernährung e.v. , Ernährungsbericht 1972, a.a.0., S. 132 und RSU, Umweltgutachten 1974, a.a.O., S. $68 \mathrm{f}$.

133) Damit soll wohl nicht nur den Schwierigkeiten bel der Ubertragung der Untersuchungsergebnisse von Tierversuchen auf den Menschen Rechnung getragen werden (vgl. Aebi, H., Die heutige Belastung der Ernährung durch Fremdstoffe, in: Universitas, 27. Jg., 1972, S. 1188), sondern wohl auch anderen Risikofaktoren, wie der Tatsache, daß der Mensch den gleichen Schadstoff nicht nur via Nahrung sondern auch via Atemluft oder Trinkwasser aufnehmen kann (vgl. Korte, F., Rückstandsprobleme, in: Natur und Landschaft, 44. Jg., 1969 , S. 227 f.).

134) In der "Verordnung über Pflanzenschutz-, Schädlingsbekämpfungs- und Vorratsschutzmittel in oder auf Lebensmitteln pflanzlicher Herkunft", der sogenannten Höchstmengenverordnung in der Neufassung vom 5.6.73 (BGB1 I S . 536) und in der "Verordnung uber Höchstmengen an DDT und 
Umweltschutzes schon bisher große Aufmerksamkeit geschenkt wurde. Indem man allerdings erst das vor dem Menschen letzte Glied in der Umweltbelastungskette schützte (nämlich die Nahrungsmittel), duldete man alle davor liegenden Umweltbelastungen.

\section{Immissionsstandards für den Lärm}

Die Festlegung von Immissionsstandards für den Lärm ist deswegen schwierig, weil Lärm subjektiv unterschiedlich störend empfunden wird. Die physiologischen und psychischen Lärmwirkungen sind außerdem noch nicht endgültig erforscht 135) Die Konsequenz darf jedoch nicht sein, keine Immissionsstandards festzulegen. Vielmehr müssen "interim standards" 136) in Form von Immissionsichtwerten formuliert werden bis die Forschung genauere Grenzwerte liefern kann.

Da durch Lärm etwa ab $25 \mathrm{~dB}(\mathrm{~A})$ Belästigungen, also Störunđen des Wohlbefindens hervorgerufen werden können, kann man eine Lärmimmission unterhalb von $25 \mathrm{~dB}(\mathrm{~A})$ als noch unschädliche Immissionsbelastung bezeichnen. $\mathrm{Ab}$ etwa $85 \mathrm{~dB}(\mathrm{~A})$ ist dagegen Lärmschwerhörigkeit als akuter Gesundheitsschaden $z u$ befürchten 137) Zwischen diesen Grenzen wird ein Immissionsrichtwert mit Hilfe von Kosten-Nutzen-Uberlegungen festgelegt werden

anderen Pestiziden in oder auf Lebensmitteln tierischer Herkunft" vom 15.11.73 (BGBI.I S. 1710) sowie in den verschiedenen Verordnungen über Lebensmittelzusatzstoffe werden für über 100 Stoffe maximal zulässige Rückstandskonzentrationen festgelegt. Entwürfe über höchstzulässige Konzentrationen von Schwermetallen, (@uecksilber, Arsen, Blei, Cadmium) sind in der Diskussion (vgl. RSU, Umweltgutachten 1974, a.a.0., S. 266).

135) Vgl. RSU, Umweltgutachten 1974, a.a.0., S. 79; Jansen, G., Nachweis von Lärmwirkungen (zuglelch ein Beitrag zur Ermittlung von Grenzwerten), in: Zentralblatt für Bakteriologie...., a.a.0., s. $315 \mathrm{ff}$. und die Ausführungen von H. Hörmann bei der öffentlichen Anhörung vor dem InnenausschuB des Deutschen Bundestages, in: Umweltschutz (III), Zur Sache 4/73, a.a.0., S. 39 .

136) U.S. Department of Commerce, The Noise Around us, a.a.O., S. 19 .

137) Vgl. RSU, Umweltgutachten 1974, a.a.0., s. 79. 
müssen. Da Lärm bei unterschiedlichen Tätigkeiten an unterschiedlichen Einwirkungsorten und zu unterschiedlichen Zeiten unterschiedlich ist, empfiehlt sich eine Differenzierung der Immissionsrichtwerte nach Tätigkeitsbereichen bzw. -merkmalen, Einwirkungsorten und Tageszeiten sowie den Lärmarten. Die Tätigkeitsbereiche bzw. -merkmale kann man noch elnmal in solche im Wohn- und Freizeitbereich und solche im Arbeitsplatzbereich unterscheiden. Es läßt sich etwa folgende Einteilung denken ${ }^{138)}$ :

Tätigkeitsberelch im Wohn- und Freizeitbereich

Schlafräume $30-35 \mathrm{~dB}(\mathrm{~A})$

Wohn- und Aufenthaltsräume 40-45 dB (A)

Balkone, Gärten $55 \mathrm{~dB}(\mathrm{~A})$

Sportstätten $60 \mathrm{~dB}(\mathrm{~A})$

Tätigkeitsmerkmale am Arbeitsplatz

Routinetätigkeit $75 \mathrm{~dB}(\mathrm{~A})$

Tätigkeit mit sehr hohen Anforderungen an die Konzentration $65 \mathrm{~dB}(\mathrm{~A})$

geistig-schematische Tätigkeiten Kommunikation $55 \mathrm{~dB}(\mathrm{~A})$ sehr hohe geistige Beanspruchung $45 \mathrm{~dB}(\mathrm{~A})$

138) Vgl. Jud, S., Lärmimmissionsrichtwerte: Zumutbare Lärmgrenzen, in: Umwelt, 1975, 2, S. 32 ; Klosterkötter, W., Immissionsrichtwerte für Lärm aus psychologisch-physiologischer Sicht, in: Umwelt, 1973, 1, S. $30 \mathrm{f}$. und die Immissionsrichtwerte nach der VDI-Richtilnie 2719, abgedruckt in: RSU, Umweltgutachten 1974, a.a.0., s. 80. 
Die Technische Anleitung zum Schutz gegen Lärm (TA Lärm) unterteilt in sechs Einwirkungsorte und zeitlich in Tag- und Nachtwerte:

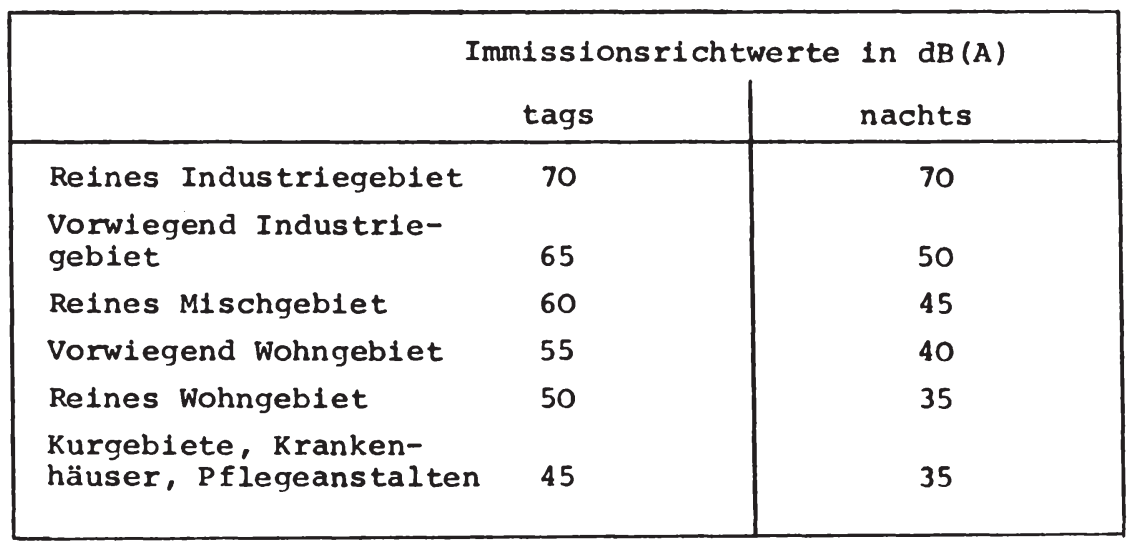

Zum Schutz vor Verkehrslärm sieht das Gesetz zum Schutz gegen Fluglärm zwe1 Lärmschutzzonen vor; in der ersten darf der Immissionsrichtwert $75 \mathrm{~dB}(\mathrm{~A})$ und in der zweiten $67 \mathrm{~dB}(\mathrm{~A})$ nicht uberschreiten 140) Der Entwurf für eine Verordnung zur Eindämmung des Verkehrslärms (Straßen-Schallschutzverordnung) gemäB $\S 43,1$ BImschG sleht vor, daß tagsüber 60 bis 75 dB(A) und nachts 50 bis $65 \mathrm{~dB}(\mathrm{~A})$ nicht überschritten werden dürfen

139) Vgl. TA Lärm vom 16.7.68, in: Bellage zum Bundesanzeiger Nr. 137 vom 26.7.1968, Ziffer 321. Die TA Lärm bezieht sich auf Gewerbelärm. Diese Vorschriften sind sehr differenziert. Es ergeben sich zwölf verschiedene Immissionsrichtwerte. Nach einem Urteil des Verwaltungsgerichts Berlin (AZ: VG XIII A $112 / 72$ vom 21.3.73) müssen gar noch $\mathrm{Zwischenwerte}$ festgelegt werden, wenn in einem Wohngebiet der zulässige Immissionsrichtwert deshalb uberschritten wird, weil es neben einem Gewerbegeblet mit einem höheren zulässigen Lärmpegel liegt (vgl. die auszugswelse Wiedergabe des Urte1ls in: Umwelt, 1973, 5, S. 68).

140) Vgl. $\S 2$ (2) des Gesetzes zum Schutz gegen Fluglărm vom $30.3 .71, \mathrm{BGBl}$. I, S. 282 .

141) Vgl. Landesverband Bürgerinitiative Umweltschutz Nordrhein-Westfalen e.V., Weniger Geld - mehr Lärm, a.a.o., S. 40 . 
Für reine Wohngebiete liegen die vorgeschlagenen Werte tags allerdings um mindestens $10 \mathrm{~dB}(\mathrm{~A})$ und nachts um mindestens $15 \mathrm{~dB}(\mathrm{~A})$ über den Immissionsrichtwerten der TA-Lärm; sie lassen sich nur als "ökonomisch begründeter Vorschlag zur Begrenzung von Entschädigungsverpflichtungen" 142) des staates verstehen. Aus gesundheitlichen Gründen sind sie u.E. nicht zu rechtfertigen.

In diesem Kapitel haben wir versucht zu zeigen, wie operationale ziele des Umweltschutzes bestimmt werden können (und auch bereits wurden). Aufgrund der operationalen ziele in Form der Immissionsstandards sowie unter Berücksichtigung der Diagnose und Prognose der Umweltsituation, mit denen wir uns im I. Kapitel befaBt haben, kann der Umweltschutz zielorientiert geplant werden. Mit den Grundlagen und einigen Möglichkeiten der Planung des Umweltschutzes werden wir uns im folgenden Kapitel beschäftigen.

142) RSU, Umweltgutachten 1974, a.a.0., S. 82; vgl. auch FuBnote 101 dieses Kapitels. 


\begin{abstract}
"Bei allem, was geschieht, um ein ziel zu erreichen, bei dem man dazu verschiedene Wege einschlagen kann, ist es notwendig, gerade jenen Weg zu bestimmen, durch den man ohne Umweg das verlangte ziel errelcht".

(Thomas von Aquin, Uber die Herrschaft der Fursten)
\end{abstract}

III. Grundzuge einer rationalen Planung des Umweltschutzes

A. Forderungen an elne rationale Umweltschutzplanung

Die Umweltschutzplanung gehört in den Bereich der Aufgabenplanung 1) Sie besteht in der Planung von MaBnahmen des Umweltschutzes, die zur Elnhaltung der Immissionsstandards erforderlich sind. Die Planung der UmweltschutzmaBnahmen ist also zielorientiert ${ }^{2}$; sie muB daruber hinaus unter Beachtung des ökonomischen Prinzips erfolgen, damit sie auch das Rationalltătskriterium erfullt.

Auf den ersten Blick scheint es sich hier um ein "technokratisch-dezision 1 stisches Planungskonzept" ${ }^{3)} \mathrm{zu}$ handeln, bei dem die ziele allein politisch (und exogen) determiniert sind und

1) Inwieweit der Umweltschutz eine offentliche Aufgabe ist, werden wir im nächsten Kapitel untersuchen.

2) Vgl. Thoss, R., Zur Planung des Umweltschutzes, in: Raumforschung und Raumordnung, 30. Jg., 1973, S. 190; derselbe, Ziel, Ansatz und Methoden eines integrierten Optimierungsmodelis zur Verbesserung des Umweltschutzes, in: Seminarbericht Nr. 8 der Geselischaft für Regionalforschung, Münster 1974, S. 3; Hahn, H.H., Umweltplanung. Gemeinsame Aufgabe von Wissenschaftler, Ingenleur und Politiker, in: Umwelt, 1971, 1, S. 18; Roth, Chr.., Programe aber keine Pläne. Zum Planungsbegriff am Beispiel Umweltproblematik, In: Umwelt, $1972,4,5.14$.

3) Wille, E., Mittel- und langfristige Finanzplanung, in: F. Neumark (Hrsg.), Handbuch der Finanzwissenschaft, 3. Auflage, Bd. 1, Tübingen 1976 , S. 457. 
die Planungs-Technokraten ausschließl1ch die optimale Lösung zu suchen haben. Vergegenwärtigen wir uns jedoch das Vorgehen bel der Able1tung der Immissionsstandards, so stellen wir fest, das sie zwar letztlich aufgrund politischer Entscheidungen festgelegt werden, diese $\mathrm{zielsetzungsentscheldungen} \mathrm{aber} \mathrm{auf}$ Informationen aus der realen (natürlichen und sozioökonomischen) Umwelt, Expertenbefragungen und Bürgeranhörungen beruhen, da $\beta$ sie also durchaus in dem sinne rückgekoppelt sind, das sie revidiert werden können, wenn sich die Umweltverhăltnisse im weltesten Sinne ändern. Tatsächlich handelt es sich also bel unserem formal getrennten Ansatz zwischen zlelbestimmung und Umweltschutzplanung um ein integriertes Planungskonzept 4) wie es auch (u.a. vom RSU) gefordert wird 5) Wegen der Komplexität der Umweltprobleme und der Vielzahl der betroffenen Disziplinen kann man unterschiedliche Konsequenzen hinsichtlich der Umweltschutzplanung ziehen. Einerseits kann man vor den Schwlerigkeiten kapitulieren, die Komplexität und Multidisziplinarităt der Umweltprobleme bel der Planung des Umweltschutzes deshalb hervorrufen, well eine allen Umweltproblemen gerecht werdende Umweltschutzplanung ebenso komplex und möglichst interdisziplinär ${ }^{6)}$ sein soll. Die Folge wäre notwendigerweise eine Umweltschutzpolitik des "muddling through" 7 ? Auch diese kann rational sein, wenn nămlich die Kosten der sicher komplizierten Umweltschutzplanung größer wăren als deren Nutzen. Andererselts müste man - würde man von den planungs-

4) Vgl. Wille, E., Mittel- und langfristige Finanzplanung, a.a.0., S. $459 \mathrm{f}$.

5) Vgl. RSU, Umweltgutachten 1974, a.a.0., S. 9; Buchwald, K., Umweltschutz und Gesellschaft, in: Naturschutz- und Naturparke, Bd. 60, 1, 1971, S. 12 .

6) Vgl. Hahn, H.H., Umweltplanung. Gemeinsame Aufgabe von Wissenschaftler, Ingenleur und Politiker, a.a.0., S. 19 ; Genscher, H.-D.. Umwelt als Gesamtheit sehen, in: U, 1973, 2 , S. $8 \mathrm{f}$.

7) Vgl. Hansmeyer, K.-H., Volkswirtschaftliche Kosten des Umweltschutzes, a.a.0., S. 101; RSU, Umweltgutachten 1974, a.a.0., s. 166 . 
technischen Schwierigkeiten und den Kosten der Umweltschutzplanung absehen - eine "comprehensive planning" fordern 8), denn nur eine solche kann allen interdependenten (interregionalen, intermediären) und interdisziplinăren Umweltproblemen gerecht werden, indem sie z.B. Teilplanungen für elnzelne Umweltmedien oder -regionen, deren Verwirklichung zu negativen Einflussen in anderen Umweltmedien oder - regionen fuhren würde 9), ausschließt. Die planungstechnischen Schwierigkelten und die Kosten des "comprehenslve approach" sind jedoch so groß, daß er für die Praxis der Umweltschutzplanung nicht in Frage kommt 10)

Da aber "ohne Umweltplanung wirksamer Umweltschutz langfristiq nicht denkbar (1st)" 11) kann die Alternative zum "comprehensive approach" nicht die Kapitulation - also keine Planung - sein, sondern nur elne möglichst umfassende Umweltschutzplanung auf einer systemtheoretischen Basis 12), die ihre Grenze dort haben muB, wo ihre Grenzertrăge beginnen, von ihren Grenzkosten uberkompensiert $z u$ werden. Die Umweltschutzplanung kann auch durchaus heute noch mehr oder weniger utopisch erscheinende Maßnahmen in thre Uberlegungen einbeziehen 13)

Mit der Forderung nach elner möglichst umfassenden planung des

8) Vgl. Dror, Y., Comprehensive Planning: Common Fallacies versus Preferred Features, in: F. von Schagen (Hrsg.), Essays in Honour of Professor Jac.P. Th1jsse, Paris 1967, S. $85 \mathrm{ff}$.

9) Vgl. Maldonado, T., Umwelt und Revolte. Zur Dialektik des Entwerfens im Spätkapitalismus, Reinbek 1972, S. 46 ; Vester, F.. Der Weg des vorausschauenden Steuerns. Prinzip und Bedeutung kybernetischer Technologien, In: Umwelt, 1974, 1, S. 11 .

10) Vgl. Dror, Y., Comprehensive Planning: Common Fallacies versus Preferred Features, a.a.0., S. $86 \mathrm{f}$.

11) Strelbl, M.. Umweltpolitik in der Bundesrepublik Deutschland, In: Horn, Chr./von Walterskirchen, M.P./Wolff, I. (Hrsq.), Umweltpolitik in Europa, a.a.0., S. 235.

12) Solche systemtheoretischen Ansatze stellen z.B. die Projekte UMPLIS und USCHI dar; vgl. dazu Kitschler, W. , Aufbau eines Informationssystems für Umweltplanung, in: Aligemeines Statistisches Arch1v, Bd. 59, 1975, S. 25 ff. und Günther, U. U.a., Umweltschutz-Informations - und steuerungssystem, a.a.0.

13) Vgl. Roth, Chr., Programme aber kelne Pläne, a.a.0., s. 14. 
Umweltschutzes und der Mitberilcks1cht1gung futuristischer Maßnahmen des Umweltschutzes ist keineswegs die Notwendigkeit verknüpft, die slch als optimal erwelsenden Maßnahmen sofort und in vollem Umfang durchzufuhren, was wegen der nur begrenzt zur Verfugung stehenden realen und finanziellen Ressourcen wohl auch gar nicht oder nur unter Inkaufnahme großer Friktionen mög$11 \mathrm{ch}$ wăre. Die Durchsetzung der geplanten Umweltschutzmaßnahmen kann durchaus inkremental erfolgen 14); sle 1st kaum ein planerisches, vielmehr ein juristisches und vor allem finanzielles Problem 15) Die 1deale Lösung lautet unseres Erachtens: mögl1chst umfassend (systemtheoretisch) planen - Inkremental durchsetzen ${ }^{16}$ ) Daraus ergeben sich zwel weltere Forderungen an elne rationale Planung des Umweltschutzes: Wenn die notwendigen UmweltschutzmaBnahmen nicht kurzfristig (und wahrscheinlich auch nicht mittelfristig) durchsetzbar sind, muß die Umweltschutzplanung langfristig sein 17), und es müssen zeltliche Prioritäten in bezug auf die Durchsetzung der verschiedenen geplanten Umweltschutzmaßnahmen festgesetzt werden; denn inkrementale Durchsetzung bedeutet zeltliche streckung und Vertellung der durchzuführenden Umweltschutzprojekte. Bel der Festlegung der Prioritäten kann man von einem Vergleich der Immissionsstandards (Sollwerte) mit den tatsächlichen Immissionskonzentrationen (Istwerte) der jewelligen Schadstoffe ausgehen, die man aus den Immissionskatastern erhält. Dort wo die tatsächlichen Immissionskonzentrationen prozentual am weltesten über den Immissionsstandards liegen, ist die Durchsetzung der geplanten Umweltschutzmaßnahmen am dringlichsten; hierbel handelt es sich sowohl un Maßnahmen der Umweltsanierung als auch des vorbeugenden Umweltschutzes. Danach sind MaBnahmen zu ergreifen, die

14) Vgl. RSU, Umweltgutachten 1974, a.a.0., S. 5.

15) Vgl. Roth, Chr., Programme aber keine Pläne, a.a.0., S. 15.

16) Ähnlich vgl. Wille, E., Mittel- und langfristige Finanzplanung, a.a.0., S. 474 .

17) Vgl. Picht, G., Umweltschutz und Politik, a.a.0., s. 82; RSU, Umweltgutachten 1974, a.a.0., s. 9 . 
Immissionskonzentrationen verringern, die näher an die Immissionsstandards herankommen.

\section{B. Die Maßnahmen des Umweltschutzes}

1. Zur Systematisierung der UmweltschutzmaBnahmen

$\mathrm{Zu}$ Beginn des I. Kapitels hoben wir bereits die Bedeutung umweltrelevanter Informationen fur eine rationale Planung des Umweltschutzes hervor. Dort hatten wir allerdings Informationen uber Art und Umfang der Schadstoffbelastung, Herkunft der Schadstoffe, thre Wirkungen und die Ursachen der Umweltbelastung Im Auge, also Informationen diagnostischer (und prognostischer) Art. Von ebenso großer Bedeutung für eine rationale planung des Umweltschutzes sind Informationen über die Möglichkelten der Umwelttherapie, also die Maßnahmen des Umweltschutzes. Nur wenn den Planern des Umweltschutzes alle zum Schutz der Umwelt verfügbaren Maßnahmen bekannt sind, können sle den Umweltschutz optimal planen.

Die "box of tools" des Umweltschutzes ist äuBerst heterogen und wird von vielen Disziplinen "bestückt", weshalb wohl nur ein multidisziplinăr zusammengesetztes Team, das interdisziplinăr zusammenarbeltet, optimal planen kann.

Bevor wir die Maßnahmen des Umweltschutzes darstellen, wollen wir sie systematisieren:

Da sind zunăchst die Maßnahmen, die uberhaupt erst die Grundlagen dafür schaffen, daB Umweltschutz betrieben werden kann, nämlich die Forderung der Umweltforschung und die Ausbildung von Umwelt(schutz) experten jeglicher Art.

Dann gibt es Maßnahmen, die ergriffen werden können, um die von uns als nicht primär bezelchneten Ursachen der Umweltbelastuna, also Bevölkerungswachstum, ungezügeltes produktionswachstum, die Konsummentalitat des Ex und Hopp und den technischen Fortschritt zu verändern. Dies sind: Geburtenkontrolle, die Schaffung elnes (neuen) UmweltbewuBtseins und die Kontrolle des technischen Fortschritts.

Nach diesen nicht-technischen Maßnahmen des Umweltschutzes 
wollen wir die Bedeutung der Raumordnung für den Umweltschutz untersuchen.

Im Mittelpunkt stehen jedoch die technischen, juristischen und Ökonomischen Maßnahmen des Umweltschutzes, well wohl nur mit ihnen die elgentliche Ursache der Umweltbelastung, nämlich die nicht erfolgte oder nur ungenugende Internalisierung der Umweltexternalităten, beseltigt werden kann, ohne daß auf wachsenden wohlstand verzichtet werden muB, wenn es auch sicher ein qualitativ anderer Wohlstand 1st, der nach erfolgrelcher Internal1slerung der externen Effekte errelcht werden wird. In diesem Kapitel wollen wir uns aus dieser vierten Maßnahmengruppe nur mit den technischen Masnahmen befassen; die juristischen und okonomischen Maßnahmen schildern wir - bedeutungshalber - Im Kapltel über die Durchsetzung der geplanten Umweltschutzmaßnahmen.

2. Darstellung der Umweltschutzmaßnahmen

a) Nicht-technische Maßnahmen des Umweltschutzes

aa) Die Förderung der Umweltforschung

Die Umweltforschung 1st die wohl wichtigste Voraussetzung einer rationalen Planung des Umweltschutzes ${ }^{18)}$, da allein die Umweltforschung einerseits Informationen uber Ursachen, Verbreitung und Wirkungen der Umweltbelastung liefern und anderérselts auch Möglichkelten der Beseltigung, Reduzlerung und Vermeldung der Umweltbelastungen aufzeigen kann.

Umweltforschung ist also zunächst elnmal "diejenige Forschung, die sich mit den Lebensbedingungen des Menschen und deren Veränderungen durch menschliche Eingriffe befaßt" 19) Darluber

18) Vgl. UNESCO, Use and Conservation of the Blosphere. Proceedings of the Intergovernmental Conference of Experts on the Scientiflc Basis for Rational Use and Conservation of the Resources of the Blosphere, Paris 4.-13. Sept. 1968, Paris 1970, S. $198 \mathrm{ff.;}$ Forschungsbericht IV, BT-Drucksache $\mathrm{VI} / 3251, \mathrm{Z} .278$.

19) Speer, J., Zur Definition, Systematik und Organisation der Umweltforschung, in: DFG-Mitteilungen 4/73, S. 4; vgl. auch Scholder, K., Zur Definition und Systematislerung der Umweltforschung, in: Umwelt, $1972,6,5.36$. 
hinaus ist es aber auch Aufgabe der Umweltforschung, umweltneutrale Technologien $z u$ erforschen und die MeBtechnik weiterzuentwickeln; ferner muB sie sich um die Erforschung sozialökonomischer Probleme der Umweltbelastung und des Umweltschutzes bemühen. Der Systematisierung der Deutschen Forschungsgemeinschaft (DFG) folgend wollen wir die Umweltforschung in eine funktionale, prohibitive, systemale und normative Umweltforschung unterteilen 20). Die funktionale Umweltforschung befast sich mit intakten okosystemen, um ihr Funktionieren $z u$ erforschen. Die prohibitive Umweltforschung widmet sich zunächst den Möglichkeiten des exakten wissenschaftlichen Nachweises von Schadstoffwirkungen auf Mensch und Umwelt und ist insoweit - ebenso wie die funktionale Orosystemforschung - Grundlagenforschung 21). Die Grundlagenforschung ist allgemein der "Humusboden" für die angewandte Forschung 22), und das gilt auch für die Umweltforschung, denn die zweite Komponente der prohibitiven Umweltforschung, die Erforschung von Möglichkeiten zur Begrenzung der Umweltbelastung durch umweltneutrale Produktionsverfahren und Produkte, baut als angewandte Forschung auf den Erkenntnissen der ökologischen und humanökologischen Grundlagenforschung auf. Die prohibitive Umweltforschung hat wohl die größte Bedeutung für die aktuelle Umweltpolitik, denn sle liefert sowohl die Informationen für die Formulierung der ziele als auch die technischen Maßnahmen zur Erreichung der ziele ${ }^{23}$ ). Die

20) Vgl. hierzu und $z u$ den folgenden Ausführungen die belden in Fußnote 19) angegebenen Beiträge.

21) Vgl. Finke, w., Umweltforschung - eine multi- und interdisziplinăre Aufgabe, in: Schultze, H. (Hrsg.), Umwelt-Report, a.a.0., S. $64 \mathrm{f}$.

22) Vgl. Forschungsbericht IV, a.a.0., s. 27.

23) Vgl. Environmental Pollution. A Challenge to Science and Technology. Report of the Subcommittee on Science, Research, and Development to the Committee on Science and Astronautics, U.S.G.P.O., Washington, D.C. 1966, S. 6; 0.V., Mehrjähriqe Forschungsprogramme der Gemeinschaft: Vorschläge der Kommission, in: Bulletin der EG, 8. Jg., 1975, H. 7/8, S. 19; Aktionsprograme für die Politik im wissenschaftlich-technologischen Bereich, Beilage $14 / 73$ des Bulletin der EG, S. $45 \mathrm{ff}$. 
systemale Umweltforschung versucht mit systemanalytischen Methoden zukunftsorientierte synthetische Gesamtmodelle ähnlich denen von Forrester und Meadows zu entwickeln. Die normative Umweltforschung hat dagegen $z u$ erforschen, was sein soll (z.B. Erforschung sozlaler Indikatoren und dessen, was Lebensqualităt sein könnte) und wie das Sein-Sollende durchzusetzen 1st (z.B. Erforschung neuer Internalisierungsstrategien).

Die Ausführungen im I. Kapitel haben gezeigt, daß die Umweltforschung berelts respektable Erfolge vorweisen kann, was sicher eine Folge der umfangrelchen und vielseitig geförderten Aktivitäten der Umweltforschung ist 24) Das gilt vor allem für die angewandte Umweltforschung - deren Erfolge auf meßtechnischem Geblet wir bereits kennengelernt haben 25) und deren erstaunliche Ergebnisse wir auf dem Geblet des technischen Umweltschutzes noch erfahren werden 26) - aber in weltaus geringerem Umfang für die bkosystemforschung und die humanökologische Forschung, also die Grundlagenforschung. Viele 8ko-

24) Vgl. 2.B. Die Forderungsaktivităten der DFG auf dem Gebiet der Umweltforschung, in: DFG-Mittellungen 2/74, S. 5 ff. und $2 / 73$, S. 6 f. sowle den vom Umweltbundesamt herausgegebenen Umweltforschungskatalog '75, Berlin 1975, der eine Fülle laufender und abgeschlossener Forschungsprojekte enthält. Auch an Anregungen für weltere Forschungen fehlt es nicht: vgl. UNESCO, Use and Conservation of the Biosphere, a.a.0., S. $209 \mathrm{ff}$., wo sehr detalllierte Empfehlungen für Umweltforschungen gegeben werden, ebenso wie in U.S. Department of Health, Education and Welfare. Man's Health and the Environment. Some Research Needs, Washington, D.C. 1970, S. $11 \mathrm{ff.} \mathrm{Zur} \mathrm{internationalen} \mathrm{interdisziplinären} \mathrm{Um-}$ weltforschung anregen will SCOPE; vgl. dazu: Graßhoff, K./ Meyl, A.H., Geschichte und Aufgaben des "Scientific Committee on Problems of the Environment" (SCOPE), in: DFG-M1ttellungen 2/73, S. 69. Vgl. ferner: Research into Environmental Pollution. Report of Five WHO Scientific Groups, WHO Technlcal Report Series No. 406, Genf 1968, worin ein Uberblick über den stand und die offenen Fragen der Umweltforschung gegeben wird.

25) Siehe vor allem FuBnote 13 des I. Kapitels.

26) Siehe unten S. $240 \mathrm{ff}$. 
logische $\mathrm{Z}$ usammenhänge sind noch unerforscht, und die wirkungen vieler Schadstoffe auf den Menschen und seine natürliche Umwelt sind noch weitgehend unbekannt ${ }^{27}$, oder es existieren bestenfalls Vermutungen oder Spekulationen über thre Schädlchkeit. Als besonderer Mangel wird die fehlende Kenntnis über Kombinations- und Langzeitwirkungen empfunden denn diesen kann wahrscheinlich bei der Ableitung von einzelnen Immissionsstandards nicht Rechnung getragen werden. Vor allem hier könnten nähere Kenntnisse einen großen Beitrag zur rationalen Umweltpolitik leisten. Die Förderung der ökologischen und humanökologischen Grundlagenforschung und die Weiterentwicklung ihrer Methoden (vor allem der Epidemiologie 29), gehört deshalb zu den wichtigsten Maßnahmen des Umweltschutzes 30 ?

Aber auch die angewandte Umweltforschung bedarf der (welteren) Förderung 31) denn es 1st zwar auf dem Gebiet des Umweltschutzes technisch sehr vieles möglich, aber - aus Kostengründen - durch-

27) Vgl. RSU, Umweltgutachten 1974, a.a.0., S. 117 f.; Congress and the Nation's Environment. Environmental Affairs of the 92st Congress, U.S.G.P.O., Washington, D.C. 1971, S. 7.

28) Vgl. Bär, F., Die toxikologische situation in der modernen Zivilisation, a.a.0., S. 191; Stelte, W., Die Kontamination von Lebensmitteln mit schädlichen Stoffen aus der Umwelt, a.a.O., S. 66; Henschler, D., Versuch einer Zuordnung von chemischen Noxen und Schäden beim Menschen, a.a.O., S. 59; Forschungsber1cht IV, a.a.0., Z. 76 und 278; U.S. Department of Health, Education and Welfare, Man's Health and the Environment, a.a.0., S. $15 \mathrm{f}$.

29) Vgl. U.S. Department of Health, Education and Welfare, Man's Health and the Environment, a.a.O., S. $14 \mathrm{ff}$.; Schaefer, H., Der Mensch in der technisch veränderten welt, a.a.o., s. 35 und S. 43; Dubos, R., Promises and Hazards of Man's Adaptability, a.a.0., s. 35 .

30) Vgl. Forschungsbericht IV, a.a.0., z. 79.

31) Vgl. ebenda, 2. 80 und 81; Hartkopf, G., Umweltpolitik aus der Sicht der Bundesreglerung, in: Glagow, M. (Hrsg.), Umweltgefährdung und Gesellschaftssystem, a.a.0., S. 185; Die Energiepolitik der Bundesreglerung, a.a.0., Z. 45 und Z. 75, 76,78 . 
aus nicht alles realisierbar ${ }^{32}$ ? Aufgabe der angewandten Umweltforschung ist also primär die Erforschung kostengünstiger(er) Umweltschutztechnologien.

bb) Ausbildung von Umwelt(schutz) fachleuten Neben der Umweltforschung ist die Ausbildung von Fachkräften des Umweltschutzes eine weltere wichtige Voraussetzung für eine rationale Umweltpolitik. Das gilt vor allem für die Bereiche der Umwelttechnik und der Umweltkontrolle. Der mengenmäBige Bedarf an "Umweltschützern" ist in den Industriestaaten sowohl im öffentlichen Bereich als auch in der Privatwirtschaft in den letzten Jahren sprunghaft angestiegen 33 ! . Er konnte zunächst durch traditionell ausgebildete Fachkräfte ohne spezifische Umwelt(schutz)kenntnisse gedeckt werden. Je umfangreicher und differenzierter die Umweltschutzanforderungen aufgrund der einsetzenden Welle von Umweltschutzgesetzen und je komplizierter die Umweltschutztechniken jedoch werden, um so nachteiliger machen sich die mangelnden Umweltschutzkenntnisse, vor allem der Ingenieure, bemerkbar.

Ihr Ausbildungsstand wurde teilweise zum Engpaßfaktor bei der Realisierung eines wirksamen Umweltschutzes ${ }^{34}$ ! Es wurde des-

32) Vgl. U.S. Department of Commerce, The Noise Around Us, a.a.0., S. 19; Coenen, R. U.a., Alternativen zur Umweltmisere, a.a.0., s. 157.

33) Vgl. Manpower and Training Needs for Air Pollution Control. Report of the Secretary of Health, Education and Welfare to the Congress of the United States, U.S.G.P.O., Washington, D.C. 1970, Böhnke, B., Volkswirtschaftlicher Aufwand für die Wasserversorgung sowie für die Beseltigung flissiger und fester Abfalistoffe bis zum Jahre 2000, in: Umweltschutz - eine Aufgabe unserer Zeit, hrsg. v. Institut für Gewerbliche Wasserwirtschaft und Luftreinhaltung e.V., Köln 1971, S. $91 \mathrm{ff.;}$ U.S. Council on Environmental Quality, Environmental Quality, The First Annual Report, a.a.o., S. 227 ff.

34) Vgl. Environmental Pollution. A Challenge to Science and Technology, a.a.0., S. 6; U.S. Department of Commerce, The No1se Around Us, a.a.O., s. 19; Böhnke, B., Volkswirtschaftlicher Aufwand für die Wasserversorgung ..., a.a.0., s. 93; RSU, Umweltgutachten 1974, a.a.0., S. 51. 
halb immer mehr die Förderung einer umwelt(schutz)bezogenen Ausbildung vor allem der Ingenteure gefordert ${ }^{35}$ ). Eine Umfrage zeigte, daß bei bestimmten Wirtschaftszweigen und bei der öffentlichen Verwaltung auch tat'sächlich ein Bedarf an Umweltfachleuten besteht. In dieser Umfrage wurde u.a. ermittelt, daß z.B. bei der Mineraloelindustrie von Hochschulabsolventen, die sich dem betrieblichen Umweltschutz zuwenden wollten, folgende umweltrelevanten Kenntnisse erwartet wurden ${ }^{36}$ ?

\begin{tabular}{|c|c|}
\hline Luftreinhaltung & $13,8 \%$ \\
\hline Müll- und Abfallbeseltigung & 12,28 \\
\hline Wasserreinhaltung & 12,28 \\
\hline Lärmminderung & 11,48 \\
\hline Umweltrecht und Umweltpolitik & 11,48 \\
\hline Umweltfreundliche Technologien & 7,38 \\
\hline Umweltfreundliche Energien & 6,58 \\
\hline EDV & 5,78 \\
\hline Umweltökonomie & 4,98 \\
\hline Sonstige Kenntnisse (jeweils unter 48 ) & 14,68 \\
\hline
\end{tabular}

35) Die Forderung nach der Ausbildung von "environmental health engineers" wurde bereits 1967 von der WHO erhoben; vgl. The Education of Engineers in Environmental Health. Report of a WHO Expert Committee, WHO-Technical Report Series No. 376, Genf 1967, S. 7 ff.

36) Vgl. Kenner, E., Wie soll der Umweltfachmann aussehen?, in: Umwelt, 1976,3, S. 228. 
Es stellt sich nun die entscheidende Frage, ob ein neuer Ausbildungsgang geschaffen werden sollte, der dem studierenden eln breltes Wissen über alle umweltrelevanten Fragen vermittelt, um ihn zu einer Art "Diplom-Umweltschützer" 37) zu machen, oder ob man den traditionellen Ausbildungsgang des Ingenieurs beibehalten und nur um eine ökologische und humanökologische Komponente ergänzen soll. Den ersten Weg scheint man inzwischen z.B. in Belgien zu gehen, wo man dem "Ingenieur en Pollution et Environnement" eine "breite Ausbildung" 38) angedeihen lassen wi11. In der Bundesrepublik Deutschland wird dieser Weg weitgehend abgelehnt, well man wohl einerseits befürchtet, auf diese Weise nur eine "Ausbildung von Alleswissern und Nichtskönnern" 39) $z u$ erreichen und andererseits die Gefahr sieht, daß das Berufsbild des Ingenteurs Schaden erleidet, wenn es zwei diametral entgegengesetzte Ausbildungsgänge gibt: den des - etwas überspitzt formuliert - "hehren" Umweltschüzers auf der einen Seite, auf der anderen Selte den des traditionellen Ingenieurs, der als "Handlanger" wirtschaftlicher Interessen die umweltfeindlichen Techniken baut und betreibt, wobei sich belde gar noch "einander in die Hand arbeiten" 40)

Den VDI haben aber wohl weniger berufspolitische Interessen als vielmehr die Befurchtung, daß breit ausgebildete Umwelt-Ingenieure für viele Spezialaufgaben auf dem Gebiet des Umweltschutzes überfordert sind, zu dem Schluß kommen lassen, daß den Anforderungen an eine umweltproblembezogene Ausbildung am besten dadurch entsprochen werden kann, daB man das Fachstudium des Ingenleurs in ökologischer und humanökologischer Hinsicht er-

37) Vgl. Best, W., Umweltschutz aus der sicht der Länder unter besonderer Berücksichtigung des Landes Hessen, in: Umweltschutz - eine Aufgabe unserer Ze1t, a.a.0., S. 25.

38) Delmon, B., Umweltingenieure von der belgischen Universität Löwen, in: Umwelt 1973, 6, S. 44.

39) Claussen, E., Gesundheitsingenteur - ein Schmalspurmediziner?, in: U 1973, 1, S. 21.

40) Heinemann, J., Zwelerlei Ingenleure?, In: Umwelt, 1972, 5, S. 16 . 
gänzt 41) Auch die Industrie will wohl den Ingenieur traditionellen Typs belbehalten, befürwortet aber ein umweltbezogenes Aufbau- und Kontaktstudium für mit Umweltschutzproblemen beauftragte Naturwissenschaftler und Ingenieure 42) Was für deren Ausbildung gilt, kann man auch auf andere Diszilinen übertragen. Vor allem in der medizinischen, biologischen, juristischen, ökonomischen und pädagogischen Hochschulausbildung sind Aspekte des Umweltschutzes $z u$ berücksichtigen, ohne daB dabei die spezielle Disziplin "verwässert" wird.

\section{cc) Geburtenkontrolle}

Diejenigen, welche die Umweltbelastung u.a. auf das Bevölkerungswachstum zurückführen, fordern konsequenterweise als wichtige Maßnahme zum Schutz der Umwelt die Begrenzung des Bevölkerungswachstums durch Geburtenkontrolle 43)

Dies ist U.E. eine Maßnahme des Umweltschutzes, die bestenfalls in Entwicklungsländern Anwendung finden solite 44), da dort weder die geeigneten Arbeitskräfte noch die ausreichenden finanziellen Mittel vorhanden sind, um einen technischen Umweltschutz zu betreiben, wie ihn sich die hochindustrialisierten Länder lelsten können.

41) Vgl. VDI-Aktionszentrum Technik und Umwelt, Umweltstudium ist abzulehnen, in: Umwelt, 1973, 6, S. 45.

42) Vgl. Kenner, E., Wie soll der Umweltfachmann aussehen?, a.a.0., S. 230. Diese Anregung hat wohl die Fachhochschule Lübeck aufgegriffen, wo graduierte Ingenieure ein dreisemestriges zusatzstudium "Technisches GesundheitswesenStudiengang Umwelt- und Hygienetechnik" absolvieren und sich nach AbschluB - verkürzt - Gesundheltsingenieure nennen können; vgl. Ohrenschall, A., Umweltfachmann durch Zusatzstudium, in: U $1976,4, \mathrm{~S} .14 \mathrm{f}$.

43) Vgl. Hardin, G., Die Tragik der Allmende, a.a.0., S. 48; Forrester, J.W., Der teuflische Regelkreis, a.a.O., S. 105; Meadows, D. U.a., Die Grenzen des Wachstums, a.a.0., S. 143 ff.

44) In vielen hochindustrialisierten Ländern - zumindest der westlichen Hemisphäre - hat sich das Bevölkerungswachstum in den letzten Jahren so stark abgeschwächt, das es als "Ursache" der Umweltbelastung sowieso nicht mehr in Frage kommt; vgl. 
dd) Die Schaffung eines (neuen) Umweltbewußtseins Wenn jeder einzelne staatsbürger - sei es als Produzent oder Konsument - das "richtige" Umweltbewußtsein hätte, gäbe es das Umweltproblem nicht, denn "richtig" ist das Umweltbewußtsein, das $z \mathrm{u}$ keinen umweltbelastenden Aktivitäten der Produzenten und Konsumenten führt. Diese tautologische Definition hilft uns nicht weit, doch wir können sie konkretisieren, indem wir als umweltbewuBtes Verhalten dasjenige bezeichnen, das zu einer f r e 1 w 1 l 1 i g e $n$ Einhaltung der Immissionsstandards führt. Notwendige Voraussetzung dafür ist, daß die Produzenten und Konsumenten wissen, welche Maßnahmen sie ergreifen (oder unterlassen) müBten, damit ihre Aktivitäten $z u$ keinen Umweltbelastungen führen würden. Das ist jedoch noch keine hinrelchende Bedingung für ein (freiwillig) umweltgerechtes Verhalten: Es müssen noch der wille und die Fähigkelt der Produzenten und Konsumenten hinzukommen, umweltbelastende Aktivitäten $z u$ vermeiden.

Wie steht es in der Realität mit den Voraussetzungen für ein (freiwillig) umweltkonformes Verhalten der Produzenten und Konsumenten?

Die notwendige Voraussetzung der Kenntnis des "Wie" ist noch keineswegs im erforderlichen Umfang erfültt. Viele Umweltbelastungen entstehen aus mangelnder Kenntnis alternativer Produktions- und Konsummoglichkeiten oder einfach deshalb, weil Produzenten und Konsumenten nicht die umweltbelastenden Folgen ihrer Aktivitäten bedenken. Diese Umweltbelastungen sind unter Umständen nur die Folge von Gedankenlosigkeit und Ignoranz 45) und ihre Verursacher würden sle vielleicht vermelden, wenn sie

Fisher, A.C., Population and Environmental Quality, in: Public Policy, vol. 19, 1971, S. $19 \mathrm{ff}$.

45) Vgl. Schwabe, G.H., Die Rolle des Menschen - Anmerkungen zu einer kritisch angewandten రkologie, in: Steubing, L./ Kunze, C./Jäger, J. (Hrsg.), Belastung und Belastbarkeit von bkosystemen, a.a.0., S. 244; Bragdon, C., The Community Noise Problem - Factors Affecting its Management, a.a.O., S. 717. 
die Folgen ihrer Handlungen und die alternativen Möglichkeiten der Produktion und des Konsums kennen würden. Man kann sagen, daß bei solchen Produzenten und Konsumenten ein Umweltbewußtsein gar nicht vorhanden ist; wäre es vorhanden, hätten sie vielleicht sogar den Willen, sich umweltgerecht $z u$ verhalten. Diese Voraussetzung aber, daB nämlich der wille zu umweltgerechten Verhalten vorhanden ist, trifft wohl noch weniger zu, vor allem dann nicht, wenn er mit einem Verzicht verbunden 1st 46): bel den Produzenten in Form niedrigerer Gewinne, bei den Konsumenten in Form liebgewonnener Konsumgüter und Konsumgewohnhelten. Diese Produzenten und Konsumenten haben das

"falsche" (alte) Umweltbewustsein.

Die dritte Voraussetzung schlieblich, die Fähigkeit, Umweltbelastungen $z u$ vermeiden, ist eigentlich immer gegeben, und sei es, daB dieser Weg auch - etwa wegen fehlender technischer oder finanzieller Lösungsmöglichkeiten - "Raumschiffokonomie" 47) oder gar "Retournez à la nature" heiße. Vor allem letzterer Weg wäre allerdings mit hohen Verzichtkosten verbunden. Ein umweltgerechtes Verhalten lieBe sich also grundsätzlich herbeifuhren, wenn es gelänge, die beiden ersten Voraussetzungen $\mathrm{zu}$ realisieren, was konkret heiBt, bel den Produzenten und Konsumenten, die kein Umweltbewußtsein haben, dieses zu bilden - und zwar gleich das "richtige" - und denen, die noch in den alten Kategorien denken, daß die Natur quas 1 alles verdaut, dieses "falsche" Umweltbewußtsein auszutreiben.

All dies kann man - so hoffen zumindest diejenigen, die in dem mangelnden oder falschen UmweltbewuBtsein die elgentliche Ursache der Umweltbelastung sehen - durch sachliche Information

46) Vgl. Coenen, R. u.a., Alternativen zur Umweltmisere, a.a.0., S. $177 \mathrm{ff}$. Die Hoffnung auf Verzlcht bel Unternehmern und Verbrauchern, auf "Triebsublimierung" (Picht, G., Wir brauchen neue Uberzeugungen, in: Schlemmer, J. (Hrsg.), Neue Ziele für das Wachstum, München 1973, S. 137 f.) ist auch unseres Erachtens 1dentisch mit der Annahme "ökonomischer Selbstmordkandidaten" (Ronge, V., Die Umwelt im kapitalistischen System, a.a.0., s. 113).

47) Boulding, K.E., The Economics of the Coming Spaceship Earth, in: Jarrett, H: (Hrsg.), Environmental Quality in a Growing Economy, a.a.0., s. $3 \mathrm{ff}$. 
und Aufklärung, vor allem aber durch Erziehung errelchen

Aber auch Regierungen

49) und nicht als unverbesserliche Opti-

misten hinsichtlich der BeeinfluBbarkeit des Menschen geltende Wissenschaftler ${ }^{50}$ ) halten die Schaffung eines (neuen) UmweltbewuBtseins für eine strategle, die zumindest langfristig die Durchsetzung des Umweltschutzes begünstigen kann 51) und

48) Vgl. Swan, J.A., Attitudes and Values and Environmental Education, in: Armstrong, T.R. (Hrsg.), Why Do we Still Have an Ecological Crisis?, Englewood Cliff, N.J., 1972, S. $124 \mathrm{ff.;}$ Arthur, D.R., Education and Human Environment, in: International Journal of Environmental studies, vol. 1,1971 , s. 315 ff.

49) Vgl. The New Environmental Education Program of the Office of Education, hrsg. v. U.S. Department of Health, Education and Welfare, U.S.G.P.O., Washington, D.C. 1971;

Environmental Protection Agency, Toward a New Environmental Ethic, U.S.G.P.O., Washington, D.C. 1971; Ford, W.D., A National Program for Environmental Education, in: Armstrong, T.R. (Hrsg.), Why Do we still Have an Ecological Crisis?, a.a.0.. S. $140 \mathrm{ff}$. In den USA haben sich die Bemühungen um die "environmental education" bereits in einem Gesetz nledergeschlagen, dem Environmental Education Act vom 9.9.1970. In der Bundesrepublik Deutschland ist man noch nicht so welt, doch vertraut man offenbar auch hier auf positive Wirkungen der Umwelterziehung (vgl. Umweltprogramm der Bundesregierung, a.a.0., S. 59 ff.; Menke-Glückert, P., Das Umweltprogramm der Bundesreglerung, a.a.0., S. 129). Die Kultusministerkonferenz der Länder hat sich für Umweltbildung und -erziehung in allen Bildungsstufen ausgesprochen (vgl. Siebert, A., Jugend und Umweltschutz, Materialien Umweltschutz und Raumordnung, Heft 8 , hrsg. v. der Akademie für Raumforschung und Landesplanung, Hannover 1974, S. $41 \mathrm{ff.}$ )

Auch internationale Organisationen, vor allem die UNESCO, plädleren für die Erzlehung zum Umweltbewußtsein (vgl. UNESCO, Use and Conservation of the Blosphere, a.a.O., s. $217 \mathrm{ff.})$

50) Vgl. U.S.C.E.Q., Environmental Quality, The First Annual Report, a.a.0., S. 222 ("Environmental education is a key to making this a livable world"); DFG, Umweltforschung. Aufgaben und Aktivitäten der DFG 1950 bis 1970, Bonn-Bad Godesberg, 1971, S. 14; Frey, R.L., Umweltschutz als wirtschaftspolitische Aufgabe, a.a.0., s. 470; Seidenfus, H.S., Umweltschutz, politisches system und wirtschaftliche Macht, a.a.0., S. 827 .

51) Vgl. Herrmann, G., Probleme der bewußten Beherrschung von Wechselwirkungen zwischen Mensch und natürlicher Umwelt, in: Umwelt, 1973, 2, S. 18 . 
zwar nicht nur deswegen, weil etwa Konsumenten und Produzenten zur höheren Einsicht gelangen könnten, sondern vor allem deswegen, well sich die von Umweltbelastungen betroffenen Bürger aufgrund sachgerechter Informationen und Aufklärungsaktionen $z u$ argumentationsstarken Interessengruppen des Umweltschutzes formieren und so elne countervaling power zur "VerschmutzungsLobby" darstellen können, die auf ihrer Selte die gewiB wichtigen Produktions- und Beschäftigungsinteressen (mit Ausnahme der Umweltschutzindustrie) hat 52 ?

Die Hoffnung auf diesen Effekt sachgerechter Information, Aufklärung und Erziehung ist durchaus realistisch, wie die inzwischen zahlreichen Bürgerinitiativen auf dem Gebiet des Umweltschutzes 53) - und vor allem deren Erfolge 54) - eindrucksvoll bewelsen, und sie hat nichts $z$ u tun mit der vagen und wohl auch weltfremden Hoffnung, durch Erziehung den guten Menschen, der beseelt ist von einem "futurologischen Ethos" 55) oder einer "neuen ökologischen Ethik" 56), formen zu können. Wir leugnen nicht, das es bereits heute z.B. den soziales Verantwortungsbe-

52) Vgl. Everett, M., The Role of Formal Education in Environmental Movements, in: Journal of Economic Issues, vol. 6, 1972, S. $90 \mathrm{f}$.

53) Vgl. Bramigk, D., Warten auf Initialzündungen, in: U 1975, 4, S. 16; ders., Bürgerinitiativen zum Umweltschutz, in: U 1973,5, S. 19.

54) Wyhl und Brokdorf, geplante Kernkraftwerksstandorte, sind heute Namen, die fast jeder in der Bundesrepublik Deutschland kennt. Hier gelang es Bürgerinitiativen, den Bau von Kernkraftwerken - jedenfalls zunächst einmal - zu stoppen. Ihr Erfolg ist u.E. allerdings wenlger in dieser Tatsache zu sehen als vielmehr darin, daB sich nun breite Bevölkerungsschichten und Politiker mit der Frage auselnandersetzen, wie gefährlich Kernkraftwerke sind.

55) Schaefer, H., Die Okologie als Problem des Umweltschutzes, a.a.O., S. 128 .

56) Mc Hale, J., Der ökologische Kontext, a.a.o., S. 47; vgl. ferner: Schăfer, W., Der kritische Raum. Uber den Bevölkerungsdruck bel Tier und Mensch, Frankfurt/Main 1971, S. 110 ff.; Swan, J.A., Att1tudes and Values and Environmental Education, a.a.0., S. 126; Budowsk1, G., Conservation and the Future Environment of Mankind, in: Sioli, H. (Hrsg.), Okologie und Lebensschutz in internationaler sicht, a.a.0., S. $439 \mathrm{ff}$. 
wußtsein zeigenden Unternehmer gibt, der freiwillig Umweltschutz betreibt ${ }^{57}$ ), wobel durchaus offen 1st, ob er dies aus Altruismus oder aus Gründen der Public Relations tut ${ }^{58}$, und

57) Vgl. Hönig, W., Die soziale Verantwortung des Unternehmers, In: NZZ vom 10.2.19.71; Dierkes, M., Qualität des Lebens und unternehmerische Entscheidung, Ansätze zu einem gesellschaftsbezogenen Rechnungslegungs- und Planungssystem, in: Battelle-Information 17, Frankfurt 1973, S. $27 \mathrm{ff}$. und die Im Literaturverzeichnis angegebenen Werke über "Corporate Social Accounting".

58) Vgl. Gysin, H., Marketing und Umweltschutz, in: von Waltersk1rchen, M.P., Umweltschutz und Wirtschaftswachstum, a.a.O., S. 195; Hönig, W., Die sozlale Verantwortung des Unternehmers, a.a.o., Odrich, P., Schütze die Umwelt und rede darüber, in: Blick durch die Wirtschaft v. 12.7.1971. Dieser Devise folgen viele Unternehmen und ihre Repräsentanten; vgl. z.B. BASF, Werk und Umwelt, Ludwigshafen o.J., deren Geschäftsbericht für 1971, S. 21 f. und die Ausführungen von $B$. Timm im Bericht über die 20 . ordentliche Hauptversammlung am 28.6.1972, S. $8 \mathrm{ff}$.; Wir und der Umweltschutz, in: Mannesmann AG (Hrsg.), Rohrpost 53; Henkel, P.. Unser Beitrag zum Umweltschutz, in: Bayer-Berichte, H. 27, 1971, S. $26 \mathrm{ff}$., World Wildilfe Fund (Hrsq.), Wasserund Luftverschmutzung, Lärm, Abfälle. Was tut die Industrie?, Bonn 1969; O.V., Das Beisplel Bosch, in: Die Wirtschaftswoche Nr. 39 v. 24.9.1971; Koszo, E., Umweltschutz bei Hoechst, in: U 1975, 5, S. 42 ff.; Haltrich, W./Malle, K.-G., Wasserreinhaltung: Die Rolle der chemischen Industrie, in: Umwelt, 1975, 5, S. 25 ff.; Graf Schlippenbach, S., Viele Milliarden fließen für sauberes wasser und reine Luft, in: Blick durch die Wirtschaft vom 21.6.71; Henkel, P., Der Graben zwischen Chemie und రffentlichkeit, in: Umwelt, 1973, 3, S. 4; Timm, B., Leben mit der Chemie - heute und morgen, in: Universitas, 28. Jg., 1973, S. $1201 \mathrm{ff}$.; Schnelder, Lothar, Umweltschutz in Ludwigshafen, in: Der Städtetag, 1971,4, S. $194 \mathrm{ff} . ;$ Schaafhausen, J., Industrie zu Maßnahmen bereit, in: Wirtschaftsdienst, 50. Jg., 1970, s. $639 \mathrm{f}$. ; Mommsen, E.W., Wirtschaft und Umwelt, in: Vortragsreihe des Deutschen Industrie-Instituts, Bd. 21, 1971, H. 4, S. $1 \mathrm{ff.}$; Schaafhausen, J., Wasser- und Luftreinhaltung aus der Sicht der Industrie, in: Wasser und Abwasser, 1968, S. $9 \mathrm{ff.}$; Schackmann, H., Umwelt und Wirtschaft im Ruhrgebiet, Schriften der Volks- und Betriebswirtschaftlichen Vereinigung im Rheinisch-Westfälischen Industriegebiet, 14. Sonderveröffentlichung, Düsseldorf 1972 .

Aber auch die Furcht vor Systemveränderern, die die Umweltbelastung als Vehikel für ihre umstürzlerischen Absichten benutzen könnten, veranlassen die Unternehmer vielleicht zu umweltbewußterem Verhalten (vgl. Schrafl, A.E., రffentliche Verantwortung des Unternehmers, in: NZZ, FA Nr. 111 
ebensowenig bestreiten wir daß bereits heute einige Konsumenten umweltbewußt kaufen 59), und wir schließen auch nicht aus, daß die $\mathrm{Zahl}$ der (freiwillig) umweltfreundlich agierenden Produzenten und Konsumenten - aus welchen Grüden auch immer - in Zukunft steigen wird; wir meinen jedoch, daß ein Vertrauen auf diese Entwicklung fahrlässig wäre. Tritt sie ein, so ist sie in unseren Augen ein positiver Nebeneffekt der Informationen, Aufklärungskampagnen und Erzlehungsmaßnahmen, die primär darauf abzielen, die von der Umweltbelastung betroffenen und am Umweltschutz interessierten Bürger instandzusetzen, sich durch Organisierung in einer Bürgerinitiative, durch eine gerichtliche Klage oder mit anderen legalen Mitteln wirksam zur Wehr zu setzen 60 ?

Es stellt sich a: lerdings die Frage, wer den Bürgern die Informationen vermitteln soll, die sie zu umweltbewußtem Handeln befähigen. Es bieten sich drel Institutionen an: Das Erziehungs-

v. $25.4 \cdot 1971$ ).

Ferner kann eine bereits eingetretene oder erwartete Wandlung des Umweltbewußtselns der Verbraucher die Unternehmen veranlassen, umweltfreundliche Produkte herzustellen. Hier ergibt sich eine neue Aufgabe fur die Marketing-Abtellungen der Unternehmen; vgl. dazu Kassarjian, H.H., Incorporating Ecology into Market Strategy: The Case of Air Pollution, in: Journal of Marketing, vol. 35 (July 1971), S. 61 ff.; Gysin, H., Marketing und Umweltschutz, a.a.0., S. 187 ff.

59) Dazu, wie z.B. in den USA Verbraucher zu umweltbewußten Kaufentscheldungen angeregt werden, vgl. 0.V., Der Verbraucher von morgen tut nicht mehr alles, Vorschläge an amerikanische Konsumenten, in: Blick durch die Wirtschaft v. 7.10 .71 .

In der Bundesrepublik Deutschland erwägt man die Einführuna eines Umweltgutezelchens für umweltfreundliche Produkte (vgl. Hartkopf, G., Umweltpolitik aus der Sicht der Bundesreglerung, a.a.O., S. 187; von Walterskirchen, M.P., Bemerkungen zur Umweltpolitik, a.a.0., S. 12).

60) Vgl. Everett, M., The Role of Formal Education in Environmental Movements, a.a.0., S. 91; Kohl, D.H., The Environmental Movement: What Might it Be?, in: Natural Resources Journal, vol. 15, 1975, S. 351. 
wesen, die Massenmedien und die Bürgerinitiativen selbst. Das Erziehungswesen ist u.E. aus drei Gründen am besten dazu geeignet, das Umweltbewußtsein $z u$ bilden: (1) Es ist von den dre1 genannten Institutionen am ehesten in der Lage, sachgerechte Informationen $z u$ vermitteln; (2) es erlaubt eine interdisziplinäre Unterrichtung der Schüler, was für das Verständnis der komplexen Umweltprobleme und deren Lösung von großer Wichtigkeit ist 61) und (3) kann es - wenn uberhaupt - am ehesten zur B1ldung eines "ökologischen Ethos" beitragen, vor allem dann, wenn die Erziehung in einem Alter beginnt, in dem die Verhaltensweisen des Menschen geprägt werden. So wird denn auch konsequenterwelse gefordert, daß die Umwelterziehung bereits im Vorschulalter beginnt - etwa mit einem "environmental sesame street"-Programm 62) - und sich durch alle Bildungsstufen bis hin zur Universität fortsetzt 63); auch die Erwachsenenbildung soll sich Umweltproblemen widmen 64) Wichtiaste Voraussetzung für die Umwelterzlehung in den verschiedenen stufen des B1ldungssystems 1st, daß die Erzieher selbst über die vielfältigen Umweltprobleme Bescheid wissen. Hierdurch entsteht eine neue Ausbildungsaufgabe für die Universitäten und pädagogischen Hoch-

61) Vgl. U.S.C.E.Q., Environmental Quality, The First Annual Report, a.a.O., S. 221 ; Ford, W.D., A National Program for Environmental Education, a.a.O., S. 142; Hall, R.H., University Education and the Natural Environment: Are they Compatible?, in: International Journal of Environmental Economics, vol. 2, 1971, S. 48 f.; 0.V.. Environmental Education in the Universities. A Key to "Relevance", In: OECD-Observer No. 68,1974, S. 27.

62) Vgl. Swan, J.A., Att1tudes and Values and Environmental Education, a.a.0., S. 135 .

63) Vgl. Congress and the Nation's Environment, a.a.o., s. 54; U.S.C.E.Q., Environmental Quality, The First Annual Report, a.a.O., S. $222 \mathrm{f}$; UNESCO, Use and Conservation of the Blosphere, a.a.0., S. $220 \mathrm{ff.;}$ Ford, W.D., A National Program for Environmental Education, a.a.O., S. 141; Seldenfus, H.S., Uimweltschutz, politisches system und Macht, a.a.0., s. 830; Kapp, K.W., Zur Praxis der Umweltpolitik und Umweltplanung, a.a.o., S. 166 .

64) Vgl. Ford, W.D., A National Program for Environmental Education, a.a.o., S. 143; Congress and the Nation's Environment, a.a.o., S. 55 . 
schulen 65) Weiterhin müssen für die verschiedenen Bildungsstufen Curricula und Lehrbücher geschrieben und praktisches Lehrmaterial zur Verfügung gestellt werden 66) Bevor dies geschehen kann, muß aber bereits entschieden sein, ob es ein neues Fach, das etwa "Okologie und Umweltschutz" he1Ben könnte, geben soll oder ob die Umwelt(schutz)probleme in anderen Fächern (mit-) behandelt werden sollen. Wie wir sehen, sind die Probleme der Umwelterziehung vielfältig, und es kann wohl nur die Erfahrung zelgen, wie deren Organisation am besten vorgenommen wird.

Die Aufgabe der Massenmedien besteht vor allem in der sachgerechten Information über Umweltbelastungen und die Möglichkeiten des Umweltschutzes 67) Zu Beginn der Umweltschutzwelle und vor

65) Vgl. U.S.C.E.Q., Environmental Quality, The First Annual Report, a.a.0., S. 222; Ford, W.D., A National Program for Environmental Education, a.a.0., S. 143; Swan, J.A., Attitudes and Values and Environmental Education, a.a.0., S. 127; Rodi, D., Umweltschutz in Forschung und Lehre im Bereich der Biologle an Pädagogischen Hochschulen, in: Steubing, L./Kunze, C./Jäger, J. (Hrsg.), Belastung und $\mathrm{Be}-$ lastbarkeit von Ökosystemen, a.a.0., S. 207.

66) Vgl. U.S.C.E.Q., Environmental Quality, The First Annual Report, a.a.O., S. 222; Ford, W.D., A National Program for Environmental Education, a.a.0., S. 143.

Recht ausführlich berichten über die Möglichkelten der Vermittlung ökologischer Lehrinhalte in Schulen und Universitäten verschiedene Beiträge, in: Steubing, I./Kunze, C./ Jäger, J. (Hrsg.), Belastung und Belastbarkeit von Okosystemen, a.a.0., S. $181 \mathrm{ff}$. In der Bundesrepublik Deutschland gibt es inzwischen ein reichhaltiges Angebot an Schulbüchern, oder für den Schulunterricht geeigneten Büchern über Umwelt und Umweltschutz; vgl. z.B. folgende vier Bücher, die das Umweltproblem didaktisch jeweils anders nahezubringen versuchen: Engelhardt, W., Umweltschutz. Gefährdung und Schutz der natürlichen Umwelt des Menschen, München 1973; Bäuerle, D./Hornung, G., Umwelt. Blologisch - sozlalkundliche Arbeitshefte, Opladen 1972; Palmstierna, L. und H., Unsere geplünderte Welt, Weinhelm und Basel 1972; Dörge, F.W. (Hrsg.), Qualität des Lebens. Ziele und Konflikte sozialer Reformpolitik didaktisch aufbereitet, Opladen 1973.

67) Vgl.U.S.C.E.Q., Environmental Quality, First Annual Report, a.a.o., S. 223 . 
allem auf threm Höhepunkt, haben Presse, Rundfunk und Fernsehen ausglebig über alle möglichen Umweltprobleme berichtet, und es ist vor allem ihnen zu verdanken, daß die Bevölkerung wachgerüttelt wurde und bei vielen Bürgern erste Ansätze eines (neuen) Umweltbewußtseins entstanden sind. Dabei mußte in Kauf genommen werden, daß manche Informationen über Umweltbelastungen und vor allem deren Gefahren nicht sachgerecht waren und thre Lieferanten auf die Sensationsgier vieler Leser und Fernsehzuschauer spekulierten. Das ständige Bombardement mit Meldungen über Tankerunfälle, Fischsterben, Quecksilbervergiftungen, illegale Giftmülldeponien, DDT-geschädigte Pelikaneier, aussterbende Schmetterlingsarten, die Entlaubung von wäldern, bleivergiftete Kühe, lärmgeschädigte Flughafenanlieger, co-vergiftete Verkehrspolizisten, pestizid-haltige Muttermilch, antibiotikahaltiges Kalbfleisch, detergentlenbelastete Flüsse, den Zerfall historischer Bauwerke, nitrithaltigen Spinat usw. war teilwelse allein vom Umfang her so stark, daB es keine sachgerechte Information mehr war und so weniger das Umweltbewußtsein weckte, als eine Art Umwelthysterie hervorrief. Die Blirger mußten sich von lauter Umweltgefahren umgeben fühlen, und es verwundert nicht, daB z.B. Krankheiten und die MiBbildung von Säuglingen ohne jeden konkreten Bewels auf Umweltbelastungen zurückgeführt wurden und daß krimlnelle Delikte z.B. mit Lärmbelästigungen entschüldigt wurden. Diese Verkrampfung der Menschen vor lauter Umweltbezogenhelt bezelchnet man auch als Okospasmus 68); dieser Zustand beschränkt die Aufnahmefähigkeit der Menschen für sachliche Informationen und behindert somit die Möglichkeit, umweltdienliche Informationen $z u$ vermitteln. Diese von Schoeck aufgezeigte Gefahr sehen wir ebenfalls. In einem anderen Punkt glauben wir aber, 1hm widersprechen zu müssen. Schoeck konstatiert - offenbar miBbilligend - daß durch Informationen über die möglichen Wirkungen der Umweltbelastung sich Bürger zur Wehr setzen; er bedauert sichtlich - in Anspielung auf die Ansiedlung der Firma

68) Vgl. Schoeck, H., Ökospasmus oder die Angst vor Kernkraftwerken, 1n: Umwelt, 1976, 2. S. $126 \mathrm{f}$. 
Reynolds in Hamburg -, daß heute der kleinste Anrainer multinationale Konzerne blockleren kann, und er weist darauf hin, daß solches in den USA bereits zum Sport geworden sei 69) Schoeck mag recht haben, daß diejenigen, die sich daraus einen sport machen, publizitätsheischende oder ein Machterlebnis auskostende Kleinbürger sind - wieso geben aber stadtväter oder sonstige für die Ansiedlung zuständige Institutionen (oder auch die Unternehmen selbst) nach, oder warum geben den armen Anrainern, wenn es zum Prozeß kommt, die Gerichte recht? Wir möchten doch bestreiten, daß auch sie berelts alle umweltneurotisch sind. Zur Rolle der Massenmedien wollen wir abschließend festhalten, daß diese auf schockierende Effekte bei ihren Meldungen über Umweltbelastungen (heute) verzichten können (aus Gründen des Wachrüttelns mag es dafür früher eine gewisse Berechtigung aegeben haben) und dann am besten ihre Aufgabe erfüllen, wenn sie sachlich über Umweltbelastungen und die Möglichkeiten ihrer Bekämpfung informieren.

Abschließend wollen wir noch untersuchen, inwleweit Bürgerinitiativen bei der Entstehung oder Veränderung des Umweltbewußtseins mitwirken können 70) In der Bundesrepublik Deutschland gibt es inzwischen wohl einige hundert Bürgerinitiativen, die sich mit Umweltschutz befassen. Einige von ihnen haben sich auf Dauer institutionalisiert, andere lösen sich nach Erfüllung (oder Nichterfüllung) ihres Zwecks wieder auf. Manche widmen sich speziellen Umweltproblemen, manche allen Problemen, die mit dem Umweltschutz zu tun haben. Eines haben aber alle Bürgerinitiativen gemeinsam: Sie wollen möglichst viele Verbündete gewinnen,

$69)$ Vgl. Schoeck, H., Okospasmus oder die Angst vor Kernkraftwerken, a.a.0., S. 73. In den USA wird sogar von offizieller Selte, nämlich der Environmental Protection Agency dazu aufgerufen, sich zu organisieren, und es werden Ratschläge ertellt über die Möglichkeiten, zu seinem Recht auf eine saubere Umwelt zu kommen (vgl. U.S. Environmental Protection Agency, Don't Leave it All to the Experts, The Citizen's Role in Environmental Decision Making, U.S.G.P.O., Washington, D.C. 1972).

70) Vgl. Zilleßen, H., Ansprüche und Arbeit der Bürgerinitiativen, in: Umwelt, 1974, 2, S. 41; Bramigk, D., Warten auf Initialzündungen, a.a.0., S. 17 . 
und das gelingt nur auf dem Weg uber Information und Aufklärung der von der jeweiligen Umweltbelastung betroffenen (oder auch der nichtbetroffenen) Bevollkerung. Es ist nun $z u$ bezwelfeln, ob diese Informationen immer objektiv sind (oder in manchen Făllen sein können), da die Informationen ja dem Zweck der Bürgerinitiativen (z.B. der Verhinderung einer Bauqenehmigung für ein Kernkraftwerk) dienen sollen.

Ferner 1st $z u$ berückslchtigen, das manche Bürgerinitlativen offenbar elne gewisse Anzlehungskraft auf Vereinsmeler haben und ein beliebtes Tummelfeld für 1deologische Eiferer sind 71) Auch das läBt es als geboten erscheinen, Informationen und Aufklärungskampagnen von Bürgerinitiativen kritisch - nicht ablehnend! - gegenüberzustehen. Relativ unbefangen kann man wohl gegenüber den Informationen und Aufklärungsaktionen sein, die von Bürgerinitiativen vorgenommen und durchgeführt werden, die slch nur Information und Aufklärung zum ziel gesetzt haben. Vor allem sie können, wenn sie verantwortungsvoll handeln, elne wichtige Rolle bei der Bildung eines (neuen) Umweltbewußtseins spielen.

ee) Die Kontrolle des technischen Fortschritts Bereits bei der Ursachendiagnose haben wir der Ansicht widersprochen, daß der technische Fortschritt an sich Umwéltbelastungen hervorrufe, und festgestellt, daß es vielmehr die umweltfelndliche Anwendung des technischen Wissens ist, die sich in der Entwicklung umweltbelastender Technologien manifestiert ${ }^{72}$. Die Ambivalenz des technischen Fortschritts haben inzwischen auch die Ingenieure und Techniker selbst erkannt ${ }^{73}$, und es

71) Vgl. Bramigk, D., Bürgerinitiativen zum Umweltschutz, a.a.0., S. 19 .

72) Siehe oben S. 115 ff.

73) Vgl. dazu vor allem die verschledenen Beiträge in einem vom VDI herausgegebenen Sammelband: VDI (Hrsg.), Wirtschaftliche und gesellschaftliche Auswirkungen des technischen Fortschritts, Düsseldorf 1971 und Steinbuch, K., Mensch, Technik, Zukunft. Basiswissen für Probleme von morgen, stuttgart 1971 . 
gibt kaum noch wissenschaftler und Politiker, die grundsätzlich gegen eine Kontrolle des technischen Fortschritts sind, zumal damit keineswegs eine Kontrolle der Forschung selbst, sondern nur der Anwendung der wissenschaftlichen Erkenntnisse der Forschung gemeint ist 74)

Voraussetzung für eine Kontrolle des techn1schen Fortschritts ist eine Bewertung von dessen sozialen, ökonomischen und - worum es uns $1 \mathrm{~m}$ besonderen geht - ökologischen und gesundhe1tlichen Auswirkungen. Gegen Ende der sechziger Jahre wurden in den USA auf Kosten-Nutzen-Analysen aufbauende Methoden mit stark systemtheoretischem Einschlag zur Bewertung des technischen Fortschritts entwickelt, die man unter dem - inzwischen auch in der Bundesrepublik Deutschland gebrăuchlichen - Begriff "Technology Assessment" zusammengefaßt hat 75) "Unter Technology Assessment ... versteht man die umfassende Bewertung gegenwărtiger und auch noch zu realisierender Technologien (Produkte, Verfahren und z.T. auch Sozialtechnologien) hinsichtlich der mit ihnen verbundenen ökologischen, sozialen und politischen Konsequenzen" 76) Diese Definition unterscheidet sich von den meisten anderen 77) dadurch, daB sie auch die Bewertung von So-

74) Vgl. OECD, Science, Growth and Society. A New Perspective, Report of the Secretary General's Ad Hoc Group on New Concepts of Science Policy, Paris 1971, S. 77; La Porte, T.R., The Context of Technological Assessment: A Changing Perspective for Public Organization, in: Public Administration Review, 1971, S. 64.

75) Vgl. Staudt, E./Metze, G., Technology Assessment, 1n: Wirtschaftsstudium, 5. Jg., 1976, S. 81 f. Ausführliche Darstellungen des Technology Assessment sind: Hetman, F., Soclety and the Assessment of Technology, Paris 1973, mit dem Schwergewicht auf methodischen Problemen, und Kasper, R.G. (Hrsg.), Technology Assessment. Uhderstanding the Social Consequences of Technological Applications, New YorkWashington-London 1972, mit dem Schwergewicht auf institutionelien Problemen.

76) Staudt, E./Metze, G., Technology Assessment, a.a.0., S. 81 f.

77) Vgl. Fischer, D.W./Muller, F.G., Ein Beitrag zur Erfassung von Elnwirkungen auf die Umwelt, in: Zeitschrift für die gesamte Staatswissenschaft, Bd. 130, 1974, S. 722; Bartocha, B., Technology Assessment-Verfahren zu einer TechnologieBewertung, in: Umwelt, 1972, 6, S. 31 . 
zialtechnologien, also z.B. politischen Maßnahmen und Gesetzen, umfaßt, woran uns deswegen gelegen 1st, weil wir im folgenden auch die Möglichkeiten prüfen wollen, die Umweltverträglichkeit öffentlicher Maßnahmen 78) - und nicht nur die von Produkten und Produktionsverfahren - zu ermitteln. Aus der Definition geht weiterhin hervor, daB Technology Assessment mehr ist als nur eine Bewertung der Umweltverträglichkelt; auch die sozialen, ökonomischen und politischen Auswirkungen des technischen Fortschritts müssen berücksichtigt werden. Nur auf diese Weise kann man erreichen, daß der technische Fortschritt auf ein gesamtwirtschaftliches Optimum und nicht etwa nur ein ökologisches optimum hingelenkt wird 79 ?

Technology Assessment scheint auf den ersten Blick so etwas zu sein wie eine Kosten-Nutzen-Analyse des technischen Fortschritts. Was die methodischen Probleme angeht, bestehen auch viele Gemeinsamkeiten: Es müssen Informationen über die vielfältigen Wirkungen des technischen Fortschritts oder politischer Maßnahmen gewonnen werden, und diese Wirkungen müssen bewertet werden. Das Technology Assessment bedient sich bei der Bewertung sogar zunächst der Kosten-Nutzen-Analyse, geht dann aber uber sie hinaus, indem es auch "weiche" Daten, das

78) Vgl. RSU, Umweltgutachten 1974, a.a.0., S. 13; Swoboda, H., Probleme der Umweltpolitik, in: Wirtschaft und Gesellschaft, 1.Jg., 1975, S. 83; Pätzold, E., Verbindliche Bewertungskriterien, in: U 1975, 5, S. 6; Otto, K., Umweltschutz überfordert Städte, In: Umwelt, 1975, 5, S. 14; Obenhaus, W., Uberlegungen für eine Konzeption zur Prüung der Umweltverträglichkeit öffentlicher Maßnahmen, in: Umwelt, 1973,5, S. $58 \mathrm{ff.:}$ Grawe, J., Sichere Energieversorgung und Umweltschutz. Wle der zielkonflikt bewalltigt wird, in: Umwelt, 1974, 2, S. 37; Menke-Glückert, P., Das Umweltprogramm der Bundesregierung, a.a.0., s. 129.

79) von Dobschütz, L./Mathias, W., Umweltverträglichkeitsprüfung öffentlicher Maßnahmen, in: Umwelt, 1973, 2, S. 37; Fischer, D.W./Muller, F.G., Ein Beitrag zur Erfassung von Elnwirkungen auf die Umwelt, a.a.0., S. 720; Schumacher, D. Hilfsmittel für die Umweltplanung, in: U 1973, 5, S. 24. 
sind nicht quantifizierbare Wirkungen, berücksichtigt ${ }^{80}$ ! DaB Technology Assessment damit eher eine Kunst als eine Wissenschaft ist ${ }^{81)}$ und dem Wissenschaftler auch noch eine Verantwortung aufbürdet, die dieser in manchen Fällen kaum tragen kann, mag das Belspiel DDT bewelsen. Die Erfindung des DDT führte zwelfellos zu einem der gröBten technischen Fortschritte im Agrarberelch, nämlich einer äuBerst effizienten und relativ billigen Schädlingsbekämpfung, die Millionen von Menschen vor dem Hungertod bewahrt hat. Heute wissen wir, daß DDT ökologische Schäden hervorrufen kann, und gesundheltsschädliche Wirkungen werden vermutet ${ }^{82}$ ? Was hätte wohl ein Wissenschaftler(team) nach der Erfindung des DDT hinsichtlich dessen Anwendung empfohlen, wenn ihm ökonomisch, sozial und politisch nützliche und ökologisch schädliche wirkungen bekannt gewesen wären? Ganz so schlimm, wie es auf den ersten Blick scheint, wäre ein bereits damals erfolgtes Verbot der industrielien Verwertung der DDT-Erfindung für die Hunger leldenden Völker nlcht gewesen, denn die Pesticidforschung hatte viellelcht schon damals ein umweltfreundliches (schnell abbaubares) schädlingsbekämpfungsmittel erfunden. Das zeigt, daß Technology Assessment kein "Technology Arrestment" 83) ist, sondern neben einer regulierenden Wirkung für die Anwendung des technischen Wissens auch eine stimulierende Wirkung auf die Forschung haben kann, indem sie zur Erforschung von mehr Alternativen anregt 84)

80) Vgl. Sachs, I., Development, Environment and Technology Assessment, in: International Social Science Journal, vol. 25, 1973, S. 276; Bartocha, B., Technology Assessment Verfahren zu elner Technologie-Bewertung, a.a.0., S. 32 ; Staudt, E./Metze, G., Technology Assessment, a.a.0., s. 82; Green, H.P., The Role of Government in Environmental Conflict, in: Wolozin, H., The Economics of Pollution, a.a.0., S. 307 .

81) Vgl. OECD, Science, Growth and Society, a.a.0., s. 84 .

82) Siehe oben S. $98 \mathrm{f}$.

83) Dierkes, M., Technology Assessment in der BRD - EIne Stellungnahme, in: Battelle - Information 19, 1974, S. 27.

84) Vgl. OECD, Sclence, Growth and Society, a.a.0., s. 82. 
Nun wollen wir noch untersuchen, wie und ob das Technology Assessment institutionalisiert werden kann und soll. In den USA gibt es seit 1972 ein "Office for Technology Assessment" 85), und die Bundesbehörden müssen für alle Projekte, die von ihnen finanziert oder geplant werden, nach $\S 102$ des National Environmental Policy Act von 1969 Umweltverträglichkeitsprüfungen vornehmen ${ }^{86}$ ? Für die Bundesrepublik Deutschland war einige Zeit ein dem Bundestag zugeordneter Ausschuß für Technology Assessment im Gespräch, um der Legislative bezüglich Sachverstand nachzuhelfen, da sie sich bel Projekten der Exekutive mit technologischem Einschlag (z.B. dem Bau von Kernkraftwerken) zur Abstimmungsmaschinerie degradiert fühlte ${ }^{87}$ ) Bisher ist es jedoch in der Bundesrepublik Deutschland noch zu keiner dem "Office for Technology Assessment" vergleichbaren Institution gekommen - vielleicht weil man glaubt, mit den bisherigen Möglichkeiten auszukommen, technologische Entwicklungen und öffentliche Maßnahmen von möglicher Umweltrelevanz kontrollieren zu können, (z.B. mit dem Genehmigungsverfahren nach $\S 16$ GewO, dem atomrechtlichen Genehmigungsverfahren nach $\S 7$ AtomG, den verschledenen Planfeststellungsverfahren im Baurecht, der Umweltverträglichkeitsprüfung von Waschmitteln, arznei- und lebensmittelrechtlichen Kontrollverfahren und den zulassungsbestimmungen für Kraftfahrzeuge) ${ }^{88}$. Vielleicht ist

85) Vgl. Staudt, E./Metze, G., Technology Assessment, a.a.0., S. 81 .

86) Vgl. Fischer, D.W./Müller, F.G., Ein Beitrag zur Erfassung von Einwirkungen auf die Umwelt, a.a.0., S. 722; Bartocha, B., Technology Assessment - Verfahren zu einer TechnologieBewertung, a.a.0., S. 31.

87) Vgl. Schumacher, D., Hilfsmittel für die Umweltplanung, a.a.0., S. 26; Zur allgemeinen Problematik des komplizierter gewordenen Entscheidungsprozesses vgl. La Porte, T.R., The Context of Technology Assessment: A Changing Perspective, a.a.0., S. 67.

88) Vgl. Obenhaus, W., Uberlegungen für eine Konzeption zur Uberprưffung der Umweltverträglichkeit öffentlicher Maßnahmen, a.a.O., S. 59; OECD, Science, Growth and Society, a.a.O., S. 79; Karsten, D., Soziale Kontrolle für techn1schen Fortschritt, in: U 1972, 4, S. 19. In diesem Zusammenhang se1 auch der Vorschlag erwähnt, die "Stiftung 
das Technology Assessment bei uns aber auch deshalb nicht institutionalisiert, weil man sich nicht uber die organisationsform der Technologie-Kontrolle einigen konnte 89) Der Grund kann schließlich auch sein, daß man einfach befürchtet, keine geeigneten Wissenschaftler für diese äußerst schwierige und höchstes Verantwortungsbewußtsein verlangende Aufgabe $z u$ finden; diese müßten darüber hinaus auch noch bereit sein "to expend their talents on perpetual negativism" 90) Wir möchten für die Einrichtung einer dem "Office for Technology Assessment" entsprechenden Institution in der Bundesrepublik Deutschland plädieren, nicht well wir in dem euphorischen Glauben leben, daB "Technology Assessment is a healthy first step toward mastering the tiger of technology" 91) sondern we11 wir uns vorstellen können, daß es in naher zukunft technologische Entwicklungen $\mathrm{zu}$ bewerten gilt, bei denen die üblichen Methoden - durch die Bürokratie und die Politiker angewandt - nicht mehr ausreichen.

Abschließend wollen wir auf einen U.E. sehr interessanten Vorschlag eingehen, der durch seine Einfachheit und mögliche Effizienz besticht, weshalb wir seine Verwirklichung für zumindest erwägenswert halten: Der "Erfinder" dieses Vorschlags geht davon aus, daß das heutige Patentsystem, das den technischen Fortschritt unabhängig von dessen Umwelteffekten honoriert, durch

Warentest" solle die getesteten Produkte auch hinsichtlich ihrer Umweltfreundlichkeit beurteilen (vgl. Otto, K., Warenbeurteilung nach ökologischen Aspekten, in: Umwelt, 1973, 4, S. $46 \mathrm{f.}$ ).

89) Vgl. Dierkes, M., Technology Assessment in der BRD, a.a.O., S. $23 \mathrm{ff.}$; dagegen gibt es seit dem 10.10.75 die "Grundsätze für dle Prüfung der Umweltverträglichkelt öffentlicher Maßnahmen des Bundes" wonach die Bundesbehörden verpflichtet sind, in der Sachregelungskompetenz des Bundes liegende Maßnahmen hinsichtlich ihrer Umweltauswirkungen $z u$ prüfen und gegen andere Belange abzuwägen (vgl. GMBI. 1975, S. 717).

90) Green, H.P., The Role of Government in Environmental Conflict, a.a.0., s. 310 .

91) 0.V., Technology Assessment Harnessing Science to Social Goals, in: OECD-Observer Nr. 67, 1973, S. 31. 
eine Änderung des Patentgesetzes dahingehend modifiziert werden sollte, daB nur noch die Erfindungen als technischer Fortschritt anerkannt und damit patentiert werden, die umweltneutral sind

b) Raumordnung als Maßnahme des Umweltschutzes

Als Raumordnung wollen wir die strukturierende Gestaltung eines Raumes im Hinblick auf wirtschaftliche, soziale, kulturelle und gesellschaftliche ziele bezeichnen ${ }^{93}$ ). Wir verwenden den Begriff "Raumordnung" also nicht zur Beschreibung eines strukturrăumlichen $z$ u $s$ a $n d e s$, sondern "zur Charakterislerung

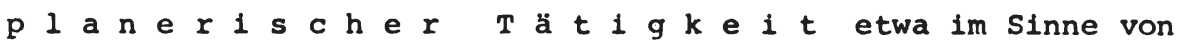
Raumplanung" 94), weshalb wir im folgenden die Begriffe "Raumordnung" und "Raumplanung" synonym gebrauchen wollen.

In der Bundesrepublik Deutschland ist die gesetzliche Grundlage für Umweltschutz via Raumordnung $\S 2$ (1), z 7 Raumordnungsgesetz (ROG), wonach es Aufgabe der Raumordnung ist, für den Schutz der Landschaft, die Reinhaltung des Wassers, die Reinhaltung der Luft und den Schutz vor Lärmbelästigungen zu sorgen.

Die Bedeutung der Raumordnung für den Umweltschutz wird von verschiedenen Selten stark hervorgehoben: "Raumordnung (ist) für den Umweltschutz konstitutiv" 95) "Raumordnung ist die Mutter des Umweltschutzes" 96)

Aber auch nüchterne Bewertungen betonen die wichtige Rolle, die die Raumplanung beim Umweltschutz spielt, was vor allem daran liegt, daß mit raumplanerischen Maßnahmen eln präventiver Um-

92) Vgl. Zipse, E., Patentierung - Hilfe für den Umweltschutz, in: U 1973, 2, S. 28 f.; der Autor dieses Artikels schlägt welterhin vor, im Patentrecht Anreize für die Erfindung umweltfreundicher Produkte und Produktionsverfahren vorzusehen (vgl. ebenda, S. 29).

93) Vgl. Malz, F., Taschenwörterbuch der Umweltplanung, München 1974, S. 432 .

94) Ebenda.

95) Picht, G., Umweltschutz und Politik, a.a.0., S. 85.

96) Hartkopf, G., Umweltgestaltung durch Raumordnung, in: Raumforschung und Raumordnung, 30. Jg., 1972, S. 146. 
weltschutz betrieben werden kann 97) Dies erhofft zumindest auch die Ministerkonferenz für Raumordnung (MKRO), denn sie glaubt, daß der zusätzliche Flächenbedarf in der Bundesrepublik Deutschland bis 1985 von über 2 \& der Gesamtfläche des Bundesgebietes für Industrieansiedlung, Wohnungsbau und den Bau von Verkehrs- und Versorgungseinrichtungen $\mathrm{zu}$ kelnen zusätzlichen Umweltbelastungen führen wird, wenn die entsprechenden Bauprojekte räumlich richtig verteilt werden 98 ?

Damit 1st bereits angedeutet, wie Raumordnung zum Umweltschutz beitragen kann, nämlich durch eine umweltgerechte räumliche Vertellung und Zuordnung der funktionalen Ansprüche wie Produzieren, Wohnen, Erholen usw. Im Gesamtraum. Die Raumordnung muß hinsichtlich des Umweltschutzes dafür sorgen, daß die Industrie-, Wohn-, Naherholungs- und Freize1t-, Fremdenverkehrs-, Landschaftsschutz-, Naturschutz-, Wasserschutz- und andere Gebiete so im Gesamtraum verteilt und einander zugeordnet sind, daß allein durch diese räumliche ordnung möglichst keine (oder nur möglichst geringe) Umweltbelastungen entstehen 99) Eine solche umwelt(schutz) gerechte Raumordnung läßt sich für ein relativ dicht besiedeltes und hochindustrialisiertes Land wie die Bundesrepublik Deutschland nur inkremental betreiben. Die uber Jahrhunderte allmählich entstandene Raumordnung kann nicht wegen des seit ein paar Jahren akuten Umweltproblems total umgekrempelt werden. Die Raumordnung kann jedoch gegenwärtige und vor allem zukünftige potentiell Umweltbelastungen hervor-

97) Vgl. Lendi, M., Die Raumplanungsgesetzgebung und ihre Konsequenzen für die Unternehmung, in: von Walterskirchen, M.P., Umweltschutz und Wirtschaftswachstum, a.a.0., S. 206; Antoine, S., Die Qualität der Umwelt und die Raumordnung, in: Aufgabe zukunft - Qualität des Lebens, Bd. 4: Umwelt, a.a.0., S. 80; Denkschrift des Hauptausschusses der Ministerkonferenz für Raumordnung "Raumordnung und Umweltschutz" hrsg. v. Bundesministerium für Raumordnung, Bauwesen und Städtebau, 0.0. und 0.J., S. 17 (im folgenden zitiert als Denkschrift der MKRO).

98) Vgl. Denkschrift der MKRO, a.a.O., s. 19.

99) Vgl. ebenda, S. 17; Bundesraumordnungsprogramm v. 3.4.75, BT-Drucksache 7/3584, S. 7; Waterkamp, R. . Interventionsstaat und Planung. Raumordnung, Regional- und Strukturpolitik, Köln 1973, S. $101 \mathrm{f}$. 
rufende Maßnahmen wie die Industrieansiedlung, den Bau von Wohngebieten, die Trasslerung von Autobahnen, den Bau von Kernkraftwerken und Flupplätzen usw. so planen, daß die tatsächlichen Umweltbelastungen möglichst gering gehalten werden.

Nach diesen allgemeinen Ausführungen wollen wir uns mit den gesetzlichen Grundlagen des Umweltschutzes durch Raumordnung befassen, bevor wir uns konkreten raumplanerischen Umweltschutzmaßnahmen zuwenden.

In föderativen Staaten wie der Bundesrepublik Deutschland entstehen wegen der Vertellung der Kompetenzen auf selbständige Gebietskörperschaften gerade im Hinblick auf die Raumordnung besondere Probleme. Damit auch die Länder und vor allem die Gemeinden den zielen der Raumordnung (in unserem Fall also vor allem den in $\S 2(1), z, 7$ ROG fixierten umweltrelevanten zielen der Raumordnung) nachkommen, bedarf es einer Bindung der Länder und Gemeinden an die ziele des ROG und damit auch einer Orientierung der raumbedeutsamen Landes- und Kommunalplanung an der Raumordnungsplanung des Bundes. Obwohl der Bund nach Art. 75, 2. 4 GG nur die Rahmenkompetenz für die Raumordnung hat und die Länder nach $\S 5(1)$ ROG elgene Raumordnungspläne aufstellen müssen, gibt es ein von Bund und Ländern gemeinsam formuliertes Bundesraumordnungsprogramm. Es wurde von der nach $\S 8$ (1) ROG gebildeten MKRO aufgestellt und hat vor allem die Aufgabe, die Ziele des ROG mit denen der Landesplanungen zu koordinieren 100), was auch nach $\S 5(2)$ ROG vorgeschrieben ist. Für die zwecke des Umweltschutzes ist besonders wichtig, daß sich das Bundesraumordnungsprogramm "in seinen umweltbezogenen $\mathrm{zlelsetzungen} \mathrm{mit}$ dem Umweltprogramm der Bundesreglerung und den entsprechenden 2lelvorstellungen der Länder (deckt)"101). Es wird damit neben $\S 2(1), z, 7$ ROG zur Grundlage eines mit Mitteln der Raumordnung betriebenen Umweltschutzes. Wenn für dle Planung der Länder eine

100) Vgl. Bundes raumordnungsprogramm, a.a.O., s. 3. 101) Ebenda, S. $4 \mathrm{f}$. 
regionale Gliederung in Tellräume erforderlich 1st, können dies die Länder nach $\S 5(3)$ ROG durch Gesetz tun. Die Regionalplanung kann dann durch regionale Planungsgemeinschaften, die aus den Gemeinden und Gemeindeverbänden der Region bestehen, vorgenommen werden. "Die Regionalplanung (schlägt) die Brücke zur Bauleitplanung" 102) für die nach $\S 2$ (1) BBaug die Gemeinden zuständig sind. Die Bauleitpläne bestehen nach $\S 1(2)$ BBauG aus dem Flächennutzungsplan und dem Bebauungsplan; im allgemeinen hat sich letzterer an ersterem zu orientieren $(\S 8(2)$ BBauG). Beide sind nach $\oint 1(3)$ BBaug den zielen der Raumordnung und Landesplanung anzupassen. Elne allgemelne zielbindung aller an der Raumplanung beteiligten stellen findet sich in $\S 5(4)$ ROG. Damit soll sichergestellt werden, daß den Erfordernissen des Umweltschutzes durch alle Träger der Raumplanung entsprochen wird. Das soll noch einmal mit dem Schema auf S. 224 verdeutlicht werden 103)

DaB die Gemeinden bei der Bauleitplanung den Erfordernissen des Umweltschutzes genügetun müssen, ergibt sich auch bereits aus $\S 1(4)$ und (5) BBaug, wonach Bauleitpläne Belange der Gesundheit der Bevölkerung und des Natur- und Landschaftsschutzes berücksichtigen müssen.

Nach diesen allgemeinen Ausfuhrungen uber die gesetzlichen Grundlagen des raumplanerischen Umweltschutzes wollen wir nun konkrete MaBnahmen der Raumordnung zwecks Vermeldung von Umweltbelastungen betrachten. Ausgangspunkte fur raumordnende Maßnahmen des Umweltschutzes sollten die Daten aus den Emlssions- und Immissions-

102) Denkschrift der MKRO, a.a.0., S. 28.

103) Zusammengestellt nach: Malz, F., Taschenwörterbuch der Umweltplanung, a.a.0., S. 443; Stahl, K./Curdes, G., Umweltplanung in der Industriegesellschaft, Hamburg 1970, S. 14; Stelger, H., Umweltschutz durch planende Gestaltung, in: Zeitschrift für Rechtspolitik, 1971, S. 137; Denkschrift der MKRO, a.a.O., S. $27 \mathrm{ff}$. 


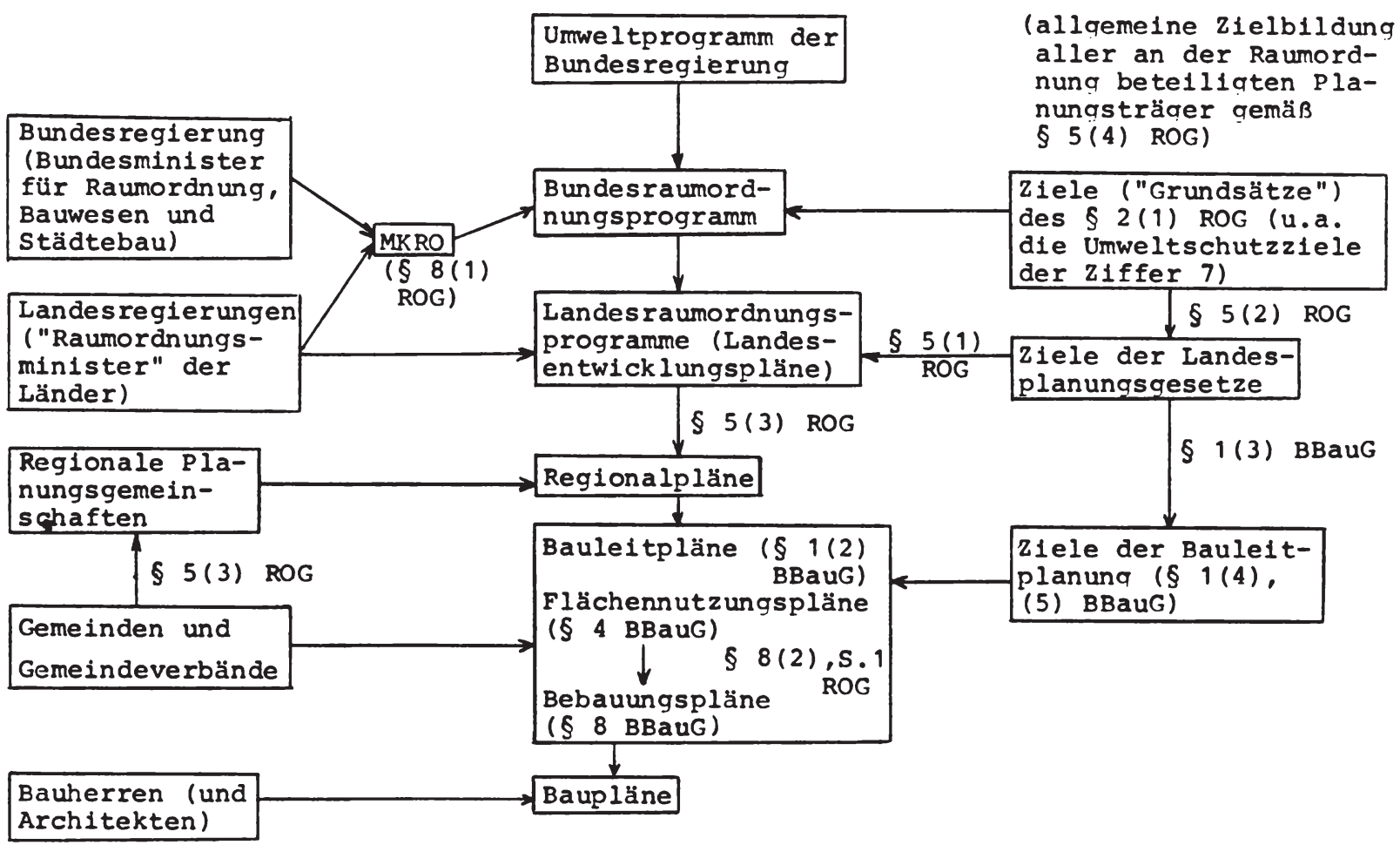


katastern sein 104). Die Emissionskataster geben die standorte der Emissionsquelien sowie Art und Menge der emittierten Schadstoffe an, und die Immissionskataster informieren über die Schadstoffbelastung des Raumes. Diese Informationen lassen nun zusammen mit den natürlichen standortfaktoren wie Klima und Topographie 105) sowie Emissionsprognosen 106) Schlüsse über den unter Umweltgesichtspunkten optimalen Standort emissionsträchtiger Objekte (z.B. Industrieansiedlungen, Kernkraftwerksbauten 107), Flugplätze) und immissionsgefährderter Projekte (z.B. Wohnsiedlungen, Fischzuchtbetriebe, Landwirtschaft) zu. Viele der bisherigen Umweltbelastungen hätten sich allein durch eine Umweltgesichtspunkte berücksichtigende Raumordnung vor allem in der Bauleitplanung der Gemeinden vermeiden lassen 108)

$104)$ Vgl. Denkscrrift der MKRO, a.a.O., S. 25; RSU, Umweltgutachten 1974, a.a.O., S. 96; Glück, K. u.a., Lärmkarten als Hilfsmittel für die Stadtplanung, Schriftenrelhe des Bundesministers flir Raumordnung, Bauwesen und städtebau, BonnBad Godesberg 0.J.

105) Vgl. Dreyhaupt, F.J., Luftreinhaltung als Faktor der stadt und Regionalplanung, a.a.O., S. 59 ff.; Georgil, H.-W., Wir brauchen eine "Luftreinhaltungsstrategie". Wie die meteorologische Wissenschaft bel der standortplanung helfen kann, in: Blick durch die Wirtschaft v. 23.9.71; Stockmann, H.-U., Die natürlichen Faktoren in der Planung, a.a.0., S. $369 \mathrm{f}$.; McCormick, R.A., Meteorological Aspects of Air Pollution in Urban and Industrial Districts, a.a.0., s. 3.

106) Vgl. Kuhlmann, A., Ein Instrument der Raumplanung: Vorsorge durch Emissionsprognose, in: Umwelt 1974, 6, S. 30 ff.; Schlipköter, H.-W., Industrleansiedlung und Umweltschutz, in: Raumforschung und Raumordnung, 30. Jg., 1972, S. 204.

107) Für die umweltgerechte Standortplanung von Kernkraftwerken haben Bund und Länder gemeinsam ein Verfahren ausgearbeitet. Mit $\mathrm{H} 1 \mathrm{lfe}$ nuklearspezifischer Standortbewertungsdaten soll der optimale Standort für Kernkraftwerke ermittelt werden. Dieses Verfahren ersetzt aber nicht die standortprufung im atomrechtlichen Genehmigungsverfahren (vgl. Umwelt, $1975,2, \mathrm{~S} .16)$.

108) Vgl. Häberle, M., Industrielärm: Versagen die Vorschriften?, In: Umwelt, 1975, 2, S. 27; Krane, D., Lärmschutz: Unberechtigte Vorwurfe gegen die "TA Lärm", in: Umwelt, 1975, 5, S. 38; Müller, Walter, Umweltschutz gegen Industrieansiedlung: Die Grenzen kommunaler Planungshohe1t, in: Juristische Schulung 1975, 4, S. $228 \mathrm{ff}$. 
Wenn Gemeinden den Bau von Sledlungen neben Autobahnstraßen, die Ansiedlung von Industrieunternehmen in Wohngebieten oder den Bau von Schulen an verkehrsreichen Straßen zulassen, sind das Verstöße gegen $\S 1(4)$ BBauG und gegen $\S 2(1) 2$. 7 ROG sowie die entsprechenden Bestimmungen der Landesplanungsqesetze.

Elne den Umwelterfordernissen gerecht werdende Raumordnung muB Gewerbe- und Wohngeblete so elnander zuordnen, daß die Immissionsbelastung der Wohnbevölkerung möglichst gering ist 109). Die Möglichkieten dazu haben die Gemeinden, indem sie in ihren Flächennutzungsplänen wohngeblete und Gewerbegebiete nach $\S 5$ BBaug im notwendigen Abstand voneinander ausweisen und möglichst elne grüne schutzzone zwlschen diesen Gebleten einplanen 110); Art und Maß der baulichen Nutzung werden dann in den Bebauungsplänen festgelegt $(\S 9(1), Z$. 1 BBauG); die Nutzung der von Bebauung frelzuhaltenden Flächen erfolgt nach den Möglichkeiten des $\S 9(1), \mathrm{Z}$. 2 BBauG. Zur Lösung eines speziellen Immissionsschutzproblems, nämlich dem Schutz von Flugplatzanwohnern vor Lärmimmissionen, ist nach $\S 2(2)$ des Gesetzes zum Schutz gegen Fluglärm ${ }^{111)} \mathrm{v}$. 30.3.71 eine Einteilung der Flugplatzumgebung in zwei Lärmschutzzonen vorgeschrieben; nach $\S 5(2)$ dürfen in Lärmschutzzone 1 kelne Wohnungen neugebaut werden, und in Schutzzone 2 dürfen nach $\S 6$ nur Wohnungen erstellt werden, wenn sie bestimmten Schallschutzanforderungen nach $\S 7$ entsprechen.

109) Dies sieht ergänzend zu den Vorschriften des BBauG auch $\S 50$ BImSchG vor. Es ist eine auch im Interesse der Emittenten liegende Forderung (vgl. Gässler, W., Zur Standortproblematik aus der Sicht der Industrie, in: Raumforschung und Raumordnung, 30. Jg. , 1972, S. 202). Vgl. ferner die $\mathrm{Z}$ iffern 10 und 12 der Entschließung der MKRO "Raumordnung und Umweltschutz" v. 15.6.72, In: Raumordnung und Umweltschutz, a.a.0., s. 10; Dreyhaupt, F.J.. Luftreinhaltung als Faktor der stadt- und Regionalplanung, a.a.0., s. 56 .

110) Vgl. Denkschrift der MKRO, a.a.O., S. 25; Dreyhaupt, F.J., Luftreinhaltung als Faktor der Stadt- und Regionalplanung, a.a.o., s. $71 \mathrm{ff}$.

111) BGBl. I, S. 282 . 
Die bisher dargestellten Maßnahmen der Raumordnung sind präventive Maßnahmen des Umweltschutzes. Selt Verabschiedung des Städtebauförderungsgesetzes (StBFG) besteht aber auch die Mögl1chke1t, ex post raumordnende Masnahmen aus Grunden des Umweltschutzes $z u$ ergreifen. Nach $\S 10$ stBFG kommt eine Verleguna von Betrieben und Wohnungen als raumordnende Maßnahme der Luftreinhaltung und des Lärmschutzes infrage. Damit ist grundsätzlich auch elne Entzerrung von gemischten Wohn- und Industriesiedlungen mögl1ch 112)

Abschliebend wollen wir noch auf die Grenzen - oder gar Gefahren - eines mit Mitteln der Raumordnung betriebenen Umweltschutzes eingehen. Betriebe man den Umweltschutz überwiegend mit Hilfe der Raumordnung, würde das in einem dichtbesiedelten und hochindustrialisierten Gebiet wie der Bundesrepublik Deutschland langfristig dazu füren, daß wegen der aus Umweltschutzgründen erforderlichen Trennung zwischen emissionsintensiven Gebieten und Wohngebieten die frelen Flächen, die vor allem als ökologische Ausglelchsrăume dienen, abnehmen würden. Es kăme zur Zersiedlung der Landschaft, was eindeutig nicht Raumordnungsvorstellungen entspricht 113). Die funktionelle Elnteilung in Zonen (Wohngebiet, Industriegebiet, Naturschutzgebiet usw.) führt zu einer Monotonie, die u.a. aus psychosozialen Gründen unerwünscht ist 114). Die strikte Trennung der Wohn-, Industrie- und Erholungsgeblete verursacht beträchtliche Verkehrsprobleme 115),

112) Vgl. Denkschrift der MKRO, a.a.O., S. 33; Steiger, H., Umweltschutz durch planende Gestaltung, a.a.0., S. 137. Uber ein solches in die gewachsene Raumordnung eingreifendes Instrumentarium verfügten bereits in der Vergangenhelt vor allem die Zentralverwaltungswirtschaften Osteuropas (val. Höhmann, H.-H./Seldenstecher, G./Vajna, T., Umweltschutz und ökonomisches System in Osteuropa, a.a.O., S. 63 und S. 164).

113) Vgl. Denkschrift der MKRO, a.a.O., S. 21; Hartkopf, G., Umweltgestaltung durch Raumordnung, a.a.0., S. 146 .

114) Vgl. Antoine, S., Die Qualitat der Umwelt und die Raumordnung, a.a.O.. S. 81. Nach der Denkschrift der MKRO ist "eine gewisse Funktionsmischung ... für eine erlebnisreiche Umwelt erwinscht" (vgl. dort S. 20).

115) Vgl. Baumberger, H., Wirtschaftliche Probleme elner um- 
die ihrerselts wiederum starke Umweltbelastungen hervorrufen. Um die Immissionsgrenzwerte elnhalten zu können, muß ein überwiegend auf die Raumordnung vertrauender Umweltschutz die Industrieansiedlungen breit streuen 116); auch das beansprucht viel Raum und wirft zusätzliche Verkehrsprobleme auf, die ihrerselts wieder Umweltbelastungen verursachen.

In allen diesen Fällen gerăt die Raumordnung, wenn sie dem Umweltschutz dienen w111, mit (anderen) elgenen zielen - wie etwa der Vermeldung elner zersledelten Landschaft - in Konflikt. Aus diesem Dilemma kann die Raumordnungspolitik nur befreit werden, wenn sie von der Aufgabe des Umweltschutzes so weit entlastet wird, daß sie thren anderen $\mathrm{z}$ ielen in genügendem Umfang entsprechen kann. Die Raumordnung muß vor allem durch Maßnahmen des technischen Umweltschutzes ergänzt und dadurch entlastet werden. Je mehr Emissionen technisch reduziert und je mehr Immissionen durch technische Maßnahmen verhindert werden können, um so dichter können potentielle Emittenten und Bevölkerung răumlich zusammenrücken oder gar in elnem M1schgeblet verflochten sein und um so mehr wird Platz sein für Naherholungs-, Freizeit-, Natur- und Landschaftschutzgebiete.

c) Technische Maßnahmen des Umweltschutzes

aa) Technik und Umweltschutz

DaB die Umweltbelastung in der Vergangenhelt teilweise die Folge einer Umweltbelange miBachtenden technolog1schen Entwicklung war, scheint unbestreitbar zu sein. Wir haben jedoch gesehen, das

weltkonformen Energleversorgung, In: von walterskirchen, M.P. (Hrsg.), Umweltschutz und Wirtschaftswachstum, a.a.0., S. 239; Schaefer, H., Die Okologie als Problem des Umweltschutzes, a.a.0., S. 137 .

116) Vgl. Vogl, J., Bayerns Luftmeßsystem, In: U 1974, 2, S. 6; Binswanger, H.C. E Ene umweltkonforme Wirtschaftsordnung, a.a.0.. S. 139; auch aus Gründen des wirtschaftlichen Recycling von Abfallstoffen ist es z.B. günstig, wenn abfallverursachende und abfallverwertende Industrien verkehrsgünstig (nahe) beleinanderliegen (vgl. Swoboda, H., Probleme der Umweltpolitik, a.a.0., S. 83). 
durch eine Kontrolle der technologischen Entwicklung negative Umweltauswirkungen zumindest begrenzt werden können. Die gezielte Förderung der technologischen Forschung hat außerdem in einem derartigen Umfang zur Entwicklung umweltfreundlicher Technologien und Produkte sowie der Vervollkommnung der Rückhaltetechnologien (dem "klassichen" technischen Umweltschutz) beigetragen 117), daß man heute wohl sagen kann, daß sich das Verhältnis zwischen Umwelt und Technik grundlegend gewandelt hat: Die Technik und die weitere technologische Entwicklung sind zur wesentlichen Voraussetzung des Umweltschutzes geworden; nicht nur weil die Umwelttechnik relativ kurzfristig wirksam ist - etwa im Gegensatz zur Umwelterziehung, sondern auch well sie Umweltschutz ohne elne radikale Begrenzung des Wachstums und Wohlstands ermöglicht.

bb) Ansatzpunkte des technischen Umweltschutzes

Um die grundsätzlichen Möglichkeiten des technischen Umweltschutzes darzustellen, gehen wir von der Annahme aus, daB Umweltbelastungen entstehen

(1) durch die mit der Güterproduktion verbundene Emission von Schadstoffen (die emittierten Schadstoffe sind also Kuppelprodukte der produzierten Guter 118), ,

(2) durch die mit dem Konsum der Güter verbundene Emission von Schadstoffen,

(3) durch die umweltbelastenden Elgenschaften mancher Produkte, wobel $\mathrm{zu}$ unterscheiden ist zwischen Produkten, die

117) Das Angebot der Umweltschutzindustrie an umweltfreundilchen Verfahrenstechniken, Recycling-Techniken und Rückhaltetechniken, ist ebenso beeindruckend wie die meßtechnischen Entwicklungen der letzten Jahre. Aber auch die ubrige Industrie 1st bemulht, ihren Beitrag - zumindest von der Seite der Herstellung umweltfreundlicher Produkte - zum Umweltschutz zu leisten. Um einen Eindruck davon $z u$ erhalten, was es heute alles an Umwelttechniken gibt, sehe man in die Sonderausgabe von $U$ vom Dezember 1976 "Umweltmarkt von A bis $\mathrm{Z}$ " .

118) Vgl. Thoss, R., Zur Planung des Umweltschutzes, in: Raumforschung und Raumordnung, 30. Jg., 1972, S. 180. 
(a) Faktorinputs in der Güterproduktion sind,

(b) konsumiert werden.

Diese Grundannahmen über die Möglichkelten der Entstehung von Umweltbelastungen verknüpfen wir mit der weiteren Annahme, daß zwel Emissionssektoren bestehen, und zwar der Emissionssektor der Produzenten EP (in dem vor allem die Sektoren Industrie, Handwerk, Handel, Verkehr und Landwirtschaft enthalten sind) und derjenige der Konsumenten EK.

Darüberhinaus soll es einen Entsorgungssektor und ein Immissionsgebiet geben, In dem die Schadstoffe zur Umweltbelastung führen. In der folgenden schematischen Darstellung (Abb. 15) wollen wir zunächst zelgen, welche umweltbelastungsrelevanten Güter- und Schadstoffstrome zwischen den einzelnen Sektoren und dem Immissionsgeblet flieBen. Sie sind mit kleinen Buchstaben bezeichnet. Der Strom a bezelchnet den gesamten Güteroutput. Er kann als Faktorinput an den Produktionssektor zurückflieBen $\left(a_{1}\right)$, in den Konsumsektor gehen $\left(a_{2}\right)$ oder als Faktorinput im Entsorgungsbereich dienen $\left(a_{3}\right)$.

Es gilt die Bezlehung

(1) $a=a_{1}+a_{2}+a_{3}$.

Es kann sein, daß $a=a_{1}$; dann handelt es sich um reine Investitlonsgüter (z.B. Baukräne). Wenn dagegen $a=a_{2}$, handelt es sich um reine Konsumgüter (z.B. Apfel), und wenn $a=a_{3}$, so ist es ein Entsorgungsgut (z.B. eine Müllverbrennungsanlage). Der Güterstrom a kann aber auch an zwel oder an alle drel Sektoren gehen (z.B. Heizöl).

Der Strom b glbt den bel der Produktion von a entstehenden Schadstoffoutput wieder, wobel gelten soll, daß

(2) $b=b(a)$

und

(2a) $\frac{\frac{d b}{b}}{\frac{d a}{a}}=1$;

es liegt also starre Kuppelproduktion vor.

Der Schadstoffoutput kann nun zurlickgewonnen werden $\left(b_{1}\right)$, und zwar innerbetrieblich, innerhalb des gleichen Produktionssektors 


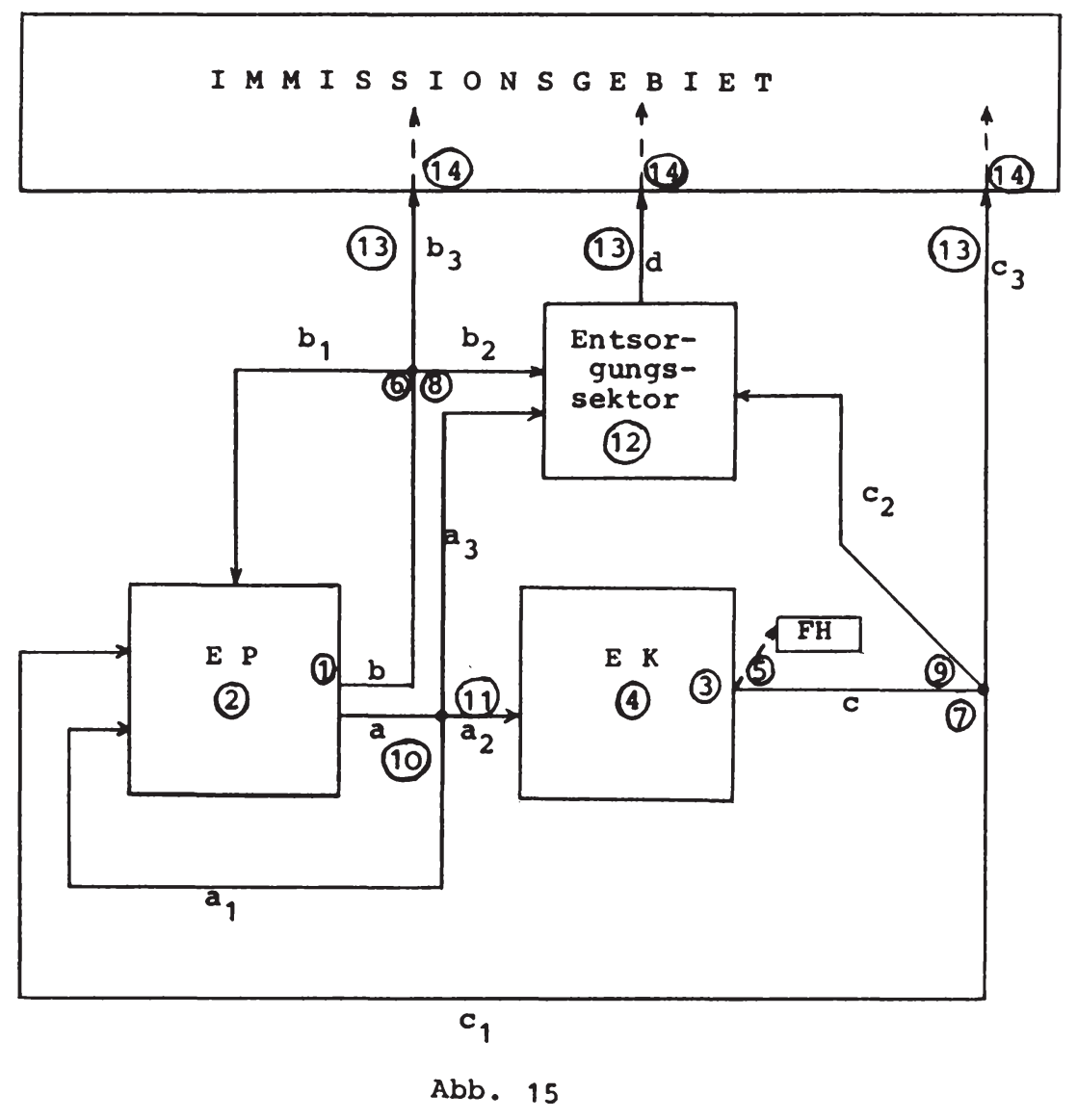

oder von einem anderen Produktionssektor; er kann auch in den Entsorgungssektor fließen $\left(b_{2}\right)$ oder zur Immissionsbelastung führen $\left(b_{3}\right)$. Auch alle Kombinationen sind möglich. Es gilt also: (3) $b=b_{1}+b_{2}+b_{3}$.

Für den Schadstoffstrom $c$ aus dem Konsumsektor gilt das gleiche wie für b: Er kann im Produktionssektor rezyklislert werden $\left(c_{1}\right)$, in den Entsorgungsbereich flleBen $\left(c_{2}\right)$, oder zur Immissionsbelastung führen $\left(c_{3}\right)$. Es sind ebenfalls alle Kombinationen dieser Möglichkeiten denkbar, so daß gilt:

(4) $c=c_{1}+c_{2}+c_{3}$. 
Der Schadstoffoutput d des Entsorgungssektors resultiert zum einen aus seiner Entsorgungsaktivität und ist insofern ein Kuppelprodukt der Schadstoffbeseitigung oder -vernichtung; zum anderen besteht er aus bei gegebener Entsorgungstechn1k nicht weiter reduzierbaren schadstoffmengen, der sogenannten Restverschmutzung.

Die Gesamtbelastung $\mathbf{S}$ des Immissionsgebietes mit Schadstoffen ist nun

(5) $s=b_{3}+c_{3}+d$.

Das ziel der Umweltpolitik, die Einhaltung der Immissionsstandards, ist offenbar gefährdet, wenn die Gesamtbelastung anstelgt. Der technische Umweltschutz muB deshalb dafür sorgen, $\mathrm{daB}$ die Komponenten $\mathrm{b}_{3}, \mathrm{c}_{3}$ und $\mathrm{d}$ möglichst gering sind. Die ersten Ansatzpunkte des technischen Umweltschutzes kann man erkennen, wenn man die Gleichungen (3) und (4) nach $b_{3}$ bzw. $c_{3}$ auflöst; man erhält dann

(6) $b_{3}=b-b_{1}-b_{2}$

und

(7) $c_{3}=c-c_{1}-c_{2}$.

Aus Gleichung (6) folgt:

Die Immissionsbelastung infolge des Schadstoffoutputs der Produzenten $\mathrm{b}_{3}$ ist um so geringer,

(I) je geringer die gesamten Schadstoffemissionen aus dem Produktionssektor b sind,

(II) je gröBer die davon rückgewonnenen schadstoffmengen $b_{1}$ sind,

(III) je größer die dem Entsorgungssektor zugeführten Schadstoffmengen $b_{2}$ sind.

Aus Gleichung ( 7 ) folgt:

Die Immissionsbelastung infolge der schadstoffemissionen der Konsumenten $c_{3}$ ist um so geringer,

(IV) je geringer der gesamte Schadstoffoutput der Konsumente c ist,

(V) je größer die davon im Produktionssektor wiederverwendeten oder wiederverwerteten Schadstoffmengen $c_{1}$ sind, 
(VI) je mehr von den Schadstoffmengen dem Entsorgungssektor $c_{2}$ zufließen.

Die Folgerungen I bis IV implizieren für die Umweltpolitik bzw. die umwelttechnologische Entwicklung folgende Maßnahmen:

Wegen I und IV: Nutzung und Verbesserung aller bekannten technischen Maßnahmen und Entwicklung neuer Technologien zur Reduzierung der Schadstof femissionen im Produktions- und Konsumsektor (Maßnahmenkategorie A);

wegen II und $\mathrm{V}$ : Nutzung und Verbesserung aller bekannten und Entwicklung neuer Techniken des Recycling (Maßnahmenkategorie B);

wegen III und VI: Nutzung und Verbesserung aller bekannten und Entwicklung neuer Techniken der Schadstoffzufuhr zum Entsorgungsbereich (Maßnahmenkategorie C).

Den Maßnahmekategorien $A$ b1s $C$ ist gemeinsam, daB sie sich auf eine unmittelbare Reduzierung der Emissionen beziehen. Wir bezeichnen sie deshalb als schadstoffoutput-orientierte technische Maßnahmen.

Manche Schadstoffemissionen entstehen nun wegen der umweltbelastenden Elgenschaften bestimmter Güter. So kann z.B. die Beziehung gelten, daB der Schadstoffoutput $b$ der Produzenten von ihrem Faktorinput $a_{1}$ abhängt:

(8) $b=f\left(a_{1}\right)$

wobel gilt, daß

(8 a) $\frac{d b}{d a},>0$.

Ferner ist es denkbar, daß der Schadstoffoutput $c$ der Konsumenten von deren Güterinput $a_{2}$ abhängt, al'so gilt:

(9) $c=c\left(a_{2}\right)$,

wobe1

(9 a) $\frac{d c}{d a_{2}} \quad 0$.

Schlieblich kann auch der Schadstoffoutput d des Entsorgungssektors von dessen Faktorinput $a_{3}$ abhängen. Es gilt: 
(10) $d=d\left(a_{3}\right)$

Hierbel sind zwel Fälle zu unterscheiden: $a_{3}$ kann ein Energieträger $a_{31}$ sein, dessen vermehrter Einsatz - wie in den anderen Sektoren auch - die Schadstoffemissionen erhöht. Es gilt dann: (10 a) $\frac{\mathrm{dd}}{\mathrm{da}}>0$.

Es kann sich beim Faktorinput $a_{3}$ aber auch um ein Entsorgungsgut $a_{32}$ handeln, das von der Umweltschutzindustrie bezogen wurde. Dieses wird den Schadstoffoutput des Entsorgungssektors (in Form der Restverschmutzung) reduzieren, so daB gilt:

(10 b) $\frac{d d}{d a_{32}}<0$.

Aus den Beziehungen (8a) und (10a) folgt, daß die Schadstoffemissionen des Produktions- und Entsorgungssektors um so gröBer sind, je umweltbelastender die Eigenschaften der als Faktorinputs verwendeten Güter sind (Fall VII).

Aus Beziehung (9a) folgt, daB die Schadstoffemissionen aus dem Konsumsektor um so größer sind, je umweltbelastender die Eigenschaften der Konsumgüter sind (Fall VIII). SchlieBlich folgt noch aus der Beziehung (10b), das die Schadstoffemissionen des Entsorgungssektors (in Form der Restverschmutzung) um so geringer sind, je effizienter die beschafften Entsorgungstechniken sind (Fall IX).

Daraus kann man als umwelttechnische Aufgabe und für die Entwicklung der Umwelttechnologie ableiten:

Wegen der Fälle VII und VIII:

Anwendung und Verbesserung aller technischen Möglichkeiten, um die bisher produzierten Güter - glelchguiltig, ob sie als Faktorinputs oder Konsumgüter verwendet werden - umweltfreundlicher zu gestalten, und Entwicklung neuer umfreundlicher Substitutionsgüter (Maßnahmenkategorie D)

und wegen IX: Einsatz und Verbesserung aller bekannten und Entwicklung neuer Entsorgungstechniken (Maßnahmenkategorie E). 
Wir wollen die Maßnahmen der Kategorie $D$ als produktorientierte technische Maßnahmen bezeichnen und die der Kategorie $E$ als entsorgungsorientierte technische Maßnahmen.

Abschließend sel noch auf die Fälle eingegangen, in denen der Schadstoffoutput von dem Schadstoffinput in Form von Produktionsund Konsumrückständen abhängt. Das ist vor allem im Entsorgungssektor der Fall, wenn - bel gegebener Entsorgungstechn1k - der Schadstoffoutput d (in Form der Restverschmutzung) mit zunehmendem Schadstoffinput $b_{2}$ und $c_{2}$ aus dem Produktions- bzw. Konsumsektor zunimmt, also gilt:

(11) $d=e\left(b_{2}, c_{2}\right)$,

wobei

(11 a) $\frac{d d}{d b_{2}}>0$.

und

(11 b) $\frac{d d}{d c_{2}}>0$

Aber auch durch Recycling, also einen Schadstoffinput im Produktionssektor, kann der Schadstoffoutput b zunehmen, und zwar sowohl bei einer Rückgewinnung produktionsbedingter Schadstoffoutputs $b_{1}$ als auch bei der konsumbedingter schadstoffoutputs $c_{1}$, so dab gilt:

(12) $b=g\left(b_{1}\right)$,

wobei

(12 a) $\frac{d b}{d b_{1}}>0$.

und

(13) $b=h \quad\left(c_{1}\right)$,

wobei

(13 a) $\frac{d b}{d c_{1}}>0$.

Die Beziehungen (11a) und (11b) kann man so interpretieren, daß bei gegebener Entsorgungstechnik die Schadstoffemissionen aus dem Entsorgungsberelch um so größer sind, je umweltbelastender 
die Eigenschaften der Schadstoffe sind (Fall $\mathrm{X}$ ).

Die Beziehungen (12a) und (13a) kann man so interpretieren, daB das Recycling von Schadstoffen um so mehr schadstoffemissionen hervorruft, je umweltbelastendere Elgenschaften die rezyklisierten schadstoffe haben (Fall XI).

Aus den Fällen $X$ und XI kann man die Forderung ableiten, daß die Umwelttechnik und die umwelttechnologische Entwicklung dafür sorgen müssen, daß die Schadstoffe entsorgungsgerechter und recycling-freundlicher werden, was wohl nur bedeuten kann, daß die Güter (Faktorinputs und Konsunguter), die sich (teilweise) in die Schadstoffe verwandeln, entsorgungsgerechtere und recycling-freundichere Elgenschaften erhalten.

$\mathrm{DaB}$ der Schadstoffoutput des Produktionssektors von den recycling-freundichen Elgenschaften der produkte abhängt, kann man sich folgendermaßen verdeutlichen:

Wir lösen zunächst die Gleichungen (3) und (4) nach b, und $c_{1}$ auf und erhalten dann:

(14) $b_{1}=b-b_{2}-b_{3}$

und

(15) $c_{1}=c-c_{2}-c_{3}$.

In die Gleichungen (14) und (15) setzen wir nun die Gleichungen

(8) bzw. (9) ein und erhalten dann:

(16) $b_{1}=f\left(a_{1}\right)-b_{2}-b_{3}$

und

(17) $c_{1}=c\left(a_{2}\right)-c_{2}-c_{3}$.

Nun setzen wir die Gleichungen (16) und (17) in die Gleichungen (12) bzw. (13) ein und erhalten dann:

$(18) b=g\left[f\left(a_{1}\right)-b_{2}-b_{3}\right]$,

wobe1

(18 a) $\frac{d b}{d b_{1}} \cdot \frac{d b_{1}}{d a_{1}}>0$

und

(19) $b=h\left[c\left(a_{2}\right)-c_{2}-c_{3}\right]$, wobei 
(19 a) $\frac{d b}{d c_{1}} \cdot \frac{d c_{1}}{d a_{2}}>0$.

Schließlich kann man auch noch zeigen, daß die Restverschmutzung im Entsorgungssektor indirekt von der Entsorgungsgerechtigkeit der Güter abhängt:

Man löst zunächst die Gleichungen (3) und (4) nach $b_{2}$ und $c_{2}$ auf und erhält dann:

(20) $b_{2}=b-b_{1}-b_{3}$

und

(21) $c_{2}=c-c_{1}-c_{3}$.

Setzt man in (20) und (21) nun die Gleichungen (8) bzw. (9) ein, so erhält man:

(22) $b_{2}=f\left(a_{1}\right)-b_{1}-b_{3}$

und

(23) $c_{2}=c\left(a_{2}\right)-c_{1}-c_{3}$.

Durch Elnsetzen der Glelchungen (22) und (23 in Gleichung (11) erhält man:

(24) $d=e\left[f\left(a_{1}\right)-b_{1}-b_{3}, c\left(a_{2}\right)-c_{1}-c_{3}\right]$,

wobel gilt:

(24 a) $\frac{d d}{d b_{2}} \cdot \frac{d b_{2}}{d a_{1}}>0$

und

(24 b) $\frac{\mathrm{dd}}{\mathrm{dc_{2 }}} \cdot \frac{\mathrm{dc}}{\mathrm{da_{2 }}}>0$.

Mit der entsorgungsgerechten und recycling-freundlichen Gestaltung der Schadstoffe via umweltfreundliche Gestaltung der Produkte, also mit den Fällen $X$ und $X I$, haben wir elne weitere Maßnahmenkategorie $F$, die wir als rückstandsorientierte technische Maßnahmen bezeichnen wollen.

Die bisher geschilderten Maßnahmen des Umweltschutzes haben gemeinsam, dab sie die Umweltbelastung mehr oder weniger an der Wurzel bekämpfen, nämlich im Emissionsbereich oder zumindest im Entsorgungssektor, der möglichst ohne "undichte" Stellen mit dem Emissionsberelch verbunden sein sollte. Wir wollen diese Maßnahmen als emissions-orientierte technische Maßnahmen des Um- 
weltschutzes bezeichnen.

Es gibt aber auch noch andere Möglichkeiten, Immissionsbelastungen $z u$ vermeiden, und zwar nachdem die Schadstoffe bereits emittiert wurden. Vergegenwärtigen wir uns noch einmal die Ausbreitung der Schadstoffe von der Emission zur Immission, so zeigen sich die Möglichkeiten, die Immissionsbelastungen auch dadurch $z u$ verhindern, daß man durch technische Maßnahmen die Transmission der emittierten Schadstoffe beeinfluBt (also die Schadstoffströme $b_{3}, c_{3}$ und $d$ in $A b b .15$ "verdünnt") oder daB man die emittierten Schadstoffe durch technische Maßnahmen daran hindert, ins Immissionsgebiet zu gelangen. W1r bezeichnen diese beiden weiteren Möglichkeiten des technischen Umweltschutzes als transmissionsorientierte technische Maßnahmen des Umweltschutzes (Maßnahmenkategorie G) und als immissionsorientierte technische MaBnahmen des Umweltschutzes (Maßnahmenkategorie $H$ ). Diese beiden Maßnahmenkategorien sind in der Regel wenlger wirksam als die emissionsorientierten technischen Maßnahmen. Die "Verdünnung" der Schadstoffemissionen während der Transmission verteilt die Schadstoffe auf gröBerem Raum und zögert damit, ceteris paribus, die Umweltbelastung bestenfalls hinaus. Der Nachteil der immissionsorientierten technischen Maßnahmen besteht in ihrer nur selektiven Wirksamkeit: nicht belastet wird lediglich das Gebiet, das sich hinter einer "Immissionsschutzwand" oder unter einer "Immissionsschutzhaube" befindet.

Trotz dieser Einwände gegen die Wirksamkeit der Maßnahmenkategorien $\mathrm{G}$ und $\mathrm{H}$ wollen wir sie als Immissionsbelastungen vermeidende technische Maßnahmen des Umweltschutzes bezeichnen. Sie gehören zusammen mit den emissionsorientierten technischen Maßnahmen (Maßnahmenkategorien A bis F) sowie den Maßnahmen der Emissions- und Immissionsmessung (MaBnahmenkategorie 0 ) zum präventiven technischen Umweltschutz. Von ihm unterscheldet sich die nachträgliche Beseitigung von Umweltschäden, die Umweltsanierung. Auf die technischen Möglichkeiten der Umweltsanierung wollen wir nicht weiter eingehen, aber doch so viel dazu sagen: Die U.E. beste Möglichkeit der Umweltsanierung ist 


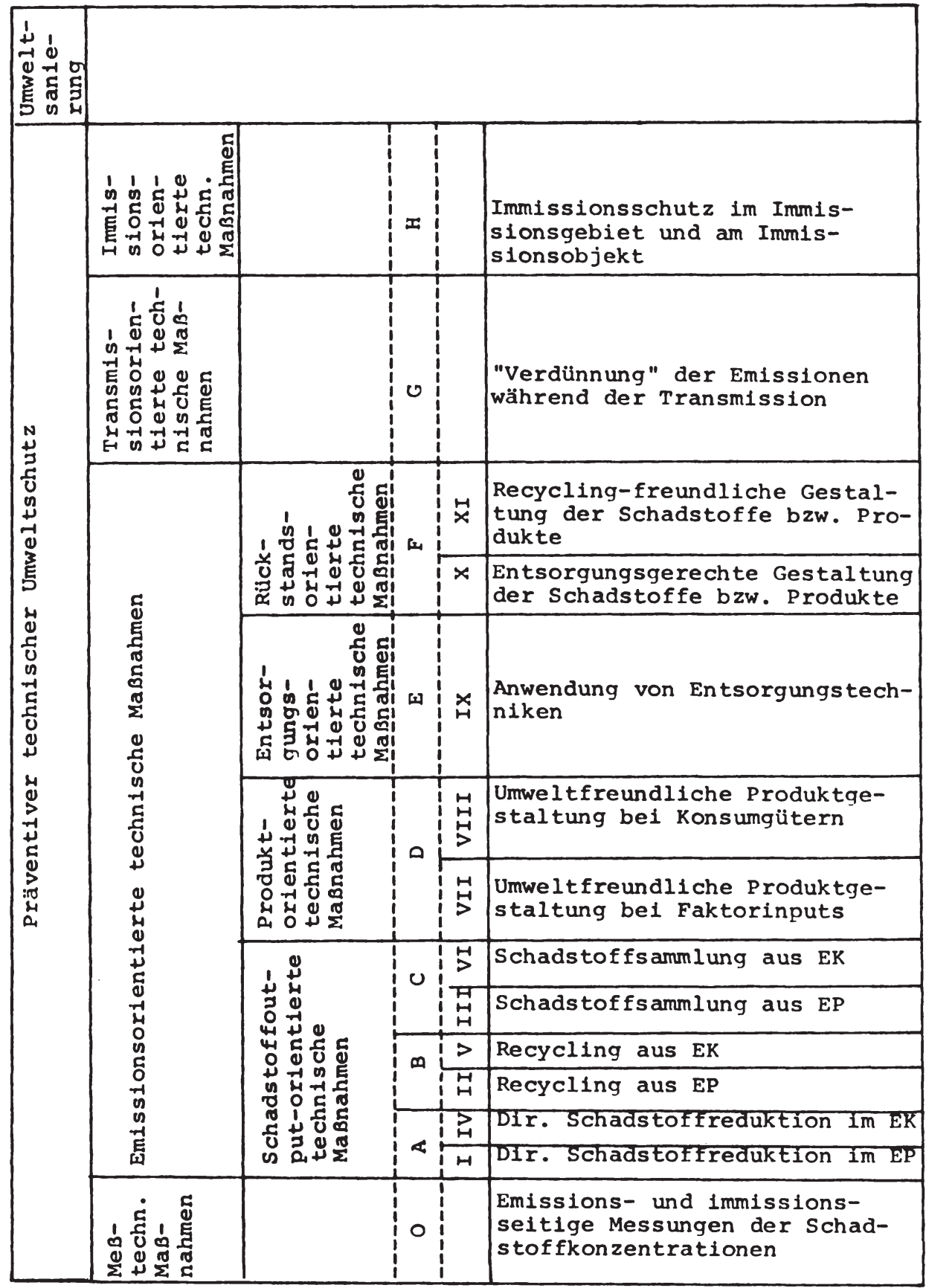


ein verstärkter präventiver technischer Umweltschutz, der es dem zu sanierenden Umweltmedium wieder ermöglicht, seine Selbstreinigungskräfte zu mobilisieren. Eine z.B. einmal im Jahr bei Niedrigwasser von der Bundeswehr oder Pfadfindern durchgeführte "Rheinputzete" ist keine solche Maßnahme.

In der Tabelle auf S. 239 ist noch einmal die in diesem Abschnitt vorgenommene Kategorisierung der technischen Maßnahmen des Umweltschutzes zusammengestellt.

cc) Konkrete Maßnahmen des präventiven technischen Umweltschutzes

(1.) Emissionsorientierte technische Maßnahmen

(1.1.) Schadstoffoutputorientierte technische Maßnahmen

(1.1.1.) Direkte Schadstoffreduktion ...

(1.1.1.1.) ... Im Emissionssektor der Produzenten

Zur direkten Reduktion des Schadstoffstroms b in Abb. 15 gibt es grundsätzlich zwei technische Möglichkelten:

(a) Die Schadstoffe werden an der Emissionsquelle (Schornstein, Auspuff, Abwasserrohr) reduziert (Rückhaltetechnologie) (1) 119)

(b) Der Produktionsproze $B$ wird effizienter gestaltet oder durch einen anderen substituiert (2).

$\mathrm{Zu} \mathrm{(a):}$

Die Rückhaltetechnologien sind die klassischen Techniken des Umweltschutzes. Es handelt sich bel der Luftreinhaltung um sogenannte Abscheidetechniken 120). Die Abscheldung fester und flüssiger luftbelastender Schadstoffe kann vor allem durch mechanische Entstaubung (z.B. in Absetzkammern und Zyklonen), durch Elektronenentstauber, durch NaBabscheider (z.B. Venturier-

119) Die Ziffern in den Klammern entsprechen hier und im folgenden den $\mathrm{Ziffern}$ in $\mathrm{Abb}$. 15 und sollen die Einsatzpunkte der Umwelttechniken zeigen.

120) Vgl. Moll, W.L.H., Taschenbuch für Umweltschutz I: Chemische und technologische Informationen, Darmstadt 1973, S. $29 \mathrm{ff.;}$ Krist, T., Grundwissen Umweltschutz. Zahlen, Daten, Fakten, Bestimmungen, Darmstadt 1974, S. 79 ff.: Klein, A., Relne Luft, Karlsruhe 1971, S. 86 ff.; Umweltmarkt von A bis $Z$, Sonderheft von U, Dezember 1976, S. $120 \mathrm{f}$. 
wäsche, Berieselungswäsche) und durch Flächenfilter (z.B. Schlauchfilter, Taschenfilter) erfolgen. Zur Abscheidung gasförmiger Schadstoffe bedient man sich neben Absorption, Adsorption und der Naßwäsche verschiedener chemischer, thermischer und katalytischer Verfahren.

Innerbetriebliche Rückhaltetechnologien in der Abwasserreinigung sind vor allem Filter; aber auch kühltürme zur Reduzierung der thermischen Belastung der Gewässer gehören i.w.S. zu den Rückhaltetechnologien. Die Kläranlagen, die man auch hier einordnen könnte, wollen wir bei den entsorgungsorientierten Maßnahmen behandeln.

Als Rückhaltetechniken für Lärm seien Schalldämpfer (z.B. an dem Auspuff von Motorrädern) genannt.

Der Nachteil der Rückhaltetechniken vor allem bel der Luft- und Gewässerreinhaltıng ist, daB - teilweise hochgiftige - Reinigungsrückstände anfallen, die, wenn sie nicht rezyklisiert oder entsorgt werden, wiederum zur Umweltbelastung (als Abfall, Klärschlamm usw.) beitragen können.

$\mathrm{Zu}(\mathrm{b})$ :

Diesen Nachteil haben die technischen Maßnahmen nicht, die via Verbesserung oder substitution des Produktionsprozesses die Emissionen $z u$ reduzieren versuchen, da hier bereits der innerbetriebliche Schadstoffanfall verringert wird. Ganz geringe technische Maßnahmen, wie z.B. die richtige Einstellung des Vergasers oder der Einspritzpumpe beim Kraftfahrzeug, können die Abgasemissionen beträchtlich reduzieren. Bei industriellen Produktionsprozessen werden solche Verbesserungs-Maßnahmen wohl relativ weniger "bringen". Dafür kann aber eine Änderung des Produktionsprozesses (ProzeBsubstitution) dazu führen, daß wentger gefährliche Schadstoffe anfallen.

In der Regel wird das daran liegen, daß mit der Prozeßsubstitution auch eine Inputsubstitution erfolgt: z.B. wirkt der Wechsel vom Otto-Motor zum Dieselmotor beim $\mathrm{Kfz}$ deswegen schadstoffreduzierend, well in Dieselöl weniger (gefährliche) Schadstoffe enthalten sind als in Benzin; es kann aber auch sein, daß bel gleichen Inputs die ProzeBsubstitution $z u$ einem geringeren 
Schadstoffanfall führt, weil der neue Produktionsproze $B$ technisch effizienter ist. ProzeBsubstitutionen in Richtung auf umweltfreundlichere, d.h. weniger schadstoffintensive Produktionsverfahren zählen U.E. zu den wichtigsten Maßnahmen des technischen Umweltschutzes.

(1.1.1.2.) ... Im Emissionssektor der Konsumenten Grundsätzlich entsprechen die Probleme der direkten Schadstoffreduktion im Konsumsektor denen im Produktionssektor; allerdings sind zum elnen die technischen Probleme der schadstoffreduktion beim e $1 \mathrm{n} z$ e $1 \mathrm{n}$ e $\mathrm{n}$ Haushalt wegen der relativ geringen Vielfalt und der relativ geringen Schädlichkeit der Emissionen mit weltaus weniger technischem Aufwand lösbar als bei den Produzenten.

Die große Bedeutung des Emissionssektors der Konsumenten für die Umweltbelastung ergibt sich aus der vielzahl der (jeweils relativ unbedeutenden) Emissionsquellen.

Rückhaltetechniken für die vielen einzelnen Emissionsquellen im Konsumsektor (3) kommen bis auf einige Ausnahmen (z.B. die Schalldämpfung von Motor-Rasenmähern) nlcht in Betracht. Um das Hauptproblem der Emissionen aus dem Konsumsektor, den Schadstoffoutput aus den vielen Hausfeuerstellen (vor allem $\mathrm{SO}_{2}$ ), zu reduzleren, kommen vor allem zwel schadstoffoutputorientierte Möglichkelten in Frage:

(a) Eine effizientere Gestaltung des Heizungsprozesses (4),

(b) Eine Zentralisierung und damit Reduzierung der Emissionsquellen (5).

$\mathrm{Zu}(\mathrm{a})$ :

Vor allem bei రlfeuerungshelzungen ließen sich die $\mathrm{SO}_{2}$-Emisslonen stark reduzieren, wenn der Brenner richtig eingestellt wäre. Dadurch könnte bei gleicher Heizleistung nicht nur der రlverbrauch gesenkt werden, sondern das $\measuredangle 1$ würde auch vollständlger verbrennen, so daß aus zwel Gründen die $\mathrm{SO}_{2}$-Belastung zurückginge.

$\mathrm{Zu} \mathrm{(b)} \mathrm{:}$

Da Rückhaltetechnologien sich an den einzelnen Hausfeuerstellen 
nicht lohnen, könnte man den Helzvorgang zentralisieren und die einzelnen Wohngebäude über ein Leitungsnetz mit Wärme versorgen. In einem solchen Fernhelzwerk FH (vgl. Abb. 15) kann dann die bestmögliche Umwelttechnik den Schadstoffoutput begrenzen 121). Man könnte diesen Fall als "Ex ante-Entsorgung" bezeichnen.

\section{(1.1.2.) Recycling}

Man kann vier Fälle des Recycling unterscheiden, und zwar die Rückgewinnung, die Wiederverwendung, die Weiterverwertung und die Weiterverwendung 122). In Abb. 15 entspricht der Schadstoffstrom $b_{1}$ der Rückgewinnung und Schadstoffstrom $c_{1}$ den anderen drei Arten des Recycling.

\section{(1.1.2.1.) Das Recycling innerhalb des Produktionssektors}

Diese Art des Recycling entspricht also der Rückgewinnung von "Schadstoffen" (wir sprechen jetzt besser von Produktionsrückständen). Es kann innerbetrieblich aber auch zwischen verschiedenen Betrieben des gleichen Produktionssektors oder auch intersektoral erfolgen.

In der Bundesrepublik Deutschland etablierten slch inzwischen verschiedene Abfallbörsen (z.B. die des Verbandes der Chemischen Industrie und die des DIHT), an denen rezyklisierbare Schadstoffe gehandelt werden 123). Besonders fortgeschritten ist vor allem die Rückgewinnung von Edelmetallen - sicher nicht zuletzt wegen Ihres Wertes - aber auch von Eisen und Stahl sowie von Nichteisenmetallen 124) Ein gut zum Bild des Recycling passendes

121) Vgl. Baumberger, H., Wirtschaftliche Probleme einer umweltkonformen Energieversorgung, a.a.O.. S. 245 f.; Siegrist, H.R., Die Auswirkungen der Energiewirtschaft auf die Umwelt, in: Leibundgut, H. (Hrsg.), Schutz unseres Lebensraums, a.a.0., s. 58 .

122) Vg1. L1chtwer, L., Zukunftsaspekte der Wiederverwendung, in: U 1975, 4, S. 42 ff.

123) Vgl. ebenda S. 42; Haenert, F., Abfallbörse der Industrieund Handelskammern, in: Umwelt 1974, 5, S. $23 \mathrm{f}$.; Wahl, K., Recycling: Systematisches Vorgehen bel Abfallstoffen, in: Umwelt 1975, 5, S. 43.

124) Vgl. Jobst, J., Recycling-Entwicklung und gegenwärtiger Stand, in: U 1975, 4, S. 37. 
Beispiel ist die kreislaufartige Versorgung von Produktionsprozessen mit Kühlwasser.

(1.1.2.2.) Das Recycling aus dem Konsumsektor (7) Hierbei kann es sich handeln um 125)

(a) Wiederverwendung: hier wird der Schadstoff (wir sprechen jetzt wohl besser von Konsumrückstand) nicht mehr produziert, sondern nur "renoviert"; so wird z.B. die Mehrwegflasche nicht neu produziert, sondern nur gereinigt, gefült und eventuell neu etikettiert und verpackt, bevor sie wieder in den Konsumsektor gelangt.

(b) Weiterverwendung: hier werden Konsumrückstände in den Produktionssektoren wiedereingesetzt, aus denen sie stammen, wie Glas in der Glasindustrie, Altpapier in der Paplerindustrie usw.

(c) Welterverwendung: hierunter versteht man schließlich den Einsatz von Konsumgüterrückständen in nicht güterspezifischen Produktionssektoren, also z.B. die Verwendung von Glasscherben zur Herstellung von Glasphalt, der als Straßenbelag Verwendung findet.

Die verschiedenen Arten des Recycling sind u.E. sehr wichtige technische Maßnahmen des Umweltschutzes, deren Förderung wir nicht nur wegen des Umweltschutzes, sondern auch wegén der Rohstoffverknappung für notwendig halten. Die verschiedenen Möglichkeiten des bereits anwendungsreifen Recycling lassen nur ahnen, welche Möglichkelten es vor allem in bezug auf die Welterverwendung geben kann 126)

\section{(1.1.3.) Schadstoffsammlung}

Die realen Mögl1chkeiten der Schadstoffsammlung zwecks Transport in den Entsorgungssektor beschränken sich auf die flüssigen und

125) Zum folgenden vgl. Lichtwer, L., Zukunftsaspekte der Wiederverwendung, a.a.0., S. 42 .

126) Vgl. den ausführlichen Bericht über die Möglichkelten und Verfahren des Recycling, den der Splegel-Verlag herausgegeben hat: Recycling in der Materialwirtschaft, Expandierende Märkte, Bd. 5, Hamburg 1975. 
festen Schadstoffoutputs, also auf Abwässer und Abfälle.

(1.1.3.1.) Die Sammlung von Schadstoffen aus dem Produktionssektor (8)

Eine umweltgerechte Entsorgung der produktionsspezifischen $\mathrm{Ab}-$

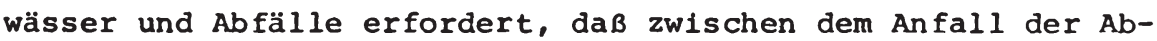

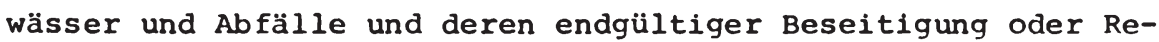
duktion im Entsorgungssektor keine Lücken entstehen, die zu Emissionen führen können. Deshalb müssen Sammlung und Transport der Schadstoffe möglichst lückenlos ineinander übergehen. Die Abwässer des Produktionssektors gelangen in der Regel über die kommunale Kanalisation lückenlos in die Kläranlage, während bei der Sammlung und dem Transport der produktionsbedingten $\mathrm{Ab}-$ fälle - z.B. beim Umladen und bel Transportunfällen - eher Lücken entstehen können. Die technischen Möglichkeiten der Abwassersammlung beschränken sich im wesentlichen auf die Verbesserung der Kanalisation, etwa die Einführung einer Trennkanalisation oder einer Ringkanalisation.

Mehr technische Möglichkeiten gibt es bei der Abfallsammlunq und dem Abfalltransport. Sie reichen von der volumenreduzierung der Abfälle bei der Sammlung durch Zerkleinerung oder Pressung, über die Sortierung, den Transport in Müllcontalnern und die Einrichtung von Umladestationen bis hin zur wünschenswertesten Art der Abfallsammlung und des Abfalltransports: der Einrichtuna eines geschlossenen $\mathrm{Abfallentsorgungssystems} \mathrm{durch} \mathrm{eine} \mathrm{Abfa} 11-$ Pipeline vom Produktionssektor bis zur Abfallbeseitigungsanlaqe.

(1.1.3.2.) Die Sammlung von Schadstoffen aus dem Konsumsektor

Die Abwassersammlung aus dem Konsumsektor erfolgt durch die kommunale Kanalisation. Die Sammlung und der Transport der $A b-$ fälle wirft vor allem wegen des großen Abfallvolumens Probleme auf.

Die technischen Lösungsmöglichkeiten sind die gleichen wie im Falle der produktionsspezifischen Abfälle. Geschlossene Entsorgungssysteme - wie sie bereits in einigen großen Wohnsiedlungen für eine lückenlose Abfallbeseitigung in Gebrauch sind - 
schelnen die beste technische Lösung zu sein, wenn die Abfallbeseitlgungsanlage (in der Regel eine Müllverbrennungsanlage) möglichst emissionsarm ist.

(1.2.) Produktorientierte technische Maßnahmen des Umweltschutzes Die meisten Arten der Umweltbelastung sind die Folge umweltschädlicher Elgenschaften der Produkte, die in den Produktionsproze $B$ als Input oder in den Konsumtionsproze $B$ als Verbrauchsguter eingehen. Die Faktorinputs gehen durch den Produktionsproze $B$ in irgendelner Form in die Endprodukte ein, werden als Schadstoffe emittiert und/oder wandeln sich in wärmeenergie um, die - wenn sie nicht genutzt wird, was eine Form des Recycling wäre - als Abwärme an die Umweltmedien Luft oder Wasser abgegeben wird und so zur thermischen Belastung der Umwelt führt. Sehr wichtig ist auch die mögliche Nutzungsdauer des Faktorinputs. Ist der Inputfaktor z.B. eine Maschine, so ist diese, ceteris paribus, um so umweltfreundlicher, je länger ihre Lebensdauer ist, denn um so geringer ist der schadstoffanfall (hier in Form von Schrott) je Zeiteinheit. Die Konsumgüter sind die Inputs der privaten Haushalte; was sich während des Konsumtionsprozesses nicht in Energie umsetzt, fällt als Schadstoff an. Ein Faktorinput ist nun um so weniger umweltbelastend, je vollständiger er in das Endprodukt eingeht, je wenlger er sich also in Schadstoffe und unerwünchte wärmeenergle aufspaltet (quantitative Komponente) und je unschädlicher eine Mengeneinheit des Schadstoffes für die Umwelt ist (qualitative Komponente).

Unter letzterem Aspekt ist auch die Nutzungsdauer der Konsumgüter für deren Umweltelgenschaften von großer Bedeutung. Je langlebiger die konsumgüter sind, desto geringer ist der schadstoffanfall (z.B. Schrott) je zeiteinheit.

(1.2.1.) Technische Maßnahmen zur umweltfreundlichen Gestaltung der Faktorinputs (10)

Es bestehen grundsätzlich drel Möglichkeiten, die Faktorinputs umweltfreundicher zu gestalten: 
(a) Man verbessert die bisher verwendeten Inputs hinsichtlich ihrer Umweltfreundlichkeit (durch Reduktion der potentiellen Schadstoffanteile).

(b) Man substitulert sie durch andere - umweltfreundlichere Inputs (Faktorsubstitution).

(c) Man verlängert die Lebensdauer der Faktoren, sofern sie Investitionsgüter sind.

$\mathrm{zu}$ (a):

Technische Lösungen zur umweltrelevanten Verbesserung der Inputs bestehen z.B. darin, die Bestandtelle aus thnen zu entfernen, die - durch den Produktionsproze $B$ bedingt - als Schadstoffe frei werden. Beispiele für solche technischen Maßnahmen sind die Entschwefelung von leichtem und vor allem von schwerem Heizöl und die Herstellung blelfreien Benzins. $\mathrm{Zu}$ (b):

Ist eine solche Entfernung der potentiellen Schadstoffe ex ante technisch (noch) nicht möglich, können die umweltschädlichen Inputs durch andere (umweltfreundlichere) ersetzt werden, was in den meisten Fällen auch eine Änderung des Produktionsprozesses erfordert. Man kann die Inputs gegen bereits bekannte (und verfügbare) umweltfreundlichere Inputs substituieren - z.B. Heizöl gegen Erdgas, DDT gegen schneller abbaubare Pesticlde - oder nach neuen Inputs suchen bzw. serienreif gestalten (z.B. Sonnenenergle, biologische Schädlingsbekämpfungsmittel).

$\mathrm{zu}$ (c):

Handelt es sich bei den Inputs um Investitionsgüter, so sind alle technischen Möglichkelten zur Verlängerung ihrer Lebensdauer zu nutzen (z.B. Rostschutz, Verwendung verschleiBarmer Werkstoffe). Es ist dabel darauf $z u$ achten, daB die längere Lebensdauer des Anlagegutes nicht mit zeitlich zunehmender Emissionsintensität erkauft wird.

(1.2.2.) Technische Maßnahmen zur umweltfreundlichen Gestaltung der Konsumgüter (11)

Wenn die Faktorinputs umweltfreundlicher gestaltet werden, wirkt sich das auch auf die umweltfreundlichere Gestaltung der Konsum- 
güter aus, und zwar zum einen deswegen, weil manche Güter sowohl als Faktorinputs als auch als konsumgüter dienen können (z.B. Heizöl), zum anderen deswegen, weil manche Schadstoffe nicht bei der produktion, sondern erst beim konsum freigesetzt werden (z.B. Rückstände in Lebensmitteln, die sogar bei der Produktion absichtlich zugesetzt werden). Durch (agrar-)technische Maßnahmen wie etwa den biologischen Anbau von pflanzlichen Produkten kann eine umweltfreundliche Gestaltung von Konsumgütern realisiert werden. Zur Reduktion des Schadstoffstroms $c$ in Abb. 15 wirde auch eine substitution der Konsumguter (etwa der detergentienhaltigen in umweltfreundliche Waschmittel) beitragen. Von großer Umweltrelevanz ist ferner die Verlängerung der Lebensdauer der Konsumgüter - auch aus Gründen der Rohstoffknappheit -, etwa durch die Verwendung stabileren Materials, oder einfach die Unterlassung der geplanten Obsoleszenz - doch letzteres ist kein technisches Problem.

Insgesamt gesehen halten wir die technischen Maßnahmen des Umweltschutzes, welche auf eine umweltfreundlichere Gestaltung der Güter (Faktorinputs und Konsumgüter) zielen, für die förderungswürdigsten, da sie bereits den Schadstoffanfall reduzieren und so gar keine Recycling- oder Beseitigungsprobleme durch Rückhaltetechnologien oder Entsorgungsmaßnahmen aufkommen lassen.

(1.3.) Entsorgungsorientierte technische Maßnahmen des Umweltschutzes (12)

Einige entsorgungstechnische Maßnahmen haben wir mit der Schadstoffsammlung und dem Schadstofftransport in den Entsorgungssektor bereits behandelt. Nun geht es un die Frage der eigentlichen Entsorgung, also der möglichst wenig Umweltbelastungen hervorrufenden Beseitigung von potentiell umweltbelastenden Schadstoffen. Die Aufgaben des Entsorgungssektors bestehen in der möglichst vollkommenen Klärung der Abwässer aus dem Produktions- und dem Konsumsektor sowie der möglichst vollständigen Beseitigung von produktions- und konsumspezifischen Ab- 
fällen.

Die Kläranlagen können aus drei stufen bestehen 127) : der mechanischen, biologischen und chemischen Stufe. In der (ersten) mechanischen Stufe können lediglich Abwassergrobstoffe (z.B. durch Rechen) und feinere Stoffe durch Absetzen im Vorklärbecken entfernt werden. In der (zweiten) biologischen stufe werden vor allem die organischen Verschmutzungen durch Bakterien im belüfteten Belebtschlammbecken beseltigt. Es kommt zu einer Mineralisierung der organischen Schadstoffe, der sogenannten Flockung, so daß durch Sedimentation eine mechanische Trennung vom Wasser erfolgen kann. Wegen der starken organischen Belastung der Abwässer sollte jede Kläranlage zumindest eine biologische Reinigungsstufe besitzen. Vor allem die toxischen Schadstoffe in den Abwässern machen jedoch die technisch aufwendigste Lösung der Abwasserreinigung, die (dritte) chemische Stufe, immer notwendiger, wenn eine toxische Belastung der Gewässer vermieden werden soll. In der chemischen Stufe werden die toxischen Schadstoffe im Abwasser chemisch umgewandelt und zwar so, daß sie mechanisch vom Wasser getrennt werden können. Um in der dritten Reinigungsstufe auch pathogene Mikroorganismen mit Sicherheit aus dem Abwasser beseitigen zu können, wird das Abwasser über Aktivkohle geleitet und durch Ozon oder Erhitzung sterilisiert.

Mit dieser technisch sehr aufwendigen Reinigungsstufe kann das Abwasser aber doch nur bis $\mathrm{zu} 608$ von Quecksilber und bis $\mathrm{zu}$ 808 von cadmium gereinigt werden, ein sicher noch nicht idealer Wert, so daß hier elne wichtige Aufgabe für die technologische Forschung besteht.

Nach der Abwasserrelnigung stellt sich für, den Entsorgungssektor ein Entsorgungsproblem: die aus dem Abwasser geklärten Schad-

127) Vgl. Moll, W.L.H., Taschenbuch für Umweltschutz I, a.a.0., S. $106 \mathrm{ff.:}$ Krist, T., Grundwissen Umweltschutz, a.a.0., S. $239 \mathrm{ff.;}$ Klee, O.. Reinigung industrieller Abwässer. Grundlagen und Verfahren, Stuttgart 1970, S. 24 ff.: Martz, G. Methoden der Abwasserreinigung, in: U $1976,3, \mathrm{~S} .22 \mathrm{f}$.; Müller, Wilhelm J., Probleme der Reinhaltung von Gewässern, Bielefeld 1973, S. 29 ff.; Umweltmarkt von A bis Z, a.a.O., S. $48 \mathrm{ff}$. 
stoffe, der sogenannte Klärschlamm muß beseitigt werden. Dafür kommen alle Möglichkeiten der ordnungsgemäßen Abfallbeseitigung in Frage - womit wir bei der nächsten Aufgabe der Entsorgung sind.

Es gibt grundsätzlich vier Möglichkeiten einer umweltgerechten Abfallbeseitigung 128): die geordnete Deponie, die Abfallverbrennung, die Pyrolyse und das - uns bereits bekannte Recycling. Hinzu kommen für besonders gefährliche Abfälle wie Giftmüll und radioaktive Rückstände spezielle Beseltigungs- oder Ablagerungsverfahren.

Die Standorte für geordnete Deponien müssen vor allem unter Beachtung des Grundwasserschutzes ausgesucht werden. Die Ablagerung der Abfälle aus dem Produktions- und Konsumsektor erfolgt dann schichtweise. Der Abfall wird - nach eventuell vorangegangener Zerkleinerung und Verrottung in Mieten - mit Planierraupen verteilt und am besten auch (z.B. mit StampffuB- oder Messerwalzenverdichtern) verdichtet. Auf diese Abfallschicht wird dann eine Schicht unzersetzbaren Materials wie Bauschutt oder Erdaushub aufgebracht - in Ermangelung solchen Materials kann auch eine Abdeckung mit Schaum erfolgen -, bevor die nächste Abfallschicht aufgetragen wird. Nach Erschöpfung der Ablagerungskapazität, wird die Deponie abgeschlossen, und das Gelände kann - z.B. für Freizeitzwecke - rekultiviert werden. Die geordnete Deponie wird auch in $\mathrm{Zukunft}$ ihre Bedeutung haben - zumindest für die Rückstände aus den anderen Abfallbeseltigungsverfahren.

Ein weiteres Verfahren der Abfallbeseitigung ist die Abfallverbrennung. Es gibt Verbrennungsanlagen für spezielle Abfallarten (z.B. Klinikabfälle, radioaktlve Abfälle) aber auch solche, die fast alles - von den Küchenabfällen bis zum Giftmüll - verbrennen. Damit die Abfallverbrennung nicht $z u$ Belastungen der Atmosphäre führt, müssen alle prozeBtechnischen und rückhaltetechnologischen Möglichkeiten der Emissionsreduktion bel der Abfallverbrennung genutzt werden. Die Verbrennungsrückstände müssen

128) Vgl. Moll, W.L.H., Taschenbuch für Umweltschutz I, a.a.O., S. $209 \mathrm{ff}$; Krist, T., Grundwissen Umweltschutz, a.a.0., S. $264 \mathrm{ff.;}$ Umweltmarkt von A bis $\mathrm{Z}, \mathrm{a.a.0.,} \mathrm{S.} 12 \mathrm{ff}$. 
geordnet deponiert werden.

Die Pyrolyse, die Entgasung oder auch Vergasung von Abfällen, ist ein in den letzten Jahren neu entwickeltes technisches Verfahren der Abfallbeseitigung 129), dessen Vor- und Nachteile vor allem gegenüber der Abfallverbrennung wohl erst die praktische Erprobung zeigen kann.

Das Recycling von Abfällen haben wir bereits kennengelernt, allerdings handelte es sich dabei um ein direktes Recycling ohne Zwischenschaltung des Entsorgungssektors. Es ist nun auch möglich, daß Abfälle die in den Entsorgungssektor gelangen - oder aber erst dort entstehen -, rezyklisiert werden. Im ersten Fall übernimmt der Entsorgungssektor eine Verteilerfunktion (eventuell nach vorheriger Sortierung); im zweiten Fall stellt er aus den rezyklisierbaren Entsorgungsrückständen selbst (neue) Produkte her.

Ein Beispiel für den ersten Fall ist die Aussortierung von Glas oder Altpapler aus dem Müll und dessen Weiterleitung an die Glas- oder Papierindustrie. Im Entsorgungssektor entstehen aber auch Entsorgungsrückstände wie Klärschlamm bei der Abwasserbeseitigung, Abfallberge bel der geordneten Deponie und Abwärme bel der Verbrennung und Pyrolyse der Abfälle, die rezyklistert werden können.

Wenn der Klärschlamm vor allem von den toxischen Metallbestandteilen und den pathogenen M1kroorganismen befreit werden kann, besteht die Möglichke1t, ihn zusammen mit Hausmüll zu kompostieren 130). Man kann damit drei Umweltprobleme gleichzeitig lösen: die Hausmüllbeseitigung, die Klärschlammbeseitigung und

129) Vgl. Fichtel, K., Pyrolyse von Hausmüll: Vier Verfahren könnten erprobt werden, in: Umwelt, 1975, 4, S. 42 ff.; Tabasaran, 0., Abfallbeseitigung: Pyrolytische Behandlung von Hausmüll und Abwasserschlamm, in: Umwelt, 1976, 2, S. $84 \mathrm{ff}$. ; Flchtel, K., Entgasen oder Vergasen?, In: U 1975, 3, S. $28 \mathrm{ff} . ;$ Nowak, F., Entgasung und Vergasung kombiniert, in: U $1976,4, S .28$ ff.

130) Vgl. Stegmaier, W., Dünger aus Klärschlamm, in: U 1976, 3, S. $24 \mathrm{ff} . ;$ O.V., Dünger aus Klärschlamm, in: U 1974, 2, S. 20 f.; Pätschke, H., Lösung mit zukunft: Kompostierung, in: U 1974, 1, S. 28 ff.; Straub, H., Kompostlerung der Abfälle, sinnvollste Methode, in: Umwelt, 1972, 3, S. $56 \mathrm{ff}$. 
die umweltfreundlichere Gestaltung der Düngung. Die Nutzung geschlossener und rekultivierter Abfalldeponien zu Freizeitund Erholungszwecken 131)

Die bei der Verbrennung und Pyrolyse entstehende Abwärme kann ebenfalls rezyklisiert werden, indem sie zur Versorung der Bevölkerung mit Heizwärme genutzt wird. Hier bietet sich die Möglichkeit, ein Fernheizwerk an den Entsorgungssektor zu koppeln und auf diese Art zwel Umweltprobleme gleichzeltig zu lösen: die Reduktion der Abwärme aus dem Entsorgungssektor und die Reduktion der Schadstoffe aus den Kleinfeuerungsanlagen der Wohngebäude via zentrale Wärmeversorgung.

Große Probleme wirft die Beseitigung besonders gefährlicher Abfälle auf. Die Versenkung von toxischen stoffen in spezialbehältern im Meer oder die Ablagerung von radioaktiven Abfällen in Stollen von ausgedienten Salzbergwerken sind letztlich keine endgültigen Lösungen, da sie im Grunde genommen keine B es e $i$ t $i$ u n $g$ der Abfälle darstellen, so daß die Gefahren, die von ihnen ausgehen können zwar nicht mehr akut, aber doch noch latent vorhanden sind.

\section{(1.4.) Schadstofforientierte technische Maßnahmen}

Die umweltbelastenden Eigenschaften einiger Schadstoffe äußern sich u.a. darin, daß sie diese erst bei der ordnungsgemäßen Beseitigung oder beim Recycling offenbaren. Dem kann dadurch begegnet werden, daß man die Produkte, die sich teilwelse oder ganz in Schadstoffe verwandeln, so gestaltet, daß wenigstens ihre ordnungsgemäße Beseitigung oder ihr Recycling nicht zu Umweltbelastungen führt.

(1.4.1.) Die entsorgungsgerechte Gestaltung der Schadstoffe (bzw. Güter) $(=10,11)$

Bel der Produktgestaltung sollte man nicht nur darauf achten, daß die produkte an sich schon möglichst geringe Umweltbelastungen

131) Vgl. Olschowy, G., Camping auf Müllwiesen, In: U 1972, 1, S. 26 ff.; Bickel, E., Müllkippen als künftige Erholungsgeblete in Holland, in: Müll und Abfall, 4. Jg., 1972, H. 3, S. 100 . 
hervorrufen, sondern auch darauf, daß die Produkte, wenn sie schon nach Durchlaufen des Produktions- und Konsumprozesses zu Schadstoffen werden, dann wenigstens relativ leicht beseitigt werden können.

In der Vergangenheit haben vor allem kunststoffe, die immer mehr in Produktion und Konsum (z.B. als Verpackungsmaterial, Tragetaschen) Verwendung finden, deshalb zu einem Beseitigungsproblem geführt, weil sie entweder biologisch nicht abbaubar waren oder weil sie bei der Verbrennung wegen ihres hohen Chlorgehaltes - wie bei Polyvinylchlorid (PVC) - zur Bildung von Chlorwasserstoffemissionen, aber auch zur Korrosion in den Feuerungsanlagen führten 132) Das Problem der Abbaubarkeit von Kunststoffen ist heute durch die Verwendung von Photooxidantien bei der Kunststoffherstellung praktisch gelöst. Sie bewirken, daß die Kunststoffe unter Einwirkung der ultravioletten Sonnenstrahlung zerfallen und dadurch mikrobiell abbaubar werden 133)

(1.4.2.) Die recycling-freundliche Gestaltung der Schadstoffe (bzw. Güter) $(=10,11)$

Bei der Produktgestaltung sollte man auch daran denken, daß die Produkte, wenn sie Abfall geworden sind, eventuell gut rezyklisierbar sind und dabei nicht auch noch Umweltbelastungen hervorrufen.

Das Recycling von Altpapier ist tellweise dadurch sehr erschwert, daB sich manche Druckfarben nur unter großem Aufwand entfernen lassen - man spricht von "de-inken" -, was auch zu einem beträchtlichen Gewässerverschmutzungsproblem führt, so daß das Recycling von Altpapier vor allem bei den Auswaschverfahren

132) Vgl. Pfennig, N., Verhalten von Kunststoffen bei der Abfallbeseitigung, in: Probleme der Umweltforschung, a.a.0., S. $140 \mathrm{ff} . ;$ Rasch, R., Kunststoffe in der Müllverbrennung, in: U 1971, 4, S. 20 ff.; derselbe, Emissionen aus Müllverbrennungsanlagen bei Müll- und Kunststoffverbrennung, in: Müll und Abfall, 4. Jg., 1972, H. 3, S. 88 ff.

133) Vgl. Jeschke, H., Verpackung - ein teurer Service, in: $\mathrm{U} 1973,3, \mathrm{~S} .32 \mathrm{ff}$; : Rasenack, D., Kommen die abbaubaren Kunststoffe?, in: Blick durch die Wirtschaft v. 30.8.71; Pfennig, N., Verhalten von Kunststoffen bel der Abfallbeseitigung, a.a.0., S. $146 \mathrm{f}$. 
"oft mehr Umweltprobleme (aufwirft) als es löst" 134). Seit längerer Zeit gibt es das recht komplizierte De-Inking durch Flotation, das umweltfreundlicher ist; der dabei entstehende Schmutzschaum wird entwässert und anschließend deponiert oder verbrannt 135). Eine weltaus bessere Lösung wäre jedoch die Verwendung recycling-freundlicherer Druckfarben.

\section{(2.) Transmissionsorientierte technische Maßnahmen (13)}

Diese Maßnahmen zielen darauf ab, die Emissionen zu "verdünnen". Das kann 2.B. durch "Filterung" der Emissionen während der Transmission geschehen. Solche "Filter" sind wälder und Grünzonen, welche z.B. Staubpartikel und $\mathrm{SO}_{2}$ aus der Luft absorbieren und so bestimmte Immissionsgebiete schützen. Eine Filterwirkung gegen Lärm haben ebenfalls wälder und vor allem Lärmschutzwälle. Eine Verdünnung von Emissionen kann man aber auch durch die Ausnutzung natürlicher Faktoren wie wind und strömung erreichen. $\mathrm{Zu}$ den klassischen Maßnahmen des technischen Umweltschutzes gehört die Errichtung möglichst hoher Kamine, um die stärkeren Luftströmungen in der Höhe zur besseren Verteilung der Emissionen zu nutzen 136). Auch die Freihaltung der Durchlüftungszonen in Städten und eine entsprechende Ausrichtung der Straßen bel dem Bau neuer siedlungen will die Verteilung der Schadstoffe in der luft fördern. Dies ist allerdings primär eine Aufgabe der Baule1tplanung. Auch die strömungsverhältnisse in Gewässern kann man zur schnelleren Diffusion der Abwässer und der Kühlwässer nutzen 137) Hier kann die Raumordnung (konkreter: die standort-

134) Recycling in der Marktwirtschaft, a.a.0., s. 61; vgl. auch Lichtwer, L., Zukunftsaspekte der Wiederverwendung, a.a.0., S. 44.

135) Hierbel handelt es sich um den Fall einer substitution des Recycling-Prozesses. Eine solche ProzeBsubstitution ist auch in den Fällen anzustreben, in denen das Recycling sehr viel Energle beansprucht (vgl. Jobst, J., Recycling - Entwicklung und gegenwärtiger Stand, a.a.0., S. 36).

136) Vgl. Moll, W.L.H., Taschenbuch für Umweltschutz I, a.a.O., S. 30 .

137) Vgl. Naudascher, E., Strömungsforschung im Dienste des Umweltschutzes, in: Schultze, H. (Hrsg.), Umwelt-Report, 
planung) technische Maßnahmen unterstützen, indem z.B. Einleiter von Abwässern an besonders strömungsstarken Stellen elnes Flusses angesiedelt werden.

\section{(3.) Immissionsorientierte technische Maßnahmen (14)}

Dies sind Maßnahmen, die unmittelbar in der Nähe oder gar direkt am immissionsgefährdeten Objekt vorgenommen werden. Von großer praktischer Bedeutung ist die Wasseraufbereitung $\mathrm{zu}$ Trinkwasser und der immissionsseitige Lärmschutz durch schalldämmende Fenster und AuBenwände von Gebäuden. Aber auch bereits ans Makabre grenzende technische Maßnahmen wie die Verwendung von Gasmasken zum Schutz vor der Abgasbelastung in den Straßenschluchten der Großstädte, gehören $z u$ bereits praktizierten Maßnahmen des "passiven Immissionsschutzes" 138)

dd) Beurteilung der technischen Maßnahmen des präventiven Umweltschutzes

Nimmt man das Wort "präventiv" bei der Beurteilung der technischen Maßnahmen des Umweltschutzes als Maßstab und abstrahiert von allen Kosten der jeweiligen Maßnahmen, so wird man $\mathrm{zu}$ folgender Prioritätenbildung kommen:

Die besten technischen Maßnahmen des Umweltschutzes sind diejenigen, die bereits das Entstehen von Schadstoffen in dem Produktions- und Konsumsektor durch Produktgestaltung vermeiden oder reduzieren oder die Produkte zumindest entsorgungsgerecht bzw. recycling-freundlich gestalten, also vor allem Produktverbesserung und Produktsubstitution (also 10 und 11 in $\mathrm{Abb}$. 15), aber auch Verfahrensverbesserung oder Verfahrenssubstitution (also 2 und 4). Die nächste Priorität genießen u.E. die technischen Maßnahmen, welche es zu keiner oder nur zu einer stark

a.a.0., S. $131 \mathrm{ff.;} \mathrm{Gewässern} \mathrm{kann} \mathrm{auch} \mathrm{wieder} \mathrm{künstlich}$ "auf die Beine" geholfen werden, z.B. durch Flußbelebung via künstliche sauerstoffanrelcherung (vgl. Müller, Wilhelm J., Probleme der Reinhaltung von Gewässern, a.a.0., S. 38 ff.; Meyer, Olaf, Frische Luft für kranke Seen, Sanierung durch Sauerstoffanreicherung, in: Umwelt, 1973, 4, S. $18 \mathrm{ff}$ )

138) Knabe, W./Luckat, S., Passiver Immissionsschutz gegen Luftverunreinigungen, in: Umwelt, $1974,4, \mathrm{~S} .28 \mathrm{ff}$. 
reduzlerten Schadstoffemission kommen lassen, also Recyclina, Rückhaltetechnologien und Entsorgung wobei wir mit dieser Reihenfolge auch eine Rangfolge ausdrücken wollen. Das Recycling (also 6 und 7) ist - obwohl es in den meisten Fällen Rückhaltetechnologien voraussetzt - den Rückhaltetechnologien und der Entsorgung vorziehen, da es auch noch eine ressourcensparende Komponente hat, während die Rückhaltetechnologien und die Entsorgung viele Ressourcen beanspruchen. Die Rückhaltetechnologien (also 1, 3 und 5) sind u.E. der Entsorgung überlegen, da sle durch die innersektorale Emissionsreduktion mögliche Emissionen während des Transports ausschlleßen. Unter den Rückhaltetechnologien des Konsumsektors genieBt die Zentralisierung der Emissionsquellen (also 5) noch Priorität gegenüber individuellen RlickhaltemaBnahmen und soll auf eine Stufe mit den Rückhaltetechnologien im Produktionssektor gestellt werden.

Bei der Entsorgung als letzte Stufe der emissionsorientierten technischen Maßnahmen (also 8,9 und 12) kann man ebenfalls sagen, daß Maßnahmen des Recycling (z.B. Kompostierung) anderen Maßnahmen wie Verbrennung oder Deponie vorzuziehen sind. Alle emissionsorientierten technischen Maßnahmen (also 1 bis 12) sind allen passiven technischen MaBnahmen des präventiven Umweltschutzes (also 13 und 14) vorzuziehen, da letztere keinen breit gestreuten Immissionsschutz bieten und sowohl regional als auch personell so selektiv sein können, daß dadurch große interregionale und interpersonelle Ungleichheiten entstehen können. Die passiven Maßnahmen des technischen Umweltschutzes bergen außerdem eine große Gefahr in sich: Sie reduzieren die Anstrengungen beim emissionsorientierten technischen Umweltschutz. Es gibt u.E. nur elnen Grund, transmissions- oder immissionsorientierte technische Maßnahmen des Umweltschutzes zu ergreifen, und das ist der, daB es keine technisch realisierbaren oder nur unverhältnismäßig teure emissionsorientierte technische Maßnahmen gibt.

Wir erhalten also - bei Nichtbeachtung der Kosten - folgende Prioritätenliste: 


\begin{tabular}{|c|c|c|}
\hline Priorität & Technische Maßnahme & Nummer in Abb. 15 \\
\hline 1 & $\begin{array}{l}\text { Produktgestaltung (Faktorinputs } \\
\text { und Konsumgüter) }\end{array}$ & 10 und 11 \\
\hline 2 & ProzeBgestaltung & 2 und \\
\hline 3 & Recycling & 6 und \\
\hline 4 & $\begin{array}{l}\text { Rückhaltetechnologie im } \\
\text { Produktionssektor und } \\
\text { Fernheizung }\end{array}$ & 1 und \\
\hline 5 & $\begin{array}{l}\text { Rückhaltetechnologien im } \\
\text { Konsumsektor }\end{array}$ & 3 \\
\hline 6 & $\begin{array}{l}\text { Entsorgung (Sammlung, Transport, } \\
\text { Beseitigung) }\end{array}$ & 8,9 und 12 \\
\hline 7 & $\begin{array}{l}\text { Passiver Immissionsschutz } \\
\text { (transmissions- und immissions- } \\
\text { orlertiert) }\end{array}$ & 13 und 14 \\
\hline
\end{tabular}

Diese Prioritätenliste kann als Basis für eine die Kosten berücksichtigende optimale Planung des Umweltschutzes dienen 139)

\section{Ansätze einer optimalen Planung des Umweltschutzes}

1. Planung des Umweltschutzes nach Gossen?

Im vergangenen Abschnitt haben wir eine Fülle von nicht-technischen, technischen und Raumordnungsmaßnahmen kennengelernt, mit denen sich die Umweltbelastung vermeiden oder reduzieren läßt. Es stellt sich die Frage, welche Maßnahme oder Maßnahmenkombinationen man wählen soll, um die Vielzahl der Umweltschutzziele in Form der Immissionsstandards einzuhalten oder zu erreichen.

Da Kriterium für eine rationale Planung des Umweltschutzes der optimale Einsatz der zur Verfügung stehenden Maßnahmen ist, muß nach dem ökonomischen Prinzip vorgegangen werden. Seine alternativen Versionen in bezug auf den Umweltschutz lauten:

(1) Die (im Sinne von Tinbergen) einseitig fixierten Ziele

139) Vgl. dazu unten S. 265 ff.

140) Vgl. Tinbergen, J., Centralization and Decentralization in Economic Policy, Amsterdam 1954, S. $7 \mathrm{ff}$. 
des Umweltschutzes in Form der Immissionsstandards sind mit einem Minimum an gesamtwirtschaftichen Ausgaben $z u$ realisieren.

(2) Mit elner bestimmten gesamtwirtschaftlichen Ausgabensumme sind die Ziele des Umweltschutzes in Form der Schadstoffreduktion zu maximieren.

Wir wollen von der zweiten Version des ökonomischen Prinzips ausgehen, da sle die realistische Prämisse enthält, daß der finanzlelle Ressourcenrahmen für den Umweltschutz vorgegeben 1st. Das impliziert, daß uber die Priorität des Umweltschutzes berelts entschieden ist.

Das Planungsproblem besteht nun darin, die in elner Periode (z.B. vier Jahren) für den Umweltschutz zur Verfügung stehende gesamtwirtschaftliche Ausgabensumme $A$ so auf die $n$ Maßnahmen $j(j=$ $1, \ldots, n)$ zu vertellen, daB die Reduktion der m Schadstoffe 1 $(1=1, \ldots, m)$ und damit das $z i e l$ des Umweltschutzes maximiert wird.

Nehmen wir an, das ziel des Umweltschutzes lasse sich in einem Umweltindikator quantifizieren, den wir als Umweltnutzen $U$ bezeichnen wollen, so gilt, daB der Umweltnutzen eine Funktion der reduzierten Menge $r_{1}$ der $m$ Schadstoffe 1 ist, also

(1) $U_{1}=U_{i}\left(r_{i}\right)$ für alle $1=1, \ldots, m$

(2) $U=\sum_{i} U_{i}\left(r_{1}\right)$

Nehmen wir weiterhin an, daB die Reduktion jedes Schadstoffs 1 von den Einsatzmengen $m_{i j}$ aller Maßnahmen $j$ abhängt, so qilt

(3) $r_{i}=\sum_{j} r_{i j}\left(m_{i j}\right)$.

Setzen wir Glelchung (3) in Gleichung (2) ein, so erhalten wir die zielfunktion

(4) $U=\sum_{i} U_{i}\left[\sum_{j} r_{1 j}\left(m_{i j}\right)\right]$.

Es soll nun weiterhin gelten, daß der Umweltnutzen mit steigender Schadstoffreduktion $r_{1}$ infolge zunehmenden Elnsatzes der jewe1- 
ligen Schadstoffreduktionsmaßnahmen $m_{i j}$ nur unterproportional zunimmt. Es gilt also:

(4 a) $\frac{\partial u}{\partial r_{1 j}} \frac{d r_{1 j}}{d m_{i j}}>0$

und

(4 b) $\frac{\partial^{2} u}{\partial r_{1 j}} \frac{d r_{1 j}}{d m_{i j}}<0$

Wir unterstellen also einen abnehmenden Grenznutzen der Schadstoffreduktionsmaßnahmen, was dem 1. Gossenschen Gesetz entspricht.

Da die Ausgaben für den Umweltschutz begrenzt sind, kann U nur unter der Nebenbedingung der Ausgabenrestriktion maximiert werden. Bezeichnen wir die Ausgaben (den Preis) für jede Mengeneinhelt jeder Maßnahme j zur Reduktion des Schadstoffs $i$ mit $a_{1 j}$, so lautet die Nebenbedingung:

(5) $A=\sum_{i j} \sum_{i j} m_{i j}$,

wenn wir unterstellen, daß die Preise unabhängig von den nachgefragten Maßnahmeneinheiten sind.

Mit Hilfe des Lagrange-Ansatzes können wir nun die optimalen Einsatzmengen der verschiedenen Maßnahmen bestimmen 141):

(6) $L=\sum_{i} U_{i}\left[\sum_{j} r_{i j}\left(m_{i j}\right)\right]-\lambda \sum_{i j} \sum_{i j} m_{i j}$

(7) $\frac{\partial L}{\partial r_{i j}} \frac{d r_{i j}}{d m_{i j}}=\frac{\partial U_{i}}{\partial r_{i j}} \frac{d r_{i j}}{d m_{i j}}-\lambda a_{i j}=0$ für alle 1 und $j$

(8) $\frac{1}{a_{i j}} \frac{\partial u_{i}}{\partial r_{i j}} \frac{d r_{i j}}{d m_{i j}}=\lambda$

141) Vgl. z.B. Stöwe, H./Härtter, E.. Lehrbuch der Mathemat1k für Volks- und Betriebswirte, Göttingen 1967, S. 103 f. 
Gleichung ( 8 ) besagt, daß der Umweltnutzen $U$ dann maximal ist, wenn die Ausgabensumme $A$ so auf die $j$ Maßnahmen verteilt wird, daß die dadurch jeweils ermöglichte schadstoffreduktion $r_{i j}$ einen jeweils gleich großen (monetären) Grenzumweltnutzen in Höhe von $\lambda$ hervorruft. Es handelt sich hierbei um nichts anderes als das Gesetz vom Ausgleich der Grenznutzen (2. Gossensches Gesetz).

Inwieweit kann das 2. Gossensche Gesetz in der Praxis als Grundlage der Umweltschutzplanung dienen?

Erste Voraussetzung wäre eine Spezifikation der Zielfunktion (4), also auch der Gleichungen (2) und (3). Um Gleichung (2) spezifizieren zu können, müßte bekannt sein, wie sich der Umweltnutzen ändert, wenn die jeweiligen schadstoffmengen reduziert werden. Welche schwierigkeiten es bereiten kann, die Nutzen von Schadstoffreduktionen $z u$ bestimmen, haben wir oben dargestellt 142)

Mindestens genau so schwierig scheint es uns in vielen Fällen $z u$ sein, die Funktionen der Gleichung (3) zu spezifizieren. Es müßte $z$.B. bekannt sein, um wieviel die $\mathrm{sO}_{2}-$ Belastuna sinkt, wenn die Ausbildung der Diplomingenieure umweltbezogener wird; oder es müßte bekannt sein, um wieviel der $\mathrm{BSB}_{5}$ eines Gewässers sinkt, wenn die Bevölkerung umweltbewußter wird; oder man müßte wissen, um wieviel der Fluglärm sinkt, wenn die Technik des Flugzeugbaus durch ein "Amt für Technology Assessment" kontrolliert würde. Dies sind sicher extreme Beispiele, doch müßte man solche Beziehungen quantitativ ermitteln können, damit bekannt ist, welcher Teil der zur Verfügung stehenden finanziellen Ressourcen auf die jeweiligen Maßnahmen verteilt werden soll. SchlieBlich ist es zumindest in den Fällen, in denen kein Markt für die Unweltschutzmaßnahmen besteht, kaum möglich, die Preise für die Maßnahmen zu bestimmen. Wie soll man z.B. bestimmen, was der Preis für einen Bebauungsplan ist, der in einem Wohngebiet die Lärmimmission unter dem Immissionsstandard beläßt? Es gibt natürlich auch Beispiele, in denen sich sowohl die Zielfunktion

142) Siehe oben S. $154 \mathrm{ff}$. 
als auch die Nebenbedingung relativ gut spezifizieren läßt. Der Nutzen von Umweltschutzmaßnahmen läßt sich dann relativ gut bestimmen, wenn er in einem verhinderten Schaden besteht, für dessen allfällige Beseitigung ein Marktpreis existiert. In manchen Fällen läßt sich auch die Wirksamkeit umweltschützender Maßnahmen hinsichtlich der Schadstoffreduktion relativ leicht spezifizieren. So ist es z.B. relativ einfach $z u$ berechnen, wie sich der Sauerstoffgehalt eines Gewässers, ceteris paribus, ändert, wenn ein, zwei, drei, vier... Kläranlagen mit biologischer Reinigungsstufe und bestimmter Kapazität entlang eines Flusses gebaut werden. Schlieblich existiert auch für viele Umweltschutzmaßnahmen, z.B. für Kläranlagen, ein Markt und somit auch ein Preis. Die positiven Beispiele für eine mögliche Spezifikation der Zielfunktion und der Nebenbedingung ändern allerdings nichts an der Tatsache, daß eine optimale Gesamtplanung - zumindest auf diese Art - nicht möglich ist. Was dagegen mit diesem Ansatz möglich scheint, ist die Optimierung von Teilproblemen, und zwar vor allem dann, wenn der Umweltschutznutzen in einem $\mathrm{zu}$ Marktpreisen bewertbaren verhinderten Schaden besteht und wenn die zur Schadstoffreduktion eingesetzten Maßnahmen technischer Art sind.

2. Das Optimierungsmodell von Thoss

Thoss geht von der ersten Version des ökonomischen Prinzips aus, indem er die Einhaltung der Immissionsstandards als Hauptziele bezeichnet. Für die Belastung B einer Region durch einen Schadstoff $s(s=1, \ldots, s)$, also $B_{s}$, gilt demnach

(1) $B_{S} \leq \bar{B}_{S}$.

In diesen $S$ Hauptzielfunktionen stellen die $\bar{B}_{S}$ die Immissionsstandards dar, die nicht überschritten werden dürfen. Diese Zielfunktionen sind unter der Nebenbedingung $z u$ realisieren,

143) Vgl. Thoss, R., Ziel, Ansatz und Methoden eines inteqrierten Optimierungsmodelis zur Verbesserung des Umweltschutzes, in: Seminarbericht Nr. 8 der Gesellschaft für Regionalforschung, Münster 1974, S. $3 \mathrm{ff}$. 
da $B$ die Produktion $x$ der Güter $i(i=1, \ldots, I)$ maximiert wird:

(2) $\sum_{i} x_{i} \rightarrow \operatorname{Max}$ !

Die Belastung einer Region durch einen Schadstoff s setzt sich nach Thoss nun zusammen aus

- der Anfangsbelastung zu Beginn einer Periode $\left(A_{S}\right)$,

- den Emissionen der betrachteten Periode ( $\left.E_{s}\right)$.

Davon sind die Schadstoffmengen abzuziehen, die entfernt wurden durch

- Entsorgungsmaßnahmen der Menschen $\left(M_{s}\right)$,

- ökologische Regenerationsvorgänge $\left(R_{s}\right)$.

Es gilt also:

(3) $B_{s}=A_{s}+E_{s}-M_{s}-R_{s}$.

Die Anfangsbelastung kann durch Messung der Schadstoffmengen oder -konzentrationen ermittelt werden.

Die Emissionen der Schadstoffe hängen nach Thoss gemäß elnem Koeffizienten $d_{s i}$ von der Produktionsmenge $a b$ :

(4) $E_{s}=\sum_{i} d_{s i} x_{i}$

Die Entsorgung durch den Menschen ist nach Thoss gemäß elnem Koeffizienten $c_{s i}$ ebenfalls eine Funktion der produzierten Gütermenge :

(5) $M_{s}=\sum_{i} c_{s i} x_{i}$

Die natürlichen Regenerationsvorgänge führen ebenfalls zu einer Schadstoffreduktion. Sie hängt nach Thoss von bestimmten ökologischen Merkmalen $w_{k}(k=1, \ldots, k)$ einer Region ab, wie z.B. der strömung des Gewässers oder der hauptsächlichen Windrichtung einer Region, und zwar gemäß einem Koeffizienten $c_{s k}$ ' der angibt, wieviele Mengeneinheiten eines schadstoffs $s$ durch eine Einhelt eines ökologischen Merkmals $k$ abgebaut werden:

(6) $R_{s}=\sum_{k} c_{s k} W_{k}$.

Setzt man die Gleichungen (4) bis (6) in die Gleichung (3) ein, so erhält man 
(7) $B_{s}=A_{s}+\sum_{i} d_{s i} x_{i}-\sum_{i} c_{s i} x_{i}-\sum_{k} c_{s k} W_{k} \cdot$

Setzt man schlieblich die Gleichung (7) in die Gleichung (1) ein, so erhält man die $S$ Hauptzielfunktionen

(8) $A_{s}+\sum_{i} d_{s i} x_{i}-\sum_{i} c_{s i} x_{i}-\sum_{k} c_{s k} W_{k} \leqslant \bar{B}_{s}$,

die es - wie berelts gesagt - unter der Nebenzielfunktionen (2) zu realisieren gilt.

Die Lösung dieses Modells mit Hilfe der linearen Planungsrechnung gibt diejenige Kombination von wirtschaftlichen Nutzungen, bei der einerseits die Immissionsstandards $\left(\bar{B}_{S}\right)$ nicht überschritten werden, "bei der aber andererseits auch nicht unnötia auf die Befriedigung wirtschaftlicher Ansprüche verzichtet wird" 144)

Die Anwendung dieses Modells für die praktische Planung des Umweltschutzes hängt weitgehend davon $a b$, inwieweit die Koeffizienten $d_{s i}, c_{s i}$ und $c_{s k}$ bestimmt werden können. Die Hoffnung, daß dies überhaupt gelingen kann, ist bei den technisch determinierten $d_{s i}$ am größten. Um die $c_{s i}$ bestimmen zu können, müßte man bestimmte Verhaltensannahmen über die Entsorgungstätigkeit der Menschen bei steigender Güterproduktion machen, was uns kaum einfacher erschelnt als die Bestimmung der ökologischen Koeffizienten $c_{s k}$. Die grundsätzlichen Schwierigkeiten bei der Spezifikation der Koeffizienten schließen nicht aus, daB Teilprobleme, bei denen z.B. besonders einfach zu quantifizierende Relationen (4) bis (6) vorliegen, mit diesem Modellansatz sehr gut gelöst werden können. Eine Optimierung des gesamten auf technischen und raumordnenden Maßnahmen beruhenden Umweltschutzes halten wir jedoch mit diesem Modell - wie mit jedem anderen auch - für unmöglich. Die Chancen für die Anwendbarkeit der Optimierungsmodelle in der Planungspraxis steigt mit deren Beschränkung auf bestimmte Teilprobleme, wie regionale Abfall-

144) Vgl. Thoss, R., Ziel, Ansatz und Methoden eines integrierten Optimierungsmodells zur Verbesserung des Umweltschutzes, a.a.0., s. 8 . 
145) Vgl. z.B. die zahlreiche Literatur über die Optimierung des Gewässerschutzes; beispielhaft seien erwähnt: Loucks, D.P./ Revelle, C.E./Lynn, W.R., Linear Programming Models for Water Pollution Control, in Management Science, vol. 14, 1967; S. B-166 ff.; Mendiratta, A.K./Davidson, B., Best Combination of Waste Treatment and Spatially Distributed Discharge of Effluent, in: Water Resources Research, vol. 8, 1972, S. 565 ff.; Ecker, J.G./McNamara, J.R.. Geometric Programming and the Preliminary Design of Industrial Waste Treatment plants, in: Water Resources Research, vol. 7, 1971, S. $18 \mathrm{ff.;}$ Hirohide Hinomoto, Dynamic Programming of Capacity Expansion of Municipal Water Treatment System, in: Water Resources Research, vol. 8, 1972, S. 1178 ff.; Young, H.P. / Thompson, R.G., Least-Cost Allocation and Valuation Model for Water Resources, in: Water Resources Research, vol. 9, 1973, S. $1186 \mathrm{ff.;} \mathrm{Riordan,} \mathrm{C.,} \mathrm{Multistage} \mathrm{Marginal}$ Cost Model of Investment Pricing Decisions: Application to Urban Water Supply Treatment Facilities, in: Water Resources Research, vol. 7, 1971, S. 463 ff.; Heaney, J.P./Carter jr., B.J./Pyatt, E.E., Costs for Equipment Upstream Reduction in Waste Water Discharges, in: Water Resources Research, vol. 7, 1971, S. 458 ff.; Abendt, R., Anwendungen der linearen Optimierung im Gewässerschutz, in: Hahn, H. (Hrsq.), Operations Research und selne Anwendung in der Siedlungswasserwirtschaft, Bielefeld 1972, S. $97 \mathrm{ff.;} \mathrm{Orth,} \mathrm{H./}$ Cembrowicz, R., Anwendungen der nicht-linearen Programmierung, ebenda, S. $217 \mathrm{ff}$.; Wiik, K., Ein multiregionales Entscheldungsmodell zur Optimierung der Abwasserbelastung, in: Seminarbericht Nr. 8 der Gesellschaft für Regionalforschung, a.a.0., s. $53 \mathrm{ff}$.

Mit der optimalen Lösung des Abfallproblems befassen sich u.a. Russell, C.S./Spofford jr., W.O., A Quantitative Framework for Residuals Management Decisions, in: Kneese, A.V./Bower, B.T., Environmental Quality Analysis, Baltimore 1972; Russell, C.S./Vaughan, W.J., A Linear Programming Model of Residuals Management for Integrated Iron and Steel Production, in: Journal of Environmental Economics and Management, vol. 1, 1974, S. 17 ff.; Russell, C.S., Models for Investigation of Industrial Response to Residuals Management Actions, in: Swedish Journal of Economics, vol. 73, 1971, S. $134 \mathrm{ff.}$; Smith, Vernon, L., Dynamics of Waste Accumulation: Disposal Versus Recycling, in: Quarterly Journal of Economics, vol. 86, 1972, S. 600 ff.; Plourde, C.G. A Model of Waste Accumulation and Disposal, in: Canadian Journal of Economics, vol. 5, 1972, S. $119 \mathrm{ff.:}$ Lusky, R. , A Model of Recycling and Pollution Control, in: Canadian Journal of Economics, vol. 9, 1976, S. $91 \mathrm{ff}$.: Brasse, P. . Optimierung der Müllbelastung, in: Seminarbericht Nr. 8 der Gesellschaft für Regionalforschung, a.a.O., 
3. Darstellung eines elnfachen Planungsansatzes

Im folgenden wollen wir darstellen, wie mit einem relativ einfachen Ansatz die Reduktion zunächst eines Schadstoffs durch technische Maßnahmen optimal erfolgen kann. Zur Verdeutlichung der Darstellung verwenden wir ein Ablaufschema (Abb. 16). Ausgangspunkt der Planung ist ein Vergleich der tatsächlichen Immissionskonzentration eines schadstoffs $s_{i}^{t}$ mit dem Immissionsstandard dieses Schadstoffs $s_{i}^{g}$. ergibt der Vergleich, daß $s_{i}^{t}>s_{i}^{g}$, so muß eine schadstoffreduktion erfolgen. Als grundsätzliche Möglichkeiten der technischen Schadstoffreduktion wollen wir nun die in der Prioritätenliste auf S. 257 aufgeführten Maßnahmen der Reihe nach hinsichtlich ihrer technischen Realisierbarkeit prüfen und diejenigen aussortieren, die nicht technisch realisierbar sind. Sollte sich herausstellen, daß keine technische Möglichkeit besteht, die tatsächliche Immissionsbelastung so weit $z u$ reduzieren, daß sie unter den Immissions-

S. $29 \mathrm{ff}$.

Relativ wenige Untersuchungen befassen sich mit einer optimalen Lösung des Luftreinhalteproblems; vgl. Aguilar, R. u.a., A Model for the Evaluation of Alternative Policies for Atmospheric Pollutant Source Emissions, in: 5 th Conference on Optimization Techniques, Part II, Lecture Notes in Computer Science, hrsg. von G. Goos und J. Hartmanis, Berlin-Heidelberg-New York 1973; Hall jr., O.P.l Licari, J.A., On the Regional Implications of Air Pollution Control, in: The Annals of Regional Science, vol. 6, 1972, S. $26 \mathrm{ff} . ;$ Atkisson, S.E./Lewis, D.H., A Cost-Effectiveness Analysis of Alternative Air Quality Control Strategies, in: Journal of Environmental Economics and Management, vol. 1, 1974 , S. 237 ff.; Döllekes, P., Die Optimierung der Abgasbelastung in der Bundesrepublik Deutschland, in: Seminarbericht Nr. 8 der Gesellschaft für Regionalforschung, a.a.O., S. $89 \mathrm{ff}$. Zur optimalen Raumordnung hinsichtlich des Umweltschutzes seien zwei Beiträge erwähnt: Førsund, F.R., Allocation in Space and Environmental Pollution, in: Swedish Journal of Economics, vol. 74,1972 , S. $19 \mathrm{ff}$.; Brasse, P./Thoss, R./ Könnecke, U., Ein aktıvitätsanalytisches Umweltmodell zur Flächennutzungsplanung, in: Seminarbericht $\mathrm{Nr} .8$ der Gesellschaft für Regionalforschung, a.a.0., S. $15 \mathrm{ff}$. Die in den jeweiligen Absätzen dieser Fußnote am Ende aufgeführten Beiträge sind von Thoss-Mitarbeitern, welche hier die Submodelle des integrierten Gesamtmodells darstellen. 


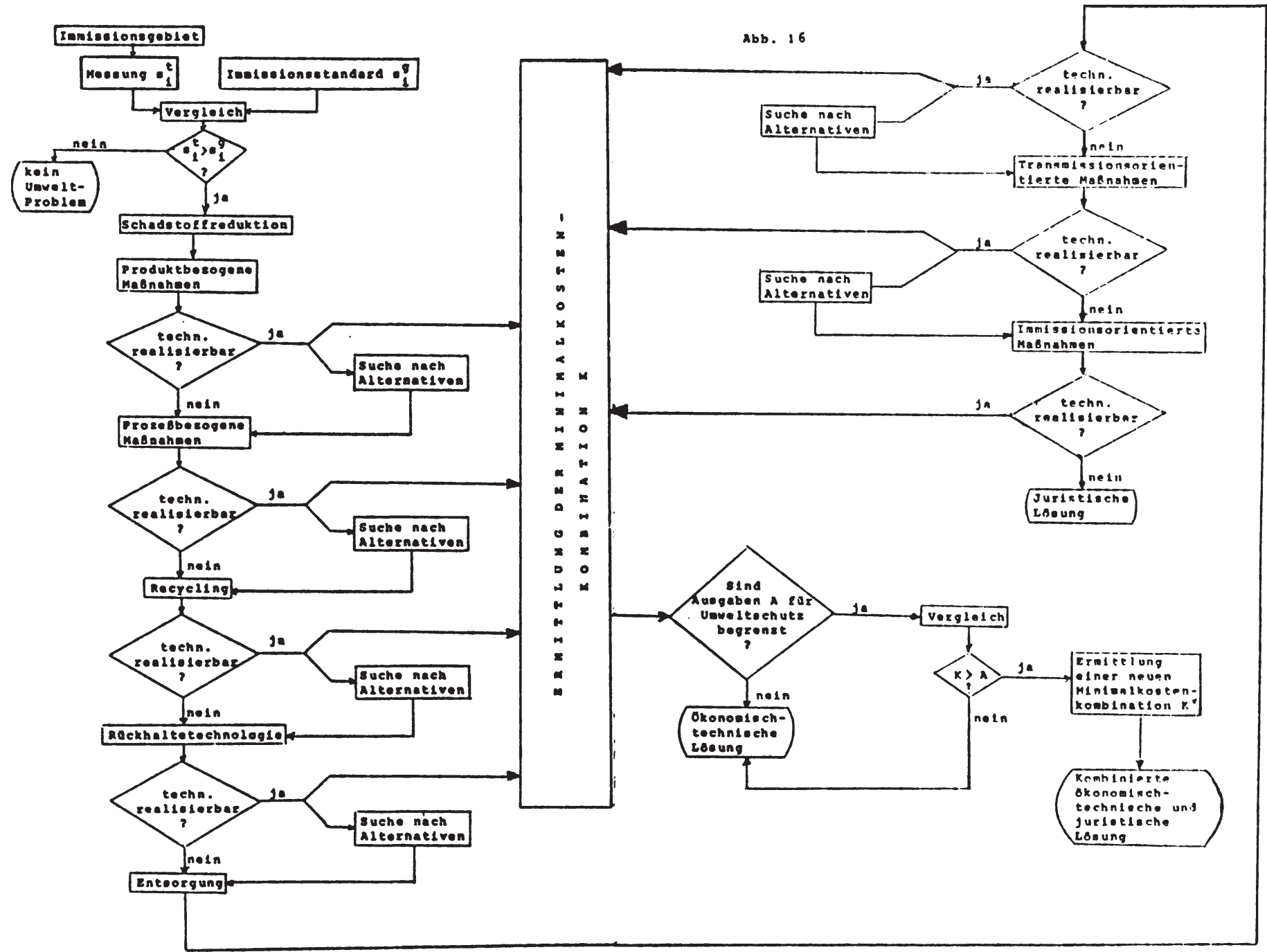


standard sinkt, bleibt nur eine juristische Lösung übrig: eine rechtlich verordnete Emissionsreduktion oder gar ein Emissionsverbot, was gleichbedeutend ist mit einer Produktionseinschränkung bzw. -einstellung. Ist dagegen eine oder sind gar mehrere technische Maßnahmen zur Schadstoffreduktion möglich, so ist die kostengünstigste Maßnahme oder Maßnahmenkombination, also die Minimalkostenkombination $\mathrm{K} z \mathrm{z}$ bestimmen. Reichen die finanziellen Ressourcen in Höhe von A, die für Zwecke des Umweltschutzes eingeplant sind aus, um diese kostenminimale technische Lösung $z u$ realisieren, so sind die entsprechenden technischen Maßnahmen zu ergreifen. Das Problem der Schadstoffreduktion ist ökonomischtechnisch gelöst. Ist dagegen $K>A$, so ist die gesamte Ausgabensumme für die damit technisch realisterbare optimale Lösung auszugeben; es ist also eine neue Minimalkostenkombination $\mathrm{K}^{\mathrm{k}}$ unter der Nebenbedingung $K=A z u$ bestimmen, die wegen der Unteilbarkeit mancher Umweltschutzmaßnahmen ganz anders aussehen kann. Mit dieser ökonomisch-technischen Lösung wird aber noch keine Schadstoffreduktion im gewünschten Umfang möglich seln; die noch notwendige Schadstoffreduktion ist durch juristische Maßnahmen wie gesetzlich verordnete Emissionsbeschränkungen zu initileren. Das hier für einen Schadstoff geschilderte Planungsverfahren zur Bestimmung des optimalen Faktoreinsatzes kann für jeden Schadstoff 1 , dessen tatsächliche Immissionskonzentration über dem jeweiligen Immissionsstandard liegt, durchgeführt werden. Man erhält dann soviele Minimalkostenkombinationen wie es Fälle gibt, in denen $s_{i}^{t}>s_{i}^{g}$. Besteht keine Begrenzung des finanziellen Ressourcenrahmens, so lassen sich alle Minimalkostenkombinationen und damit auch die Einhaltung aller Immissionsstandards auf ökonomisch-technische Art realisieren. Ist dagegen $K>A$, so ist der Schadstoff zuerst $z u$ reduzieren, dessen Immissionskonzentration prozentual am weitesten über dem entsprechenden Immissionsstandard liegt; dann wird derjenige reduziert, dessen Immissionskonzentration relativ am zwelthöchsten über dem entsprechenden Immissionsstandard liegt usw., bis die zur Verfügung stehenden finanziellen Ressourcen aufgebraucht sind. Dieses Problem entspricht der Struktur nach dem sogenannten Knapsackproblem, dessen 
Lösung mit Hilfe der linearen Planungsrechnung in diesem Fall relativ einfach wäre, wenn die technischen Maßnahmen der Schadstoffreduktion und damit die für sie aufzuwendenden Ausgaben teilbar wären 146) Da dies in der Regel nicht der Fall ist, entsteht das Knapsackproblem mit Unteilbarkeitsbedingung, dessen Lösung komplizierter ist 147)

Das Hauptproblem des von uns vorgeschlagenen Ansatzes für eine optimale Planung des Umweltschutzes liegt darin, die Schadstoffreduktionsfunktionen $z u$ spezifizieren, was unbedingt notwendig ist, um die Minimalkostenkombination zu bestimmen. In vielen Fällen, wie z.B. der $\mathrm{SO}_{2}$-Reduktion, ist es durchaus mörlich $z$ u ermitteln, wie sich die $\mathrm{SO}_{2}$-Emissionen reduzieren, wenn man z.B. von schwerem $z u$ leichtem Heizöl wechselt (Inputsubstitution), das Heizöl vorher entschwefelt (Inputverbesserung) oder das $\mathrm{SO}_{2}$-Rauchgas absorbiert (Rückhaltetechnologie).

Den Vorteil des von uns vorgeschlagenen Ansatzes einer optimalen Planung des Umweltschutzes sehen wir gegenüber den beiden anderen dargestellten Ansätzen darin, daß er $z u$ einem umfassenderen Ansatz i $n t$ e $g i$ e $r t$ werden $k$ a $n n$, wenn die Spezifikation der Schadstoffreduktionsfunktionen möglich ist, während die anderen umfassenden Ansätze $d$ e $s$ i $t$ e $g r i$ e $t$ werden $m$ ü s $s$ e $n$, wenn sie der Planungspraxis des Umweltschutzes dienlich sein sollen.

D. Grenzen einer rationalen Planung des Umweltschutzes

Zum AbschluB dieses Kapitels wollen wir uns noch mit den Grenzen einer rationalen Planung des Umweltschutzes befassen, die aus der Vielzahl der Planungsträger resultieren, die sich mit Um-

146) Vgl. Müller-Merbach, H., Operations Research. Methoden und Modelle der Optimalplanung, Berlin und Frankfurt 1969, S. $143 \mathrm{ff}$.

147) Vgl. ebenda, S. $341 \mathrm{ff}$. 
weltschutz befassen.

Die Vielzahl der Planungsträger in einem Land wie der Bundesrepublik Deutschland ist die Folge der föderativen und demokratischen Staatsform und der marktwirtschaftlichen ordnung, die zwangsläufig mit einer Dezentralisierung der Entscheidungen und damit auch den Entscheidungsvorbereitungen (den Planungen) verbunden sind.

Die Folge des Föderalismus ist eine horizontale und vertikale Vertellung der planungskompetenzen für den Umweltschutz auf die Gebietskörperschaften.

Die Folge der demokratischen staatsform ist zumindest das Bestreben vieler Bürger qua gewählte Vertreter in Gemeinden, Land und Bund und - sofern dies keinen Erfolg verspricht, weil die Repräsentation der Volksvertreter ungenügend erscheint - durch Bürgerinitiativen an umweltrelevanten planungen beteiligt $z u$ werden.

Die Folge der marktwirtschaftlichen ordnung ist, da diese vor allem auf die Elgeninitiative der Unternehmer vertraut, die individuelle unternehmerische Planungsbefugnis hinsichtlich des betrieblichen Umweltschutzes, der im Bereich des technischen Umweltschutzes die größte Bedeutung zukommt. Die sich aus Staatsform und Wirtschaftsordnung ergebende Zersplitterung der Planungskompetenzen vervielfacht sich noch durch das Ressortprinzip, das gerade bei einer interdisziplinären (Planungs-)Aufgabe wie dem Umweltschutz dazu führt, daß fast jedes Ressort zumindest auf Bund- und Länderebene betroffen 148) d.h. für unweltrelevante Planungen verantwortlich ist.

Eine derartige Dezentralisierung der Planungskompetenzen scheint ein großer Nachteil für eine rationale Planung des Umweltschutzes zu sein. Tatsächlich ist sie aber unseres Erachtens eine weitere Voraussetzung für eine rationale Planung, denn nur elne dezentrale planung kann den regional und auch lokal sehr unterschiedlichen natürlichen Bedingungen, die einen großen

148) Vgl. Raumordnung und Umweltschutz, a.a.O., s. $60 \mathrm{f} .$, wo die Zuständigkeiten der Ressorts für die Bereiche des Umweltschutzes in Bund und Ländern zusammengestellt sind. 
Einfluß auf das Ausmaß der Umweltbelastungen und damit auf den notwendigen Umweltschutz haben können, Rechnung tragen. Worauf es allerdings bei einer dezentralisierten Planung ankommt, damit man sie auch als rational bezeichnen kann, ist eine Koordination der dezentralen Planungen. Gelingt keine ausreichende Koordination der dezentralen Planungen, ist wahrscheinlich doch eine zentrale planung rationaler 149). Im Falle der Planung des Umweltschutzes ist für den öffentlichen Sektor eine vertikale Koordination wegen der föderativen struktur und eine horizontale Koordination der Ressorts innerhalb einer Exekutive ${ }^{150)}$ und $z w i s c h e n$ Ländern bzw. Gemeinden erforderlich. Die vertikale Koordination der Planung kann gesetzlich vorgeschrieben sein, wie wir das beim ROG in Verbindung mit dem BBaug gesehen haben 151), ist aber auch ohne gesetzliche Grundlage möglich wie z.B. in der Bund-Länder-Umweltministerkonferenz oder anderen Bund-Länder-Ausschüssen 152) In den regionalen Planungsgemeinschaften kann sowohl eine vertikale (nämlich zwischen Ländern und Gemeinden) als auch horizontale Koordination (nämlich unter den Gemeinden) erfolgen.

Die horizontale Koordination zwischen den Ressorts im Bund und in den Ländern ist sowohl auf Kabinettsebene ("Umweltkabinett") als auch darunter möglich. Das Bundesinnenministerium hat z.B. einen Ausschuß der Abteilungsleiter im Bereich Umweltplanung eingerichtet ${ }^{153}$ ) Eine horizontale koordination zwischen den Ländern kann in der Ministerpräsidentenkonferenz oder in Fachministerkonferenzen der Länder erfolgen. Auch regionale Planungsgemeinschaften können ihre Pläne über Ländergrenzen hinweg koordinieren. Für die Koordination zwischen privatem und öffent-

149) Vgl. Flickinger, H.-G./Summerer, S., Voraussetzungen erfolgreicher Umweltplanung in Recht und Verwaltung, Göttingen 1975 , S. $39 \mathrm{f}$.

150) Vgl. ebenda, S. $115 \mathrm{ff}$.

151) Siehe oben S. 224.

152) Vgl. Flickinger, H.-G./Summerer, S., Voraussetzungen erfolgreicher Umweltplanung in Recht und Verwaltung, a.a.0., S. 125 .

153) Vgl. ebenda, S. 116. 
lichem Bereich ist eine Mitwirkung von Bürgerinitiativen am Planungsproze $B$ günstig, denn so $k$ a $n$ eher ein Konsensus zwischen dem staat und seinen Bürgern in umweltplanungsrelevanten Fragen hergestellt werden.

Schwierig 1st die Koordination der Planung zwischen dem öffentlichen Sektor (vor allem den Gemeinden) und den privaten Unternehmen einerseits, sowie den privaten Unternehmen unterelnander andererseits. Die Vorgabe von Emissionsstandards für die verschiedenen von den Unternehmen emittierten Schadstoffe, die aus den Immissionsstandards unter Berücksichtiqung der natürlichen Faktoren abgeleitet werden können 154), stellen u.E. ein geeiqnetes Instrument dar, um sowohl die Umweltschutzplanung der Gemeinden mit derjenigen der privaten Unternehmen als auch die Umweltschutzplanungen zwischen den privaten Unternehmen $z u$ koordinieren; jedes Unternehmen weiß jetzt, was es tun muß, um die Einhaltung der Immissionsstandards, also die überbetrieblichen ziele des Umweltschutzes, nicht zu gefährden (vertikale Koordinationswirkung der Emissionsstandards), ohne sich dabei mit anderen Unternehmen über die betriebsspezifischen Schadstoffemissionsraten einigen zu müssen (horizontale Koordinationswirkung der Emissionsstandards).

In manchen Fällen sind also die Koordinationsmöqlichkeiten bereits vorhanden, in anderen Fällen müssen sie noch geschaffen oder verbessert werden.

Die Rationalität und damit auch die Qualität der Umweltschutzplanung hängt wesentlich davon $a b$, wie gut die Koordination zwischen den Planungsträgern funktioniert. Die Erfahrung zeigt, daß vor allem bei den Gemeinden einerselts zu viel auf Kosten anderer geplant wird - seien es die eigenen Bürger oder die Nachbargemeinden; andererseits macht sich aber auch ein Planungsdefizit hinsichtlich des Umweltschutzes bemerkbar 155)

154) Siehe unten S. 329.

155) Vgl. Flickinger, H.-G./Summerer, S., Voraussetzungen erfolgreicher Umweltplanung in Recht und Verwaltung, a.a.o., S. $128 \mathrm{ff}$. 
Mit einem Planungsdeflzit ist aber bereits der Grundstein zu dem viel beschworenen Vollzugsdefizit im Umweltschutz gelegt, mit dem wir uns im nächsten Kapitel befassen werden 156)

156) Siehe unten S. $290 \mathrm{ff}$. 
"Bei der Regierung eines wohlgeordneten Staates sollte man dem Beispiel der Natur folgen, die stets langsam voranschreitet, Schritt für schritt".

(Jean Bodin, Uber den Staat)

IV. Trăger und Instrumente elner rationalen Umweltpolitik

A. Zur Allokation der Umweltschutzaufgabe zwischen privatem und öffentlichem Bereich

1. Zur Aufgabe einer rationalen Umweltpolitik

Aufgabe elner rat:onalen Umweltpolitik ist die Durchsetzung der Ziele des Umwelts hutzes durch Realisierung der optimal deplanten Maßnahmen-(Koubinationen) des Umweltschutzes. Als von uns zusätzlich geforderte Nebenbedingung kommt hinzu, daß umweltpolitische ziele so durchgesetzt werden, daß der öffentliche Haushalt möglichst wenig belastet wird.

Die notwendigen Voraussetzungen, um diese Aufgabe erfüllen zu können, haben wir in den ersten drei Kapiteln kennengelernt. Es zeigte sich, daß zwar noch viele Informationen vor allem über die Wirkungen der Schadstoffe fehlen, was besonders die Normlerung der Immissionsstandards erschwert; andererseits liegen jedoch genügend Informationen vor, um eine Reihe von Immissionsstandards festzulegen und in einzelnen Bereichen die vielfältig zur Verfügung stehenden Maßnahmen des Umweltschutzes zielorientiert zu planen. Vor allem die technischen Möglichkeiten des Umweltschutzes bieten in Kombination mit der Raumordnung ausreichende Möglichkeiten, die Einhaltung der Immissionsstandards zu gewährleisten. "Die Technik (kann) alles lösen, was an Aufgaben denkbar ist ... Für mich ist das Umweltproblem ... ein rein ökonomisches und gesetzgeberisches Problem" 1)

1) Diese Äußerung Ludwig Bölkows findet sich in einem Artikel der Wirtschaftswoche, 26. Jg., 1972, H. 8, S. 70. Vgl. ähnliche Äußerungen in: Henke, W., Umwelt, Technik, 
Die Realisierung der umweltpolitischen ziele ist mit anderen Worten in erster Linie ein Durchsetzungsproblem. Diese Feststellung haben wir bereits bei der Ursachendiagnose getroffen. als wir anführten, daß die ungenügende Internalisierung der externen Effekte die eigentliche Ursache der Umweltbelastung ist. Die ungenügende Internalisierung der Umweltexternalitäten ist aber nichts anderes als die direkte Folge eines unzureichenden Durchsetzungswillens oder -vermögens der umweltschutzrelevanten Entscheidungsinstanzen 2)

Es könnte sein, daß es die falschen Instanzen sind, denen die umweltschutzrelevanten Entscheidungen obliegen und daß es Instanzen gibt, die sowohl den willen als auch die Fähigkeit besitzen, die für elnen wirksamen Umweltschutz notwendigen Entscheidungen $z u$ treffen und durchzusetzen. In diesem Fall würde eine Reallokation der Umweltschutzaufgabe das Durchsetzungsproblem lösen. Das schlagen z.B. diejenigen vor, welche die Existenz von Umweltexternalitäten - zu Recht - auf Mängel des Marktmechanismus zurückführen und deshalb die Umweltschutzaufgabe via öffentliche Planung realisiert sehen wollen. Daß dies aber kein Allheilmittel ist, zeigten die Ausführungen über die Raumordnung ${ }^{3)}$ und über die Grenzen einer rationalen planung 4 ) Weder die Entscheidungsträger im privaten noch diejenigen im öffentlichen Bereich sind a priori besonders dafür geeignet, die ziele des Umweltschutzes zu realisieren. Tullock zieht daraus den etwas fatalistischen Schluß, daß wir "deshalb die Wahl

Natur, Kultur, a.a.0., S. 32 f.; Coenen, R. u.a., Alternativen zur Umweltmisere, a.a.0., S. 157 f. Als konkretes Beispiel sei die Entschwefelung von Heizöl und die Rauchgasentschwefelung genannt. Sie ist technisch mönlich, ökonomisch aber noch ein Problem (vgl. Meyer-Abich, K.M., Umweltbeeinträchtigung durch den wirtschaftlich-technischen Proze $\beta$

- Beispiel: Energieumwandlungsprozesse, in: Giersch, H.

(Hrsg.), Das Umweltproblem in ökonomischer sicht, a.a.o., S. $14 \mathrm{f.}$ )

2) Siehe oben S. $122 \mathrm{ff}$.

3) Siehe oben S. $222 \mathrm{ff}$.

4) Siehe oben S. $268 \mathrm{ff}$. 
zwischen einem privaten Markt haben, der systematisch verzerrte Entscheidungen liefert, und einer Regierung, die schlecht durchdachte Entscheldungen fällt" 5). Es wäre U.E. jedoch falsch, es deshalb als gleichgültig anzusehen, inwieweit der Umweltschutz privater Initiative überlassen bleiben oder inwieweit er dem Staat als öffentliche Aufgabe übertragen werden soll, denn es gibt Ordnungs- und Effizienzkriterien, die eine Beurteilung darüber erlauben, in welchem Umfang Aufgaben vom privaten oder vom öffentlichen Bereich wahrgenommen werden sollen. Die Ordnungskriterien sind dabel u.E. den Effizienzkriterien überzuordnen - allerdings nur insoweit, wie mangelnde Effizienz nicht die Ordnung selbst zu gefährden droht. Diese Gefahr besteht vor allem dann, wenn das dogmatische Festhalten an ordnungspolitischen Vorstellungen zu ordnungsgefährdenden Effizienzeinbußen führt.

In einem ideal funktionierenden marktwirtschaftlichen system ist die allokative Effizienz gewährleistet, da das freie spiel von Angebot und Nachfrage via Preismechanismus automatisch zur pareto-optimalen Allokation führt ${ }^{6}$ ). Gibt es jedoch sogenannte freie Güter, die keinen Preis oder keinen ihrer Knappheit entsprechenden Preis haben, weil für sie kein Markt existiert, wie das z.B. bei den Umweltgütern reine Luft, saubere Gewässer, saubere Landschaft und Ruhe der Fall ist, so ist das marktwirtschaftliche system aus eigener kraft heraus nicht in der Laqe, das Allokationsoptimum zu realisieren 7). Der staat muß regu-

5) Tullock, G., Öffentliche Entscheidungen als öffentliche Güter, in: Frey, B.S./Meißner, W. (Hrsg.), Zwei Ansätze der polltischen ökonomie. Marxismus und ökonomische Theorle der Politik, Frankfurt/Main 1974, S. 104; vgl. auch Pennance, F.G., The Polluters: Industry or Government?, The Institute of Economic Affairs, London 1972, S. 42.

6) Vgl. Schlieper, U., Pareto-Optimum, externe Effekte und die Theorie des Zweltbesten, Köln u.a. 1969, S. 13 ff.; Sohmen, E., Allokationstheorie und Wirtschaftspolitik, Tübingen 1976 , besonders 4. Kapitel.

7) Vgl. Kneese, A.V., Pollution and the Profit Motive, a.a.O., S. $221 \mathrm{ff.;}$ Issing, 0., Zerstörung der Umwelt - ein Versagen des marktwirtschaftlichen Systems?, a.a.0., s. 132; Jacoby, N.H., The Polluters: Industry or Government?, a.a.o., s. $21 \mathrm{f}$. 
lierend in das Marktgeschehen eingreifen ${ }^{8}$ ), und zwar möglichst so, daB das Allokationsoptimum erreicht wird, ohne daß allzu negative distributive Wirkungen entstehen. Das gilt in besonderem Maße für eln System der $s$ o 1 a 1 e $n$ Marktwirtschaft. Wie und in welchem Umfang der staat in das Marktgeschehen eingreifen oder es gar substituieren muß, hängt von der Anpassungsbereitschaft und - fähigkeit der Marktpartner - man kann auch sagen: der Privatinitiative von Produzenten und Konsumenten - $a b$. Auf den Umweltschutz bezogen, heiBt das konkret: In einem demokratischen staat mit einer sozial verpflichteten marktwirtschaftlichen Ordnung wie der Bundesrepublik Deutschland sollte der Umweltschutz primär eine private Aufgabe sein. Nur dann, wenn privat initiierter Umweltschutz nicht effizient genug ist oder negative distributive Wirkungen hat, sollte der staat eingreifen, und zwar nicht nur um den Umweltschutz selbst, sondern auch um die bel ungenügendem Umweltschutz gefährdete demokratische und soziale marktwirtschaftliche Ordnung zu sichern.

Umweltschutz wird so einmal zum Prüfstein für die Bereitschaft und Fählgkelt der Bürger eines demokratischen staates mit einer sozialen marktwirtschaftlichen Ordnung das Umweltproblem durch Privatinitiative zu lösen ${ }^{9}$ ) und sofern das - aus welchen Gründen

Binswanger, H.C., Eine umweltkonforme Wirtschaftsordnung, a.a.O.. S. $129 \mathrm{f}$.

8) Selbst als liberal bekannte Wissenschaftler und Politiker bestreiten das nicht: vgl. Friedman, M., The Role of Government in a Free Society, in: Goldman, M.I., Ecology and Economics, a.a.O.. S. 122; von Mises, L., Human Action, London-New York 1949, S. 650; Hayek, F.A., Der Weg zur Knechtschaft, Erlenbach und Zürich 1952, S. 61 f.; Erhard, L./Müller-Armack, A. (Hrsg.), Soziale Marktwirtschaft, a.a.0., S. 303 ff.; Hamm, W. , Zur ökonomischen Bedeutung und ordnungstheoretischen Begründung der Rolle des staates in Marktwirtschaften, in: Staat und moderne Marktwirtschaft, hrsg. vom Landespersonalamt Hessen, Wlesbaden 1975, S. 23; Streibl, M., Umweltpolitik in der Bundesrepublik Deutschland, a.a.O., S. 242 .

9) Auf die "Soctal Responsibility of Business-Bewegung" und das Corporate Social Accounting wurde bereits hingewiesen (siehe oben S. $207 \mathrm{ff.l}$.

Es soll dadurch die kapitalistisch-marktwirtschaftliche Ordnung vor Systemzerstörern bewahrt werden (vgl. Dierkes, M., Qualität des Lebens und unternehmerische Entscheidung, a.a.0., 
auch immer - nicht möglich ist, wird der Umweltschutz zum Prüfstein für den staat, die demokratische und soziale marktwirtschaftliche Ordnung aufrecht zu erhalten 10), und dies nicht nur dadurch, $d$ a $\beta$ er die Realisierung der Umweltschutzziele durchsetzt, sondern auch dadurch, wi e er sie durchsetzt, nämlich mit einem möglichst marktkonformen und distributive Wirkungen berücksichtigenden umweltpolitischen Instrumentarium. Aufgabe einer rationalen Umweltpolitik in diesem Sinn ist es also, die nicht durch Privatinitiative erreichbaren Ziele des Umweltschutzes zu verwirklichen, und zwar so, daß dabei die öffentlichen Haushalte möglichst wenig belastet werden. Dieser Nebenbedingung kann um so mehr entsprochen werden, je mehr die Umweltschutzziele durch Privatinitiative angestrebt werden. Auch ein möglichst marktkonformes umweltpolitisches Instrumentarium scheint für die Einhaltung der Nebenbedingung günstig zu sein. Das gilt jedoch nur, wenn die marktkonformen Instrumente auch im Einklang mit den distributiven Zielen sind und nicht zu einer distributiven Verschlechterung führen, welche staatliche Kompensationszahlungen erfordern, also zusätzlich Budgetbelastungen bewirken würden 11)

Im nächsten Abschnitt wollen wir nun untersuchen, ob und inwie-

S. 27), denn die "Umweltkrise (w1rd) bewußt ideologisiert und zum Kampfmittel gegen das System der Marktwirtschaft umfunktioniert" (von Walterskirchen, M.P., Bemerkungen zur Umweltpolitik, a.a.0., s. 11).

10) Vgl. Rupp, H.H., Umweltschutz und Demokratie, in: Umweltschutz - aber wie?, a.a.O., S. 60 ff.; Seldenfus, H.S., Umweltschutz, politisches System und wirtschaftliche Macht, a.a.O., S. 828 f.; Littmann, K., Finanzierung von kollektiven Gütern, in: Aufgabe $\mathrm{zukunft}$ - Qualität des Lebens, Bd. 7, a.a.O.., S. 13; Genscher, H.-D., Sozialisierunq löst die Umweltprobleme nicht, in: Die Wirtschaftswoche Nr. 23, v. 1.6.1973, S. 19; Hartkopf, G., Wirtschaftspolitische Aspekte der Umweltpolitik, in: Schultz, H. (Hrsq.), UmweltReport, a.a.O., S. 19; derselbe, Umweltpolitik aus der Sicht der Bundesreglerung, a.a.0., S. 183 .

11) Vgl. Zimmermann, Klaus, Die Last des Umweltschutzes: Uberlegungen zum Konzept der "Volkswirtschaftlichen Kosten" des Sachverständigenrats für Umweltfragen, in: Kyklos, Bd. 27 , 1974 , S. $858 \mathrm{ff}$.; derselbe, Vorausschätzung staatlicher Umweltkosten, in: Umwelt 1976, S. 133. 
welt die Umweltschutzziele durch Privatinitiative erreicht werden können.

2. Umweltschutz durch Privatinitiative?

a) Mögliche Träger privater Umweltschutzinitiativen

Umweltschutz durch Privatinitiative ist aus zwei Gründen möglich: aufgrund höherer Einsicht und aus Elgeninteresse. DaB ersteres - zumindest in dem Umfang, daB die Umweltschutzziele erreicht werden - der Fall sein könnte, halten wir für sehr unwahrschein11ch. Als realistische Möglichkeit privater Umweltschutzinitiativen ist der Fall interessanter, in dem aus Eigeninteresse Umweltschutz betrieben wird 12) Initiatoren des Umweltschutzes können dabel einzelne Bürger als Konsumenten, Produzenten, (potentielle) Verursacher und (potentielle) Träger der Umweltbelastungen, aber auch homogene Gruppen der jeweiligen "Initiatorengattung" oder heterogene Gruppen sein, die sich aus Vertretern verschiedener "Initiatorengattungen" zusammensetzen. In der Regel vereinigen die Initiatoren mehrere Kennzeichen in sich: zumindest sind sie Bürger und Konsumenten; darüber hinaus können die glefchen Inftlatoren aber auch noch Produzenten und Verursacher oder Träger elner Umweltbelastung sein. In welcher Eigenschaft ein Individuum die Initiative zu privatem Umweltschutz ergreift, hängt davon $a b$, in welcher Elgenschäft er sich

12) Wir denken dabei nicht an Fälle, in denen Unternehmen deshalb "freiwillig" Umweltschutz betreiben, well sonst die in der Umgebung wohnenden Arbeitskräfte abwandern würden; dies wäre auch eine - allerdings etwas makabre - Form der Internalisierung (vgl. Ferrar, T.A., A Rationale for a Corporate Air Pollution Abatement Pollcy, in: American Journal of Economics and Soclology, vol. 33, 1974, S. 233 ff.; Siebert, H., Environment and Regional Growth, in: zeitschrift für Nationalökonomie, Bd. 33, 1973, S. 83 f.; Ronge, V., Die Umwelt Im kapitalistischen System, a.a.0., S. 103; Glagow, M., Zur staatlichen Regulierung von Umweltschäden, in: derselbe, Umweltgefährdung und Geselischaftssystem, a.a.O., S. 193). Wenn es dazu kommen sollte, daß die Arbeltskräfte wegen der Umweltbelastung abzuwandern beginnen, düfte die Umweltbelastung bereits welt über den Immissionsstandards liegen. 
durch die (potentiellen) Umweltbelastungen betroffen fühlt. Will ein Individuum, das Verursacher einer Umweltbelastung ist, aktiv werden, kann die Initiative zum Umweltschutz im eigenen Berelch erfolgen, ist es dagegen Träger einer Umweltbelastung, besteht die Privatinitiative darin, den Verursacher $z u$ veranlassen die umweltbelastenden Tätigkelten $z u$ beschränken oder ganz einzustellen. Da sowohl produzenten als auch Konsumenten Verursacher und Träger von Umweltbelastungen sein können, kann man vier Arten von Umweltexternalitäten unterscheiden, die in der folgenden Tabelle zusammengestellt sind:

\begin{tabular}{|c|c|c|}
\hline $\begin{array}{r}\text { Verur- } \\
\text { sacher }\end{array}$ & Produzent & Konsument \\
\hline Pröger & 1 & 2 \\
\hline Konsument & 3 & 4 \\
\hline
\end{tabular}

Im ersten Fall spricht man von "production-to-production effects", 1m zweiten von "consumption-to-production effects", im dritten von "production-to-consumption effects" und im vierten von "consumption-to-consumption effects" 13). In jedem dieser vier Fälle kann die Initiative zur Internalisierung entweder a) vom Verursacher oder b) vom Träger ausgehen, so da $B$ wir acht Fälle privater Umweltschutzinitiativen unterscheiden können, die in der folgenden Tabelle noch einmal zusammengestellt sind:

13) Vgl. Peters, G.H., Cost-Benefit-Analyse und staatliche Aktivität, Hamburg 1968, S. 24 f. 


\begin{tabular}{|c|c|c|c|c|}
\hline $\begin{array}{l}\text { Art der } \\
\text { Ini- Umwelt } \\
\text { tia- ext. } \\
\text { tor }\end{array}$ & $\begin{array}{l}\text { production } \\
\text { - to-produc- } \\
\text { tion } \\
\text { effects } \\
\quad 1\end{array}$ & $\begin{array}{l}\text { consump- } \\
\text { tion-to- } \\
\text { produc- } \\
\text { tion } \\
\text { effects } \\
\quad 2\end{array}$ & $\begin{array}{l}\text { produc- } \\
\text { tion-to- } \\
\text { consump- } \\
\text { tion } \\
\text { effects } \\
\quad 3\end{array}$ & $\begin{array}{l}\text { cons umption } \\
\text { - to-cons ump- } \\
\text { tion } \\
\text { effects } \\
\quad 4\end{array}$ \\
\hline$\frac{\text { a) }}{\text { Verursacher }}$ & $1 \mathrm{a}$ & $2 a$ & $3 a$ & $4 a$ \\
\hline $\begin{array}{c}\text { b) } \\
\text { Träger }\end{array}$ & $1 \mathrm{~b}$ & $2 b$ & $3 b$ & $4 b$ \\
\hline
\end{tabular}

Wir wollen im folgenden stellvertretend für alle Effekte die "production-to-production effects" näher betrachten 14), und zwar zunächst für den Fall, daß nur ein Verursacher und ein Träger existieren.

b) Möglichkeiten privater Umweltschutzinitiativen Die Konstellation, daB ein Produzent negative externe Effekte verursacht, die ein anderer produzent trägt, ist genau die, die dem berühmten Beispiel von Coase zugrundeliegt, und mit dem dieser beweist, daß negative externe Effekte ohne staatliche Maßnahmen allein aufgrund von Verhandlungen zwischen den betroffenen Partelen, also dem Verursacher $\mathrm{V}$ und dem Träger $\mathrm{T}$ beseitigt oder vermieden werden können 15)

14) Die anderen Fälle ergeben keine grundsätzlich anderen Ergebnisse. Wenn Haushalte als Verursacher oder Träger der Umweltbelastungen beteiligt sind, erschwert sich allerdings das Bewertungsproblem (vgl. Buchanan, J.H./Stubblebine, W.C., Externality, in: Staaf, R./Tannian, F. (Hrsg.), Externalities. Theoretical Dimensions of Political Economy, New York-London, 0.J., S. 278).

15) Vgl. Coase, R.H., The Problem of Social Cost, in: Staaf, R./ Tannian, F. (Hrsg.), Externalities, a.a.O., S. 119 ff.; Turvey, R., On Divergences between social cost and private cost, in: Economica, N.S., vol. 30, 1963, S. 309 ff.; Knappe, E., Möglichkeiten und Grenzen dezentraler Umweltschutz- 
Von wem die Initiative zur Verhandlung und damit zur Beseitigung bzw. Vermeldung der negativen externen Effekte ausgeht, hängt davon $a b$, ob für den Verursacher eine rechtliche Verpflichtung (und damit für den Trăger eine Klagemöglichkeit) besteht, die negativen externen Effekte zu verhindern oder zu beseitigen. Besteht keine solche Verpflichtung, wird der Trăger initiativ werden (Fall $1 \mathrm{~b}$ ) und den Verursacher $z u$ "bestechen" versuchen, die die Produktion von $T$ negativ beeinträchtigende Aktivität zu begrenzen. Die Bestechungssumme, die T maximal bereit sein wird $z u$ bieten, wird bis $z u$ der Schadenssumme gehen, die er $z u$ tragen hat, wenn $V$ keinen Umweltschutz betrelbt. Ist die (potentielle) Schadenssumme bel $T$ hober als die Vermeidungskosten bei $V$, so wird sich eine Losung ergeben, denn $T$ wird dem $V$ die summe zahlen, die für die Vermeldungsmaßnahmen notwendig ist. $V$ wird sich dann nicht schlechter und $T$ wird sich gegenüber der situation ohne Vermeidungsmaßnahmen besser stellen. In diesem Fall handelt es sich un sogenannte pareto-relevante negative externe Effekte 16). Ist dagegen die Schadenssumme niedriger als die Vermeidungskosten, kann $T$ im Falle marginaler negativer externer Effekte den $V$ durch Zahlung der Bestechungssumme nur zur schadstoffreduzierung und damit zur Schadenssenkung bringen; den verbleibenden Schaden muB T tragen. Im Fall inframarginaler negativer externer Effekte - das sind solche, die sich wegen ihrer Unteilbarkeit nur entweder vollig oder überhaupt nicht beseitigen oder vermeiden lassen - ist es für T vorteilhafter, den gesamten Schaden zu tragen 17)

Zusammenfassend kann man bis zu dieser stelle sagen: Die rein private Verhandlungslösung füht also nur dann zur Beseitigung oder Vermeidung der Umweltbelastung, wenn pareto-relevante Um-

politik.Bekämpfung externer Nachteile durch Verhandlungen, Berlin 1974, S. 39 ff.; Littmann, K., Umweltbelastung sozialbkonomische Gegenkonzepte, Gottingen 1974, S. 57 ff. und S. $95 \mathrm{ff}$.

16) Vgl. Buchanan, J.M./Stubblebine, W.C., External1ty, a.a.0., S. 280.

17) Zur Unterscheldung zwischen marginalen und inframarginalen extermen Effekten, vgl. ebenda, S. $278 \mathrm{f}$. 
weltexternalitäten vorliegen. Da die Kosten der Schadensvermeldung allein der Träger der Umweltbelastung aufbringt, ändert sich die Einkommensvertellung zugunsten des Verursachers der Umweltbelastung. Die Privatinitiative des $T$ hat also nur zur allokativen Effizienz geführt, wohl aber nicht zur distributiven Effizienz. Eine rein private Lösung ist zwar - zumindest unter den Annahmen von Coase - möglich, aber nicht mit den auf Vertellungsgerechtigkeit bedachten Grundsätzen einer sozialen marktwirtschaftlichen ordnung vereinbar. Ist $V$ nicht gesetzlich zur Unterlassung umweltbelastender Aktivitäten verpflichtet, so kann er das gar zu einer Erpressung des $T$ ausnutzen: $V$ kann dem $T$ mit umweltbelastenden (Schein-) Aktivitäten drohen, um von $T$ eine Bestechungssumme zwecks Unterlassung der gar nicht ernsthaft in Erwägung gezogenen Aktivitäten zu erhalten ${ }^{18}$ ?

Wir wollen nun die Bedingung aufgeben, das für den Verursacher keine rechtliche Verpflichtung besteht, Umweltschäden $z u$ vermeiden. Dies impliziert bereits elne staatliche Aktivităt: Die Legislative muB ein Gesetz erlassen, das umweltbelastende Tätigkeiten verbletet oder - etwa in Form von Emissionsstandards einschränkt; die Verwaltung muB die Einhaltung des Verbotes oder der Standards kontrollieren und die Judikative muB VerstöBe gegen das Verbot oder die Uberschreitung der Standards ahnden. Bel einer solchen ebenfalls von Coase untersuchten Konstellation wird die - zwelfellos ebenfalls private, wenn auch durch die Rechtsordnung erzwungene - Initiative zur Vermeldung oder Beseitigung der Umweltbelastung vom Verursacher ausgehen (Fall 1a). Der Verursacher wird die bei $1 \mathrm{hm}$ entstehenden Vermeldungskosten mit der Entschädigungssumme, die der Trăger fordert, wenn die Umweltbelastung nicht vermieden oder beseltigt wird, vergleichen und dann die für ihn billigste Maßnahme ergrelfen, also entweder die Schadstoffreduktion durchfüren oder dem Träger die Entschädigung zahlen. Die Kosten der Vermeldung, Beseitigung

18) Vgl. Möblus, K., Das Umweltproblem aus wirtschaftllcher Sicht, Kiel 1971, S. $11 \mathrm{f}$. 
oder Entschädigung trägt jetzt der Verursacher, so das man sagen kann, das neben der allokativen auch der distributiven Effizienz entsprochen wird. Eine solche Lösung würde den Ordnungsvorstellungen einer sozialen Marktwirtschaft am ehesten entsprechen. Sie ist - vom Ergebnis her - mit dem Verursacherprinzip identisch.

Bei "production-to-production effects" ist unter bestimmten Voraussetzungen auch eine andere auf Privatinitiative beruhende Lösung mögl1ch, nämlich eine Fusion zwischen dem Verursacher und Träger der Umweltexternalităt 19) Eine solche Lösung bietet sich vor allem dann an, wenn die Produktion von $V$ in irgendeiner Weise von $T$ abhängig 1st, sel es, das $T$ Vorprodukte für $V$ produziert oder Abnehmer der Produkte von $\mathrm{V}$ ist. Die bel getrennter Gewinnmaximierung auftretenden von $V$ verursachten negativen externen Effekte werden durch die Fusion nicht nur formal internalisiert, sondern es kommt bei verbundener Gewinnmaximierung auch zu einer materiellen Beseitigung der die Produktion von $T$ beeinträchtigenden Umweltbelastung. Mit einer Fusion kann man also eine Marktunvollkommenhelt beseltigen, eine andere allerdings hervorrufen, nämlich - wegen der durch die Fusion bewirkten Monopolisierung eine Elnschränkung des Wettbewerbs 20) Marktunvollkommenheiten sollte man aber nicht mit anderen Marktunvolikommenhelten $z u$ bekämpfen versuchen 21) - vor allem nicht in einem marktwirtschaft11 chen System.

C) Grenzen des Umweltschutzes durch Privatinitiative

Die unter den Annahmen von Coase verwirklichte Beseitigung der Umweltbelastungen aufgrund privater Inltiative 1st nur in einem Fall mit den Grundsätzen einer sozlalen Marktwirtschaft verein-

19) Vgl. Coase, R.H., The Problem of Soclal Cost, a.a.O., S. 132 ff.: Davis, O.A./Whinston, A., Externalities, Welfare, and the Theory of Games, in: Journal of Political Economy, vol. 70, 1962, S. $244 \mathrm{ff}$.; Schlleper, U., Pareto-Opt1ma, externe Effekte und die Theorie des Zweitbesten, a.a.0., S. $45 \mathrm{ff}$.

20) Vgl. Littmann, K., Umweltbelastung - soz1alökonomische Gegenkonzepte, a.a.0., S. 56 .

$21)$ Vgl. Sohmen, E:, Allokationstheorie und Wirtschaftspolitik, a.a.O., S. 247 
bar, und in diesem Fall hat der staat bereits eine wichtige Funktion: Er muß eine Rechtsordnung schaffen, in der Gesetze die Umweltbelastungen verbieten oder begrenzen, und er muß deren Elnhaltung kontrollieren und Verstoße gerichtllch ahnden, damit die (potentiellen) Verursacher die Umweltbelastungen vermeiden oder zumindest die Träger der Umweltbelastungen entschädigen, sofern diese bereit sind, ihr Recht auf eine saubere Umwelt zu verkaufen.

Bereits daraus wird ersichtlich, daB Umweltschutz in einer sozialen Marktwirtschaft a $u c h$ eine staatliche Aufgabe ist, und zwar 1st die Erfüllung dieser staatllchen Aufgabe konstitutiv für einen Umweltschutz, der in einer dem Verursacherprinzip entsprechenden Internalisierung der negativen externen Effekte besteht und nicht in einer "Ausbeutung der rechtlich Schwächeren" 22) die bel coase leicht zustandekommen kann, wenn keine Gesetze die Umweltbelastungen verbieten; sie führt zwar auch zum Allokationsoptimum, nicht aber zum Distributionsoptimum. "... when negotiation is possible, the case for government intervention is one of justice not of economic efficiency" 23). Die Grenzen privater Umweltschutzinitiativen werden noch deutlicher, wenn wir nur einige dem Coase-Theorem zugrundeliegende Prämissen auf thren Realitätsgehalt hin überprüfen.

Die Analyse von Coase, nach der allein durch Verhandeln der an der Umweltbelastung beteiligten Parteien das Allokationsoptimum zustandekommt, beruht auf der Annahme, daB keine Verhandlungskosten bestehen. Solche Verhandlungskosten fallen aber zwangsläufig an, z.B. für die Beschaffung von Informationen über die Höhe der Vermeidungskosten und - was, wie wir wissen, u.U. äußerst schwierig ist - uber die Höhe der Schäden, welche die Umweltbelastungen hervorrufen können. Ferner entstehen Kosten für die Organisation und Durchführung der Verhandlungen. Alle diese Kosten können so hoch sein, daß sie den Nutzen, den die

22) Littmann, K., Umweltbelastung - sozialökonomische Gegenkonzepte, a.a.0., s. 247.

23) Turvey, R., On Divergences between Social cost and Private Cost, a.a.o., s. 313 . 
Beseitigung der Umweltexternalitäten stiften würde, uberkompensieren 24) Coase selbst weist auf diesen Tatbestand hin, gibt allerdings $z u$ bedenken, daB alternative staatliche Masnahmen keineswegs geringere Kosten implizieren müssen: "All solutions have costs and there is no reason to suppose that government regulation is called for simply because the problem is not well handled by the market .... 25)

Der Haupteinwand gegen das Coase-Theorem 1st aber wohl der, daß private Initiativen zum Umweltschutz gar nicht zustandekommen werden, da es bis auf wenige Fälle - und die sind in der Regel relativ unbedeutend - eine Vielzahl von Verursachern und vor allem Trägern einer Vielzahl verschiedener Umweltbelastungen gibt, die sich in threr wirkung addieren, potenzieren oder auch neutralisieren können oder deren Wirkung erst nach Jahren oder gar Generationen eintritt, so daß weder die Verursacher noch die Träger - jedenfalls nicht durch private Initiative - zwelfelsfrei identifiziert noch die Schäden elndeutig ermittelt werden können 26)

24) Vgl. Head, J.G., Public Goods and Public Welfare, Durham, N.C., 1974, S. 201.

25) Coase, R.H., The Problem of Social Cost, a.a.O., s. 135. Vgl. dazu auch Calabres1, G. , Transaction Costs, Resource Allocation, and Liability Rules, in: Dorfman, R./Dorfman, N.S. (Hrsg.), Economics of the Environment, a.a.0., S. 197 f. der staatliche Eingriffe in den Marktmechanismus be fürwortet, wenn durch diese Eingriffe der Allokationsmechanismus wieder so funktionsfähig gemacht werden kann, daß er das Allokationsoptimum effizienter erreicht, also ohne staatliche Maßnahmen, und wenn neben dem Allokationsziel auch andere $\mathrm{zlele}$ (z.B. das Vertellungsziel) gefährdet sind.

26) Vgl. Schlieper, U., Pareto-Optima, externe Effekte und die Theorle des Zweltbesten, a.a.0., S. 63; Pennance, F.G., The Polluters: Industry or Government, a.a.0., s. 36; Head, J. G., Public Goods and Public Welfare, a.a.O., s. 193 f.: Hyman, D.N., The Economics of Governmental Activity, a.a.0., S. 44 f.: Green, H.P., The Role of Government in

Environmental Conflict, a.a.0., S. 302; Hofmann, U., Rechtswidrige Tatbestände - aber selten nachweisbare Schuld, in: Blick durch die Wirtschaft v. 6.9.71. Berelts Pigou hat darauf hingewiesen, daB bei externen Effekten, die "nicht nur auf eine kleine, leicht erfaBbare $\mathrm{Zahl}$ von Personen beschränkt sind"-der staat auf dem Wege fiskalischer MaBnah- 
Auch die besten Gesetze, welche elne Umweltbelastung verbieten, helfen nichts, wenn der Verursacher in der Anonymitat untertauchen kann, und die wohlwollendsten Richter können keinem Träger der Umweltbelastungen helfen, wenn sie nicht wissen, wer der Verursacher ist. Für manche schäden kommen auch elne vielzahl von Verursachern in Frage, deren individueller Anteil aber minimal 1st; das Problem 1st die "Masslerung gleichartigen Verhaltens ... durch die Addition ... tausender Infinitesimaler Schädigungspotentiale" 27) Einsichtige Träger von Umweltbelastungen werden sich unter solchen Bedingungen erst gar nicht zu einer gerichtlichen Klage entschließen, denn der ProzeB wird thnen nur Kosten und Muhe bringen 28) Einige Träger von Umweltbelastungen hoffen vielleicht auch, in den GenuB einer Free-rider-Position zu kommen, well andere Träger der Umweltbelastungen eventuell klagen. Denken alle so, geschleht gar nichts 29) In manchen Fällen wird sich der Träger elner Umweltbelastung auch dann nicht zur Klage entschlieben, wenn der Verursacher bekannt ist, well er sich keine Chance ausrechnet, seinen individuell vielleicht groBen, im Verhältnis zu den Produktions- und Beschäftigungsinteressen des verursachenden Produzenten und dessen Angestellten jedoch relativ kleinen schaden geltend machen zu können 30 ? "The

men elngreifen muB (vgl. Pigou, A.C., Volkswirtschaftlicher und privatwirtschaftilcher Reinertrag und die Lehre von der Maximalbefriedigung, in: Mayer, H./Fetter, F.A./Reisch, R. (Hrsg.), Die Wirtschaftstheorie der Gegenwart, 3. Bd., Wien 1928, S. 47. f.)

27) Schăfer, Dieter, Soziale Schäden, soziale Kosten und soziale Sicherung, Berlin 1972, S. 130.

28) Vgl. Rupp, H.H., Popularklage Im Umweltschutzrecht?, In: Zeltschrift für Rechtspolitik, 5. Jg.. H. 2, S. 32; siebert, H. . Das produzierte Chaos. Okonomie und Umwelt. Stuttgart u.a. 1973, S. $145 \mathrm{ff}$.

29) Vgl. Bernholz, P., Grundlagen der politischen Okonomie, 1. Bd. Tubingen 1972, S. 178; Arnold, V., Kuppelprodukte, offentliche Unguter und externe Effekte, in: Zeitschrift für die gesamte Staatswissenschaft, Bd. 132, 1976, S. 101; Schlieper, U.. Pareto-Optima, externe Effekte und die Theorie des Zweitbesten, a.a.0.. S. 64.

30) Vgl. Kneese, A.V., Environmental Pollution: Economics and Policy, In: American Economic Review, vol. 61. 1971, papers and proceedings, S. 154. 
litigation is a David v. Gollath affair" 31)

Coase selbst sieht das Problem, das sich aus der vielzahl der Beteiligten ergibt; und in solchen Fällen gesteht er dem staat durchaus eine Aufgabe zu: "... there is no reason why, on occasion, (such) governmental administrative regulation should not lead to an improvement in economic efficlency ... particularly when, as is normally the case with the smoke nuisance, a large number of people are involved and in which therefore the costs of handling the problem through the market... may be high" 32)

3. Die umweltpolitischen Aufgaben des Staates

a) Zur Eintellung der urmweltpolitischen Aufgaben des Staates Die Ausfuhrungen des letzten Abschnitts haben gezelgt, daB sich die Umweltbelastungen nicht oder nur zu elnem sehr geringen Teil durch Privatinitiative vermelden oder beseltigen lassen, da die meisten Arten der Umweltbelastung den Charakter von "public bads" haben, von deren Duldung keiner ausgeschlossen werden kann 33), oder umgekehrt formuliert: Saubere Gewässer, reine Luft, saubere Landschaft und Ruhe sind offentliche (Umwelt-) Güter, von deren GenuB kein Bürger ausgeschlossen werden kann ${ }^{34}$ ). Die Erhaltung oder Wiederherstellung einer bestimmten Umweltqualităt 1st somit

31) Green, H.P., The Role of Government in Environmental Conflict, a.a.0., S. 303; vgl, auch Rehblnder, E., Argumente fur die Verbandsklage im Umweltrecht, in: Zeitschrift für Rechtspolitik, 9. Jg., 1976, S. 159.

32) Vgl. Coase, R.H., The Problem of Social cost, a.a.O., S. 134.

33) Vgl. Arnold, V., Kuppelprodukte, offentliche Unguter und externe Effekte, a.a.0., S. 91; Frey, R.L., Umweltschutz als wirtschaftspolit1sche Aufgabe, a.a.0., s. 471; Buchanan, J.M., Jolnt Supply, Externality and Optimality, in: Economica, N.S., Bd. 33, 1966, S. 404 ff.; Slebert, H., Das produzierte Chaos, a.a.O., s. $128 \mathrm{ff}$.

34) Vgl. Blair, R.D., The Clean Air Act of 1970, a.a.0., S. 261; Bonus, H., Uber Schattenpreise von Umweltressourcen, in: Jahrbuch für Sozialwissenschaft, Bd. 23, 1972, S. 342. 
eine öffentliche Aufgabe 35! Damit ist allerdings noch keineswegs gesagt, daB sle auch in vollem Umfang vom staat finanziert werden muß, und es 1st auch nicht notwendig, daß der staat die konkrete Durchführung der Maßnahmen, die für die Realisierung der Umweltschutzziele notwendig sind, in vollem Umfang ubernimmt. In vielen Fällen wird es genügen, wenn der staat elnen Rahmen setzt oder einen AnstoB zur privaten Durchfuhrung der notwendigen Umweltschutzmaßnahmen gibt. Ihm steht dazu ein reichhaltiges juristisches und ökonomisches Instrumentarium zur Verfügung 36 ! Es dient vor allem elner Korrektur oder Ergănzung des Allokationsmechanismus Markt, um diesen (wieder) in die Lage zu versetzen, aus elgener Kraft die Allokation der Umweltguter in optimaler Weise vorzunehmen, also Umweltbelastungen durch eine Ex-ante-Internalisierung der (potentiellen) Umweltexternal1täten zu vermelden.

Der Allokationsmechanismus Markt kann u.U. bereits durch das Schaffen und Verbessern der Rahmenbedingungen, die für private Umweltschutzinitiativen erforderlich sind, funktionsfähig gemacht werden. Wenn dies nicht ausreicht, um einen zieladăquaten Umweltschutz zu gewährleisten, muB der staat zusätzlich in das Marktgeschehen eingrelfen, um mit elnem möglichst marktkonformen umweltpolitischen Instrumentarium die Erreichung der umweltpolitischen ziele durchzusetzen.

Die umweltpolitischen Aufgaben des staates bestehen also darin

1. die Rahmenbedingungen vor allem für die privaten Umweltschutzmaßnahmen zu schaffen und zu verbessern,

2. die innerhalb dieses Rahmens nicht durch privatinitiative wahrgenommenen Umweltschutzmaßnahmen mit Hilfe staatlicher Instrumente durchzusetzen 37 ?

35) Vgl. RSU, Umweltgutachten 1974, a.a.0., S. 8 und S. 168 f.; Hansmeyer, K. -H., Volkswirtschaftliche Kosten des Umweltschutzes, a.a.0., S. 108 .

36) Siehe unten S. $310 \mathrm{ff}$.

37) Vgl. Saladin, P., Glelchgewichtssicherung als staatsaufgabe, in: NZZ, FA Nr. 195 v. 22./23.8.1976. 
b) Zur schaffung und Verbesserung der umweltpolitischen Rahmenbedingungen

Wie die Ausfuhrungen über das Coase-Theorem gezeigt haben, kommt ein gerechter Umweltschutz auf privater Basis nur zustande, wenn die Rechtsordnung dem Träger einer Umweltbelastung das Recht einrăumt, den Verursacher auf Unterlassung oder Entschădigung zu verklagen. Neben der rechtlichen Institutionalisierung dieses dem Verursacherprinzip entsprechenden Grundsatzes bedarf es vor allem rechtspolitischer Maßnahmen, um dieses Prinzip auch in der Praxis anwenden zu können. Die juristische Realisierung des Verursacherprinzips scheiterte bisher vor allem daran, daß die Verursacher kaum zu identifizieren waren, die Schăden kaum bewertet werden konnten und die Trăger der Schäden aus verschiedenen Grüden einen ProzeB scheuten.

Durch die folgenden drei rechtspolitischen Maßnahmen könnte man die "Funktionsbedingungen für das Wirksamwerden des Coase-Theorems verbessern" 38):

(1) die Umkehr der Beweislast,

(2) die Inst1tutionalisierung der Verbandsklage,

(3) die Schaffung von Elgentumsrechten an den Umweltmedien.

$\mathrm{Zu}(1)$ :

Gegenwărtig muß der Trăger elner Umweltbelastung als Kläger vor Gericht beweisen, daß bestimmte Schadstoffe, die von einem oder mehreren Verursachern emittiert wurden, seine Gesundheit oder sein Elgentum geschädigt haben. Die Bewelslast liegt also beim Träger der Umweltbelastung. Würde man die Beweislast umdrehen so müBte der vermeintliche Verursacher beweisen, das er nicht für den vom Träger erlittenen Schaden verantwortlich ist. Diese Umkehr der Bewelslast würde die ohnehin schwache stellung des

38) RSU, Umweltgutachten 1974, a.a.O., S. 157.

39) Vgl. Green, H.P., The Role of Government in Environmental Conflict, a.a.0., S. 302; Congress and the Nation's Environment, a.a.0., S. 45; wăldle, T., Bürgerklage und Umweltverträglichkeltsprufung, in: Zeitschrift fur Rechtspolitik, 8. Jg., 1975, S. 107; Kapp, K.W.. Zur Praxis der Umweltpolitik und der Umweltplanung, a.a.O.. S. 165. 
Klägers sowohl in prozessualer als auch finanzleller Hinsicht stärken. Allerdings bedeutet diese Maßnahme auch nur dann elne Verbesserung, wenn die Anzahl der möglichen Verursacher relativ gering ist.

Zu $(2)$ :

Als elgentliche Ursache der Umweltbelastung haben wir mehrfach die ungenügende Durchsetzung der gesetzl1chen Vorschriften durch die Behörden bezelchnet: Es ist ein Vollzugsdefizit entstanden, und zwar nicht zuletzt deshalb, well die Behörden infolge der zunehmenden offentlichen Daseinsvorsorge auch Aufgaben des Umweltschutzes ubernommen haben 40) - und wohl auch ubernehmen muBten -, für deren Durchsetzung sie weder technisch noch personell hinreichend ausgestattet waren 41) Das vollzugsdefizit ist zumindest tellwelse also auch die Folge objekt1ver Unzulänglichkeiten in der offentlichen Verwaltung.

M1t zunehmender Umweltbelastung wurden - zunächst in den USA die offentlichen Verwaltungen, deren Aufgabe es gewesen wăre, die Umweltbelastungen $z u$ unterbinden, in zunehmendem MaBe von elnzelnen Bürgern, aber vor allem privaten Organisationen verklagt, und zwar glelchgultig, ob es sich um materlell von der jeweillgen Umweltbelastung betroffene Bürger oder Organisationen handelte oder nicht. "Such lawsuits are Indicators of a citizens revolt against official protectors of the public interest" 42 ) Die Gerichte in den USA wiesen zunăchst die Klagen ab, die von

40) Vgl. Schafer, Dieter, Soziale Schäden, soziale Kosten und soziale stcherung, a.a.0., s. 138.

41) Vgl. Schmldt, Helmut, Wirtschaftspol1t1sche Moglichke1ten, Programme und Masnahmen zum Umweltschutz, in: Kapp, K.W./ Vilmar, F., Sozialisierung der Verluste?, a.a.0., S. 181; RSU, Umweltgutachten 1974, a.a.0., S. 181. Symptomat1sch dafür 1st z.B. die ungenügende Anzahl gut ausgeb1ldeter Fachkräfte 1m kerntechnischen Berelch (vgl. dazu den Ministerbericht zur Reaktorsicherheit, der auszugsweise abgedruckt 1st, in: Umwelt 1974, 6, s. 11 ff.).

42) Sax, J.L., Legal Redress of Environmental Disruption in the United States: The Role of Courts, in: Tsuru, S. (Hrsg.), Proceedings of the International Symposium on Environmental Disruption, a.a.0., s. 224. 
Nicht-Betroffenen im offentlichen Interesse geführt wurden

allmahlich setzt sich jedoch die Ansicht durch, daB auch solche Klage formen berechtigt sind 44) Besonders Naturschutzverbände wie der Sierra Club fuhrten "im öffentlichen Interesse" viele Prozesse gegen offentliche Institutionen und die Verursacher von Umweltbelastungen 45) Der Ruf nach der Institutionalisierung einer solchen Klagemöglichke1t wurde laut: "The job of lawyers is to fashion tools that will make the public interest into public rights" 46)

Auch in der Bundesrepublik Deutschland wurde die Forderung nach einer Verbesserung der gerlchtlichen Vollzugskontrolle der Verwaltung zwecks Intensivierung des Umweltschutzvollzugs erhoben ${ }^{47}$ )

43) Vgl. Sax, J.L., standing to Sue: A Critical Review of the Mineral King Decision, in: Natural Resources Journal, vol. 13, 1973, s. $76 \mathrm{ff}$.

44) Vgl. Lev1, D.R./Colyer, D., Some Legal and Economic Aspects of Citizen-Initiated Legal Mechanisms for Solving Environmental Quality Problems, in: Intermountain Economic Review, vol. 3 (2), 1972, S. $40 \mathrm{ff}$.

45) Vgl. ebenda, S. $40 \mathrm{f}$. und Sax, J.L., standing to sue: A Critical Review of the Mineral king Decision, a.a.0., s. 76 ff.

46) Sax, J.L.. Legal Strategies Applicable to Environmental Quality Management Decisions, in: Kneese, A.V./Bower, B.T., Environmental Quality Analysis, a.a.0., S. 343; vgl. auch Congress and the Nation's Environment, a.a.0., s. 45. Inzwischen wurde die Verbandsklage in den USA institutional1siert. Sie kann sich auch auf die gerlchtliche Kontrolle der "environmental impact statements" erstrecken, die den Umweltvertrăglichkeitsprüfungen in der Bundesrepublik Deutschland entsprechen (vgl. Wäldle, T., Bürgerklage und Umweltverträglichkeitsprüfung, a.a.0., S. 109).

47) Vgl. Rupp, H.H., Popularklage Im Umweltschutzrecht?, a.a.O., S. 32 ff.; ders., Mobilisierung des Umweltschutzrechts durch Beteiligung der Aktivburgerschaft an seinem Vollzug, Manuskript eines unveröffentlichten Vortrags im Rahmen der Gemelnschaftsvorlesung "Umweltschutz und Umweltforschung" an der Johannes Gutenberg-Universität Malnz, Mainz 1976; ders.. Rechtsgutachten zur Intensivierung des Umweltschutzes durch Verstärkung der Initiative Privater und zur Harmonisierung des Umweltschutzrechts der Mitgliederstaaten in den Europä1schen Gemeinschaften, Manuskript, Mainz 1974/75; Rehbinder, E., Argumente für die Verbandsklage im Umweltrecht, 
Die Vorschläge relchen von der Einführung eines Grundrechts auf eine saubere Umwelt in der Verfassung uber die Institutionalislerung elnes Ombudsmanns für Umweltschutz oder elnes Umweltschutz-staatsanwalts, also elner "Reakt1vierung behördlicher Beanstandungsklagen" 48), bis hin zur Popularklage und Verbandsklage, also elner "Mobilisierung des Klagetyps individueller Beanstandung" 49 ?

Die Einfuhrung eines Grundrechts auf eine saubere Umwelt, wie es u.a. der RSU empfiehlt 50), das sich aber im Prinzip bereits aus den Art. 1 und 2 GG ableiten ließe 51), würde wohl nur eine (zusätzliche) moralische Verpflichtung der Exekutive zu einem besseren Vollzug bewirken, aber kaum eine zusätzliche Klagemöglichkelt schaffen, da sowohl in der Literatur als auch in der Rechtsprechung elne erhebliche zurückhaltung besteht, aus grundrechtlichen Garantien einen einklagbaren Ausspruch des Bürgers gegen den staat abzuleiten 52 )

Das Ombudsmann-System kann seinen sinn als $z$ e $t r$ a $l$ e $r$, nur dem Parlament verantwortlicher Mobilisierungsstelle in einem föderativen staat nur unzureichend nachkommen 53). Der Umwelt-

a.a.0., S. $157 \mathrm{ff.;}$ ders., Grundfragen des Umweltrechts, in: Zeltschrift für Rechtspolit1k, 3. Jg., 1970, S. 250 ff.; wäldle, T., Bürgerklage und Umweltverträglichkeitsprüfung, a.a.0., S. 105 ff.; RSU, Umweltgutachten 1974, a.a.0., S. $172 \mathrm{ff}$.

48) Rupp, H.H., Popularklage Im Umweltschutzrecht?, a.a.0., s.33.

49) Ebenda, S. 34 .

50) Vgl. RSU, Umweltgutachten 1974, a.a.0., s. 173.

51) Vgl. Rupp, H.H., Die verfassungsrechtliche Seite des Umweltschutzes, in: Juristenzeitung Nr. 13 v. 2.7.1971, S. 402 .

52) Vgl. ebenda und Rehbinder, E., Umweltschutz: Die Rolle von Gesetzgebung und Rechtsprechung, In: Umwelt, 1971, 1, S. 27. Vielversprechender scheint die Möglichkeit zu sein, den Umweltschutz uber die Sozialpflichtigkeit des Eigentums nach Art. 14 (2) GG auf verfassungsrechtlichem wege $z u$ intensivieren (vgl, Kimminich, O., Das Recht des Umweltschutzes, München 1972 , S. $57 \mathrm{ff.})$.

53) Vgl. Rupp, H.H., Popularklage Im Umweltschutzrecht?, a.a.O., S. 33 . 
schutz-Staatsanwalt wäre wegen seiner Welsungsgebundenheit immer dann in einem Interessenkonflikt, wenn der staat bei industriellen Umweltbelastungen wegen mangelnden Vollzugs mitverantwortlich ist. Ein weisungsgebundener Umweltschutz-Staatsanwalt wäre im Rechtssystem der Bundesrepublik Deutschland ein zu großer Fremakörper 54)

Die Popularklage, die nicht der Durchsetzung subjekt1ver Rechte, sondern des objektiven Rechts dient, hat gerade beim Umweltschutz beträchtliche Nachteile, weil sie wegen der Weite des Umweltschutzbereichs und der Vielzahl differenzierter Klagegründe zu elner Fülle von Klagen führen könnte, die "das ganze hergebrachte System des Rechtsschutzes in Mitleidenschaft ziehen (würde)" 55) Als empfehlenswerte Möglichkelt gerichtlicher Vollzugskontrolle gilt - allerdings nicht unumstritten 56) - die Verbandsklage 57) In der Bundesrepublik Deutschland kennt man diese klageform vor allem im Wettbewerbsrecht (vgl. $\S 13$ UWG und $\S 35$ (2) GWB). In der Schwelz hat man mit ihr auch auf dem Gebiet des Umweltschutzes bereits gute Erfahrungen gemacht ${ }^{58}$ ) Man kann die egoistische und die ideelle Verbandsklage unterscheiden 59! Während

54) Vgl. Rupp, H.H., Popularklage im Umweltschutzrecht?, a.a.O.. S. $33 \mathrm{f}$.

55) Ebenda, S. 34.

56) Vgl. Redeker, K., Verfahrensrechtliche Bedenken gegen die Verbandsklage, in: Zeitschrift für Rechtspolitik, 9. Jg., 1976 , S. $163 \mathrm{f}$.

57) Vgl. Rupp, H.H., Rechtsgutachten zur Intensivierung des Umweltschutzes ...., a.a.0., S. 63 ff.; Rehblnder, E., Argumente für die Verbandsklage im Umweltrecht, a.a.0., S. $157 \mathrm{ff}$.; RSU, Umweltgutachten 1974, a.a.0., S. 178. Die Verbandsklage bezieht sich auf verfassungsrechtliche VerstöBe; der RSU schlägt darüber hinaus vor, auch zu prüfen, inwleweit Umweltschutzverbände das Recht zur Einleitung von Anklageerzwingungsverfahren nach $\S \S 172 \mathrm{ff}$. StPo eingeräumt werden kann (vgl. RSU, Umweltgutachten 1974, a.a.0., S. 179).

58) Vgl. Rehbinder, E., Grundfragen des Umweltsechts, a.a.0., S. 255; ders.. Argumente für die Verbandsklage im Umweltrecht, a.a.O., S. 163 .

59) Vgl. Rehbinder, E., Argumente für die Verbandsklage im Umweltrecht, a.a.o.. s. 158. 
erstere der "Bündelung individueller Rechte" 60) dient, um wohl vor allem die ProzeBkosten auf möglichst viele Verbandsmitglieder zu vertellen, bezweckt die ideelle Verbandsklage die Kontrolle des Vollzugs der Umweltgesetze und ist somit ein Instrument objekt1ver Rechtskontrolle 61)

Das gröBte Problem besteht wohl darin, welche Verbände als klageberechtigt anerkannt werden sollen 62) W11l man eine Uberlastung der Gerichte vermeiden, kann man wohl nur z.B. In einem behördlichen Anerkennungsverfahren besonders legitimierten Verbänden das Recht zur Verbandsklage einräumen und nicht etwa allen bestehenden oder slch ad hoc blldenden Bürgerinitiativen, die sich dem Umweltschutz verschrieben haben. Mitgliederstărke, repräsentative organisation und Sachverstand sind wichtige Kriterien, die bel der Anerkennung berücksichtigt werden mussen 63) Problematisch scheint es uns allerdings zu sein, das Anerkennungsverfahren von Behorden durchfuhren zu lassen, denn es sind u.U. gerade diese Behörden, welche dann von den Verbänden verklagt werden sollen. Unseres Erachtens bletet ein gerichtliches Anerkennungsverfahren eine bessere Gewăhr fur die Unabhăngigkeit der Verbände.

Kurzfristig wird die Wirkung der Elnführung einer Verbandsklage $\mathrm{zu}$ einer stärkeren Stellung der Umweltschutzinteressen $1 \mathrm{~m}$ P $r \circ z$ e $B$ füren, langfristig versprechen sich ihre Befürworter eine präventive Kontrollfunktion 64): Die Umweltschutzverwaltung wird versuchen, möglichst gar kein Vollzugsdefizit

60) Rehbinder, E., Argumente für die Verbandsklage im Umweltrecht, a.a.0., s. 158 .

61) Vgl. ebenda.

62) Vgl. Redeker, K., Verfahrensrechtliche Bedenken gegen die Verbandsklage, a.a.0., S. 164.

63) Vgl. Rehbinder, E., Argumente für die Verbandsklage im Umweltrecht, a.a.0., s. 161 .

64) Vgl. ebenda: Rupp, H.H., Mobilisierung des Umweltschutzrechts durch Betelligung der Aktivbürgerschaft an seinem Vollzug, a.a.O.. S. 11; Caponera, D.A., Towards a New Methodological Approach in Environmental Law, in: Natural Resources Journal, vol. 12. 1972, S. 148. 
entstehen zu lassen, was wohl auch die Privatinitiative der potentiellen Verursacher erhöhen dürfte.

Die Hauptgefahr, die durch eine Institutionalisierung der Verbandsklage befürchtet wird, ist elne Blocklerung oder zumindest Verzögerung wichtiger offentlicher und indirekt auch privater Großprojekte und damit letztlich eine Gefährdung des wirtschaftswachstums 65) Solche Gefahren ließen sich vermindern, wenn die Umweltschutzverbände neben dem Klagerecht auch ein Anhörungsund Mitwirkungsrecht bei der Planung offentlicher Großprojekte (Bau von Kernkraftwerken, Staudămmen, Autobahntrassierungen u.a.) zugestanden erhielten, so dạ zwischen den beteiligten Gruppen bereits bel der Entscheldungsvorbereltung ein Konsens hergestellt werden konnte 66)

Es wird auch auf die Gefahr hingewiesen, daB sich mehrere zugelassene Umweltschutzverbände Konkurrenz machen könnten 67) Dies könnte, weil sich die Verbände gegenseitig übertrumpfen wollen, leicht zu Maximalforderungen hinsichtlich des Umweltschutzes führen, die gesamtwirtschaftlich nachtellige Folgen haben könnten. In solchen Fällen wird es darauf ankommen, das slch die offentliche Verwaltung nicht von den Umweltschutzverbänden einschulchtern läBt und das $r e c h t e \quad M$ a $B$ an Umweltschutz durchsetzt und dabel - wenn es zum prozeB kommt - auch von den Gerichten unterstützt wird 68?

65) Vgl. Rehbinder, E., Argumente für die Verbandsklage Im Umweltrecht, a.a.O., S. 162; Rupp, H.H., Mobilislerung des Umweltschutzrechts durch Beteiligung der Akt1vburgerschaft an seinem Vollzug, a.a.0., s. $9 \mathrm{f}$.

66) Vgl. Rupp, H.H., Mobilisierung des Umwaltschutzrechts durch Betelligung der Aktivbürgerschaft an seinem Vollzug, a.a.0., S. $6 \mathrm{f} . ;$ Wäldle, T., Bürgerklage und Umweltverträglichkeitsprüfung, a.a.0., s. 105; Saladin, P., Glelchgewichtssicherung als Staatsaufgabe, a.a.O.: Z1lleBen, H., Ansprüche und Arbeit der Bürgerinitiativen, a.a.0.. s. 43.

67) Vgl. Redeker, K., Verfahrensrechtliche Bedenken gegen die Verbandsklage, a.a.O., S. 164 .

68) Vgl. RSU, Umweltgutachten 1974, a.a.0., s. 178. 
Zu (3):

Wir haben bereits angedeutet, daB die "Ubernutzung" der Umweltmedien Luft, Wasser und Boden die Folge davon ist, daß sie zumindest tellweise als freie Güter angesehen werden, die von jedem genutzt werden können, ohne einen Prels für die Nutzung zu zahlen. Daß diese Umweltgüter kostenlos genutzt und damit be 1 ihrer Ubernutzung geschädigt werden können, liegt daran, daß für sie keine Elgentumsrechte bestehen. Es handelt sich bel ihnen um "unrestricted common property" 69! um "communal ownership" 70), um Gemeineigentum von der Art der Allmende. Das vorgeschlagene Rezept für elne kostengerechte Bew1rtschaftung der Umweltressourcen besteht nun darin, die Umweltmedien in privates Eigentum $z u$ überführen oder zumindest privaten die Nutzungsrechte an den Umweltgütern zu ubertragen 71) Man verspricht sich davon eine Internalisierung der externen Effekte, denn die jeweiligen Elgentümer werden ihr Eigentum vor Umweltbelastungen $\mathrm{zu}$ bewahren versuchen, indem sie ihre eigenen Umweltressourcen nicht überbeanspruchen und indem sie Beeinträcht1gungen ihrer Umweltgüter durch andere - jetzt genau definierte Elgentiumer auf dem Verhandlungswege im Sinne von Coase zu vermelden versuchen oder Entschädigungen dafür verlangen 72) Da die Schaffung von Elgentumsrechten nicht nur zur Berechtigung führt, die elgenen Umweltressourcen zu nutzen, sondern auch zur Verpflichtung, die Umweltressourcen der anderen nicht zu schädigen, stellt sich bei genau definierten Elgentumsrechten an den Umweltgütern, wie bei Coase, automatisch eine Internalisierung der externen Effekte ein - vorausgesetzt es sind die glelchen Annahmen erfüllt, die auch dem Coase-Theorem zugrunde-

69) Dales, J.H., Pollution, Property and Prices. An Essay in Policy-making and Economics, Toronto 1968, S. 63.

70) Demsetz, H., Toward a Theory of Property Rights, in: American Economic Review, vol. 57, 1967, papers and proceedings, S. 354 .

71) Vgl. ebenda, S. $347 \mathrm{ff}$.

72) Vgl. ebenda, S. 356. 
liegen: Vor allem die Annahme, daß keine Verhandlungskosten bestehen, 1st wichtig für eine $v \circ 1$ l $s \mathrm{t} a \mathrm{n} d i \mathrm{~g} e$ Internalisierung der externen Effekte, also die automatische Realisierung des Allokationsoptimums.

Gibt es jedoch Verhandlungskosten, so kommt es allein aufgrund von Privatinitiativen nicht zur vollständigen Internalisierung der externen Effekte. Demsetz weist jedoch $z u$ Recht darauf hin, da $\beta$ durch die Schaffung privater Eigentumsrechte die Verhandlungskosten gegenüber der situation reduziert worden sind, in der bei Gemeineigentum eine gemeinschaftliche Verminderung der Umweltbelastung versucht wirde ${ }^{73}$ ), da nicht mehr jeder einzelne der Gemeinschaft als Verursacher einer bestimmten Umweltbelastung in Frage kommt, sondern nur noch ein bestimmter oder mehrere bestimmte Eigentiumer. Dieser Vortell reduziert sich allerdings um so mehr, je mehr verursacher und Träger an einer bestimmten Umweltbelastung betelligt sind. Die entstehenden Schwierigkeiten sind die gleichen wie beim Coase-Theorem: Eine privat initilerte Internalisierung wăre $z u$ teuer ${ }^{74}$. Hier spinnt Demsetz den Faden nicht weiter: Er prüt nicht - obwohl er die Moglichkeit erwähnt -, inwieweit eine Internalisierung der Umweltexternalitäten dadurch möglich oder zumindest verbessert wird, daB das Gemelneigentum in staatliches Eigentum überführt wird, so daB der staat mit seinem Instrumentarium die Internalisierung erzwingen kann ${ }^{75}$ ?

73) Vgl. Demsetz, н., Toward a Theory of Property Rights, a.a.o., S. 356 .

74) Vgl. ebenda, S. 357.

75) Vgl. Borchardt, K., Volkswirtschaftliche Kostenrechnung und E1gentumsverteilung. Bemerkungen zum Problem der Sozialkosten, in: Jahrbücher für Nationalökonomie und Statistik, Bd. 178, 1965, S. 77.

Der Gedanke, das Gemeineigentum an den Umweltgütern in staatliches Eigentum zu überführen und vom Staat so bewirtschaften $z u$ lassen, daß keine Ubernutzung eintritt - was nichts anderes bedeutet als daB die Immissionsstandards eingehalten werden -, wird Im AnschluB an Demsetz von Krier und Montgomery aufgenommen (vgl. Krier, J.E./Montgomery, W.D., Resource Allocation, Information cost and the Form of Government Intervention, in: Natural Resources Journal, 
Aufgrund der Schaffung von Eigentumsrechten allein kann wohl nur unter sehr günstigen Voraussetzungen elne mehr oder weniger automatische Internalisierung gelingen. Allerdings erleichtert die Schaffung von privaten und/oder staatlichen Elgentumsrechten die Durchsetzung einer rationalen Umweltpolitik. Ob Private oder Staat Elgentumer der jeweiligen Umweltmedien werden sollen, hängt zunächst von der Wirtschaftsordnung ab. In einem marktwirtschaftlichen system sollten die Umweltmedien, soweit dies technisch möglich ist, in privateigentum überfuhrt werden. Eigentumer sollten allerdings nur solche Privatpersonen oder privaten Institutionen werden, die ein Interesse an der Erhaltung der jewelligen Umweltmedien haben. So sollten z.B. nicht potentielle Abwassereinleiter Eigentumer von FluBabschnitten werden, sondern Wasserwerke oder Fischzuchtbetriebe, Angelsportverelne oder das Fremdenverkehrsgewerbe. Es müBte allerdings auch sichergestellt werden, daB die privaten Eigentümer ihr E1gentumsrecht nicht an potentielle Verursacher von Umweltschäden veräußern oder die Nutzung thres Eigentums durch potentielle Umweltverschmutzer in $z u$ groBem Umfang zulassen, well die Kaufpreise oder die Mieten und Pachten, die lhnen die potentielien Verschmutzer bieten, hobher sind als die Erträge, die sie mit Ihrer umweltadäquaten Nutzung der Umweltmedien erzielen können. Bei den Umweltmedien Luft und groBen Gewässern wird es aber schon aus technischen Gründen praktisch unmöglich sein, sie in Privatelgentum zu überführen, und zwar wegen Ihrer Untellbarkeit und der damit verbundenen kaum möglichen AusschlieBbarkeit 76) Die Umweltgüter Luft und Gewässer sind typisch öffentliche Güter, weshalb sie aus dem Gemelneigentum in das Eigentum des Staates überführt werden müssen. Damit ist allerdings noch keineswegs gewährleistet, daB der staat die Umweltgüter auch optimal - d.h.

vol. 13, 1973, S. 89 ff.l.

Mit dem Vorschlag von Dales, daB der staat als Eigentümer der Umweltressourcen deren Nutzung via Verkauf von Verschmutzungsilzenzen regelt, werden wir uns noch befassen (vgl. unten S. $380 \mathrm{ff.}$ ).

76) Vgl. Borchardt, K., Volkswirtschaftliche Kostenrechnung und Elgentumsvertellung, a.a.0., s. $76 \mathrm{f}$. 
so, daß die Immissionsstandards eingehalten werden - bewirtschaftet. In zentralverwaltungswirtschaftlich organisierten Staaten mit öffentlichem Eigentum an den Umweltressourcen sind die Umweltbelastungen nicht geringer als in anderen staaten, da dort die Umweltressourcen ebenfalls als frele Guter behandelt werden 77) eine Folge der marxistischen Auffassung vom Warenwert, der nur aus der Arbelt erklärt wird 78) Daraus allerdings den Schluß zu ziehen, die Umweltguter grundsätzlich nicht in staatliches Eigentum zu überführen 79), halten wir für verfehlt, da zumindest in demokratischen staaten die staatlichen Instanzen gezwungen werden können, die in ihrem Elgentum befindlichen Umweltgüter auch im bffentlichen Interesse zu nutzen.

Ein weiterer Grund, der für staatliches Eigentum an den Umweltgutern spricht, ist die Existenz intertemporaler negativer externer Effekte; nur der staat kann - sofern das wegen der Schwierigkeit, die sozialen Wohlfahrtsfunktionen zuküftiger Generationen vorauszuschätzen, überhaupt möglich ist - für eine opt1male zeitliche Nutzung der Umweltgüter sorgen 80), also dafür, daB wir zukünftigen Generationen nicht die natürlichen Lebensgrundlagen (z.B. Grundwasser, landwirtschaftliche Anbau-

77) Vgl. Issing, 0., Zerstörung der Umwelt - ein Versagen des marktwirtschaftlichen Systems?, a.a.0., s. 136; Goldman, M.I. , The Convergence of Environmental Disruption, a.a.0., S. 302 f.: Altvater, E., Gesellschaftliche Produktion und ökonomische Rationalität. Externe Effekte und zentrale Planung im Wirtschaftssystem des Sozialismus, Frankfurt/Main und Wien 1969, S. 156.

78) Vgl. Binswanger, H.C., Eine umweltkonforme Wirtschaftsordnung, a.a.O., S. 134; Höhmann, H.-H./Seldenstecher, G./ Vajna, T., Umweltschutz und ökonomisches System in Osteuropa, a.a.0., S. 29.

79) Binswanger, H.C., Umrisse einer umweltkonformen Wirtschaftsordnung, in: Schlemmer, J. (Hrsg.), Neue Ziele für das Wachstum, a.a.0., s. 128 .

80) Vgl. Frey, B.S./Schwödiauer, G., Uber die zeltliche Nutzung der Natur, in: Schmollers Jahrbuch, 91. Jg., 1971, S. 696; Schlieper, U., Pareto-Optima, externe Effekte und die Theorie des Zweitbesten, a.a.0., S. 65 . 
flächen) zerstören. Man kann allerdings der Auffassung sein, daß dies eher eine ethische Verpflichtung als eine ökonomische Aufgabe des staates ist, da zukünftige Generationen nichts für die Umweltschutzlelstungen der vorangegangenen Generationen zahlen müssen, außer diese wären durch langfristige Verschuldung in der Gegenwart finanziert 81 ?

Zusammenfassend können wir sagen, daß die Umwandlung des Gemeinelgentums (communal ownership) in privates (private ownership) oder staatliches Eigentum (state ownership) eine wichtige $\mathrm{V} \circ \mathrm{r}$ a $\mathrm{s} s$ e $t \mathrm{z} \mathbf{n} \mathrm{g}$ für eine private oder staatlich initiierte Internalisierung (negativer) externer Effekte und damit für eine optimale Allokation 1st. $\mathrm{Zu}$ bedenken ist allerdings auch hier, daß die Schaffung von Eigentumsrechten zwar die Allokationseffizienz erhöht, die Distributionseffizienz aber unter Umständen verringert. Die Verteilung der Eigentumsrechte muB daher auch unter distributiven Aspekten vorgenommen werden.

Nach dieser relativ ausführlichen Darstellung verschiedener n e u e r Möglichkeiten, den umweltpolitischen Rahmen zu verbessern, wollen wir noch auf die eher traditionellen oder zumindest schon institutionalisierten vom staat geschaffenen Rahmenbedingungen eingehen. Wir können uns dabel kurz fassen, weil diese Rahmenbedingungen bereits im Verlauf der Arbeit geschildert wurden und dabei nur mehr oder weniger offen blieb, wer diese für private und staatliche Umweltschutzmasnahmen notwendigen Rahmenbedingungen zu setzen hat. Es handelt sich dabel vor allem um die Normierung der Immissionsstandards möglichst vieler Schadstoffe. Solange "sich das Parlament (dieser) elementaren Aufgabe entzieht" 82) scheint eine rationale Umweltpolitik nicht möglich.

Ferner ist die Messung der Umweltbelastung eine wichtige staat11 che Aufgabe. Dies gilt in erster Linie für die Messung der

81) Siehe dazu unten S. $396 \mathrm{f}$.

82) Rehbinder, E.. Argumente für die Verbandsklage im Umweltrecht, a.a.0., S. 160 . 
Immissionskonzentrationen; mit der Messung der Emissionen und Immissionen in der Umgebung industrieller Anlagen kann der staat die Emittenten beauftragen ${ }^{83)} ;$ er muß diese MeBergebnisse aber zumindest stichprobenweise kontrollieren. Die Aufstellung der Emissions-, Immissions- und Wirkungskataster muB dagegen der staat wieder selbst übernehmen.

Immissionsstandards und statistische Daten luber die tatsächliche Umweltbelastung bllden den Ausgangspunkt für Planung und Durchführung der Umweltschutzmaßnahmen.

Der staat muß dabei den Rahmenplan aufstellen, in dem auch die Aufgabenallokation zwischen privatem und offentlichem Sektor erfolgen muB. Sodann muB er die sich selbst zugeteilten konkreten Umweltschutzaufgaben und deren Finanzierung planen.

Rein staatliche Aufgaben sind die Raumordnung und die Umweltverträglichkeitsprufungen offentlicher Maßnahmen. Aufgaben, die nach allgemeiner Auffassung 84) uberwiegend vom staat wahrgenommen werden mulssen, sind die Forderung der Umweltforschung

83) Dies geschieht z.B. durch die $\S \S 26$ ff. BImSchG.

84) Vgl. Rathjens, G.W., National Environmental Pollcy: Goals and Priorities, a.a.o., S. $41 \mathrm{f}$; : The Annual Report of the Council of Economic Advisers, 1966, Washington, D.C. 1966, S. 174; The Economics of Clean A1r, 1972, a.a.0., S. 2-7 ff.; Sobers, D.G., Planning for the Environmental Revolution, in: Armstrong, T.A. (Hrsg.), Why Do We Still Have an Ecological Crisis?, a.a.0., S. 68; Gruhl H., Wer soll für die Umwelt zahlen?, in: Marktwirtschaft, 3. Jg. Nr. 7, 1971, S. 14.

85) Würgler welst allerdings $z u$ Recht darauf hin, daB staatliche Normen, wie die Emissionsgrenzwerte für Kraftfahrzeuge, erhebliche private Forschungen induzieren können (vgl. Würgler, H., రkonomische und politische Ursachen der Umweltveränderungen, a.a.O., S. 99). Die Grundlagenforschung, z.B. Uber die Umwelteffekte der Schadstoffe, mus dagegen nach Ansicht Würglers "In einer Wirtschaftsordnung, die dem Marktmechanismus vertraut" (ebenda, S. 101) eine bffentliche Aufgabe sein, In dieser apodiktischen Form kann man das u.E. nicht akzeptieren. In der Bundesrepubik Deutschland sind z.B. private Stiftungen erheblich an der Forderung der Grundlagenforschung beteiligt (vgl. Forschungsbericht IV, a.a.0., 2. 29). 
die Ausbildung von Umwelt(schutz-)experten in verschiedenen Disziplinen, die Kontrolle der Technik durch Technology Assessment 86 ) und die Erziehung $z u$ einem (neuen) Umweltbewubtsein.

Die Erfüllung dieser Aufgaben ermöglicht die Schaffung eines umweltpolitischen Rahmens, innerhalb dessen sich der privat initilerte (freiwilifge), staatlich erzwungene oder vom staat selbst durchgefühte technische Umweltschutz vollziehen kann. Abschließend sel noch auf die ureigenste staatliche Aufgabe hinsichtlich der Rahmenbedingungen hingewiesen: die Gesetzgebung. Die direkten staatlichen Umweltschutzmaßnahmen können nur aufgrund von Gesetzen durchgefüht werden, und auch die staatlichen Maßnahmen, welche die Privaten zum Umweltschutz anregen oder zwingen sollen, bedürfen einer gesetzlichen Grundlage. Je vollkommener die umweltpolitischen Rahmenbedingungen erfullt sind, um so weniger bedarf es staatlicher Interventionen, denn um so eher werden die Privaten mehr oder weniger freiwillig bereit sein, die zur Einhaltung der Immissionsstandards notwendigen MaBnahmen des Umweltschutzes zu ergreifen. Sind die Rahmenbedingungen dagegen unzureichend oder unvollständig, muß der staat zur Durchsetzung der Umweltschutzziele sein umweltpolitisches Instrumentarium stärker einsetzen. Es besteht also zumindest tellwelse eine substitutionsmöglichke1t zwischen dem Setzen umweltpolitischer Rahmenbedingungen und dem Einsatz des umweltpolitischen Durchsetzungsinstrumentariums.

Bevor wir uns mit dem umweltpolitischen Durchsetzungsinstrumen-

86) Technology Assessment kann auch innerbetrieblich erfolgen, und zwar nicht nur aus sozialer Verantwortung der Unternehmer (vgl. Dierkes, M. , Qualität des Lebens und unternehmerische Entscheldung, a.a.0., S. 27; Schumacher, D., Hilfsmittel für die Umweltplanung, a.a.0., S. 26), sondern auch u.U. deshalb, weil umweltfelndliche produktionsverfahren oder Produkte zu staatlichen Sanktionen fuhren oder umweltbewubte kăufer der Konkurrenz zutrelben können, die umweltfreundlicher produziert (vgl. Gramm, W.P., A Theoretical Note on the Capacity of the Market System to Abate Pollution, in: Land Economics, vol. 45, 1969, S. 365 ff. und Stephens, I.K.. "A Theoretical Note on the Capacity of the Market System to Abate Pollution": A Reply, in: Land Economics, vol. 48, 1972, S. 304 f.). 
tarlum im elnzelnen befassen, wollen wir im nächsten Abschnitt noch prüfen, welche grundsätzlichen staatlichen Möglichkeiten es gibt, eine rationale Umweltpolitik durchzusetzen.

c) Strateglen zur Durchsetzung der Umweltpolitik

Unter Durchsetzung wollen wir die Durchfuhrung und Finanzierung der zur Einhaltung der Immissionsstandards notwendigen - vor allem technischen - Maßnahmen des Umweltschutzes verstehen. Für die Durchsetzung einer rationalen Umweltpolitik sind vor allem zwel Fragen von Bedeutung:

(1) Wer soll die technischen Umweltschutzmaßnahmen durchführen?

(2) Wer soll die Durchführung der technischen Maßnahmen des Umweltschutzes finanzieren?

$\mathrm{Zu}(1)$ :

Wer die technischen Umweltschutzmaßnahmen durchfüren soll, muB unter Effizienzgesichtspunkten entschieden werden. Vergegenwärtigen wir uns die Elntellung der präventiven Maßnahmen des technischen Umweltschutzes, so kann man sagen, das dann, wenn sich emissionsorientierte technische Maßnahmen als am effizientesten erwelsen, der Emittent, den wir mit dem Eigentümer der Emissionsquelle gleichsetzen und als Verursacher bezeichnen wollen, die notwendigen Maßnahmen zur Emissionsvermeldung durchführen muB. Stellen sich dagegen immissionsorientierte technische MaBnahmen als effizienter heraus, so müsen die potentiellen Träger der Umweltbelastung die Immissionsschutzmaßnahmen durchfuhren. Ergibt die optimale planung, daB die transmissionsorientierten technischen Maßnahmen am effizientesten sind, müssen die emittierenden Verursacher die technischen Maßnahmen durchführen, wenn die "Verdünnung" der Schadstoffströme durch emisslonsseitige Masnahmen, wie etwa den Bau hoher Schornsteine, optimal erreicht werden kann. Erfolgt die "Verdünnung" der Schadstoffe wăhrend der Transmission (z.B. durch die strömung eines Gewässers), muB wohl der staat die notwendigen technischen Maßnahmen (hier z.B. die Regulierung des Flußbettes) durchfuhren. Der staat kann aber auch noch aus anderen Effizienzgrúnden mit der Durchführung der technischen Umweltschutzmaßnahmen beauf- 
tragt werden. Die Praxis des technischen Umweltschutzes hat gezelgt, daß manche umwelttechnischen Maßnahmen erhebliche "economies of scale" aufweisen. Um in den Genuß möglichst hoher Skalenerträge zu kommen, müssen die Umweltschutzanlagen eine gesamtwirtschaftlich optimale Betriebsgröße haben. Einzelne private Wirtschaftseinhelten sind nun in der Regel nicht in der Lage, gesamtwirtschaftlich optimal dimensionierte Anlagen auch optimal auszulasten. Das standardbeispiel hierfür sind Kläranlagen 87) Um die "economies of scale" voll ausnutzen zu können, müssen die Kläranlagen Kapazitäten haben, die In der Regel nicht von einzelnen Unternehmen ausgelastet werden können. Eine Unterauslastung großer Kläranlagenkapazitäten ist aber in der Regel teurer als eine Vollauslastung kleinerer Anlagen. Aus einzelwirtschaftlichen Uberlegungen heraus wäre deshalb u.U. eine kleinere, aber optimal ausgelastete Kläranlage günstiger. Unter gesamtwirtschaftlichen Effizienzgesichtspunkten ist aber eine größere Kläranlage wegen der größeren Skalenerträge günstiger - vorausgesetzt sie ist ihrerseits optimal ausgelastet. $\mathrm{Zu}$ einer solch optimalen Auslastung kann es u.U. durch Kooperation privater Unternehmen kommen; wahrscheinlicher ist jedoch, daß Unternehmen mit den Kommunen kooperieren, zumal die Gemeinden auch das Kanalisationsnetz für die Sammlung der industriellen Abwässer zur Verfügung stellen 88 )

87) Vgl. U.S. Department of the Interior/Federal Water Pollution Control Administration, The Economics of Clean Water, Summary Report, Washington, D.C., 1970, S. 130; dieselben, The Cost of Clean Water, vol. II: Detailed Analyses, Washington, D.C., 1968, S. 39 ff.; U.S.E.P.A., The Cost of Clean Water, vol. II: Cost Effectiveness and Clean Water, Washington, D.C., 1971, S. 71 ff. und S. 117: Littmann, K., Umweltbelastung - sozialökonomische Gegenkonzepte, a.a.0.. S. $45 \mathrm{ff}$.

88) Ein Musterbelspiel für die gemeinsame Abwasserklärung von privaten Unternehmen und Kommunen, das in der ganzen Welt Nachahmung gefunden hat, sind die Ruhrgenossenschaften (vgl. Kneese, A.V./Bower, B.T., Die Wassergütewirtschaft, München und Wien 1972, S. 261 ff.; Klevorick, A.K./Kramer, G.H. Social Cholce on Pollution Management: The Genossenschaften, in: Journal of Public Economics, vol. 2, 1973, S. $101 \mathrm{ff.l}$. 
Ahnliche Uberlegungen gelten auch für die Abfallbeseitigung ${ }^{89)}$ und die zentralisterung der häuslichen $\mathrm{SO}_{2}$-Emissionsquellen durch den Bau von Fernheizwerken. Vor allem die Entsorgungsaktivitäten scheinen also unter Effizlenzgesichtspunkten - teilweise auch aus Sicherheltsgründen, wie bel der Entsorgung von Kernkraftwerken - am besten vom staat durchgefüht zu werden 90). Unter dem Aspekt einer effizienten Durchführung der technischen Maßnahmen des Umweltschutzes kommen also (potentielle) Verursacher, (potentielle) Träger und der staat in Frage. Da Verursacher und Träger von Umweltbelastungen private Haushalte, private Unternehmen und der Staat sein können, ist es also auch möglich, daß der staat in seiner Eigenschaft als

89) Vgl. Hösel, J., Kosten elner befriedigenden Neuordnung der Abfallbeseitigung, in: Schultze, H. (Hrsg.), Umwelt-Report, a.a.0., S. 198 .

90) Demgegenüber sieht der Wissenschaftliche Beirat beim Bundesfinanzministerium gerade im kommunalen Entsorgungsbereich eine Möglichkeit der materiellen Verlagerung öffentlicher Dienstleistungen auf den privaten Bereich, und zwar entweder mit weiterhin vollständiger öffentlicher Finanzierung, tellweiser öffentlicher Finanzierung oder gar mit vollständiger privater Finanzlerung ( $\mathrm{vgl}$. Gutachten des Wissenschaftlichen Beirats beim Bundesministerium der Finanzen zur Lage und Entwicklung der Staatsfinanzen in der Bundesrepublik Deutschland, Bonn 1975, S. $23 \mathrm{ff.}$ ). Der Wissenschaftliche Beirat beim BFM empfiehlt eine solche Verlagerung, um das strukturelle Defizit des öffentlichen Haushalts zu verringern. Wie eine Untersuchung des Deutschen Instituts für Urbanistik in Zusammenarbeit mit dem Deutschen Städtetag ergeben hat, ist jedoch gerade bei den Entsorgungsleistungen der Gemeinden ein sehr hoher Kostendeckungsgrad realisiert; er beträgt bei der Abwasserbeseitigung durchschnittlich 938 und bei der Abfallbeseitigung durchschnittlich 998 (vgl. Deutsches Institut für Urbanistik / Deutscher Städtetag (Hrsg.), Kommunale Gebuhrenhaushalte, Bd. 1: Kalkulationsverhalten und Deckungsgrade, Berlin 1976, s. $11 \mathrm{f}$. ). Obwohl Kostendeckungsgrade über 1008 in manchen Gemeinden deshalb zustandekommen, weil nicht die kalkulatorischen Kosten berücksichtigt werden (vgl. ebenda), ist nicht auszuschlieBen, daß manche Gemeinden an der Entsorgung sogar "verdienen" und mit diesen Uberschüssen andere Defizitpositionen z.B. im kulturbereich reduzieren.

Das ändert natürlich nichts an der Aussage, daß eine Ver lagerung der Entsorgung zum privaten Sektor dann erfolgen sollte, wenn sie dort effizienter wahrgenommen werden kann. 
(potentleller) Verursacher oder (potentieller) Träger einer Umweltbelastung die notwendigen Umweltschutzmaßnahmen durchführen muB.

$\mathrm{Zu} \mathrm{(2):}$

Mit der Aufgabenallokation, also der Bestimmung derjenigen, welche die Umweltschutzaufgaben $d$ u $r$ h $f$ u h $r$ e $n$ sollen, ist u.E. noch nicht die Frage beantwortet, wer die Umweltschutzaufgaben $f$ i $n$ a $z i$ e $r$ soll. Während für die Aufgabenallokation ausschlieBlich Effizienzaspekte maBgeblich sein sollten, müssen nach unserer Ansicht bel der Bestimmung derjenigen, welche die Umweltschutzaufgaben finanzieren sollen, auch Vertellungsaspekte - und damit bestimmte Gerechtigkeitsvorstellungen - berücksichtigt werden. So können U.E. die Träger von Umweltbelastungen nicht direkt zur Finanzierung der Umweltschutzmaßnahmen verpflichtet werden, und zwar auch dann nicht, wenn sie von ihnen aus Effizienzgründen durchgeführt werden müssen 91 ) Aus Gründen der Gerechtigkeit und aus ordnungspolitischen Gründen sollten in einer sozialen Marktwirtschaft die Verursacher die technischen Maßnahmen des Umweltschutzes finanzieren 92), und zwar gleichgultig, ob sie von lhnen selbst, von den (potentiellen) Trägern der Umweltbelastung oder vom staat aus Effizienzgründen durchgeführt werden. Diesen Grundsatz bezeichnet man als Verursacherprinzip 93! Aus sozialen und aus Effizienzgründen kann es allerdings auch oder gerade in einer sozialen Marktwirtschaft notwendig werden, daß der staat die Finanzierung übernimmt, und zwar wieder unabhängig davon, ob er die

91) Indirekt zahlen natürlich auch die (potentiellen) Träger der Umweltbelastungen elnen Teil der Umweltschutzkosten, entweder über höhere Preise oder über höhere Steuern.

92) Vgl. Menke-Glückert, P., Das Umweltprogramm der Bundesregierung, a.a.0., S. 128; derselbe, Vom Umweltschutz zur Umweltpolitik, in: Umwelt 1972, 1, S. 5; Schäfer, Friedrich, Das Verursacherprinzip in der Umweltschutzpolitik, in: Umwelt, 1972, 2, S. 4 ff.

93) Vgl. RSU, Umweltgutachten 1974, a.a.O., S. 10 f. und S. $155 \mathrm{ff.;}$ Hansmeyer, K.-H., Volkswirtschaftliche Kosten des Umweltschutzes, a.a.0., S. 108. 
Maßnahmen selbst durchführt oder ob dies durch die privaten Verursacher oder Träger der potentiellen Umweltbelastungen geschieht. Soziale Gründe könnten z.B. seln, daB die (potentiellen) Verursacher sozial schwache Bürger sind oder aber Grenzbetriebe, die aus beschäftigungspolitischen Grüden erhalten werden sollen. Aus Effizienzgründen wird der staat die Finanzierung übernehmen müssen, wenn die Anwendung des Verursacherprinzips $z u$ hohe Kosten z.B. für die Identifikation der Verursacher oder die $\mathrm{Zurechnung}$ der jeweiligen Schadensantelle auf dieselben erfordert 94). In den Fallen, in denen der staat die Finanzierung der Umweltschutzmaßnahmen übernimmt, spricht man von Gemelnlastprinzip 95)

Sehen wir von dem nicht wünschenswerten Fall ab, daB die (potentiellen) Träger der Umweltbelastungen zur direkten Finanzierung des Umweltschutzes verpflichtet werden sollen, so kann man sechs Kombinationen zwischen der Durchfuhrung und Finanzierung der technischen Umweltschutzmaßnahmen unterscheiden. Sie sind in der folgenden Matrix-Tabelle zusammengestellt:

\begin{tabular}{|l|c|c|c|}
\hline $\begin{array}{l}\text { Fi- Durch- } \\
\text { nanzie- } \\
\text { rung }\end{array}$ & Verursung \\
\hline Verursacher & 1 & Trăger & Staat \\
\hline Staat & 4 & 2 & 3 \\
\hline
\end{tabular}

94) Vgl. Ackermann, K./Geschka, H./Karsten, D., Die wirtschaftspolitische Lösung: Verursacherprinzip, a.a.0., S. 32; dieselben, Gutachten zur Gesamtbelastung der Volkswirtschaft durch das Umweltprogramm der Bundesreglerung, a.a.0., S. $602 \mathrm{ff.}$; RSU, Umweltgutachten 1974, a.a.O., S. 155.

95) Vgl. RSU, Umweltgutachten 1974, a.a.0., S. 155; Hansmeyer, K. - H. , Volkswirtschaftliche Kosten des Umweltschutzes, a.a.o., S. 108 . 
Die Fälle 1 bis 3 entsprechen dem Verursacherprinzip, die Fälle 4 bls 6 dem Gemeinlastprinzp 96) Inwieweit durch die verschiedenen Fälle das öffentliche Budget belastet wird, hängt von der Budgetrelevanz der Instrumente ab, mit denen die Einhaltung der Immissionsstandards durchgesetzt werden soll. Wir müssen also zunächst die umweltpolitischen Instrumente des Verursacher- und Gemeinlastprinzips näher betrachten, um diese danach hinsichtlich 1hrer Budgetrelevanz und threr Effizienz zur Erhaltung der Immissionsstandards beurteilen zu können.

\section{B. Instrumente zur Durchsetzung der Umweltpolitik}

1. Zur Eintellung und Bewertung der umweltpolitischen Instrumente Interpretieren wir die Matrix-Tabelle auf S. 307 als "box" der umweltpolitischen Instrumente, so können wir diese einteilen in die Instrumente zur Durchsetzung des Verursacherprinzips (mit den drei Instrumentengruppen $1,2,3)$ und in die Instrumente zur Durchsetzung des Gemeinlastprinzips (mit den drei Instrumentengruppen 4,5,6). Am vielfältigsten ist zweifellos das Instrumentarium der "box" 1. Es enthält alle die Instrumente, welche die Verursacher zur Emissionsreduktion und ihrer Finanzierung anregen oder zwingen sollen. Bei der Darstellung dieser Instrumente gehen wir von dem klassischen Instrument der Internalisierung negativer externer Effekte aus: der Pigou'schen Steuer. Ihre Ablehnung als praktikables Instrument zur Internalisierung negativer externer Effekte in der Form von Umweltschäden fuhrt uns dann zur Analyse weiterer Instrumente, die ebenfalls dem Verursacherprinzip entsprechen: Wir untersuchen zunächst Auflagen, und zwar sowohl in bezug auf produkte, Technologien und Standorte als auch

96) In der Literatur ist die Trennung zwischen Verursacherprinzip und Gemeinlastprinzip nicht immer klar. Das gilt vor allem für eine kostendeckende Finanzierung öffentlich durchgeführter Maßnahmen durch Gebühren. Nach unserer Definition wirde dies eindeutig dem Verursacherprinzip entsprechen. Nach Klaus zimmermann gehort elne solche Finanzierung, da sie "In staatlichen Budgets erscheint" (Zimmermann, Klaus, Vorausschätzung staatlicher Umweltkosten, a.a.0., S. 130) zum Gemeinlastprinzip. 
in bezug auf Emissionen. Die Auflagen können die Form von Geboten, Verboten und Standards haben. Neben den Auflagen enthălt "box" 1 als weltere Instrumentenkategorle Abgaben, die als Bemessungsgrundlage nicht, wie die Pigou-steuer, den Schaden haben, sondern die Produktmenge, den Faktorinput oder die Schadstoffemissionen. Wir werden uns vor allem mit der Emissionsabgabe befassen und auch auf elne besondere Version davon, nämlich auf Emissionszertifikate, eingehen.

Die Instrumente der "box" 2 bestehen aus Maßnahmen, welche die potentiellen Träger von Umweltbelastungen dazu veranlassen, Immissionsschutzmaßnahmen durchzufuhren, die jedoch nicht von thnen, sondern von den Verursachern bezahlt werden müssen. Solche Instrumente sind z.B. die Schaffung von Eigentums- und Klagerechten, mit denen wir uns bereits befaBt haben.

Die "box" 3 enthält die Instrumente, die dem staat zur Verfügung stehen, um die Kosten der von ihm aus Effizienzgründen durchgeführten Umweltschutzmaßnahmen auf die potentiellen Verursacher der Umweltbelastungen umzulegen, also vor allem Gebühren, z.B. für die Inanspruchnahme der staatlichen (oder kommunalen) Abfall- und Abwasserbeseitigungsanlagen.

Nach den Instrumenten zur Durchsetzung des Verursacherprinzips wenden wir uns denen zur Durchsetzung des Gemeinlastprinzips zu. In "box" 4 sind alle die Finanzierungsinstrumente enthalten, die der staat dazu verwenden kann, bei den Verursachern Maßnahmen zur Emissionsreduktion zu initiieren. Es handelt sich dabei um Subventionen aller Art (Darlehen, Zuschüsse, Steververgünstigungen, Sonderabschreibungen u.a.). Die "box" 5 enthält die gleichen Instrumente, nur daß sie in diesen Fällen aus Effizienzgründen den potentiellen Trägern der Umweltbelastungen zur Durchführung von ImmissionsschutzmaBnahmen gewährt werden. In "box" 6 schließlich befinden sich die Instrumente, die der staat zur Verfügung hat, um die von ihm durchgeführten Maßnahmen des Umweltschutzes zu finanzieren, nämlich steuern und Kredite. Da wir die umweltpolitischen Instrumente im nächsten Abschnitt nicht nur darstellen, sondern auch hinsichtlich ihrer Eignung zur Realisierung der umweltpolitischen zlele analysieren wollen, 
müssen wir die Instrumente auch bewerten und zwar nicht nur hinsichtlich ihrer theoretischen Allokationseffizienz, sondern auch in bezug auf thre praktische Anwendbarke1t. Es wird sich dabel zeigen, daB die Anwendbarkeit gerade der Instrumente, die theoretisch eine groBe Allokationseffizienz haben, oft nicht praktikabel ist, well die dafur erforderlichen Informationen und der administrative Aufwand $z u$ groB sind. Auf die Bewertung der Instrumente hinsichtlich ihrer ordnungspolitischen Konformität und ihrer Wirkungen auf andere wirtschaftspolit1sche Ziele werden wir im Verlauf dieses Kapitels eingehen.

Erst dann können wir die möglichen Budgetbelastungen durch die jewelligen Instrumente abschätzen, denn diese ergeben sich nicht nur aus der Anwendung der Instrumente selbst und den Informations- und Administrationskosten, welche die Anwendung hervorruft, sondern auch aus den Folgekosten und Folge $n$ u $t z e n$ der Anwendung der Instrumente hinsichtlich der Zielerreichungsgrade anderer (wirtschafts)politischer ziele.

2. Darstellung und krit1sche wüdigung der umweltpolitischen Instrumente

a) Die Instrumente zur Durchsetzung des Verursacherprinzips aa) Zur Internalisierung negativer externer Effekte durch eine Pigou'sche Steuer

Im Umweltprogramm der Bundesregierung helBt es: "Jeder, der die Umwelt belastet oder sie schädigt, soll für die Kosten dieser Belastung oder schädigung aufkommen" 97? Diese Version des Verursacherprinzips ist kongruent mit der Forderung nach einer Internalisierung negativer externer Effekte. Von P1gou stammt der Vorschlag, die Internalisierung negativer externer Effekte dadurch vorzunehmen, daB man die Produktion des Gutes besteuert, bel der die negativen externen Effekte entstehen, und zwar in Hohe der negativen externen Effekte, also der Differenz zwischen privaten und sozialen Kosten 98). In marginalen GröBen

97) Das Umweltprogramm der Bundesregierung, a.a.0., S. 27.

98) Vgl. Pigou, A.C., The Economics of Welfare, Reprint der 4. Auflage von 1932 , London 1952, S. 224 f. und die vor allem durch den Coase-Aufsatz von 1960 (a.a.O.) ausgelösten Dis- 
und auf die Umweltbelastung bezogen kann man das auch so formulieren: Negative marginale externe Effekte in Form marginaler Umweltschäden lassen sich dadurch internalisieren, daB man die Produktion, bel der die Schadstoffe emittiert werden, mit einer Steuer belegt, deren marginaler Satz gleich dem marginalen Umweltschaden im Allokationsoptimum 1st. Um die Internalisierungssteuer bzw. deren marginalen satz ermitteln zu können, muB man also das Allokationsoptimum und die (marginale) Schadensfunktion kennen. Wir wollen nun für den einfachsten denkbaren Fall eines negativen externen Effektes den marginalen Satz der Internalisierungssteuer bestimmen 99?

Wir gehen davon aus, $\operatorname{daB}$ zwei Unternehmen je ein Gut, nămlich $x_{1}$ und $x_{2}$, herstellen, das sle zum preis von $p_{1} b z w . p_{2}$ verkaufen. Bel der Produktion von $x_{1}$ entsteht nun als Kuppelprodukt ein Schadstoff, dessen Emissionsmenge $e_{1}$ die Produktion der Unternehmung 2 negativ beeinfluBt, also für Unternehmung 2 einen (kontrollexternen) Kostenfaktor darstellt. Die Unternehmung 2 verursacht keine externen Effekte bei Unternehmung 1, und der negative externe Effekt, der von Unternehmung 1 verursacht wird, beeinflusse nur die Gesamtkosten der Unternehmung 2, nicht aber deren Grenzkosten. Wir unterstellen also einen sowohl nicht-

kussionen über die Internalisierung externer Effekte. Einen sehr guten Uberblick über diese Diskussionen geben Mishan (vgl. Mishan, E.J., The Postwar Literature on Externalities: An Interpretative Essay, in: Journal of Economic Literature, vol. 9, 1971, S. $1 \mathrm{ff.l}$ und mit besonderem Bezug zum Umweltproblem Fisher und Peterson (vgl. Fisher, A.C./Peterson, F.M., The Environment in Economics: A Survey, in: Journal of Economic Literature, vol. 14, 1976, s. 1 ff.).

99) Zur folgenden Darstellung vgl. Jürgensen, H./Jaeschke, K.P. Allokationseffekte der Social Costs im Umweltschutz - Untersuchung zur Anwendung des Verursacherprinzips. Unveröffentlichtes Gutachten für das Bundesministerium des Innern, Hamburg 1972, S. $43 \mathrm{ff}$; Weber, W. , Wohlfahrtstheoretische Aspekte der Besteuerung: Zielkonflikte und Theorie des Zweitbesten, in: Haller, H. U.a. (Hrsg.), Theorie und Praxis des finanzpolitischen Interventionismus, Fritz Neumark zum 70. Geburtstag, Tübingen 1970, S. $198 \mathrm{ff.;}$ Head, J.G., Public Policies and Pollution Problems, in: Finanzarchiv, Bd. 33, 1974, S. $1 \mathrm{ff}$. 
reziproken als auch separablen negativen externen Effekt 100) Beide Unternehmen sollen Gewinnmaximierung betreiben. Es gelten dann folgende Beziehungen:

Der Gewinn der Unternehmung 1 ist

(1) $G_{1}=p_{1} x_{1}-K_{1}\left(x_{1}\right)$

Die Kosten der Unternehmung 2 sind außer von der produzierten Menge $x_{2}$ auch abhängig von der Emissionsmenge $e_{1}$, so daß gilt:

(2) $k_{2}=k_{2}\left(x_{2}, e_{1}\right)$.

Da die emittierten Schadstoffmengen $e_{1}$ von der hergestellten Produktmenge $x_{1}$ abhăngen, also gilt:

(3) $e_{1}=e_{1}\left(x_{1}\right)$

ergibt sich durch Einsetzen von (3) in (2)

(4) $K_{2}=K_{2}\left[x_{2}, e_{1}\left(x_{1}\right)\right]$.

Der Gewinn der Unternehmung 2 beträgt dann

(5) $G_{2}=p_{2} x_{2}-k_{2}\left[x_{2}, e_{1}\left(x_{1}\right)\right]$.

Der marginale (kontrollexterne) Verlust der Unternehmung 2 beträgt nun

(6) $\frac{\partial G_{2}}{\partial x_{1}}=-\frac{\partial K_{2}}{\partial e_{1}} \cdot \frac{d e_{1}}{d x_{1}}<0$.

Da im Allokationsoptimum die Gesamtproduktion maximiert sein muß, können wir die Bedingungen für eln solches Optimum ableiten, indem wir die Gewinnfunktionen der beiden Unternehmen zusammenfassen und maximieren. Bei gemeinsamer Gewinnmaximierung ergibt sich als Gewinnfunktion

(7) $G=G_{1}+G_{2}=p_{1} x_{1}+p_{2} x_{2}-K_{1}\left(x_{1}\right)-K_{2}\left[x_{2}, e_{1}\left(x_{1}\right)\right]$

und als Maximierungsbedingungen erhalten wir

100) Bei separablen negativen externen Effekten ist deren Höhe allein vom Aktivitätsniveau des Verursachers abhängig. Es bleibt damit die Grenzkostenfuktion des Trăgers unbeeinflust (vgl. Davis, 0.A./Whinston, A., Externalities, Welfare, and the Theory of Games, a.a.O., S. $294 \mathrm{ff.})$ 
(8) $\frac{\partial G}{\partial x_{2}}=p_{2}-\frac{\partial k_{2}}{\partial x_{2}}=0$

und die uns vor allem interessierende Bedingung

(9) $\frac{\partial G}{\partial x_{1}}=p_{1}-\frac{\partial K_{1}}{\partial x_{1}}-\frac{\partial K_{2}}{\partial e_{1}} \cdot \frac{d e_{1}}{d x_{1}}=0$

oder umgeformt:

(10) $p_{1}-\frac{\partial k_{1}}{\partial x_{1}}=\frac{\partial k_{2}}{\partial e_{1}} \cdot \frac{d e_{1}}{d x_{1}}$

Der Ausdruck auf der linken Seite von Gleichung (10) ist der interne Grenzgewinn der Unternehmung 1 und der Ausdruck auf der rechten Seite entspricht dem in Glelchung (6), ist also der externe Grenzverlust der Unternehmung 2, so daß im Allokationsoptimum gelten muB, daB der interne Grenzgewinn des Verursachers der Umweltbelastung gleich dem externen Grenzverlust des Trägers der Umweltbelastung sein muB:

(11) $\frac{\partial G_{1}}{\partial x_{1}}=-\frac{\partial G_{2}}{\partial x_{1}}$

Wir wollen diese Bedingung auch noch graphisch darstellen (vgl. Abb. 17) :

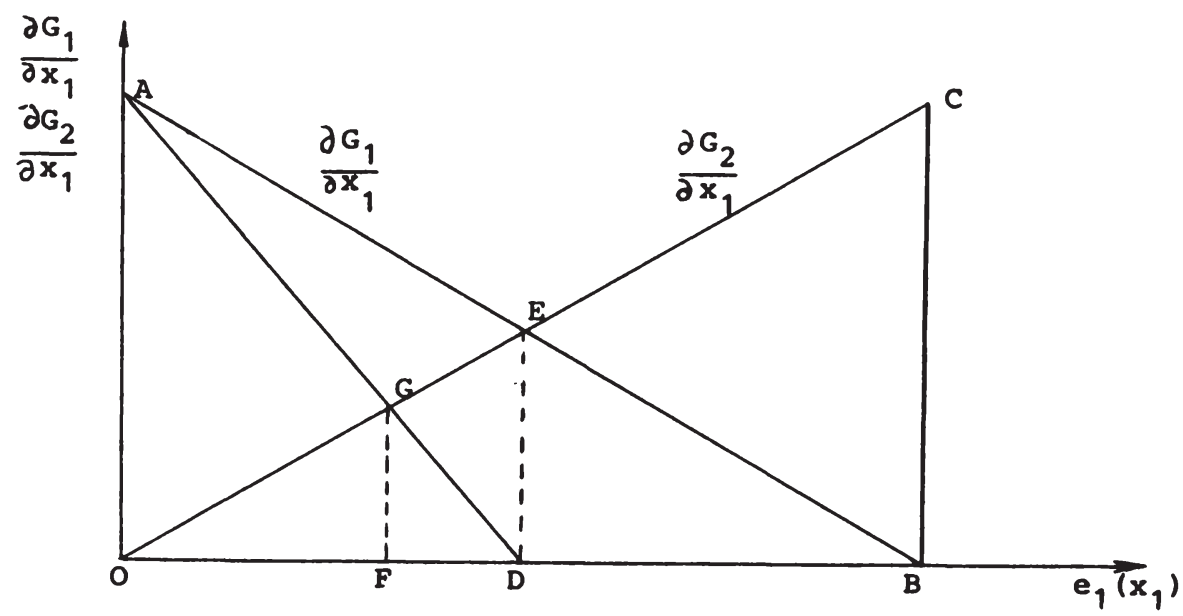

Abb. 17 
Die Gerade $A B$ stellt die Grenzgewinnfunktion der Unternehmung 1 dar. Bel getrennter Gewinnmaximierung wird die Unternehmung 1 die zu B gehörende Güter- und Schadstoffmenge produzieren, wo ihr Grenzgewinn gleich Null ist. Die Gerade OC ist die Grenzverlustfunktion der Unternehmung 2. Unternehmung 2 erleidet bei getrennter Gewinnmaximierung einen externen Verlust in Höhe des Flächeninhalts des Dreiecks OBC.

Das Allokationsoptimum liegt aber bel einer Güter-SchadstoffKombination, die durch den Punkt $D$ gekennzeichnet ist, über dem sich in $\mathrm{E}$ die Grenzgewinnkurve der Unternehmung 1 und die Grenzverlustkurve der Unternehmung 2 schneiden. Um den Verursacher $\mathrm{zu}$ einer dem Punkt $\mathrm{D}$ entsprechenden Produktion $\mathrm{zu}$ zwingen, muB man seine Produktion mit einer steuer belegen, die zumindest im Allokationsoptimum einen marginalen steuersatz $t$ in Höhe von ED hat, also dem externen Grenzverlust der Unternehmung 2 entspricht.

Man kann die Steuerhöhe bzw. deren Satz auch leicht analytisch bestimmen. Nach Steuer hat die Gewinnfunktion der Unternehmung 1 folgendes Aussehen:

$$
G_{1}=p_{1} x_{1}-k_{1}\left(x_{1}\right)-T\left(x_{1}\right)
$$

Unternehmen 1 maximiert seinen Gewinn, wenn

$$
\frac{\partial G_{1}}{\partial x_{1}}=p_{1}-\frac{\partial K_{1}}{\partial x_{1}}-\frac{\partial T}{\partial x_{1}}=0
$$

oder umgeformt:

$$
p_{1}-\frac{\partial k_{1}}{\partial x_{1}}=\frac{\partial T}{\partial x_{1}} \text {. }
$$

Aus den Gleichungen (10) und (14) folgt

$$
\text { (15) } \frac{\partial T}{\partial x_{1}}=\frac{\partial K_{2}}{\partial e_{1}} \cdot \frac{d e_{1}}{d x_{1}} \text {. }
$$

Der Steuerbetrag, den Unternehmung $1 \mathrm{zu}$ bezahlen hat, damit elne volle Internalislerung erfolgt, beträgt 


$$
T=\int_{0}^{x_{1}} \frac{\partial k_{2}}{\partial e_{1}} \cdot \frac{d e_{1}}{d x_{1}} \cdot d x_{1} \cdot
$$

Hierbei wird also unterstellt, daB die Grenzsteuerfunktion identisch ist mit der Grenzverlustfunktion der Unternehmung $2^{101}$. Dadurch wird garantiert, daB der Steuerbetrag Im Allokationsoptimum immer gleich dem externen verlust ist. Die marginale Nettogewinnfunktion der Unternehmung 1 wird dann durch die Gerade $A D$ wiedergegeben. Man erhält sie durch vertikale Subtraktion der Grenzsteuerfunktion von der Grenzgewinnfunktion der Unternehmung 1 .

An ihr orientiert sich die Unternehmung 1 nach Steuer, so daB sie ihren Gewinn dort maximiert, wo der marginale Nettogewinn gleich Null ist, also bel einer Produktmengen-Schadstoff-Komb1nation, die dem Punkt $D$ entspricht. Der externe Verlust der Unternehmung 2 hat sich jetzt verringert; er entspricht dem Inhalt des Drelecks ODE, also genau dem in Gleichung (16) angegebenen Steuerbetrag. Der Steuerbetrag muB an den Träger des externen Verlusts, also Unternehmen 2, als Entschädigung transferiert werden, weil sonst nicht das Allokationsoptimum realisiert wird, sondern eine suboptimale Produktionsmengen-Schadstoff-Kombination $O F$, und zwar deshalb, well belde Unternehmen Ihre Gewinnsituation nach Steuer verbessern können, wenn sie sich durch Ver-

101) Im Fall einer (proportionalen) Stücksteuer mit einem konstanten marginalen satz $t$, wäre die Grenzsteuerfunktion eine Parallele zur Abszisse in Hobhe von DE, und die marginale Nettogewinnfunktion des Unternehmens 1 wäre eine parallele zu $A B$ durch $D$. In diesem Fall wäre die Internalisierungsstever

$\mathrm{T}=\frac{\partial \mathrm{K}_{2}}{\partial \mathrm{e}_{1}} \cdot \frac{\mathrm{de}}{\mathrm{dx_{1 }}} \cdot \mathrm{x}_{1} \cdot$

Dieses Aufkommen 1st offenbar gröBer als im oben zugrundegelegten Fall, so daB für die Unternehmung 1 eine Mehrbelastung entsteht, obwohl das gesamtwirtschaftliche Allokationsoptimum ebenfalls realisiert wird. Der staat würde nach voller Kompensation des Verlustes der Unternehmung 2 noch einen Tell der Steuer als (Netto-)Einnahme verbuchen konnen. 
handlungen einigen, daß Unternehmung 1 nur noch die Punkt F entsprechende Menge produziert, über dem sich marginale Nettogewinnkurve der Unternehmung 1 und marginale externe Verlustkurve von Unternehmung 2 schneiden. $\mathrm{Zu}$ einer solchen Entschädigung des Trägers der verbleibenden externen Verluste muß es dann nicht kommen, wenn Verursacher und Träger der externen Effekte nicht miteinander verhandeln können, weil die Träger nlcht identifizierbar sind, wie dies bei den meisten Arten der Umweltbelastung der Fall ist. Dann wird die allokationsoptimale Menge OD realisiert, allerdings ohne daß die Träger der externen Verluste entschädigt werden müssen, was nach unserer Ansicht aus distributiven Gründen negativ zu beurteilen ist. Sind die Träger der externen Verluste nicht identifizierbar, wird man aber auch keine Schadensfunktion aufstellen können, wodurch der Realisierung der Pigou' schen Steuerlösung des Internalisierungsproblems sowleso die Grundlage entzogen 1 st.

Zur Pigou-steuer als Instrument zur Internalisierung negativer externer Effekte läBt sich abschlieBend folgendes sagen: Ihre Allokationseffizienz ist im Modell sehr groB, und zwar grober als bel jeder anderen Maßnahme zur Internalisierung externer Effekte 102). Damit allerdings durch die Internalisierung das Allokationsoptimum erreicht werden kann, müssen die marginalen Gewinn-bzw. Nutzenfunktionen der Verursacher und die marginalen Schadensfunktionen der Träger der Umweltbelastung für alle Produktionsalternativen des Verursachers bekannt sein 103) denn sonst kann die allokationsoptimale Internalisierungssteuer nicht ermittelt werden. Zusätzlich müssen auch die einzelnen Träger

102) Vgl. Zerbe, R.O., Theoretical Efficiency in Pollution Control, in: Western Economic Journal, vol. 8, 1970, S. 371; Gerhardt, P.H., Air pollution Control: Benefits, Costs, and Inducements, in: Mushkin, S. (Hrsg.), Public Prices for Public Products, Washington, D.C., 1972, S. 161; Littmann, K., Umweltbelastung - sozialökonomische Gegenkonzepte, a.a.0., S. 64 .

103) Vgl. Turvey, R., On Divergences between Social Cost and Private Cost, a.a.0., S. 312 . 
der Umweltbelastung bekannt sein, damit sie in Höhe ihrer externen Verluste entschädigt werden können. In unserem einfachen Beispiel mit nur zwel Unternehmen, von denen das eine nicht-reziproke, separable negative externe Effekte verursacht, ist es relativ einfach, die erforderlichen Informationen zu erlangen. Jedoch gerade im Fall der Umweltexternalitäten, wo viele Verursacher und Träger einer Vielzahl von Umweltbelastungen vorhanden sind und reziproke sowie nicht-separable negative externe Effekte eher die Regel als die Ausnahme sind, läBt sich den Informationserfordernissen für eine Pigou'sche Schadensteuer - wenn überhaupt - nur zu extrem hohen Kosten nachkommen 104). Außerdem wäre der für die Administration einer solchen Steuer notwendige Apparat kaum erschwinglich. Die gesellschaftlichen Kosten zur Realislerung dieser Version des Verursacherprinzips wurden auch in einem nicht annähernd akzeptablen Verhältnis zum gesellschaftlichen Nutzen stehen, den ein so realisiertes Allokationsoptimum hervorrufen würde. Die Tatsache, daB die Pigou'sche Steuerlösung für die umweltpolitische Praxis also kaum von Bedeutung ist, impliziert keineswegs, daB auch das Verursacherprinzip nicht angewendet werden kann 105) denn letzteres läßt sich - wie wir im folgenden sehen

104) Vgl. Schenk, K.E., Externe Effekte und rationale Wirtschaftspolitik, in: Hamburger Jahrbuch für Wirtschaftsund Gesellschaftspolitik, 13. Jg., 1968, S. 285 ff.; Head, J.G., Public Goods and Public Welfare, a.a.0., S. $201 \mathrm{ff}$.; Gerhardt, P.H., Air Pollution Control: Benefits, Costs, and Inducements, a.a.O., S. 161 f.; Hansmeyer, K.-H., Die Abwasserabgabe als Versuch einer Anwendung des Verursacherprinzips, in: Issing, 0 . (Hrsg.), Okonomische Probleme der Umweltschutzpolitik, Berlin 1976, S. 70 f.; Littmann, K., Umweltbelastung - sozialökonomische Gegenkonzepte, a.a.o., S. $90 \mathrm{ff}$.

105) So sehen manche Autoren als Alternative zum "Verursacherprinzip durch Abgabenerhebung entsprechend den sozialen Zusatzkosten" nur Kooperative Lösungen, ohne zu prüfen, ob es nicht auch andere Möglichkeiten zur Durchsetzung des Verursacherprinzips gibt als die Pigou'sche Steuerlösung (vgl. Jansen, P., Was können Systemanalysen zur praktischen Verwirklichung von Umweltschutz beitragen?, in: Zur Problematik des Verursacherprinzips, a.a.0., S. 18 ; Thoss, R., Zur Planung des Umweltschutzes, a.a.0., S. 183). Allerdings 
werden - mit Hilfe von anderen finanzwirtschaftlichen oder juristischen Instrumenten oder Kombinationen zwischen diesen in allokationstheoretisch allerdings weniger anspruchsvoller Welse durchsetzen. Angestrebt wird mit diesen Ansäzen nicht mehr das Allokationsoptimum, sondern die Einhaltung der Immissionsstandards, die letztlich durch politische Entscheidung festgelegt wurden. Die Realisierung dieser Immissionsstandards wäre übrigens dann mit der Errelchung des Allokationsoptimums identisch, wenn sie in Höhe der optimalen Immissionskonzentration festgelegt würden, und wenn die Bestimmung der optimalen Immissionskonzentrationen aufgrund vollständiger Information über Verme1dungs-, Opportunitäts- und Schadenskosten "richtig" erfolgt wäre - eine wahrhaft heroische Annahme 106) Die modifizierten Versionen des Verursacherprinzips, wie sie nun geschildert werden sollen, beruhen nlcht mehr darauf, die Schadenskosten zu internalisieren, sondern darauf, den Verursachern die Verme1dungskosten der (potentiellen) Umweltbelastungen anzulasten. Nur diese Version des Verursacherprinzips entspricht ubrigens dem Grundsatz elnes präventiven Umweltschutzes. Die P1gou'sche Internalisierungslösung impliziert nämlich wegen der notwendigen

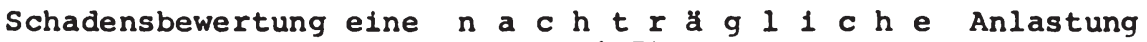
der negativen externen Verluste ${ }^{107}$ ) denn nicht entstandene

meinen auch wir, daß die Durchsetzung des Verursacherprinzips kein ziel ist, sondern nur ein Mittel zur Realisierung der Immissionsstandards (vgl. Meyer-Abich, K.M., Umweltbeeinträchtigungen durch den wirtschaftlich-technischen Proze B, a.a.0., S. 16; Thoss, R., Verursacherprinzip Kompensation oder Verhinderung von Umweltschäden?, in: Schultze, H. (Hrsg.), Umwelt-Report, a.a.O., S. 23). "Easy slogans like 'polluters must pay' may win hearts and votes but cost dear in disguising the need in every case to compare alternatives for their full economic effects on incentive, choice, and efficiency" (Pennance, F.G.' The Polluters: Industry or Government?, a.a.0., s. 47).

106) Siehe oben S. 148 ff.

107) Vgl. RSU, Umweltgutachten 1974, S. 11; Thoss, R., Zur Planung des Umweltschutzes, a.a.0., S. 181; ders., Verursacherprinzip - Kompensation oder Verhinderung von Umweltschäden?, a.a.0., s. 21 . 
(oder nur potentielle) Schäden können nicht zur Bemessungsgrundlage einer steuer gemacht werden. Die Anlastung der Vermeldungskosten 1st dagegen durchaus ex ante möglich, so daß diese Version des Verursacherprinzips bel konsequenter Anwendung zu einer Exante-Internalisierung der (potentielien) Umweltexternalitaten fürt. Die Allokationsaufgabe besteht nun nicht mehr darin, eine Internalisierungssteuer $z u$ finden, die zum Allokationsoptimum füht, sondern darin, juristische und/oder finanzwirtschaftliche Maßnahmen zu finden, welche die Verursacher veranlassen, Emissionsvermeidungs- oder Emissionsreduktionsmaßnahmen $z u$ ergreifen, die zu gesamtwirtschaftlich minimalen Kosten die Einhaltung der Immissionsstandards gewährle1sten 108)

bb) Auflagen als Instrument zur Durchsetzung des Verursacherprinzips

Auflagen sind (neben Subventionen und offentlichen Investitionen) das traditionelle Instrument staatlicher Umweltpolitik. Man kann die Auflagen in Verbote, Gebote und standards einteilen. Gegenstand der Umweltschutzauflagen können die Produkte, die umweltschutzrelevanten Technologien, der standort und die Emissionen sein. Alle Auflagen können räumlich, zeitlich und sachl1ch differenztert werden.

Der Freiheltsgrad für die von den Auflagen betroffenen Verursacher ist in der Regel bel den Geboten am geringsten und bei den standards am gröBten. Gebote kann man als Verbote von Alternativen bezelchnen. Existieren allerdings nur wenige Alternativen, können Verbote einen ebenso geringen Freiheltsgrad belassen wie Gebote. In bezug auf den Gegenstand der Auflagen ist der Fre1heltsgrad dann am gröbten, wenn diese die Emissionen betreffen, denn dann können die betroffenen Verursacher zwischen allen mög-

108) Vgl. Jürgensen, H./Jaeschke, K.-P., Allokationseffekte der Soclal Costs im Umweltschutz, a.a.0., S. 75 f.; Nowotny, E., Wirtschaftspolitik und Umweltschutz, Freiburg 1974, S. 193; Baumol, W.J./Oates, W.E., The Use of Standards and Prices for Protection of the Environment, in: Swedish Journal of Economics, vol. 73, 1971, S. $44 \mathrm{f}$. 
Systematisierung der Auflagen

\begin{tabular}{|c|c|c|c|c|c|}
\hline \multicolumn{3}{|c|}{$\begin{array}{l}\begin{array}{l}\text { Gegenstand } \\
\text { der Auflagen }\end{array} \\
\text { Auflagen der }\end{array}$} & Gebote & $\begin{array}{l}\text { Verbote } \\
\text { (Null-Standards) }\end{array}$ & $\begin{array}{l}\text { Standards } \\
\\
\text { Produkt- } \\
\text { standards }\end{array}$ \\
\hline \multirow{4}{*}{ 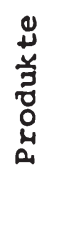 } & \multirow{2}{*}{$\begin{array}{l}\text { Produktions- } \\
\text { mittel } \\
\text { (Inputs) }\end{array}$} & Herstellung & Herstellungsgebot & \multirow{2}{*}{$\begin{array}{l}\text { Herstellungsverbot } \\
\text { Verwendungsverbot }\end{array}$} & \multirow{4}{*}{$\begin{array}{l}\text { Produkt- } \\
\text { standards }\end{array}$} \\
\hline & & Verwendung & Verwendungsgebot & & \\
\hline & \multirow{2}{*}{ Konsumgüter } & Herstellung & Herstellungsgebot & Herstellungsverbot & \\
\hline & & Gebrauch & Gebrauchsgebot & Gebrauchsverbot & \\
\hline \multirow{4}{*}{ 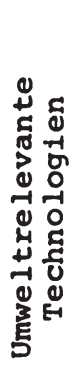 } & \multicolumn{2}{|l|}{$\begin{array}{l}\text { Produktions- } \\
\text { verfahren }\end{array}$} & Verfahrensgebot & Verfahrensverbot & \multirow{4}{*}{ - } \\
\hline & \multicolumn{2}{|l|}{$\begin{array}{l}\text { Rückhalte- } \\
\text { techrologien }\end{array}$} & $\begin{array}{l}\text { Einbaugebot für } \\
\text { Rückhal te techno- } \\
\text { logien }\end{array}$ & - & \\
\hline & \multicolumn{2}{|l|}{ Recycling } & Recycling-Gebot & - & \\
\hline & \multicolumn{2}{|l|}{ Entsorgung } & Entsorgungsgebot & - & \\
\hline \multicolumn{3}{|c|}{ Standort } & Standortgebot & Standortverbot & - \\
\hline \multicolumn{3}{|c|}{ Emissionen } & - & Emissionsverbot & $\begin{array}{l}\text { Emissions- } \\
\text { standards }\end{array}$ \\
\hline
\end{tabular}


lichen Maßnahmen der Emissionsvermeldung oder -reduktion wăhlen, die sich im Produktionsproze $B$ im weltesten sinne - also von der Beschaffung der Produktionsfaktoren bis zum Absatz der Produkte ergeben. In der Tabelle auf Seite 320 unternehmen wir den Versuch einer Systematisierung der Umweltschutzauflagen. Der Freiheitsgrad der Entscheidungen nimmt in der Tabelle (tendenziell) von links nach rechts und von oben nach unten $z u$.

Wir wollen nun die Auflagen im einzelnen betrachten.

(1) Gebote ...

(a) ... In bezug auf produkte

Solche Gebote können sich auf die Herstellung oder Verwendung ganz bestimmter produktionsmittel oder Konsumgüter beziehen oder auch nur ein allgemeines Postulat darstellen, z.B. daß nur umweltfreundliche Produkte hergestellt oder verwendet werden dürfen.

Gebote, bestimmte Produkte aus Umweltschutzgründen herzustellen, kommen wohl nicht in Frage. Dagegen wăre es denkbar, daß die Verwendung (in der Produktion) und/oder der Verbrauch (im Haushalt) ganz bestimmter Produkte (z.B. umweltfreundlicher Energietrăger wie Erdgas) vorgeschrieben werden. Auch den AnschluBzwang an ein Fernhelzwerk kann man als Gebot, Fernwärme zu verwenden, bezeichnen. Solche Gebote stellen zwar elnen beträchtlichen Eingriff in die persönliche Entscheldungsfreiheit von Unternehmen und Haushalten dar, sind aber, wenn sie elngehalten (durchgesetzt) werden, sehr wirksam.

(b) ... in bezug auf umweltrelevante Technologien Hierbel kann es sich um Vorschriften hinsichtlich der Produktionsverfahren oder hinsichtlich der konkreten umwelttechnischen Maßnahmen handeln, die ergriffen werden müssen, um Emissionen $z u$ vermeiden oder zu reduzieren. Solche Gebote erfordern für die Behörden viele Informationen über den stand der Technik 109), denn es können nur technisch erprobte (und finanziell zumutbare) umweltschützende Technologien vorgeschrieben werden. Damit

109) Vgl. Wohlgemuth, R., Umweltgebühren als Alternative zur Auflage, in: Umwelt, 1974, 6. S. 46; RSU, Umweltgutachten 1974, a.a.0., S. 161 
wird der ErlaB wirksamer Gebote äuBerst schwierig und langwierig, denn daruber, was als Stand der Technik zu bezeichnen ist, kann es zwischen der Behörde und den Unternehmen zu langwierigen Auseinandersetzungen kommen, die letztlich in einem Kompromis enden werden 110). In bezug auf Produktionsverfahren und Rückhaltetechnologien halten wir Gebote für kein geeignetes Instrumentarium. Hinsichtlich des Recycling und vor allem der Entsorgung sind sie jedoch von großer Bedeutung, well Gebote U.E. das wirksamste Instrumentarlum des Verursacherprinzips sind, das zu Recycling und Entsorgung toxischer Schadstoffe führen kann, wodurch sich Herstellungs- oder Verwendungsverbote der Güter, bel deren Produktion oder Konsum diese toxischen Schadstoffe anfallen, vermeiden lassen. Das sei an zwei Beispielen erläutert: Wenn es nicht gelingt, durch Recycling z.B. toxische Schwermetalle aus den Abwässern oder der Abluft zurückzugewinnen, oder wenn es nlcht gelingt, radioaktive Abfälle mit dem geringstmöglichen Risiko zu entsorgen - und beides ist, solange es nicht rentabel ist, nur durch Gebote zu erreichen -, wird aus Gründen des Umweltschutzes ein Verbot der Aktivitäten notwendig sein, bei denen toxische Schwermetallverbindungen und radioaktive $A b f a ̈ l l e ~ e n t s t e h e n$.

DaB es auch bei Geboten in bezug auf Recycling- und Entsorgungstechnologien Auseinandersetzungen über den Stand der Technik geben w1rd, die gerade wegen der Neuartigkeit dieser Technologien besonders schwierig sein werden, läbt sich nicht vermelden. Damit keine zu großen Verzögerungen und auch keine faulen Kompromisse entstehen, kann in diesen Fällen eine tellweise Durchbrechung des Verursacherprinzips erfolgen, indem den von den Recycling- und Entsorgungsgeboten betroffenen Unternehmen Finanzierungshilfen gewährt werden 111 !

(cc) ... in bezug auf den standort

Durch Gebote kann auch die Standortwahl vor allem von Unterneh-

110) Vgl. White, L.J., Effluent Charges as a Faster Means of Achieving Pollution Abatement, In: Public Policy, vol. 24, 1976 , S. 112 .

111) Siehe dazu unten S. $389 \mathrm{ff}$. 
men und Verkehrsanlagen vorgeschrieben werden. Das wird in erster Iinie Unternehmen betreffen, die (potentiell) besonders gefährliche Schadstoffe emittieren (wie Kernkraftwerke), oder Anlagen, von denen nur schwer vermeidbare Emissionen ausgehen (wie Flugplätze). Auch solche Gebote sind sehr wichtig, well es zu ihnen U.E. keine wirksamen Alternativen gibt.

(2) Verbote

Verbote scheinen auf den ersten Blick strengere Maßnahmen darzustellen als Gebote. Das ist jedoch dann nicht der Fall, wenn es $z u$ den verbotenen Produkten oder Verfahren gleichwertige oder zumindest akzeptable Alternativen gibt. In bezuq auf die Emissionen stellen Verbote allerdings die strengste Maßnahme dar. Herstellungsverbote können slch auf Produktionsmittel und Konsumgüter beziehen. Bei Produktionsmitteln sind Herstellungsverbote vertretbar, wenn von den Produktionsmitte in ausqehende Gefahren nicht durch technologische Verfahren (wie Rückhaltetechnologien, Recycling und Entsorgung) vermieden werden können. Das ist z.B. bei bestimmten Bioziden (wie DDT) der Fall 112) Bei Konsumgütern sind Verbote angebracht, wenn die Konsumgüter gesundheitsschädliche Folgen haben, ökologische Schäden oder schwerwiegende Entsorgungsprobleme hervorrufen können, die sich durch andere Instrumente aus ökonomischen Gründen nlcht vermeiden oder bewältigen lassen. $\mathrm{Zu}$ denken wäre hier $\mathrm{z}$. B. an bleihaltiges Kinderspielzeug (gesundheitsschädlich), Waschmlttel, die harte Detergentien enthalten 113) (ökologische Schäden) und Einwegflaschen (Entsorgungsproblem).

Verwendungs - und Gebrauchsverbote sind immer entweder zeltlich oder räumlich begrenzt, denn sonst wären Herstellungsverbote

112) Vgl. die Verordnung ubber Anwendungsverbote und Beschränkungen der Pflanzenschutzmittel v. 23.7.71 (BGBl. I, S. 1117) in der Fassung der Bek. vom 31.5.74 (BGBI. I, S. 1204) und das Gesetz über den Verkehr mit DDT in der Fassung $v$. 2.3.74 (BGBI. I, S. 469).

113) Vgl. das Gesetz über die Beschränkung und Verwendunq bestimmter Detergentien in Wasch- und Reinigungsmitte in v. 26.5.72 (BGB1. II, S. 553); Rehbinder, E., Grundfragen des Umweltschutzes, a.a.0., S. 254 . 
das adäquate Instrument. Zeitlich begrenzte Verwendungsverbote von bestimmten Produktionsmitteln können in bestimmten kritischen Umweltbelastungssituationen notwendig werden. So kann z.B. bei Inversionswetterlagen zur Verhinderung einer Smog-Katastrophe die Verwendung schwefelhaltiger Brennstoffe verboten werden 114) Räumlich und ze1tlich begrenzte Gebrauchsverbote in Form elnes Fahrverbots für Kraftfahrzeuge in Innenstädten sind aus dem gleichen Grunde denkbar 115) Der zu bestimmten Tageszeiten und in der Nacht verbotene Gebrauch von Rasenmähern ist eln Beispiel für ein zeitlich begrenztes Gebrauchsverbot. Verfahrensverbote sind dann vertretbar, wenn durch das Verfahren bedingte Emissionen nicht auf andere Art (Rückhaltetechnologie, Recycling, Entsorgung) vermieden oder reduziert werden können.

Das Verbot, bestimmte standorte $\mathrm{zu}$ wăhlen, ist wohl dann eine unvermeidliche Maßnahme, wenn es keine anderen technisch und finanziell zumutbaren Möglichkeiten gibt, besondërs schutzwürdige Regionen oder Gebiete wie z.B. Wasserschutzgebiete, ökologische Ausgleichsräume, Erholungsgebiete, Kurgeblete oder die Umgebung von Krankenhäusern vor einer $\mathrm{zu}$ hohen Immissionsbelastung zu schützen. Emissionsverbote haben gegenüber den b1sher geschilderten, die produktionsmittel und -verfahren betreffenden Verbote einen großen Vortell: Sle überlassen es dem Unternehmer als (potentiellem) Verursacher, wie er die Emissionen verhindert: Er kann die emissionsverursachende Aktivität (Faktoreinsatz, Produktionsverfahren) substitutieren, umwelttechnische Maßnahmen einsetzen (Rückhaltetechnologien, Recycling, Entsorgung) oder die Produktion einstellen. Emissionsverbote sind insofern grundsätzlich den anderen, die Produktionsmittel

114) Vgl. $§ 49$ (1), Z. 4 und $\S 49$ (2) Satz 2, Z. 2 BImSchG, wonach in besonders schutzbedürtigen Gebieten oder bel austauscharmen Wetterlagen, emissionsintensive Brennstoffe nicht oder nur beschränkt verwendet werden dürfen.

115) Vgl. $\S 40$ und $\S 49$ (1), Z. 1 und 3 BImSchG, wonach bei Inversionswetterlagen Verkehrsbeschränkungen möglich sind. 
und - verfahren betreffenden Verboten vorzuziehen.

Für die Konsumgüter betreffenden Verbote gibt es u.E. allerdings keine Alternativen.

Alle Verbote haben vor allem zwel Vorteile:

Sie gewährleisten mit großer sicherheit die Vermeidung von Schäden und sind somit ein ausgezeichnetes Mittel des präventiven Umweltschutzes 116) Die Administration und Kontrolle ist relativ einfach 117)

Dem steht allerdings ein schwerwiegender Nachtell gegenüber. Mit dem Verbot umweltbelastender Aktivitäten oder Produkte ist immer ein Nutzenverlust verbunden 118), der un so größer ist, je wenlger technisch und finanziell gleichwertige Alternativen es $\mathrm{zu}$ den verbotenen Aktivitäten und Produkten gibt. Verbote sollten deshalb nur im Falle akuter Gefahren für die menschliche Gesundheit und irreversibler Schäden an Gesundheit und Umwelt angewendet werden, also bei der Herstellung, Verwendung oder Emission toxischer oder irreversible Schäden hervorrufender Produkte bzw. Schadstoffe 119)

(3) Standards

Standards kann man auch als Gebote oder Verbote betrachten. Es sind Gebote, bestimmte Qualitätsnormen (Produktstandards) oder Emissionsgrenzwerte (Emissionsstandards) einzuhalten, oder es

116) Vgl. Littmann, K., Umweltbelastung - sozlalökonomische Gegenkonzepte, a.a.0., S. 54 .

117) Vgl. Cansier, D., Okonomische Grundprobleme der Umweltpolitik, Berlin 1975, S. 83.

118) Vgl. Domsch, K.H., Zum Problem der Biozide, a.a.O., S. 148.

119) Vgl. Cansier, D., Okonomische Grundprobleme der Umweltpolitik, a.a.0., S. 85; Oberhauser, A., Abgrenzung des Verursacherprinzips und seine Einordnung in die Umweltpolitik, in: Das Verursacherprinzip und seine Instrumente, Berlin 1974, S. 39; Rincke, G., Anwendbarkeit des Verursacherprinzips am Beispiel der Wassergütewirtschaft, in: Das Verursacherprinzip und seine Instrumente, a.a.0., S. 145; Zander, H. , Trinkwasser - ein kostbares Lebensmittel, in: U 1975, 3, S. 22; Schultze, C.L./Fried, E.R./Rivlin, A.M./Teeters, N.H., Setting National Priorities, The 1973 Budget, a.a.O., S. 373; Schultze, C.L. U.a., Setting National Priorities, The 1972 Budget, a.a.O., s. 241 . 
sind Verbote, solche Qualitätsnormen oder Emissionsgrenzwerte zu überschre1ten.

\section{(a) Produktstandards}

Produktstandards können Produkteigenschaftsnormen 120) oder Begrenzungen des Schadstoffgehalts in Produkten 121) sein. Cansier zählt auch die produktbezogenen Emissionsgrenzwerte, z.B. die Grenzwerte für Lărm- und Abgasemissionen der Kraftfahrzeuge, zu den Produktstandards 122) Aus systematischen Gründen sind sie u.E. aber zu den Emissionsstandards $z u$ rechnen.

Produkteigenschaftsnormen können sich z.B. auf die Lebensdauer, die Recycling-"Freundilchkeit" und die Entsorgungsgerechtigkeit beziehen. So könnte man z.B. normieren, daB besonders rostgefährdete Teile an Kraftfahrzeugen durch nlcht-rostendes Material ersetzt werden, wodurch natürlich die anderen Elgenschaften, wie vor allem die sicherheit der Kraftfahrzeuge, nicht beeintrachtiat werden durfen. Man kann auch eine recycling-freundliche produktgestaltung normieren, indem man z.B. das De-Inking erleichternde Druckfarben vorschrelbt ${ }^{123}$ ). Für elne entsorgungsgerechte Produktgestaltung kann man z.B. zersetzbare Kunststoffe bei der Herstellung von Verpackungsmaterial fordern 124). Von gröBerer prakt1scher Bedeutung 1st U.E. die Begrenzung des Schadstoffgehalts in den Produkten (Produktionsmitteln und Konsumgüter). So kann man z.B. fur den Blelgehalt in Benzin 125), den Phosphatgehalt in

120) Vgl. Schatz, K.-W., Administrative und marktwirtschaftliche Maßnahmen zur Lösung des Umweltschutzproblems, in: Giersch, H. (Hrsg.), Das Umweltproblem aus ökonomischer Sicht, a.a.O., S. 126 .

121) Vgl. Delmhorst, B., Das Verursacherprinzip: Der kategorische Imperativ des Umweltschutzes, in: Die Neue Gesellschaft 1972 , H. 10 , S. 760 .

122) Vgl. Cansier, D., Okonomische Grundprobleme der Umweltpol1tik, a.a.0., s. 83 .

123) Siehe dazu oben S. 254.

124) Siehe dazu oben S. 253.

125) Vgl. das Gesetz zur Verminderung von Luftverunreinigungen durch Bleiverbindungen in Ottokraftstoffen für Kraftfahrzeugmotoren in der Fassung v. 25.10 .75 (BGBl. I, S. 2919). 
Waschmitteln 126) und den Schwefelgehalt in Heizöl 127) Höchstgrenzen angeben. Auf diese Weise kann man durch Begrenzung des Schadstoffgehalts (indirekt) beim Hersteller der schadstoffhaltigen Produkte zur Emissionsreduktion des jeweiligen Schadstoffs bei den (potentiellen) Verursachern (Emittenten) beitragen. Dies hat vor allem im Falle vieler Kleinemittenten (Kraftfahrzeuge, Haushalte) einen großen Vorteil, da es die Emissionsveranlagung und Kontrolle der vielen elnzelnen Emissionsquellen erübrigt 128) Es wäre allerdings dann eine gesamtwirtschaftlich nachteilige Maßnahme, wenn andere Arten der Emissionsreduktion billiger wären. Bel den aufgezählten Beisplelen (Ble1, Phosphate, Schwefel) kämen als erwägenswerte alternative MaBnahmen zur Schadstoffbegrenzung (in Benzin, Waschmitteln, Heizöl) nur elne Inputsubstitution oder Verfahrensverbesserung in Frage. Rückhaltetechnologien, Recycling und Entsorgung scheiden wegen der Vielzahl der Emissionsquellen aus. Produktstandards soliten elnheltilch sein 129), da sich bei unterschiedlichen standards, die sich in unterschiedlich hohen Produktpreisen äußern würden, die gesamte Nachfrage auf die billigsten, d.h. schadstoffhaltigsten Produkte konzentrieren würde.

(b) Emissionsstandards

Emissionsstandards sind gesetzlich vorgeschriebene Höchstgrenzen für die Emission von Schadstoffen aus den verschiedensten Quellen. Sie können in der Form von Emissionsraten, also Emissionsmengen je zeiteinheit, oder in der Form prozentualer Emissions-

126) Vgl. das Gesetz über die Umweltverträglichkeit von Waschund Reinigungsmitteln v. 20.8.75 (BGBI.I, S. 2255).

127) Vgl. die Verordnung über Schwefelgehalt von leichtem Helzöl und Dieselkraftstoff (3. BImSchV) v. 15.1.75 (BGBI. I, S. 264).

128) Vgl. Cansier, D., Ökonomische Grundprobleme der Umweltpolitik, a.a.0., s. 88 .

129) Vgl. Siebert, H., Regional planning - land use approaches to environmental quality management, Discussion paper $67 / 75$ des Instituts für Volkswirtschaftslehre und statistik der Universität Mannheim, S. 5 . 
reduktionserfordernisse (Reinheitsgraden) angegeben werden. Nehmen wir an, die Emissionsstandards für jeden Schadstoff sollen für alle Emittenten einheitlich sein 130) so würde die Standardsetzung in Form elnheitlicher Emissionsraten, also absoluter Höchstmengenbegrenzungen der Schadstoffemissionen, eine erhebliche Benachteiligung der Großemittenten bedeuten 131); während die Kleinemittenten vielleicht überhaupt keine oder nur geringe EmissionsreduktionsmaBnahmen ergreifen müBten, um die Emissionsstandards einzuhalten, müBten die Großemittenten hohe Summen investieren, um die Emissionsgrenzwerte einhalten zu können. Dies ist besonders dann ungerecht, wenn die summe der Schadstoffemissionen der vielen Kleinemittenten größer ist als die der wenigen Großemittenten. Im Extremfall müßten bei einer solchen Regelung die Großemittenten die gesamte Last der Emissionsreduktion tragen, obwohl die Kleinemittenten in ihrer Gesamtheit mehr zur Immissionsbelastung beitragen 132) Würden, als anderes Extrem, von jedem Emittenten gleich große Emissionsreduktionsraten gefordert, wäre das eine erhebliche Benachteiligung der Kleinemittenten, denn sie müßten unter Umständen eine vollständige Emissionsreduktion vornehmen, während Großemittenten nur einen relativ kleinen Tell ihrer Emissionen reduzieren müBten. Als bessere Lösung ist ein $p r o z$ e $t$ u a $l$ gleicher Reinigungsgrad als Emissionsstandard anzusehen 133 ?

130) Die Implikationen elnheitlicher Emissionsstandards untersuchen wir weiter unten (siehe dazu S. $333 \mathrm{ff.}$ ).

131) Vgl. Cansier, D., Okonomische Grundprobleme der Umweltpolitik, a.a.0., s. 86 .

132) Das sel an einem Beispiel erläutert. Nehmen wir an, daß 100 Kleinemittenten jeweils 50 schadstoffeinheiten (SE) je Zeiteinhelt (ZE) emittieren und ein GroBemittent $2000 \mathrm{SE} / \mathrm{ZE}$. Der Emissionsstandard betrage $60 \mathrm{SE} / \mathrm{ZE}$ für jede Emissionsquelle. In diesem Fall müBten die Klelnemittenten keine Maßnahmen zur Emissionsreduktion ergreifen, obwohl sie $5000 \mathrm{SE} / \mathrm{ZE}$ emittieren und der GroBemittent muB seine Emissionen un $1940 \mathrm{SE} / \mathrm{ZE}$ reduzieren, obwohl er weniger emittiert als die vielen kleinen Emittenten.

133) Vgl. Cansier, D., Okonomische Grundprobleme der Umweltpol1tik, a.a.0., S. 86; U.S. Council of Economic Advisers, The 1971 Report, a.a.0., S. 116; U.S. Council on Environmental Quality, Environmental Quality, The Second Annual Report, a.a.0., S. 136 . 
Nun wollen wir uns mit dem zweck der Emissionsstandards befassen. Beim studium vor allem der umweltökonomischen und umweltplanerischen Literatur 134) muB man, bis auf wenige Ausnahmen 135) den Eindruck gewinnen, als diene die Festlegung der Emissionsstandards dem ziel, die Immissionsstandards zu r e a 1 i s 1 er e n. Dieser Eindruck drängt sich vor allem auf, wenn man liest, wie Emissionsstandards aus den Immissionsstandards mit Hilfe von Diffusionsmodellen abgeleitet werden 136) Diese mechanistische Betrachtung geht von der Höhe der Immissionsstandards aus und berechnet unter $\mathrm{Zugrundelegung} \mathrm{der} \mathrm{meteorologischen,} \mathrm{topogra-}$ phischen und sonstigen Umweltmedien-Verhaltnisse für die răumlich verteilten stationären Emissionsquellen die maximal zulässigen Emissionsgrenzwerte (nun wieder in Form von Emissionsraten), die wegen der unterschiedlichen natürlichen Verhältnisse und der unterschiedlichen Entfernung der verschiedenen Emissionsquellen vom Immissionsgebiet verschieden hoch sein müssen 137) Würde man die so abgeleiteten Emissionsgrenzwerte zu Emissionsstandards machen, würde man sowohl den Zweck der Immissionsals auch den der Emissionsstandards gründlich verkennen: Immis-

134) Vgl. U.S. Council of Economic Advisers, The 1971 Report, a.a.0., S. 116; Blatr, R.D., The Clean Air Act of 1970, a.a.O., S. 266; The Economics of Clean Air (1972), a.a.0., S. 1-15 f.: U.S. Council on Environmental Quality, Environmental Quality, The Second Annual Report, a.a.o., S. 136 ; Günther, U. U.a., Umweltschutz-Informations- und Steuerungssystem, a.a.0., s. 32 .

$135) \mathrm{Vgl}$. vor allem RSU, Umweltgutachten 1974, a.a.0., S. 13.

136) Vgl. Gorr, W.L./Kortanek, K.O., Optimal Control Strategies for Air Quality Standards, Manuskript, Pittsburgh 1971 ; Kohn, R.E., A Cost-Effectiveness Model for Air Pollution Control with a Single Stochastic Variable, in: Journal of the American Statistical Association, vol. 67, 1972, S. 20.

137) Vgl. Rembold, G., Rohstoffverknappung und Umweltbelastbarkeit als Determinanten des regionalen, sektorspezifischen Wirtschaftswachstums - ein regionalisierter, multisektoraler Modellansatz -, in: Wirtschaftstheorle, Wirtschaftspolitik und Planung öffentlicher Ausgaben, Karlsruhe 1975, S. 123; McCormick, R.A., Meteorological Aspects of Air Pollution in Urban and Industrial Districts, a.a.0., s. 3 . 
sionsstandards sind keine anzustrebenden ziele, sondern Normen, die nicht überschritten werden dürfen, und Emissionsstandards sind keine Maßnahmen zur $R$ e a $l i s$ e $r$ u $g$ der Immissionsstandards, sondern Auflagen zur Durchsetzung des Standes der Technik 138), um auf diese Weise zur E i $\mathrm{n} \mathrm{h}$ a l$t$ u $\mathrm{g}$ der Immissionsstandards beizutragen.

Würden Immissions- und Emissionsstandards nicht in dieser Welse interpretiert, würde das z.B. folgendes bedeuten: In einem ländlichen Geblet mübte ein GroBemittent als einziger Ansiedler keine EmissionsreduktionsmaBnahmen ergreifen, weil seine Emissionen nicht "ausreichen" den Immissionsstandard des von thm emittierten Schadstoffs zu "realisieren".

In einem Ballungsgebiet, wo alle Emittenten gerade so viel emittieren dürften, daB die Immissionsstandards für die verschiedenen Schadstoffe "realisiert" werden, müBten bel jeder Neuansiedlung (Abwanderung) von Emittenten die Emissionsstandards nach unten (oben) revidiert werden. Diese beiden Beispiele mögen genügen, um $z u$ zeigen, daß Emissionsstandards weitaus niedriger (also strenger) festgelegt werden müssen, als die durch Ausbreitungsrechnungen ermittelten Emissionsgrenzwerte, und zwar müssen sie so festgelegt werden, daB sie (durch die Emittenten) dem Stand der Technik entsprechend realisiert werden können 139?

Wird nach diesem Prinzip verfahren, ergibt sich im allgemeinen

138) Vgl. RSU, Umweltgutachten 1974, a.a.0., s. 13.

139) Nach diesem Prinzip wird in der Bundesrepublik Deutschland verfahren. In der TA Luft und in der TA Lärm werden Emissionsstandards gemäB dem stand der Technik für die unter $\S 4$ BImSchG bzw. $\S 16$ GewO fallenden Anlagen festgelegt (vgl. DreiBigacker, H.-L./Surendorf, F./Weber, E., Zum Entwurf der TA zur Reinhaltung der Luft. Dem stand der Technik angepaßt, in: Umwelt, 1974, 3, S. 20; Gilbert, T., Emissionsbegrenzungen und EmissionsmeBwerte - ein Vergleich, in: Umwelt, 1973, 6, s. 32). Der Emissionsstandard für KfzLärm wird in $\S 49$ Stvzo und den Richtlinien dazu festgelegt. Für die Gewässer werden nach $\S 7$ a WHG einheitliche Emissionsstandards, wie sie nach dem stand der Technik erreichbar sind, festgelegt (vgl. Riegel, R., Gewässergutewirtschaft: Immissions- oder Emissionsstandards?, a.a.0., s. 41). 
eine mehr oder weniger weit unter den Immissionsstandards liegende Umweltbelastung, so daß noch Reserven für die Ansiedlung neuer Emittenten vorhanden sind, ohne daB wieder gleich eine Anpassung aller relevanten Emissionsstandards erfolgen muß. Will man allerdings auch in Ballungsgebieten weitere Emittenten ansiedeln, sind unter Umständen häufigere Anpassungen der Emisslonsstandards nach unten notwendig 140 ?

Eine Anpassung der Emissionsstandards ist aber auch dann notwendig, wenn sich der Stand der Technik verbessert 141) Uber die Strenge der Emissionsstandards gehen die Meinungen weit auseinander. Während Rehbinder z.B. meint, die Emissionsstandards müßten - zeitlich abgestuft - gegenwärtig noch unerfüllbare technische und wirtschaftliche Normen darstellen, um die Entwicklung in die rechtspolitisch erwinschte Richtung zu lenken befürchten andere "UbermaBeffekte" 143)

Es dürfte u.E. feststehen, daß die widerstände der potentiellen Emittenten gegen die Emissionsstandards um so grober sein werden, je strenger sie sind. Der Widerstand düfte sich noch verstärken, wenn die Emissionsstandards, den unterschiedllchen natürlichen Bedingungen entsprechend, für die verschiedenen Emittenten unterschiedich hoch festgelegt werden, da hieraus Wett-

140) Ist das aus technischen oder finanziellen Gründen nicht mehr möglich, können die Immissionsstandards nur noch eingehalten werden, wenn die Emissionsquellen begrenzt werden. Beisplele dafür sind die Beschränkungen des Autoverkehrs in den Innenstädten oder auch die Begrenzung der Anzahl von Motorbooten auf Binnengewässern (vgl. Tourlsmus in der Bundesrepublik Deutschland, a.a.o., s. 34 ).

141) Vgl. Jürgensen, H./Jaeschke, K.-P., Allokationseffekte der Social Costs im Umweltschutz, a.a.0., S. 80.

142) Vgl. Rehbinder, E., Grundfragen des Umweltrechts, a.a.o., S. 253; dies kann man als dle "kallfornische Lösung" des Emissionsreduktionsproblems bezelchnen. Dort wurden so strenge Emissionsstandards für die Abgase von Kraftfahrzeugen aufgestellt, die in einem uber Jahre gehenden zeitplan stufenweise zu realisieren waren, daB sie zunächst für technisch kaum realisierbar oder zumindest für wirtschaftlich unzumutbar empfunden wurden. Belde Befürchtungen stellten sich als unbegründet heraus.

143) Riegel, R., Gewässergütewirtschaft: Immissions- oder Emissionsstandards?, a.a.0., S. 44. 
bewerbsnachtelle befürchtet werden.

Für die Behörden, welche die Emissionsstandards festlegen müssen, ergeben sich somit nicht nur die meBtechnischen Probleme der Identifizierung der Emittenten und der Zurechnung der Emissionen, sondern vor allem die Probleme der Durchsetzung der von ihr als notwendig erachteten Emissionsstandards. Das Identifizierungsund Zurechnungsproblem läBt sich umso besser lösen, je vollständiger die Emissions- und Immissionskataster sind, denn umso einfacher läßt sich von einer Umweltbelastung auf die Emissionsquelle schlleBen, womlt erst die Voraussetzung für die Durchsetzung der Emissionsstandards geschaffen wird ${ }^{144}$ ) Sodann muß die Behörde die Nachwelse führen, daB (1) die Emissionen zu Schäden führen (können) (wobel Wirkungskataster sehr von Nutzen sind), (2) technische Vermeidungs- oder Reduktionsmöglichkeiten vorhanden und (3) finanziell für den Emittenten zumutbar sind 145). Vor allem der Nachweis der technischen Vermeidungs- oder Reduktionsmöglichkelt im zur Realisierung des Emissionsstandards erforderlichen Umfang, ist für die Behörden äußerst schwierig, da sie in der Regel zu wenig Detailkenntnisse uber die technischen Möglichkeiten haben; mit anderen Worten: Der Stand der Technik 1st für die Behörden sehr schwierig festzulegen 146). Die Durchsetzung der Emissionsstandards wird zu einem langwierigen Pro$z e B$, in dem die Unternehmen nicht nur Exkulpationsversuche starten, sondern auch Verzögerungstaktiken anwenden und nicht zuletzt ihre Machtposition als Gewerbesteuerzahler und für Arbeltsplätze sorgende Ansiedler ausspielen. Das Ergebnis ist bekannt: zu lasche Emissionsstandards - man könnte hier von einem Durchsetzungsdefizit sprechen -, die oft nicht einmal eingehalten werden (Vollzugsdefizit). Die Durchsetzungsprobleme

144) Vgl. Schultze, C.L. u.a., Setting National Priorities, The 1973 Budget, a.a.o., s. 378 f.

145) Vgl. White, L.J., Effluent Charges as a Faster Means of Achieving Pollution Abatement, a.a.0., S. 112; Hansmeyer, K. -H., Die Abwasserabgabe als Versuch einer Anwendung des Verursacherprinzips, a.a.0., S. 76 .

146) Vgl. Wohlgemuth, R., Umweltgebuhren als Alternative zur Auflage, a.a.0., s. 46. 
ließen sich reduzieren, wenn einheitliche Emissionsstandards in Form von Reinigungsgraden festgelegt würden. Wie wir im folgenden zeigen werden, entsprechen einheitliche Emissionsstandards aber in zwelerlei Hinsicht nicht der Allokationseffizienz. Wir unterstellen, das zwel Emittenten I und II den gleichen Schadstoff emittieren; sle sollen unterschiedliche Grenzvermeldungskostenfunktionen, aber identische Immissionskoeffizienten $\alpha_{I}$ und $\alpha_{\text {II }}$ $(0<\alpha<1)$ haben. Immissionskoeffizienten geben im allgemeinen an, wie groB die Immissionskonzentrationen infolge der Emission einer Schadstoffelnheit je Zeiteinheit sind ${ }^{147}$. Wir wollen unter den Immissionskoeffizienten $\alpha_{I}$ und $\alpha_{I I}$ Größen verstehen, die angeben, wie hoch die Immissionsbelastungen $s_{I}$ und $s_{I I}$ bel alternativen Emissionsreduktionsgraden $e_{I}$ und $e_{I I} b z w$. Emissionsgraden $\left(100-e_{I}\right)$ und $\left(100-e_{I I}\right)$ sind. Identische Immissionskoeffizienten implizieren in der Regel 148) identische Ausbreitungsbedingungen für die Schadstoffemissionen belder Emittenten. In Abb. 18 ergänzen wir eine Darstellung von Bea 149) um die zwe1 unteren Quadranten, in denen wir die Immissionsbelastungsfunktionen

(1) $s_{I}=\alpha_{I}\left(100-e_{I}\right)$ und

(2) $s_{I I}=\alpha_{I I}\left(100-e_{I I}\right)$ eintragen.

Es wird also vom Ursprung aus nach rechts (links) der Emissions-

147) Vgl. Rincke, G., Anwendbarkeit des Verursacherprinzips am Beispiel der Wassergütewirtschaft, a.a.0., S. 116; Jürgensen, H./Jaeschke, K. -P., Operationale Verfahren zur Anwendung des Social Costs-Prinzips im Umweltschutz, Gutachten für das Bundesministerium des Innern, Hamburg 1971, S. $34 \mathrm{ff.;}$ RSU, Umweltgutachten 1974, a.a.0., S. 49.

148) Es könnte rein zufällig allerdings sein, daß günstıge Diffusionsbedingungen durch die geringe Entfernung von der Emissionsquelle zum Immissionsgeblet gerade so kompensiert werden, daB der gleiche Immissionskoeffizient zustandekommt wie im Falle ungüntiger Diffusionsbedingungen und großer Entfernung der Emissionsquelle vom Immissionsgeblet.

149) Vgl. Bea, F.X., Die Verteilung der Lasten des Umweltschutzes nach dem Verursacherprinzip, in: Wirtschaftsstudium, 2. Jg., 1973 , S. 455 . 


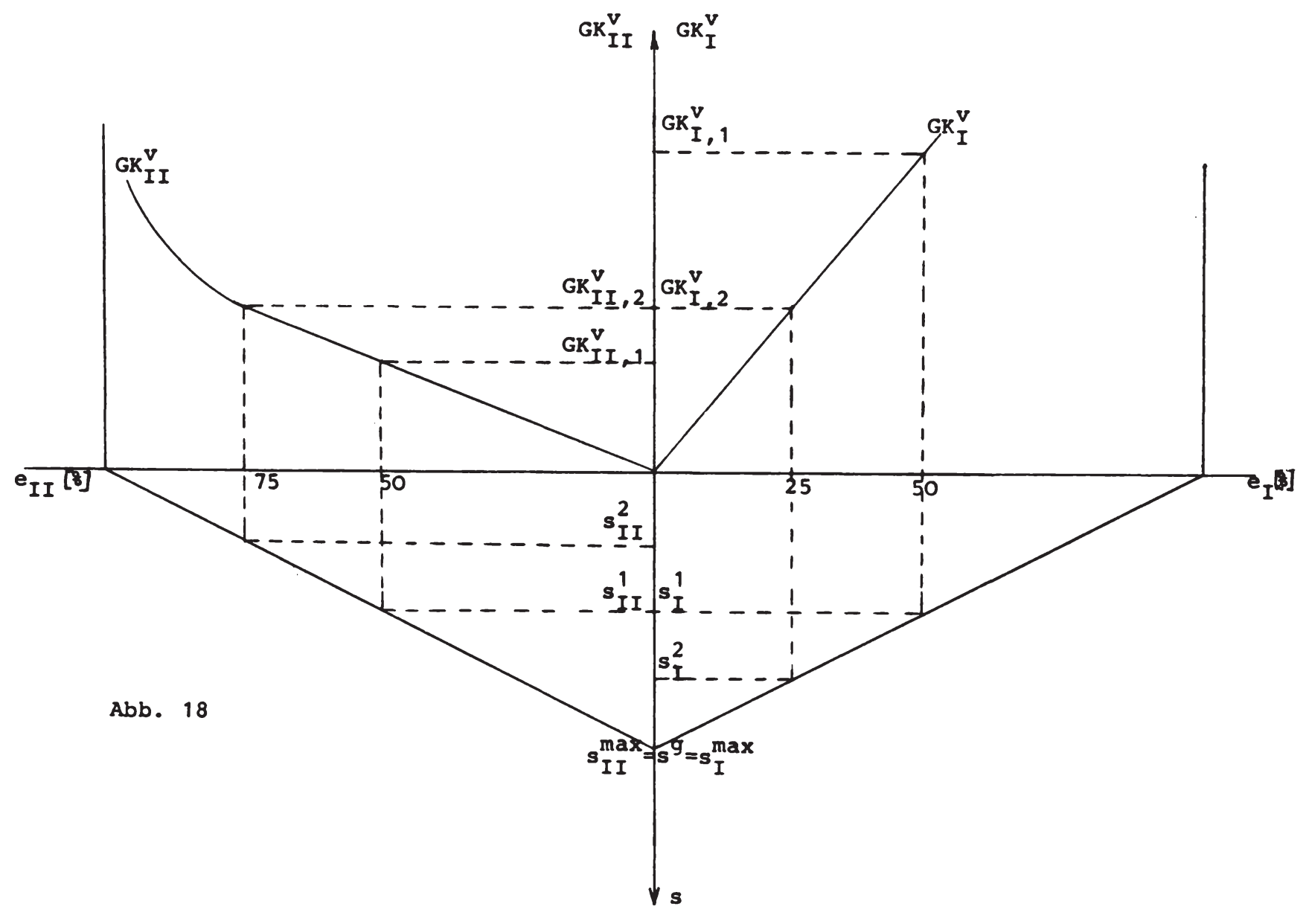


reduktionsgrad des Emittenten I (II) abgetragen. In den ersten und zweiten Quadranten sind die (unterschiedlichen) Grenzvermeldungskostenfunktionen beider Emittenten elngetragen. Dabel sel unterstellt, daß bel jedem Reinheltsgrad die Grenzvermeidungskosten von Emittent I größer sind als die von Emittent II.

Ist der Immissionsstandard $s^{g}$ bekannt, kann man die einheitlichen Emissionsstandards bestimmen, die notwendig sind, um den Immissionsstandard einzuhalten 150?

Man kann den Emissionsstandard für jeden Emittenten aus der Umkehrung der jewelligen Immissionsbelastungs funktion ermitteln. Für Emittent I ergibt sich:

(3) $100-e_{I}=\frac{1}{\alpha_{I}} \cdot s_{I}$.

W1e man aus Abb. 18 entnehmen kann, 1st der Immissionskoeffizient

(4) $\alpha_{I}=\frac{s_{I}^{\max }}{100}$.

Daraus, daB die Immissionsbelastungsfunktionen identisch sind, folgt, daß auch die "Belträge" der Emittenten I und II zur Immissionsbelastung, also $s_{I}$ und $s_{I I}$, Identisch sein müssen, wenn sie einheitliche Emissionsstandards realisieren:

(5) $s_{I}=s_{I I}$

Es gilt also wegen

(6) $s_{I}+s_{I I}=s^{g}$

(7) $s_{I}=\frac{s^{g}}{2}$.

Durch Einsetzen von (4) und (7) erhalten wir für (3)

150) Aus Vereinfachungsgründen unterstellen wir im folgenden auch, daB der Immissionsstandard "angestrebt" werden soll. 
(8) $100-e_{I}=\frac{1}{\frac{s_{I}^{\max }}{100}} \cdot \frac{s^{g}}{2}$

und durch Umstellung $e_{I}=100-\frac{100}{s_{I}^{\max }} \cdot \frac{s^{g}}{2} \cdot$

Be1 $n$ Emittenten $i(1=1, \ldots, n)$ gilt allgemein:

$$
e_{1}=100-\frac{100}{s_{1}^{\max }} \cdot \frac{1}{n} s^{g}
$$

In unserem Beispiel erhalten wir für $s_{I}^{\max }=5$ und $s^{g}=5$ : $e_{I}=100-\frac{100}{5} \frac{5}{2}=50$.

Für den Emissionsstandard $e_{I I}$ ergibt sich ebenfalls 508. Sind nun solche einheitlichen Emissionsstandards allokationsoptimal? Der Immissionsstandard $\mathbf{s}^{9}$ wird zwar eingehalten, da aber die $\mathrm{GK}^{\mathrm{V}}$ bel dem jewell1gen Emissionsreduktionsgrad von 508 unterschiedlich hoch sind, liegt kein Allokationsoptimum vor, denn dieses erfordert Gleichheit der marginalen Emissionsreduktionskosten.

Emittent II könnte einen höheren Relnigungsgrad als $508 \mathrm{zu}$ niedrigeren Grenzkosten realisieren als Emittent I einen niedrigeren Reinigungsgrad. Offenbar ist es sinnvoll, den Reinigungsgrad von Emittent II so lange $z u$ erhöhen und den von Emittent I so lange $\mathrm{zu}$ senken, bis sich die Grenzvermeldungskosten angeglichen haben 151) Das ist bel einem Reinigungsgrad von $75 \%$ be1 Emittent II und von 258 bei Emittent I der Fall. Dies resultiert in einer Reduktion der durch Emittent II hervorgerufenen Immissionsbelastung auf $s_{I I}^{2}$ und in einer Erhöhung der durch Emittent I bewirkten Immissionsbelastung auf $s_{I}^{2}$. Es gilt aber

151) Vgl. Nowotny, E., Wirtschaftspolitik und Umweltschutz, a.a.O., S. 200 E.: Cansier, D., Okonomische Grundprobleme der Umweltpolitik, a.a.O., S. 99; Krier, J.E./Montgomery, W.D., Resource Allocation, Information Cost and the Form of Government Intervention, a.a.0., S. 97; U.S. Council on Environmental Quality, Environmental Quality, The Second Annual Report, a.a.O., S. 136; Schultze, C.L. u.a., Setting National priorities, The 1973 Budget, a.a.0., s. $370 \mathrm{f}$. 
weiterhin, daB der Immissionsstandard gerade eingehalten wird: $s^{g}=s_{I}^{2}+s_{I I}^{2}$. Dieses Beisplel zeigt, daB die Allokationseffizienz wegen der unterschiedlichen Grenzvermeidungskostenfunktionen unterschiedlich hohe Emissionsstandards erfordert. In einem nächsten Schritt unterstellen wir nicht mehr identische Immissionskoeffizienten, sondern identische Grenzvermeidungskostenfunktionen und unterschiedliche Ausbreitungsbedingungen (also unterschiedliche Immissionskoeffizienten). Emittent I soll welter vom Immissionsgeblet entfernt sein und deshalb einen niedrigeren Immissionskoeffizienten als Emittent II haben: $\alpha_{\text {I }}<\alpha_{\text {II }}$ (vgl. Abb. 19).

Bei einer einheltlichen Auflage in Hobhe von 508 wird offenbar die Bedingung erfül1 ., daß die $G^{\mathbf{V}}$ belder Emittenten übereinstimmen. Auch wird bei dissem Reinigungsgrad der Immissionsstandard gerade eingehalten, denz $s^{g}=s_{I}^{1}+s_{I I}^{1}$. Es gilt nun aber offenbar, daß elne zusätzliche Emissionsreduktion durch Emittent II um einen Prozentpunkt die Immissionskonzentration um mehr senken würde als eine Verminderung der Emissionsreduktion um einen Prozentpunkt bel Emittent I die Immissionskonzentration anstelgen lassen würde. Das impliziert, daB Emittent I seine Emissionsreduktion um mehr als einen Prozentpunkt senken kann, wenn Emittent II seine Emissionsreduktion um einen Prozentpunkt erhöht, ohne daß der Immissionsstandard überschritten wird. Das sel an Abb. 19 erläutert. Wenn Emittent II seine Emissionsreduktion um 10 Prozentpunkte auf 608 steigert, senkt er die von ihm verursachte Immissionskonzentration von $s_{I I}^{1}$ auf $s_{I I}^{2}$. Dies kostet 1 hn einen der Fläche A II $_{\text {II }}$ enprechenden Betrag. Das inplizlert, daß Emittent I seinen "Beitrag" zur Immissionsbelastung von $s_{I}^{1}$ auf $s_{I}^{2}$ erhöhen kann, ohne daß der Immissionsstandard uberschritten wird. Er kann fola$11 \mathrm{ch}$ seine Emissionsreduktion um 20 Prozentpunkte (!) auf 308 senken. Dadurch spart Emittent I Kosten in Höhe der Fläche $A_{I}$. Ist $A_{I}>A_{I I}$, und das ist in unserem Beispiel der Fall, hat sich die zusätzliche Emissionsreduktion durch Emittent II (um 10 Prozentpunkte) und die überproportional gesenkte Emissionsreduktion von Emittent I (um 20 Prozentpunkte) gelohnt. Die durchschnittliche gesamtwirtschaftliche Emissionsreduktion konnte 


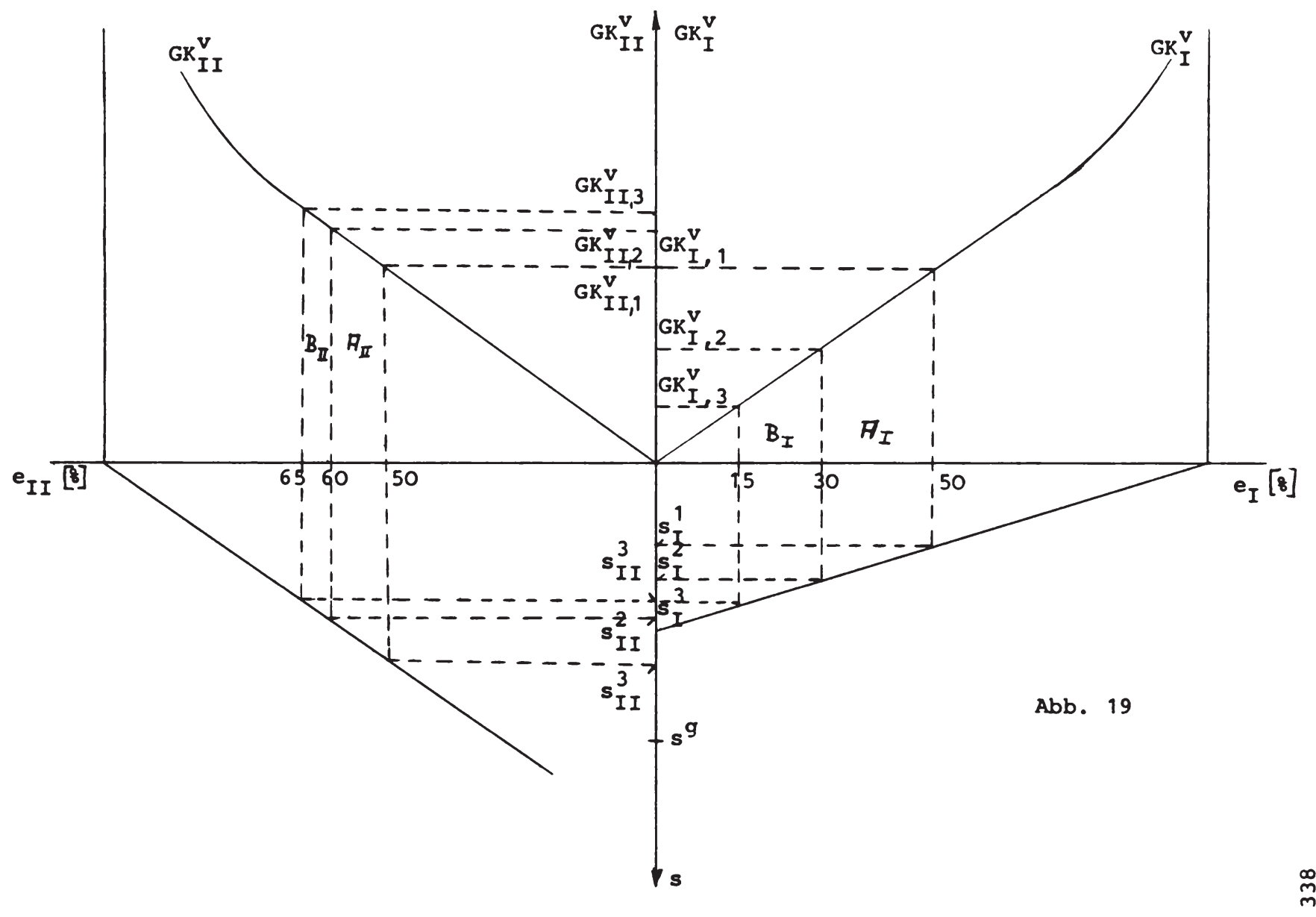


von 508 auf 458 gesenkt werden, ohne daß der Immissionsstandard überschritten wurde, wodurch sich eine gesamtwirtschaftliche Kostenersparnis in Höhe von $A_{I}-A_{I I}$ ergeben hat.

Erhöht Emittent II seine Emissionsreduktion auf 658 so sinkt sein Beitrag zur Immissionsbelastung auf $\mathbf{s}_{\mathrm{II}}^{3}$; derjenige des Emittenten I kann also auf $s_{I}^{3}$ steigen, ohne daB $s^{g}$ überschritten wird. Dies ermöglicht Emittent I aber seine Emissionsreduktion um 15 Prozentpunkte auf $158 \mathrm{zu}$ senken. Wir müssen nun wieder die zusätzlichen Emissionsreduktionskosten von Emittent II in Höhe von B $_{\text {II }}$ mit den Kostenersparnissen von Emittent I in Höhe von $B_{I}$ vergleichen, um sagen zu können, ob sich diese weitere Differenzierung der Emissionsstandards gelohnt hat. Unser Beispiel ist so gewählt, $d a B B_{I I}=B_{I}$, so daß das Allokationsoptimum also dort realisiert 1st, wo die Konstellation $e_{I I}: e_{I}=65: 15$ erreicht ist. Das ist dort der Fall, wo die "Beitrăge" der beiden Emittenten zur Immissionsbelastung gleich groB sind, wo also $s_{I}^{3}=s_{I I}^{3}$. Im Fall einheitlicher Grenzkostenfunktionen der Emissionsreduktion und unterschiedlicher Immissionsbelastungsfunktionen lautet die allokationsoptimale Bedingung also, daB die Emissionsstandards dort liegen müssen, wo die "Beiträge" der Emittenten zur Immissionsbelastung, $s_{I}$ und $s_{I I}$ gleich groß sind und wo außerdem gilt, daB $s_{I}+s_{I I}=s^{9}$, also der Immissionsstandard eingehalten wird.

Sind die Immissionsbelastungsfunktionen der Emittenten bekannt, so können die optimalen Emissionsstandards leicht ermittelt werden. GemäB Gle1chung (9) ergibt sich für Emittent I

$e_{I}=100-\frac{100}{3} \cdot \frac{5}{2} \approx 158$

und für Emittent II

$e_{I I}=100-\frac{100}{7} \cdot \frac{5}{2} \approx 658$

Aus Gründen der Allokationseffizienz ist also auch bel unterschiedlichen Immissionskoeffizienten eine Differenzierung der Emissionsstandards erforderlich 152)

152) Vgl. Rincke, G., Anwendbarkeit des Verursacherprinzips am Beispiel der Wassergutewirtschaft, a.a.0., S. 116; RSU, 
In einem nächsten Schritt können wir nun annehmen, daß sich sowohl die Grenzvermeidungskostenfunktion als auch die Immissionskoeffizienten der beiden Emittenten unterscheiden. Ob sich aus der Kombination unterschiedlicher Grenzvermeidungskostenfunktionen und unterschiedlicher Immissionskoeffizienten aus Gründen der Allokationseffizienz extrem unterschiedliche oder dicht zusammenliegende (im Extremfall gar 1dentische) Emissionsstandards ergeben, hängt von der jeweiligen Konstellation ab. Hat der Emittent mit den höheren Grenzvermeldungskosten auch den geringeren Immissionskoeffizienten, der Emittent mit den niedrigeren Grenzvermeidungskosten also den höheren Immissionskoeffizienten, so werden sich extrem auseinanderliegende Emissionsstandards ergeben. Liegt dagegen die Konstellation vor, daB der Emittent mit den niedrigeren (höheren) Grenzvermeldungskosten auch den niedrigeren (höheren) Immissionskoeffizienten hat, so ergibt sich eine tendenzielle Annäherung der allokationsoptimalen Emissionsstandards - zufälligerwelse vielleicht gar eine Identität.

Wir können abschlleßend zusammenfassen:

Aus Gründen der Allokationseffizienz wird es notwendig sein, die Emissionsstandards wegen unterschiedlicher Grenzvermeidungskostenfunktionen und wegen unterschiedlicher Immissionskoeffizienten der Emittenten $z u$ differenzieren. Solche differenzierenden (allokationsoptimalen) Emissionsstandards lassen sich aber nur schwer ermitteln, denn dazu müssen alle Grenzvermeidungskostenfunktionen und alle Immissionskoeffizienten bekannt sein. Die Informationserfordernisse sind also sehr hoch 153). Aber selbst wenn ihre Ermittlung gelingen würde, wăre es sehr schwierig, die allokationsoptimalen Emissionsstandards durchzusetzen. Eine dem Verursacherprinzip entsprechende Anlastung der Vermeidunaskosten

Umweltgutachten 1974, a.a.0., S. 49; Slebert, H., Regional planning - land use approaches to environmental quality management, a.a.0., s. 5 .

153) Vgl. Krier, J.E./Montgomery, W.D., Resource Allocation, Information Cost and the Form of Government Intervention, a.a.0., S. 98; Head, J.G., Public Policies and Pollution Problems, a.a.0., S. 23, Frey, B.S., Umweltökonomie, a.a.0., S. 114 f.; Blair, R.D., The Clean Air Act of 1970, a.a.0., s. 266 . 
würde $z u$ extrem unterschiedlichen Belastungen der potentiellen Emittenten führen, die viellelcht nur daher rühren, daß manche Emittenten einen sehr hohen Immissionskoeffizienten haben, weil sie an einem zum Immissionsgebiet ungünstig liegenden standort angesiedelt wurden, wofür sie unter Umständen überhaupt nichts können 154) In einem solchen Fall scheint eine Durchbrechung des Verursacherprinzips geboten. Die Emissionsstandards können durchaus allokationsoptimal festgelegt werden, doch sollte ihre Realisierung bei den Emittenten, die unverschuldet hohe Verme1dungskosten zu tragen hätten, durch staatliche Finanzhilfen erleichtert werden 155)

cc) Marktwirtschaftliche Instrumente zur Durchsetzung des Verursacherprinzips

Da auch die juristische Durchsetzung des Verursacherprinzips letztlich die glelchen Probleme aufwirft wie die Pigou'sche Steuerlösung, daß nămlich die allokationsoptimale Realisieruna kaum erreichbare Anforderungen an die Informationsbeschaffuna und Durchsetzung stellt - oder ungekehrt formuliert: nur eine nicht allokationsoptimale Durchsetzung praktisch möglich scheint -, wollen wir von neuem die Frage aufwerfen, ob nicht andere ökonomische Maßnahmen (als die der Internalisierung durch eine Besteuerung der Produktmenge in Höhe des externen Verlustes) zum gewünschten Ziel, also der kostenminimalen Einhaltung der Immissionsstandards fuhrt. Die Pigou'sche Schadensteuer - von der theoretischen Konzeption her die optimale Lösung scheitert bekanntlich vor allem daran, daß die (marginalen) Schadensfunktionen kaum bestimmt werden können. Es liegt nun der Gedanke nahe, nach einer anderen Bemessungsgrundlage als dem Schaden, der durch die Emissionen eintritt, zu suchen, und durch eine Besteuerung dieser GröBe indirekt den Schaden zu

154) Vgl. Bullinger, M., Rechtsfragen des Verursacherprinzips beim Umweltschutz, in: Das Verursacherprinzip und seine Instrumente, a.a.0., S. $93 \mathrm{f}$.

155) Vgl. ebenda. 
reduzieren. Eine solche Ersatz-Bemessungsgrundlage für den Schaden ist um so besser geelgnet, in je engerer Beziehung sie sich zur Schadensentstehung befindet. Von den drei in Frage kommenden Bemessungsgrundlagen Produktmenge, Faktorinput und Emissionsmenge ist das offenbar letztere, denn der (potentielle) Schaden 1st direkt von der Emissionsmenge und - wenn uberhaupt nur indirekt von Produktmenge oder Faktoreinsatz abhängig 156) Das soll im folgenden näher erlautert werden. Wir betrachten nacheinander die Eignung einer Besteuerung der Produktmenge, des Faktorinputs und einer Emissionsabgabe hinsichtlich einer kostenminimalen Einhaltung der Immissionsstandards.

(1) Die Besteuerung der Produktmenge

Die Besteuerung der Produktmenge kann erfolgen, weil das betreffende Produkt selbst $z u$ elner Umweltbelastung fuhrt (z.B. Einwegflaschen) oder weil die mit der Produktion dieses Gutes als Kuppelprodukt entstehende Schadstoffmenge auf diese Weise indirekt reduziert werden soll.

In Abb. 20 betrachten wir zunächst den ersten Fall. Auf dem Markt für Einwegflaschen herrsche Gleichgewicht bei einem Preis von DM 0,60 und einer entsprechenden Produktion von 10 Mio. Flaschen. Die maximal zulässige Anzahl von produzierten Einwegflaschen pro Jahr soll aus Gründen des Umweltschutzes auf 7 Mio. gesenkt werden. Diesen Produktionsrückgang kann der Staat erzwingen, indem er die Produktion von Elnwegflaschen mit elner Mengensteuer belegt, die einen Satz von $t=0,50$ DM hat. Zum neuen Prels von DM 0,95 werden dann genau die gerade noch $z$ lässigen Elnwegflaschen in Höhe von 7 Mio. produziert. Um den richtigen Steuersatz zu finden, müssen die Angebotsund Nachfragekurve bzw. deren Elastizitäten im relevanten Be-

156) In noch größerer Nähe zum Schaden als die Emissionen 1st natürlich die Immissionsbelastung, doch kann sie ebenso wie der Schaden selbst wegen der Zurechnungsprobleme nicht zur Bemessungsgrundlage für eine steuer werden. Außerdem wäre auch sie keine Maßnahme des präventiven Umweltschutzes, denn die Immissionsbelastung müBte tatsächlich eintreten, damit die steuer angewendet werden kann. 


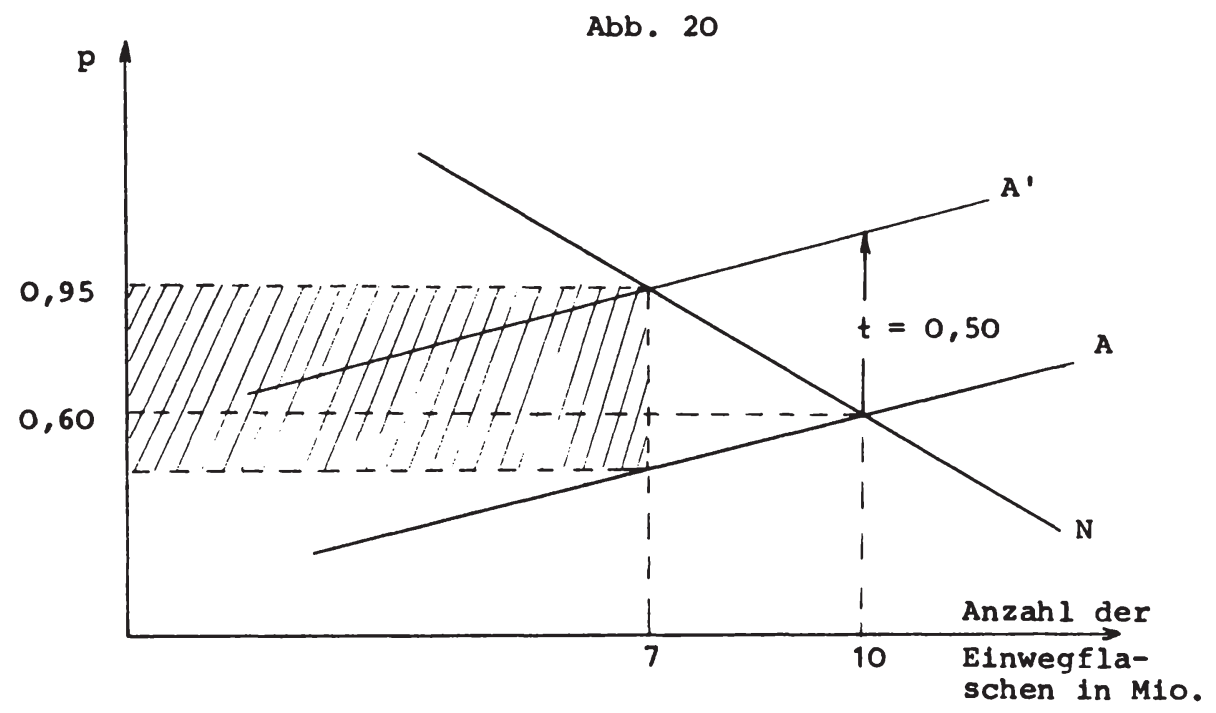

reich bekannt sein. Ist das nicht der Fall, kann versucht werden, sich iterativ an den richtigen steuersatz heranzutasten. Das steueraufkommen in Höhe der schraffierten Fläche $(3,5$ Mio. DM) sollte zweckgebunden für die ordnungsgemäBe Beseltigung der verbleibenden 7 Mio. Einwegflaschen verwendet werden, und sofern davon noch etwas übrig bleibt, ebenfalls Zwecken des Umweltschutzes zugeführt werden (z.B. Umweltforschung).

In diesem Fall ist die Besteuerung der Produktmenge eine ăußerst effiziente Maßnahme, denn das produkt selbst ist in diesem Falle auch gleichzeitig der "Schadstoff".

Nun wollen wir den anderen Fall betrachten, daB also via Produktmengenbesteuerung das schädliche Kuppelprodukt im erforderlichen Umfang reduziert werden soll (vgl. Abb. 21).

Im ersten Quadranten sind die Marktbeziehungen dargestellt. Im Marktgleichgewicht wird die Produktmenge $x_{1}$ zum Preis $p_{1}$ hergestellt. Dabei entsteht gemäB der im zweiten Quadranten dargesteliten Emissionsfunktion $e=e(x)$ eine Emissionsmenge in Höhe von $e_{1}$, die gemäB einer im dritten Quadranten wiedergegebenen Immissionsbelastungsfunktion (mit konstantem Immissionskoeffi- 


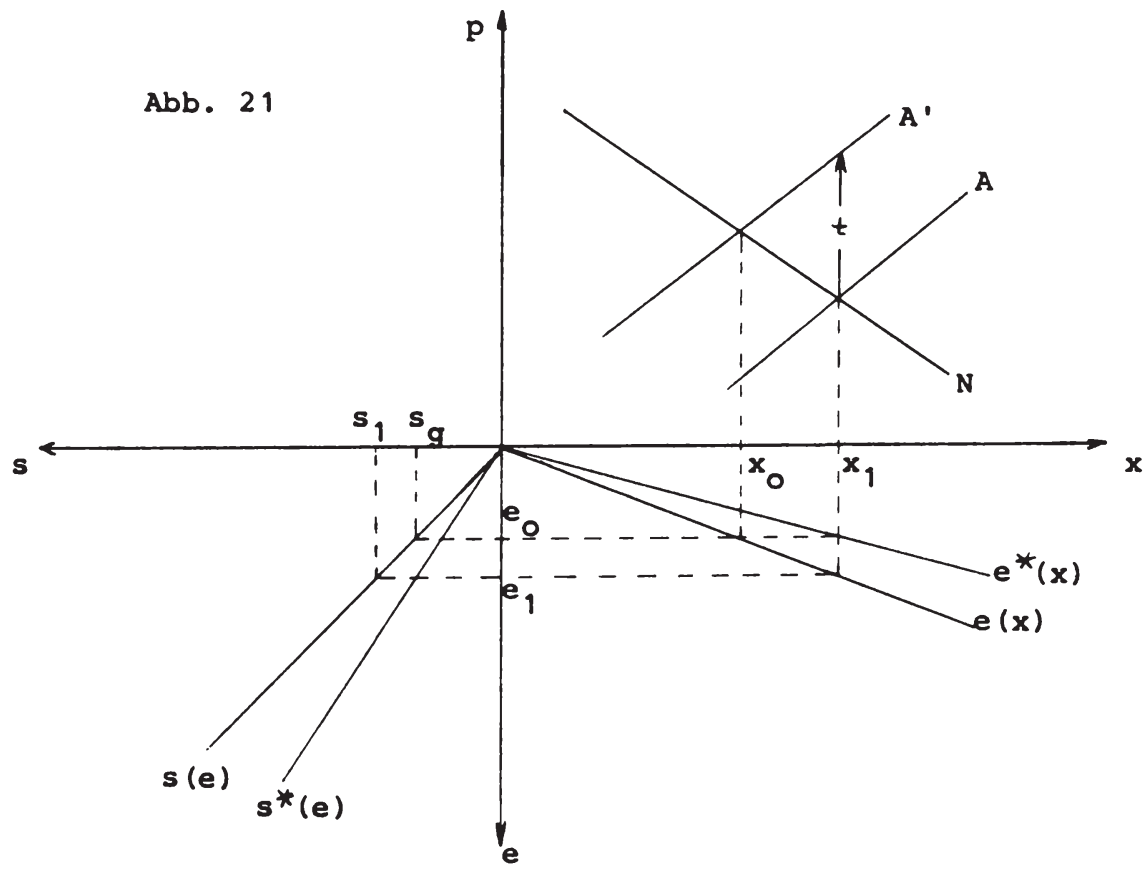

zienten) $s=s(e)$ zu einer Immissionsbelastung in Höhe von $s_{1}$ führt. Der Immissionsstandard soll nun aber $s_{g}$ betragen, so daß $s_{g}<s_{1}$

Die Immissionsbelastung kann gesenkt werden, wenn die Produktion des Gutes $x$ mit einer Mengensteuer, deren Satz $t$ ist, belegt wird. Der dadurch bewirkte Rückgang der Produktionsmenge auf $x_{0}$ bewirkt nämlich gemäß $e=e(x)$ einen Rückgang der Emissionen auf $e_{O^{\prime}}$ die gemäB der Immissionsbelastungsfunktion gerade noch $z u$ einer zulässigen Immissionsbelastung in Höhe von $\mathbf{s}$ führen 157) Das Umweltschutzziel wurde also erreicht. Die Informationserfordernisse bestehen darin, die Angebots- und Nachfragefunktion sowle die Emissions- und Immissionsbelastungsfunktion zu kennen, was im einzelnen sicher nicht einfach, doch 157) Wir nehmen aus Vereinfachungsgründen wieder an, daB s $\mathrm{g}$ an-
gestrebt werden soll. 
durchaus möglich erscheint. Eventuell kann wiederum durch einen iterativen Proze $B$ der richtige Steuersatz ermittelt werden. Die entscheidende Frage ist nun aber die, ob sg kostenminimal erreicht wurde. Der Nutzen, den die Verringerung der Immissionsbelastung bewirkte, ist, weil die Schadensfunktion unbekannt ist, nicht (genau) bestimmbar. Die Kosten lassen sich jedoch auf den ersten Blick feststellen; sie bestehen real in einem Rückgang der Produktion von $x_{1}$ auf $x_{0}$. Der staat kann mit seinen steuereinnahmen allerdings andere, unter Umständen nützlichere Güter zusätzlich zur Verfügung stellen, so daß dieser Produktionsrückgang von $x$ durch die staatlichen Leistungen viellelcht sogar überkompensiert wird.

Es stellt sich jedoch die Frage, ob der Immissionsstandard nicht auch hätte erreicht werden können, ohne das die produktion des Gutes $x$ eingeschränkt worden wäre. Offenbar wäre das durch zwe1 Maßnahmen oder eine Kombination dieser beiden möglich gewesen. Es wäre nämlich unter Umständen möglich gewesen, die Emissionsintensität der Produktion von $x$ so weit $z u$ senken, daß die neue Emissionsfunktion $e^{\star}=e^{\star}(x)$ bereits zur Einhaltung von $s_{g}$ führen würde. Eine solche geringere Emissionsintensität wäre unter Umständen durch eine Input- oder Verfahrenssubstitution oder -verbesserung $z u$ erreichen, deren gesellschaftliche Kosten geringer wären als die der Produktmengenreduktion. Statt durch emissionsorientierte technische Maßnahme die Emissionsintensität der Produktion zu senken, hätte man aber auch durch transmissionsorientierte und/oder immissionsorientierte technische Maßnahmen den Immissionskoeffizienten soweit senken können, daß z.B. die neue Immissionsbelastungsfunktion $s^{*}=s^{*}(e)$ gerade zur Einhaltung des Immissionsstandards geführt hätte. Solche Alternativen werden nicht erwogen, wenn die Produktmenge besteuert wird. Von einer Produktmengenbesteuerung gehen also keine incentives zur Emissionsintensitätsreduktion oder zu Immissionsschutzmaßnahmen aus, die unter Umständen gesamtwirtschaftlich billiger sind als der Rückgang der Produktion ${ }^{158}$ ?

158) Vgl. Zerbe, R.O., Theoretical Efficiency in Pollution 
Vergegenwärtigt man sich, daß sich der umwelttechnische Fortschritt in einer Verringerung der Emissionsintensität der Produktion äußert, kann man auch den Schluß ziehen, daß eine Produktmengenbesteuerung keinen incentive zur umwelttechnischen Forschung liefert, denn warum sollen die Unternehmen nach Möglichkeiten forschen, die Emissionsintensität $z u$ verringern, wenn ihre Produkte besteuert werden?

Wir wollen nun noch auf zwei weitere Einwände eingehen, welche die Besteuerung der Produktmenge als ein noch fragwürdigeres Instrument des Verursacherprinzips zur Einhaltung der Immissionsstandards erscheinen lassen. Der erste Elnwand ist wohlfahrtstheoretischer Art und wurde von Buchanan vorgetragen 159): Im Falle eines Monopols, wo aus Gründen der optimalen Allokation elne Produktionsausdehnung erforderlich wäre, führt eine aus Gründen der Emissionsreduktion erhobene Steuer auf die Produktmenge weiter weg vom Allokationsoptimum 160) Von Plott stammt der Einwand, daß durch eine Besteuerung der Produktmenge in dem Fall, in dem ein inferiorer Produktionsfaktor für die Schadstoffemission verantwortlich ist, die Emissionsmenge zunehmen kann 161) Das sei an folgender Abb. 22 demonstriert 162 ?

Control, a.a.0., s. 370; Littmann, K., Umweltbelastung sozialökonomische Gegenkonzepte der Umweltpolitik, a.a.0., S. 76 f.; Nowotny, E., Wirtschaftspolitik und Umweltschutz, a.a.O., S. 191; Siebert, H., Gebuhren als Instrument der Umweltpolitik, in: WISU, 3. Jg., 1974, S. 13; Jürgensen, H./ Jaeschke, K.-P., Allokationseffekte der Social Costs im Umweltschutz, a.a.0., S. 84 .

159) Vgl. Buchanan, J.M., External Diseconomies, Corrective Taxes, and Market Structure, in: Staaf, R.J./Tannian, F.X. (Hrsg.), Externalities, a.a.0., S. 269 ff. Buchanan bezog seinen Elnwand zwar auf die Pigou'sche Steuer, doch gilt er hier in glelcher Weise.

160) Vgl. auch Head, J.G., Public Policies and Pollution Problems, a.a.0., S. 16 .

161) Vgl. Plott, C.R., Externalities and Corrective Taxes, in: Staaf, R.J./Tannian, F.X. (Hrsg.), Externalities, a.a.0., S. $261 \mathrm{ff}$. Auch Plott bezieht sich auf die Pigou-Steuer; jedoch gilt sein Einwand hier in gleicher Weise.

162) Vgl. ebenda, S. 262. 


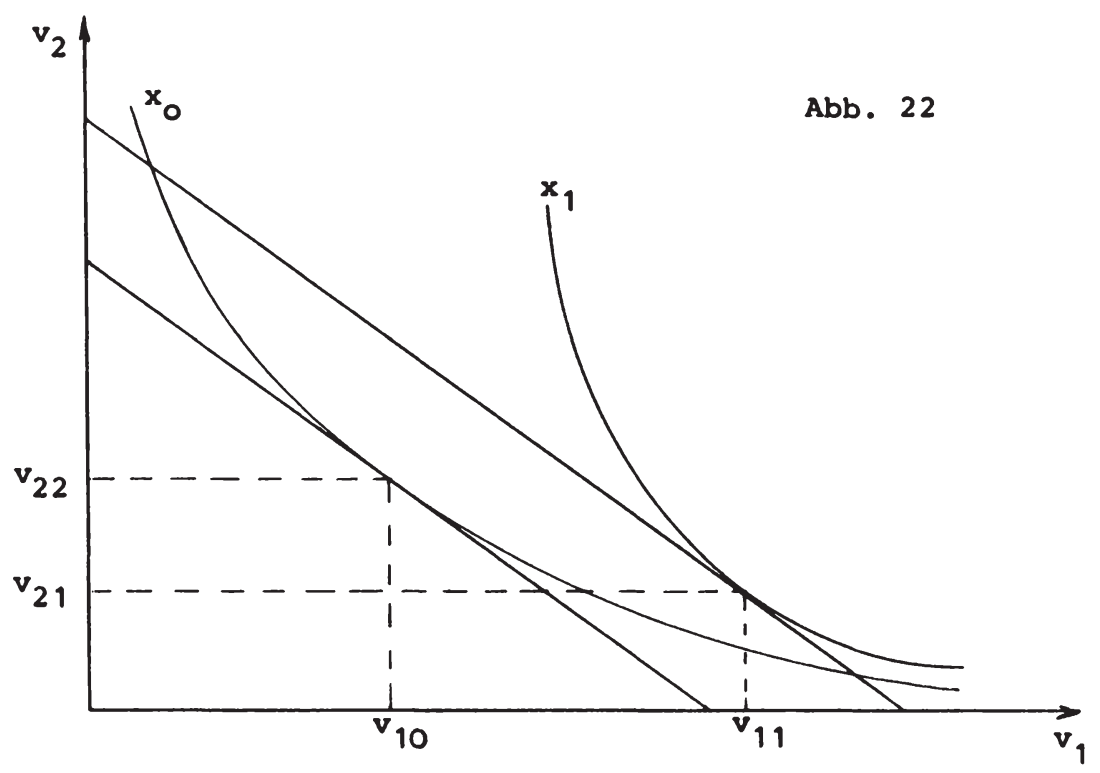

$v_{1}$ und $v_{2}$ seien die Produktionsfaktormengen, die zur Herstellung von $x$ benötigt werden. Der Faktor 2 sei für die Emissionen verantwortlich, da er elnen schadstoff enthalte, der während des Produktionsprozesses freigesetzt wird. Die Produktion in Höhe von $x_{1}$ soll besteuert werden, damit die durch die Verwendung von $v_{21}$ hervorgerufene Emission verringert wird. Man glaubt, die erforderliche Emissionsreduktion zu erreichen, indem man die Produktion durch eine steuer auf $x$ bis $x_{0}$ senkt. Der Rückgang der Produktionsmenge äußert sich nun aber nicht in einer Emissionsreduktion, sondern im Gegente11: Da der schadstoffverursachende Input inferior ist und folglich von $v_{21}$ auf $v_{22}$ steigt, erhöhen sich auch die Emissionen.

Die Durchsetzung des Verursacherprinzips via Besteuerung der Produktmenge erweist sich als ein "unduly clumsy approach to pollution control" 163) Das gilt allerdings - wie bereits ge-

163) Zerbe, R.O., Theoretical Efficiency in Pollution Control, a.a.0., s. 370 . 
sagt - nicht für die Fälle, in denen Produkte selbst $z u$ Umweltbelastungen führen. Für diese Fälle wurden in den USA einige interessante Vorschläge der Produkt-Besteuerung gemacht; wir wollen sie kurz darstellen, obwohl wir uns der Bewertung dieser Vorschläge durch Solow anschließen möchten: "Even it cannot be done, it is a good guide to thinking" 164)

Diese Vorschläge haben gemeinsam, daß sie sich in der Regel auf langlebigere Konsumgüter beziehen, die während ihrer Nutzung $z u$ Emissionen führen (z.B. Kraftfahrzeuge) und/oder nach threr Zweckerfüllung Abfallbeseitlgungsprobleme aufwerfen (vor allem Schrott aller Art) 165) Eine Besteuerung der Emissionen oder der Abfallmengen ist wegen der Vielzahl der Emittenten kaum möglich. Aus diesem Grunde wird vorgeschlagen, Produkte beim Hersteller mit elner Steuer in der Höhe zu belasten, in der diese zu Umweltschäden führen können, wenn von thnen der denkbar schädlichste Gebrauch gemacht wird. So könnte man z.B. für jeden Kraftfahrzeugtyp, der keine Umweltschutzvorrichtungen eingebaut hat, die Abgasemissionsraten und die Lärmemissionen messen und unter zugrundelegung der durchschnittlichen Lebensdauer und durchschnittlich zurückgelegten kilometerzahl annäherungsweise den Schaden $z u$ bestimmen versuchen, den dieser Kraftfahrzeugtyp durch Abgas- und Lärmemissionen hervorrufen kann. Die Höhe dieses geschätzten Schadens bildet die elne Komponente der Bemessungsgrundlage für die Steuer. Die andere Komponente besteht aus den Kosten der ordnungsgemäBen Beseitigung des ausgedienten Kraftfahrzeugs, wovon bei einem eventuellen Recycling bestimmter Teile

164) Solow, R.M., The Economist's Approach to Pollution and its Control, in: Sclence, vol. 173, No. 3996 vom 6.8.1971, S. 503.

165) Vgl. ebenda,; Schultze, C.I. u.a., Setting National Priorities, The 1973 Budget, a.a.O., S. 393; Cansier, D., Okonomische Grundprobleme der Umweltpolitik, a.a.0., s. 79 ff.; Nowotny, E., Wirtschaftspolitik und Umweltschutz, a.a.O., S. 220 f.; Schellhaaß, H.M., Kfz-Steuer - Für eine marktgerechte Lösung, in: Die Wirtschaftswoche Nr. 28, 1971 , S. 24; Wittmann, W. , Einführung in die Finanzwissenschaft, Bd. 2: Die öffentlichen Einnahmen, 2. Aufl., Stuttgart 1975, S. 188 . 
des Autos die Erlöse aus dem Verkauf der rezyklisierten Stoffe abgezogen werden können. Nehmen wir an, die summe der beiden Komponenten ergäbe einen steuerbetrag von DM 1.000,-- für jedes Auto eines bestimmten Typs und dieser Betrag werde in voller Höhe (offen ausgewiesen) auf den Käufer überwälzt.

Der Käufer kann sich nun zwischen drei Möglichkeiten entscheiden: 1. Er kauft das Auto nicht mehr, weil es ihm zu teuer geworden ist (es tritt insoweit keine Umweltbelastung ein); 2 . er kauft das Auto und zahlt die Steuer von DM 1.000,--, wofür er Abgase und Lärm emittieren und das ausgediente Auto am Straßenrand stehen lassen darf, oder 3. der Käufer entschließt sich a) eine Abgasentgiftungsanlage zum Preis von DM 300,-- einbauen zu lassen, wofür er als "Belohnung" DM 400,-- an Steuer zurückerhält, b) eine Schalldämpferanlage am Auspuff $z$ um Preis von DM 150,-- anbringen zu lassen, wofür er eine weitere steuerersparnis von DM 250,-- kassieren kann, c) das ausgediente Auto in eine Shredder-Anlage zu bringen, wofür er die restliche von ihm gezahlte steuer in Höhe von DM 350,-- erhält.

Eine solche Besteuerung wirkt auf den ersten Blick bestechend. Dadurch daB die Komponenten der Steuer für Abgas- und Lärmemissionen sowie die ordnungsgemäBe Beseitigung des Abfallprodukts höher sind als die entsprechenden Aufwendungen des Käufers zur Vermeidung der (geschätzten) Schäden, besteht ein starker Anreiz für den Käufer, die umweltschützenden Maßnahmen vorzunehmen. Faktisch llegt nun auch die Beweislast, daß die Umwelt nicht belastet wird, beim potentiellen Verursacher 166) Man fragt sich allerdings, ob sich der administrative Aufwand einer solchen Besteuerung lohnt, denn sie müßte aus Wettbewerbsgründen auf alle kraftfahrzeuge und eventuelle substitutionsgüter, wie öffentliche Nahverkehrsmittel, ausgedehnt werden. Den gleichen Effekt könnte man sicherer und billiger durch Herstellungsgebote oder Emissionsstandards erreichen, die von den Herstellern zu erfüllen wären. Den Autokäufern würde dadurch lediglich die

166) Vgl. Solow, R.M., The Economist's Approach to Pollution and its Control, a.a.O., S. 503 . 
Freiheit genommen, für einen bestimmten Preis (in unserem Beispiel DM $1.000,--)$ die Umwelt zu belasten.

Allein auf die Reduzierung des Abfallproblems läuft der Vorschlag einer Amortisationssteuer hinaus 167) Danach soll eine Steuer auf die Güter erhoben werden, die nach ihrer Zweckerfüllung (Abfall-)Beseltigungsprobleme aufwerfen, und zwar soll deren Satz mit zunehmender Lebensdauer der besteuerten Produkte abnehmen. Es wird zunächst eine Soll-Lebensdauer für jenes Gut festgelegt und sodann der steuerbetrag bestimmt, der beim Kauf des Gutes zu entrichten 1st. Erreicht das Produkt die SollLebensdauer, so wird der gesamte steuerbetrag zurückerstattet. Fällt es früher als Abfall an, wird nur ein Tell der steuer zurückerstattet, da die steuersätze so gestaffelt sind, daB sie mit zunehmender Lebensdauer abnehmen. Das sei an Abb. 23 verdeutlicht.

Der gesamte steuerbetrag entspricht bei einer Soll-Lebensdauer von 10 Jahren der gesamten schraffierten Fläche.

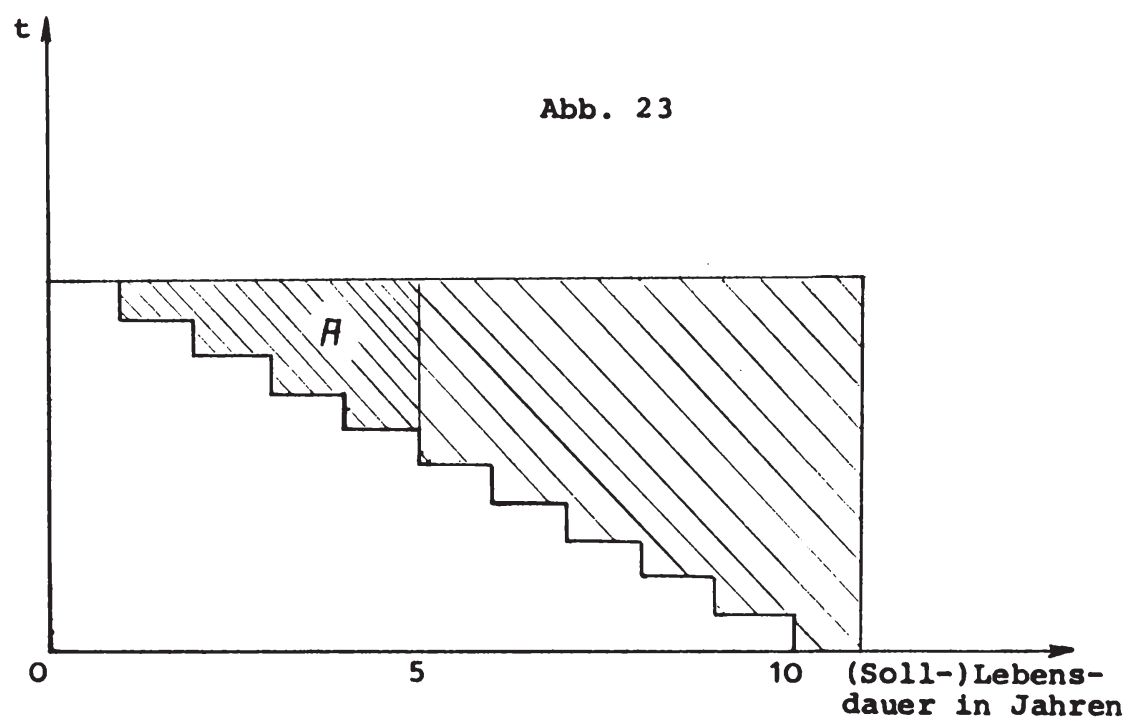

167) Vgl. Goldsmith, E./Allen, R., Planspiel zum Uberleben, Stuttgart 1972, S. $31 \mathrm{f} . ;$ Wittmann, W., Einführung in die Finanzwissenschaft, Bd. 2, a.a.0., s. 189 . 
Fallt das Produkt mit einer Soll-Lebensdauer von $10 \mathrm{~J}$ ahren berelts vor Ablauf des ersten Jahres als Abfall an, wird keine Steuer zurückerstattet. Fällt es z.B. zwischen dem vierten und füften Lebensjahr als Abfall an, so erhält der Käufer Steuern in Höhe der schrafflerten Fläche $A$ zurïck. Wird das Produkt erst nach dem zehnten Jahr zum Abfall, erhält der Käufer die gesamte Stever zurück.

Der Vortell einer solchen Steuer wäre, daß die Kăufer einen incentive hätten, das Produkt zumindest bis zu seinem Soll-Alter zu nutzen.

Einen Anrelz für die Produzenten, auch langlebigere Produkte herzustellen, liebe sich errelchen, wenn man die im voraus zu zahlenden Steuerbeträge $T$ mit zunehmender Soll-Lebensdauer senkt (vgl. Abb. 24). Hat z.B. ein Pkw eine Soll-Lebensdauer von 4 Jahren, kann man thn mit DM 450,-- besteuern. Wird dagegen die SollLebensdauer auf 8 Jahre erhöht, beträgt die zu entrichtende Steuer nur noch DM 250,--.

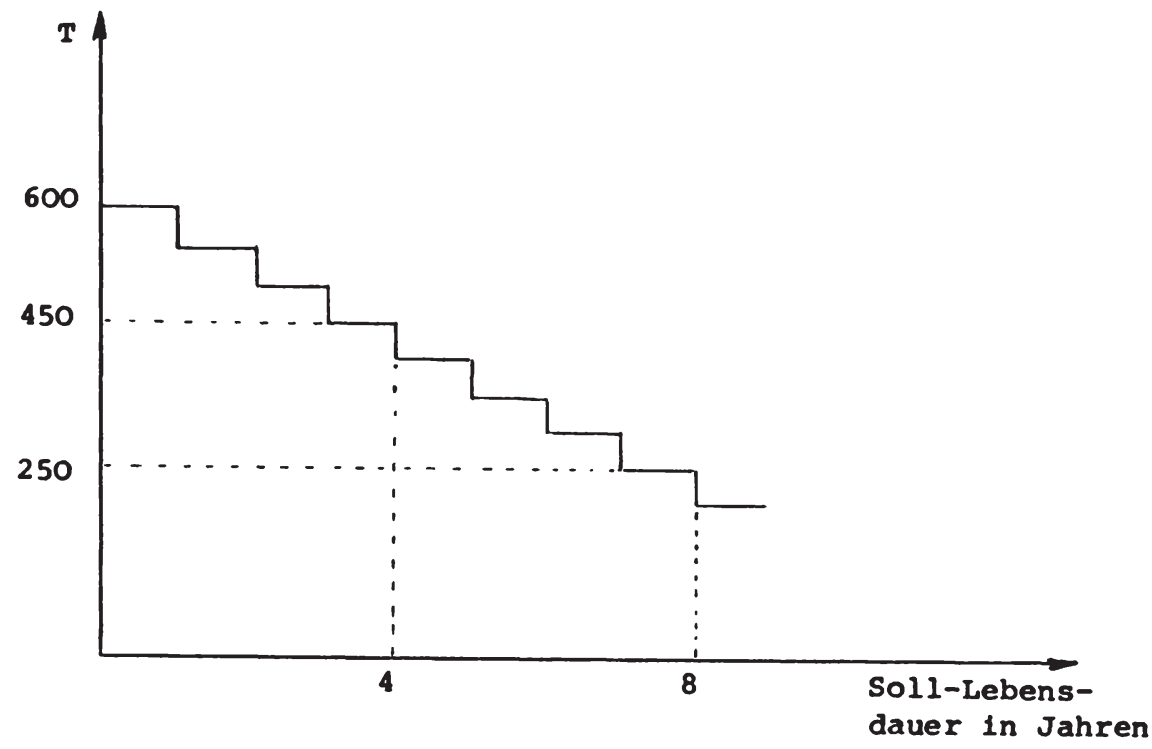

Abb. 24 
Jedoch ist auch eine solche der "planned obsolescence" und Weqwerfmentalität entgegenwirkende Besteuerung der Produkte kaum praktikabel. Die Festlegung der Soll-Lebensdauer für elne Fülle von Produkten und die Zurückerstattung der Steuerbetrăge würde einen erheblichen Informations- und Administrationsaufwand erfordern. Um vor allem Konsumgüter langlebiger zu gestalten - sofern das uberhaupt erwünscht ist - scheinen Herstellungsgebote besser geeignet zu sein.

(2) Die Besteuerung von Produktionsfaktoren

Wir haben bereits mehrfach erwähnt, daB für viele schadstoffemissionen der Schadstoffgehalt der Produktionsmittel verantwortlich ist. Es liegt deshalb nahe, in solchen Fällen die Faktorinputs je nach deren Schadstoffgehalt zu besteuern. So könnte man z.B. Heizöl je nach dessen Schwefelgehalt oder Benzin je nach dessen Bleigehalt oder Waschmittel je nach deren Phosphatgehalt besteuern. Zweck einer solchen Besteuerung wäre es, entweder die Schadstoffentfernung oder -reduzierung aus den Produktionsmitteln vor deren Einsatz (z.B. Heizölentschwefelung) oder die vollständige oder teilweise Substitution dieser Inputs zu erreichen, um auf diese Weise auch die Schadstoffemissionen zu reduzieren. Eine solch indirekte Methode der Emissionsreduktion empfiehlt sich vor allem in den Fällen, in denen viele kleine Emissionsquellen vorhanden sind (private Haushalte, Kraftfahrzeuge), deren direkte Emissionsbesteuerung aus administrativen Gründen äußerst schwierig und kostsplelig wäre. Die Besteuerung der schadstoffhaltigen Produkte - die in nachgelagerten Unternehmen als Produktionsfaktoren eingesetzt werden - bei den Herstellern 1st nämlich administrativ relativ einfach ${ }^{169}$.

168) Unter Produktionsfaktoren seien in diesem Abschnitt auch in der "häuslichen Produktion" eingesetzte Faktoren, wie Helzöl, Benzin, Waschmittel u.a. verstanden.

169) Vgl. Ackermann, K./Geschka, H./Karsten, D., Gutachten zur Gesamtbelastung der Volkswirtschaft durch das Umweltprogramm der Bundesregierung, a.a.O., S. 602 f.; Solow, R.M., The Economist's Approach to Pollution and its Control, a.a.0., S. 502; Zerbe, R.O., Theoretical Efficiency in Pollution Control, a.a.O., S. 370; Cansier, D., Okonomische Grundprobleme der Umweltpolitik, a.a.0., s. 77; Nowotny, E., Wirt- 
Sie stellt bei den Produzenten einen incentive zur Beseitigung der Schadstoffe aus den produkten vor deren Verkauf dar. Denn die Produzenten entgehen nun der steuer, die ihre produkte eventuell mehr verteuern würde als die schadstoffentfernungsmaßnahmen, was sie weniger wettbewerbsfähig machen würde. Für die Verursacher (Emittenten) entsteht durch die verteuerten Produktionsmittel ein incentive zur Inputsubstitution.

Die Besteuerung schadstoffhaltiger Inputs hat allerdings auch erhebliche Nachteile.

Nicht alle Inputmengen des glelchen Faktors führen, obwohl sie den gleichen Schadstoffgehalt haben, immer zur gleichen Emissionsrate je Inputeinheit; die gleichen Inputs haben also unterschiedliche Emissionsintensitäten 170). Ein Beispiel dafür ist die Verbrennung von Helıöl. Heizöl mit völlig identischem Schwefelgehalt kann - wie air wissen - zu ganz unterschiedlichen $\mathrm{SO}_{2}$-Emissionsraten führen, je nachdem wie gut der olbrenner eingestellt ist. Besteuert man das Heizöl nach dessen Schwefelgehalt, so wird derjenige Helzölverbraucher bestraft, der seinen olbrenner gut warten läBt, denn er muB für einen vermeintlichen schaden zahlen, den er gar nicht verursacht, weil er nur unbedeutende Mengen $\mathrm{SO}_{2}$ emittiert. Eine Besteuerung des Faktorinputs kann also dem Verursacherprinzip widersprechen. Weiterhin läBt die Inputbesteuerung, ähnllch der Produktmengenbesteuerung, die Möglichkeit eventuell billigerer Emissionsreduktionsmaßnahmen, wie die Umstellung oder Verbesserung der Produktionsprozesse, Rückhaltetechnologien und Recycling auBer Acht 171) Ein großes Problem entsteht vor allem dadurch, daß a 11 e Inputfaktoren, die irgendwelche schadstoffe enthalten und als

schaftspolitik und Umweltschutz, a.a.0., S. 218.

170) Vgl. Cansier, D., Okonomische Grundprobleme der Umweltpolitik, a.a.0., S. 78; Jürgensen, H./Jaeschke, K.-P., Allokationseffekte der Social Costs im Umweltschutz, a.a.0.. S. 84 .

171) Vgl. Siebert, H., Gebühren als Instrument der Umweltpolitik, a.a.0., S. 14; Arnold, V., Kuppelprodukte, bffentliche Ungüter und externe Effekte, a.a.0., S. 103; Zerbe, R.0., Theoretical Efficiency in Pollution Control, a.a.o., s. 370 . 
Substitutionsprodukt in Frage kommen, besteuert werden mussen, da sonst elntretende substitutionsprozesse nach der Besteuerung eines Produktionsmittels nur zu einer substitution der Schadstoffemissionen führen würde 172) Würde man z.B. nur Helzöl besteuern, könnte es c.p. seln, daß auf Helzölbasis strom erzeugende Kraftwerke durch Kernkraftwerke substitutiert würden und folglich an die stelle der $\mathrm{SO}_{2}$-Belastung der Luft eine verstärkte thermische Belastung der Gewăsser und schwierige Beseitiqungsprobleme für radioaktive Abfälle treten würden. Die insgesamt resultierende Umweltbelastung kann durch eine lückenhafte Besteuerung schadstoffhaltiger Produktionsfaktoren also größer werden und somit welter vom Allokationsoptimum wegfuhren. Aber auch wenn der besteuerte produktionsfaktor inferior ist, können die Emissionen (jetzt allerdings des gleichen schadstoffs) durch seine Besteuerung zunehmen 173) Das sei an Abb. 25 erläutert.

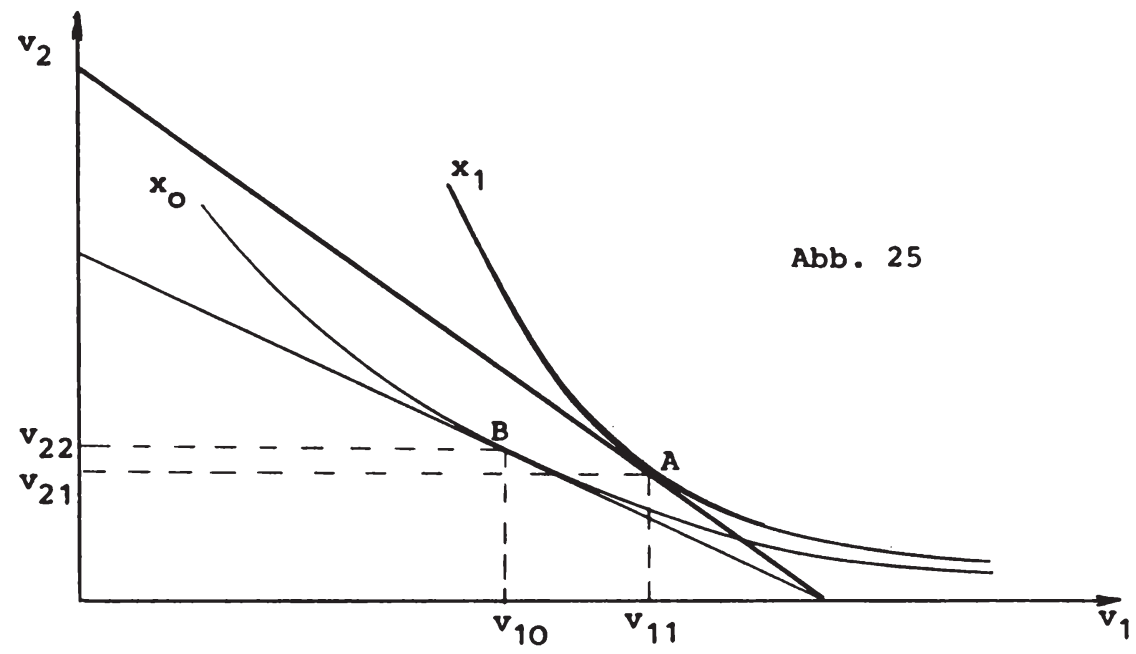

172) Vgl. Watson jr..,W.D., Cost of Air Pollution Control in the Coal-Fired Electric Power Industry, in: Quarterly Review of Economics and Business, vol. 12, 3, 1973, S. 79 ; Cansier, D., Okonomische Grundprobleme der Umweltpolitik, a.a.0., S. 78 f.: Littmann, K., Umweltbelastung - sozialökonomische Gegenkonzepte, a.a.0., S. 62. f.

173) Vgl. Jürgensen, H./Jaeschke, K.-P., Allokationseffekte der Social Costs im Umweltschutz, a.a.O., S. $84 \mathrm{f}$. 
Durch die Besteuerung des schadstoffhaltigen Produktionsfaktors $v_{2}$ verändert sich die Minimalkostenkombination von A nach B, wo bel geringerer Produktion von $x$ mehr von $v_{2}$ eingesetzt wird. Folglich wird c.p. auch mehr von dem schadstoff emittiert, der in $v_{2}$ enthalten ist.

Obwohl sich also auch die Besteuerung der Produktionsmittel als sehr problematisch erwelst, wird sie in den Fällen eine brauchbare Lösung darstellen können, in denen wegen der Vielzahl der Emissionsquellen direkte Emissionsmengenbesteuerungen ausscheiden. Allerdings "konkurriert" die Inputbesteuerung in solchen Fällen mit ebenfalls administrativ relativ einfach durchsetzbaren Produktstandards.

(3) Die Emissionsabgabe

Fast alle bisher geschilderten Instrumente des Verursacherprinzips zur Durchsetzung einer rationalen Umweltpolitik haben mehr oder weniger große Nachteile. Wenn sie eine theoretisch aroße Allokationseffizienz haben, wie reglonal und kostenmäßig differenzierte Emissionsstandards oder wie die Pigou'sche Schadensteuer, erwelsen sie sich vor allem aus Gründen der Informationsbeschaffung und der Administration als kaum realisierbar. Lösungen, die administrativ relativ einfach sind und relativ wenige Informationen erfordern, wie einheitliche Emissionsstandards, Emissionsverbote, Produktsteuern oder Produktionsfaktorsteuern zeigen dagegen eine mehr oder minder geringe Allokationseffizienz. Ein Instrument des Verursacherprinzips, das sowohl relativ allokationseffizient als auch administrativ relativ einfach anwendbar ist, haben wir bisher noch nicht kennengelernt.

Ob die Emissionsabgabe ein solches Instrument ist, wollen wir im folgenden untersuchen; wir vergleichen sie dabei mit den wohl im Rahmen des Verursacherprinzips allein als Alternative in Fraqe kommenden Emissionsstandards.

Ausgangspunkt unserer Analyse sei die Betrachtung dreier Versionen einer Emissionsabgabe 174) Eine Emissionsabgabe kann lediq-

174) Vgl. Oberhauser, A., Abgrenzung des Verursacherprinzips und seine Einordnung in die Umweltpolitik, a.a.0., S. 45. 
lich den Zweck haben, die Vermeldungskosten (Emissionsreduktionskosten), die notwendig sind, um die Immissionsstandards einzuhalten, zu internalisieren. Hat die Emissionsabgabe diesen Zweck erfüllt, wird sle hinfällig; sle hat also nur eine ordnungsfunktion (Ordnungssteuer). Die zweite Version einer Emisslonsabgabe will neben der Internalisierung der Emissionsreduktionskosten auch noch die nach der erforderlichen Emissionsreduktion verbleibenden Schäden besteuern, und die dritte Version will neben der verursachungsgemäBen Anlastung der Emissionsreduktionskosten auch noch die sogenannte Restverschmutzung erfassen und somit auch noch eine Art Benutzungsgebühr für die Umweltmedien darstellen. Diese drei Versionen seien an Abb. 26 näher betrachtet.

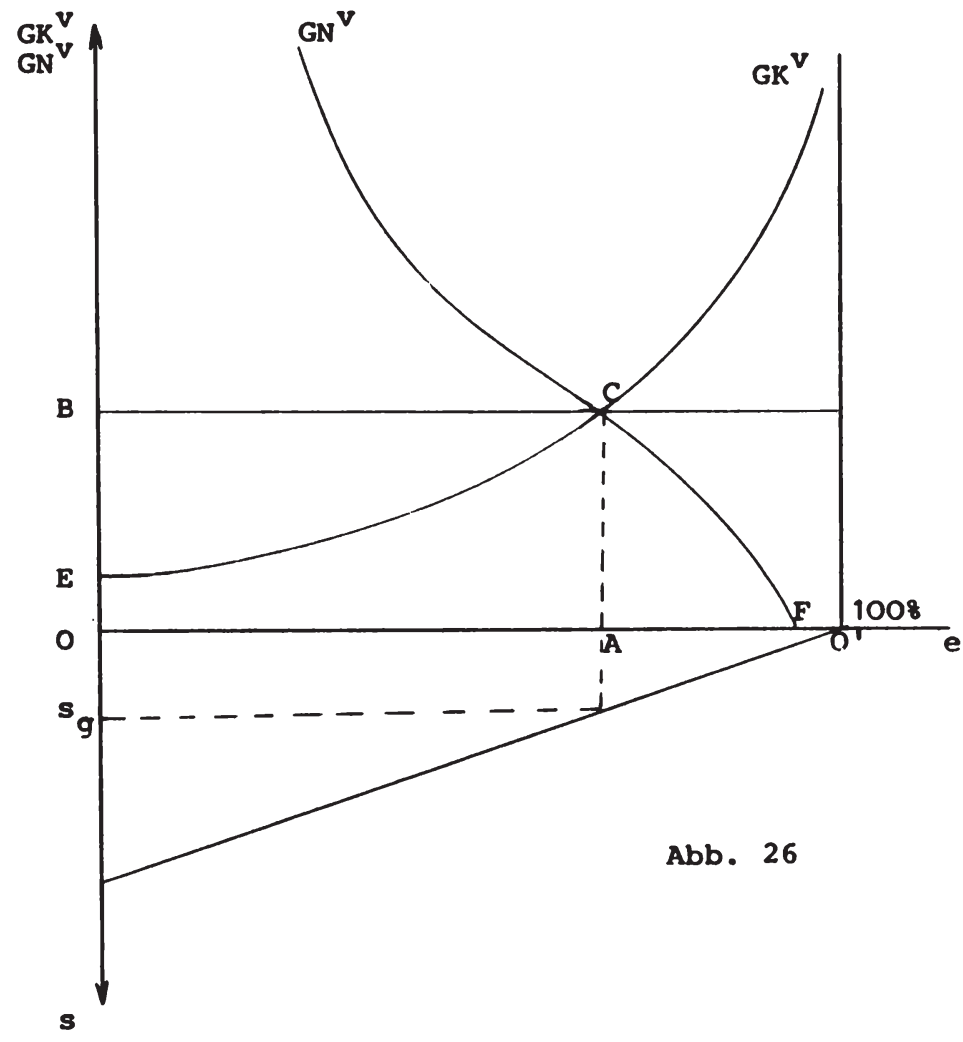


Auf der Abszisse sind von links nach rechts die Emissionsreduktionsgrade (in umgekehrter Richtung also der Verschmutzungsgrad) eingetragen, auf der positiven Ordinatenachse die Grenzkosten und der Grenznutzen der Emissionsreduktion eines Emittenten sowie der Emissionsabgabensatz und auf der negativen ordinatenachse die Immissionsbelastung s. Im ersten Quadranten liegt die allokationsoptimale Emissionsreduktion dort, wo sich Grenznutzen und Grenzkosten der Emissionsreduktion schneiden, also bei OA. Nehmen wir an, bel dieser Emissionsreduktion des einzigen Emittenten werde der Immissionsstandard gerade eingehalten. Der allokationsoptimale Satz einer Emissionsabgabe ist nun derjenige, der den Verursacher veranlaßt, seine Emissionen um OA zu reduzieren, also nur noch O'A zu emittieren. Dieser allokationsoptimale Abgabensatz muß offenbar die Höhe $O B=A C$ haben, denn der Emittent wird seine Emissionsreduktionsmaßnahmen so lange ausdehnen wie die Grenzkosten der Emissionsreduktion niedriger sind als der marginale Emissionsabgabensatz. Durch eine Emissionsabgabe mit einem marginalen Satz in Höhe von OB wird also die allokationsoptimale Emissionsreduktion in Höhe von OA erreicht 175)

Wenn der Verursacher keine Emissionsreduktionsmaßnahmen durchführt, muß er nach der oben geschilderten ersten Version eine Abgabe in Höhe von OACB entrichten. Nach der zweiten Version muB er zusätzlich noch den Schaden AFC zahlen, der auch dann noch verbliebe, wenn er die erforderliche Emissionsreduktion durchführen würde, also insgesamt $\mathrm{OACB}+\mathrm{AFC}=\mathrm{OFCB}$. Nach der dritten Version schließlich würde das Abgabenaufkommen am

175) Vgl. Oberhauser, A., Abgrenzung des Verursacherprinzips und seine Einordnung in die Umweltpolitik, a.a.0., s. $40 \mathrm{f}$.; Head, J.G., Public Policies and Pollution Problems, a.a.0., S. 24 ; Siebert, H., Erfolgsbedingungen elner Abgabenlösund (Steuern/Gebuhren) in der Umweltpolitik, in: Issing, 0. (Hrsg.), Ókonomische Probleme der Umweltschutzpolitik, a.a.0., S. 40; Swoboda, H., Probleme der Umweltpolitik, a.a.O.. S. 86; Cansier, D. , Okonomische Grundprobleme der Umweltpolitik, a.a.0., S. 67 f.; Schultze, C.L. u.a., Setting National priorities, The 1973 Budget, a.a.O., S. 372; Rincke, G., Anwendbarke1t des Verursacherprinzips am Beispiel der Wassergütewirtschaft, a.a.0., S. 45. 
gröBten sein und $O O^{\prime} D B$ betragen. Ein rational handelnder Verursacher, der seine Kosten minimiert, wird aber bei allen drei Versionen die Emissionen bis zum Optimum OA reduzieren. Nach uer ersten version muß er dann überhaupt keine Abgabe mehr zahlen, hat also nur die Kosten für die Emissionsreduktion in Höhe von OACE zu tragen. Gegenüber der Nicht-Reduktion spart er also eine summe in Höhe von $O A C B$ - $O A C E=E C B$. Diese summe spart er bel optimaler Emissionsreduktion auch bel den anderen beiden Versionen. Bei der zweiten Version muß er aber zu den Vermeidungskosten auch den verbleibenden schaden in Höhe von AFC als Emissionsabgabe abfuhren; insgesamt hat er dann also Kosten in Höhe von $\mathrm{OACE}+\mathrm{AFC}=\mathrm{OFCE}$. Bel der dritten Version muB der Verursacher zu seinen Emissionsreduktionskosten auch noch für die Restverschmutzung in Höhe von $O^{\prime A}$ den vollen Abgabesatz zahlen, also $A O^{\prime} D C$, und somit insgesamt $O A C E+A O^{\prime} D C=O O^{\prime} D C E$. Wir wollen im folgenden von der dritten Version ausgehen, da wir in der ersten Version gegenüber einer Emissionsstandard-Lösung keinen besonderen Vorteil sehen und die zweite Version wegen der Schadensbewertung und-zurechnung Ausgaben entstehen läßt, die wahrscheinlich die ganzen Einnahmen aus der Emissionsabgabe in Höhe von AFC bel weltem übertreffen würden. Gehen wir aber realistischerweise davon aus, daß die Grenzschadensfunktion (bzw. Grenznutzenfunktion der Emissionsreduktion) unbekannt ist, scheint auch die Grundlage für die Bestimmung der optimalen Höhe der Abgabesätze zu fehlen. Es gibt jedoch eine Möglichkeit, trotzdem den allokationsoptimalen 176) Abgabesatz zu bestimmen. Wenn die Immissionsbelastungsfunktion $s=s(e)$ unbekannt ist, kann der Abgabesatz, der zur Einhaltung des Immissionsstandards erforderlich 1st, durch ein iteratives Verfahren ermittelt werden 177), das unter Umständen allerdings erhebliche Kosten verur-

176) Wir unterstellen $1 \mathrm{~m}$ folgenden, der Immissionsstandard entspreche elner allokationsoptimalen Immissionskonzentration. Dann sind Maßnahmen, die zur Realisierung dieses Immissionsstandards zu minimalen Kosten führen, allokationseffizient (oder allokationsoptimal).

177) Vgl. Baumol, W.J./Oates, W.E., The Use of Standards and Prices for Protection of the Environment, a.a.0., S. $44 \mathrm{f}$; 
sacht 178). Es wird dabei zunächst ein Abgabesatz festgelegt, von dem man annimmt, daß er der richtige ist, um zur Einhaltung des Immissionsstandards zu führen. Sinkt die Immissionsbelastung nicht bis auf oder unter die Höhe des Immissionsstandards wird der Abgabesatz so lange in kleinen Schritten erhöht, bis der Immissionsstandard erreicht wird. Erweist es sich, daB der Abgabesatz zu hoch festgelegt wurde und der Immissionsstandard erheblich unterschritten wird, muB der Abgabesatz gesenkt werden. Man kann sich lelcht vorstellen, daß ein solcher iterativer pro$z e B$, wenn er nicht nach wenigen Iterationen bereits $z$ um richtiqen Abgabesatz führt, erhebliche Friktionen hervorrufen kann. Wäre die Immissionsbelastungsfunktion $s=s(e)$ bekannt, so könnte der dem Immissionsstandard entsprechende Emissionsstandard eines Emittenten abgeleitet werden, und so bei Kenntnis der Grenzkostenfunktion der Emissionsreduktion der optimale Abgabesatz direkt bestimmt werden. In Abb. 27 wollen wir das näher erläutern.

Nehmen wir an, im Ausgangszustand sei die Reinigungsleistung des Emittenten 08. Dann entsteht gemä $\beta$ der Immissionsbelastungsfunktion die maximal mögliche Immissionsbelastung in Höhe von $s_{\max }{ }^{*}$ Der Immissionsstandard soll jedoch niedriger sein und sg betragen. Aus ihm läßt sich nun mit Hilfe der Immissionsbelastungsfunktion die mindestens erforderliche Emissionsreduktion $e_{1}$, also ein Emissionsstandard von 508 bestimmen. Der allokationsoptimale Abgabesatz ist nun offenbar der, der zur $e_{1}$ entsprechenden Emissionsreduktion in Höhe von 508 führt, also $t=O A=e_{1} B$.

Siebert, H., Erfolgsbedingungen einer Abgabenlösung, a.a.O., S. 44; Freeman III, M.A./Haveman, R.H., Residuals Charges for Pollution Control: A Pollcy Evaluation, in: Science, vol. 177, No. 4046, v. 28.7.72, S. 324 ; Hass, J.E., Optimal Taxing for the Abatement of Water Pollution, in: Water Resources Research, vol. 6, 1970, S. 353 ff.

178) Vgl. Dorcey, A.H.J., Effluent Charges, Information Generation and Bargaining Behavior, in: Natural Resources Journal, vol. 13, 1973, S. 127 f.; Wohlgemuth, R., Umweltgebủhren als Alternative zur Auflage, a.a.0., s. 46. 


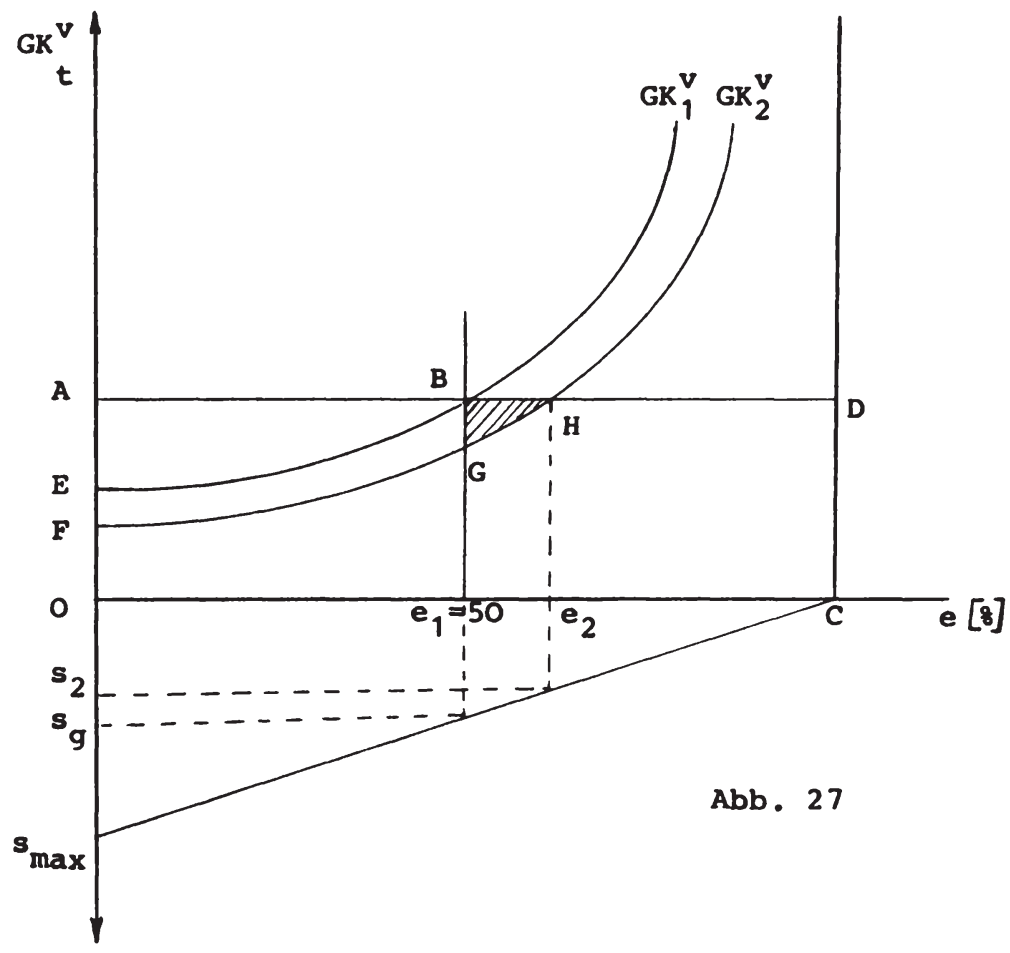

Die Informationserfordernisse bestehen in der Ermittlung des Immissionskoeffizienten und der $\mathrm{GK}^{\mathrm{V}}$ des Emittenten. Auf den ersten Blick scheint in der Abb. 27 kein Unterschied hinsichtlich der Allokationswirkung einer reinen Auflagenlösung in Form eines Emissionsstandards und der kombinierten AbgabenStandard-Lösung $2 u$ bestehen. In beiden Fällen würde durch eine Emissionsreduktion von 508, die den Verursacher Oe ${ }_{1}$ BE kostet, der Immissionsstandard erreicht werden können. Der erste Unterschied besteht jedoch darin, daB der Emittent im Falle der Abgabenlösung auch für die Restverschmutzung zahlen muß. Es entstehen $1 \mathrm{hm}$ also neben den Emissionsreduktionskosten auch noch Kosten in Höhe der Emissionsabgabe für die Restverschmutzung in Höhe von $e_{1} C D B$. Dies scheint gegenüber der reinen Auflagenlösung zunächst nichts anderes $z u$ bedeuten als eine Umverteilung zugunsten des Staates. Tatsächlich ist dies jedoch einer der wesentlichsten Vortelle der Abgabenlösung - manche meinen der 
Vorteil überhaupt - gegenüber allen bisher geschilderten Instrumenten des Verursacherprinzips, denn die Besteuerung der Restverschmutzung stellt einen ständigen Anreiz dar, den technischen Fortschritt auf dem Gebiet der Emissionsreduktion anzuwenden, der sich in einer Senkung der Emissionsreduktionskosten ăußert. Man könnte sagen, daß die Besteverung der Restverschmutzung nahezu automatisch zur Realisierung des Standes der Umwelttechnik führt 179). Das soll ebenfalls an Abb. 27 demonstriert werden: Nehmen wir an, der umwelttechnische Fortschritt äußere sich in einer Reduzierung der Emissionsreduktionskosten von $\mathrm{GK}_{1}^{\mathrm{V}}$ auf $\mathrm{GK}_{2}^{\mathrm{V}}$. Im Fall der Auflagenlösung schlägt sich das lediglich in einer Verminderung der Emissionsreduktionskosten und damit einer Erhöhung des Gewinns des Emittenten um FGBE nieder. Im Fall der Abgabenlösung aber wird die Emissionsreduktion ausgedehnt bis $e_{2}$, wo die neue marginale Emissionsreduktionskostenfunktion $\mathrm{GK}_{2}^{\mathrm{v}}$ die Grenzsteuerfunktion schneidet (in $\mathrm{H}$ ). Auch in diesem Fall reduziert der umwelttechnische Fortschritt die Gesamtkosten des Emittenten, und zwar sinken sie sogar um die schraffierte Fläche GHB mehr als die im Fall der Auflagenlösung, denn der Emittent spart neben den gesunkenen Emissionsreduktionskosten ${ }^{180}$ ) auch noch Emissionsabgaben in Höhe von $e_{1} e_{2} \mathrm{HB}$ infolge seiner reduzierten Restverschmutzung. Die auf $e_{2}$ erhöhte Emissionsreduktion bewirkt einen Rückgang der Immissionsbelastung unter den Immissionsstandard auf $s_{2}$ ' wo-

179) Vgl. Zerbe, R.O., Theoretical Efficiency in Pollution Control, a.a.0., S. 371 f.; Orr, L., Incentive for Innovation as the Basis for Effluent Charge strategy, in: American Economic Review, vol. 66, 1976, papers and proceedings, S. 442; Head, J.G., Public Policies and Pollution Problems, a.a.0., S. 24; Nowotny, E., Wirtschaftspolitik und Umweltschutz, a.a.0., S. 202 ff.; Wenders, J.T., Methods of Pollution Control and the Rate of Charge in Pollution Abatement Technology, in: Water Resources Research, vol. 11, 1975, S. 393 ff.; Cansier, D., రkonomische Grundprobleme der Umweltpolitik, a.a.0., s. 100; Schultze, C.L. u.a., Setting National Priorities, The 1973 Budget, a.a.O., s. 372; dieselben, Setting National Priorities, The 1972 Budget, a.a.O., S. 242.

180) Die Emissionsreduktionskosten sind im Fall der Abgabenlösung gesunken, well FGEB > $e_{1} e_{2} \mathrm{GH}$. 
durch Reserven für eine Produktionsausdehnung geschaffen werden. Infolge des umwelttechnischen Fortschritts wäre es auch möglich gewesen, entweder den Abgabensatz auf $e_{1} G z u$ senken, um so den Immissionsstandard weiterhin nur zu erreichen (und nicht $z u$ unterschreiten), und es wäre auch möglich gewesen, den Immissionsstandard auf $s_{2} z u$ senken (also $z u$ verschärfen). Die Senkung des Abgabensatzes halten wir nicht für sinnvoll, denn daraus resultiert lediglich eine Verbesserung der Gewinnsituation des Emittenten - genau wie bei der Auflagenlösung. Die Anpassung des Immissionsstandards an den technischen Fortschritt halten wir dagegen - allerdings nur in größeren Zeltintervallen - für durchaus wünschenswert. Er sollte nicht bel jeder realisierten Emissionsreduktion erfolgen, da hierdurch Emissions- und das heiBt Produktionswachstumsreserven allzusehr beschränkt würden.

Nachdem wir die Uberlegenheit der Emissionsabgabe hinsichtlich der incentives zur Anwendung des umwelttechnischen Fortschritts - man könnte von einer besonders großen dynamischen Allokationseffizienz der Emissionsabgabe sprechen,- dargestellt haben, wollen wir uns nun mit der traditionellerwelse besonders hervorgehobenen überlegenen statischen Allokationseffizienz der Emissionsabgabe befassen, die darin bestehen soll, daß die Emissionsabgabe im Falle mehrerer Emittenten mit unterschiedlichen marginalen Emissionsreduktions funktionen automatisch zu einer allokatlonsoptimalen Aufteilung der erforderlichen Emissionsreduktionen unter den Emittenten führt, wobel bekanntlich bei der Auflagenlösung groBe Informationsbeschaffungsprobleme entstanden. Zur Demonstration bedienen wir uns wieder des von uns auf vier Quadranten erwelterten Schemas von Bea, wobei wieder zwei Emittenten I und II unterstellt seien, die zunächst identische Immissionsbelastungsfunktionen aber unterschiedliche marginale Kostenfunktionen der Emissionsreduktion haben sollen (vgl. Abb. 28). Wir verwenden in Abb. 28 die glelchen Grenzkosten- und Immissionsbelastungsfunktionen wie in $A b b$. 18, so daB sich das gleiche allokationsoptimale Ergebnis ergibt: Emittent I muß seine Emissionen um 25 Prozentpunkte und Emittent II seine Emissionen um 75 Pro- 


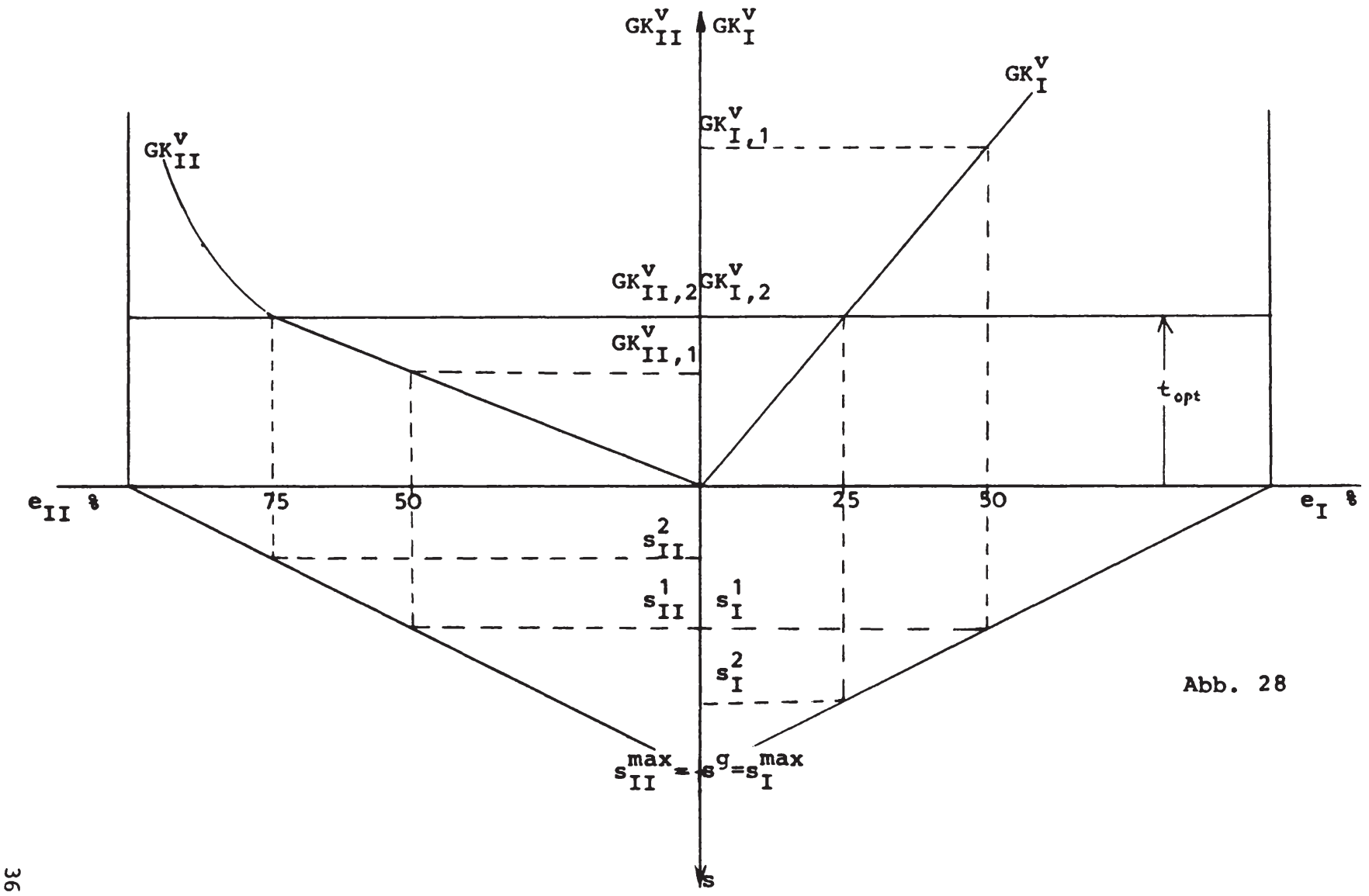

a 
zentpunkte reduzieren, un den Immissionsstandard in Höhe von $s_{g}$ einhalten zu können. Im Fall einer Auflagenlösung müßten Informationen über den Verlauf der (identischen) Immissionsbelastungsfunktionen und der (unterschiedlichen) marginalen Kostenfunktionen der Emissionsreduktion vorliegen, um zur allokationsoptimalen Aufteilung der Emissionsreduktionsquoten auf beide Emittenten zu gelangen. Die allokationsoptimale Bedingung, daß die marginalen Grenzkosten der Emissionsreduktion für alle Emittenten gleich sein müssen, erforderte im Falle der Auflagenlösung also die Kenntnis der jeweiligen Grenzkostenfunktionen der Emissionsreduktion.

Es ist nicht mehr als eine Tautologie, wenn nun behauptet wird, bei einer Abgabenlösung sei die Kenntnis dieser Grenzkostenfunktionen nicht erforderlich, da das Allokationsoptimum durch eine Emissionsabgabe mit einheitlichem Satz automatisch erreicht würde, denn alle Emittenten würden ihre Individuell verschiedenen marginalen kosten der Emissionsreduktion an den marqinalen Satz der Abgabe anpassen und somit allokationsoptimale Emissionsreduktionen vornehmen. Dies würde nichts anderes bedeuten, als daB das Allokationsoptimum bei jedem Abgabesatz realislert wäre. Das 1st jedoch nicht der Fall; im Gegenteil: Es gibt nur einen Abgabensatz bei dem sowohl die Bedingung $G_{I}^{V}=G_{I I}^{V}=t$ erfüllt ist als auch der Immissionsstandard gerade eingehalten wird, und das ist ein steuersatz in Höhe von topt. Um ihn auf Anhieb festlegen zu können, sind genau die gleichen Informationen erforderlich wie bei einer allokationsoptimalen Auflagenlösung: Es müssen sowohl die Grenzkostenfunktionen der Emissionsreduktion beider Emittenten als auch die Immissionsbelastungsfunktionen bekannt sein. Man könnte $1 \mathrm{~m}$ Falle der Abgabenlösung allerdings versuchen, den optimalen Abgabesatz wieder durch einen iterativen Proze $\beta$ $z u$ ermitteln. Die Uberlegenheit der Abgabenlösung gegenüber der Auflagenlösung hinsichtlich der (theoretischen) statischen Allokationseffizienz würde dann davon abhängen, ob der iterative SuchprozeB nach dem optimalen Abgabesatz höhere Kosten erfordert als die Suche nach den Grenzkostenfunktionen der Emissionsreduktion und nach den Immissionsbelastungsfunktionen. 
Hinsichtlich der Administration dürfte sich allerdings die Abqabenlösung als überlegen erweisen 181), da sie - sofern ihre Institutionalisierung in optimaler Höhe gelungen ist - ein idealer "24-hour automatic enforcer" 182) ist, während die Auflagenlösung ständiger Kontrollen bedarf.

Im Falle einheitlicher Immissionsbelastungsfunktionen und unterschiedlicher Grenzkostenfunktionen der Emissionsreduktion sind die Informationserfordernisse für eine optimale Abgabenlösung in etwa die gleichen wie für eine Auflagenlösung; hinsichtlich der administrativen Kosten hat aber die Abgabenlösung eindeutige Vorteile gegenüber der Auflagenlösung.

Nun wollen wir noch den Fall untersuchen, daB die Emittenten unterschiedliche Immissionsbelastungs funktionen, aber einheitliche Grenzkostenfunktionen der Emissionsreduktion haben (vgl. Abb. 29). Die Funktionen der Abb. 29 seien identisch mit denen der Abb. 19.

Ein einheitlicher Steuersatz in Höhe von $t_{0}$ scheint nun allokationsoptimal zu sein, denn er führt wie die einheitliche Auflage in Höhe von 508 in $\mathrm{Abb} .19$ sowohl zur Erfüllung der Bedinguna $\mathrm{GK}_{I}^{\mathrm{V}}=\mathrm{GK}_{\mathrm{II}}^{\mathrm{V}}=\mathrm{t}_{\mathrm{O}}$ als auch zur Realisierung des Immissionsstandards, denn $s^{g^{\circ}}=s_{I}^{1}+s_{I I}^{1}$. Im Falle unterschiedlicher Immissionskoeffizienten ist aber ein einheitlicher Abgabensatz nicht mehr effizient, denn die gesamtwirtschaftlichen Kosten können gesenkt werden, wenn der Emittent II mit dem hohen Immissionskoeffizienten seine Emissionsreduktion auf 658 ausdehnt, denn dann kann Emittent I mit dem geringeren Immissionskoeffizienten seine Emissionsreduktion auf 158 senken, ohne daß der Immissionsstan-

181) Vgl. Freeman III, M.A./Haveman, R.H., Residuals Charges for Pollution Control: A Policy Evaluation, a.a.o., S. 328; White, L.J., Effluent Charges as a Faster Means of Achieving Pollution Abatement, a.a.o., s. $111 \mathrm{ff}$.; Solow, R.M., The Economist's Approach to Pollution and its Control, a.a.o., S. 500; Nowotny, E., Wirtschaftspolitik und Umweltschutz, a.a.o., s. 211.

182) U.S. Council on Environmental Quality, The Second Annual Report, a.a.o., S. 137. 


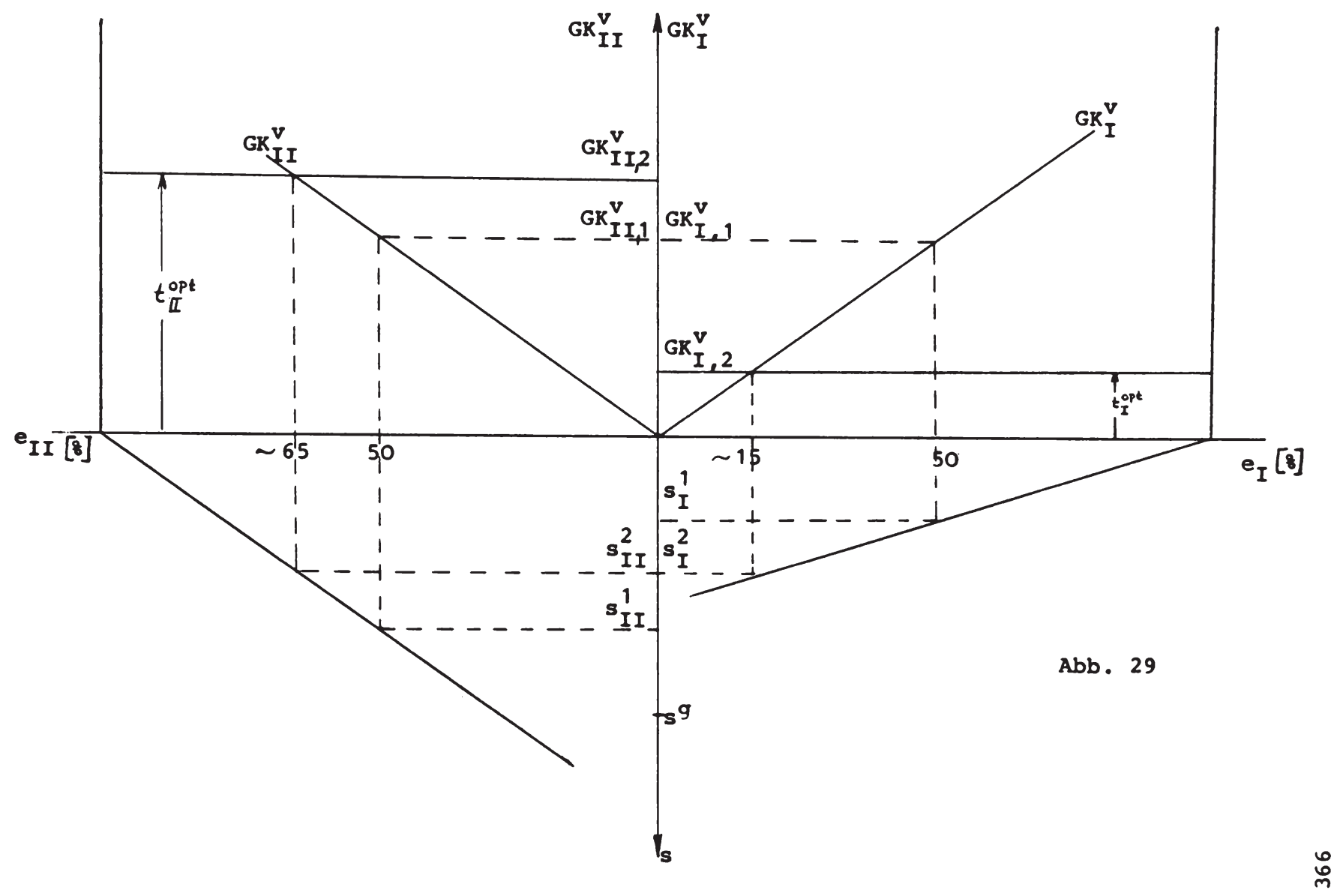


dard überschritten wird. Elne allokationsoptimale Abqabenlösung verlangt jetzt offenbar eine Differenzierung der Abgabensätze 183) Der optimale Abgabensatz für Emittent II muß $t_{\text {II }}^{\text {opt betragen und }}$ größer sein als der von Emittent $I$ in Höhe von $t_{I}^{\text {opt }}$. Die optimale Differenzierung der Abgabensätze erhält man, wenn man von gleich hohen "Beiträgen" zur Immissionsbelastung $s_{I}=s_{I I}$ ausgeht, die zusammen gerade zur Einhaltung von $s^{g}$ führen. Daraus lassen sich die optimalen Emissionsstandards $e_{I}$ und $e_{I I}$ ableiten. Setzt man sie in die $G K_{I}^{V}$ bzw. $G K_{I I}^{V}$ ein, so erhält man $t_{I}^{\text {Opt }}$ bzw. $t_{I I}^{\text {Opt }}$. Im Falle, daß unterschiedliche Abgabesätze aus Gründen der Allokationseffizienz notwendig sind, kehrt'sich der administrative Vortell der Abgabenlösung in einen Nachteil um, denn es 1st wohl einfacher, Emissionsstandards emittentenindividuell zu differenzieren als Abgabesätze 184). Im Fall unterschiedlicher Immissionsbelastungsfunktionen und unterschledlicher Grenzkostenfunktionen

183) Vgl. Hansmeyer, K. $-\mathrm{H} .$, Die Abwasserabgabe als Versuch einer Anwendung des Verursacherprinzips, a.a.0., s. $76 \mathrm{f}$.; Schultze, C.L.,u.a., Setting National Priorities, The 1973 Budget, a.a.0., S. 388 . Eine regionale Differenzierung der Abgabesätze kann auch schon deshalb erforderlich sein, weil in verschiedenen Regionen Z.B. unterschiedliche Gewässergüteziele angestrebt werden sollen (vgl. Rincke, G., Anwendbarkeit des Verursacherprinzips am Beispiel der Wassergütewirtschaft, a.a.o., S. 139). Vgl. auch die Kontroverse zwischen Lerner und Stein über die regionale Differenzierung der Abqabesätze; dabei scheint es allerdings mehr um ein MiBverständnis zwischen belden zu gehen: Stein plädiert für einen auf nationaler Ebene einheitlichen Abgabesatz pro $s \mathrm{c} h$ a $d$ e $n$ seinheit, während Lerner den Abgabesatz pro Schadstof fmengeneinheit regionalisieren will (vgl. Lerner, A.P., The 1971 Report of the President's Council of Economic Advisers: Priorities and Efficlency, in: American Economic Review, vol. 61, 1971 , S. $527 \mathrm{ff.}$; Stein, J.L., The 1971 Report of the President's Council of Economic Advisers: Microeconomic Aspects of Public Policy, a.a.0., S. $531 \mathrm{ff.;}$ Kneese, A.V., Pollution and Pricing, in: American Economic Review, vol. 62, 1972, S. 958; Peltzman, S./Tideman, T.N., Local Versus National Pollution Control: Note, ebenda, S. $959 \mathrm{ff} . ;$ Lerner, A.P., Priorities and Pollution: Comment, in: American Economic Review, vol. 64, 1974, S. 715 ff.; Stein, J.L., Priorities and Pollution: Reply, ebenda, S. $718 \mathrm{ff.}$.)

184) Vgl. Cansier, D., రkonomische Grundprobleme der Umweltpolitik, a.a.0., s. 100. 
der Emissionsreduktion können sich die optimalen Abgabesätze angleichen oder welter auseinanderstreben. In diesem komplexen der Realität entsprechenden Fall mit vielen Emittenten und Schadstoffarten ist aus Gründen der statischen Allokationseffizienz wohl weder die Abgabenlösung der Auflagenlösung noch umgekehrt die Auflagenlösung der Abgabenlösung überlegen, denn die Informationserfordernisse sind nahezu die gleichen und die administrativen Vorteile der Abgabenlösung bei der Realisierung des Allokationsoptimums im Falle unterschiedlicher Grenzkostenfunktionen der Emissionsreduktion gehen zumindest verloren, wenn auch noch unterschiedliche Immissionskoeffizienten unterstellt werden.

Was allerdings in jedem Fall bestehen bleibt, ist die uberlegene dynamische Allokationseffizienz der Abgabenlösung, weshalb wir sie im Berelch der Gewässergütewirtschaft und der Luftreinhaltung der Auflagenlösung als überlegen bezeichnen möchten 185). Aus Gründen der Praktikabilität kann der Abgabesatz zumindest nicht für jeden Emittenten unterschiedlich gestaltet werden. Es empfiehlt sich jedoch eine gewisse Regionalisierung der Abgabesätze.

Die aus Gründen der Allokationseffizienz notwendige Differenzierung der Abgabesätze im Falle unterschiedlicher Immissionskoeffizienten wird vor allem von Tietenberg betont 186) Er kritisiert den Baumol-Oates-Approach, weil dieser keine unterschiedlichen Immissionskoeffizienten, die allein realitätsnah sind, berücksichtigt. Tietenberg kommt aber nicht auf elne Auflagenlösung als beste Maßnahme, sondern er modifiziert den Baumol-Oates-Ansatz, um die Abgabenlösung zu retten, zu einem

185) Zur Durchsetzung von Lärmschutzmaßnahmen und dem Schutz der Lebensmittel vor Schadstoffen sind Emissions- und Produktstandards sicher besser qeeignet(siehe unten s. $405 \mathrm{ff.})$

186) Vgl. Tietenberg, T.H., On Taxation and the Control of Externalities: Comment, in: American Economic Review, vol. 64,1974, S. 462 ff. 
"Zonal Price and Standards"-Ansatz 187). Ausgangspunkt seiner Analyse ist die Tatsache, daB die Emittenten eine unterschiedliche Immissionsbelastungsfunktion haben; Tietenberg bezeichnet sie als "production function which summarizes the relationship between average pollutant concentrations and average emission rates", und folglich bezeichnet er den Immissionskoeffizienten als "the marginal physical product of a unit of emissions". offenbar hängt nun der Immissionskoeffizient jedes Emittenten von dessen standort und den zwischen Standort und Immissionsqebiet vorhandenen sehr unterschiedlichen natürlichen Faktoren (Windrichtung, Strömung, Topographie, Temperatur) ab. Die Folge werden zwangsläufig unterschiedliche Immissionskoeffizienten sein. "Since this is true, unless more than one emitter occupies the same location a minimum cost and standards pollcy would have to impose a different tax rate on all emitters. This would be administratively difficult at best and politically infeasible at worst" 188). Eine administrativ mögliche und politisch eventuell durchsetzbare "Rettung" der Abgabenlösung könnte darin bestehen, ein Ballungsgebiet in bestimmte Zonen einzuteilen. Die Emittenten in jeder Zone haben nun ähnliche Immissionskoeffizienten.

Die Zonen können nach Immissionskoeffizienten abgestuft werden. Für die Emittenten jeder Zone werden die Abgabesätze gemăß den in der jeweiligen Zone herrschenden durchschnittlichen Immissionskoeffizienten festgelegt. Die Emittenten in der zone mit dem höchsten durchschnittlichen Immissionskoeffizienten müssen den höchsten Abgabesatz je Emissionseinheit zahlen, diejenigen mit dem niedrigsten Immissionskoeffizienten den niedrigsten Abgabesatz. Bei $n$ zonen gilt es also $n$ optimale Abgabesätze $z u$ bestimmen. Je größer $n$ wird, um so mehr wird dem Einzelfall

187) Vgl. Tietenberg, T.H., On Taxation and the Control of Externalities: Comment, in: American Economic Review, vol. 64 , 1974, S. $464 \mathrm{f}$; d diesen Ansatz wollen wir im folgenden schildern.

188) Ebenda. 
Rechnung getragen und um so näher wird die Lösung dem Allokationsoptimum sein. Je größer $n$ wird, um so größer werden jedoch auch die Kosten der Administration. "Conceptually the optimum number of zones would be that number of which the marginal saving from achieving a better resource allocation by increasing the spatial detail is equal to the marginal cost of administering that number of zones" 189?

Die Hauptschwierigkeit des Tietenberg-Ansatzes liegt wohl in der funktionsgerechten Abgrenzung der Zonen, die für jeden Schadstoff, dessen Emissionen besteuert werden sollen, wohl anders aussehen müBte. Doch solche Schwierigkeiten würden sich in einem erhöhten administrativen Aufwand äußern und damit, gemaß der oben zitierten Optimumbedingung, zu einer geringeren Anzahl von zonen führen.

Bevor wir zu einer abschließenden Würdigung der Emissionsabaabe kommen, wollen wir noch auf den Vorwurf eingehen, eine Emissionsabgabe stelle elne "license to pollute" dar, und sodann soll noch kurz untersucht werden, was mit dem Aufkommen aus der Emissionsabgabe geschehen soll. Es wird manchmal der abwertend gemeinte Einwand erhoben, die Emissionsabgabe sei eine "license to pollute" 190) Diese abschätzige Klassifizierung rührt wohl daher, daB für die Restverschmutzung eine Abgabe erhoben wird, also z.B. die Nutzung der Gewässer als Abwasserkanal mit einer Abgabe belastet wird. Ist der Abgabesatz allokationsoptimal festgelegt, entspricht die Restverschmutzung dem Allokationsoptimum, und die Tatsache, daß der staat fur die Restverschmutzung einen Preis verlangt, darf thn unter der Voraussetzung einer optimalen Abgabesatzgestaltung nicht in den Verdacht bringen, aus fiskalischen Gründen etwa eine neue Elnnahmequelle anzapfen zu wollen. Dieser Verdacht taucht allerdings dann $z u$ Recht auf, wenn der Abqabesatz

189) Tletenberg, T.H., On Taxation and the Control of Externalities: Comment, in: American Economic Review, vol. 64,1974, S. 465 .

190) Vgl. Freeman III, M.A./Haveman, R.H., Residuals Charges for Pollution Control: A Policy Evaluation, a.a.0., S. 325 ; Schultze, C.L. U.a., setting National Priorities, The 1972 Budget, a.a.0., s. 242 . 
zu niedrig festgesetzt wird, so daß das Allokationsoptimum in Form des Immissionsstandards total verfehlt wird, der staat aber eine hohe Abgabe aus der Besteuerung der "Rest"verschmutzung erzielt. Das sel an $\mathrm{Abb} .30$ illustriert wo $\mathrm{GK}^{\mathrm{V}}$ die gesamtwirtschaftliche (gewichtete) Grenzkostenfunktion der Emissionsreduktion sei.

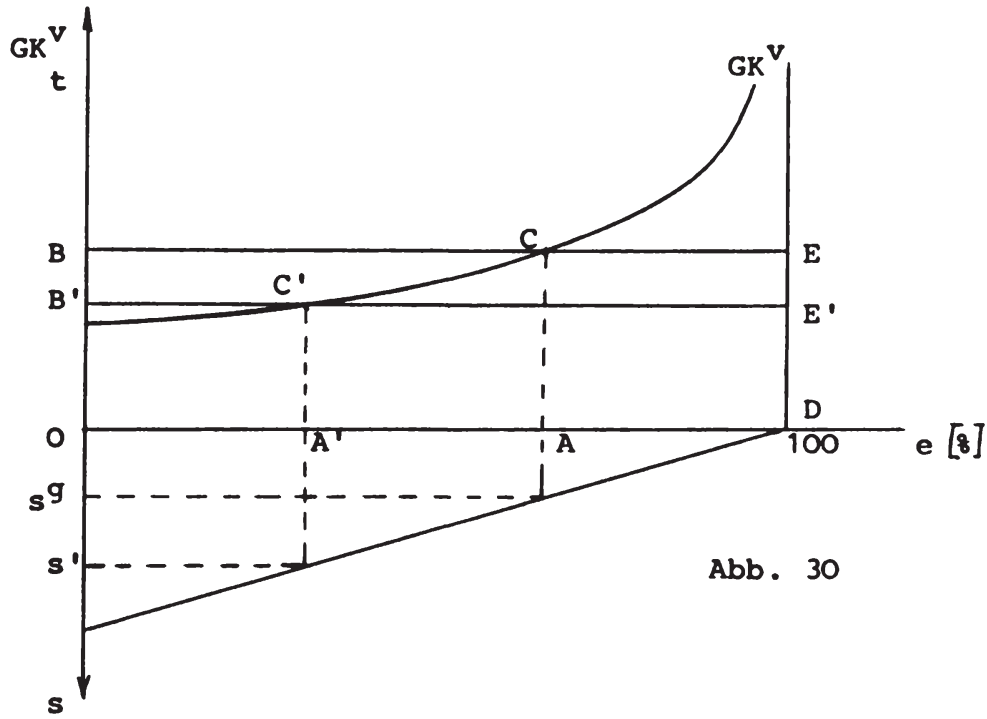

Der allokationsoptimale Abgabesatz wäre offenbar $O B=A C$. Wird eine Abgabe mit diesem Satz erhoben, beträgt die Restverschmutzung $A D$ und die Emissionsabgabe entspricht der Fläche ADEC. Setzt der Staat den Satz nun aber in der geringeren Höhe $O B^{\prime}=A^{\prime} C^{\prime}$ fest, so steigt im neuen "Optimum" nicht nur die Immissionsbelastung beträchtlich über den Immissionsstandard auf $s^{\prime}$ infolce der großen "Rest"-Verschmutzung in Höhe von A'D, sondern die Einnahmen aus der Emissionsabgabe steigen beträchtlich auf $A^{\prime} D E^{\prime} C^{\prime}$. Die Staatsfinanzen werden auf Kosten der Umweltqualität aufqebessert 191)

191) Vgl. The Economics of National Priorities, Hearings Before the Subcommittee on Priorities and Economy in Government, Part I, U.S.G.P.O. , washington, D.C., 1971, S. 228;

Hansmeyer, K.-H., Die Abwasserabgabe als Versuch einer Anwendung des Verursacherprinzips, a.a.0., S. 83; Rincke, G., Die Abwasserabgabe als mögliches Optimierungsinstrument aus der Sicht der Wassergütewirtschaft, in: Issind, O. (Hrsa.), ökonomische Probleme der Umweltschutzpolitik, a.a.0., s. 112. 
In einem solchen Fall verdient eine Emissionsabqabe allerdings das abschätzlg gemeinte prädikat "license to pollute".

Die in der Bundesrepublik Deutschland ab $1981 \mathrm{zu}$ erhebende Abwasserabgabe stellt unseres Erachtens tatsächlich nlchts anderes dar als eine solche "license to pollute"; wir glauben allerdings nicht, daß der im ersten Jahr geltende Abgabesatz von $12 \mathrm{DM} /$ Schadstoffeinheit und von $6 \mathrm{DM} / \mathrm{Schadstoffeinheit} \mathrm{für} \mathrm{die} \mathrm{Rest-}$ verschmutzung (sofern ein Reinigungsverfahren 1.S. des $\S 7$ a WHG angewendet wird) 192) deshalb weit unter dem als optimal erachteten Niveau festgelegt wurde, well der staat sich dadurch eine ergiebige Einnahmequelle verschaffen wollte (die staatlichen Folqekosten dieses niedrigen Satzes werden enorm sein!), sondern deshalb, well sich der staat gegenüber den (potentiellen) Emittenten (Industrie und Kommunen) nicht durchsetzen konnte. Ist darin bereits der erste schritt zu einem "Valet dem Verursacherprinzip" 193) zu sehen? Das könnte sehr wohl der Fall sein, denn wenn sich z.B. der Gewässerzustand weiter verschlechtert - und die der Bundesregierung und dem Parlament "aufgezwungene" Lösung der Abwasserabgabe kann dies mit sicherheit nicht verhindern, zumal sie erst $a b 1981$ in Kraft tritt, wenn der Abgabesatz wegen der bis dahin eingetretenen inflationären Entwicklung sowieso keinen Anrelz zu Abwasserbeseitigungsmaßnahmen mehr schaffen dürfte -, dann wird dem staat nichts anderes übrig bleiben, als durch wesentlich ineffizientere MaBnahmen wie subventionen und öffentliche Investitionen, also Instrumenten des Gemeinlastprinzips, für elne einigermaßen befriedigende Gewässergüte zu sorgen. Da die Abwasserabgabe aber besteht, wird man sagen, sie habe versagt 194) Auf diese Welse ist das Verursacherprinzip diskriminiert, und die Interessenvertreter können bel zukünftiq

192) Zur Verwässerung des Abwasserabgabe-Gesetzes vergleiche die Gegenubberstellung der Entwürfe und der endgulltigen Fassung, in: Hansmeyer, K.-H., Die Abwasserabgabe als Versuch einer Anwendung des Verursacherprinzips, a.a.0., s. $92 \mathrm{ff}$.

193) Ebenda, S. 90.

$194)$ Vgl. ebenda, S. 87. 
notwendigen umweltrelevanten Investitionen wieder mit Subventionen oder schlimmstenfalls mit Auflagen rechnen; auch mit letzteren haben sie jedoch ganz gut zu leben gelernt, wie das Vollzugsdefizit zeigt. Eine Abwasser-Abgabenlösung mit einem optimalen Abgabesatz, die das Vollzugsdefizit im Gewässerschutz automatisch beseitigt hätte, haben die industriellen und kommunalen Interessenvertreter prompt und erfolgreich torpediert. Nun wollen wir noch kurz untersuchen, wozu das Aufkommen aus der Abwasserabgabe verwendet werden sollte. Aus rechtlichen Gründen ist eine Zweckbindung des Abwasserabgabe-Aufkommens notwendig 195) Es bieten sich grundsätzlich folgende Möglichkeiten an:

( $\alpha$ ) Der Staat könnte mit dem Aufkommen aus der Abwasserabgabe den Schaden der von der Restverschmutzung Betroffenen kompensieren. Dies dürfte wegen des Bewertungsproblems zu schwieria oder zumindest administrativ zu teuer sein 196)

( $\beta$ ) Es könnten mit dem Aufkommen Maßnahmen gefördert oder ganz finanziert werden, welche die Effizienz des Gewässerschutzes erhöhen können: Hierbei denken wir vor allem an die Förderung der entsorgungs- und recycling-technologischen Forschung und an die Ausbildung qualifizierten Fachpersonals (z.B. für Kläranlagen) 197) Eine solche Verwendung halten wir für sehr sinnvoll: sie kann erhebliche positive externe Effekte haben. (r) Aus dem Aufkommen aus der Abwasserabgabe könnten die mit

195) Vgl. Rincke, G., Anwendbarkelt des Verursacherprinzips am Beispiel der Wassergütewirtschaft, a.a.O., S. 152. Das scheint die Möglichkeit auszuschließen, daß der staat die Restverschmutzung aus fiskalischen Gründen möglichst hoch wünscht und deshalb einen niedrigen Abgabesatz festleat. Das ist jedoch nicht der Fall, denn der staat könnte seinen bisherigen Wasserwirtschaftsetat allein aus der Abwasserabgabe bestreiten und hätte die dort vorgesehenen Mittel dann frei zur Verfügung.

196) Vgl. ebenda; Hansmeyer, K.-H., Die Abwasserabgabe als Versuch einer Anwendung des Verursacherprinzips, a.a.0., s. 77.

197) Vgl. Rincke, G., Anwendbarkelt des Verursacherprinzips am Beispiel der Wassergütewirtschaft, a.a.O., s. 153. 
der Abgabe verbundenen Verwaltungskosten gedeckt werden 198) Auch diese Verwendung scheint uns gerechtfertigt.

( $\delta$ ) Es könnten mit dem Aufkommen diejenigen Gewässerschutzmaßnahmen intensiviert werden, welche am effizientesten sind, und so der Reinheitsgrad der Gewässer weiter erhöht werden. Es dürften aber nicht, wie dies bisher geschieht, die kleinen unrentablen Kläranlagen von kleinen Gemeinden bezuschußt werden ${ }^{199}$ ? Man hat berechnet, daß sich bei einer gezielten Aufstockung der Anrelze in Form von Subventionen in Höhe von 758 des Abgabeaufkommens bei einer Abgabenhöhe von 25 DM/SE elne Gewässerqüte realisieren ließe, die elner Abgabensatzhöhe von $60 \mathrm{DM} / \mathrm{SE}$ entspräche! 200) Eine solche Verwendung erscheint somit besonders attraktiv.

Abschließend wollen wir die Emissionsabgabe noch einmal zusammenfassend würdigen, Indem wir sie mit den Emissionsstandards vergleichen.

Hinsichtlich der statischen Allokationseffizienz kann man wohl allgemein sagen, daß die Abgabenlösung der Standard-Lösung aus Informations - und Administrationsgründen immer dann uberleqen ist, wenn ein einheitlicher Abgabesatz grundsätzlich zum Allokationsoptimum fuhrt. Das ist immer dann der Fall, Wenn die Immissionskoeffizienten der Emittenten identisch sind (Fall 1 und 2 in den folgenden Tabellen), da in diesem Fall ein elnheitlicher Abgabesatz, dessen optimale Höhe allerdings eventuell in einem iterativen Prozeß $z u$ ermitteln ist, automatisch sowohl die unterschiedlichen $G_{1}^{V}$ angleicht als auch $s^{g}$ realisiert. Die Auf-

198) Diese Verwendung sieht das Abwasserabgaben-Gesetz in $\S 15$ ausdrücklich vor. Vgl. auch Boehm, V., Die Abwasserabgabe ist ein Anfang, in: Umwelt, 1976, 4, S. 260.

199) Vgl. Hansmeyer, K.-H., Die Abwasserabgabe als Versuch einer Anwendung des Verursacherprinzips, a.a.0., S. 78 und S. 89.

200) Vgl. Rincke, G., Anwendbarkeit des Verursacherprinzips am Be1spiel der Wassergütewirtschaft, a.a.0., S. 138; derselbe, Die Abwasserabgabe als mögliches Optimierungsinstrument aus der Sicht der Wassergütewirtschaft, a.a.0., S. 109 . 
lagenlösung wäre in den Fällen der Abgabenlösung eindeutig überlegen, in denen $n$ u $r$ einheitliche Emissionsstandards für alle Emlttenten zum Allokationsoptimum führen würden. Solche Fälle gibt es aber nicht. Nur im Fall 1 führen auch einheitliche Emissionsstandards zum Allokationsoptimum, doch ist in diesem Fall die Emissionsabgabe vorzuziehen, da sie die Verursacher quasi automatisch zum allokationsoptimalen Verhalten veranlaBt.

\begin{tabular}{|l|l|l|}
\hline$\alpha_{i}$ & identisch & verschieden \\
\hline identisch & Fall 1 & Fall 2 \\
\hline verschieden & Fall 3 & Fall 4 \\
\hline
\end{tabular}

\begin{tabular}{|c|c|c|}
\hline $\mathrm{Fall}$ & $\begin{array}{l}\text { allokations- } \\
\text { optimale } t_{i}\end{array}$ & $\begin{array}{l}\text { allokations- } \\
\text { optimale } e_{i}\end{array}$ \\
\hline 1 & einheitlich & einheitlich \\
\hline 2 & einheitlich & unterschiedlich \\
\hline 3 & unterschiedlich & unterschiedlich \\
\hline 4 & unterschiedlich & unterschiedlich \\
\hline
\end{tabular}

In den Fällen 1 und 2 1st also die Emissionsabgabe der Emissionsstandard-Setzung eindeutig überlegen, aber diese Fälle sind in der Realität wohl kaum anzutreffen. Die Existenz identischer Immissionskoeffizienten ist sehr unwahrscheinlich, so daß die bisher nachgewiesene Uberlegenheit der Abgabenlösung rein akademischer Natur ist. 
Realistischer scheint Fall $3 \mathrm{zu}$ sein, denn es gibt für die Emissionsreduktion bestimmter Schadstoffe mehr oder weniger ähnliche (technische) Aggregate, die sich in ihren Grenzkostenverläufen sehr ähnlich sein können. Da eine emittentenindividuelle Abgabesatzdifferenzierung wohl nicht in Frage kommt, scheint in diesem Fall eine differenzierte Auflagenlösung die einzige Möglichkeit zu sein. Eine differenzierte allokationsoptimale standardsetzung ist jedoch mit sehr hohen Kosten für die Informationsbeschaffung und Administration verbunden, so daß der Netto-Nutzen der (anqestrebten) allokationsoptimalen Emissionsstandard-Setzung wahrscheinlich gerlnger ist, das Allokationsoptimum also letztlich mehr verfehlt, als eine nicht-differenzierende Abgabe-Lösung, die von vornherein auf das ehrgeizige Allokationsziel verzichtet. Die gleichen Uberlegungen gelten für Fall 4: Durch Differenzierung der Emissionsstandards gemäß unterschiedlicher GK $_{i}^{v}$ und $\alpha_{i}$ wäre nur unter so enormem Aufwand für Informationsbeschaffung und Administration das Allokationsziel zu erreichen, daB der Nutzen einer einheitlichen Abgaberegelung weitaus größer wäre. Es ist allerdings nicht fair, in den Fällen 3 und 4 eine a priori suboptimale, da einheitliche, Abgabelösung mit einer das optimum anstrebenden aber immens teuren Auflagen-Lösung $z u$ vergleichen. Worum es in der praktischen Wirtschaftspolitik geht, ist letzt$11 \mathrm{ch}$ die Frage, ob in der dem Fall 4 entsprechenden realen Welt ein administrativ vertretbarer Abgaben-Approach einem administrativ vertretbaren Auflagen-Approach überlegen ist oder nicht. Administrativ vertretbar ist nur eine jeweils einheitliche oder bestenfalls regionalisierte Lösung. Die eigentlich entscheidende Frage ist somit folgende: Ist im Fall 4 eine einheitliche (regional1sierte) Abgabenlösung einer einheltlichen (regionalisierten) Auflagenlösung überlegen? Der Automatismus, mit dem die einheltliche Emissionsabgabe für eine (wenn auch nicht allokationsoptimale) Einhaltung des Immissionsstandards sorgt, spricht unseres Erachtens für deren Uberlegenheit in bezug auf die statische Allokationseffizienz. Von überragender Bedeutung ist aber, wie mehrfach hervorgehoben, die Uberlegenheit der Emissionsabgabe hin- 
sichtlich ihrer "incentives for innovation" 201!

Hinzu kommt der weltere Vortell, das die Emissionsabgabe eine vollständigere Realisierung des Verursacherprinzips ermöglicht als die Auflagenlösung; denn durch das Aufkommen aus der Emissionsabgabe können auch die Kosten der Informationsbeschaffung und Administration zumindest tellweise gedeckt werden, während der Staat bel der Auflagenlösung diese Ausgaben aus dem allgemeinen Budget bestreiten muB. Die Erhebungskosten können ebenfalls den Emittenten auferlegt werden, indem man zur Selbstveranlagung übergeht 202), wodurch allerdings strengere Kontrollen notwendig sind; doch diese sind auch im Fall der Auflagenlösung erforderlich.

Bevor wir zu zwei Gründen kommen, die gegen eine Emissionsabgaben- und für elne Emissionsstandard-Lösung sprechen, wollen wir noch eine Graphik wledergeben (vgl. Abb. 31), welche die

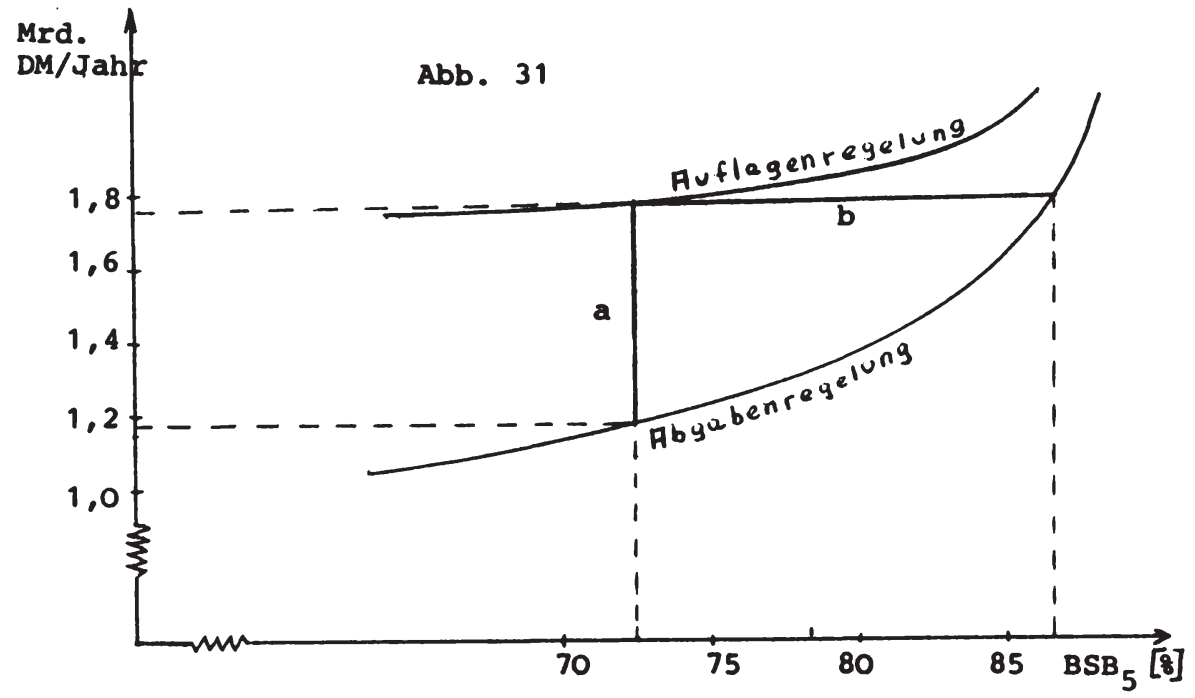

201) Orr, L., Incentive for Innovation as the Basis for Effluent Charge Strategy, a.a.0., S. 441.

202) Vgl. Freeman III, M.A./Haveman, R.H., Residuals Charges for Pollution Control: A Policy Evaluation, a.a.0., S. 328; Ackermann, K./Geschka, H./Karsten, D. , Gutachten zur Gesamtbelastung der Volkswirtschaft durch das Umweltprogramm der Bundesregierung, a.a.0., S. 602; Nowotny, E., Wirtschaftspolitik und Umweltschutz, a.a.0., s. 206. 
Überlegenheit der Abgabenlösung bei der Abwasserabgabe gegenüber der Auflagenlösung hinsichtlich der Kosten im öffentlichen Bereich eindrucksvoll darlegt 203).

Die Graphik zeigt, daß ein Abwasserreinigungsgrad von rund 72,58 für den Staat bei der Auflagenlösung um $a \approx 0,6$ Mrd. DM teurer wäre bzw. daß man durch 0,6 Mrd. öffentliche Mehrausgaben via Abgabenregelung den Reinigungsgrad auf rund $86 z$ erhöhen kann.

Nun kommen wir auf zwei Argumente für eine Auflagen- und gegen eine Abgabenregelung. Nicht rational agierende Emittenten werden durch eine Abgabe unter Umständen nicht zu einer - der eigentlichen Anreizwirkung entsprechenden - Emissionsreduktion veranlaßt, während sie beim Emissionsstandard zu dessen Einhaltung und damit zur Emissionsreduktion gezwungen werden können 204) Die Auflagenlösung weist auch im Falle der Inflation einen Vorteil auf. Noch so hohe Preissteigerungen zwingen die Emittenten, die Emissionsstandards einzuhalten. Im Fall der Abgabenregelung geht aber bei konstantem Abgabesatz mit steigender Inflation die Anrelzwirkung zu Emissionsreduktionsmaßnahmen immer mehr verloren 205). Das kann an Abb. 32 veranschaulicht werden.

Unser, wie die Jahreszahlen andeuten, polemisches Beispiel zeigt, wie die Anreizwirkung einer Abgabe durch Inflation sinken kann. Im Jahre 1978 wäre noch ein Anreiz vorhanden, der zu einer Emissionsreduktion in Höhe von $e_{3}$ führen würde. Durch Inflation steigen die $\mathrm{GK}^{\mathrm{V}}$ bis 1981 aber so weit an, daß die Anreizwirkuna über $e_{2}$ und $e_{1}$ auf einen Emissionsreduktionsgrad von 0 \& sinkt. Dem könnte man dadurch vorbeugen, daß man den Abgabesatz der inflationären Entwicklung so anpaßt, daß der ursprünglich be-

203) Vgl. Rincke, G., Anwendbarkeit des Verursacherprinzips am Beispiel der Wassergütewirtschaft, a.a.0., S. 136.

204) Vgl. Cansier, D., Okonomische Grundprobleme der Umweltpolitik, a.a.0., S. 95.

205) Vgl. ebenda, S. $97 \mathrm{f}$. 


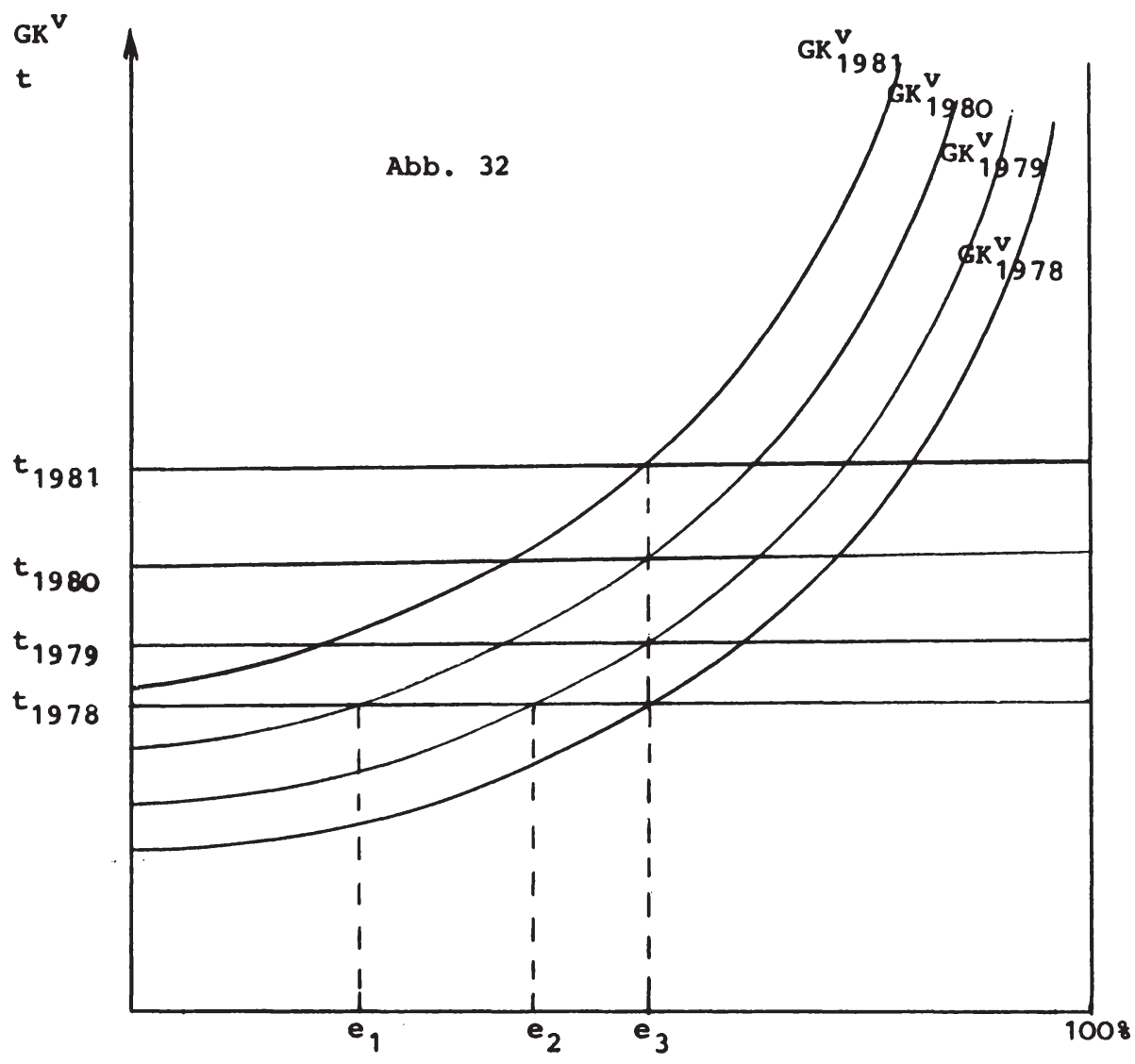

absichtigte Emissionsstandard in Höhe von $e_{3}$ bei rationalem Verhalten welterhin realisiert wird. Diese Anpassung vollzieht sich bei der Auflagenlösung allerdings automatisch.

Wir haben nun bis auf ein Instrument, nämlich die Emissionszertifikate, alle Instrumente der "box" 1 dargestellt und hinsichtlich ihrer informellen und administrativen Erfordernisse gewürdigt. Die bisher besprochenen Instrumente haben eines gemeinsam: Es liegen Erfahrungen über ihre Stärken und Schwächen vor, denn sie werden bereits alle mehr oder weniger lang in der 
praktischen Umweltpolitik angewendet. Ein neuartiges und damit von manchen mit großer Skepsis, von anderen mit nahezu euphorischen VorschuBlorbeeren bedachtes Instrument sind die Emissionszertifikate, mit deren Darstellung und Würdigung wir uns nun befassen wollen.

\section{(4) Emissionszertifikate}

Der Vorschlag, durch Emissionszertifikate zu einer Einhaltung der Immissionsstandards zu gelangen, geht auf Dales zurück 206) Die Idee ist folgende 207): Der staat (am besten eine regionale öffentliche Gebietskörperschaft) als Eigentümer der Umweltmedien bestimmt die Grenzen für deren Nutzung in Form von Immissionsstandards. Aufgrund der regionsspezifischen Besonderheiten wird annäherungsweise die Schadstoffmenge ermittelt, die pro Zeiteinheit insgesamt emittiert werden darf, ohne daB der Immissionsstandard überschritten wird.

Diese regionsspezifische Emissionsrate bestimmt zusammen mit der vorgesehenen stückelung der Emissionszertifikate die fixierte Anzahl der Emissionszertifikate. Die Emissionszertifikate stellen Berechtigungsscheine für die Nutzung der jeweiligen Umweltmedien in Form bestimmter Schadstoffemissionen dar.

Nehmen wir an, zur Einhaltung des Immissionsstandards für $\mathrm{SO}_{2}$ in einer bestimmten Region, dürften insgesamt maximal $100000 \mathrm{SE} / \mathrm{ZE}$ emittiert werden, und ein Zertifikat soll die Berechtigung erbringen $10 \mathrm{SE} / \mathrm{ZE}$ zu emittieren, so ergibt sich ein fixiertes staatliches Angebot an Emissionszertifikaten in Höhe von 10000. Diese Emissionszertifikate werden nun von den Emittenten bis $z u$ der Menge nachgefragt, in der die Grenzkosten der Emissionsre-

206) Vgl. Dales, J.H., Pollution, Property and Prices, a.a.0., s. $77 \mathrm{ff}$.

207) Vgl. Hyman, D.N., The Economics of Governmental Activity, a.a.o., S. $64 \mathrm{f} . ;$ Cansier, D., Okonomische Grundprobleme der Umweltpolitik, a.a.0., s. 56 f. und S. 101 ff.; U.S. Council of Economic Advisers, The 1971 Report, a.a.o., S. 118 . 
duktion für $10 \mathrm{SE} / \mathrm{ZE}$ größer sind als der Prels für das $10 \mathrm{SE} / \mathrm{ZE}$ gestattende Emissionszertifikat. In Abb. 33 ergibt sich bei der angenommenen Nachfragefunktion nach Emissionszertifikaten ein Preis von 300 .

Liegen die Grenzkosten der Emissionsreduktion für einen Emittenten unter 300, wird er die Emissionen reduzieren, liegen sie darüber, wird er Emissionserlaubnisscheine kaufen. Die Zertifikatlösung wirkt offenbar wie eine kombinierte Abgaben-Auflagen-Lösung. Der sich frei am Markt für Emissionszertifikate bildende Preis entspricht dem Abgabesatz der Emissionsabgabe und die

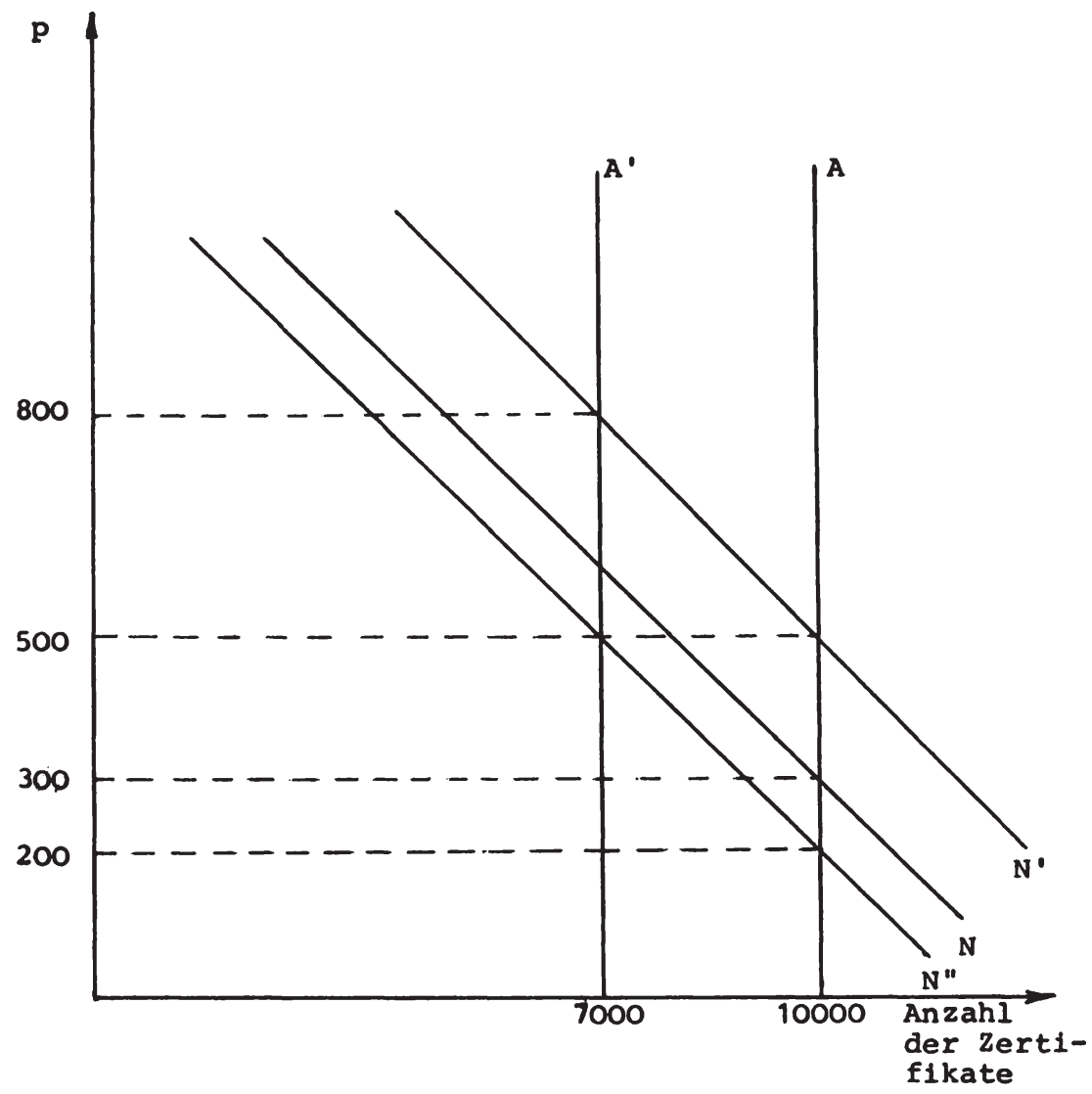

Abb. 33 
Fixierung der zulässigen Emissionsmengen entspricht der Setzung eines Emissionsstandards. Der eventuell notwendige trial and error-ProzeB zur Bestimmung des optimalen Abgabesatzes erübrigt sich. Der Markt sorgt für den optimalen preis 208) Die Zertifikat-Lösung vereinigt die Vortelle der Abgabenlösung mit den Vorteilen der Auflagenlösung. Der Preis für die Emissionszertifikate und damit die Anreize zur Emissionsreduktion werden steigen, wenn neue Emittenten angesiedelt werden, wenn die Produktion ausgedehnt wird oder wenn Inflation herrscht. Die Nachfragekurve verschiebt sich dann nach $\mathrm{N}^{\prime}$, und der Preis für die Zertifikate steigt an. Es müssen im Fall neu hinzukommender Emittenten keine neuen Emissionsstandards festgelegt werden, um die Immissionsstandards einzuhalten, da die gestiegenen Preise für die Emissionszertifikate die Emissionsreduktion aller Emittenten erhöhen werden 209?

Im Fall der Inflation muß nicht laufend der Abgabesatz angepaßt werden, da die Preise für die Nutzung des Umweltmediums wegen des rationierten Angebots an Nutzungsrechten automatisch steigen und somit ihre Anreizwirkung behalten, wenn die Nachfrage nach Emissionszertifikaten infolge gestiegener Emissionsredukt1onskosten zunimmt. Die Nachfrage nach Emissionszertifikaten kann auch zurückgehen (etwa bis N''), z.B. dann, wenn durch den technischen Fortschritt die Grenzkosten der Emissionsreduktion sinken; dann sinkt auch der Preis für die Zertifikate 210) Bisher wurde angenommen, daB Emissionszertifikate nur von potentiellen Emittenten erworben werden. Es wäre jedoch auch denkbar, $\mathrm{da} B$ an einer höheren Umweltqualität interessierte Bürger, Bürgerinitiativen oder Naturschutzverbände die frei an einer Börse

208) Head, J.G., Public Policies and Pollution Problems, a.a.0., S. 25; Frey, B.S., Umweltökonomie, a.a.O., S. 120.

209) Vgl. Head, J.G., Public Policies and Pollution Problems, a.a.0., S. 25 .

210) Vgl. Cansier, D., Ökonomische Grundprobleme der Umweltpol1tik, a.a.0., s. 103; Frey, B.S., Umweltökonomie, a.a.0., s. 122 . 
gehandelten Emissionszertifikate kaufen, und zwar um sie zu horten 211) Geschieht dies, steigt nicht nur die Nachfrage nach den Zertifikaten, z.B. wieder auf $\mathrm{N}^{\prime}$, sondern es sinkt, wegen der Hortung der Zertifikate bei den an mehr Umweltschutz Interessierten, auch das für Emittenten verfügbare Angebot. Kaufen z.B. Bürgerinitiativen 3000 Emissionszertifikate, um sie zu horten, steigt der Preis bei dem auf $A^{\prime}$ gesunkenen verfügbaren Angebot auf 800, was einen großen Anreiz bei den Emittenten zur weiteren Emissionsreduktion hervorruft. Der Immissionsstandard wird unter Umständen erheblich unterschritten. Die Möglichkeit des Hortens der Emissionszertifikate können allerdings auch die Emittenten wahrnehmen, um via Preissteigerung der Zertifikate mögliche Grenzanbieter-Konkurrenten vom Markt zu verdrängen oder Neuansiedlungen von Konkurrenten $z u$ erschweren 212). Im Fall des Hortens durch Emittenten wird auch der Ausgleich der Grenzkosten gestört, also das Allokationsoptimum verfehlt, denn wenn z.B. große Unternehmen mehr Zertifikate kaufen als sie zwecks Emission benötigen, treiben sie die Preise für die Emissionszertifikate für die anderen Unternehmen in die Höhe, so daß diese thre Emissionsreduktionsmaßnahmen uber das allokationsoptimale Maß hinaus erhöhen: Es kommt zu unterschiedlich hohen Grenzkosten der Emissionsreduktion zwischen den einzelnen Emittenten 213)

Als Einwand gegen eine Zertifikat-Lösung wird auch die Schwierlgkeit der funktionsgerechten Regionsabgrenzung vorgebracht 214 ) dieser Einwand gilt jedoch für alle regionalen Lösungen. Den Einwand, die Emissionen von Kleinemittenten (private Haushalte und Kraftfahrzeuge) ließen sich wegen der Meß- und Kon-

211) Lerner, A.P., Priorities and Pollution: Comment, a.a.O., S. 715; Hyman, D.N., The Economics of Governmental Activity, a.a.O.. S. $64 \mathrm{f}$.

212) Vgl. Jürgensen, H./Jaeschke, K.-P., Allokationseffekte der Social Costs im Umweltschutz, a.a.0., S. $68 \mathrm{f}$.

213) Vgl. ebenda; Cansier, D., Okonomische Grundprobleme der Umweltpolitik, a.a.0., S. 102 .

$214)$ Vgl. Cansier, D. Okonomische Grundprobleme der Umweltpolitik, a.a.0., s. 101 und S. 107. 
trollprobleme durch eine Zertifikat-Lösung nicht erfassen halten wir für nicht stichhaltig. Bonus hat eine recht praktikabel erscheinende Zertif1kat-Lösung für Kraftfahrzeuge beschrieben 216)

Die Kontrollkosten sind wohl kaum höher als im Falle einer Emissionsabgabe oder einer Emissionsstandardsetzung. Die Zertifikat-Lösung verursacht von den bisher geschilderten Instrumenten die geringsten Informationskosten 217), geringe Administrationskosten 218) und die geringsten gesellschaftlichen Kosten ${ }^{219)}$ ? Wir halten die Zertifikat-Lösung für wassergütewirtschaftliche und Luftrelnhaltungsprobleme für die beste denkbare Lösung, sehen allerdings auch das größte Hindernis ihrer Realisierbarke1t: Die umweltpolitische Bewährung der Zertifikat-Lösung wird wegen ihrer Neuartigkeit bei den wenig flexiblen und zum verstärkten Denken in ökonomischen Kategorien kaum bere1ten Bürokraten des Umweltschutzes wohl nicht so schnell möglich sein. Selbst elndeutige Plädoyers für die Zertifikat-Lösung durch Oronomen 220) sind wohl so schnell nicht in der Lage, die vor allem Im juristischen "Auflagen-Denken" geschulte UmweltschutzAdministration 221) von der Effizienz und Marktkonformität dieser exemplarisch ökonomischen Lösung zu überzeựen.

215) Vgl. Head, J.G., Public Policies and Pollution Problems, a.a.o., s. 25 .

216) Vgl. Bonus, H., Uber Schattenpreise von Umweltressourcen, a.a.0., S. $349 \mathrm{ff}$.

217) Vgl. Krier, J.E./Montgomery, W.D., Resource Allocation, Information Cost and the Form of Government Intervention, a.a.0., S. 101, Anmerkung 44 .

218) Vgl. Frey, B.S., Umweltökonomie, a.a.O., S. 122.

$219)$ Vgl. Schatz, K. -W., Administrative und marktwirtschaftliche Maßnahmen zur Lösung des Umweltschutzproblems, a.a.0., S. 129 .

220) Vgl. Andreae, C.A., Umweltschutz und Marktwirtschaft, in: Wirtschaft und Wettbewerb 11/12, 1971, S. 755 ff.

$221)$ Vgl. White, L.J., Effluent Charges as a Faster Means of Achieving Pollution Abatement, a.a.O., S. 120 ff. 
Da wir die Verhandlungslösung als Instrument der "box" 2 berelts behandelt haben 222) wenden wir uns nun der "box" 3 und damit dem letzten Instrument des Verursacherprinzips, den Benutzungsgebühren für offentliche Einrichtungen $z u$.

dd) Benutzungsgebühren als Instrument des Verursacherprinzips Die Instrumente der "box" 3 werden dann benötigt, wenn es darum geht, vom staat aus Effizienzgründen durchgeführte Maßnahmen des Umweltschutzes durch die Verursacher finanzieren $z u$ lassen. Das entscheidende Problem ist die verursachungsgerechte Zurechnung der staatlichen Lelstungen auf die (potentielien) Emittenten. Da die in "box" 3 befindlichen Instrumente des Verursacherprinzips dem Äquivalenzprinzip entsprechen, bietet sich als typisches Instrument des Aquivalenzprinzips die Gebührenerhebung zur verursachungsgerechten Anlastung der staatlich vorfinanzierten Umweltschutzmaßnahmen an. Verursachungsgerechte Gebühren müssen neben der richtigen zurechnung eine weltere Forderung erfüllen: Sie müssen die vollen Kosten der staatlichen Umweltschutzmaßnahmen decken 223)

Als vom staat durchgeführte Umweltschutzmaßnahmen, die grundsätzlich durch die Erhebung von Gebühren gedeckt werden können, kommen die Abwasser- und Abfallbeseitlgung, die Versorgung mit Fernwärme und der offentliche Personennahverkehr in Frage. Die beiden ersten Maßnahmen bezeichnet man als Entsorgung; sie dienen der ordnungsgemäßen Reinlgung und Beseitigung von Abwässern und Abfällen und werden deshalb dem staat ubertragen, well sie dort wegen der hohen economies of scale großer Kläranlagen- und Abfallbeseitigungsanlagenkapazitäten am effizientesten vorgenommen werden können. Die Versorgung mit Fernwărme und öffentlichen Nahverkehrsleistungen bezweckt eine drastische Verminde-

222) Siehe oben S. 280 ff.

223) Die Kommunalabgabengesetze der meisten Bundesländer sehen inzwischen elne volle Kostendeckung für Kläranlagen durch Gebuhren vor. Fur noch nicht fertiggestellte Kläranlagen können sogar angemessene Vorausleistungen erhoben werden (vgl. Mensing, W., Investitionen zur Reinhaltung der Gewässer, in: Der Landkreis 8-9, 1970, S. 332). 
rung kleiner Emissionsquellen (Hausfeuerungsanlagen und Kraftfahrzeuge), die in Ihrer Gesamtheit aber beträchtlich zur Umweltbelastung beitragen. Wenn kein freiwilliger Anschluß der Emittenten an das Fernwärmenetz erfolgt, kann man unter Umständen einen Benutzungszwang einführen. Der Zwang, auch öffentliche Nahverkehrsmittel zu benutzen, wăre ein starker Eingriff in die persönliche Freiheit und sollte nur als letztes Mittel des Umweltschutzes in Frage kommen. Die Kostendeckunasgrade be 1 der Entsorgung sind, wie wir bereits wissen, in der Bundesrepublik Deutschland durchschnittlich nahezu 1008 224), so daß bei der Abwasser- und Abfallbeseitigung die Forderung nach vollständiger Kostenanlastung als (fast) erfüllt gelten kann. Uber den Kostendeckungsgrad bei der Fernwärmeversorung liegen uns kelne $\mathrm{Z}$ ahlen vor. Beim Personennahverkehr sind sie weit unter 1008 . Unsere weiteren Ausführungen beschränken sich auf die Möglichkeiten einer verursachungsgerechten Zurechnung der Entsorgungskosten, speziell auf eine Abwassergebür.

Eine solche Abwassergebühr ist formal streng von der oben geschilderten Abwasserabgabe zu unterscheiden, obwohl sie materiell das glelche bezweckt - und auch erreichen kann: nämlich eine verursachungsgerechte Belastung der Abwassereinleiter. Die Abwassergebühr stellt eine $\mathrm{Z}$ ahlung der Abwasserproduzenten (oder Wasserkonsumenten) an den kommunalen Träger (oder Zweckverband, wie etwa die Ruhrgenossenschaften) der Abwasserentsorgung für die Benutzung der Entsorgungsanlagen (Kanalisation, Kläranlage) dar.

Die Abwasserabgabe stellt dagegen hinsichtlich der Restverschmutzung eine zahlung der Abwassereinleiter (Industrie und Kommunen) an den staat für die Benutzung der Gewässer dar 225) Durch die Gemelnden kann sozusagen eine Transformation der Abwasserabgabe in eine Abwassergebühr erfolgen, indem die Gemeinde

224) Vgl. oben S. 305, Anm. 90.

225) Vgl. Freeman III, M.A./Haveman, R.H., Residuals Charges for Pollution Control: A Policy Evaluation, a.a.0., S. 325 . 
eine Kläranlage baut und dadurch die Restverschmutzung reduziert. Dies impliziert eine Verringerung der Abwasserabgabe der Gemeinde, aber eine Erhöhung der Abwasserreinigungskosten, die nun auf die Benutzer der Kläranlage umgelegt werden können 226) Würden alle Kommunen Kläranlagen bauen, welche $z u$ einer vollständigen Abwasserreinigung führen würden, und würden sie die entsprechenden Abwasserreinigungskosten auf die Verursacher umlegen, wäre die Abwasserabgabe völlig durch eine Abwassergebühr substituiert. Die incentives zur innerbetrieblichen Abwasserreinigung, die von einer Abwassergebühr ausgehen, entsprechen denen einer Abwasserabgabe 227)

Wenden wir uns nun dem Problem $z u$, wie ein verursachungsgerechter Maßstab für die Bemessung der Abwassergebüh'r ermittelt werden kann 228) Es besteht grundsätzlich die Möolichkeit, zwischen einem sogenannten Wirklichkeitsmaßstab und einem sogenannten Wahrscheinlichkeitsmaßstab der Gebührenbemessung $z u$ wählen. Der Wirklichkeitsmaßstab soll möglichst der tatsächlichen Inanspruchnahme der öffentlichen Lelstungen durch den Verursacher entsprechen; einen solchen Maßstab bei der unterschiedlichen Quantität, Qualität und Transportlänge der Abwässer zu finden, ist zweifellos recht schwierig, doch z.B. bei relativ glelchartigen Haushaltsabwässern durchaus möglich. Die Kommunalabgabengesetze der Länder schreiben grundsätzlich elnen Wirklichkeitsmaßstab vor, doch ist auch ein Wahrscheinlichkeitsmaßstab zuläss1g, wenn sonst die Bemessung der Gebühren $z u$ schwierig und damit zu teuer wäre 229)

226) Vgl. Oberhauser, A., Abgrenzung des Verursacherprinzips und seine Elnordnung in die Umweltpolitik, a.a.O., S. 43.

227) Vgl. Jürgensen, H./Jaeschke, K.-P., Allokationseffekte der Social Costs im Umweltschutz, a.a.O., S. 77; U.S. Council of Economic Advisers, The 1966 Report, a.a.O., S. 175.

228) Vgl. Giglio, R.J./Wrightington, R., Methods for Apportionino Costs Among Participants in Regional Systems, in: Water Resources Research, vol. 8, 1972, S. 1135 ff.

229) Vgl. Kessels, K. , Verursachungsgerechte Abwassergebühren, in: $\mathrm{Zur}$ Problematik verursachungsgerechter Berechnungsschlüssel in der Abwasserableitung, VIK-Berichte Nr. 186, Essen 1974 , S. 18. 
Als Wahrscheinlichkeitsmaßstab könnte die Bemessung einer Abwassergebüh nach der Dimensionierung der einzelnen Kanalisationsanschlüsse der Grundstücke einer Gemeinde in Form der Spitzenabflüsse in [1/sec] dienen 230) Die Abwassereinleiter würden dann gemäß ihrer potentielien Inanspruchnahme der Kanalisation und Kläranlage belastet und nicht nach deren tatsächlicher Benutzung. Einleiter mit einer großen Kapazitätsbeanspruchung wären besser gestellt als kleine Abwassereinleiter, welche die Abwasserbeseltigungskapazitäten überhaupt nicht ausnutzen können. Die Länge der Transportwege, welche die Abwässer von den Grundstücken bis zur Kläranlage zurücklegen, sollte bei der Bemessung der Abwassergebühr kelne Rolle spielen, da sie mehr oder weniger zufäll1g zustandekommt 231) Zusammenfassend wollen wir feststellen, daß die Bezahlung der vom Staat aus Effizienzgründen angebotenen Entsorgungsleistungen durch Gebühren bel annähernd verursachungsgerechter Bemessung der Gebuhren eine Maßnahme ist, die bei umweltbewußt handelnden Kommunen ähnlich wirksam sein kann, wie man das von einer kombinierten Abgaben-Auflagen-Lösung oder gar Emissionszertifikaten erwarten kann, denn die Gebühren stellen einen Anreiz für die Verursacher dar, Ihre Abwässer und Abfälle betriebsintern zu reduzieren, und die verantwortungsbewußte kommunale Aufgabenerfüllung wirkt wie die Einhaltung einer Emissions-Auflage. Kommen die Kommunen ihrer Entsorgungsaufgabe allerdings nicht nach - man könnte dann von elnem (öffentlichen) Leistungsdefizit sprechen -, werden auch die incentives, die sie in Form von Gebühren an die Unternehmen (und eventuell Haushalte) weitergeben, nicht ausrelchen, das heißt zu niedrig sein, um die angestrebte Gewässergüte zu errelchen.

Es soll abschließend auch noch auf ein Problem verursachungs-

230) Vgl. Munchau, F., Grundzüge elnes gerechten Maßstabes für die Vertellung der Entwässerungskosten einer Gemeinde, in: $\mathrm{Zur}$ Problematik verursachungsgerechter Berechnungsschlüssel in der Abwasserableitung, VIK-Berichte Nr. 186, Essen 1974, s. 9 .

231) Vgl. L1ttmann, K., Umweltbelastung - sozialökonomische Gegenkonzepte, a.a.0., s. 72 . 
gerechter Gebühren hingewiesen werden. Wegen der economies of scale großer Kläranlagen kann es $z u$ erheblichen Unterschieden In der Gebührenbelastung der Abwassereinleiter zwischen aroßen Gemeinden (= großen Kläranlagen) und kleinen Gemeinden (=kleinen kläranlagen) kommen 232). Aus Gründen der interkommunalen Vertellungsgerechtigkeit können deshalb Maßnahmen des kommunalen Finanzausgleichs notwendig werden, welche die unterschiedliche Gebührenbelastung kompensieren.

Wir wenden uns nun dem anderen Finanzierungsprinzip des Umweltschutzes, dem Gemeinlastprinzip, und seinen Instrumenten zu.

b) Die Instrumente des Gemeinlastprinzips

\section{aa) Subventionen}

Subventionen können Verursachern für Maßnahmen der Emissionsreduktion ("box" 4) oder Trägern für Maßnahmen des Immissionsschutzes ("box" 5) gewährt werden. Es empfiehlt sich, zwei Kategorien von Subventionen $z u$ unterscheiden, und zwar subventionen, die nur teilweise die Emissionsreduktions- oder Immissionsschutzkosten decken und die Restfinanzierung dem Verursacher oder Träger überlassen, und Subventionen, welche die vollen Kosten der Emissionsreduktion oder des Immissionsschutzes decken. Rational handelnde Verursacher von Umweltbelastungen werden durch Subventionen der ersten Kategorie ohne zusätzliche 2 wangsmaßnahmen, wie Auflagen oder Abgaben (was eine Kombination von Verursacher- und Gemeinlastprinzip impliziert), wohl kaum dazu veranlaBt, die mit der Subvention verbundenen Emissionsreduktionsmaßnahmen durchzuführen, denn eine nicht mindestens die Kosten der Emissionsreduktion deckende Subvention verringert ihren Gewinn (bzw. Nutzen) 233) Rational handelnde Träger von

232) Vgl. U.S. Department of the Interior/Federal Water pollution Control Administration, The Cost of Clean Water, vol. I, Economic Impact on Affected Units of Government, U.S.G.P.O., Washington, D.C., 1968, S. 5 .

233) Vgl. Cansier, D., Ökonomische Grundprobleme der Umweltpol1tik, a.a.0., s. 75 .

Eine Untersuchung hat ergeben, daß in der Bundesrepublik Deutschland gewährte Sonderaubschreibungen gemä $\beta \S \S 79,82$ und $82 \mathrm{e}$ EinkStDV kaum einen Einfluß auf freiwillige Umweltschutzinvestitionen haben (vol. Heigl, A., Wie wirkt die Sonderabschrelbung?, in: Umwelt, $1975,4, \mathrm{~s} .18 \mathrm{ff}$. 
Umweltbelastungen werden dagegen solche, nicht die vollen Immissionsschutzkosten deckenden subventionen dann zum Immissionsschutz verwenden, wenn ihr verbleibender eigener Finanzierungsbeltrag geringer ist als der Verlust (bzw. Nutzenentgang) den sie erleiden würden, wenn sie keine Immissionsschutzmaßnahmen ergrelfen würden.

Die in der Bundesrepublik Deutschland für Maßnahmen des Umweltschutzes gewährten subventionen 234 ) gehören alle in die erste Kategorie, implizieren also keine volle Kompensation der Emissionsreduktions- und Immissionsschutzkosten. Da die Subventionen für Umweltschutzmaßnahmen zweckgebunden sind, kann man im allgemeinen wohl schließen, daß ihre Inanspruchnahme vor allem dann erfolgt, wenn dadurch Auflagen billiger erfült oder z.B. Abwassergebühren gespart werden können. In bestimmten Fällen können Subventionen dieser Art u.E. gerechtfertigt sein, wenn ein Verursacher aus Gründen des Allokationsoptimums sehr hohe Auflagen erfüllen oder Abgaben zahlen bzw. Emissionsreduktionen durchführen muB, weil er z.B. niedrige Grenzkosten der Emissionsreduktion und einen hohen Immissionskoeffizienten hat. Das gilt vor allem dann, wenn der hohe Immissionskoeffizient die Folge elner nicht durchdachten kommunalen Ansiedlungspolitik ist. Als weltere Gründe für Subventionen in Verbindung mit Auflagen werden genannt: die Beschleunigung von Investitionen zur Emissionsreduktion, die Erhaltung von Arbeitsplätzen und der inter-

234 ) In der Bundesrepublik Deutschland werden Steuervergünstigungen und Finanzhilfen gewährt. Die Steuervergünstigungen bestehen aus Sonderabschreibungen für Investitionen zur Verhinderung, Beseitigung oder Reduktion von Abwässern ( $\$ 79$ EStDV), Emissionen in die Luft ( $\S 82$ EStDV) und Lärm ( $\S 82 \mathrm{e} E S t D V$ ) und aus Sonderabschreibungen für bestimmte Umweltforschungsvorhaben ( $\$ 82 \mathrm{~d}$ EStDV).

Die Finanzhilfen werden aus Mitteln der Gebietskörperschaften, des ERP-Sondervermögens der Kreditanstalt für Wiederaufbau, der Europäischen Investitionsbank und der EG gewährt. Sie bestehen aus Investitionszuschüssen bzw. -zulagen (vgl. $\S 4$ InvzulG), zinsverbilligten Darlehen, Zinszuschüssen, Bürgschaften und Garantien (vgl. Dittes, H., Die Finanzierungshilfen des Bundes und der Länder an die gewerbliche Wirtschaft, Frankfurt/Main 1976, die Subventionsberichte der Bundesregierung und die Jahresberichte der an der Gewährung der Finanzhilfen beteiligten Organisationen). 
nationalen Konkurrenz fähigkeit 235 !

Die Teilsubventionierung der Emissionsreduktionskosten in Verbindung mit Auflagen hat vor allem gegenüber der Abgabenregelung erhebliche Nachteile.

Es geht, wie bei der reinen Auflagenregelung, kein Anreiz von den Subventionen aus, über den Emissionsstandard hinaus Emissionen zu reduzieren 236), womit auch die Anwendung umweltfreundlichen technischen Fortschritts kaum initiiert wird 237)

Außerdem besteht die große Gefahr, daß nicht das effizienteste Verfahren der Emissionsreduktion gewählt wird, sondern dasjenige, wofür es subventionen gibt 238 )

Es ist oft schwierig, Investitionen, die dem Umweltschutz dienen, von allgemeinen Investitionen $z u$ trennen. Es besteht die $\mathrm{Ge}^{-}$ fahr, daß mehr als Umweltschutz-Investition deklariert wird als tatsächlich aus Gründen des Umweltschutzes geschieht, um dadurch Subventionen $\mathrm{zu}$ erhalten 239 )

235) Vgl. Oberhauser, A., Abgrenzung des Verursacherprinzips und seine Einordnung in die Umweltpolitik, a.a.O., S. 44.

$236)$ Vgl. ebenda, S. 43.

$237) \mathrm{Vgl}$. Nowotny, E., Wirtschaftspolitik und Umweltschutz, a.a.0., S. 180; Miller, E., Implications of Process Change for Industrial Pollution Abatement Pollcy, in: Land Economics, vol. 48, 1972, S. 397; Jürgensen, H./Jaeschke, K.-P., Allokationseffekte der Social Costs im Umweltschutz, a.a.0., s. 71 .

238) Vgl. Müller, F.G., Finanzpolitik und Umweltverschmutzung: Die kanadischen Maßnahmen zur Kontrolle der Wasserverunreinigung, in: Finanzarchiv, Bd. 31, 1973, S. 525; U.S. Council on Environmental Quality, Environmental Quality, The Fourth Annual Report, a.a.O., S. 114; Gerhardt, P.M., Air Pollution Control: Benefits, Costs, and Inducements, a.a.O., S. 164; Nowotny, E., Wirtschaftspolitik und Umweltschutz, a.a.O., S. 184; Schneider, Wolfgang, Verursacherprinzip oder die Belastung aller?, a.a.O., S. 474.

$239)$ Vgl. Muiller, F.G., Finanzpolitik und Umweltverschmutzung: Die kanadischen Maßnahmen zur Kontrolle der Wasserverunreinigung, a.a.o., S. 523; Cansier, D., Okonomische Grundprobleme der Umweltpolitik, a.a.0., S. 75; Gerhardt, P.H., Air Pollution Control: Benefits, Costs, and Inducements, a.a.O., S. 164; Nowotny, E., Wirtschaftspolitik und Umweltschutz, a.a.0., S. 185 . 
Die Subventionen bewirken, daB sich die Preise infolge der Emissionsreduktion nicht in dem Umfang erhöhen, wie dies bei einer reinen Auflagen- oder Abgabenlösung der Fall wäre, so daß die Nachfrage nach den Produkten, bei deren Herstellung die Emissionen entstehen, nicht im allokationsoptimalen Umfang zurückgeht 240). Die Teilsubventionierung privater Emissionsreduktionskosten verschlechtert also aus verschiedenen Gründen die Allokation.

Wir wenden uns nun der zweiten Kategorie von Subventionen zu, die man auch als Umkehrung der Abgaben bezeichnen kann 241). Sie werden dann gewährt, wenn Emissionen im erforderlichen Umfang reduziert werden, und zwar in einer Höhe, daß dem Verursacher noch ein Nettogewinn verbleibt.

Das sei an Abb. 34 verdeutlicht 242)

Wenn ein Verursacher einen Emissionsreduktionsgrad von OA realisiert und wendet dabei den neuesten Stand der Emissionsreduktionstechnik an, so kann ihm als "Belohnung" dafür eine Emissionsreduktions-Subvention mit einem Satz von $O C=r$ gewährt werden. Eine solche Subvention stellt also einen Anreiz dar, den neuesten Stand der Technik anzuwenden. Sie ist insofern der Form der Teilsubventionierung überlegen. Ein Verursacher, der bis OA reinigt, erhält dafür eine Subvention in Höhe von OABC. Da seine Emissionsreduktionskosten nur OABD betragen, erzielt er aus der Emissionsreduktion einen Nettogewinn in Höhe von $O A B C-O A B D=D B C$. Die Allokationswirkung scheint formal die gleiche $z u$ sein, wie im Falle einer Emissionsabgabe mit einem Satz von $t=O C=r$.

240) Wulf, J., Umweltpolitik: Probleme, Phasen, Forschungsstrategien, in: Jahrbuch für Sozialwissenschaft, Bd. 24, 1973, S. 188 f.: Swoboda, H., Probleme der Umweltpolitik, a.a.O., S. 88 f.; Müller, F.G., Finanzpolitik und Umweltverschmutzung: Die kanadischen MaBnahmen zur Kontrolle der Wasserverunreinigung, a.a.0., S. 525; Nowotny, E., Wirtschaftspolitik und Umweltschutz, a.a.0., s. 174 .

$241)$ Vgl. Frey, B.S., Umweltökonomie, a.a.O., S. 119.

242) Vgl. Cansier, D., Okkonomische Grundprobleme der Umweltpolitik, a.a.0., s. 74 . 


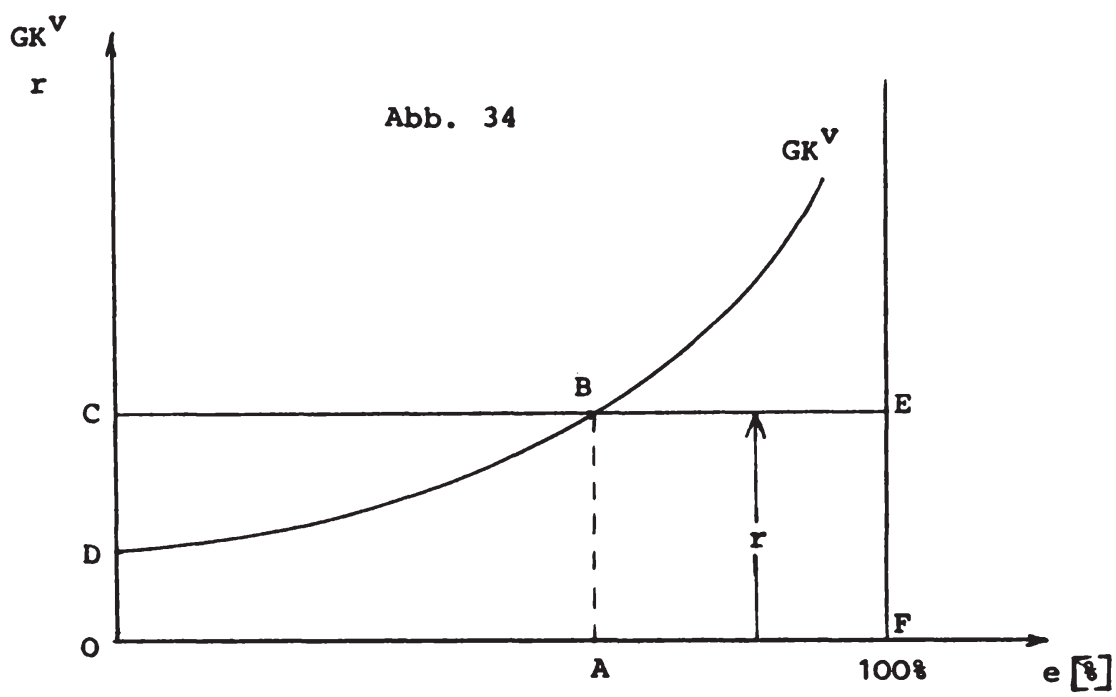

Das kann in statischer Hinsicht auch der Fall sein, nicht aberbei dynamischer Betrachtung, denn durch den Nettogewinn infolge der Emissionsreduktion kann die potentiell umweltschädliche Produktion weiter expandieren 243). Es gehen auch keine Preissteigerungseffekte von der Emissionsreduktion aus, so daB die Nachfrage nach dem Gut, dessen Produktion Emissionen hervorruft, nicht sinkt. Eine solche Subvention kann daruber hinaus zu dem "perverse result" 244) führen, daß gerade emissionsintensive Produktionstechniken angewendet werden, um via subventionen für die Emissionsreduktion einen Netto-Gewinn zu erzielen.

Alle Arten von Subventionen erweisen sich somit in allokativer Hinsicht vor allem Emissionsabgaben gegenüber als unterlegen. Aus Gründen der Allokationseffizlenz sind deshalb Emissionsabgaben gegenüber Subventionen vorzuziehen. Sollten von Emissions-

243) Vgl. Solow, R.M., The Economist's Approach to Pollution and its Control, a.a.0., S. 500 .

244 ) Ebenda; vgl. dazu auch Miller, E.. Implications of Process Change for Industrial Pollution Abatement Policy, a.a.0., S. 397 . 
abgaben - etwa via deren Uberwälzung auf die Preise - allzu negative Distributionswirkungen ausgehen, können diese durch staatliche Transfers kompensiert werden 245)

Das Argument, daß Subventionen den Weg des geringsten politischen Widerstandes darstellen 246) und deshalb Abgaben gegenüber vorzuziehen seien, können wir nicht akzeptieren. Es käme einer Kapitulation des Staates vor den Interessen der Verursacher gleich und würde die Last des Umweltschutzes auf die Allgemeinheit verteilen, obwohl sich mit Hilfe einer konsequenten Anwendung der Instrumente des Verursacherprinzips eine allokativ bessere und auch gerechtere Verteilung der Umweltschutzkosten erreichen ließe. Wir verkennen dabei nicht, daß im Falle des Verursacherprinzips via Utberwälzung e $i_{\mathrm{n}} \mathrm{s} T$ e $i \mathrm{l}$ der Umweltschutzkosten auf die Preise auch die Allgemeinheit zur Finanzierung herangezogen wird, doch wird dieser Anteil durch den $M$ a $r k$ bestimmt, während im Falle des Gemeinlastprinzips die $g$ e s a m$t e n$ Umweltschutzkosten über die ö $f e n t l i c h$ e $n$ $\mathrm{E} i \mathrm{n} \mathrm{n}$ a $\mathrm{m}$ e $\mathrm{n}$ finanziert werden. Bei den realistischen Annahmen eines leicht regressiven Effekts der Uberwälzung einer Emissionsabgabe auf die Preise und eines leicht progressiven Steuersystems scheint zwar das Gemeinlastprinzip hinsichtlich der Distributionswirkung überlegen zu sein, doch ist Gerechtigkeit eben nicht nur eine Frage der Verteilungsstruktur sondern auch des Niveaus, oder mit anderen Worten: Die Allokationseffizienz des Verursacherprinzips ist der des Gemeinlastprinzips so sehr überlegen, daß die eventuell auftretenden regressiven Effekte leicht via öffentliche Transfers kompensiert werden können, so daß sich etwa eine Distribution ergibt, wie sie nach der Finanzierung des Umweltschutzes durch das Gemeinlastprinzip zustandegekommen wäre. Gegenüber der Finanzierung nach dem Gemeinlastprinzip wäre aber zusätzlich noch - wegen der größeren Allokationseffizienz des Verursacherprinzips - ein weit höheres

245) Vgl. Solow, R.M., The Economist's Approach to Pollution and its Control, a.a.0., S. 500 .

$246)$ Vgl. Swoboda, H., Probleme der Umweltpolitik, a.a.O., S. 89. 
Wohlfahrtsniveau realisiert.

bb) Allgemeine Deckungsmittel

Die "box" 6 enthält Instrumente zur Finanzierung von Maßnahmen, die vom Staat aus Effizlenzgründen oder wegen ihres "öffentlichen Gut-Charakters" durchgeführt werden. Zur ersten Kategorie gehören die glelchen offentlichen Umweltschutzinvestitionen wie in "box" 3, mit dem Unterschied, daß sie jetzt vom Staat finanziert werden, entweder weil die Identifikation der Verursacher $\mathrm{zu}$ hohe Informationskosten erfordern würde oder weil das Verursacherprinzip aus sozialen Gründen (z.B. im Personennahverkehr) nicht angewandt werden soll.

Die zwelte Kategorie besteht aus den Maßnahmen, die wir als nicht-technische Rahmenbedingungen des technischen Umweltschutzes bezeichnen können 247 ) und deren setzung im wesentlichen eine öffentliche - via Budget zu finanzierende - Aufgabe ist 248) Betrachten wir Umweltschutz als eine neue öffentliche Aufgabe, so kommen - wenn wir von Ausgabekürzungen in anderen Berelchen absehen 249)- grundsätzlich drei Möglichkeiten zur Finanzierung des öffentlichen Umweltschutzes aus allgemeinen Deckungsmitteln in Frage: (1) die Erhöhung bereits vorhandener Steuern, (2) die Einführung einer (neuen) Umweltsteuer und (3) die Kreditaufnahme. Da die Hauptlast der öffentlichen Umweltschutzfinanzierung bei den Gemeinden liegt, spielen als allgemeine Deckungsmittel der kommunalen Haushalte in erster Linie (4) Zuwelsungen an die Gemeinden im Rahmen des vertikalen Finanzausgleichs eine wichtige Rolle 250!

$\mathrm{Zu} \mathrm{(1):}$

Da infolge der Anwendung des Verursacherprinzips wahrscheinlich

247) Siehe oben S. $196 \mathrm{ff}$.

248) Siehe oben S. $289 \mathrm{ff}$.

$249)$ Wir unterstellen, daß über die Priorität des Umweltschutzes innerhalb der politischen zielhierarchie bereits entschieden 1st.

250) Vgl. Materialien zum Bericht zur Lage der Nation 1974, a.a.o., s. 399 . 
leicht regressive Wirkungen von der tellwelsen Uberwälzung der Emissionsreduktionskosten auf die Güterpreise ausgehen werden, wirde es sich zur allfälligen Kompensation empfehlen, eine progressive Steuer $z u$ erhöhen, um die öffentlichen Umweltschutzausgaben zu finanzieren. Es käme wohl nur die Einkommensteuer in Frage. Eine lineare Anhebung des Einkommensteuertarifs auch um nur wenige Prozentpunkte dürfte jedoch wegen der bereits hohen Gesamtbelastung des Einkommens nur schwer durchsetzbar sein. $\mathrm{Zu} \mathrm{(2):}$

Die Einführung einer Umweltsteuer 251) die zum gleichen Aufkommen führen müßte wie die Erhöhung der Einkommensteuer, könnte theoretisch die gleichen Widerstände auslösen. Infolge des gestiegenen Umweltbewußtselns der Bevölkerung wăre es jedoch denkbar, daß eine zweckgebundene Umweltsteuer als notwendiges Ubel akzeptiert würde. Man könnte die Umweltsteuer in Gestalt einer Ergänzungsabgabe zur Einkommensteuer erheben. Das Aufkommen müßte zweckgebunden sein, und es würde slch empfehlen, den steuerzahler in regelmäßigen Abständen detallliert uber die Verwendung der Umweltabgabe zu unterrichten.

Die Einführung einer Umweltsteuer mit eigener Bemessungsgrundlage düfte sehr schwierig sein. Man könnte allerdings an die "Umfunktionierung" der Gewerbesteuer in eine Umweltsteuer denken, zumal mit der Gewerbesteuer auch bisher schon Umweltschutzleistungen der Gemeinden tellweise abgegolten werden sollten 252). Weder Gewerbeertrag, noch Gewerbekapital oder Lohnsumme wären jedoch U.E. äquivalente Bemessungsgrundlagen.

$\mathrm{Zu} \mathrm{(3):}$

Die Finanzierung öffentlicher Umweltschutzinvestitionen durch Kredite halten wir in den Fällen für eine adäquate Methode, in denen durch diese Investitionen vor allem die natürlichen Ressourcen geschützt werden, was nicht nur der gegenwärtigen sondern vor allem auch zukünftigen Generationen zugutekommt. So läßt

251) Vgl. RSU, Umweltgutachten 1974, a.a.0., S. 155.

252) Vgl. Bullinger, M., Rechtsfragen des Verursacherprinzips belm Umweltschutz, a.a.0., s. 74 . 
sich vor allem der Bau von Kläranlagen, aber auch von Abfallbeseitigungsanlagen und umweltfreundlichen Energieversorgungsanlagen sowie der Ausbau eines attraktiven Nahverkehrssystems durch öffentliche Kreditfinanzierung rechtfertigen. Es ist allerdings die Frage, ob auch die Inanspruchnahme dieser öffentlichen Leistungen zum Null-Tarif erfolgen sollte, oder ob mehr oder weniger kostendeckende Benutzungsgebühren (für Kapitaldienst und Betriebskosten) erhoben werden sollten 253) Der Null-Tarif ließe sich u.E. nur dann rechtfertigen, wenn die Nutzungskosten nicht zugerechnet werden könnten, die Erhebung der Benutzungsgebühren $z u$ teuer wäre oder wenn andernfalls die Leistungen nicht in Anspruch genommen, d.h. also die Umwelt weiter belastet würde. Könnte man z.B. durch den Null-Tarif für öffentliche Nahverkehrsmittel die Bevölkerung dazu bewegen, statt mit dem eigenen Pkw mit öffentlichen Nahverkehrsmitteln zum Arbeitsplatz oder Einkauf in die städte zu fahren und ließen sich dadurch die Kosten der Umweltbelastungen und Stauungen um mehr reduzieren als der Nul1-Tarif die öffentliche Hand kostet, wäre der NullTarif gerechtfertigt.

Die Entsorgungsleistungen sollten allerdings, wenn die zurechnung nicht zu teuer wird, nicht zum Null-Tarif zur Verfügung gestellt werden, da sonst keine incentives bestehen würden, innerbetriebliche Emissionsreduktionsmaßnahmen durchzuführen, die unter Umständen weitaus billiger sind 254)

$\mathrm{Zu} \mathrm{(4):}$

Die Hauptlast der Umweltschutzfinanzierung tragen die Gemeinden, vor allem auf dem Gebiet der Abwasserbeseitigung. Eine wichtige Finanzierungshilfe für den Bau kommunaler Kläranlagen stellen Zuweisungen im Rahmen des Finanzausgleichs für Zwecke der Abwasserbeseitigung dar. Damit verfügen die Zentralregierungen, welche die $\mathrm{z}$ uwelsungen verteilen, über ein wichtiges Instrument, um den Bau von Kläranlagen optimal $z u$ verteilen. Es kommt nämlich

253) Vgl. Littmann, K., Umweltbelastung - sozialökonomische Gegenkonzepte, a.a.0., s. 71.

$254)$ Vgl. Frey, B.S., Umweltökonomie, a.a.0., S. 112 f.;Fry, C.L., Pollution Control, a.a.0., s. 2 f. 
nicht darauf an, jeder Gemeinde eine Kläranlage finanzieren zu helfen, sondern darauf, die Finanzzuweisungen möglichst dorthin fließen zu lassen, wo sie den größten Nutzen für die Abwasserreinigung stiften, und das ist in der Regel bei den großen Kläranlagen der großen Gemeinden und den Gemeinschaftskläranlagen mehrerer kleiner Gemeinden der Fall. 255)

Mit der Bezuschussung kommunaler Kläranlagen ist indirekt auch eine Subventionierung der Verursacher verbunden, denn die Gemeinden werden in ihren Abwassergebühren in der Regel die $\mathrm{Zu}-$ weisungen nicht berücksichtigen. Das führt zu einer Verzerrung der Preisstruktur, die bewirkt, daß die Verursacher die "zu billigen" Leistungen öffentlicher Kläranlagen nutzen, statt eventuell gesamtwirtschaftlich effizientere interne Abwasserreduktionsmaßnahmen durchzuführen. "... firms should be required to choose the method of pollution control they will use in the light of the full costs of each alternative" 256)

Da slch die Zuweisungen auf den $B$ a $u$ der Kläranlagen beschränken, also auf Kapitalinvestitionen, bergen sie die Gefahr elner Ubberdimensionierung der Kläranlagenkapazitäten in sich. Effizienter wären in manchen Fällen sicher Zuweisungen für eine bessere Personalausstattung bestehender Anlagekapazităten 257). Allokationsoptimale Finanzzuweisungen für Kläranlagen müssen also sowohl hinsichtlich der räumlichen Verteilung der Mittel als auch im Hinblick auf eine optimale Faktorkombination erfolgen.

Zusammenfassend können wir zur allokationsoptimalen Gestaltung der allgemeinen Deckungsmittel sagen, daß sie sehr schwierig ist, weil sie nicht über den Markt erfolgt. Die Finanzierung öffentlicher Umweltschutz-Investitionen durch Kredite ist eine adäquate Finanzierungsmethode; die Benutzung oder Inanspruchnahme der öffentlichen Entsorgungs- und Versorgungseinrichtungen

255) Schultze, C.L. u.a., Setting National Priotities, The 1973 Budget, a.a.0., s. $380 \mathrm{f}$.

256) Ebenda, S. 380.

257) Vgl. U.S. Council on Environmental Quality, Environmental Quality, The Fourth Annual Report, a.a.o., s. 114. 
sollte jedoch, wenn immer möglich und aus sozialen Gründen vertretbar, nur gegen Erstattung einer kostendeckenden Gebuihr erlaubt sein. Die Finanzierung der umweltpolitischen Rahmenbedingungen muß dagegen wohl aus allgemeinen steuermitteln oder einer speziellen Umweltsteuer vorgenommen werden.

\section{Mögliche Auswirkungen einer rationalen Umweltpolitik auf das öffentliche Budget}

1. Einteilung der budgetären wirkungen einer rationalen Umweltpolitik

Unterteilen wir die Einnahmen- und Ausgabenaggregate des öffentlichen Budgets in der unten dargestellten Welse, so kann man sagen, daß sich die budgetăren Konsequenzen des Umweltschutzes, $\mathrm{B}_{U^{\prime}}$ nach folgender Gleichung ergeben:

(1) $\mathrm{B}_{U}=\left(\mathrm{A}_{\mathrm{dir}}^{+}+\mathrm{A}_{\mathrm{dir}}^{-}+\mathrm{A}_{\text {ind }}^{+}+\mathrm{A}_{\text {ind }}^{-}\right)-\left(\mathrm{E}_{\mathrm{dir}}^{+}+\mathrm{E}_{\mathrm{dir}}^{-}+\mathrm{E}_{\text {ind }}^{+}+\mathrm{E}_{\text {ind }}^{-}\right)$

Einnahmen (E)

Direkte Mehreinnahmen

Direkte Mindereinnahmen

Indir. Mehreinnahmen

Indir. Mindereinnahmen
Öffentliches Budget Edir Eirr E $\stackrel{+}{\text { ind }}$ Eind
Direkte Mehrausgaben Direkte Minderausgaben Indir. Mehrausgaben Indir. Minderausgaben
(A)

A dir

Air

Aind

A ind

Saldo ( $\mathrm{B}_{\mathrm{U}}$

10

Durch eine rationale Umweltpolitik ergibt sich eine Belastung des Budgets, wenn $\mathrm{B}_{U_{+}}>0$ und eine Entlastung, wenn $\mathrm{B}_{U}<0_{+}$. Die direkten Mehreinnahmen $\mathrm{E}_{\mathrm{dir}}^{+}$und die direkten Mehrausgaben $\mathrm{A}_{\mathrm{d} I \mathrm{r}}^{+}$ergeben sich unmittelbar aus der umweltpolitischen Aktivität des Staates. So füht z.B. die Erhebung einer Abwasserabgabe zu direkten Mehreinnahmen in Höhe des Abgabeaufkommens und zu direkten Mehraus- 
gaben in Form der Verwaltung der Abgabe, der staatlichen Erhebungskosten und der Kontrollkosten.

Ein Beispiel für direkte Mindereinnahmen $E_{\text {dir }}^{-}$sind steuervergünstigungen, die - etwa im Rahmen der Einkommensteuer - für Umweltschutzinvestitionen gewährt werden. Beispiele für direkte Minderausgaben $A_{d i r}^{-}$lassen sich in der Praxis kaum finden, weshalb wir diesen Posten im folgenden vernachlässigen können. Indirekte Mehrelnnahmen können sich daraus ergeben, daß durch den Umweltschutz bestimmte Steuerbemessungsgrundlagen gestiegen sind. So wäre es z.B. denkbar, daß durch die gestiegenen Umsätze der Umweltschutzindustrie die Umsatzsteuer steigt. Ferner kann es sein, daß durch die gesünderen Umweltbeding̣ugen infolge des Umweltschutzes die Arbeitsproduktivität und damit c.p. das Volkseinkommen stelgt, was vor allem zu einer Erhöhung des Aufkommens aus den direkten Steuern führen würde. Da Umweltschutz eine große prophylaktische wirkung in bezug auf die Gesundheit haben

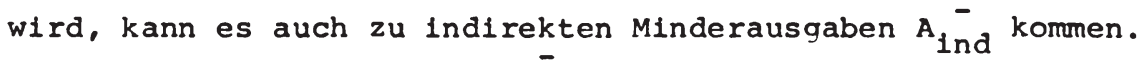
Indirekte Mindereinnahmen $E_{\text {ind }}^{-}$sind die Folge davon, daß der Umweltschutz Besteuerungsmengen reduziert hat. So können $z$. B. durch Betriebsstillegungen wegen nicht erfülibarer Umweltschutzauflagen Einkommensverluste bei den Angestellten entstehen; ganz allgemein wird Umweltschutz nach dem Verursacherprinzip in manchen Branchen Gewinneinbußen mit sich bringen; solche Entwicklungen werden c.p. $z u$ einem Rückgang der einkommens- und gewinnabhängigen steuern führen.

Indirekte Mehrausgaben ${ }^{+}{ }_{\text {ind }}^{+}$werden sich dann nicht vermeiden lassen, wenn der Umweltschutz zu sozialen Härten führt oder strukturpolitisch nachteilige Folgen hat. In vielen Fällen wird es - wie schon erwahnt - notwendig sein, aus allokationspolitischen Gründen durchgefühte Maßnahmen durch distributive zu ergänzen, damit nicht bestimmte Einkommensgruppen besonders hohe Beiträge für die Allokationseffizienz zahlen müssen.

Die bisher geschilderten budgetären Auswirkungen resultierten aus der umweltpolitischen Aktivität des Staates. Es kann aber auch deshalb zu Budgetbelastungen kommen, weil der staat Verursacher oder Träger von Umweltbelastungen ist. Der staat wird als 
(potentieller) Verursacher von Umweltbelastungen vor allem Mehrausgaben für die Umweltschutzmaßnahmen in seinem Aufgabenbereich (Straßenbau, Städtebau, Flughäfen, Bahn u.a.) haben. Als Trăger von potentiellen Umweltbelastungen wird er andererseits infologe des allgemeinen Umweltschutzes Minderausgaben z.B. für die Restauration historischer Bauwerke haben, deren Eigentümer er ist.

Mehreinnahmen kann er als Träger von Umweltbelastungen dann erhalten, wenn er die Verursacher ausfindig machen und zur Zahlung von Schadenersatz bringen kann.

Diese kursorlsche Darstellung mag genügen, um $z u$ zeigen, daß das Budget auf sehr verschiedene Art direkt oder indirekt durch eine rationale Umweltpolitik be- oder entlastet werden kann.

Im folgenden woljen wir nun darstellen, wie man versuchen kann, die budgetären Wirkungen der verschiedenen Instrumente des Verursacher- und Gemeinlastprinzips hinsichtlich ihrer budgetären Konsequenzen $z u$ beurteilen. Es soll also eln Weg aufgezeigt werden, wie eine solche Bewertung der Instrumente erfolgen kann; die definitiven budgetären Auswirkungen jedes Instrumentes lassen sich nur aufgrund empirischer Untersuchungen ermitteln.

2. Eln möglicher Weg zur Emittlung der budgetären Auswirkungen einer rationalen Umweltpolitik

Hauptaufgabe einer rationalen Umweltpolitik ist die Elnhaltuna der Immissionsstandards $s_{1}^{g}$ aller Schadstoffe $1(i=1, \ldots, n)$. Zur Durchsetzung jedes dieser $n$ Immissionsstandards stehen der Umweltpolitik $r$ ökonomische und juristische Instrumente $m_{i j}$ $(j=1, \ldots, r)$ des Verursacher- und Gemeinlastprinzips zur Verfügung. Nehmen wir an, daß grundsätzlich alle $m_{i j} z u r$ Einhaltung oder Realisierung aller $s_{i}^{g}$ geelgnet sind, so gilt, wenn wir die tatsächlichen Immissionskonzentrationen $\mathrm{mit}_{i}^{t}$ bezelchnen

(1) $s_{1}^{t}=s_{1}^{t}\left(m_{i j}\right)$, wobel gelten muß, daß

(2) $s_{i}^{t} \leqslant s_{i}^{g}$.

und wegen des instrumentellen Charakters der $m_{i j}$ für die $s_{i}^{t}$ muß 
gelten

$$
\frac{\partial s_{1}^{t}}{\partial m_{1 j}}<0
$$

Mit stelgendem Elnsatz des Instruments j sinkt also die tatsäch11che Immissionskonzentration des Schadstoffs 1 . Jedes der Instrumente $j$ ist nun unterschiedlich gut geelgnet ("effizient"), einen bestimmten Immissionsstandard elnzuhalten oder zu erreichen. Am effizientesten ist offenbar jenes Instrument, das Bedingung (2) zu den niedrigsten Kosten einhält oder realisiert; das wird jenes umweltpolitische Instrument sein, das je eingesetzter Werteinheit zur größten Reduktion der tatsächlichen Immissionskonzentration $s_{1}^{t}$ führt, bel dessen Anwendung der absolute Wert von Ausdruck (3) also am gröBten 1st. Wir wollen dieses Instrument als dasjenige mit dem höchsten Zielerreichungsgrad oder einfach als optimales Instrument $m_{1 j}^{o p t}$ bezelchnen. Man kann nun

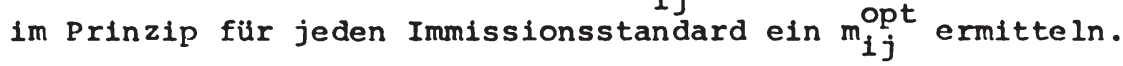
Die direkte Budgetbelastung oder -entlastung infolge der Anwendung aller $m_{1 j}^{\text {opt }}$ erhält man, wenn man die direkten Mehreinnahmen und -ausgaben, die mit der Anwendung der $m_{1 j}^{\text {opt }}$ verbunden sind, ermittelt.

Um die indirekten Budgetwirkungen $z u$ bestimmen, müssen die Zielerrelchungsgrade der anderen (wirtschafts-)politischen Ziele infolge der Anwendung der $m_{1 j}$ opt untersucht werden. Bezelchnen wir die von den umweltpolitischen Maßnahmen tangierten 1 ziele mit $z_{k}(k=1, \ldots, 1)$, so gilt:

(4) $z_{k}=z_{k}\left[s_{i}^{t}\left(m_{1 j}\right)\right]$, wobel folgende Beziehungen möglich sind:

$$
\frac{\partial z_{k}}{\partial s_{i}^{t}} \frac{d s_{1}^{t}}{d m_{i j}}>0
$$

$$
\frac{\partial z_{k}}{\partial s_{i}^{t}} \frac{d s_{i}^{t}}{d m_{1 j}}=0
$$


(7) $\frac{\partial z_{k}}{\partial s_{i}^{t}} \frac{d s_{1}^{t}}{d m_{i j}}<0$.

In (5) herrscht 2 ielkomplementarität, in (6) zielneutralität und in (7) ein zielkonflikt.

Kann man nun die verschiedenen zielerreichungsgrade quantifizieren, so lassen sich auch die indirekten Budgetwirkungen der rationalen Umweltpolitik ermitteln.

Im allgemeinen wird im Fall einer komplementären Zielbeziehung via indirekter Mehreinnahmen oder indirekter Minderausgaben eine Entlastung des Budgets entstehen und im Fall der Zielkonflikte eine Belastung via indirekte Mindereinnahmen und Indirekte Mehrausgaben.

Im Fall der zielkonflikte, kann man noch prüfen, ob nicht Second-best-Lösungen des Umweltschutzproblems, also nicht die Anwendung des Instruments $m_{i j}^{o p t}$, sondern $z$.B. die nächstbeste Maßnahme $m_{i j}^{*}$ den 2 lelkonflikt so stark reduzieren, daß die verminderte Effizienz bei der Realisierung des Immissionsstandards durch die größere 21 lelerreichung bei anderen $z i e l e n$ überkompensiert wird.

3. Versuch einer Tendenzaussage über die budgetären Konsequenzen des Verursacher- und Gemeinlastprinzips

Obwohl es eine Reihe von theoretischen 258) und ökonometrischen 259) Untersuchungen über die Wirkungen der Umweltpolitik

258) Vgl. z.B. Bender, D., Makroökonomik des Umweltschutzes, Göttingen 1976, S. 107 ff.; Nowotny, E., Wirtschaftspolitik und Umweltschutz, a.a.0., S. 229 ff.; ders., Zur gesamtwirtschaftlichen Wirkung einer Besteuerung von Urhebern externer Effekte, in: Finanzarchiv, Bd. 31, 1973, S. $420 \mathrm{ff}$; Oberhauser, A., Gesamtwirtschaftliche Wirkuncen der Anwendung des Verursacherprinzips, in: Das Verursacherprinzin und seine Instrumente, a.a.0., S. $51 \mathrm{ff}$.

259) Vgl. Fazio, A.G./Cascio, M.L., Evaluation of the Economic Effects of Anti-Pollution Public Policy: Proposal for an Econometric Analysis Model, in: OECD (Hrsg.), Problems of Environmental Economics, a.a.O., S. $143 \mathrm{ff.;}$ U.S. Council of Environmental Quality/Department of Commerce/ Environmental Protection Agency, The Economic Impact of Pollution Control, a.a.o. 
auf andere wirtschaftspolitische ziele gibt, läßt sich wohl kaum mehr als eine tendenzielle Aussage darüber machen, wie das öffentliche Budget nicht nur direkt, sondern auch indirekt durch alternative umweltpolitische Maßnahmen beeinflußt wird. Unsere Analyse der umweltpolitischen Instrumente ermöglicht uns jedoch zunächst vier tendenzielle Aussagen, wenn wir Verursacherprinzip und Gemeinlastprinzip hinsichtlich ihrer budgetären Konsequenzen beurteilen sollen.

(1) Das Verursacherprinzip führt vor allem dann, wenn es durch ökonomische Instrumente durchgesetzt wird eher zu einer direkten Entlastung des Budgets als zu einer direkten Belastung, da die direkten Mehreinnahmen aus den verschiedenen Steuern, Abgaben und Gebühren wahrscheinlich größer sind, als die Administrations- und Informationskosten.

(2) Das Gemeinlastprinzip führt seiner Natur nach zu einer unmittelbaren Belastung des Budgets via direkter Mehrausgaben in Form von Subventionen aller Art und in Form öffentlicher Investitionsausgaben .

(3) Das Verursacherprinzip führt zumindest kurzfristig wahrscheinlich $\mathrm{zu}$ einer Belastung des Budgets infolge indirekter Mindereinnahmen und indirekter Mehrausgaben, weil insgesamt wohl die Gewinne der Unternehmer geschmälert werden, besonders emissionsintensive Branchen Arbeitskräfte entlassen müssen und regressive Vertellungswirkungen infolge der Uberwälzungsvorgänge entstehen.

(4) Das Gemeinlastprinzip vermeidet die negativen Wirkungen auf Gewinne und Beschäftigung und führt somit wahrscheinlich $z u$ keiner großen indirekten Budgetbelastung; mit Sicherheit duirfte sie geringer sein als beim Verursacherprinzip.

Man könnte versucht sein, nun bereits den Schluß zu ziehen, daß dasjenige Finanzierungsprinzip überlegen sel, dessen summe aus direkten und indirekten Budgetbelastungen am geringsten ist. Dieser Schluß wäre jedoch $\mathrm{zu}$ voreilig, denn unsere Tendenzaussagen (1) bis (4) erfolgten unter der stillschweigenden Annahme, daB die Effizienz der Instrumente des Verursacher- und Gemeinlastprinzips gleich groß sind, daß also z.B. 
$\frac{\partial s_{i}^{t}}{\partial m_{i 1}}=\frac{\partial s_{i}^{t}}{\partial m_{12}}=\ldots=\frac{\partial s_{i}^{t}}{\partial m_{1 j}}=\ldots=\frac{\partial s_{i}^{t}}{\partial m_{i r}}$.

Die Analyse der Instrumente des Verursacher- und Gemeinlastprinzips hat jedoch gezelgt, das die Allokationseffizienz der Instrumente des Verursacherprinzips - und hier besonders der Emissionsabgaben und Emissionszertifikate - mit hoher Wahrscheinlichkelt gröBer ist als die der Instrumente des Gemelnlastprinzips. Die Folge davon 1st, daß die Instrumente des Verursacherprinzips viel geringer dosiert sein können als die "Gießkannen"-Instrument des Gemeinlastprinzips, um die gleiche Reduktion der Immissionskonzentration $z u$ bewirken. Der Unterschied, der sich in den direkten Budgetwirkungen zugunsten des Verursacherprinzips ergibt, wird daher beträchtlich größer sein als man dies aus (1) und (2) entnehmen konnte, und der unterschied der sich bel der indirekten Budgetbelastung zugunsten des Gemeinlastprinzips ergibt wird weitaus geringer sein als dies nach (3) und (4) schien. Die Waage neigt sich also stark zugunsten des Verursacherprinzips. Es ist mit großer Wahrscheinlichkeit nicht nur gesamtwirtschaftlich die billigste Finanzierungsmethode, sondern belastet selbst unter Berücksichtigung indirekter Budgetauswirkungen das öffentliche Budget weniger als das Gemeinlastprinzlp.

\section{Zur Auswahl der umweltpolitischen Instrumente}

In den vergangenen drei Abschnitten dieses Kapitels haben wir eine Vielzahl von Maßnahmen und Instrumenten der Umweltpolitik kennengelernt. Es waren dies Maßnahmen zur Schaffung und Verbesserung der umweltpolitischen Rahmenbedingungen und Instrumente zur Durchsetzung elner rationalen Umweltpolitik. Wir wiesen berelts daraufhin, daß zwischen den umweltpolitischen Rahmenbedingungen und dem Einsatz der umweltpolitischen Instrumente ein substitutionales Verhältnis besteht, was bedeutet, daB sich der Einsatz der umweltpolitischen Instrumente umso mehr erübrigt, je besser die umweltpolitischen Rahmenbedingungen sind, innerhalb derer sich - vor allem der privat betriebene - Umwelt- 
schutz vollzieht 260 )

Besonders in elnem marktwirtschaftlichen System ist es wichtig, die Rahmenbedingungen für den Umweltschutz so zu setzen, daB der Staat mit seinem umweltpolitischen Instrumentarium möglichst wenig eingreifen muB. Auf diese Welse wird dann auch das offent11 che Budget von Umweltschutzausgaben entlastet.

Die Schaffung und Verbesserung der umweltpolitischen Rahmenbedingungen - wie etwa die Erzeugung eines (neuen) Umweltbewustseins, die Ausbildung von Umweltschutzexperten oder die Technologiekontrolle - haben auBerdem den Vortell, daB sie der Verbesserung der Qualitat aller Umweltmedien zugute kommen.

Das gilt nicht fur die umweltpolitischen Instrumente des Verursacher- und Gemeinlastprinzips. Die Eignung der jeweiligen Instrumente, die Qualität der Umweltmedien, der Lebensmittel und der Ruhe zu verbessern ist sehr unterschiedlich. Siebert spricht in diesem $\mathrm{Z}$ usammenhang von der "ökologischen Inzidenz" 261) der umweltpolitischen Instrumente. Es liegt nun nahe, die ökologische Inzidenz als Auswahlkriterium für die umweltpolitischen Instrumente heranzuziehen. Das 1st allerdings nur dann möglich, wenn es gelingt, den "verschiedenen Instrumenten und unterschiedlichen Dosierungen der Instrumente jewells alternative Wirkungen auf die Umweltqualität zuzuordnen" 262). Eine solche Quantifizierung der Wirkungen umweltpol1tischer Instrumente auf die Umweltqualität 1st slcher auberst schwierig. Sie kann nur aufgrund empirischer Untersuchungen vorgenommen werden. Hierin liegt ein groBer "Wettbewerbs"-Nachtell für solche umweltpolitischen Instrumente, die bisher noch nicht in der umweltpolitischen Praxis erprobt worden sind, vor allem also Emissionszertifikate. Sie mögen theoretisch eine sehr hohe okologische Inzidenz haben; die Anwendung dieses Instruments - etwa zur Reduzierung der $\mathrm{SO}_{2}$-Emissionen - wird jedoch auf große Schwierigkeiten stoBen,

260) Siehe oben S. 302 .

261) Siebert, H. Analyse der Instrumente der Umweltpolitik, Göttingen 1976 , S. 111 .

262) Ebenda. 
da seine tatsächliche ökologische Inzidenz ungewiB 1st, im Gegenzu derjenigen von Emissionsstandards, dem klassischen Instrument behördlicher Emissionsbekămpfung.

Es gibt jedoch noch elne Reihe weiterer Kriterien, welche die Auswahl der umweltpolitischen Instrumente erlelchtern helfen. Das für die umweltpolitische Praxis wichtigste Kriterium ist U.E. die Allokationseffizienz der umweltpolitischen Instrumente, und zwar unter Berücksichtigung der Informations- und Administrationskosten (einschlieblich der Kontrollkosten). Instrumente mit $n$ u groBer $t h$ e r e $t$ s c h e $r$ Allokationseffizienz, wie etwa die Pigou'sche Schadensteuer, sind für die umweltpolitische praxis irrelevant, da ihr (allokationsoptimaler) Einsatz zu hohe Informations- und Administrationskosten erfordern würde. Von ganz besonderer Bedeutung 1st, wie wir bereits oben betont haben, die dynamische Allokationseffizienz, also die "incentives for innovation" der umweltpolitischen Instrumente 263) Die "incentives for innovation", die von den umweltpolitischen Instrumenten ausgehen, sollten U.E. ein besonders wichtiges kriterium bel der Auswahl der umweltpolitischen Instrumente sein. Das hat die Konsequenz, daB Emissionsabgaben und Emissionszertifikate, die als einzige umweltpolitischen Instrumente dieses Kriterium erfüllen, ein besonderes Gewicht in der umweltpolitischen Praxis zukommt. Das gilt allerdings fast ausschl1eBlich auf dem Geblet der Luft- und Gewăsserreinhaltung, da bel diesen Umweltmedien auch die (vermutete) 8kologische Inzidenz von Emissionsabgaben und Emissionszert1fikaten groB ist und die Informations- und Administrationskosten relativ gering sind.

Unter dem Aspekt der Allokationseffizienz konnen allerdings auch Benutzungsgebühren und durch öffentliche Kredite finanzierte staatliche Investitionen die am besten geelgneten Instrumente zur Realisierung der umweltpolitischen ziele der Luftrelnhaltung, vor allem aber wohl der Abwasser- und Abfall-

263) Siehe oben S. $360 \mathrm{ff}$. und S. $376 \mathrm{f}$. 
beseitigung sein.

Benutzungsgebühren haben dann eine besonders große Bedeutung, wenn economies of scale großer Umweltschutzanlagen ausgenutzt werden sollen und der Staat als einziger Investor in der Lage 1st, solche groß dimensionierten Anlagen (z.B. Kläranlagen, Abfallbeseitigungsanlagen, Fernhelzwerke) zu erstellen und auch $\mathrm{zu}$ betreiben. Benutzungsgebühren decken in solchen Fällen im allgemeinen nur die laufenden Kosten, während die Investitionskosten aus allgemeinen Deckungsmitteln finanziert werden müssen. Hier bieten sich nun in vielen Fällen aus Gründen der intertemporalen Allokationseffizienz (und unter Umständen auch der intertemporalen Verteilungsgerechtigkeit) Kredite als Finanzierungsinstrument an.

Akzeptlert man als welteres Auswahlkriterium die ordnungspol1tische Konformität der umweltpolitischen Instrumente, so sind die Instrumente des Verursacherprinzips - und unter diesen eben besonders die marktwirtschaftlichen Instrumente, also wiederum Emissionsabgaben und vor allem Emissionszertifikate - den Instrumenten des Gemeinlastprinzips vorzuziehen.

Weniger aus ordnungspolitischen als aus finanzwirtschaftlichen Gründen haben wir als Nebenbedingung einer rationalen Umweltpolitik gefordert, das das öfentliche Budget durch den Einsatz der umweltpolitischen Instrumente möglichst wenig belastet werden soll. Diese Nebenbedingung wird somit zwangsläufig zu einem weiteren Kriterium für die Auswahl der umweltpolitischen Instrumente. Wie die Ausführungen in Abschnitt $C$ dieses Kapitels gezelgt haben, sind auch hier die Instrumente des Verursacherprinzips - und unter diesen vor allem die Emissionsabgaben nicht nur wegen ihrer relativ niedrigen Informations- und Administrationskosten, sondern auch wegen ihres fiskalischen Effekts der - wenn auch zweckgebundenen - Einnahmenerzielung, den Instrumenten des Gemeinlastprinzips uberlegen.

Als letztes wichtiges Kriterium bei der Auswahl der umweltpoli- 
tischen Instrumente wollen wir noch berucksichtigen, wie diese auf andere wirtschaftspolitische ziele (wie Vollbeschäfigung, Wachstum, Preisniveaustabilität, Zahlungsbilanzgle1chgewicht, Wettbewerbsneutralität und Verteilungsgerechtigkeit) wirken. Man mübte, um dieses Kriterium anwenden zu können, wissen, wie die verschiedenen Instrumente und deren unterschiedlichen Dosierung auf die zielerreichung bei diesen zielen wirken. Die Ermittlung dieser ökonomischen Inzidenz der umweltpolitischen Instrumente 1st sicher nicht elnfacher als die der okologischen Inzidenz. Manche Autoren meinen, daB die Anwendung der Instrumente des Verursacherprinzips eher zu Konflikten mit den meisten wirtschaftspolitischen zielen füht als die Anwendung der Instrumente des Gemeinlastprinzips 264) Diese Autoren lassen u.E. allerdings unberücksichtigt, daß die Dosierung der Instrumente des Verursacherprinzips zur Realisierung der umweltpolitischen ziele wegen deren größerer Allokationseffizienz geringer sein kann als die Dosierung der Instrumente des Gemeinlastprinzips. Möglicherwelse wird auch übersehen, das von der Mittelbeschaffung für Subventionen und bffentliche Investitionen in Form von Steuern und Krediten Entzugseffekte ausgehen können, die gesamtwirtschaftlich weitaus negativere Folgen haben konnen als die ökonomische Inzidenz der Instrumente des Verursacherprinzips.

Daß Subventionen in der umweltpolitischen Praxis eine so grobe Bedeutung haben, liegt wenlger an ihrer Effizienz als wohl daran, daB sie gegenüber Auflagen und Abgaben einfacher durchzusetzen sind, doch sollte u.E. die Durchsetzbarke1t kein allzu wichtiges Kriterium für die Auswahl der umweltpolitischen Instrumente sein 265) Das könnte nämlich gesamtwirtschaftlich sehr teuer werden, weil es den weitgehenden Verzicht auf Allokationseffizienz - vor allem die "Incentives for innovation" -

264) Vgl. z.B. Siebert, H., Analyse der Instrumente der Umweltpolitik, a.a.0., s. 115 .

265) Diese Auffassung vertritt letztlich auch Siebert, wenn er die Durchsetzbarkeit auch als Kriterium erwăhnt (vgl. ebenda, S. 112). 
bedeutet.

Die bisher geschilderte Uberlegenheit der marktwirtschaftlichen Instrumente "Emissionsabgabe" und "Emissionszertifikate" gilt - wie bereits angedeutet - fast nur für die Umweltmedien Luft und Gewässer und auch hier nur für solche schadstoffe, deren meBtechnische Erfassung relativ einfach ist, also relativ geringe Informationskosten erfordert. Emissionsabgaben und Emissionszertifikate sind auch keine geeigneten Instrumente zur Regulierung toxischer Schadstoffemissionen, da die Wirksamkeit dieser Instrumente entscheidend vom Rationalitätskalkül der Verursacher abhängt. Da man kaum unterstellen kann, daB sich alle Verursacher $z u$ jedem Zeitpunkt rational verhalten, wăre das Risiko einer Abgaben- oder Zertifikatlösung bei der Begrenzung toxischer Belastungen zu groB.

Zur Eindämmung toxischer Schadstoffemissionen in Luft und Gewässer sind deshalb u.E. Auflagen - vor allem in Form von Emissionsstandards und eventuell gar in Form von Verboten (Nullstandards) das geeignete Instrument. Die Effizienz dieses Instruments hängt allerdings stark von der Intensität der Kontrollen ab; Emissionsstandards, die elne hohe ökologlsche Inzidenz haben, sind also teuer.

Auflagen sind U.E. neben Benutzungsgebuhren auch das wichtigste Instrument zur Begrenzung der Bodenbelastung durch Abfälle. Vor allem Produktstandards hinsichtlich der Konsumgütergestaltung, aber auch Herstellungsverbote (z.B. bei schwer beseitigbaren Abfällen), sind geeignete Instrumente zur Lösung des Abfallproblems. Hinsichtlich der Belastung des Bodens durch Bauten aller Art sind ebenfalls Auflagen - etwa in bezug auf den Standort, aber auch in bezug auf asthische Anforderungen - die adäquaten Instrumente.

Die Belastung der Tier- und Pflanzenwelt kann auch am besten durch Auflagen (z.B. Jagdverbote, Verwendungsauflagen für Pflanzenschutzmittel) reguliert werden, aber auch durch öffentllche 
Investitionen (z.B. die Errichtung von wildzäunen entlang von Autobahnen, die Einrichtung von Naturschutzgebieten).

Bel der Begrenzung der Lebensmittelbelastung durch Schad- und Zusatzstoffe sind Produktstandards in Form von Höchstmengenbegrenzungen (Toleranzgrenzen) U.E. das allein in Frage kommende Instrument.

Zur Lärmbekämpfung werden in der Regel ebenfalls Produktstandards als geeignetes Instrument empfohlen 266) Wir halten jedoch auch eine Lärmemissionsabgabe für ein erwägenswertes Instrument zur Reduzlerung des Lärms, wobel allerdings nicht die aktuellen Lärmemissionen der Lărmquellen (z.B. Kraftfahrzeuge, Flugzeuge, Baumaschinen, Rasenmäher) als Bemessungsgrundlage herangezogen werden soliten, da die dafür erforderlichen Messungen zu aufwendig wären, sondern die potentielien Lärmemissionen in dB(A) der jeweiligen Lärmquellen, dle relativ elnfach auf einem Prüfstand (eventuell von den Technischen Uberwachungsvereinen) festgestellt werden könnten. Die Lärmemisslonsabgabe würde also unabhängig davon, wie oft und in welcher aktuellen Lautstärke die Lärmquelle emittlert, fällig. Sie rückt damit in die Nähe der Produktstandards, unterscheidet sich aber von diesen dadurch, dab sie einen $\delta k \circ n \circ m i s c h$ e $n$ Anreiz darstellt, wenlger lärmintensive und damit weniger abgabenbelastete Produkte herzustellen, was zweifellos die Allokationseffizienz gegenüber Produktstandards erhöht.

Von den Instrumenten des Gemeinlastprinzips haben wir bisher nur offentliche Investitionen, die durch Kredite $z u$ finanzieren sind, befurwortet. Aber auch andere allgemeine Deckungsmittel sind bei der Finanzierung bestimmter Umweltschutzaufgaben gerechtfertigt. So halten wir allgemeine steuern oder eventuell auch eine (zweckgebundene) Umweltsteuer für adäquate Möglichkelten, die Schaffung und Verbesserung der umweltpolitischen

266) Vgl. z.B. Siebert, H., Analyse der Instrumente der Umweltpolitik, a.a.0., s. 117 . 
Rahmenbedingungen zu finanzieren 267 ?

Daß sich auch Subventionen zur Finanzierung des Umweltschutzes rechtfertigen lassen, haben wir bereits dargestellt 268). Neben den dort geschilderten Rechtfertigungsgründen für Subventionen zum 2 wecke des Umweltschutzes möchten wir noch auf zwei Fälle hinwelsen, in denen U.E. Subventionen durchaus gerechtfertigt sind. Es ist zum elnen der Fall positiver Umweltexternalitäten. Sie entstehen z.B. durch die Aktivitaten in der Land- und Forstwirtschaft dadurch, daB Land- und Forstwirte in vielen Fallen - sozusagen nebenbe1 - landschaftspflegerische Aufgaben erfüllen, die Ihnen nicht entgolten werden. Der gesellschaftliche Nutzen, den sie dadurch stiften, ist ihnen aus Grüden der Allokationseffizienz in Form von Subventionen zu entgelten. Auch hierbel handelt es sich um die Internalislerung (jetzt positiver) externer Effekte. Zum anderen lassen sich Subventionen in den Fällen rechtfertigen, in denen die Träger von Umweltbelastungen Maßnahmen des Emissionsschutzes ergreifen (mulssen), weil die Verursacher nicht oder nur zu hohen Kosten identifizierbar sind 269?

Die zur Bekämpfung der komplexen Arten der Umweltbelastung verfügbaren umweltpolitischen Instrumente haben - wie dieser $\mathrm{Ab}$ schnitt zelgte - fast alle elne mehr oder minder wichtige Bedeutung. Elnen optimalen policy-mix der Instrumente unter zugrundelegung der in diesem Abschnitt geschilderten Kriterien zu finden, 1st eine - auch theoretisch - nur schwer losbare Aufgabe, well vor allem die 8kologische und 8konomische Inzidenz der umweltpolitischen Instrumente nur schwer zu bestimmen ist. Letztlich hilft auch hier nur eine politische Entscheidung darüber, welche Instrumente zur Bekämpfung welcher Umweltbelastungen eingesetzt werden sollen.

267) Siehe oben S. 395 und S. 399.

268) Siehe oben S. $340 \mathrm{f}$. und S. $390 \mathrm{f}$.

269) So sollte Z.B. der Einbau schalldämmender Bauteile bei Wohngebäuden dann subventioniert werden, wenn die Verursacher des Lärms nicht identifiziert werden können. 
Die verschiedenen Arten der Umweltbelastung sind alltägliche Begleiterscheinungen der mit Produktion und Konsum verbundenen Aktivitäten. Die Wirkungen der Schadstoffimmissionen auf die menschliche Gesundheit, das ökologische Gleichgewicht und die materielle Umwelt des Menschen sind äußerst komplex. Vor allem Kombinations- und Langzeitwirkungen sind noch weitgehend unerforscht. Unter solchen Bedingungen ist eine rationale Umweltpolitik vor allem aus zwel Gründen sehr schwierig: Wegen der ungenügenden Kenntnisse über viele Schadstoffwirkungen lassen sich zum einen die ziele der Umweltpolitik in Form der Immissionsstandards nur schwer operationalisieren; naturwissenschaftliche und medizinische Forschungsergebnisse hinsichtlich der Schadstoffwirkungen liefern in vielen Fällen günstigstenfalls Grenzwerte, die als Ausgangswerte für die politische Festlegung der Immissionsstandards dienen können. Bel der Fixierung der Immissionsstandards wirken im vorparlamentatischen Raum immer mehr die verschiedenen Interessengruppen mit, was bel ausgeglichener Vertretung der potentiellen Verursacher und Träger der Umweltbelastungen positiv zu beurteilen ist. Die andere Schwierigkeit folgt aus der Komplexität der verschiedenen Arten der Umweltbelastung. Es gibt eine Vielzahl verschiedener Emissionsquellen an einer Vielzahl von standorten, an denen jeweils unterschiedliche natürliche Bedingungen $z u$ ganz verschiedenen Immissionsbelastungen führen. Eine rationale Umweltpolitik mu $\beta$ diesen unterschiedlichen Phänomenen mit differenzierten Maßnahmen des technischen Umweltschutzes $\mathrm{zu}$ begegnen versuchen. In welcher Phase des Schadstoffflusses technische Maßnahmen des Umweltschutzes eingesetzt werden sollen, muß mit Hilfe einer optimalen Planung bestimmt werden.

Die staatliche Planung sollte sich soweit wie möglich auf die Planung der umweltpolitischen Rahmenbedingungen und den optimalen policy-mix des juristischen und finanzwirtschaftichen Durchsetzungsinstrumentariums beschränken . Besonders marktwirtschaftliche Durchsetzungsinstrumente wie 
Emissionsabgaben und Emissionszertifikate überlassen die optimale Planung des technischen Umweltschutzes weitgehend den Unternehmen. Da sie sich auch als besonders effizient erweisen, sollten sie jenen Instrumenten vorgezogen werden, die interventionistische Züge haben (wie Auflagen). Dadurch wird auch der Gefahr begegnet, daß der staat durch Wahrnehmung der Umweltschutzaufgabe zum "Etat fonctionnel $\rightarrow$ wird, der Wesen und Substanz (Smend) verliert" 1)

Durch Partizipation der umweltbewußt gewordenen Bevölkerung bel umweltbedeutsamen Entscheidungs $v$ o $r$ e $r$ e $i$ u $\mathrm{g}$ e $n$ können andererseits Wesen und substanz eines demokratischen Staates sogar gestärkt werden, wenn Partizipation nicht als "Demokratisierung" des Entscheidungs $p$ r $z$ e s s e s mißverstanden wird. In der Tatsache, daB die von den verschiedenen Umweltbelastungen betroffenen Bürger nicht mehr zu den "vergessenen Gruppen, die schweigend leiden" 2) gehören, sondern daB sie sich in potenten Bürgerinitiativen organisieren, liegt eine große Chance für eine wirksame Umweltpolitik. Bürger, welche vom staat mehr Umweltschutz verlangen, müssen gewärtig sein, daß der staat von ihnen dafür Opfer abverlangt. Die Bürger haben es in der Hand, über die Art ihres opfers zu entscheiden. Umweltschutz kann z.B. darin bestehen, keine Kernkraftwerke $z u$ bauen. Das Opfer besteht dann zumindest langfristig in einem Verzicht auf Wohlstand, zivilisatorische Errungenschaften, Annehmlichkeiten aller Art, die von Energie abhängig sind. Umweltschutz kann aber auch darin bestehen, das Risiko und die Umweltbelastungen, die mit dem Betrieb und der Entsorgung von Kernkraftwerken verbunden sind, zu minimieren. Das Opfer besteht dann in hohen Aufwendungen für die Reduzierung der thermischen Belastung der Gewässer oder der Luft sowle für die Reaktorsicherheit, den möglichst gefahrlosen Transport und die möglichst slchere Lagerung des radioaktiven Mülls.

1) Saladin, P., Gleichgewichtssicherung als Staatsaufgabe, a.a.o.

2) Olson jr., M., Die Logik des kollektiven Handelns, Tübingen 1968, S. 163. 
Im Lichte dieser Alternativen wird die Entscheldung wohl zugunsten der zweiten Alternative fallen, und dies umso mehr, je mehr Vertrauen die Bürger in die Bereitschaft und Fähiakeit der Politiker haben, alles in ihrer Macht stehende zu tun, damit die Bevölkerung vor den Gefahren der radioaktiven Belastung so gut wie möglich geschützt wird.

In Zeiten großer Investitionsunlust und hoher Arbeitslosenquoten elnerseits und einer hohen Belastung des öffentlichen Budgets andererseits wird es besonders notwendig sein, die ziele des Umweltschutzes möglichst effizient zu errelchen, also einerseits die finanziellen Opfer der Bevölkerung (Unternehmer, Arbeltnehmer) in Form der Abgabenbelastung und höherer Preise für Zwecke des Umweltschutzes möglichst zu minimieren und andererseits das öffentliche Budget möglichst wenig zu belasten. Die konsequente Anwendung des Verursacherprinzips ist unseres Erachtens wegen der großen Effizienz seiner Instrumente am besten geeignet, diese Aufgabe zu lösen. Leider scheint sich in der Bundesrepublik Deutschland eine andere "Lösung" anzubahnen. Sie besteht darin, die Normen herabzusetzen, also weniger strenge Immissionsstandards festzulegen, als dies aus Gründen eines wirksamen Schutzes vor Belastungen der Gesundheit und des ökologischen Glelchgewichts notwendig wäre. Ein beredtes Belspiel dafür ist die Festlegung des Satzes für die Abwasserabgabe auf eine Höhe, die völlig unzureichend ist, um die im Schnitt angestrebte Gewässergüteklasse II zu erreichen. Ein anderes Beispiel ist die Festsetzung der Immissionsrichtwerte für Straßenverkehrslärm. Diese Standards gewährleisten keinen Schutz der Bevölkerung vor Straßenverkehrslärm. Ihr einziger Effekt ist eine Entlastung des öffentlichen Budgets, denn der Staat wäre als Eigentümer der straßen für den notwendigen Bau von Lärmschutzeinrichtungen zuständig. Es ist abzusehen, daß durch dieses schlechte Beispiel, mit dem der staat vorangeht, weitere - außer der Abwasserabgabenregelung - nach sich ziehen wird.

Eine noch so rege gesetzgeberische Tätigkeit auf dem febiet des 
Umweltschutzes ${ }^{3)}$ kann den Eindruck nicht verwischen, daß faktisch noch $\mathrm{zu}$ wenig geschieht, um den Schutz der menschlichen Gesundheit und seiner natürlichen und materielien Umwelt vor Umweltbelastungen zu schüzen.

Wir haben unsere Schlußbetrachtung mit der Feststellung begonnen, daß Umweltbelastungen alltägliche Begleiterscheinungen der Produktion und des Konsums sind. Wir können sie mit der Feststelluna beenden, daß auch der Umweltschutz Gegenstand der alltäglichen Beschäftigung von Politikern, Verbandsfunktionären, Produzenten, Konsumenten und Bürgern geworden ist - zwar nicht in dem Umfang, wie es unseres Erachtens wünschenswert und notwendig wäre und schon gar nicht in dem Umfang, wie es sich ökologisten vielleicht zu Beginn der Umweltschutz-Welle erhofft haben, aber doch mehr als viele es $1 \mathrm{~m}$ Zeichen der Energie- und Wachstumskrise vielleicht befürchteten. Der Umweltschutz ist als öffentliche Aufgabe etabliert ${ }^{4}$ ) und seinen $\mathrm{zielen}$ wird in einer funktionierenden parlamentarischen Demokratie letztlich in dem Umfang entsprochen, in dem es die Mehrzahl der Bürger wünscht. Es scheint allerdings, daß das Gemeinlastprinzip als konfliktmindernde Strategie 5) immer mehr dem Verursacherprinzip vorgezogen wird.

3) Vgl. den Tätigkeitsbericht der Bundesregierung über die Arbeit in der 7. Legislaturperiode, in: Bulletin der Bundesregierung Nr. 92 v. 5.8.76, S. 905 ff.

4) Vgl. das Mehrjährige öffentliche Investitionsproqramm zur wachstums- und umweltpolitischen Vorsorge, in: Bulletin der Bundesregierung $\mathrm{Nr} .33 \mathrm{v} .30 .3 .77$, S. $305 \mathrm{ff}$.

5) Vgl. RSU, Umweltgutachten 1974, a.a.O., S. 155. 
Abendt, R., Anwendungen der linearen Optimierung im Gewässerschutz, in: H. Hahn, Operations Research und seine Anwendung in der Siedlungswasserwirtschaft, Bielefeld 1972 , S. 97 ff.

Ackermann, K./ Geschka, H./ Karsten, D., Gutachten zur Gesamtbelastung der Volkswirtschaft durch das Umweltprogramm der Bundesreglerung, in: Materialien zum Umweltprogramm der Bundesregierung, zu BT-Drucksache VI/2710, S. 593 ff.

Ackermann, K./ Geschka, H./ Karsten, D., Die wirtschaftspolitische Lösung: Verursacherprinzip, in: Umwelt 1972, 2, S. $27 \mathrm{ff}$.

Aeb1, H.. Die heutige Belastung der Ernährung durch Fremdstoffe, in: Universitas, 27. Jg., 1972, S. $1185 \mathrm{ff}$.

Aguilar, R. u.a. A Model for the Evaluation of Alternative Policies for Atmospheric Pollutant Source Emissions, in: 5 th Conference on Optimization Techniques, Part II, Lecture Notes in Computer Science, hrsg. v. G. Goos und J. Hartmanis, Berlin - Heidelberg New York 1973

Aktionsprogramm für die Politik im wissenschaftlich-technologischen Bereich, Bulletin d. EG, Beilage 14/73

Alessio, F.J.. The Opportunity cost of Pollution Abatement in a Steady-State Expanding Economy, in: The Annals of Regional Science, vol. 5, 1971, S. $84 \mathrm{ff}$.

Altvater, E., Gesellschaftliche Produktion und 6konomische Rationalität. Externe Effekte im Wirtschaftssystem des Sozialismus, Frankfurt - Wien 1969

Anderson, R.J./Crocker, T.D., Air Pollution and Property Values: A Reply, in: Review of Economics and Statistics, vol. 54, 1972, S. $470 \mathrm{ff}$.

Anderson, R.J./ Crocker, T.D., Air Pollution and Residential Property Values, in: Urban Studies, vol. 8, 1971, S. $171 \mathrm{ff}$.

Andreae, C.A., Umweltschutz und Marktwirtschaft, in: Wirtschaft und Wettbewerb 11/12, 1971, S. $753 \mathrm{ff}$.

Anno 709 p.R., SchluBbericht der Prospektivkonferenz der Neuen Helvetischen Geselischaft, Aarau und Frankfurt 1973

Ant, H., Olverschmutzung der Meere und ihre Folgen, in: Olschowy, G. (Hrsg.), a.a.0., S. $81 \mathrm{ff}$.

Ant, H., Blologische Probleme der Verschmutzung und akuten Vergiftung von Fliebwässem unter besonderer Berücksichtigung der Rheinvergiftung im Sommer 1969, in: Schriftenreihe fur Landespflege und Naturschutz, H. 4, Bonn-Bad Godesberg 1969, S. $97 \mathrm{ff}$. 
Ant, H., Verschmutzung der FlieBgewăsser und thre Folgen - am Belspiel der Lippe und des Rheins, in: Olschowy, G. (Hrsg.), a.a.0., s. 35 ff.

Antoine, S., Qualität der Umwelt und Raumordnung, in: Aufgabe Zukunft - Qualität des Lebens, Bd. 4: Umwelt, a.a.0., S. $73 \mathrm{ff}$.

Antweiler, H., Tiere als Indikatoren der Luftverschmutzung, in: Probleme der Umweltforschung, a.a.0., S. 46 ff.

Antweiler, H./Pott, F., Tlerexperimentelle Ergebnisse über die Wirkung partikel- und gasformiger Luftverunreinigungen, in: Zentralblatt für Bakterlologie .... a.a.0., S. $263 \mathrm{ff}$.

Armstrong, T.R. (Hrsg.), Why Do We Still Have an Ecological Crisis? Englewood Cliffs, N.J., 1972

Arnold, V., Kuppelprodukte, offentliche Ungüter und externe Effekte, 1n: Zeitschrift für die gesamte staatswissenschaft, Bd. 132, 1976, S. 91 ff.

Arthur, D.R., Education and Human Environment, in: International Journal of Environmental studies, vol. 1, 1971, S. 315 ff.

Atkinson, S.E./ Lewis, D.H., A Cost - Effectiveness Analysis of Alternative Air Quality Control Strategies, in: Journal of Environmental Economics and Management, vol. 1, 1974, S. 237 ff.

Aufgabe Zukunft - Qualität des Lebens, Beitrăge zur vierten internationalen Arbeitstagung der Industriegewerkschaft Metall für die Bundesrepublik Deutschland vom 11.-14. April 1972 in Oberhausen, 10 Bde., Frankfurt am Main 1973

Aurand, K., Umweltschutz und Gesundheit, in: Aufgabe $\mathrm{zukunft}$ - Qualität des Lebens, Bd. 4: Umwelt, a.a.0., S. $37 \mathrm{ff}$.

Bär, F., Die toxikologische situation in der modernen Zivilisation, in: Sioll, H. (Hrsg.), a.a.0., S. $165 \mathrm{ff}$.

Bär, F./ Grunow, W., Hygienisch-toxikologische Aspekte der Umweltsituation, in: Schultze, H. (Hrsg.), a.a.0., S. $287 \mathrm{ff}$.

Bäuerle, D./ Hornung, G. (Hrsg.), Umwelt. Blologisch - sozialkundliche Arbeitshefte, Schülerarbeitsheft und Lehrerheft, Opladen 1972

Balke, S., Umweltbedingungen als Lebensgrundlage. Schriftenreihe der Vereinigung Deutscher Gewässerschutz EV-VDG, Nr. 28, Bonn-Bad Godesberg 1970

Ball, D.F.. The Identification and Measurement of Gaseous Pollutants, in: International Journal of Environmental Studies, vol. 1., 1971, S. $267 \mathrm{ff}$. 
Baltes, H./Nowak, W., Umweltstatistik - ein Instrument der Umweltplaung, in: Wirtschaft und Statistik, 1974, 4, S. $237 \mathrm{ff}$.

Bartelmus, P., Probleme der Entwicklung eines umweltstatistischen Systems, in: Statistische Hefte, N.F., 14. Jg., 1973 , S. $123 \mathrm{ff}$.

Bartels, H., Statistik als Hilfsmittel der Umweltpolitik, in: Allgemeines Statistisches Archiv, Bd. 59, 1975, S. $11 \mathrm{ff}$.

Bartocha, B., Technology Assessment - Verfahren zu einer Technologie-Bewertung. Lebensqualität in Mark und Pfennig, in: Umwelt 1972, 6, S. $30 \mathrm{ff}$.

BASF, Geschäftsbericht für 1971

BASF, Werk und Umwelt, Ludwigshafen 0.J.

Basler, E., Umweltprobleme aus der Sicht der technischen Entwicklung, in: NZZ, FA Nr. 132 vom 16.5.1971

Bauer, K., Umweltfreundliche Technik - ein Ziel und seine Verwirklichung, in: Umwelt 1974, 4, S. $44 \mathrm{ff}$.

Bauer, R.K., Methodische Grundprobleme der Umweltstatistik, in: Allgemeines Statistisches Archiv, Bd. 59, 1975, S. $32 \mathrm{ff}$.

Baumberger, H., Wirtschaftliche Probleme einer umweltkonformen Energieversorgung, in: von Walterskirchen, M.P. (Hrsg.), a.a.0., S. 225 ff.

Baumol, W.J./Oates, W.E., The Use of Standards and Prices for Protection of the Environment, in: Swedish Journal of Economics, vol. 73, 1971, S. $42 \mathrm{ff}$.

Bea, F.X., Die Verteilung der Lasten des Umweltschutzes nach dem Verursacherprinzip, in: Wist, 2. Jg., 1973, S. $453 \mathrm{ff}$.

Beck, E.G., Fasermengen sind schwer zu messen, in: Umwelt 1975, 4, S. $29 \mathrm{ff}$.

Becker, Herbert, Der Nachweis von Luftfremdstoffen mit abstimmbaren Lasern, in: Umwelt 1972, 1, S. $45 \mathrm{f}$.

Becker, Herbert, Gleichzeitig mehrere stoffe messen, in: Umwelt 1973, 6, S. 25 ff.

Becker, K.H./Schurath, U., Photochemie der Luftverschmutzung, in: Schultze, H. (Hrsg.), a.a.O., S. $230 \mathrm{ff}$.

Beckerman, W., Environmental Policy Issues: Real and Fictitious, in: OECD (Hrsg.), Problems of Environmental Economics, a.a.O., S. $19 \mathrm{ff}$.

van Belle, G./Schneiderman, M., Some Statistical Aspects of Environmental pollution and Protection, in:

International Statistical Review, vol. 41, 1975, S. 315 ff. 
Bender, D., Makroökonomik des Umweltschutzes, Göttingen 1976

Benecke, P., Ökologische Probleme des Wasserhaushalts auf dem Lande, in: Probleme der Umweltforschung, a.a.o., S. 112 ff.

Benthem, R.J., Stădtische Ballungen, in: Offner, H. (Hrsg.), a.a.O., S. $11 \mathrm{ff}$.

Bernholz, P., Grundlagen der politischen Okonomie, Bd. 1, Tübingen 1972

Best, $w$. , Umweltschutz aus der sicht der Länder unter besonderer Berücksichtigung des Landes Hessen, in: Umweltschutz - eine Aufgabe unserer zelt, hrsg. vom Institut für Gewerbliche wasserwirtschaft und luftreinhaltung e.V.. Köln 1971

Blckel, E., Müllkippen als künftige Erholungsgebiete in Holland, in: Müll und Abfall, 4. Jg., 1972, H. 3, S. 100

Blederbick, J./Gronwald, D., Umweltingenieur nach neun Semestern, in: Umwelt $1974,5, \mathrm{~S} .54 \mathrm{ff}$.

Binswanger, H.C., Umrisse einer umweltkonformen Wirtschaftsordnung, in: Schlemmer, J. (Hrsg.), a.a.O., S. 118 ff.

Binswanger, H.C., Eine umweltkonforme Wirtschaftsordnung, in: von Walterskirchen, M.P. (Hrsg.), a.a.0., S. 127 ff.

Birkle, M. u.a., Informationssystem Umwelt. Konzept eines Messund Planungsinformationssystems für Umweltmaßnahmen, hrsg. von der Siemens AG., München 1973

Blair, R.D., The Clean Air Act of 1970, in: Land Economics vol. 49, 1973, S. 260 ff.

Blanke, F., Der Christ und die Erde, in: Neue Helvetische Gesellschaft (Hrsg.), a.a.0.. S. $11 \mathrm{ff.}$

Blel und Umwelt, Berlin 1972

Blohm, H./Steinbuch, K. (Hrsg.), Technische Prognosen in der Praxis, Düsseldorf 1972

Bock, J., Wasser, Abwasser und Müll heute und morgen, in: Wasser und Boden, Bd. 22, 1970, H. 5, S. $117 \mathrm{fF}$.

Boehm, V., Die Abwasserabgabe ist ein Anfang, in: Umwelt 1976 , 4, S. $258 \mathrm{ff}$.

Böhnke, B., Volkswirtschaftlicher Aufwand für die Wasserversorgung sowle für die Beseitigung flüssiger und fester Abfallstoffe bis zum Jahre 2000, in: Umweltschutz eine Aufgabe unserer Ze1t, hrsg. vom Institut für Gewerbliche Wasserwirtschaft und Luftreinhaltung e.V., Koln 1971, S. 65 ff.

Böhnke, B., Was vertragen überhaupt unsere Binnengewässer an Belastungen und welcher Reinigungsgrad läBt sich hieraus für Kläranlagen ableiten?, in: Schultze, H. (Hrsg.), a.a.0., S. $95 \mathrm{ff}$. 
Böhnke, B., Siedlungswasserwirtschaftliche Probleme bei der Müllbeseitigung, in: Niedersächsiche Gemeinde, Bd. 20, 1968 , H. 4, S. $97 \mathrm{ff}$.

Bohm, P., A Note on the Problem of Estimatina Benefits from Pollution Controll, in: OECD (Hrsg.), Problems of Environmental Economics, a.a.0., S. $83 \mathrm{ff}$.

Bonus, H., Ubber Schattenpreise von Umweltressourcen, in: Jahrbuch für Sozialwissenschaft, Bd. 23, 1972, S. 342 ff.

Bora, G., Planwirtschaft als Voraussetzung einer wirksamen Umweltpolitik, in: Horn, C./von Walterskirchen, M.P./ Wolff, J. (Hrsg.), a.a.O., S. 245 ff.

Borchardt, K., Volkswirtschaftliche Kostenrechnund und Eiqentumsverteilung. Bemerkungen zum Problem der Sozialkosten, in: Jahrbücher für Nationalökonomie und Statistik, Bd. 178, 1965, S. $70 \mathrm{ff}$.

Borneff, J., Forderungen an die Reinhaltung unserer Binnengewässer aus hygienischer Sicht, in: Schultze, H. (Hrsg.), a.a.0., S. $87 \mathrm{ff}$.

Borneff, J., Hygiene. Ein Leitfaden für Studenten und Ärzte, Stuttgart 1971

Borneff, J., Schadstoffe im Wasser: Herkunft, Bedeutung und Beseitigung, in: Zentralblatt für Bakterioloqie .... a.a.0., S. $220 \mathrm{ff}$.

von Borries, D.F.W., Zur Konstruktion von Umweltindizes, in: Allgemeines Statistisches Archiv, Bd. 59, 1975, S. $41 \mathrm{ff}$.

Boulding, K.E., The Economics of the Coming Spaceship Earth, in: Jarrett, H. (Hrsg.), a.a.O., s. $3 \mathrm{ff.}$

Bragdon, C., The Community Noise Problem: Factors Affectina its Management, in: Natural Resources Journal, vol. 10, 1970, S. $687 \mathrm{ff}$.

Bramigk, D., Bürgerinitiativen zum Umweltschutz, in: U 1973, 5

Bramigk, D., warten auf Initialzündungen, in: U 1975, 4

Brasse, P., Optimierung der Müllbelastung, in: Seminarbericht 8 der Gesellschaft für Regionalforschuna, Münster 1974 , S. $29 \mathrm{ff}$.

Brasse, P./Thoss, R./Könnecke, U., Ein aktivitätsanalytisches Umweltmodell zur Flächennutzungsplanung, in: Seminarbericht 8 der Gesellschaft für Reoionalforschung, Münster $1974, \mathrm{~S} .15 \mathrm{ff}$.

Brennpunkt Müllproblem, hrsg. vom Presse- und Informationsamt der Bundesregierung, Bonn 1968

Brösse, U., Ziele in der Regionalpolitik. Zielforschund und Probleme der Realisierung von Zielen, Berlin 1972 
Bryan, M./William, T., Cause for Concern - Nolse Pollution of the Work Environment, in: International Journal of Environmental Studies, vol. 1, 1971, S. 99 ff.

Buchanan, J.M., External Diseconomies, Corrective Taxes, and Market Structure, in: Staaf, R.J./Tannian, F.X. (Hrsg.), a.a.0., s. 269 ff.

Buchanan, J.M., Joint Supply, Externality and Optimality, in: Economica, N.S., Bd. 33, 1966, S. $404 \mathrm{ff}$.

Buchanan, J.M./Stubblebine, W.C., Externality, in: Staaf, R.J./ Tannian, F.X. (Hrsg.), a.a.O., S. $272 \mathrm{ff.}$

Buchwald, K., Umweltschutz und Gesellschaft, in: Naturschutzund Naturparke, Bd. 60, 1971, H. 1, S. $1 \mathrm{ff}$.

Budowski, G., Conservation and the Future Environment of Mankind, In: Sioll, H. (Hrsg.), a.a.O., S. 439 ff.

Budyko, M.T., Das Klima der Zukunft, in: Forschung 173, Frankfurt/Main 1972, S. 85 ff.

Bullinger, M., Rechtsfragen des Verursacherprinzips beim Umweltschutz, in: Das Verursacherprinzip und seine Instrumente, a.a.0., S. $69 \mathrm{ff}$.

Bundesraumordnungsprogramm, BT-Drucksache 7/3584

Calabresi, G., Transaction Costs, Resource Allocation, and Liability Rules, in: Dorfman, R./Dorfman, N.S. (Hrsg.), a.a.0., S. $194 \mathrm{ff}$.

Cansier, D., Okonomische Grundprobleme der Umweltpolitik, Berlin 1975

Caponera, D.A., Towards a New Methodological Approach in Environmental Law, in: Natural Resources Journal, vol. 12, 1972, S. $133 \mathrm{ff}$.

Carson, R., Der stumme Frühling, München 1970

Caspers, H., Sieben Jahre Förderung des Schwerpunktprogramms "Litoralforschung - Abwăsser in Küstennähe", In: DFG-Mitteilungen, 2/73, S. $64 \mathrm{ff}$.

Cassell, E.J.. The Health Effects of Air Pollution and their Implications for Control, in: Law and Contemporary Problems, vol. 33, 1968, S. $197 \mathrm{ff}$.

Cazes, B.. Environmental Quality Indicators and Social Indicators, in: OECD (Hrsg.), Problems of Environmental Economics, a.a.0., s. $77 \mathrm{ff}$.

Claussen, E., Gesundheitsingenieur - ein Schmalspurmediziner?, in: U 1973,1

Cmelka, D., Für elne sorgsame Umwelt-Terminologie, in: Umwelt 1975,5, S. 12 und Umwelt $1976,1, S .32$

Coase, R.H., The Problem of Social Cost, in: Staaf, R.J./Tannian, F.X. (Hrsg.), a.a.0., S. $119 \mathrm{ff}$. 
Coddington, A./Opschoor, H./Pearce, D., Some Limitations of Benefit-Cost Analysis in Respect of Programmes with Environmental Consequences, in: OECD (Hrsa.), Problems of Environmental Economics, a.a.O., S. $119 \mathrm{ff}$.

Coenen, R. u.a., Alternativen zur Umweltmisere. Raubbau oder Partnerschaft, München 1972

Comar, C.L./Thompson jr., J.C., Social, Envi ronmental, and Health Costs, in: Summary Report of the Cornell Workshop on Energy and the Environment, a.a.0., S. $27 \mathrm{ff}$.

Committee on the Problem of Noise, Noise, Final Report, H.M.S.O., London 1963

Commoner, B., D1e Bedeutung der Blosphäre, in: Lohmann, M. (Hrsg.), a.a.0., S. $100 \mathrm{ff}$.

Congress and the Nation's Environment. Environmental Affairs of the 91st Congress, hrsg. von Environmental Policy Division/Congressional Research Service/Library of Congress, Washington, D.C., 1971

Corti, W.R., Mensch und Natur, in: Neue Helvetische Gesellschaft (Hrsg.), a.a.O., S. 53 ff.

The Cost of Clean Air. Second Report of the Secretary of Health, Education, and Welfare to the Congress of the United States, Doc No. 91-65, Washington, D.C. 1970

Creutz, G., Bericht uber die Diskussion der Vorträge zum Thema "Umweltschutz und Statistik" auf der 45. Jahreshauptversammlung der Deutschen Statistischen Gesellschaft am 26.9.1974 in Dortmund, in: Allgemeines Statistisches Arch 1v, Bd. 59, 1975 , S. 65 ff.

Dahmen, E., Umweltschutz und ökonomische Systeme, in: Glagow, M. (Hrsg.), a.a.0., S. $61 \mathrm{ff}$.

Dales, J. H., Pollution, Property and Prices, An Essay in Policy-making and Economics, Toronto 1968

Davis, 0.A./Whinston, A., Externalities, Welfare, and the Theory of Games, in: Journal of Political Economy, vol. 70, 1962, S. 241 ff.

Degenhardt, K.-H., Teratologische Probleme der Umweltverschmutzung, in: Schultze, H. (Hrsg.), a.a.O., S. $290 \mathrm{ff}$.

Delmhorst, B., Das Verursacherprinzip: Der kategorische Imperativ des Umweltschutzes, in: Die Neue Gesellschaft, 1972 , S. $759 \mathrm{ff}$.

Delmon, B., Umweltingenieure von der belgischen Universität Löwen, In: Umwelt 1973, 6, S. $44 \mathrm{f}$.

Demsetz, H., Toward a Theory of property Rights, in: American Economic Review, vol. 57, 1967 , papers and proceedings, S. 347 ff. 
Denkschrift des Hauptausschusses der Ministerkonferenz für Raumordnung, in: Raumordnung und Umweltschutz, a.a.O.

Deutsche Gesellschaft für Ernährung e.v., Ernährungsbericht 1973, Frankfurt am Main 1973

Deutsches Institut für Urbanistik / Deutscher städtetag, Kommunale Gebuhrenhaushalte, Teil 1: Kalkulationsverhalten und Deckungsgrade, Berlin 1976

DFG, Fluglärmwirkungen. Eine interdisziplinäre Untersuchung über die Auswirkungen des Fluglärms auf den Menschen, 3 Bde., Boppard 1974

DFG, Die Förderungsaktivitäten der DFG auf dem Gebiet der Umweltforschung, in: DFG-Mitteilungen $2 / 74,5.5$ ff.

DFG, Umweltforschung, in: DFG-Mitteilungen 2/73

DFG, Umweltforschung, Aufgaben und Aktivitäten der DFG 1950 bis 1970, Bonn-Bad Godesberg 1971

Dierkes, N., Qualität des Lebens und unternehmerische Entscheidung. Ansätze zu einem gesellschaftsbezogenen Rechnungslegungs- und Planungssystem, in: BattelleInformation 17, 1973, s. $23 \mathrm{ff}$.

Dierkes, N., Technology Assessment in der BRD - Eine Stellungnahme, in: Battelle-Information 19, 1974, S. 23 ff.

Dittes, H., Die Finanzierungshilfen des Bundes und der Länder an die gewerbliche Wirtschaft, Frankfurt am Main 1976

von Dobschütz, L./Mathias, W. , Umweltverträglichkeitsprüfung offentlicher Maßnahmen. Ein Schema zur Kontrolle, in: Umwelt 1973, 2, S. 35 ff.

Döllekes, P., Die Optimierung der Abgasbelastung in der Bundesrepublik Deutschland, in: Seminarbericht 8 der Gesellschaft für Reglonalforschung, Münster 1974, S. $89 \mathrm{ff}$.

Dörge, F.W. (Hrsg.), Qualität des Lebens, Opladen 1973

Domsch, K.H., Zum Problem der Blozide, in: Olschowy, G. (Hrsg.), a.a.0.. S. $143 \mathrm{ff}$.

Dorcey, A.H.J., Effluent Charges, Information Generation and Bargaining Behavior, in: Natural Resources Journal, vol. 13, 1973, S. $118 \mathrm{ff}$.

Dorfman, R./Dorfman, N.S. (Hrsg.), Economics of the Environment, New York 1972

Downs, A., Up and down with ecology - the "issue - attention cycle", in: Public Interest, vol. $28,1972, \mathrm{~S} .38 \mathrm{ff}$.

DreiBlgacker, H.L./Surendorf, F./Weber, E., Zum Entwurf der Technischen Anleitung zur Reinhaltung der Luft. Dem Stand der Technik angepast, in: Umwelt 1974, S. $20 \mathrm{ff}$. 
Dreyhaupt, F.J., Das Emissionskataster, in: Schultze, H. (Hrsg.), a.a.0., S. 212 ff.

Dreyhaupt, F.J., Emissionskataster als Hilfsmittel zur Luftreinhaltung, in: Umweltschutz - eine Aufgabe unserer zeit, hrsg. vom Institut für Gewerbliche Wasserwirtschaft und Luftreinhaltung e.V., Köln 1971, S. 45 ff.

Dreyhaupt, F.J., Die modellmäBige Ermittlung und Darstellung der Lärmverteilung in Stadtstrukturen, in: Deutscher Arbeitsring für Lärmbekämpfung e.V. (Hrsạ.), Verkehrslärmtagung in Bonn-Bad Godesberg vom 19./20.4. $1971, \mathrm{~S} .12 \mathrm{ff}$.

Dreyhaupt, F.J., Luftreinhaltung als Faktor der stadt- und Regionalplanung, Aachen 1970

Drobil, M., Der Lärmschutz in der Raumplanung, in: Mitteilungen des Österreichischen Instituts für Raumplanung, 1970, $143 / 144$, S. $11 \mathrm{ff}$.

Dror, Y., Comprehensive Planning: Common Fallacies Versus Preferred Features, in: F. von Schagen (Hrsg.), Essays in Honour of Professor Jac P. Thijsse, Paris 1967

Dubos, R., Man, Medicine, and Environment, Harmondsworth 1970

Dubos, R., Promises and Hazards of Man's Adaptability, in: Jarrett, H. (Hrsg.), a.a.O., S. $23 \mathrm{ff}$.

Dürr, E., Wirtschaftspolitische Ziele - ein historischer Uberblick, in: Recktenwald, H.C. (Hrsg.), a.a.O., S. 9 ff.

Ecker, J.G./Mc Namara, J.R., Geometric Programming and the Preliminary Design of Industrial Waste Treatment Plants, in: Water Resources Research, vol. 7, 1971, S. $18 \mathrm{ff}$.

The Economics of Clean Air. Annual Report of the Administrator of the Environmental Protection Agency to the Congress of the United States, March 1971, U.S.G.P.O. Washington, D.C. 1971

The Economics of Clean Air. Annual Report of the Administrator of the Environmental Protection Agency to the Congress of the United States, March 1972, U.S.G.P.O., Washington, D.C. 1972

The Economics of National Priorities. Hearings before the Subcommittee on Priorities and Economy in Government of the Joint Economic Committee, part 1, U.S.G.P.O., Washington, D.C. 1971

The Economy, Energy, and the Environment. A Background Study Prepared for the Use of the Joint Economic Committee, U.S.G.P.O., Washington, D.C. 1970

The Education of Engineers in Environmental Health, Report of a WHO Expert Committee, Technical Report Series, No. 376 , Genf 1967 
The New Environmental Education Program, hrsg. vom U.S. Department of Health, Education and Welfare/Office of Education, U.S.G.P.O., Washington, D.C., 1971

Egli, E., Die Erhaltung der Landschaft, in: Neue Helvetische Gesellschaft (Hrsg.), a.a.0., s. 19 ff.

Egli, E., Natur in Not. Gefahren der Zivilisationslandschaft, 2. Auflage, Bern und Stuttgart 1970

Eickel, K.H./Jud, S., Umweltkosten des Straßenverkehrs, in: Umwelt $1974,4, \mathrm{~S} .17 \mathrm{ff}$.

von Eiff, A.W., Mensch und Lärm, in: DFG-Mitteilungen, Jahresversammlung 71, Bonn 1971, S. $28 \mathrm{ff}$.

Einbrodt, H.J., Grenzwerte für staubförmige Luftverschmutzungsstoffe und andere Bekämpfungsmaßnahmen, in: Zentralblatt für Bakteriologie ..., a.a.0., S. $296 \mathrm{ff}$.

Ellenberg, H., Belastung und Belastbarkeit von Ckosystemen, in: Steubing, L./Kunze, C./Jäger, J. (Hrsg.), a.a.O., S. $19 \mathrm{ff}$.

Elster, H.-J., Ökologische Probleme der Binnengewässer, in: Forschung 173, Frankfurt am Main 1972, S. 205 ff.

Elster, H.-J., Forderungen an die Reinhaltung unserer Binnengewässer aus biologischer Sicht, in: Schultze, H. (Hrsg.), a.a.O., S. $93 \mathrm{ff}$.

Die Energiepolitik der Bundesregierung, BT-Drucksache 7/1057

Engelhardt, W., Umweltschutz. Gefährdung und Schutz der natürlichen Umwelt des Menschen, München 1973

Entschließung der Ministerkonferenz für Raumordnung vom 15. Juni 1972, in: Raumordnung und Umweltschutz, a.a.o.

Entwicklungstendenzen des Kraftfahrzeugverkehrs in der Bundesrepublik Deutschland bis 1980 , Schriftenreihe des Verbandes der Automobilindustrie e.V., Nr. 5, Frankfurt am Main 1970

Entwurf eines Gesetzes über Umweltstatistiken, BT-Drucksache $7 / 988$

Entwurf der gesundheitspolitischen Leitsätze, vorgelegt vom Gesundheitspolitischen Ausschuß beim SPD Vorstand, in: Langzeitprogramm 1, Bonn-Bad Godesberg, 1972, S. 191 ff.

Erhard, L./Müller-Armack, A., Soziale Marktwirtschaft, FrankfurtBerlin-Wien 1972

Eriksson, E., The Importance of Investigating Global Background Pollution, in: WMO (Hrsg.), Meteorological Aspects of Air Pollution, a.a.0., S. $31 \mathrm{ff}$.

Evans, M.K., A Forecasting Model Applied to Pollution Control Costs, in: American Economic Review, vol. 63, 1973, papers and proceedings, S. $244 \mathrm{ff}$. 
Everett, M., The Role of Formal Education in Environmental Movements, in: Journal of Economic Issues, vol. 6 , 1972 , S. 87 ff.

Ewringmann, D./Zimmermann, K., Kommunale Wirtschaftsförderung und Umweltschutz, in: Archiv für Kommunalwissenschaften, 12. Jg., 1973, S. 282 ff.

Fazio, A.G./Casclo, M.I., Evaluation of the Economic Effects of Anti-Pollution Public Policy: Proposal for an Econometric Analysis Model, in: OECD (Hrsg.), Problems of Environmental Economics, S. $143 \mathrm{ff}$.

Fedorenko, N./Gofman, K., Problems of Optimization in the Planning and Control of the Environment, in: Problems of Economics, vol. 15,12, 1973, S. $37 \mathrm{ff}$.

Ferrar, T.A., A Rationale for a Corporate Air Pollution Abatement Policy, in: American Journal of Economics and Sociology, vol. 33, 1974, S. 232 ff.

Fichtel, K., Pyrolyse von Hausmüll: Vier Verfahren könnten erprobt werden, in: Umwelt 1975, 4, S. 42 ff.

Fichtel, K., Entgasen oder Vergasen?, in: U 1975, 3

Finke, W., Umweltforschung - eine multi- und interdisziplinäre Aufgabe, In: Schultze, H. (Hrsg.), a.a.0., S. $64 \mathrm{ff}$.

Firnhaber, R.B., Patient Bodensee. Wie lange noch Trinkwasserspeicher?, in: Umwelt 1971, 3, S. $27 \mathrm{f}$.

Fischer, A., Die Struktur von Wirtschaftsräumen. Ein Beitrag zur Anwendung statistischer Methoden in der Regionalforschung, Wiesbaden 1969

Fischer, D.W./ Müller, F.G., Ein Beitrag zur Erfassung von Einwirkungen auf die Umwelt, in: Zeitschrift für die gesamte Staatswissenschaft, Bd. 130, 1974, S. 718 ff.

Fisher, A.C., Population and Environmental Quality, in: Public Pollcy, vol. 19, 1971, S. $19 \mathrm{ff}$.

Fisher, A.C./Krutilla, J.V., Valuing Long Run Ecological Consequences and Irreversibilities, in: Journal of Environmental Economics and Management, vol. 1, 1974, S. $96 \mathrm{ff}$.

Fisher, A.C./Peterson, F.M., The Environment in Economics: A Survey, in: Journal of Economic Literature, vol. 14, 1976, S. 1 ff.

Fisher, J.L., Impact of Population on Resources and the Environment, in: American Economic Review, vol. 61, 1971, papers and proceedings, S. $392 \mathrm{ff.}$

FitzRoy, F.R./von Weizsäcker, E., Einige politisch-ökonomische Fragen im Umweltschutz, in: von Weizsäcker, E. (Hrsg.), a.a.0., S. 95 ff. 
Flelscher, K., Schall als Umweltnoxe, in: Schaefer, Hans (Hrsg.), a.a.0., s. $68 \mathrm{ff}$.

Flickinger, H.-G./Summerer, S., Voraussetzungen erfolgreicher Umweltplanung in Recht und Verwaltung, Göttingen 1975

Flohn, H., Klimaschwankungen und Klimamodifikation: Fakten und Probleme, in: Universitas, 28. Jg., 1973, S. 1293 ff.

Flohn, H./Fraedrich, K., Eingriffe in das Klima, in: Umwelt $1973,5, \mathrm{~S} .20 \mathrm{f}$.

Fodor, G.G./Winneke, G. ,Belästigung durch geruchsintensive Stoffe, in: Zentralblatt für Bakteriologie .... a.a.o., S. $282 \mathrm{ff}$.

Ford, W.D., A National Program for Environmental Education, in: Armstrong, T.R. (Hrsg.), a.a.0., S. $140 \mathrm{ff.}$

Forrester, J.W., Der teufliche Regelkreis. Kann die Menschheit überleben?, Stuttgart 1972

Forschungsbericht IV, BT-Drucksache VI/3251

Forsthoff, E., Der Staat der Industriegesellschaft, 2. Auflage, München 1971

Forsund, F.R., Allocation in Space and Environmental Pollution, in: Swedish Journal of Economics, vol. 74, 1973, S. $19 \mathrm{ff}$.

Foster, C.D./Mackie, P.J., Nolse: Economic Aspects of Choice, in: Urban Studies, Bd. 7, 1970, H. 2, S. $123 \mathrm{ff}$.

Fox, I.K., Institutional Mechanisms, in: Summary Report of the Cornell Workshop on Energy and the Environment, a.a.o., S. 166 ff.

Fox, I.K./Wible, L.F., Information Generation and Communication to Establish Environmental Quality Objectives, in: Natural Resources Journal, vol. 13, 1973, S. 134 ff.

Freeman III, A.M., Air Pollution and Property Values: A Further Comment, in: Review of Economics and statistics, vol. 56, 1974, S. 554 ff.

Freeman III, A.M., Air Pollution and Property Values: A Methodological Comment, in: Review of Economics and Statistics, vol. 53, 1971, S. $415 \mathrm{f}$.

Freeman III, A.M., On Estimating Air Pollution Control Benefits from Land Value Studies, in: Journal of Environmental Economics and Management, vol. 1, 1974 , S. 74 ff.

Freeman III, A.M./Haveman, R.H., Residuals Charges for Pollution Control: A Policy Evaluation, in: Science, vol. 177, No. 4046 , v. 28.7.1972, S. $322 \mathrm{ff}$.

Frey, B.S., A Dynamic Theory of Public Goods, in: Finanzarchiv N.F., Bd. $32,1973 / 74$, S. $185 \mathrm{ff}$. 
Frey, B.S., Umweltökonomie, Göttingen 1972

Frey, B.S./ Schwödiauer, G., Uber die zeitliche Nutzung der Natur, in: Schmollers Jahrbuch, 91. Jg., 1971, S. $691 \mathrm{ff}$.

Frey, P., Giftmüll-Produzenten werden erfaßt, in: Umwelt 1974,1 , S. 20 ff.

Frey, P., Streit um den Zerfall von Baudenkmalen: Luftverunreinigung oder Verwitterung?, in: Umwelt 1973, 2, S. $22 \mathrm{f}$.

Frey, R.L., Umweltschutz als wirtschaftspolitische Aufgabe, in: Schweizerische Zeitschrift für Volkswirtschaft und Statistik, 108. Jg., 1972, S. $453 \mathrm{ff}$.

Friedman, M., The Role of Government in Free Society, in: Goldman, M.I. (Hrsg.), a.a.0., S. $117 \mathrm{ff}$.

Friedman, S., Facing Man and Society: The Challenge, in: Tsuru, S. (Hrsg.), a.a.0., S. $32 \mathrm{ff}$.

Fritsch, B., Umwelt und Grenzen des Wachstums, in: Mitteilungen der List-Gesellschaft Fasc. 8, 1973/74, Nr. 5, S. $92 \mathrm{ff}$.

Fry, C.L., Pollution Control. Proper Policies could Improve Functioning of Market Economy, in: Federal Reserve Bank of Dallas, Business Review, Oct. 1973, S. 1 ff.

Fuß, K., Gewässerschäden - Gewässerschutz, in: Der Landkreis $8-9,1970$, S. 326 ff.

Gässler, W., Zur Standortproblematik aus der Sicht der Industrie, in: Raumforschung und Raumordnung, 30. Jg., 1972, S. 200 ff.

Gardner, M.J., Using the Environment to Explain and Predict Mortality, in: Journal of the Royal Statistical Society, vol. 136, 1973, S. $421 \mathrm{ff}$.

Genscher, H.-D., Kein Abstrich beim Umweltschutz, in: U 1974, 1

Genscher, H.-D., Gesellschaftspolitische Aufgabe des Umweltschutzes, in: Bulletin der Bundesregierung $\mathrm{Nr} .178 \mathrm{v}$. 18.12.1970, S. $1945 \mathrm{ff}$.

Genscher, H.-D., Sozialisierung löst die Umwelt-Probleme nicht, in: Die Wirtschaftswoche Nr. 23 v. 1.6.1973

Genscher, H.-D., Umwelt als Gesamtheit sehen, in: U 1973, 2

Georgii, H.-W., Wir brauchen eine "Luftreinhaltungs-Strategie" . Wie die meteorologische Wissenschaft bei der Standortplanung helfen kann, in: Blick durch die Wirtschaft v. 23.9 .1971

Georgil, H.-W., Die lufthygienisch-meteorologische Modelluntersuchung im Untermaingebiet, in: Schultze, H. (Hrsq.), a.a.0., S. $216 \mathrm{ff}$. 
Gerhardt, P.H., Air Pollution Control: Benefits, Costs, and Inducements, in: Mushkin, S. (Hrsg.), Public Prices for Public Products, Washington, D.C., 1972, S. 153 ff.

Gerlach, S.A., Auswirkungen der Meeresverschmutzung auf das Leben im Meer und die Nahrungsketten, in: Schultze, H. (Hrsg.), a.a.0., S. $156 \mathrm{f}$.

Geschka, H./Lichtwer, L., Schätzungen der monetären Aufwendungen für Umweltschutz in der BRD für den zeitraum 1971 - 1975, in: Battelle Information 14, 1972, S. 4 ff.

Giersch, H. (Hrsg.), Das Umweltproblem in ökonomischer Sicht, Symposium 1973, Tübingen 1974

Giglio, R.J./Wrightington, R., Methods for Apportioning Costs Among Participants in Regional systems, in: Water Resources Research, vol. 8, 1972, S. $1133 \mathrm{ff}$.

Gilbert, T., Emissionsbegrenzungen und EmissionsmeBwerte - ein Vergleich, in: Umwelt 1973, 6, S. 32 ff.

Glagow, M., Zur staatlichen Regulierung von Umweltschäden, in: Glagow, M. (Hrsg.), a.a.O., S. $193 \mathrm{ff}$.

Glagow, M. (Hrsg.), Umweltgefährdung und Gesellschaftssystem, München 1972

Glück, K. U.a., Lärmkarten als Hilfsmittel für die Stadtplanung, Schriftenreihe des Bundesministers für Raumordnung, Bauwesen und Städtebau, Bonn-Bad Godesberg O.J.

Goldman, M.I., The Convergence of Environmental Disruption, in: Dorfman, R./Dorfman, N.S. (Hrsg.), a.a.O., S. 294 ff.

Goldman, M.I., Environmental Disruption in the Soviet Union, in: Tsuru, S. (Hrsg.), a.a.0., S. $171 \mathrm{ff}$.

Goldman, M.I. (Hrsg.), Ecology and Economics, Englewood Cliffs., N.J., 1972

Goldman, M.I/Shoop, R., What is Pollution?, in: Goldman, M.I. (Hrsg.), a.a.0., S. $102 \mathrm{ff}$.

Goldsmith, E./Allen, R., Planspiel zum Uberleben, Stuttgart 1972

Gorr, W.L./Kortanek, K.O., Optimal Control strategies for Air Quality Standards, Manuskript, Pittsburgh 1971

Grad, F.K./Rathjens, G.W./Rosenthal, A.J. (Hrsạ.), Environmental Control: Priorities, Policies, and the Law, New York und London 1971

Gräf, W., Umweltgefährdung durch kanzerogene Substanzen, in: Steubing, L./Kunze, C./Jäger, J. (Hrsg.), a.a.O., S. $115 \mathrm{ff}$.

Gräff, B./Spegele, H., Wörterbuch des Umweltschutzes, Stuttgart 1972 
Graeub, R., Die sanften Mörder. Atomkraftwerke demaskiert, Frankfurt 1974

Gramm, W.P., A Theoretical Note on the Capacity of the Market System to Abate Pollution, in: Land Economics, vol. 45, 1969 , S. 365 ff.

Grandjean, E./Gilgen, A./Bättig, K., Die Fluglärmbelastung, In: Städtehygiene, Bd. 20, 1969, Nr. 4, S. $73 \mathrm{ff}$.

Graßhoff, K./Meyl, A.H., Geschichte und Aufgaben des "Scientific Committee on Problems of the Environment" (SCOPE), in: DFG-Mitteilungen 2/73, S. $69 \mathrm{ff}$.

Grawe, J., Sichere Energieversorgung und Umweltschutz. Wie der Zielkonflikt bewältigt wird, in: Umwelt 1974, 2, S. 32 ff.

Green, H.P., The Role of Government in Environmental Conflict, in: Wolozin, H. (Hrsg.), a.a.O., S. $301 \mathrm{ff}$.

Grefen, K., Feinstäube gefährden den Städter, in: Umwelt 1974, 4, S. 22 f.

Gruhl, H., Wer soll für die Umwelt zahlen?, in: Marktwirtschaft, 3. Jg., 1971, Nr. 7, S. 12 ff.

Guderian, R., Wirkungen von Luftverunreinigungen auf Pflanzen, in: Schultze, H. (Hrsg.), a.a.O., S. $206 \mathrm{ff}$.

Günther, P., Exakte Messung - reinere Luft, in: U 1972, 4

Günther, U. u.a., Umweltschutz-Informations- und Steuerungssystem, IBM DV-Anwendung, 0.0. 1972

Gumpel, W./Keese, D. (Hrsg.), Probleme des Industriallsmus in Ost und West, Festschrift für Hans Raupach, München 1973

Gutachten des Wissenschaftlichen Beirats beim Bundesministerium der Finanzen, Zur Lage und Entwicklung der staatsfinanzen in der Bundesrepublik Deutschland, Bonn 1975

Gysin, H., Marketing und Umweltschutz, in: von Walterskirchen, M.P. (Hrsg.), a.a.O., S. $187 \mathrm{ff}$.

de Haar, U., Das MeBstellenprojekt "Reinhaltung der Luft", in: Umschau in Technik und Wissenschaft, 1970, H. 23, S. $746 \mathrm{f}$.

Häberle, M., Zur Erstellung eines Geruchskatasters der BASF Ludwigshafen, in: Umwelt 1973, 6, S. $39 \mathrm{ff}$.

Häberle, M., Wo sind die wirklichen Grenzen?, in: Umwelt 1976, 1, S. $24 \mathrm{ff}$.

Häberle, M., Industrielärm: Versagen die Vorschriften?, in: Umwelt 1975,2 , S. 26 ff.

Häfele, W., Stoffbilanzen, in: Zur Problematik des Verursacherprinzips, a.a.0., S. $22 \mathrm{ff}$.

Haenert, F., Abfallbörse der Industrie- und Handelskammern, in: Umwelt $1974,5,5.23 \mathrm{f}$. 
Hahn, H.A., Umweltplanung. Gemeinsame Aufgabe von Wissenschaftler, Ingenieur und Politiker, in: Umwelt 1971, 1 , S. $17 \mathrm{ff}$.

Hahn, J./Aehnelt, E., Die Fruchtbarkeit der Tiere als biologischer Indikator für Umweltbelastungen, in: Steubing, L./ Kunze, C./Jäger, J. (Hrsg.), a.a.O., S. 49 ff.

Hahnemann, H.W., Strahlenschäden und Strahlenschutz, in: Umwelt 1971,5, S. 25

Halbritter, G., Abgabenprinzip und Bewertungsproblematik, in: Zur Problematik des Verursacherprinzips, a.a.o., S. $36 \mathrm{ff}$.

Hall, jr. O.P./Licari, J.A., On the Regional Implications of Air Pollution Control, in: The Annals of Regional Science, vol. 6, 1972, S. 26 ff.

Hall, R.H., University Education and the Natural Environment: Are They Compatible?, in: International Journal of Environmental Studies, vol. 2, 1971, S. $47 \mathrm{ff}$.

Haltrich, W./Malle, K.-G., Wasserreinhaltuna; die Rolle der chemischen Industrie, in: Umwelt 1975, 5, S. 25 ff.

Hamm, W., Zur ökonomischen Bedeutung und ordnungstheoretischen Begründung der Rolle des Staates in Marktwirtschaften, in: Staat und moderne Marktwirtschaft, hrsa. vom Landespersonalamt Hessen, Wiesbaden 1975, S. 21 ff.

Hansmeyer, K.-H., Die Abwasserabgabe als Versuch einer Anwendung des Verursacherprinzips, in: Issing, 0 . (Hrsg.), a.a.0., S. $65 \mathrm{ff}$.

Hansmeyer, K.-H., Anforderungen der Umweltpolitik an die amtliche Statistik, in: Allgemeines Statistisches Archiv, Bd. 59, 1975, S. 1 ff.

Hansmeyer, K.-H., Volkswirtschaftliche Kosten des Umweltschutzes, in: Giersch, H. (Hrsg.), a.a.O., S. $99 \mathrm{ff}$.

Hansmeyer, K.-H./Rürup, B., Umweltgefährdung und Gesellschaftssystem, in: Wirtschaftspolitische Chronik 1973, H. 2 , S. $7 \mathrm{ff}$.

Hardin, G., Die Tragik der Allmende, in: Lohmann, M. (Hrsq.), a.a.O., S. $30 \mathrm{ff}$.

Hartkopf, G., Wirtschaftliche Aspekte der Umweltpolitik, in: Schultze, H. (Hrsg.), a.a.O., S. $15 \mathrm{ff}$.

Hartkopf, G., Umweltgestaltung durch Raumordnung, in: Raumforschung und Raumordnung, 30. Jg., 1972, s. $145 \mathrm{ff}$.

Hartkopf, G., Umweltpolitik nach Gymnich, in: U 1975, 4

Hartkopf, G., Umweltpolitik aus der Sicht der Bundesregierung, in: Glagow, M. (Hrsg.), a.a.O., S. $181 \mathrm{ff}$.

Hass, J.E., Optimal Taxing for the Abatement of Water Pollution, in: Water Resources Research, vol. 6, 1970, S. $353 \mathrm{ff}$. 
van Haut, H./Guderian, R., Pflanzen als Indikator der Luftverschmutzung, in: Probleme der Umweltforschung, a.a.0., s. $36 \mathrm{ff}$.

Hawksworth, D.L., Lichens as Litmus for Air Pollution: A Historical Review, in: International Journal of Environmental Studies, vol. 1, 1971, S. $281 \mathrm{ff}$.

Hayek, F.A., Der Weg zur Knechtschaft, Erlenbach und Zürich, 1952

Hazelton, J.E., Effluents and Affluence, in: Goldman, M.I. (Hrsg.), a.a.0., S. $134 \mathrm{ff}$.

Head, J.G., Public Goods and Public Welfare, Durham, N.C., 1974

Head, J.G., Public Policies and Pollution Problems, in:

Finanzarchiv, Bd. 33, 1974, S. 1 ff.

Heaney, J.P./ Carter jr., B.J./Pyatt, E.E., Costs for Equivalent Upstream Reduction in waste Water Discharges, in:

Water Resources Research, vol. 7, 1971, S. $458 \mathrm{ff}$.

Heigl, A., Wie w'rkt die Sonderabschreibung?, in: Umwelt 1975, 4, S. 18 :f.

Heinemann, J., Zweierlei Ingenieure?, in: Umwelt 1972, 5, $\mathrm{S} .14 \mathrm{ff}$.

Henke, W., Umwelt, Technik, Natur, Kultur, in: Recktenwald, H.C. (Hrsg.), a.a.O., S. $17 \mathrm{ff}$.

Henkel, P., Unser Beitrag zum Umweltschutz, in: Bayer-Berichte, H. 27, 1971, S. 26 ff.

Henkel, P., Der Graben zwischen Chemie und öffentlichkeit, in: Umwelt 1973,3, S. 4

Henschler, D., Versuch einer Zuordnung von chemischen Noxen und Schäden beim Menschen, in: Schaefer, Hans (Hrsg.), a.a.0., S. $46 \mathrm{ff}$.

Herder-Lexikon Umwelt, Freiburg 1973

Herrmann, G., Probleme der bewußten Beherrschung von Wechselwirkungen zwischen Mensch und natürlicher Umwelt,

in: Umwelt $1973,2, \mathrm{~S} .15 \mathrm{ff}$.

Hetman, F., Society and the Assessment of Technolocy, Paris 1973

Hettche, H.O., Gesundheit und Großstadtluft, in: Staub, Bd. 2, 1961 , S. 48 ff.

Hirohide Hinomoto, Dynamic Programming of Capacity Expansion of Municipal Water Treatment System, in: Water Resources Research, Vol. 8, 1972, S. $1178 \mathrm{ff}$.

Hirohide Hinomoto, Unit and Total Cost Functions for Water Treatment Based on Koenig's Data, in: Water Resources Research, vol. 7, 1971, S. 1064 ff. 
Hödl, E., Umweltpolitik, Die Scheuklappen der "Okkonomisten",

in: Wirtschaftswoche, 26. Jg., 1972, Nr. 8, S. $35 \mathrm{ff}$.

Höffken, F., Probleme der Abfallbehandlung, in: Olschowy, G., (Hrsg.), a.a.0., S. 222 ff.

Höhmann, H.-H./Seidenstecher, G./Vajna, T., Umweltschutz und ökonomisches System in Osteuropa, Stuttgart-BerlinKöln-Mainz 1973

Hönig, W., Die soziale Verantwortung des Unternehmens, in: $\mathrm{NZZ}$ vom 10.2.1971

Hösel, G., Kosten elner befriedigenden Neuordnung der Abfallbeseitigung, in: Schultze, H. (Hrsg.), a.a.O., S. $196 \mathrm{ff}$.

Hösel, G., Umweltkrise und Gesundheitsgefahren, in: Der Städtetag, 24. Jg., 1971, H. 2, S. 72 ff.

Hötker, D./Wierling, L., Umweltschutz im Ruhrgebiet, in: Bundesbaublatt, 20. Jg., 1971, S. $324 \mathrm{ff}$.

Hofmann, U., Rechtswidrige Tatbestände - aber selten nachweisbare Schuld, in: Blick durch die Wirtschaft vom 6.9 .1971

Horn, C./von Walterskirchen, M.P./Wolff, J. (Hrsg.), Umweltpolitik in Europa, Frauenfeld 1973

Huber, G., Geistige Implikationen der Umweltveränderung, in: Leibundgut, H. (Hrsg.), a.a.O., S. $118 \mathrm{ff}$.

Hueting, R., The Set-Up of the Statistical system within which the Deterioration of the Human Environment will be Estimated, in: OECD (Hrsg.), Problems of Environmental Economics, a.a.0., S. $93 \mathrm{ff}$.

Hyman, D.N., The Economics of Governmental Activity, New York u.a. 1973

Internationale Arbeitsgemeinschaft der Wasserwerke am Rhein (IAWR), Memorandum der IAWR, in: Gas - Wasser Abwasser, 53. Jg., 1973, Nr. 6

Infas, Die inneren Reformen im Spiegel der Bevölkerungsmeinung, Bonn 1971

Irving, R.M./Priddle, G.B. (Hrsg.), Crisis, Readings in Environmental Issues and Strategies, London 1971

Issing, O. (Hrsg.), Okkonomische Probleme der Umweltschutzpolitik, Berlin 1976

Issing, O., Zerstörung der Umwelt - ein Versagen des marktwirtschaftlichen Systems?, in: Recktenwald, H.C. (Hrsg.), a.a.0., S. $127 \mathrm{ff}$.

Istock, C.A., Modern Environmental Deterioration as a Natural Process, in: International Journal of Environmental Studies, vol. 1, 1971, S. $151 \mathrm{ff}$.

Jacoby, N.H., The Polluters: Industry or Government? The Institute of Economic Affairs, London 1972, S. $11 \mathrm{ff}$. 
Janocha, P., Verschmutzungsgrenzen für jede Region, in: Umwelt 1975,4 , S. 54 ff.

Jansen, G., Nachweis von Lärmwirkungen (zugleich ein Beitrad zur Ermittlung von Grenzwerten), in: Zentralblatt für Bakteriologie..., a.a.0., S. 315 ff.

Jansen, P., Was können Systemanalysen zur praktischen Verwirklichung von Umweltschutz beitragen?, in: Zur Problematik des Verursacherprinzips, a.a.0., s. $15 \mathrm{ff}$.

Jarrett, H. (Hrsg.), Environmental Quality in a Growing Economy, 3. Auflage, Baltimore und London 1971

Jermini, C., Die gesundheitlichen Gefährdungen durch Kohlenmonoxid, in: Universitas, 28. Jg., 1973, S. $1239 \mathrm{ff}$.

Jeschke, H., Verpackung - ein teurer Service, in: U 1973, 3

Jessel, U., Seven Years Experience in Air Pollution Monitorina in Germany, in: DFG-Mitteilungen, 2/74, S. $41 \mathrm{ff}$.

Jobst, J., Recycling - Entwicklung und gegenwärtiger stand, in: $U 1975,4$

Jud, S., Lärmimmissionsrichtwerte: Zumutbare Lärmgrenzen, in: Umwelt 1975,2 , S. $31 \mathrm{ff}$.

Jürgensen, H./Jaeschke, K.-P., Allokationseffekte der social costs $1 \mathrm{~m}$ Umweltschutz - Untersuchung zur Anwendung des Verursacherprinzips, unveröffentlichtes Gutachten für das Bundesministerium des Innern, Hamburg 1972

Jürgensen, H./Jaeschke, K.-P., Operationale Verfahren zur Anwendung des Social Costs-Prinzips im Umweltschutz, unveröffentlichtes Gutachten für das Bundesministerium des Innern, Hamburg 1971

Jürging, P., Flechten - Bioindikatoren der Luftverunreinioung?, in: Steubing, L./Kunze, C./Jäqer, J. (Hrsq.), a.a.O., S. $141 \mathrm{ff}$.

Kade, G. Okonomische und gesellschaftspolitische Aspekte des Umweltschutzes, in: Gewerkschaftliche Monatshefte, 22. Jg., 1971, S. 257 ff.

Kade, G., Marktwirtschaft und Umweltschutz, in: gdi-topies $4 / 71$, S. 5 ff.

Kade, G., Durch das Profitmotiv in die Katastrophe, in: Wirtschaftswoche Nr. 40, 1971, S. 39 ff.

Kapp, K.W., Zur Praxis der Umweltpolitik und der Umweltplanung, in: Kapp, K.W./Vilmar, F. (Hrsg.), a.a.O., S. $153 \mathrm{ff}$.

Kapp, K.W., 'Recycling' in Contemporary Chlna, in: Kyklos, Bd. 27, 1974, S. 286 ff.

Kapp, K.W./Vilmar, F. (Hrsg.), Sozialisierung der Verluste? München 1972 
Karsten, D., Soziale Kontrolle für technischen Fortschritt, in: U $1972,4, S .18 \mathrm{ff}$.

Karsten, D., Umweltpolitik - Argumente für die marktwirtschaftliche Lösung, in: Wirtschaftswoche Nr. 20, 1972, S. $37 \mathrm{ff}$.

Kasper, R.G. (Hrsg.), Technology Assessment, Understanding the Social Consequences of Technological Applications, New York-Washington-London 1972

Kassarjian, H.H., Incorporating Ecology into Marketing Strategy: The Case of Air Pollution, in: Journal of Marketing, vol. 35, July 1971, S. $61 \mathrm{ff}$.

Katz, M., Measurement of Alr Pollutants, Gulde to the Selection of Methods, Genf 1969

Kazantzis, G. , The Poison Chain for Mercury in the Environment, in: International Journal of Environmental Studies, vol. 1, 1971, S. $301 \mathrm{ff}$.

Kenner, E., Wie soll der Umwelt-Fachmann aussehen?, in: Umwelt 1976,3, S. 228 ff.

Kesse 1s, K., Verursachungsgerechte Abwassergebühren, in: Zur Problematik verursachungsgerechter Berechnungsschlüssel in der Abwasserableitung, a.a.0., S. $17 \mathrm{ff}$.

Kiese, 0., Die Rolle künstlich erzeugter wärme in der Atmosphäre, in: Steubing, L./Kunze, C./Jäger, J. (Hrsq.), a.a.0., S. $175 \mathrm{ff}$.

Kimminich, O., Das Recht des Umweltschutzes, München 1972

Kitschler, W., Aufbau eines Informationssystems für Umweltplanung, in: Allgemeines Statistisches Archiv, Bd. 59, 1975, S. 25 ff.

Klausewitz, W./Schäfer, W./Tobias, W., Umwelt 2000, Kleine Senckenbergreihe 3, Frankfurt am Main 1971

Klee, O., Reinigung industrieller Abwässer, Stuttgart 1970

Klee, O., Wie stirbt ein Fluß? in: Kosmos 1971, H. 1, s. 11 ff.

Klee, $0 .$, Eine Klärwerke-Kur für den Bodensee, in: Kosmos 1971, H. 12, S. $504 \mathrm{ff}$.

Klein, A., Reine Luft. Die Verschmutzung der Luft und die technischen und praktischen Möglichkeiten zur Wiederherstellung reiner Luft, Karlsruhe 1971

Klevorick, A.K./Kramer, G.H., Social Choice on Pollution Management: The Genossenschaften, in: Journal of Public Economics, vol. 2, 1973, S. $101 \mathrm{ff}$.

Klosterkötter, W., Immissionsrichtwerte für Lärm aus psychologisch-physiologischer sicht, in: Umwelt 1973, 1, S. $28 \mathrm{ff}$.

Klosterkötter, W. , Lärmforschung und Lärmbekämpfung, in: Universitas, 28. Jg., 1973, S. $891 \mathrm{ff}$. 
Klosterkötter, W., Lärmwirkungen und Lebensqualität, in: U 1973,3

Klosterkötter, W., Lärmwirkungen auf den Menschen, in: Schultze, H. (Hrsg.), a.a.O., S. $261 \mathrm{ff}$.

Klosterkötter, W./Gono, F., Quellen und gesundheitliche Wirkung des Lärms, in: Zentralblatt für Bakteriologie..., a.a.0., s. $300 \mathrm{ff}$.

Knabe, W./Luckat, S., Passiver Immissionsschutz gegen Luftverunreinigungen, in: Umwelt 1974, 4, S. $28 \mathrm{ff}$.

Knappe, E., Möglichkeiten und Grenzen dezentraler Umweltschutzpolitik. Bekämpfung externer Nachteile durch Verhandlungen, Berlin 1974

Kneese, A.V., Pollution and Pricing, in: American Economic Review, vol. 62, 1972, S. 958.

Kneese, A.V., Pollution and the Profit Motiv, in: Wolozin, H. (Hrsg.), a.a.O., S. 219 ff.

Kneese, A.V., Environmental Pollution: Economics and Policy, in: American Economic Review, vol. 61, 1971, papers and proceedings, S. $153 \mathrm{ff}$.

Kneese, A.V./Bower, B.T. (Hrsg.), Environmental Quality Analysis, Baltimore und London 1972

Kneese, A.V./Bower, B.T., Die Wassergutewirtschaft. Wirtschaftstheoretische Grundlagen, Technologien, Institutionen. München-Wien 1972

Kneese, A.V./Rolfe, S.E./Harned, J.W., Managing the Environment. International Economic Cooperation for Pollution Control, 2. Auflage, New York-Washington-London 1973

Knelson, J.E.. Luftqualitätskriterien und Immissionsgrenzwerte für Kohlenmonoxid in den Vereinigten Staaten, in:

VDI (Hrsg.), Kohlenmonoxid. Entstehung, Messung und Wirkungskriterien, Düsseldorf 1972

Koenig, H.-W., Thermische Belastung der Fließgewässer, in: Olschowy, G. (Hrsg.), a.a.O., S. $51 \mathrm{ff}$.

Kohl, D.E., The Environmental Movement: What Might it Be?, in: Natural Resources Journal, vol. 15, 1975, S. $327 \mathrm{ff}$.

Kohn, R.E., A Cost-Effectiveness Model for Air Pollution Control with a Single Stochastic Variable, in: Journal of the American Statistical Association, vol. 67, 1972, S. $19 \mathrm{ff}$.

Korte, F., Rückstandsprobleme, in: Natur und Landschaft, 44. Jg., 1969, H. 9, S. 225 ff.

Betriebswirtschaftliche Kosten von Umweltschutzmaßnahmen und ihre gesamtwirtschaftliche Auswirkung, Berlin 1972

Koszo, E., Umweltschutz bei Hoechst, in: U 1975, 5, S. 42 ff. 
Kovda, V.A., Boden, Mensch und Biosphäre, in: Aufgabe Zukunft - Qual1tät des Lebens, Bd. 4: Umwelt, a.a.0., S. 116 ff.

Kowalski, E., Strahlengefährdung und Umweltschutz, in: NZZ, FA Nr. 66 vom 7.3.1972

Krane, D., Lärmschutz: Unberechtigte Vorwürfe gegen die "TA Lärm", in: Umwelt 1975, 5, s. $37 \mathrm{ff.}$

Krengel, R., Die Messung der Umweltverschmutzung - Ein neuer Wohlstandsindikator, in: Gumpel, W./Keese, D. (Hrsg.), a.a.O., s. 431 ff.

Krier, J.E./Montgomery, W.D., Resource Allocation, Information Cost and the Form of Government Intervention, in: Natural Resources Journal, vol. 13, 1973, S. 89 ff.

Krist, T., Grundwissen Umweltschutz. Zahlen, Daten, Fakten, Bestimmungen. Darmstadt 1974

Kuhlmann, A., Ein Instrument der Raumplanung: Vorsorge durch Emissionsprognose, in: Umwelt 1974, 6, S. $30 \mathrm{ff}$.

Kunz, W./Rittel, H., Projekt UMPLIS (Umwelt-Planungs-Informations-System) - Aufgaben und Aufbau, in: Umwelt 1973, 3, S. $43 \mathrm{ff}$.

Kuper, G., Laser-Lidar, Was kann es wirklich?, in: Umwelt 1972, 1, S. 40 ff.

Kurir, A., Chemische Pflanzenbekämpfung - Gefahren für Mensch und Tier, in: $U 1974,4$ und 5

Lahmann, E., Literaturstudie über die ökonomischen Konsequenzen der Schäden und Belästigungen, die durch die Luftverschmutzung durch Schwefeldioxid sowohl bei Materialien und der Vegetation als auch bei Mensch und Tier hervorgerufen werden, Luxemburg 1974

LaMont Cole, C., A Race for Survival, in: Pole, N. (Hrsg.), a.a.0., S. $16 \mathrm{ff}$.

Landesverband Bürgerinitiative Umweltschutz Nordrhein-Westfalen e.V. , Weniger Geld - mehr Lärm. Schutz der Bürger vor Straßenlärm ungenügend, in: U 1976,3 ,

S. $40 \mathrm{ff}$.

Landsberg, H.E., Man-Made Climatic Changes, in: Science, vol. 170, Nr. 3964, v. 18.12.1970, S. $1265 \mathrm{ff}$.

Lange, G., Die Suche nach der gefährlichen Dosis, in: Umwelt $1973,5, \mathrm{~S} .17 \mathrm{ff}$.

Lave, L.B., Air Pollution Damage: Some Difficulties in Estimating the Value of Abatement, in: Kneese, A.V.l Bower, B.T. (Hrsg.), a.a.O., S. $213 \mathrm{ff}$.

Lave, L.B./Seskin, E.P., Air Pollution and Human Health, in: Dorfman, R./Dorfman, N.S. (Hrsg.), a.a.O., S. 356 ff.

Lave, L.B./Seskin, E.P., Health and Air Pollution, in: Swedish Journal of Economics, vol. 73, 1971, S. 76 ff. 
Lawther, P.J./Martin, A.E./Wilkins, E.T., Epidemiology of Air Pollution, Report on a Symposium, WHO Public Health Papers No. 15, Genf 1962

Leibundgut, H. (Hrsg.), Schutz unseres Lebensraumes, MünchenBern-Wien 1971

Leipert, C., Sozlale Indikatoren. Uberblick über den Stand der Diskussion, in: Konjunkturpolitik, 19. Jg., 1973, S. $204 \mathrm{ff}$.

Leitsätze für die Zentrale Trinkwasserversorung, DIN 2000

(Mai 1959), in: Wasserversorgungsnormen, DIN-Taschenbuch 12, 2. geänderte Auflage, hrsg. v. DNA, Berlin-Köln-Frankfurt 1971, S. 42 f.f.

Lendi, M., Die Raumplanungsgesetzgebung und ihre Konsequenzen für die Unternehmung, in: von Walterskirchen, M.P. (Hrsq.), a.a.0., S. 205 ff.

Lerner, A.P., Priorities and Pollution: Comment, in: American Economic Review, vol. 64, 1974, S. 715 ff.

Lerner, A.P., The 1971 Report of the President's Council of Economic Advisers: Priorities and Efficiency, in: American Economic Review, vol. 61, 1971, S. $527 \mathrm{ff}$.

Levi, D.R./Colyer, D., Some Legal and Economic Aspects of Citizien-Initiated Legal Mechanisms for Solving Environmental Quality Problems, in: Intermountain Economic Review, vol. 3 (2), 1972, S. 36 ff.

Lichtwer, L., Zukunftsaspekte der Wiederverwendung, in: U 1975, 4 , S. $42 \mathrm{ff}$.

Lichtwer, L./Schaude, G.R., Nutzen der Fernerkundung der Erde, in: Battelie-Information $18,1974, S .34 \mathrm{ff}$.

Liebmann, H., Die Wasserqualität der Oberbayerischen Seen, in: Olschowy, G. (Hrsg.), a.a.0., S. $64 \mathrm{ff}$.

Liste von MIK-Werten, in: Umwelt 1974, 6, S. $34 \mathrm{ff}$.

Littmann, K. , Finanzierung von kollektiven Gütern, in: Aufgabe Zukunft - Qualität des Lebens, Bd. 7: Qualitatives Wachstum, a.a.0., s. $11 \mathrm{ff}$.

Littmann, K., Umweltbelastung - Sozialökonomische Gegenkonzepte. Zur Internalisierung externer Nachtelle, Göttingen 1974

Litvinov, N., Water Pollution in the USSR and other Eastern European Countries, in: Bulletin of the WHO, Bd. 26, 1962 , S. 439 ff.

Lord Llewellyn-Davies/Cowan, P., Stadtplanung und Wissenschaft, in: Lohmann, M. (Hrsg.), a.a.0., S. $439 \mathrm{ff}$.

Lohmann, M. (Hrsg.), Gefährdete Zukunft - Prognosen angloamerikanischer Wissenschaftler, München 1970

Lorenz, G. , Umweltschutz für eine Großstadt, in: Umweltschutz - aber wie?, a.a.0., s. 51 ff. 
Loucks, D.P./Revelle, C.E./Lynn, W.R., Linear Programing Models for Water Pollution Control, in: Management Science, vol. 14, Application, 1967, S. B-166 ff.

Lowenstein, F.W., Man's Health as Depending on His Environment, in: Sioli, H. (Hrsg.), a.a.O., S. $245 \mathrm{ff}$.

Lüscher, E., Gesundheitsschädliche Wirkungen des Lärms und deren Ursachen, in: Zeitschrift für Präventivmedizin $12 / 1957$, S. 437 ff.

Lüth, P., Was ist Gesundhe1t? - Was ist Krankhe1t?, in: Die Neue Gesellschaft, 20. Jg., 1973, S. $831 \mathrm{ff}$.

Lusky, R., A Model of Recycling and Pollution Control, in: Canadian Journal of Economics, vol. 9, 1976, S. $91 \mathrm{ff}$.

Maihofer, W., Umweltpolitik in der Bewährung, in: U 1976, 2, S. 12

Maldague, M.E., Landwirtschaft und Forstwesen, in: Offner, H. (Hrsg.), a.a.0., s. $73 \mathrm{ff}$.

Maldonado, T., Umwelt und Revolte, Zur Dialektik des Entwerfens im Spätkapitalismus, Reinbek 1972

Malorny, G., Allgemeiner Ubberblick über die Wirkung von Kohlenmonoxid auf den Menschen, in: VDI (Hrsg.), Kohlenmonoxid, a.a.0., S. 47 ff.

Malz, F., Taschenwörterbuch der Umweltplanung, München 1974

Manpower and Training Needs for Air Pollution Control. Report of the Secretary of Health, Education, and Welfare, June 1970, Washington, D.C. 1970

Marburger, E.-A., Die ökonomische Beurteilung der städtischen Umweltbelastung durch Automobilabgase. Methoden und Quantiflzierungsversuche, Düsseldorf 1974

Marquardt, H., Die Auslösung von Erbschäden und Krebs durch Umweltfaktoren, in: Universitas, 28. Jg., 1973, S. 1313 ff.

Marquardt, H., Umweltbedingte Mutagenität, in: Schultze, H. (Hrsg.), a.a.0., S. 295 ff.

Martz, G., Methoden der Abwasserreinigung, in: U 1976, 3

Materialien zum Bericht zur Lage der Nation, 1974, BT-Drucksache $7 / 2423$

Materialienband $\mathrm{zum}$ Umweltprogramm der Bundesregierung, $z u$ BT-Drucksache VI/2710

May, H./Plassmann, E., Abgasemissionen von Kraftfahrzeugen in Großstädten und industriellen Ballungsgebieten, Köln 1973

McCormick, R.A., Meteorological Aspects of Air Pollution in Urban and Industrial Districts, in: WMO (Hrsg.), a.a.0., s. $1 \mathrm{ff}$. 
McHale, J., Der ökologische Kontext, Frankfurt 1974

McKean, R.N., Some Problems of Criteria and Acquiring

Information, in: Jarrett, H. (Hrsg.), a.a.0., S. 61 ff.

Meadows, D. U.a., Die Grenzen des Wachstums. Bericht des Club

of Rome zur Lage der Menschheit, Stuttgart 1972

Mendiratta, A.K./Davidson, B.. Best Combination of Waste Treatment and Spatially Distributed Discharges of Effluent, in: Water Resources Research, vol. 8, 1972 , S. 565 ff.

Menke-Glückert, P., Das Umweltprogramm der Bundesregierung, in: von Weizsäcker, E. (Hrsg.), a.a.0., S. 122 ff.

Menke-Glückert, P., Umweltschutz - Achillesferse oder Herausforderung für die Industriegesellschaft, in: Die Neue Gesellschaft, 1971, S. 581 ff.

Menke-Glückert, P., Vom Umweltschutz zur Umweltpolitik, in: Umwelt 1972, 1, S. 4 f.

Mensing, W., Investitionen zur Reinhaltung der Gewăsser, in: Der Landkreis 8-9, 1970, S. 331 ff.

Meyer, Olaf, Frische Luft für kranke Seen. Sanierung durch Sauerstoffanreicherung, in: Umwelt 1973, 4, S. $18 \mathrm{ff}$.

Meyer-Abich, K.M., Umweltbeeinträchtigungen durch den wirtschaftlich-technischen ProzeB - Beispiel:

Energieumwandlungsprozesse, in: Giersch, H. (Hrsg.), a.a.0., S. $3 \mathrm{ff}$.

Meyer-Abich, K.M., Was kostet die Umweltverschmutzung?, in: Umweltschutz - aber wie?, a.a.0., S. 7 ff.

Meyer-von Gonzenbach, R., Die Beanspruchung der Umwelt durch die Besiedlung, in: Leibundgut, H. (Hrsg.), a.a.0.. S. 65 ff.

Meyl, A.H., Abwässer in Küstennähe, in: DFG-Mittellungen 2/74, S. $28 \mathrm{ff}$.

Miller, E., Implications of Process Change for Industrial Pollution Abatement Pollcy, in: Land Economics, vol. 48, 1972, S. $396 \mathrm{ff}$.

Ministerbericht $z$ ur Reaktorsicherheit, Kritische Stellen, in: Umwelt 1974, 6, S. 11 ff.

von Mises, L., Human Action, London-New York 1949

Mishan, E.J., Evaluation of Life and Limb: A Theoretical Approach, in: Journal of Political Economy, vol. 79, 1971 , S. 687 ff.

Mishan, E.J., The Postwar Literature on Externalities: An Interpretative Essay, in: Journal of Economic Literature, vol. 9, 1971, S. 1 ff.

Mislin, H., Zerstörung des Lebensraumes und Umweltverantwortung, in: Leibundgut, H. (Hrsg.), a.a.0., S. $19 \mathrm{ff}$. 
Möbius, K., Das Umweltproblem aus wirtschaftlicher sicht. Kieler Diskussionsbeiträge zu aktuellen wirtschaftspolitischen Fragen, H. 14, Kiel 1971

Möller, H., Zum Begriff der Umweltschäden insbesondere im öffentlichen Sektor, in: Gumpel, W./Keese, D. (Hrsg.), a.a.0., S. $439 \mathrm{ff}$.

Moll, W.L.H., Taschenbuch für Umweltschutz I: Chemische und technologische Informationen, Darmstadt 1973

Mommsen, E.W., Wirtschaft und Umwelt, in: Vortragsreihe des Deutschen Industrieinstituts, Bd. 21, 1971, H. 4, S. $1 \mathrm{ff}$.

Moncrief, L.W., The Cultural Basis for Our Environmental Crisis, in: Dorfman, R./Dorfman, N.S. (Hrsg.), a.a.0., S. $284 \mathrm{ff}$.

Morgan, G.B./Ozolins, G./Tabor, E.C., Air Pollution Surveillance Systems, in: Science, vol. 170, 1970, S. $289 \mathrm{ff}$.

Müller, F.G., Finanzpolitik und Umweltverschmutzung: Die kanadischen Maßnahmen zur Kontrolle der Wasserverunreinigung, in: Finanzarchiv, Bd. 31, 1973, S. $519 \mathrm{ff}$.

Müller, H.-J., stadtreinigung im Rahmen des Umweltschutzes, in: Der Städtetag, 1971, H. 7, S. 405 ff.

Müller, Walter, Umweltschutz gegen Industrieansiedlung: Die Grenzen kommunaler Planungshohe1t, in: Juristische Schulung, 1975, 4, S. $228 \mathrm{ff}$.

Müller, Wilhelm J., Probleme der Reinhaltung von Gewässern, Bielefeld 1973

Müller-Merbach, H., Operations Research. Methoden und Modelle der Optimalplanung, Berlin und Frankfurt 1969

Münchau, F., Grundzüge eines gerechteren Maßstabes für die Verteilung der Entwässerungskosten elner Gemeinde, in: Zur Problematik verursachungsgerechter Berechnungsschlüssel in der Abwasserableitung, a.a.0., s. $7 \mathrm{ff}$.

Nagel, S., Umweltschutz: Neue Aufgaben der NATO, in: Umwelt 1971,4, S. 25 ff.

Naudascher, E., Strömungs forschung im Dienste des Umweltschutzes, in: Schultze, H. (Hrsg.), a.a.O., S. $132 \mathrm{ff}$.

Neue Helvetische Gesellschaft (Hrsg.), Natur - geplündert oder gehegt, Horgen 1961

Nitschke, E., Lärm, in: Schultz, U. (Hrsg.), Lebensqualität, a.a.0., S. 142 ff.

Nowak, F., Entgasung und Vergasung kombiniert, in: U 1976, 4 Nowotny, E., Wirtschaftspolitik und Umweltschutz, Freiburg 1974 
Nowotny, E., Zur gesamtwirtschaftlichen Wirkung einer Besteuerung von Urhebern externer Effekte, in:

Finanzarchiv, Bd. 31, 1973, S. $420 \mathrm{ff}$.

Nydegger, A., Die Social Costs des Umweltungleichgewichts, in: von Walterskirchen, M. P. (Hrsg.), a.a.0., S. $143 \mathrm{ff}$.

Obenhaus, W., Uberlegungen für eine Konzeption zur Prüfung der Umweltverträglichkeit öffentlicher Maßnahmen, in: Umwelt 1973,5 , S. $58 \mathrm{ff}$.

Oberhauser, A., Abgrenzung des Verursacherprinzips und seine Einordnung in die Umweltpolitik, in: Das Verursacherprinzip und seine Instrumente, a.a.0., S. 27 ff.

Oberhauser, A., Gesamtwirtschaftliche Wirkungen der Anwendung des Verursacherprinzips, in: Das Verursacherprinzip und seine Instrumente, a.a.0., s. $51 \mathrm{ff}$.

Odrich, P., Schütze die Umwelt und rede darüber, in: Blick durch die wirtschaft vom 12.7.71

Odum, E.P., Ökolog1e, München-Basel-Wien 1967

OECD, Activities of the OECD in 1972, Paris 1973

OECD, Environmental Damage Costs. Record of a Seminar held at the OECD in August 1972, Paris 1974

OECD, Effects of Traffic and Roads on the Environment in Urban Areas, Paris 1973

OECD, Eutrophication in Large Lakes and Impoundments, Uppsala Symposium, Report Prepared by C.P. Milway, Paris 1970

OECD, Methods of Measuring Air Pollution, Paris 1964

OECD, OECD's Programme on the Environment, in: OECD-Observer No. 50,1971, S. $7 \mathrm{f}$.

OECD, OECD at Work for Environment, Paris 1971

OECD, Problems of Environmental Economics, Paris 1972

OECD, Problems in Transfrontier Pollution, Paris 1974

OECD, Science, Growth and Society, A New Perspective. Report of the Secretary General's' Ad Hoc Group on New Concepts of Science Policy, Paris 1971

OECD-Environment Directorate, Analysis of Costs of Pollution Control, Paris 1973

OECD-Environment Directorate, Instances, if any, where Cost/ Benefit Analysis Has Been Applied to Environmental

Problems - Japan, Paris 1972

OECD-Environment Directorate, Survey of Pollution Control Cost Estimate Made in Member Countries, Paris 1972

offner, H. (Hrsg.), Die Zukunft der Landschaft in Europa, München 1971 
Ohrenschall, A., Umweltfachmann durch zusatzstudium, in: U 1976,4

Olschowy, G., Auswirkung der Luftverunreinigung auf den Menschen, in: Olschowy, G. (Hrsg.), a.a.0., s. $127 \mathrm{ff}$.

Olschowy, G., Zur Belastung des Bodens, in: Olschowy, G. (Hrsg.), a.a.0., S. $138 \mathrm{ff}$.

Olschowy, G., Bilanz des Abfallproblems, in: Olschowy, G. (Hrsg.), a.a.0., s. $212 \mathrm{ff}$.

Olschowy, G., Camping auf Müllwiesen, in: U 1972, 1

Olschowy, G., Katalog ökologischer Noxen und der von ihnen bedingten Schäden, in: Schaefer, Hans (Hrsg.), a.a.O., S. $158 \mathrm{ff}$.

Olschowy, G. (Hrsg.), Belastete Landschaft - Gefährdete Umwelt, München 1971

Olschowy, G., Zersiedlung der freien Landschaft, in: Olschowy, G. (Hrsg.), a.a.0., S. 327 ff.

Olschowy, G./Mrass, W./Kullmer, H.J./Bürger, K., Zur Belastung der Landschaft. Schriftenreihe für Landschaftspfleqe und Naturschutz, H. 4, Bonn-Bad Godesberg 1969

Orr, L., Incentive for Innovation as the Basis for Effluent Charge Strategy, in: American Economic Review, vol. 66 , 1976, papers and proceedings, S. $441 \mathrm{ff}$.

Orth, H./Cembrowicz, R., Anwendungen der nichtlinearen Programmierung, in: Hahn, H. (Hrsg.), Operations Research und seine Anwendung in der Siedlungswasserwirtschaft, Bielefeld 1972, S. 217 ff.

Osswald, A., Grundprobleme des Umweltschutzes, in: Hessen-Informationen, Schriftenreihe der Hessischen Landesregierung, H. 2, Wiesbaden, 0.J., S. $17 \mathrm{ff}$.

Otto, K., Umweltschutz überfordert Städte, in: Umwelt 1975, 5, s. $13 \mathrm{ff}$.

Otto, K., Warenbeurteilung nach ökologischen Aspekten, in: Umwelt 1973, 4, S. $46 \mathrm{f}$.

o.V., Das Beispiel Bosch, In: Die Wirtschaftswoche, Nr. 39 vom 24.9.1971

o.V., How to Estimate the Real Cost of Environmental Damage, in: OECD-Observer, No. 75, 1975, S. $29 \mathrm{f}$.

o.v., Der Dom im Wettlauf mit der Zeit, in: Umwelt 1972, 6, S. $38 \mathrm{f}$.

o.V., Dünger aus Klärschlamm, in: U 1974, 2

o.V., Environmental Education in the Universities, A Key to "Relevance", in: OECD-Observer, No. 68, 1974, S. $27 \mathrm{ff}$.

o.V., Environment - A New Programme of International

Co-operation, in: OECD-Observer, No. 58, 1972, S. 27 f. 
o.V., Mehrjährige Forschungsprogramme der Gemeinschaft: Vorschläge der Kommission, in: EG-Bulletin, 8. Jg., 1975, H. $7 / 8, S .17 \mathrm{ff}$.

o.V., Gewässerüberwachung in Hessen, in: U 1973, 2

o.V., Grundgesetz für die Luftreinhaltung: Die TA Luft, in: Umwelt 1974, 4, S. 12

o.V., Initiative für Bayerns Luft, in: U 1973, 5

o.V., Krebs zerstört die Akropolis, in: U 1974, 4, S. 12

o.V., Fahrendes MeBlabor für Umweltdaten, in: Umwelt 1971, 6, S. 45

o.V., Nachbarn eines Gewerbegebietes müssen mehr Lärm ertragen, in: Umwelt 1973, 5, S. 68

o.V., OECD and the Environment, in: OECD-Observer, No. 53, 1971 , S. 19 ff.

o.V., Some Recent OECD Initiatives in the Field of the Environment, in: OECD-Observer, No. 62, 1973, S. 8 ff.

o.V., Who "Exports" and Who "Imports" Pollution?, in: OECDObserver, No. 70, 1974, S. 12 f.

o.V., Der Schwerpunkt Umweltschutz: Das Milliardenrätsel, in: Die Wirtschaftswoche, 26. Jg., 1972, H. 8, S. 68 ff.

o.V., Technology Assessment Harnessing Science to Social Goals, in: OECD-Observer, No. 67, 1973, S. 29 ff.

o.V., Umweltschutz - Angst der Gewerkschaften, in: Die Wirtschaftswoche, $\mathrm{Nr} .14$ vom 30.3.1973

o.V., Umweltschutz als Mythos. Rede Borlaugs vor der Römer FAO-Konferenz, in: NZZ, FA Nr. 308 vom 10.11.1971

o.V., Wir und der Umweltschutz, in: Rohrpost 53

o.V., Der Verbraucher von morgen tut nicht mehr alles. Vorschläge an amerikanische Konsumenten, in: Blick durch die Wirtschaft vom 7.10.1971

Pätzold, E., Verbindliche Bewertungskriterien, in: U 1975, 5

Palmstierna, L. und H., Unsere geplünderte Welt, Weinheim und Basel 1972

Passino, R., Industrie und Landschaft, in: Offner, H. (Hrsg.), a.a.0., S. $39 \mathrm{ff}$.

Paul, M.E., Can Aircraft Noise Nuisance be Measured in Money?, In: Oxford Economic Papers, vol. 23, 1971, S. $297 \mathrm{ff}$.

Pearce, D. The Economic Evaluation of Nolse-Generating and Noise Abatement Projects, in: OECD (Hrsg.), Problems of Environmental Economics, a.a.0., S. $103 \mathrm{ff}$.

Pearce, D., The Limits of Cost-Benefit Analysis as a Guide to Environmental Policy, in: Kyklos, Bd. 29, 1976, S. $97 \mathrm{ff}$. 
Peltzman, S./Tideman, T.N., Local Versus National Pollution Control: Note, in: American Economic Review, vol. 62, 1972, S. 959 ff.

Pennance, F.G., The Polluters: Industry or Government?, The Institute of Economic Affairs, London 1972, S. $35 \mathrm{ff}$.

Peters, G.H., Cost Benefit Analyse und staatliche Aktivität, Hamburg 1968

Pfennig, N., Verhalten von Kunststoffen bei der Abfallbeseitigung, in: Probleme der Umweltforschung, a.a.o., S. $140 \mathrm{ff}$.

Picht, G., Wir brauchen neue Uberzeugungen, in: Schlemmer, J. (Hrsg.), a.a.0., s. 130 ff.

Picht, G., Umweltschutz und Politik, in: von Weizsäcker, E. (Hrsg.), a.a.0., S. 80 ff.

Pigou, A.C., The Economics of Welfare, Reprint der 4. Auflage von 1932, London 1952

Pigou, A.C., Volkswirtschaftlicher und privatwirtschaftlicher Reinertrag und die Lehre von der Maximalbefriedigung, in: Mayer, H./Fetter, F.A./Reisch, R. (Hrsg.), Die Wirtschaftstheorie der Gegenwart, Bd. 3, Wien 1928

Piperek, M., "Bauen als Umweltzerstörung". Eine visuelle Bankrotterklärung, in: Umwelt 1974, 1, S. $28 \mathrm{f}$.

Plaggemeier, W., Zivildienst: Modellversuche mit Umweltschutz, in: Umwelt 1975, 2, S. $41 \mathrm{f}$.

Plott, C.R., Externalities and Corrective Taxes, in: Staaf, R.J./Tannian, F.X. (Hrsg.), a.a.0., S. $261 \mathrm{ff}$.

Plourde, C.G., A Model of Waste Accumulation and Disposal, in: Canadian Journal of Economics, vol. 5, 1972, S. 119 ff.

Pötschke, H., Lösung mit Zukunft: Kompostierung, in: U 1974, 1 Pole, N. (Hrsg.), Environmental Solutions, Cambridge 1972

Environmental pollution. A Challenge to Science and

Technology. Report of the Subcommittee on

Science, Research, and Development to the

Committee on Science and Astronautics,

U.S.G.P.O., Washington, D.C. 1966

La Porte, T.R., The Context of Technology Assessment: A Changing Perspective for Public Organization, in: Public Administration Review, 1971, S. $63 \mathrm{ff}$.

Preuschen, G., Der Einfluß der Landwirtschaftsformen auf die Änderung in der natürlichen Umwelt, in: Sioli, H. (Hrsg.), a.a.0., S. 269 ff.

Preussmann, R., Analytik und Entstehung von N-Nitroso-Verbindungen, in: DFG-Mitteilungen 2/74, S. $21 \mathrm{ff}$. 
Zur Problematik verursachungsgerechter Berechnungsschlüsse 1 in der Abwasserableitung, VIK-Berichte $\mathrm{Nr}$. 186, Essen 1974

Zur Problematik des Verursacherprinzips, Berlin 1972

Probleme der Umweltforschung, Berlin 1973

Randall, A./Ives, B./Eastman, C., Bidding Games for Valuation of Aesthetic Environmental Improvements, in: Journal of Environmental Economics and Management, vol. 1, 1974 , S. $132 \mathrm{ff}$.

Randers, G., NATO's International Governmental Cooperation on Environmental Management, in: Kneese, A.V./Rolfe, S.E./ Harned, J.W. (Hrsg.), a.a.O., S. $343 \mathrm{ff}$.

Rasch, R., Emissionen aus Müllverbrennungsanlagen bel Müllund Kunststoffverbrennung, in: Müll und Abfall, 4. Jg., 1972 , H. 3, S. $88 \mathrm{ff}$.

Rasch, R., Kunststoffe in der Müllverbrennung, in: U 1971, 4

Rasenack, D., Kommen die abbaubaren Kunststoffe?, in: Blick durch die Wirtschaft vom 20.8.1971

Der Rat von Sachverständigen für Umweltfragen, Umweltgutachten 1974, Stuttgart und Mainz 1974

Der Rat von Sachverständigen für Umweltfragen, Umweltprobleme

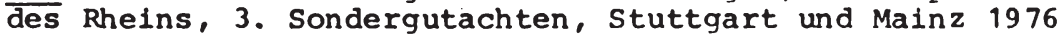

Rathjens, G.W., National Environmental Policy: Goals and Priorities, in: Grad, F.K./Rathjens, G.W./Rosenthal, H.J. (Hrsg.), a.a.O., S. $11 \mathrm{ff}$.

Raumordnung und Umweltschutz, hrsg. vom Bundesministerium für Raumordnung, Bauwesen und Städtebau, 0.0 . und $\mathrm{J}$.

Recktenwald, H.C. (Hrsg.), Das Umweltproblem aus ökonomischer und juristischer Sicht, Göttingen 1975

Recycling in der Materialwirtschaft, hrsg. vom SPIEGEL-Verlag, Hamburg 1975

Redeker, K., Verfahrensrechtliche Bedenken gegen die Verbandsklage, in: Zeitschrift für Rechtspolitik, 9. Jg., 1976 , S. $163 \mathrm{f}$.

Reglerungserklärung des Bundeskanzlers vor dem Deutschen Bundestag vom 28.10.1969, in: Bulletin des Presse- und Informationsamtes der Bundesregierung, Nr. 132 vom 29.10.1969

Rehbinder, E., Argumente für die Verbandsklage im Umweltrecht, in: Zeitschrift für Rechtspolitik, 9. Jg., 1976, S. $157 \mathrm{ff}$.

Rehbinder, E., Grundfragen des Umweltrechts, in: Zeitschrift für Rechtspolitik, 3. Jg., 1970, S. $250 \mathrm{ff}$.

Rehbinder, E., Umweltschutz: Die Rolle von Gesetzgebung und Rechtsprechung, in: Umwelt 1971, 1, S. $23 \mathrm{ff}$. 
Reichardt, R., Approaches to the measurement of environment, in: International Social Science Journal, vol. 22, 1970 , S. $661 \mathrm{ff}$.

Rembold, G., Rohstoffverknappung und Umweltbelastbarkeit als Determinanten des regionalen, sektorspezifischen Wirtschaftswachstums - ein regionalisierter, multisektoraler Modellansatz -, in: Wirtschaftstheorie, Wirtschaftspolitik und Planung öffentlicher Ausgaben, Karlsruhe 1975, S. $123 \mathrm{ff}$.

Repenning, K., Das Verursacherprinzip, in: Zur Problematik des Verursacherprinzips, a.a.0., S. 9 ff.

Research into Environmental Pollution, Report of Five WHO Scientific Groups, WHO-Technical Report Series, No. 406, Genf 1968

Ridker, R.G., Economic Costs of Air Pollution. Studies in Measurement, New York-Washington-London 1967

Ridker, R.G./Henning, J.A., The Determinants of Residential Property Values with Special Reference to Air Pollution, in: The Review of Economics and Statistics, vol. 49, 1967 , S. $246 \mathrm{ff}$.

Riederer, J., Plädoyer für die Verwitterung. Steinschäden auch ohne Rauchgas, in: Umwelt 1974, 1, S. 42 f.

Riegel, R., Gewässergütewirtschaft: Immissions-oder Emissionsstandard?, in: Umwelt $1976,1, \mathrm{~s} .41 \mathrm{ff}$.

Rincke, G., Die Abwasserabgabe als mögliches Optimierungsinstrument aus der sicht der Wassergütewirtschaft, in: Issing, O. (Hrsg.), a.a.0., S. $99 \mathrm{ff}$.

Rincke, G., Anwendbarkeit des Verursacherprinzips am Beispiel der Wassergütewirtschaft, in: Das Verursacherprinzip und seine Instrumente, a.a.0., S. 109 ff.

Riordan, C., Multistage Marginal Cost Model of Investment Pricing Decisions: Application to Urban Water Supply Treatment Facilities, in: Water Resources Research, vol. 7, 1971, S. $463 \mathrm{ff}$.

Rodi, D., Umweltschutz in Forschung und Lehre im Bereich der Biologie an Pädagogischen Hochschulen, in: Steubing, L./ Kunze, C./Jäger, J. (Hrsg.), a.a.0., S. 207 ff.

Röntcke, G., Die Atmosphäre, thre Entwicklung und gegenwărtige Situation. Änderungen durch den Einfluß des Menschen, in: Sioli, H. (Hrsg.), a.a.0., s. $35 \mathrm{ff}$.

Rönicke, G., Messung der Luftverunreinigung im Rahmen eines Netzes von Backgroundstationen, in: Olschowy, G. (Hrsg.), a.a.0., S. $107 \mathrm{ff}$.

Rokosch, P., Mit Flugzeug und Kamera, in: U 1972, 5, s. 32 ff. Ronge, V., Die Umwelt im kapitalistischen System, in: Glagow, M. (Hrsg.), a.a.0., S. $97 \mathrm{ff}$. 
Roth, C., Programme, aber keine Pläne. Zum Planungsbegriff am Beispiel Umweltproblematik, in: Umwelt 1972,4, S. $14 \mathrm{ff}$.

Rupp, H.H., Mobilisierung des Umweltschutzrechts durch Beteiligung der Aktivbürgerschaft an seinem Vollzug; unveröffentlichtes Manuskript eines Vortrags an der Johannes Gutenberg-Universität Mainz, Mainz 1976

Rupp, H.H., Popularklage im Umweltschutzrecht?, in: Zeitschrift für Rechtspolitik, 5. Jg., 1972, H. 2, S. 32 ff.

Rupp, H.H., Rechtsgutachten zur Intensivierung des Umweltschutzes durch Verstärkung der Initiative Privater und zur Harmonisierung des Umweltschutzrechts der Mitgliedstaaten in den Europäischen Gemeinschaften, Mainz 1974/75

Rupp, H.H., Die verfassungsrechtliche Selte des Umweltschutzes, in: Juristenzeitung $\mathrm{Nr} .13 \mathrm{vom} 2$. Juli 1971

Rupp, H.H. Umweltschutz und Demokrat1e, 1n: Umweltschutz aber wie?, a.a.0., S. $60 \mathrm{ff}$.

Russe11, C.S., Mt dels for Investigation of Industrial Responsc to Residuals Management Actions, in: Swedish Journal of Economics, vol. 73, 1971, S. $143 \mathrm{ff}$.

Russel1, C.S./Spofford jr. W.O., A Quantitative Framework for Residuals Management Decisions, in: Kneese, A.V./ Bower, B.T. (Hrsg.), a.a.O., S. $115 \mathrm{ff}$.

Russell, C.S./Vaughan, W.J., A Linear Programming Model of Residuals Management for Integrated Iron and Steel Production, in: Journal of Environmental Economics and Management, vol. 1, 1974, S. $17 \mathrm{ff}$.

Sachs, I., Development, Environment and Technology Assessment, in: International Social Science Journal, vol. 25, 1973 , S. 273 ff.

Der Sachverständigenrat zur Begutachtung der gesamtwirtschaftlichen Entwicklung, Vor dem Aufschwung, Jahresgutachten $1975 / 76$, Stuttgart und Mainz 1975

Saladin, P., Gleichgewichtssicherung als Staatsaufgabe, in: NZZ, FA Nr. 195, vom 22./23.8.1976

Salgo, H., The Obsolescence of Growth: Capitalism and the Environmental Crisis, in: Review of Radical Political Economics, vol. 5, H. 3, 1973, S. $26 \mathrm{ff}$.

Sander, J., Ernährung und Krebs, in: Schultze, H. (Hrsg.), a.a.o., S. $301 \mathrm{ff}$.

Sandscheper, G., Luftüberwachungsnetz für die Niederlande, in: Umwelt $1971,6, \mathrm{~S} .34 \mathrm{f}$.

Sax, J.L., Legal Redress of Environmental Disruption in the United States: The Role of Courts, in: Tsuru, S. (Hrsg.), a.a.0., S. 223 ff. 
Sax, J.L., Standing to Sue: A Critical Review of the Mineral

King Decision, in: Natural Resources Journal, vol. 13, 1973, S. 76 ff.

Sax, J.L., Legal Strategies Applicable to Environmental

Quality Management Decisions, in: Kneese, A.V./Bower, B.T. (Hrsg.), a.a.0., s. $333 \mathrm{ff}$.

Schaafhausen, J., Industrie zu Maßnahmen bereit, in: Wirtschaftsdienst, 50. Jg., 1970, S. $639 \mathrm{f}$.

Schaafhausen, J., Wasser- und Luftreinhaltung aus der Sicht der Industrie, in: Wasser und Abwasser, 1968, S. $9 \mathrm{ff}$.

Schackmann, H., Umwelt und Wirtschaft im Ruhrgebiet. Schriften der Volks- und Betriebswirtschaftlichen Vereinigung im Rheinisch-Westfälischen Industriegebiet, 14. Sonderveröffentlichung, Düsseldorf 1973

Schäfer, Dieter, Soziale Schäden, soziale Kosten und soziale Sicherung, Berlin 1972

Schäfer, Friedrich, Das Verursacherprinzip in der Umweltschutzpolitik, in: Umwelt $1972,2,5.4 \mathrm{ff}$.

Schaefer, Gerhard, Kybernetische Modelle als Hilfsmittel zur Darstellung ökologischer Zusammenhänge, in:

Steubing, L./Kunze, C./Jäger, J. (Hrsg.), a.a.O., S. $181 \mathrm{ff}$.

Schaefer, Hans (Hrsg.), Folgen der Zivilisation. Therapie oder Untergang?, Frankfurt 1974

Schaefer, Hans, Hinweise auf Umweltschäden aus Lebenserwartung, spezifischen Sterblichkeiten, Sterbeziffern und Krankheitshäufigkeiten, in: Schaefer, Hans (Hrsg.), a.a.O., S. $72 \mathrm{ff}$.

Schaefer, Hans, Der Mensch in der technisch veränderten Welt, in: Schaefer, Hans (Hrsg.), a.a.0., S. $22 \mathrm{ff}$.

Schaefer, Hans, Die Okologie als Problem des Umweltschutzes, in: Schaefer, Hans (Hrsg.), a.a.0., S. $95 \mathrm{ff}$.

Schäfer, Wilhelm, Der kritische Raum. Uber den Bevölkerungsdruck bei Tier und Mensch. Kleine Senckenbergreihe 4 , Frankfurt am Main 1971

Schär, M., Der Mensch als Nutznießer und Opfer der technischen Entwicklung, in: Leibundgut, H. (Hrsg.), a.a.O., S. $28 \mathrm{ff}$.

van Schaik, R.J., The Impact of the Economic Situation on Environmental Policies, in: OECD-Observer, No. 79, 1976 , S. 25 f.

Schatz, K.-W., Administrative und marktwirtschaftliche Maßnahmen zur Lösung des Umweltschutzproblems, in: Giersch, H. (Hrsg.), a.a.0., S. $125 \mathrm{ff.}$ 
Schellhaaß, H.M., Kraftfahrzeugsteuer - Für eine marktgerechte Lösung, in: Wirtschaftswoche Nr. 28, 1971, S. $22 \mathrm{ff}$.

Schenk, K.E., Externe Effekte und rationale Wirtschaftspolitik, in: Hamburger Jahrbuch, 13. Jg., 1968, S. $283 \mathrm{ff}$.

Scherrer, H.U., Gesamtwertung der Umweltbelastung, in: NZZ, FA Nr. 131, vom 14.5.1972

Schlemmer, J. (Hrsg.), Neue Ziele für das Wachstum, München 1973

Schlieper, U., Pareto-optima, externe Effekte und die Theorie des Zweltbesten, Köln-Berlin-Bonn-München 1969

Schlipköter, H.-W., Auswirkung von Autoabgasen auf die Stadtbevölkerung, in: Forschung '74, Frankfurt 1973, S. $30 \mathrm{ff}$.

Schlipköter, H.-W., Belastungen des Menschen durch die Umweltverschmutzung, in: Probleme der Umweltforschung, a.a.0., S. $54 \mathrm{ff}$.

Schlipköter, H.-W., Industrieansiedlung und Umweltschutz, in: Raumforschung und Raumordnung, 30. Jg. 1972, S. 202 ff.

Schlipköter, H. -W., Die Luftverunreinigung als gesundheitliches Problem, in: Schultze, H. (Hrsg.), a.a.O., S. $199 \mathrm{ff}$.

Schlipköter, H.-W., MIK-Werte dienen dem Schutz der Bevölkerung, in: Umwelt $1973,5, \mathrm{~S} .36 \mathrm{ff}$.

Schlipköter, H.-W., MIK-Wert-Vorschläge für die Schwermetalle Blei, Zink und Cadmium, in: Umwelt 1973, 2, S. $12 \mathrm{ff}$.

Schlipköter, H.-W., Krank von der Stadtluft, in: U 1973, 2

Schlipköter, H.-W./Beck, E.G., Die zellschädigende Wirkung von Blei, in: Umwelt 1972, 5, S. $50 \mathrm{ff}$.

Schlipköter, H.-W./Dolgner, R., Epidemiologische Untersuchuncen im $\mathrm{Z}$ usammenhang mit lufthygienischen Fragen, in: Zentralblatt für Bakteriologie ..., S. $272 \mathrm{ff}$.

Schlippenbach, S. Graf v., Viele Milliarden fließen für sauberes Wasser und reine Luft, in: Blick durch die Wirtschaft vom 21.6.1971

Schmid, R., Gefährdete Pflanzen in der heutigen welt, in: Universitas, 28. Jg., 1973, S. 1351 ff.

Schmidt, Ferdinand, Krebsursache: Abqas oder Tabakrauch, in: Unwelt $1971,6, \mathrm{~S} .28 \mathrm{ff}$.

Schmidt, Helmut, Wirtschaftspolitische Möqlichkeiten, Proaramme und Maßnahmen zum Umweltschutz, in: Kapp, K.W./ Vilmar, F. (Hrsg.), a.a.O., s. $171 \mathrm{ff}$.

Schmidt, Helmut, Umweltzerstörung und Sozialkosten, in: Kapp, K.W./Vilmar, F. (Hrsg.), a.a.O., S. $79 \mathrm{ff}$. 
Schmidt, Kurt, Zur politischen Reaktion auf Nachfragewogen in der Staatswirtschaft, in: Finanzarchiv, Bd. 33, 1974 , S. $30 \mathrm{ff}$.

Schmidt-Burbach, G.M., Klimaänderung im urbanen Bereich, in: $\mathrm{U} 1973,4, \mathrm{~S} .20 \mathrm{ff}$.

Schneider, Lothar, Umweltschutz in Ludwigshafen, in: Der Städtetag, 1971, 4, S. $194 \mathrm{ff}$.

Schneider, Wilhelm, Verschmutzende Saubermacher. Bedrohliche Phosphatverschmutzung unserer Gewässer, in: Umwelt 1971, 3, S. 26 f.

Schneider, Wolfgang, Verursacherprinzip oder die Belastung aller?, In: Gumpel, W./Keese, G. (Hrsg.), a.a.O., S. $457 \mathrm{ff}$.

Schoeck, H., రkospasmus oder die Angst vor Kernkraftwerken, in: Umwelt 1976,2 , S. $125 \mathrm{ff}$.

Scholder, K., Zur Definition und Systematisierung der Umweltforschung, in: Umwelt 1972, 6, S. $36 \mathrm{ff}$.

Schrafl, A.E., Öffentliche Verantwortung des Unternehmers, in: NZZ, FA Nr. 111 vom 25.4.1971

Schreiber, M., Kosten der Luftreinhaltung, in: Wasser, Luft und Betrieb, Bd. 15, 1971, H. 3, S. $105 \mathrm{ff.,} \mathrm{H.} \mathrm{4,}$ S. $145 \mathrm{ff}$. und H. 5, S. $179 \mathrm{ff}$.

Schütz, H., Umweltschutz - Meßwagen, Einzelbericht L $3474 \mathrm{zu}$ $\mathrm{H}$ \& B-Meßwerte, 0.O. und $\mathrm{J}$.

Schulte, H.J., Maximale Emissions-Konzentrationen für 20 Stoffe erarbeltet, in: Umwelt 1973, 6, S. $37 \mathrm{f}$.

Schultz, U. (Hrsg.), Lebensqualität, Frankfurt/Main 1975

Schultz, U. (Hrsg.), Umwelt aus Beton oder Unsere unmenschlichen Städte, Reinbek 1971

Schultze, C.L. u.a., Setting National priorities, The 1973 Budget, Washington, D.C. 1972

Schultze, C.I. u.a., setting National Priorities, The 1972 Budget, Washington, D.C. 1971

Schultze, H. (Hrsg.), Umwelt-Report. Unser verschmutzter Planet, Frankfurt 1972

Schultze, P.M., Region und Informationssystem, unveröffentlichte Habilitationsschrift, Mainz 1975

Schumacher, D., Hilfsmittel für die Umweltplanung, in: U 1973,5

Schwabe, G.H., Die Rolle des Menschen - Anmerkungen zu einer kritisch-angewandten రkologie, in: Steubing, L./ Kunze, C./Jäger, J. (Hrsg.), a.a.O., S. $239 \mathrm{ff}$.

Schwarzes Kollektiv, Okologie und Macht, in: Kursbuch 33, Berlin 1973, S. $95 \mathrm{ff}$. 
Schwoerbel, J., Biologie des Wassers, in: Schröder, B. (Hrsg.), Wasser, Frankfurt/Main 1970, s. 177 ff.

Seldenfus, H.S., Umweltschutz, politisches System und wirtschaftliche Macht, in: Schneider, H.K./Watrin, C. (Hrsg.), Macht und ökonomisches Gesetz, Bd. 2, Berlin 1972, S. 809 ff.

Siebert, A., Jugend und Umweltschutz. Materialien Umweltschutz und Raumordnung, H. 8, hrsg. von der Akademie für Raumforschung und Landesplanung, Hannover 1974.

Siebert, H., Analyse der Instrumente der Umweltpolitik, Göttingen 1976

Siebert, H., Das produzierte Chaos, రkonomie und Umwelt, Stuttgart-Berlin-Köln-Mainz 1973

Siebert, H., Environment and Regional Growth, in: Zeitschrift für Nationalökonomie, Bd. 33, 1973, S. 79 ff.

Siebert, H., Erfolgsbedingungen einer Abgabenlösung (Steuern/ Gebuhren) in der Umweltpolitik, in: Issing, O. (Hrsq.), a.a.O., S. $35 \mathrm{ff}$.

Siebert, H., Gebuhren als Instrument der Umweltpolitik, in: WISU, 3. Jg., 1974, S. $13 \mathrm{ff}$.

Slebert, H., Probleme von Nutzen-Kosten-Analysen umweltschützender Maßnahmen, in: Wirtschaftsdienst, 53, Jg., 1973, S. $131 \mathrm{ff}$.

Slebert, H., Regional Planning - land use approaches to environmental quality management, Discussion paper $67 / 75$ des Instituts fur Volkswirtschaftslehre und statistik der Universităt Mannheim

Siegrist, H.R., Die Auswirkungen der Energiewirtschaft auf die Umwelt, in: Lelbundgut, H. (Hrsg.), a.a.0., S. $51 \mathrm{ff}$.

Siemens AG, Forschung und Entwicklung, München 0.J., S. 40 ff.

Simonis, W., Eutrophierung, in: Schaefer, Hans (Hrsg.), a.a.0., S. $169 \mathrm{ff}$.

Simonis, W., Pestizide und thre Akkumulation in den "Nahrungsketten", In: Schaefer, Hans (Hrsg.), a.a.0., S. $256 \mathrm{ff}$.

Siol1, H. (Hrsg.), రkologle und Lebensschutz in internationaler Sicht, Freiburg 1973

Sinth, R.E., Political Control of Technology to Preserve Human Values, in: The Engineering Economist, vol. 15, 1970, S. 217 ff.

Smith, V.L., Dynamics of Waste Accumulation: Disposal Versus Recycling, in: Quarterly Journal of Economics, vol. 86, 1972 , S. $600 \mathrm{ff}$.

Sobers, D.G., Planning for the Environmental Revolution, In: Armstrong, T.R. (Hrsg.), a.a.O., S. $63 \mathrm{ff}$.

Sohmen, E., Allokationstheorie und Wirtschaftspolitik, Tübingen 1976 
Solow, R.M., The Economist's Approach to Pollution and its Control, in: Sclence, vol. 173, No. 3996 v. 6.8.1971

Sontheimer, H., Untersuchungen zur Belastung des Rheins mit organischen Stoffen, in: Gas- und Wasserfach. GWF, Wasser 111, 1970, 8, S. $420 \mathrm{ff}$.

Speer, J., Zur Definition, Systematik und Organisation der Umweltforschung, in: DFG-Mitteilungen $4 / 73$, S. 3 ff.

Sprenger, R.-U., Struktur und Entwicklung von Umweltschutzaufwendungen in der Industrie, Berlin und München 1975

Staaf, R.J./Tannian, F.X. (Hrsg.), Externalities. Theoretical Dimensions of Political Economy, New York-London 0.J.

Stahl, K./Curdes, G., Umweltplanung in der Industriegesel1schaft. Lösungen und ihre Probleme, Hamburg 1970

Staudt, E./Metze, G., Technology Assessment, in: Wist, 5. Jg., 1976 , S. $81 \mathrm{f}$.

Steckert, U., Umweltschutz als kommunale Aufgabe, in: Umwelt 1971,6 , S. $15 \mathrm{f}$.

Steckeweh, P., Umweltschutz und Städtebau, in: Bundesbaublatt, Bd. 20, 1971, S. 70 ff.

Stegmaier, W., Dünger aus Klärschlamm, in: U 1976, 3

Stehfest, H., Standards, in: Problematik des Verursacherprinzips, a.a.0., s. $43 \mathrm{ff}$.

Steiger, H., Umweltschutz durch planende Gestaltung, in: Zeltschrift für Rechtspolitik, 4. Jg., 1971, S. $133 \mathrm{ff}$.

Steln, J.L., Priorities and Pollution: Reply, in: American Economic Review, vol. 64, 1974, S. 718 ff.

Stein, J.L., The 1971 Report of the President's Council of Economic Advisers: Microeconomic Aspects of Public Policy, in: American Economic Review, vol. 61, 1971, S. $531 \mathrm{ff}$.

Steinbuch, K., Die Kontrolle der Technik und die probleme unserer Gesellschaft in Gegenwart und $\mathrm{Zukunft}$, in: Universitas, 27. Jg., 1972, S. $229 \mathrm{ff}$.

Steinbuch, K., Mensch, Technik, Zukunft. Basiswissen für Probleme von morgen, stuttgart 1971

Stelte, W., Die Kontamination von Lebensmitteln mit schädlichen Stoffen aus der Umwelt, in: Schaefer, Hans (Hrsg.), a.a.0.. S. 60 ff.

Stephens, J.K., "A Theoretical Note on the Capacity of the Market System to Abate Pollution": A Reply, in: Land Economics, vol. 48, 1972, S. $304 \mathrm{f}$.

Steubing, L./Kunze, C./Jtiger, J. (Hrsg.), Belastung und Belastbarkeit von ökosystemen. Tagungsbericht der Gesellschaft für ökologie, Gießen 1972 
Stockmann, H.-U., Die natürlichen Faktoren in der Planung, in: Institut für Raumforschung, Informationen, Bd. 20, 1970 , S. 365 ff.

Stöwe, H./Härtter, E., Lehrbuch der Mathematik für Volks- und Betriebswirte, Göttingen 1967

Stratmann, H., Emissionsüberwachung und Immissionsschutz, IBMSeminar "Umweltschutz und Datenverarbeitung" vom

7. bis 9. März 1973 in Bad Liebenzell

Straub, H., Kompostierung der Abfälle, sinnvollste Methode, in: Umwelt 1973, 3, S. 56 ff.

Streibel, M., Umweltpolitik in der Bundesrepublik Deutschland, in: Horn, C./von Walterskirchen, M.P./Wolff, J.

(Hrsg.), a.a.O., S. $233 \mathrm{ff}$.

Summary Report of the Cornell Workshop on Energy and the Environment, U.S.G.P.O., Washington, D.C. 1972

Swan, J.A., Attitudes and Values and Environmental

Education, in: Armstrong, T.R. (Hrsg.), a.a.O., S. $124 \mathrm{ff}$.

Swoboda, H., Probleme der Umweltpolitik, in: Wirtschaft und Gesellschaft, 1. Jg., 1975, 1, S. $77 \mathrm{ff}$.

Tabasaran, O., Abfallbeseitigung: Pyrolytische Behandlung von Hausmüll und Abwasserschlamm, in: Umwelt 1976, 2 , S. $81 \mathrm{f}$.

Tax Foundation Inc., Pollution Control: Perspectives on the Government Role, A Summary, New York 1971

Taylor, G.R., Das Selbstmordprogramm. Zukunft oder Untergang der Menschheit, Frankfurt 1971

Thom, G.C.lOtt, W.R., Air Pollution Indices. A Compendium and Assessment of Indices Used in the United States and Canada, U.S.G.P.O., Washington, D.C. 1975

Thomas, H.A., Ein mathematisches Modell zur Diskussion von Umwelt-Gütenormen, in: Umwelt 1973, 2, s. $37 \mathrm{ff}$.

Thoss, R., Zur Planung des Umweltschutzes, in: Raumforschung und Raumordnung, 30. Jg., 1972, S. $180 \mathrm{ff}$.

Thoss, R., Verursacherprinzip - Kompensation oder Verhinderung von Umweltschäden?, in: Schultze, H. (Hrsg.), a.a.0., S. $21 \mathrm{ff}$.

Thoss, R., Ziel, Ansatz und Methoden eines integrierten Optimierungsmodells zur Verbesserung des Umweltschutzes, in: Seminarberichte 8 der Gesellschaft für Regionalforschung, Münster $1974, \mathrm{~s} .3 \mathrm{ff}$.

Tietenberg, T.H., On Taxation and the Control of Externalities: Comment, in: American Economic Review, vol. 64, 1974 , S. 462 ff.

Timm, B., Ausführungen auf der 20. ordentlichen Hauptversammlung der BASF am 28.6.1972 
Timm, B., Leben mit der Chemie - heute und morgen, in: Universitas, 28. Jg., 1973, S. 1201 f.f.

Tinbergen, J., Centralization and Decentralization in Economic Policy, Amsterdam 1954

The Torrey Canyon, Report of the Committee of Scientists on the Scientific and Technological Aspects of the Torrey Canyon Desaster, H.M.S.O., London 1967

Tourismus in der Bundesrepublik Deutschland - Grundlagen und Ziele, BT-Drucksache 7/3840'

Tschumi, P.A., Bevölkerungswachstum, Wirtschaft und Umweltschutz, in: NZZ, FA Nr. 236 vom 29.8.1971

Tsuru, S. (Hrsg.), Proceedings of the International Symposium on Environmental Disruption, Tokio 1970

Tullock, G., Öffentliche Entscheidungen als öffentliche Güter, in: Frey, B.S./MeiBner, W. (Hrsg.), Zwei Ansätze der politischen Ökonomie, Marxismus und ökonomische Theorie der Politik, Frankfurt am Main 1974, S. $97 \mathrm{ff}$.

Turvey, R., On Divergences between Social Cost and Private Cost, in: Economica, N.S., Bd. 30, 1963, S. 309 ff.

Uhrhammer, C., Umweltverschmutzung durch Profitinteressen?, in: Dörge, F. (Hrsg.), a.a.0., S. $69 \mathrm{ff}$.

Ui Jun, Der japanische Kapitalismus als Vorhut der ökologischen Katastrophe, in: Kursbuch 33, Berlin 1973, S. $163 \mathrm{ff}$.

Ulrich, B., Die Filterfunktion von Böden, in: Steubing, L./ Kunze, C./Jäger, J. (Hrsg.), a.a.O., S. $169 \mathrm{ff}$.

Umwelt-ABC, Wiesbaden 1972

Umweltforschungskatalog 175, Berlin 1975

Umweltmarkt von A bis Z, Sonderausgabe von U, Dezember 1976

Das Umweltprogramm der Bundesregierung, 3. Auflage, stuttgartKöln-Berlin-Mainz 1973

Umweltschutz (1): Wasserhaushalt, Binnengewässer, hohe See und Küstengewässer, Aus den öffentlichen Anhörungen des Innenausschusses und des Ausschusses für Jugend, Familie und Gesundheit des Deutschen Bundestages, Zur Sache 3/71

Umweltschutz (2): Luftreinhaltung und Abfallbeseitigung, Aus den öffentlichen Anhörungen des Innenausschusses des Deutschen Bundestages, Zur Sache 3/72

Umweltschutz (3): Lärmbekämpfung, Bundes-Immissionsschutzgesetz, Aus der öffentlichen Anhörung des Innenausschusses des Deutschen Bundestages, Zur Sache 4/73

Umweltschutz (4): Das Risiko Kernenergie, Aus der öffentlichen Anhörung des Innenausschusses des Deutschen Bundestages, Zur Sache 2/75 
Umweltschutz - aber wie? Rechtliche Hindernisse, rechtliche Möglichkeiten. Schriften der evangelischen Akademie in Hessen und Nassau, H. 95, Frankfurt 1972

UNESCO, Use and Conservation of the Biosphere. Proceedings of the Intergovernmental Conference of Experts on the Scientific Basis for Rational Use and Conservation of the Resources of the Biosphere, Paris 4-13 Sept. 1968, Paris 1970

U.S. Council of Economic Advisers, The Annual Report of the Council of Economic Advisers, 1966, Washington, D.C. 1966

U.S. Council of Economic Advisers, The Annual Report of the Council of Economic Advisers, 1971, Washington, D.C. 1971

U.S. Council on Environmental Quality, Environmental Quality, The First Annual Report, Washington, D.C. 1970

U.S. Council on Environmental Quality, Environmental Quality, The Second Annual Report, Washington, D.C. 1971

U.S. Council on Environmental Quality, Environmental Quality, The Third Annual Report, Washington, D.C. 1972

U.S. Council on Environmental Quality, Environmental Quality, The Fourth Annual Report, Washington, D.C. 1973

U.S. Council on Environmental Quality/Department of Commerce/ Environmental Protection Agency, The Economic Impact of Pollution Control, U.S.G.P.O., Washington, D.C. 1972

U.S. Department of Commerce, The Noise Around us. Findings and Recommendations, Sept. 1970, U.S.G.P.O., Washington, D.C. 1971

U.S. Department of Health, Education, and Welfare, Man's Health and the Environment. Some Research Needs, Washington, D.C. 1970

U.S. Department of the Interior/Federal Water Pollution Control Administration, The Cost of Clean Water, 2 Bde., U.S.G.P.O., Washington, D.C. 1968

U.S. Department of the Interior/Federal Water Pollution Control Administration, The Cost of Clean Water, Summary Report, Washington, D.C. 1968

U.S. Department of the Interior/Federal Water Pollution Control Administration, The Economics of Clean Water, 2 Bde., U.S.G.P.O., Washington, D.C. 1970

U.S. Department of the Interior/Federal Water Pollution Control Administration, The Economics of Clean Water, Summary Report, U.S.G.P.O., Washington, D.C. 1970

U.S. Environmental Protection Agency, The Cost of Clean Water, Annual Report of the Administrator of the Environmental protection Agency to the Congress of the United States, U.S.G.P.O., Washington, D.C. 1971 
U.S. Environmental Protection Agency, Effects of Noise on People, U.S.G.P.O., Washington, D.C. 1971

U.S. Environmental Protection Agency, Toward a New Environmental Ethic, U.S.G.P.O., Washington, D.C. 1971

U.S. Environmental Protection Agency, Don't Leave it All to the Experts. The Citizen's Role in Environmental Decision Making, U.S.G.P.O., Washington, D.C. 1972

U.S. Environmental Protection Agency, The Economic Impact of Noise, U.S.G.P.O., Washington, D.C. 1971

U.S. Environmental Protection Agency, Research and Monitoring, U.S.G.P.O., Washington, D.C. 1972

Valentin, H., Arbeitsmedizinische Untersuchungen von Berufstätigen, in: Der Städtetag 1971, 3, S. $177 \mathrm{f}$.

Vaughan, R.D., Environmental Quality - A National Goal, in: Gluckman, L.A. (Hrsg.), Planning for Solid Waste Management, U.S.G.P.O., Washington, D.C. 1971, S. $111 \mathrm{f}$.

VDI (Hrsg.), Wirtschaftliche und gesellschaftliche Auswirkungen des technischen Fortschritts, Düsseldorf 1971

VDI (Hrsg.), Kohlenmonoxid. Entstehung, Messung und Wirkungskriterien, Düsseldorf 1972

VDI-Aktionszentrum Technik und Umwelt, Umweltstudium ist abzulehnen, in: Umwelt 1973 , 6 , S. 45

Verband der Deutschen Feinmechanischen und Optischen Industrie e.V. (Hrsg.), UmwsltmeBtechnik. Instrumente der Feinmechanik und Optik, Köln 1974

Das Verursacherprinzip und seine Instrumente, Berlin 1974

Vester, F., Der Weg des vorausschauenden Steuerns. Prinzip und Bedeutung kybernetischer Technologien, in: Umwelt $1974,1, \mathrm{~S} .11 \mathrm{ff}$.

Villiers, A.J. de, The Effects of Air Pollution on Health, in: Irving, R.M/Priddle, G.B.(Hrsg.), a.a.O., S. $112 \mathrm{ff.}$

Vilmar, F., Vergeudungskapitalismus oder Wirtschaftsdemokratie, in: Kapp, K.W./Vilmar, F. (Hrsg.), a.a.0., S. $12 \mathrm{ff}$.

Vogl, J. Bayerns LuftmeBsystem, in: U 1974, 3

Wachsmann, F., Gefahren durch ionisierende Strahlungen, in: Schultze, H. (Hrsg.), a.a.O., S. 335 f.

Wäldle, T., Bürgerklage und Umweltverträglichkeitsprüfung, in: Zeitschrift für Rechtspolitik, 8. Jg., 1975, S. $105 \mathrm{ff}$. 
Wahl, K., Recycling: Systematisches Vorgehen bei Industrieabfällen, in: Umwelt 1975, 5, S. $42 \mathrm{f}$.

Waller, R.A., Environmental Quality, its Measurement and Control, in: Regional Studies, vol. 4, 1970, H. 2, S. $177 \mathrm{ff}$.

Walser, B., Sind Atomreaktoren gefährlich?, in: NZZ, FA Nr. 134 vom 18.5.1971

von Walterskirchen, M.P., Bemerkungen zur Umweltpolitik, in: Horn, C./von Walterskirchen, M.P./Wolff, J. (Hrsg.), a.a.O., S. $9 \mathrm{ff}$.

von Walterskirchen, M.P.(Hrsg.), Umweltschutz und Wirtschaftswachstum. 1. Symposium für wirtschaftliche und rechtliche Fragen des Umweltschutzes an der Hochschule St. Gallen vom 19. bis 21. Okt. 1971, Frauenfeld und stuttgart 1972

Waterkamp, R., Interventionsstaat und Planung. Raumordnung, Regional- und Strukturpolitik, Köln 1973

Watson jr., W.D., Costs of Air Pollution Control in the Coal-Fired Electric Power Industry, in: Quarterly Review of Economics and Business, vol. 12, 3 . 1972 , S. $63 \mathrm{ff}$.

Watson jr., W.D., Costs and Benefits of Fly Ash Control, in: Journal of Economics and Business, vol. 26, 3, 1974 , S. $167 \mathrm{ff}$.

Weber, Wilhelm, Wohlfahrtstheoretische Aspekte der Besteuerung: Zielkonflikte und Theorie des Zweitbesten, in: Haller, H. u.a. (Hrsg.), Theorie und Praxis des finanzpolitischen Interventionismus, Fritz Neumark zum 70. Geburtstag, Tübingen 1970, S. 185 ff.

von Weizsäcker, E. (Hrsg.), Humanökologie und Umweltschutz, Studien zur Friedensforschung, Bd. 8, Stuttgart und München 1972

Wellenstein, G., Chemie aus dem Hubschrauber, in: U 1974, 2 Wenders, J.T., Methods of Pollution Control and the Rate of Change in Pollution Abatement Technology, in: Water Resources Research, vol. 11, 1975, S. $393 \mathrm{ff}$.

White, L.J., Effluent Charges as a Faster Means of Achievina Pollution Abatement, in: Public Policy, vol. 24, 1976, S. $111 \mathrm{ff}$.

White jr., L.T., Die historischen Wurzeln unserer ökologischen Krise, in: Lohmann, M. (Hrsg.), a.a.0., S. $20 \mathrm{ff.}$

WHO, Measurement of Levels of Health. Report of a study Group, Technical Report Series No. 137, Genf 1957

WHO, European Standards for Drinking-Water, 2. Auflage, Genf 1970 
Wieand, K.F., Air Pollution and Property Values: A Study of the Saint Louis Area, in: Journal of Regional Science, vol. 13, 1, 1973, s. $91 \mathrm{ff}$.

Wiik, K., Ein multiregionales Entscheidungsmodell zur Optimierung der Abwasserbelastung, Seminarbericht 8 der Gesellschaft für Regionalforschung, Münster 1974, S. 53 ff.

Wille, E., Mittel- und langfristige Finanzplanung, in: Neumark, F. (Hrsg.), Handbuch der Finanzwissenschaft, 3. Auflaqe, Bd. 1, Tübingen 1976 , S. 427 ff.

Wille, E., Planung und Information, Berlin 1970

Wittmann, W., Einführung in die Finanzwissenschaft, Bd. 2, Die öffentlichen Einnahmen, 2. Auflage, Stuttgart 1975

WMO, Air Pollutants, Meteorology, and Plant Injury, Technical Note No. 96, Genf 1968

WMO, (Hrsg.), Meteorological Aspects of A1r Pollution, Technical Note No. 106, Genf 1970

WMO, Meteorology and the Human Environment, WMO-No. 313, Genf 1971

Wohlgemuth, R., Umweltgebühren als Alternative zur Auflage, in: Umwelt 1974,6 , S. 46

Wolozin, H. (Hrsg.), The Economics of Pollution, Morristown, N.J., 1974

Würgler, H., Ökonomische und politische Ursachen der Umweltveränderung, in: Leibundgut, H. (Hrsg.), a.a.O., S. $96 \mathrm{ff}$.

Wulf, J., Umweltpolitik: Probleme, Phasen, Forschungsstrategien, in: Jahrbuch für Sozialwissenschaft, Bd. 24, 1973, S. $180 \mathrm{ff}$.

WWF-Deutschland (Hrsg.), Wasser- und Luftverschmutzung, Lärm, Abfälle - Was tut die Industrie?, Bonn 1969

Young, H.P./Thompson, R.G., Least-Cost Allocation and Valuation Model for Water Resources, in: Water Resources Research, vol. 9, 1973, S. $1186 \mathrm{ff}$.

Zander, H., Trinkwasser - Ein kostbares Lebensmittel, in: U 1975,3

Zentralblatt für Bakteriologie, Parasitenkunde, Infektionskrankheiten und Hygiene, I. Abt. Orig. B., Bd. 155, H. 3, 1971

Zerbe, R.O., Theoretical Efficiency in Pollution Control, in: Western Economic Journal, vol. 8, No. 4, 1970, S. 364 ff.

Letkin/Schaldach, wörterbuch der Medizin, 5. Auflage, München 1974

Zilleßen, H., Ansprüche und Arbeit der Bürgerinitiativen, in: Umwelt 1974, 2, S. $40 \mathrm{ff}$. 
Zimmermann, Klaus, Die Last des Umweltschutzes: Uberlecungen zum Konzept der "Volkswirtschaftlichen Kosten" des Sachverständigenrates für Umweltfragen, in: Kyklos, Bd. 27, 1974, S. $840 \mathrm{ff}$.

Zimmermann, Klaus, Vorausschätzung staatlicher Umweltkosten, in: Umwelt 1976,2 , S. $130 \mathrm{ff}$.

Zipse, E., Patentierung - Hilfe für den Umweltschutz, in: U 1973, 2

Zschocke, H., Ungelöstes Problem der Umweltgestaltung in Westdeutschland, in: Glagow, M. (Hrsg.), a.a.O., S. 142 ff.

Zuckerman, S., Technologie und Gesellschaft. Eine Herausforderung an die Privatwirtschaft. Grundsatzbericht vor dem XXIII. Kongress der IHK Wien, 0.0. 1971 



\section{FINANZWISSENSCHAFTLICHE SCHRIFTEN}

Band 1 Prof. Dr. Werner Steden: Finanzpolitik und Einkommensverteilung. Ein Wachstums- und Konjunkturmodell der Bundesrepublik Deutschland. (In Vorbereitung)

Band 2 Rainer Hagemann: Kommunale Finanzplanung im föderativen Staat. 173 S., 1976.

Band 3 Klaus Scherer: Maßstäbe zur Beurteilung von konjunkturellen Wirkungen des öffentlichen Haushalts. 242 S., 1977.

Band 4 Brita Steinbach: „Formula Flexibility“ - Kristische Analyse und Vergleich mit diskretionärer Konjunkturpolitik. 370 S., 1977.

Band 5 Hans-Georg Petersen: Personelle Einkommensbesteuerung und Inflation. Eine theoretisch-empirische Analyse der Lohn- und veranlagten Einkommensteuer in der Bundesrepublik Deutschland. 330 S., 1977.

Band 6 Friedemann Tetsch: Raumwirkungen des Finanzsystems der Bundesrepublik Deutschland. Eine Untersuchung der Auswirkungen der Finanzreform von 1969 auf die Einnahmenposition der untergeordneten Gebietskörperschaften und ihrer regionalpolitischen Zieladäquanz. 384 S., 1978.

Band 7 Wilhelm Pfähler: Normative Theorie der fiskalischen Besteuerung. Ein methodologischer und theoretischer Beitrag zur Integration der normativen Besteuerungstheorie in die Wohlfahrtstheorie. 224 S., 1978.

Band 8 Wolfgang Wiegard: Optimale Schattenpreise und Produktionsprogramme für öffentliche Unternehmen. Second-Best-Modelle im finanzwirtschaftlichen Staatsbereich. 222 S., 1978.

Band 9 Hans P. Fischer: Die Finanzierung des Umweltschutzes im Rahmen einer rationalen Umweltpolitik. 464 S., 1978. 


